\title{
RESPIRATORY DISEASE
}

\section{IN AGRICULTURAL WORKERS:}

\section{MORTALITY AND MORBIDITY STATISTICS}

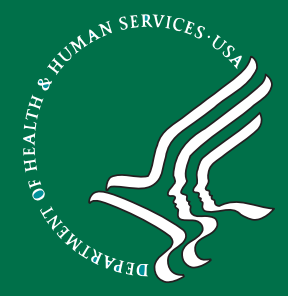

DEPARTMENT OF HEALTH AND HUMAN SERVICES Centers for Disease Control and Prevention National Institute for Occupational Safety and Health

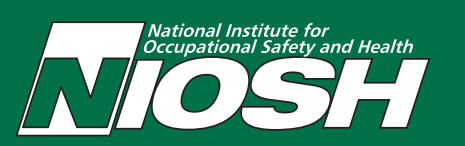





\section{Respiratory Disease in Agricultural Workers: Mortality and Morbidity Statistics}

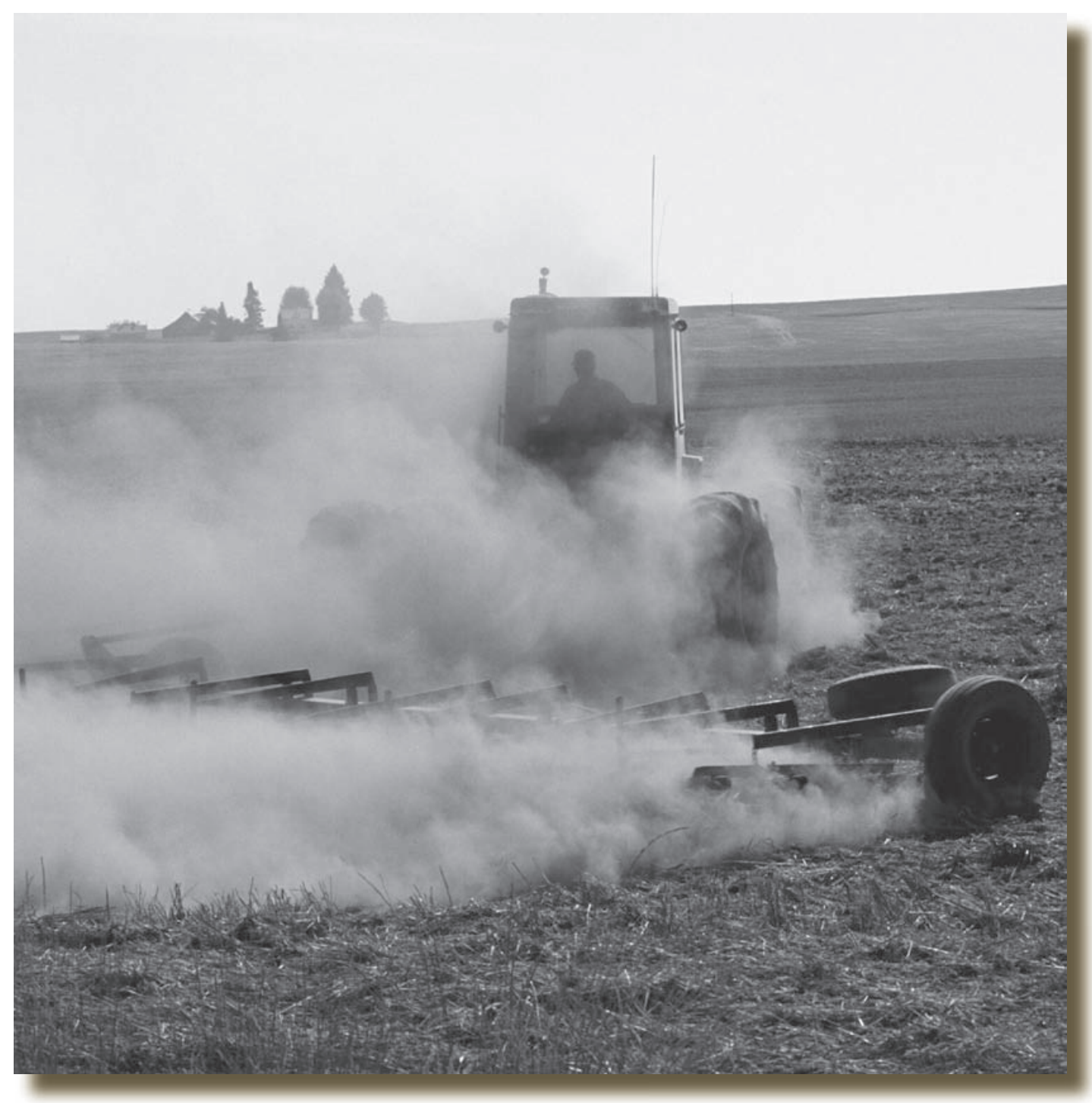

February 2007

DEPARTMENT OF HEALTH AND HUMAN SERVICES

Centers for Disease Control and Prevention National Institute for Occupational Safety and Health 


\section{DISCLAIMER}

Mention of any company or product does not constitute endorsement by the National Institute for Occupational Safety and Health (NIOSH). In addition, citations to Web sites do not constitute NIOSH endorsement of the sponsoring organizations or their programs or products. Futhermore, NIOH is not responsible for the content of these Web sites.

All Web addresses referenced in this report were accessible as of the date this manuscript was prepared for publication.

\section{ORDERING INFORMATION}

To receive documents or other information about occupational safety and health topics, contact NIOSH at

\section{NIOSH}

Publications Dissemination

4676 Columbia Parkway

Cincinnati, OH 45226-1998

Telephone: 1-800-35-NIOSH (1-800-356-4674)

Fax (513) 533-8573

e-mail: pubstaft@cdc.gopbv

or visit the NIOSH web at www.cdc.gov/niosh

\section{This document is in the public domain and may be freely copied or reprinted.}

DHHS (NIOSH) Publication Number 2007-106

February 2007 


\section{Table of Contents}

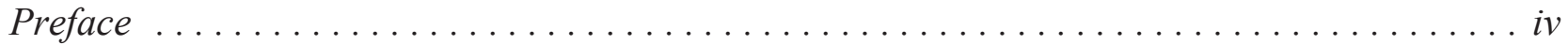

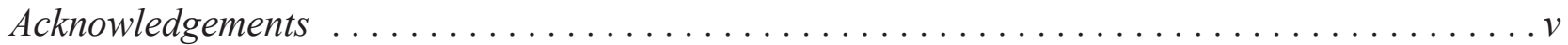

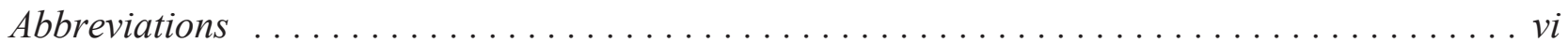

List of Tables and Figures $\ldots \ldots \ldots \ldots \ldots \ldots \ldots \ldots \ldots \ldots \ldots \ldots \ldots \ldots \ldots \ldots$

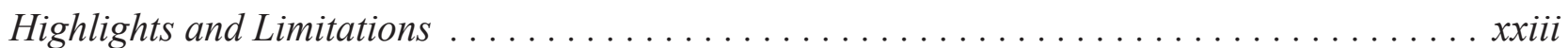

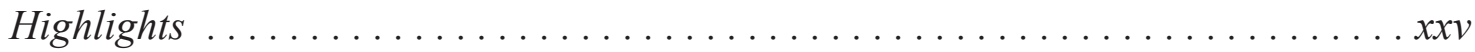

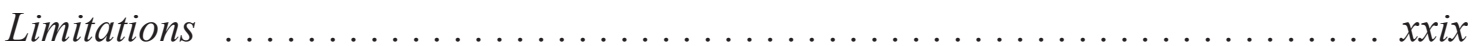

Section 1. Demographics . . . . . . . . . . . . . . . . . . . . .

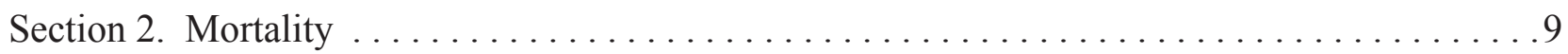

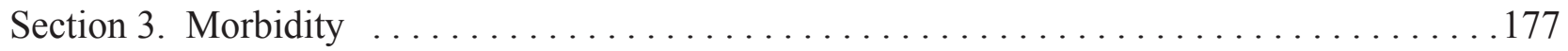

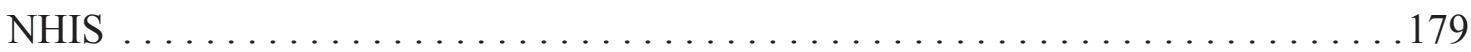

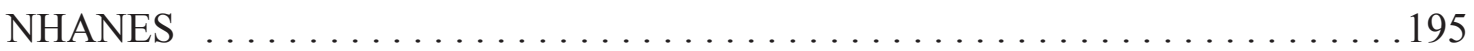

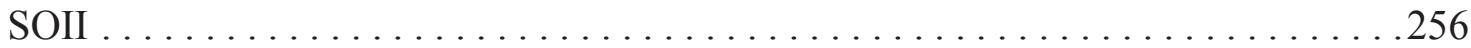

Section 4. Recommendations for Future Studies . . . . . . . . . . . . . . . . . . . . . . 259

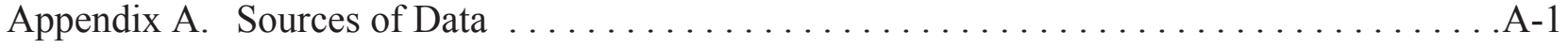

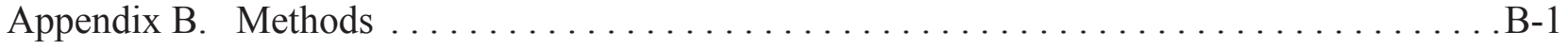

Appendix C. ICD-9 Codes and Descriptions for Respiratory Diseases Included in the Mortality Analysis . . . . . . . . . . . . . . . . . . . . C-1

Appendix D. States and Years with Industry and Occupation Codes from Death

Certificates Used in the Mortality Analysis, 1988-1998 . . . . . . . . . . . . . . D-1

Appendix E. Agricultural Groups Used in the Mortality Analysis and Their Derivation from the U.S. Bureau of Census Industry and Occupation Codes . . . . . . . . . . E-1

Appendix F. Agricultural Groups Used in the Morbidity Analysis and Their Derivation from the National Health and Interview Survey (NHIS) Industry and Occupation Codes . F-1

Appendix G. Agricultural Groups Used in the Morbidity Analysis and Their Derivation from the Third National Health and Nutrition Examination Survey (NHANES III) Industry and Occupation Codes . . . . . . . . . . . . . . . . . . 


\section{Preface}

Respiratory Disease in Agriculture: Mortality and Morbidity Statistics presents summary tables and figures of occupational respiratory disease surveillance data focusing on various occupationally relevant respiratory diseases for the Agriculture, Forestry, and Fishing industries. The report has seven major sections that provide the following data: (1) highlights and data usage limitations; (2) demographic statistics for agricultural workers; (3) mortality statistics for agricultural workers, including by sex and race/ ethnicity; (4) morbidity statistics for agricultural workers, including by sex, race/ethnicity, smoking status, and source of data; (5) recommendations to fill research gaps for respiratory disease in agriculture; and (6) appendices with descriptions of data sources, methods, and other supplementary information.

Data contained in this report originate from various publications, reports, data files, and tabulations provided by the National Center for Health Statistics (NCHS) and the Bureau of Labor Statistics (BLS). Details on the major data sources and on the methods used to compute specific statistics can be found in Appendices A and $\mathrm{B}$, respectively.
Interpreted with appropriate caution, the information contained in this report can help to establish priorities for research and respiratory disease prevention in agriculture. To increase the utility of future surveillance of occupational respiratory disease in agriculture, comments on the report, descriptions of how the information could be used, and suggestions of other data for inclusion in future reports are invited.

Send comments, suggestions, and other correspondence to:

Respiratory Disease in Agricultural Workers Public Health Surveillance Team

Surveillance Branch

Division of Respiratory Disease Studies

NIOSH

1095 Willowdale Road

Morgantown, WV 26505-2888

Phone: 304-285-5754

FAX: 304-285-6111 


\section{Acknowledgements}

This report was prepared under contract (\#2002001-08009) by Michael Koontz and Laura Niang of GEOMET Technologies, LLC, a subsidiary of Versar, Inc. Marc Schenker at the University of California-Davis provided helpful consultation.

Special appreciation is expressed to Gregory R. Wagner, former Director, Division of Respiratory Disease Studies (DRDS), and Frank J. Hearl, former Deputy Director, DRDS for initiating the effort that resulted in this report.

The following NIOSH staff contributed to this report: Michael D. Attfield, Ki Moon Bang, Robert
M. Castellan, Mark F. Greskevitch, Bret L. Jackson, Gregory J. Kullman, Jacek Mazurek, Stephen A. Olenchock, Teri Palermo, and Cathy J. Rotunda.

Drafts of this report were provided for review and comment to epidemiologists, physicians, industrial hygienists, agricultural health experts, and representatives of industry and labor associations. Their comments have been considered in preparing the final version of this report. 


\section{Abbreviations}

BLS Bureau of Labor Statistics

CDC Centers for Disease Control and Prevention

DHHS Department of Health and Human Services

$\mathrm{FEV}_{1} \quad$ forced expiratory volume in one second

FVC forced vital capacity

ICD International Classification of Diseases

L liters

LCL lower confidence limit

LLN lower limit of normal

$\mathrm{L} / \mathrm{sec} \quad$ liters per second

NCHS National Center for Health Statistics

NHANES National Health and Nutrition Examination Survey

NHIS National Health Interview Survey

NIOSH National Institute for Occupational Safety and Health

PEF peak expiratory flow

PMR proportionate mortality ratio

PR prevalence ratio

SD standard deviation

SOII Survey of Occupational Injuries and Illnesses

UCL upper confidence limit 


\section{List of Tables and Figures}

\section{Demographics}

Table 1-1. Demographic characteristics of employed U.S. agricultural workers by agricultural group and

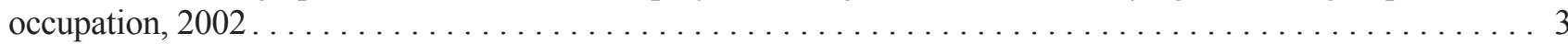

Figure 1-1. Distribution of employed U.S. agricultural workers by sex, race, and ethnicity in comparison to

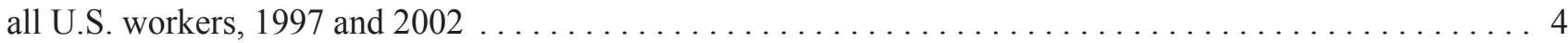

Figure 1-2. Distribution of employed U.S. agricultural groups by sex, race, and ethnicity, $1997 \ldots \ldots$. . . . 5

Figure 1-3. Distribution of employed U.S. agricultural groups by sex, race, and ethnicity, $2002 \ldots \ldots \ldots$

Table 1-2. Distribution of employed U.S. agricultural workers by state, $2002 \ldots \ldots \ldots \ldots \ldots \ldots \ldots$

\section{Mortality}

\section{Mortality by Disease Category within Agricultural Group}

Table 2-1. Crop farm workers: Proportionate mortality ratio (PMR) adjusted for age, sex, and race/ethnicity by disease category, U.S. residents age 15 and over, selected states, $1988-1998 \ldots \ldots \ldots \ldots \ldots \ldots \ldots$

Table 2-2. Livestock farm workers: Proportionate mortality ratio (PMR) adjusted for age, sex, and race/ ethnicity by disease category, U.S. residents age 15 and over, selected states, $1988-1998 \ldots \ldots \ldots \ldots \ldots 12$

Table 2-3. Farm managers: Proportionate mortality ratio (PMR) adjusted for age, sex, and race/ethnicity by disease category, U.S. residents age 15 and over, selected states, $1988-1998 \ldots \ldots \ldots \ldots \ldots \ldots \ldots \ldots 13$

Table 2-4. Landscape and horticultural workers: Proportionate mortality ratio (PMR) adjusted for age, sex, and race/ethnicity by disease category, U.S. residents age 15 and over, selected states, 1988-1998 . . . . 14

Table 2-5. Forestry workers: Proportionate mortality ratio (PMR) adjusted for age, sex, and race/ethnicity by disease category, U.S. residents age 15 and over, selected states, $1988-1998 \ldots \ldots \ldots \ldots \ldots \ldots \ldots$

Table 2-6. Fishery workers: Proportionate mortality ratio (PMR) adjusted for age, sex, and race/ethnicity by disease category, U.S. residents age 15 and over, selected states, $1988-1998 \ldots \ldots \ldots \ldots \ldots \ldots \ldots \ldots$

\section{Mortality by Agricultural Group within Disease Category}

Figure 2-1. Tuberculosis: Proportionate mortality ratio (PMR) adjusted for age, sex, and race/ethnicity by

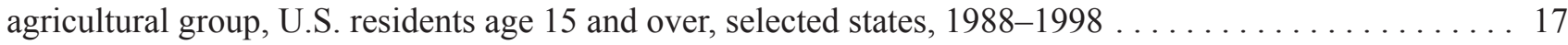

Figure 2-2. Mycoses: Proportionate mortality ratio (PMR) adjusted for age, sex, and race/ethnicity by agricultural group, U.S. residents age 15 and over, selected states, $1988-1998 \ldots \ldots \ldots \ldots \ldots \ldots \ldots$

Figure 2-3. Sarcoidosis: Proportionate mortality ratio (PMR) adjusted for age, sex, and race/ethnicity by agricultural group, U.S. residents age 15 and over, selected states, $1988-1998 \ldots \ldots \ldots \ldots \ldots \ldots$

Figure 2-4. Malignant neoplasms of trachea/bronchus/lung/pleura: Proportionate mortality ratio (PMR) adjusted for age, sex, and race/ethnicity by agricultural group, U.S. residents age 15 and over, selected states,

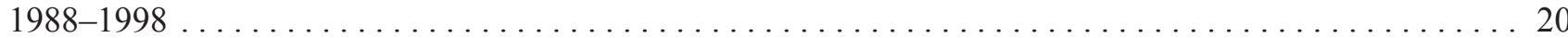

Figure 2-5. Acute respiratory infections: Proportionate mortality ratio (PMR) adjusted for age, sex, and race/ ethnicity by agricultural group, U.S. residents age 15 and over, selected states, 1988-1998 . . . . . . . . 21

Figure 2-6. Other diseases of upper respiratory tract: Proportionate mortality ratio (PMR) adjusted for age, sex, and race/ethnicity by agricultural group, U.S. residents age 15 and over, selected states, 1988-1998 . 22 


\section{List of Tables and Figures}

Figure 2-7. Pneumonia and influenza: Proportionate mortality ratio (PMR) adjusted for age, sex, and race/ ethnicity by agricultural group, U.S. residents age 15 and over, selected states, $1988-1998 \ldots \ldots \ldots \ldots$

Figure 2-8. Chronic obstructive pulmonary disease and allied conditions: Proportionate mortality ratio (PMR) adjusted for age, sex, and race/ethnicity by agricultural group, U.S. residents age 15 and over, selected

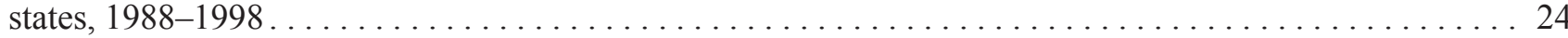

Figure 2-9. Pneumoconioses and other lung diseases-external agents: Proportionate mortality ratio (PMR) adjusted for age, sex, and race/ethnicity by agricultural group, U.S. residents age 15 and over, selected states,

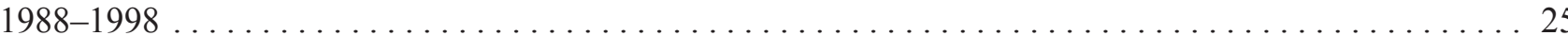

Figure 2-10. Other diseases of respiratory system: Proportionate mortality ratio (PMR) adjusted for age, sex, and race/ethnicity by agricultural group, U.S. residents age 15 and over, selected states, 1988-1998 . . . . 26

\section{Mortality by Disease Category within Agricultural and Sex Group}

Table 2-7. Crop farm workers, males: Proportionate mortality ratio (PMR) adjusted for age and race/ ethnicity by disease category, U.S. residents age 15 and over, selected states, 1988-1998 . . . . . . . . . 27

Table 2-8. Livestock farm workers, males: Proportionate mortality ratio (PMR) adjusted for age and race/ ethnicity by disease category, U.S. residents age 15 and over, selected states, $1988-1998 \ldots \ldots \ldots \ldots \ldots$

Table 2-9. Farm managers, males: Proportionate mortality ratio (PMR) adjusted for age and race/ethnicity by disease category, U.S. residents age 15 and over, selected states, $1988-1998 \ldots \ldots$. . . . . . . . . . . . . 29

Table 2-10. Landscape and horticultural workers, males: Proportionate mortality ratio (PMR) adjusted for age and race/ethnicity by disease category, U.S. residents age 15 and over, selected states, 1988-1998 . . . 30

Table 2-11. Forestry workers, males: Proportionate mortality ratio (PMR) adjusted for age and race/ethnicity by disease category, U.S. residents age 15 and over, selected states, $1988-1998 \ldots \ldots \ldots \ldots \ldots \ldots \ldots$

Table 2-12. Fishery workers, males: Proportionate mortality ratio (PMR) adjusted for age and race/ethnicity by disease category, U.S. residents age 15 and over, selected states, $1988-1998 \ldots \ldots \ldots \ldots \ldots \ldots \ldots$

Table 2-13. Crop farm workers, females: Proportionate mortality ratio (PMR) adjusted for age and race/ ethnicity by disease category, U.S. residents age 15 and over, selected states, 1988-1998 . . . . . . . . 33

Table 2-14. Livestock farm workers, females: Proportionate mortality ratio (PMR) adjusted for age and race/ ethnicity by disease category, U.S. residents age 15 and over, selected states, $1988-1998 \ldots \ldots \ldots \ldots$. . . . 34

Table 2-15. Farm managers, females: Proportionate mortality ratio (PMR) adjusted for age and race/ethnicity by disease category, U.S. residents age 15 and over, selected states, $1988-1998 \ldots \ldots \ldots \ldots \ldots \ldots$

Table 2-16. Landscape and horticultural workers, females: Proportionate mortality ratio (PMR) adjusted for age and race/ethnicity by disease category, U.S. residents age 15 and over, selected states, 1988-1998 . . . 36

Table 2-17. Forestry workers, females: Proportionate mortality ratio (PMR) adjusted for age and race/ ethnicity by disease category, U.S. residents age 15 and over, selected states, $1988-1998 \ldots \ldots \ldots \ldots$. . . 37

Table 2-18. Fishery workers, females: Proportionate mortality ratio (PMR) adjusted for age and race/ ethnicity by disease category, U.S. residents age 15 and over, selected states, 1988-1998 . . . . . . . . 38

\section{Mortality by Agricultural Group and Sex within Disease Category}

Figure 2-11. Tuberculosis: Proportionate mortality ratio (PMR) adjusted for age and race/ethnicity by agricultural group and sex, U.S. residents age 15 and over, selected states, 1988-1998. 


\section{List of Tables and Figures}

Figure 2-12. Mycoses: Proportionate mortality ratio (PMR) adjusted for age and race/ethnicity by agricultural group and sex, U.S. residents age 15 and over, selected states, 1988-1998 . . . . . . . . . . 40

Figure 2-13. Sarcoidosis: Proportionate mortality ratio (PMR) adjusted for age and race/ethnicity by agricultural group and sex, U.S. residents age 15 and over, selected states, $1988-1998 \ldots \ldots \ldots \ldots \ldots 1$

Figure 2-14. Malignant neoplasms of trachea/bronchus/lung/pleura: Proportionate mortality ratio (PMR) adjusted for age and race/ethnicity by agricultural group and sex, U.S. residents age 15 and over, selected

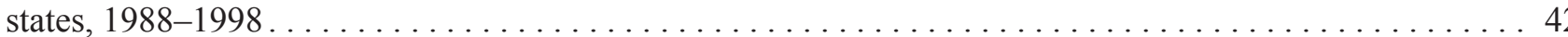

Figure 2-15. Acute respiratory infections: Proportionate mortality ratio (PMR) adjusted for age and race/ ethnicity by agricultural group and sex, U.S. residents age 15 and over, selected states, 1988-1998 . . . . . 43

Figure 2-16. Other diseases of upper respiratory tract: Proportionate mortality ratio (PMR) adjusted for age and race/ethnicity by agricultural group and sex, U.S. residents age 15 and over, selected states, 1988-1998 . 44

Figure 2-17. Pneumonia and influenza: Proportionate mortality ratio (PMR) adjusted for age and race/ ethnicity by agricultural group and sex, U.S. residents age 15 and over, selected states, 1988-1998. . . . . 45

Figure 2-18. Chronic obstructive pulmonary disease and allied conditions: Proportionate mortality ratio (PMR) adjusted for age and race/ethnicity by agricultural group and sex, U.S. residents age 15 and over,

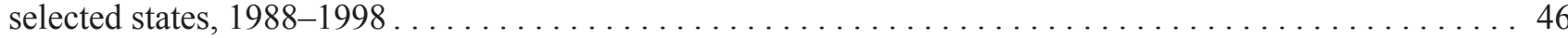

Figure 2-19. Pneumoconioses and other lung diseases-external agents: Proportionate mortality ratio (PMR) adjusted for age and race/ethnicity by agricultural group and sex, U.S. residents age 15 and over, selected

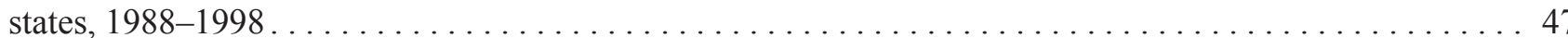

Figure 2-20. Other diseases of respiratory system: Proportionate mortality ratio (PMR) adjusted for age and race/ethnicity by agricultural group and sex, U.S. residents age 15 and over, selected states, 1988-1998 . . 48

\section{Mortality by Disease Category within Agricultural and Race/Ethnicity Group}

Table 2-19. Crop farm workers, white, non-Hispanic: Proportionate mortality ratio (PMR) adjusted for age and sex by disease category, U.S. residents age 15 and over, selected states, 1988-1998 _. . . . . . . . . 49

Table 2-20. Livestock farm workers, white, non-Hispanic: Proportionate mortality ratio (PMR) adjusted for age and sex by disease category, U.S. residents age 15 and over, selected states, $1988-1998 \ldots \ldots \ldots \ldots 50$

Table 2-21. Farm managers, white, non-Hispanic: Proportionate mortality ratio (PMR) adjusted for age and sex by disease category, U.S. residents age 15 and over, selected states, 1988-1998 . . . . . . . . . . 51

Table 2-22. Landscape and horticultural workers, white, non-Hispanic: Proportionate mortality ratio (PMR) adjusted for age and sex by disease category, U.S. residents age 15 and over, selected states, 1988-1998 . 52

Table 2-23. Forestry workers, white, non-Hispanic: Proportionate mortality ratio (PMR) adjusted for age and sex by disease category, U.S. residents age 15 and over, selected states, $1988-1998 \ldots \ldots \ldots \ldots \ldots \ldots$

Table 2-24. Fishery workers, white, non-Hispanic: Proportionate mortality ratio (PMR) adjusted for age and sex by disease category, U.S. residents age 15 and over, selected states, $1988-1998 \ldots \ldots \ldots \ldots \ldots \ldots$

Table 2-25. Crop farm workers, black, non-Hispanic: Proportionate mortality ratio (PMR) adjusted for age and sex by disease category, U.S. residents age 15 and over, selected states, $1988-1998 \ldots \ldots \ldots \ldots \ldots$

Table 2-26. Livestock farm workers, black, non-Hispanic: Proportionate mortality ratio (PMR) adjusted for age and sex by disease category, U.S. residents age 15 and over, selected states, 1988-1998 . . . . . . 56 


\section{List of Tables and Figures}

Table 2-27. Farm managers, black, non-Hispanic: Proportionate mortality ratio (PMR) adjusted for age and sex by disease category, U.S. residents age 15 and over, selected states, $1988-1998 \ldots \ldots \ldots \ldots \ldots \ldots$

Table 2-28. Landscape and horticultural workers, black, non-Hispanic: Proportionate mortality ratio (PMR) adjusted for age and sex by disease category, U.S. residents age 15 and over, selected states, 1988-1998 . . 58

Table 2-29. Forestry workers, black, non-Hispanic: Proportionate mortality ratio (PMR) adjusted for age and sex by disease category, U.S. residents age 15 and over, selected states, 1988-1998 _ . . . . . . . . . 59

Table 2-30. Fishery workers, black, non-Hispanic: Proportionate mortality ratio (PMR) adjusted for age and sex by disease category, U.S. residents age 15 and over, selected states, $1988-1998 \ldots \ldots \ldots \ldots \ldots$

Table 2-31. Crop farm workers, Hispanic: Proportionate mortality ratio (PMR) adjusted for age and sex by disease category, U.S. residents age 15 and over, selected states, $1988-1998 \ldots \ldots \ldots \ldots \ldots \ldots \ldots \ldots 6$

Table 2-32. Livestock farm workers, Hispanic: Proportionate mortality ratio (PMR) adjusted for age and sex by disease category, U.S. residents age 15 and over, selected states, $1988-1998 \ldots \ldots \ldots \ldots \ldots \ldots$

Table 2-33. Farm managers, Hispanic: Proportionate mortality ratio (PMR) adjusted for age and sex by disease category, U.S. residents age 15 and over, selected states, $1988-1998 \ldots \ldots \ldots \ldots \ldots \ldots \ldots \ldots$

Table 2-34. Landscape and horticultural workers, Hispanic: Proportionate mortality ratio (PMR) adjusted for age and sex by disease category, U.S. residents age 15 and over, selected states, 1988-1998 . . . . . . . 64

Table 2-35. Forestry workers, Hispanic: Proportionate mortality ratio (PMR) adjusted for age and sex by disease category, U.S. residents age 15 and over, selected states, $1988-1998 \ldots \ldots \ldots \ldots \ldots \ldots \ldots 65$

Table 2-36. Fishery workers, Hispanic: Proportionate mortality ratio (PMR) adjusted for age and sex by disease category, U.S. residents age 15 and over, selected states, $1988-1998 \ldots \ldots \ldots \ldots \ldots \ldots \ldots \ldots 66$

\section{Mortality by Agricultural Group and Race/Ethnicity within Disease Category}

Figure 2-21. Tuberculosis: Proportionate mortality ratio (PMR) adjusted for age and sex by agricultural group and race/ethnicity, U.S. residents age 15 and over, selected states, 1988-1998

Figure 2-22. Mycoses: Proportionate mortality ratio (PMR) adjusted for age and sex by agricultural group and race/ethnicity, U.S. residents age 15 and over, selected states, $1988-1998 \ldots \ldots \ldots \ldots \ldots \ldots \ldots 68$

Figure 2-23. Sarcoidosis: Proportionate mortality ratio (PMR) adjusted for age and sex by agricultural group and race/ethnicity, U.S. residents age 15 and over, selected states, $1988-1998 \ldots \ldots \ldots \ldots \ldots \ldots$

Figure 2-24. Malignant neoplasms of trachea/bronchus/lung/pleura: Proportionate mortality ratio (PMR) adjusted for age and sex by agricultural group and race/ethnicity, U.S. residents age 15 and over, selected

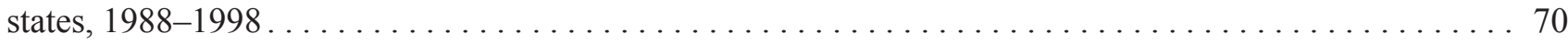

Figure 2-25. Acute respiratory infections: Proportionate mortality ratio (PMR) adjusted for age and sex by agricultural group and race/ethnicity, U.S. residents age 15 and over, selected states, $1988-1998 \ldots \ldots \ldots 71$

Figure 2-26. Other diseases of upper respiratory tract: Proportionate mortality ratio (PMR) adjusted for age and sex by agricultural group and race/ethnicity, U.S. residents age 15 and over, selected states, 1988-1998. . 72

Figure 2-27. Pneumonia and influenza: Proportionate mortality ratio (PMR) adjusted for age and sex by agricultural group and race/ethnicity, U.S. residents age 15 and over, selected states, 1988-1998 . . . . . 73 
Figure 2-28. Chronic obstructive pulmonary disease and allied conditions: Proportionate mortality ratio (PMR) adjusted for age and sex by agricultural group and race/ethnicity, U.S. residents age 15 and over,

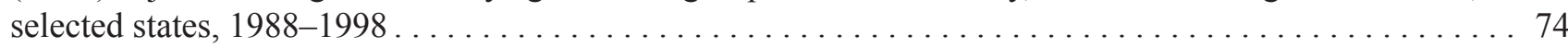

Figure 2-29. Pneumoconioses and other lung diseases-external agents: Proportionate mortality ratio (PMR) adjusted for age and sex by agricultural group and race/ethnicity, U.S. residents age 15 and over, selected

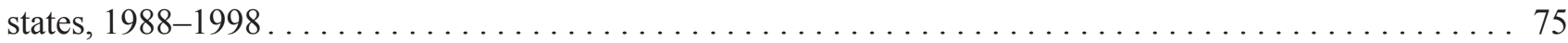

Figure 2-30. Other diseases of respiratory system: Proportionate mortality ratio (PMR) adjusted for age and sex by agricultural group and race/ethnicity, U.S. residents age 15 and over, selected states, 1988-1998 . . 76

\section{Tuberculosis Mortality within and by Agricultural Group}

Table 2-37. Crop farm workers: Proportionate mortality ratio (PMR) adjusted for age, sex, and race/ethnicity for tuberculosis, U.S. residents age 15 and over, selected states, $1988-1998 \ldots \ldots \ldots \ldots \ldots \ldots \ldots \ldots 77$

Table 2-38. Livestock farm workers: Proportionate mortality ratio (PMR) adjusted for age, sex, and race/ ethnicity for tuberculosis, U.S. residents age 15 and over, selected states, $1988-1998 \ldots \ldots \ldots \ldots \ldots \ldots 78$

Table 2-39. Farm managers: Proportionate mortality ratio (PMR) adjusted for age, sex, and race/ethnicity for tuberculosis, U.S. residents age 15 and over, selected states, $1988-1998 \ldots \ldots \ldots \ldots \ldots \ldots \ldots \ldots \ldots$

Table 2-40. Landscape and horticultural workers: Proportionate mortality ratio (PMR) adjusted for age, sex, and race/ethnicity for tuberculosis, U.S. residents age 15 and over, selected states, $1988-1998 \ldots \ldots \ldots 80$

Table 2-41. Forestry workers: Proportionate mortality ratio (PMR) adjusted for age, sex, and race/ethnicity for tuberculosis, U.S. residents age 15 and over, selected states, $1988-1998 \ldots \ldots \ldots \ldots \ldots \ldots \ldots \ldots$

Table 2-42. Fishery workers: Proportionate mortality ratio (PMR) adjusted for age, sex, and race/ethnicity for tuberculosis, U.S. residents age 15 and over, selected states, $1988-1998 \ldots \ldots \ldots \ldots \ldots \ldots \ldots \ldots$.

Figure 2-31. Pulmonary tuberculosis: Proportionate mortality ratio (PMR) adjusted for age, sex, and race/ ethnicity by agricultural group, U.S. residents age 15 and over, selected states, $1988-1998 \ldots \ldots \ldots \ldots 3$

Figure 2-32. Other respiratory tuberculosis: Proportionate mortality ratio (PMR) adjusted for age, sex, and race/ethnicity by agricultural group, U.S. residents age 15 and over, selected states, 1988-1998 _ . . . . 84

Figure 2-33. Tuberculosis of meninges and central nervous system: Proportionate mortality ratio (PMR) adjusted for age, sex, and race/ethnicity by agricultural group, U.S. residents age 15 and over, selected states,

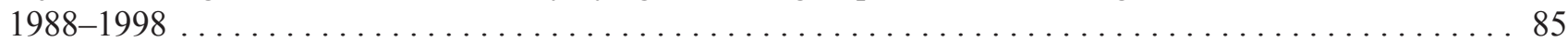

Figure 2-34. Tuberculosis of bones and joints: Proportionate mortality ratio (PMR) adjusted for age, sex, and race/ethnicity by agricultural group, U.S. residents age 15 and over, selected states, 1988-1998 _ . . . 86

Figure 2-35. Tuberculosis of other organs: Proportionate mortality ratio (PMR) adjusted for age, sex, and race/ethnicity by agricultural group, U.S. residents age 15 and over, selected states, $1988-1998 \ldots \ldots \ldots 87$

Figure 2-36. Miliary tuberculosis: Proportionate mortality ratio (PMR) adjusted for age, sex, and race/ ethnicity by agricultural group, U.S. residents age 15 and over, selected states, $1988-1998 \ldots \ldots \ldots$. . . 88

\section{Mycoses Mortality within and by Agricultural Group}

Table 2-43. Crop farm workers: Proportionate mortality ratio (PMR) adjusted for age, sex, and race/ethnicity for mycoses, U.S. residents age 15 and over, selected states, $1988-1998 \ldots \ldots \ldots \ldots \ldots \ldots \ldots$ 


\section{List of Tables and Figures}

Table 2-44. Livestock farm workers: Proportionate mortality ratio (PMR) adjusted for age, sex, and race/ ethnicity for mycoses, U.S. residents age 15 and over, selected states, $1988-1998 \ldots \ldots \ldots \ldots \ldots \ldots$

Table 2-45. Farm managers: Proportionate mortality ratio (PMR) adjusted for age, sex, and race/ethnicity for mycoses, U.S. residents age 15 and over, selected states, $1988-1998 \ldots \ldots \ldots \ldots \ldots \ldots \ldots \ldots \ldots$

Table 2-46. Landscape and horticultural workers: Proportionate mortality ratio (PMR) adjusted for age, sex, and race/ethnicity for mycoses, U.S. residents age 15 and over, selected states, 1988-1998 . . . . . . . 92

Table 2-47. Forestry workers: Proportionate mortality ratio (PMR) adjusted for age, sex, and race/ethnicity for mycoses, U.S. residents age 15 and over, selected states, $1988-1998 \ldots \ldots \ldots \ldots \ldots \ldots$. . . . . . . . 93

Table 2-48. Fishery workers: Proportionate mortality ratio (PMR) adjusted for age, sex, and race/ethnicity for mycoses, U.S. residents age 15 and over, selected states, 1988-1998 . . . . . . . . . . . . . . . . . 94

Figure 2-37. Candidiasis: Proportionate mortality ratio (PMR) adjusted for age, sex, and race/ethnicity by agricultural group, U.S. residents age 15 and over, selected states, $1988-1998 \ldots \ldots \ldots \ldots \ldots \ldots$

Figure 2-38. Histoplasmosis: Proportionate mortality ratio (PMR) adjusted for age, sex, and race/ethnicity by agricultural group, U.S. residents age 15 and over, selected states, $1988-1998 \ldots \ldots \ldots \ldots \ldots \ldots \ldots$

Figure 2-39. Blastomycotic infection: Proportionate mortality ratio (PMR) adjusted for age, sex, and race/ ethnicity by agricultural group, U.S. residents age 15 and over, selected states, 1988-1998 . . . . . . . 97

Figure 2-40. Other mycoses: Proportionate mortality ratio (PMR) adjusted for age, sex, and race/ethnicity by agricultural group, U.S. residents age 15 and over, selected states, $1988-1998 \ldots \ldots \ldots \ldots \ldots \ldots \ldots$

\section{Malignant Neoplasm of Trachea/Bronchus/Lung/Pleura Mortality within and by Agricultural Group}

Table 2-49. Crop farm workers: Proportionate mortality ratio (PMR) adjusted for age, sex, and race/ethnicity for malignant neoplasms of trachea/bronchus/lung/pleura, U.S. residents age 15 and over, selected states,

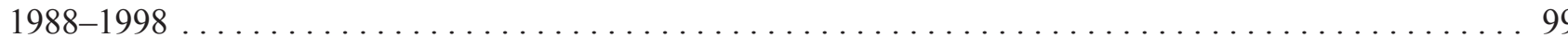

Table 2-50. Livestock farm workers: Proportionate mortality ratio (PMR) adjusted for age, sex, and race/ ethnicity for malignant neoplasms of trachea/bronchus/lung/pleura, U.S. residents age 15 and over, selected

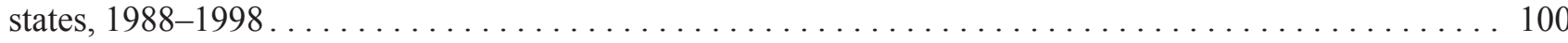

Table 2-51. Farm managers: Proportionate mortality ratio (PMR) adjusted for age, sex, and race/ethnicity for malignant neoplasms of trachea/bronchus/lung/pleura, U.S. residents age 15 and over, selected states, 1988-

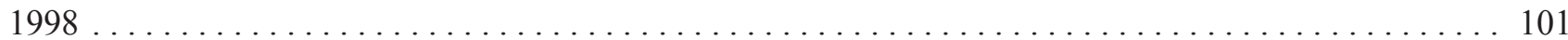

Table 2-52. Landscape and horticultural workers: Proportionate mortality ratio (PMR) adjusted for age, sex, and race/ethnicity for malignant neoplasms of trachea/bronchus/lung/pleura, U.S. residents age 15 and over,

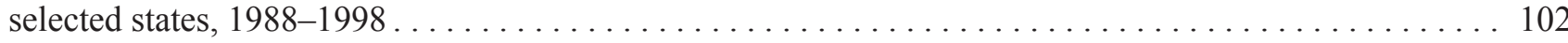

Table 2-53. Forestry workers: Proportionate mortality ratio (PMR) adjusted for age, sex, and race/ethnicity for malignant neoplasms of trachea/bronchus/lung/pleura, U.S. residents age 15 and over, selected states,

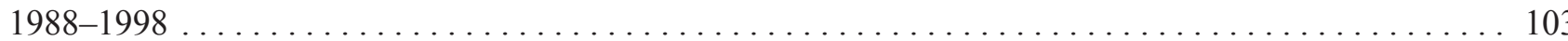

Table 2-54. Fishery workers: Proportionate mortality ratio (PMR) adjusted for age, sex, and race/ethnicity for malignant neoplasms of trachea/bronchus/lung/pleura, U.S. residents age 15 and over, selected states,

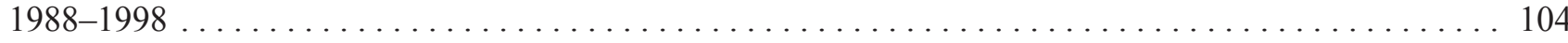


Figure 2-41. Malignant neoplasm of trachea, bronchus, and lung: Proportionate mortality ratio (PMR) adjusted for age, sex, and race/ethnicity by agricultural group, U.S. residents age 15 and over, selected states, 1988-1998 105

Figure 2-42. Malignant neoplasm of pleura: Proportionate mortality ratio (PMR) adjusted for age, sex, and race/ethnicity by agricultural group, U.S. residents age 15 and over, selected states, 1988-1998 _ . . . 106

\section{Acute Respiratory Infection Mortality within and by Agricultural Group}

Table 2-55. Crop farm workers: Proportionate mortality ratio (PMR) adjusted for age, sex, and race/ethnicity for acute respiratory infections, U.S. residents age 15 and over, selected states, 1988-1998 _ . . . . . 107

Table 2-56. Livestock farm workers: Proportionate mortality ratio (PMR) adjusted for age, sex, and race/ ethnicity for acute respiratory infections, U.S. residents age 15 and over, selected states, 1988-1998 _ . 108

Table 2-57. Farm managers: Proportionate mortality ratio (PMR) adjusted for age, sex, and race/ethnicity for acute respiratory infections, U.S. residents age 15 and over, selected states, $1988-1998 \ldots \ldots \ldots \ldots$. . . 109

Table 2-58. Landscape and horticultural workers: Proportionate mortality ratio (PMR) adjusted for age, sex, and race/ethnicity for acute respiratory infections, U.S. residents age 15 and over, selected states, 1988-1998 . . . 110

Table 2-59. Forestry workers: Proportionate mortality ratio (PMR) adjusted for age, sex, and race/ethnicity for acute respiratory infections, U.S. residents age 15 and over, selected states, 1988-1998 _ . . . . . 111

Table 2-60. Fishery workers: Proportionate mortality ratio (PMR) adjusted for age, sex, and race/ethnicity for acute respiratory infections, U.S. residents age 15 and over, selected states, 1988-1998 . . . . . . 112

Figure 2-43. Acute laryngitis and tracheitis: Proportionate mortality ratio (PMR) adjusted for age, sex, and race/ethnicity by agricultural group, U.S. residents age 15 and over, selected states, $1988-1998 \ldots \ldots \ldots 113$

Figure 2-44. Acute upper respiratory infections of multiple or unspecified sites: Proportionate mortality ratio (PMR) adjusted for age, sex, and race/ethnicity by agricultural group, U.S. residents age 15 and over, selected states, 1988-1998 . . . . . . . . . . . . . . . . . . . . . . . . . . . . . . . . . . . . . . . . 114

Figure 2-45. Acute bronchitis and bronchiolitis: Proportionate mortality ratio (PMR) adjusted for age, sex, and race/ethnicity by agricultural group, U.S. residents age 15 and over, selected states, 1988-1998 . . . 115

\section{Other Diseases of Upper Respiratory Tract Mortality within and by Agricultural Group}

Table 2-61. Crop farm workers: Proportionate mortality ratio (PMR) adjusted for age, sex, and race/ethnicity for other diseases of upper respiratory tract, U.S. residents age 15 and over, selected states, 1988-1998 . 116

Table 2-62. Livestock farm workers: Proportionate mortality ratio (PMR) adjusted for age, sex, and race/ ethnicity for other diseases of upper respiratory tract, U.S. residents age 15 and over, selected states, 1988-

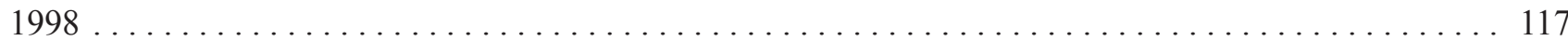

Table 2-63. Farm managers: Proportionate mortality ratio (PMR) adjusted for age, sex, and race/ethnicity for other diseases of upper respiratory tract, U.S. residents age 15 and over, selected states, 1988-1998 . . . 118

Table 2-64. Landscape and horticultural workers: Proportionate mortality ratio (PMR) adjusted for age, sex, and race/ethnicity for other diseases of upper respiratory tract, U.S. residents age 15 and over, selected states, $1988-1998$ 


\section{List of Tables and Figures}

Table 2-65. Forestry workers: Proportionate mortality ratio (PMR) adjusted for age, sex, and race/ethnicity for other diseases of upper respiratory tract, U.S. residents age 15 and over, selected states, 1988-1998 . 120

Table 2-66. Fishery workers: Proportionate mortality ratio (PMR) adjusted for age, sex, and race/ethnicity for other diseases of upper respiratory tract, U.S. residents age 15 and over, selected states, 1988-1998 . 121

Figure 2-46. Chronic sinusitis: Proportionate mortality ratio (PMR) adjusted for age, sex, and race/ethnicity

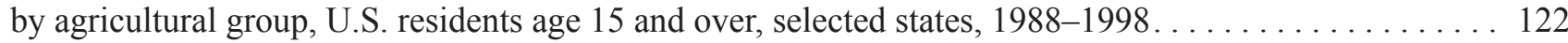

Figure 2-47. Other diseases of upper respiratory tract: Proportionate mortality ratio (PMR) adjusted for age, sex, and race/ethnicity by agricultural group, U.S. residents age 15 and over, selected states, 1988-1998 . 123

\section{Pneumonia and Influenza Mortality within and by Agricultural Group}

Table 2-67. Crop farm workers: Proportionate mortality ratio (PMR) adjusted for age, sex, and race/ethnicity for pneumonia and influenza, U.S. residents age 15 and over, selected states, $1988-1998 \ldots \ldots \ldots \ldots$

Table 2-68. Livestock farm workers: Proportionate mortality ratio (PMR) adjusted for age, sex, and race/ ethnicity for pneumonia and influenza, U.S. residents age 15 and over, selected states, 1988-1998 . . . . 125

Table 2-69. Farm managers: Proportionate mortality ratio (PMR) adjusted for age, sex, and race/ethnicity for pneumonia and influenza, U.S. residents age 15 and over, selected states, $1988-1998 \ldots \ldots \ldots \ldots \ldots$

Table 2-70. Landscape and horticultural workers: Proportionate mortality ratio (PMR) adjusted for age, sex, and race/ethnicity for pneumonia and influenza, U.S. residents age 15 and over, selected states, 1988-1998 . 127

Table 2-71. Forestry workers: Proportionate mortality ratio (PMR) adjusted for age, sex, and race/ethnicity for pneumonia and influenza, U.S. residents age 15 and over, selected states, 1988-1998 . . . . . . . . 128

Table 2-72. Fishery workers: Proportionate mortality ratio (PMR) adjusted for age, sex, and race/ethnicity for pneumonia and influenza, U.S. residents age 15 and over, selected states, $1988-1998 \ldots \ldots \ldots$. . . . 129

Figure 2-48. Viral pneumonia: Proportionate mortality ratio (PMR) adjusted for age, sex, and race/ethnicity by agricultural group, U.S. residents age 15 and over, selected states, $1988-1998 \ldots \ldots \ldots \ldots \ldots \ldots$

Figure 2-49. Pneumococcal pneumonia: Proportionate mortality ratio (PMR) adjusted for age, sex, and race/ ethnicity by agricultural group, U.S. residents age 15 and over, selected states, 1988-1998 . . . . . . . 131

Figure 2-50. Other bacterial pneumonia: Proportionate mortality ratio (PMR) adjusted for age, sex, and race/ ethnicity by agricultural group, U.S. residents age 15 and over, selected states, 1988-1998 . . . . . . 132

Figure 2-51. Pneumonia due to other specified organism: Proportionate mortality ratio (PMR) adjusted for age, sex, and race/ethnicity by agricultural group, U.S. residents age 15 and over, selected states, 1988-1998 . . . 133

Figure 2-52. Bronchopneumonia, organism unspecified: Proportionate mortality ratio (PMR) adjusted for age, sex, and race/ethnicity by agricultural group, U.S. residents age 15 and over, selected states,

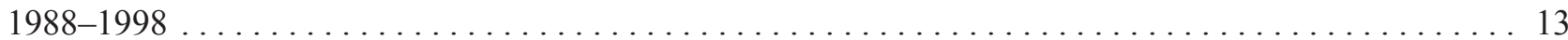

Figure 2-53. Pneumonia, organism unspecified: Proportionate mortality ratio (PMR) adjusted for age, sex, and race/ethnicity by agricultural group, U.S. residents age 15 and over, selected states, $1988-1998 \ldots \ldots 135$

Figure 2-54. Influenza: Proportionate mortality ratio (PMR) adjusted for age, sex, and race/ethnicity by agricultural group, U.S. residents age 15 and over, selected states, $1988-1998 \ldots \ldots \ldots \ldots \ldots \ldots$ 


\section{COPD Mortality within and by Agricultural Group}

Table 2-73. Crop farm workers: Proportionate mortality ratio (PMR) adjusted for age, sex, and race/ethnicity for chronic obstructive pulmonary disease and allied conditions, U.S. residents age 15 and over, selected

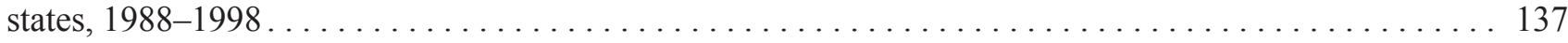

Table 2-74. Livestock farm workers: Proportionate mortality ratio (PMR) adjusted for age, sex, and race/ ethnicity for chronic obstructive pulmonary disease and allied conditions, U.S. residents age 15 and over,

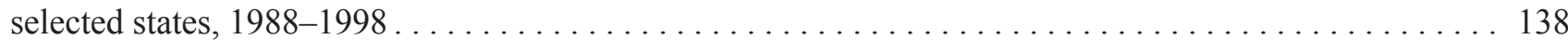

Table 2-75. Farm managers: Proportionate mortality ratio (PMR) adjusted for age, sex, and race/ethnicity for chronic obstructive pulmonary disease and allied conditions, U.S. residents age 15 and over, selected states,

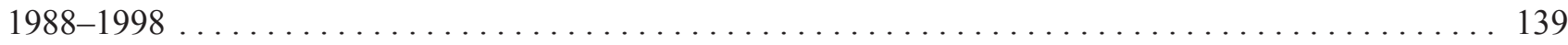

Table 2-76. Landscape and horticultural workers: Proportionate mortality ratio (PMR) adjusted for age, sex, and race/ethnicity for chronic obstructive pulmonary disease and allied conditions, U.S. residents age 15 and

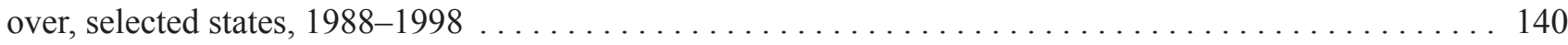

Table 2-77. Forestry workers: Proportionate mortality ratio (PMR) adjusted for age, sex, and race/ethnicity for chronic obstructive pulmonary disease and allied conditions, U.S. residents age 15 and over, selected

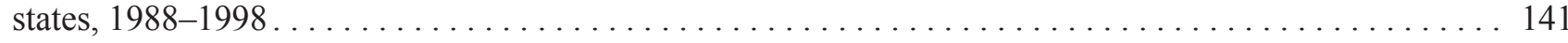

Table 2-78. Fishery workers: Proportionate mortality ratio (PMR) adjusted for age, sex, and race/ethnicity for chronic obstructive pulmonary disease and allied conditions, U.S. residents age 15 and over, selected

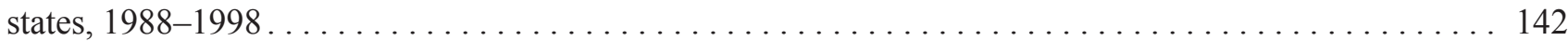

Figure 2-55. Bronchitis, not specified as acute or chronic: Proportionate mortality ratio (PMR) adjusted for age, sex, and race/ethnicity by agricultural group, U.S. residents age 15 and over, selected states, 1988-1998 . . 143

Figure 2-56. Chronic bronchitis: Proportionate mortality ratio (PMR) adjusted for age, sex, and race/ ethnicity by agricultural group, U.S. residents age 15 and over, selected states, 1988-1998 . . . . . . . 144

Figure 2-57. Emphysema: Proportionate mortality ratio (PMR) adjusted for age, sex, and race/ethnicity by agricultural group, U.S. residents age 15 and over, selected states, $1988-1998 \ldots \ldots \ldots \ldots \ldots \ldots \ldots$

Figure 2-58. Asthma: Proportionate mortality ratio (PMR) adjusted for age, sex, and race/ethnicity by agricultural group, U.S. residents age 15 and over, selected states, 1988-1998 . . . . . . . . . . . . 146

Figure 2-59. Bronchiectasis: Proportionate mortality ratio (PMR) adjusted for age, sex, and race/ethnicity by agricultural group, U.S. residents age 15 and over, selected states, $1988-1998 \ldots \ldots \ldots \ldots \ldots \ldots \ldots$

Figure 2-60. Hypersensitivity pneumonitis: Proportionate mortality ratio (PMR) adjusted for age, sex, and race/ethnicity by agricultural group, U.S. residents age 15 and over, selected states, 1988-1998 _ . . . 148

Figure 2-61. Chronic airway obstruction, not elsewhere classified: Proportionate mortality ratio (PMR) adjusted for age, sex, and race/ethnicity by agricultural group, U.S. residents age 15 and over, selected states,

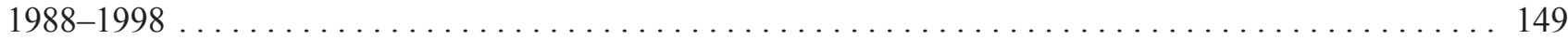

\section{Pneumoconiosis and Other Lung Disease Mortality within and by Agricultural Group}

Table 2-79. Crop farm workers: Proportionate mortality ratio (PMR) adjusted for age, sex, and race/ethnicity for pneumoconiosis and other lung diseases-external agents, U.S. residents age 15 and over, selected states,

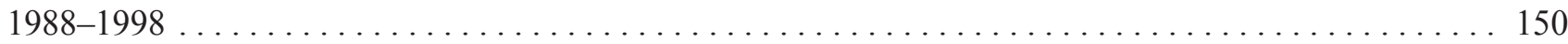




\section{List of Tables and Figures}

Table 2-80. Livestock farm workers: Proportionate mortality ratio (PMR) adjusted for age, sex, and race/ ethnicity for pneumoconiosis and other lung diseases-external agents, U.S. residents age 15 and over, selected

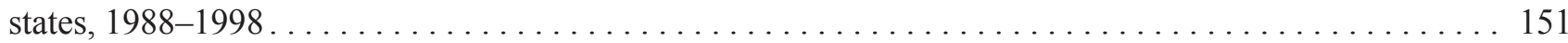

Table 2-81. Farm managers: Proportionate mortality ratio (PMR) adjusted for age, sex, and race/ethnicity for pneumoconiosis and other lung diseases-external agents, U.S. residents age 15 and over, selected states,

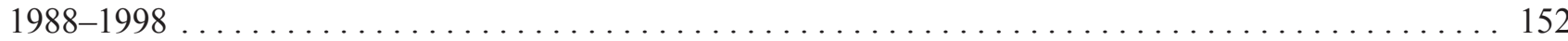

Table 2-82. Landscape and horticultural workers: Proportionate mortality ratio (PMR) adjusted for age, sex, and race/ethnicity for pneumoconiosis and other lung diseases-external agents, U.S. residents age 15 and

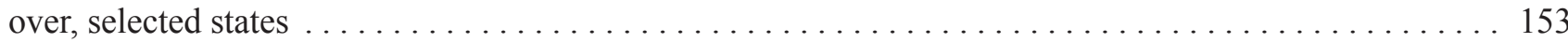

Table 2-83. Forestry workers: Proportionate mortality ratio (PMR) adjusted for age, sex, and race/ethnicity for pneumoconiosis and other lung diseases-external agents, U.S. residents age 15 and over, selected states,

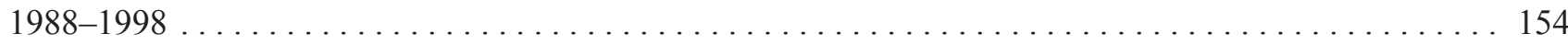

Table 2-84. Fishery workers: Proportionate mortality ratio (PMR) adjusted for age, sex, and race/ethnicity for pneumoconiosis and other lung diseases-external agents, U.S. residents age 15 and over, selected states,

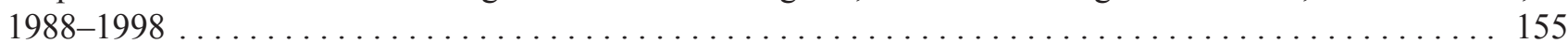

Figure 2-62. Coal workers' pneumoconiosis: Proportionate mortality ratio (PMR) adjusted for age, sex, and race/ethnicity by agricultural group, U.S. residents age 15 and over, selected states, 1988-1998 . . . . . 156

Figure 2-63. Asbestosis: Proportionate mortality ratio (PMR) adjusted for age, sex, and race/ethnicity by agricultural group, U.S. residents age 15 and over, selected states, $1988-1998 \ldots \ldots \ldots \ldots \ldots \ldots \ldots$

Figure 2-64. Pneumoconiosis due to other silica or silicates: Proportionate mortality ratio (PMR) adjusted for age, sex, and race/ethnicity by agricultural group, U.S. residents age 15 and over, selected states,

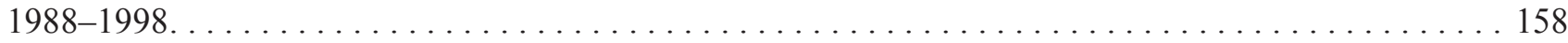

Figure 2-65. Pneumoconiosis, unspecified: Proportionate mortality ratio (PMR) adjusted for age, sex, and race/ethnicity by agricultural group, U.S. residents age 15 and over, selected states, 1988-1998 . . . . . 159

Figure 2-66. Pneumonitis due to solids and liquids: Proportionate mortality ratio (PMR) adjusted for age, sex, and race/ethnicity by agricultural group, U.S. residents age 15 and over, selected states, 1988-1998 . 160

Figure 2-67. Respiratory conditions due to other and unspecified external agents: Proportionate mortality ratio (PMR) adjusted for age, sex, and race/ethnicity by agricultural group, U.S. residents age 15 and over,

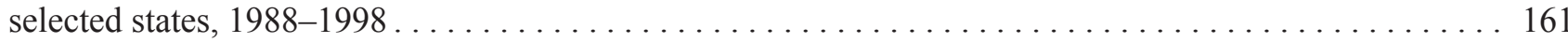

\section{Other Diseases of Respiratory System Mortality within and by Agricultural Group}

Table 2-85. Crop farm workers: Proportionate mortality ratio (PMR) adjusted for age, sex, and race/ethnicity for other diseases of respiratory system, U.S. residents age 15 and over, selected states, 1988-1998 _ . . 162

Table 2-86. Livestock farm workers: Proportionate mortality ratio (PMR) adjusted for age, sex, and race/ ethnicity for other diseases of respiratory system, U.S. residents age 15 and over, selected states, 19881998

Table 2-87. Farm managers: Proportionate mortality ratio (PMR) adjusted for age, sex, and race/ethnicity for other diseases of respiratory system, U.S. residents age 15 and over, selected states, 1988-1998 . . . . 164

Table 2-88. Landscape and horticultural workers: Proportionate mortality ratio (PMR) adjusted for age, sex, and race/ethnicity for other diseases of respiratory system, U.S. residents age 15 and over, selected states,

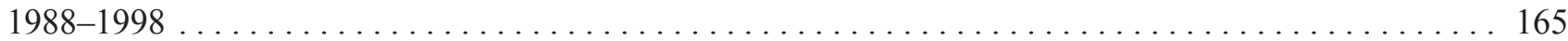


Table 2-89. Forestry workers: Proportionate mortality ratio (PMR) adjusted for age, sex, and race/ethnicity for other diseases of respiratory system, U.S. residents age 15 and over, selected states, 1988-1998 _ . . 166

Table 2-90. Fishery workers: Proportionate mortality ratio (PMR) adjusted for age, sex, and race/ethnicity for other diseases of respiratory system, U.S. residents age 15 and over, selected states, 1988-1998 _ . . 167

Figure 2-68. Empyema: Proportionate mortality ratio (PMR) adjusted for age, sex, and race/ethnicity by agricultural group, U.S. residents age 15 and over, selected states, $1988-1998 \ldots \ldots \ldots \ldots \ldots \ldots \ldots$

Figure 2-69. Pleurisy: Proportionate mortality ratio (PMR) adjusted for age, sex, and race/ethnicity by agricultural group, U.S. residents age 15 and over, selected states, $1988-1998 \ldots \ldots \ldots \ldots \ldots$. . . . . . 169

Figure 2-70. Pneumothorax: Proportionate mortality ratio (PMR) adjusted for age, sex, and race/ethnicity by

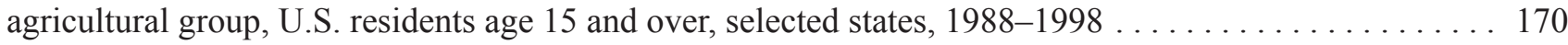

Figure 2-71. Abscess of lung and mediastinum: Proportionate mortality ratio (PMR) adjusted for age, sex, and race/ethnicity by agricultural group, U.S. residents age 15 and over, selected states, 1988-1998 . . . 171

Figure 2-72. Pulmonary congestion and hypostasis: Proportionate mortality ratio (PMR) adjusted for age, sex, and race/ethnicity by agricultural group, U.S. residents age 15 and over, selected states, 1988-1998 . 172

Figure 2-73. Postinflammatory pulmonary fibrosis: Proportionate mortality ratio (PMR) adjusted for age, sex, and race/ethnicity by agricultural group, U.S. residents age 15 and over, selected states, 1988-1998 . 173

Figure 2-74. Other alveolar and parietoalveolar pneumonopathy: Proportionate mortality ratio (PMR) adjusted for age, sex, and race/ethnicity by agricultural group, U.S. residents age 15 and over, selected states, 1988-1998

Figure 2-75. Other diseases of the lung: Proportionate mortality ratio (PMR) adjusted for age, sex, and race/ ethnicity by agricultural group, U.S. residents age 15 and over, selected states, 1988-1998 . . . . . . . 175

Figure 2-76. Other diseases of respiratory system: Proportionate mortality ratio (PMR) adjusted for age, sex, and race/ethnicity by agricultural group, U.S. residents age 15 and over, selected states, 1988-1998 . . . 176

\section{Morbidity}

\section{Morbidity by Agricultural Group within Respiratory Condition-NHIS}

Table 3-1. Hayfever (past year): Estimated prevalence and prevalence ratio (PR) adjusted for age, sex, race/ ethnicity, and smoking status by agricultural group and survey year, U.S. residents age 18 and over, 19971999

Table 3-2. Sinusitis (past year): Estimated prevalence and prevalence ratio (PR) adjusted for age, sex, race/ ethnicity, and smoking status by agricultural group and survey year, U.S. residents age 18 and over, 19971999

Table 3-3. Chronic bronchitis (past year): Estimated prevalence and prevalence ratio (PR) adjusted for age, sex, race/ethnicity, and smoking status by agricultural group and survey year, U.S. residents age 18 and over, 1997-1999

Table 3-4. Emphysema (ever): Estimated prevalence and prevalence ratio (PR) adjusted for age, sex, race/ ethnicity, and smoking status by agricultural group and survey year, U.S. residents age 18 and over, 19971999 


\section{List of Tables and Figures}

Table 3-5. Asthma (ever): Estimated prevalence and prevalence ratio (PR) adjusted for age, sex, race/ ethnicity, and smoking status by agricultural group and survey year, U.S. residents age 18 and over, 19971999

Table 3-6. Lung cancer (ever): Estimated prevalence and prevalence ratio (PR) adjusted for age, sex, race/ ethnicity, and smoking status by agricultural group and survey year, U.S. residents age 18 and over, 19971999

Figure 3-1. Respiratory conditions: Prevalence ratio (PR) adjusted for age, sex, race/ethnicity, and smoking status by agricultural group, U.S. residents age 18 and over, $1997-1999 \ldots \ldots \ldots \ldots \ldots \ldots$. . . . . . . . 185

\section{Morbidity by Respiratory Condition and Sex within Agricultural Group-NHIS}

Figure 3-2. Farm workers: Prevalence ratio (PR) adjusted for age, race/ethnicity, and smoking status by respiratory condition and sex, U.S. residents age 18 and over, $1997-1999 \ldots \ldots \ldots \ldots \ldots \ldots \ldots \ldots$

Figure 3-3. Farm managers: Prevalence ratio (PR) adjusted for age, race/ethnicity, and smoking status by respiratory condition and sex, U.S. residents age 18 and over, 1997-1999 . . . . . . . . . . . . . . . . 187

Figure 3-4. Forestry/fishery workers: Prevalence ratio (PR) adjusted for age, race/ethnicity, and smoking status by respiratory condition and sex, U.S. residents age 18 and over, $1997-1999 \ldots \ldots \ldots \ldots \ldots$

\section{Morbidity by Respiratory Condition and Race/Ethnicity within Agricultural Group-NHIS}

Figure 3-5. Farm workers: Prevalence ratio (PR) adjusted for age, sex, and smoking status by respiratory condition and race/ethnicity, U.S. residents age 18 and over, $1997-1999 \ldots \ldots \ldots \ldots \ldots \ldots \ldots \ldots \ldots$

Figure 3-6. Farm managers: Prevalence ratio (PR) adjusted for age, sex, and smoking status by respiratory condition and race/ethnicity, U.S. residents age 18 and over, $1997-1999 \ldots \ldots \ldots \ldots \ldots \ldots \ldots \ldots$

Figure 3-7. Forestry/fishery workers: Prevalence ratio (PR) adjusted for age, sex, and smoking status by respiratory condition and race/ethnicity, U.S. residents age 18 and over, 1997-1999.

\section{Morbidity by Respiratory Condition and Smoking Status within Agricultural Group-NHIS}

Figure 3-8. Farm workers: Prevalence ratio (PR) adjusted for age, sex, and race/ethnicity by respiratory condition and smoking status, U.S. residents age 18 and over, $1997-1999 \ldots \ldots \ldots \ldots \ldots$. . . . . . . . . 192

Figure 3-9. Farm managers: Prevalence ratio (PR) adjusted for age, sex, and race/ethnicity by respiratory condition and smoking status, U.S. residents age 18 and over, $1997-1999 \ldots \ldots \ldots \ldots \ldots \ldots \ldots \ldots$

Figure 3-10. Forestry/fishery workers: Prevalence ratio (PR) adjusted for age, sex, and race/ethnicity by respiratory condition and smoking status, U.S. residents age 18 and over, 1997-1999 . .

\section{Morbidity by Agricultural Group within Respiratory Condition-NHANES III}

Table 3-7. Wheezing, apart from a cold (current): Estimated prevalence and prevalence ratio (PR) adjusted for age, sex, race/ethnicity, and smoking status by agricultural group, U.S. residents age 17 and over, 1988-

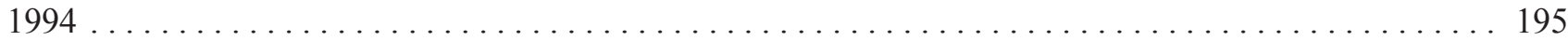

Table 3-8. Cough (current): Estimated prevalence and prevalence ratio (PR) adjusted for age, sex, race/ ethnicity, and smoking status by agricultural group, U.S. residents age 17 and over, 1988-1994 . . . . . 196

Table 3-9. Phlegm (current): Estimated prevalence and prevalence ratio (PR) adjusted for age, sex, race/ ethnicity, and smoking status by agricultural group, U.S. residents age 17 and over, 1988-1994 . . . . . 197 


\section{List of Tables and Figures}

Table 3-10. Shortness of breath (current): Estimated prevalence and prevalence ratio (PR) adjusted for age, sex, race/ethnicity, and smoking status by agricultural group, U.S. residents age 17 and over, 1988-1994. 198

Table 3-11. Stuffy, itchy, runny nose (past year): Estimated prevalence and prevalence ratio (PR) adjusted for age, sex, race/ethnicity, and smoking status by agricultural group, U.S. residents age 17 and over,

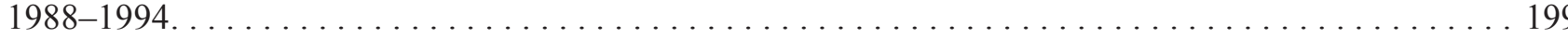

Table 3-12. Cold or flu (past year): Estimated prevalence and prevalence ratio (PR) adjusted for age, sex, race/ethnicity, and smoking status by agricultural group, U.S. residents age 17 and over, 1988-1994 _. . 200

Table 3-13. Sinusitis (past year): Estimated prevalence and prevalence ratio (PR) adjusted for age, sex, race/ ethnicity, and smoking status by agricultural group, U.S. residents age 17 and over, 1988-1994 _ . . . . 201

Table 3-14. Pneumonia (past year): Estimated prevalence and prevalence ratio (PR) adjusted for age, sex, race/ethnicity, and smoking status by agricultural group, U.S. residents age 17 and over, 1988-1994 . . . 202

Table 3-15. Wheezing (past year): Estimated prevalence and prevalence ratio (PR) adjusted for age, sex, race/ethnicity, and smoking status by agricultural group, U.S. residents age 17 and over, 1988-1994 . . . 203

Table 3-16. Asthma (ever): Estimated prevalence and prevalence ratio (PR) adjusted for age, sex, race/ ethnicity, and smoking status by agricultural group, U.S. residents age 17 and over, 1988-1994 . . . . . 204

Table 3-17. Chronic bronchitis (ever): Estimated prevalence and prevalence ratio (PR) adjusted for age, sex, race/ethnicity, and smoking status by agricultural group, U.S. residents age 17 and over, 1988-1994 _ . . 205

Table 3-18. Emphysema (ever): Estimated prevalence and prevalence ratio (PR) adjusted for age, sex, race/ ethnicity, and smoking status by agricultural group, U.S. residents age 17 and over, 1988-1994 . . . . . 206

Table 3-19. Hayfever (ever): Estimated prevalence and prevalence ratio (PR) adjusted for age, sex, race/ ethnicity, and smoking status by agricultural group, U.S. residents age 17 and over, 1988-1994 . . . . . 207

Figure 3-11. Respiratory conditions (current): Prevalence ratio (PR) adjusted for age, sex, race/ethnicity, and smoking status by agricultural group, U.S. residents age 17 and over, 1988-1994 . . . . . . . . . . . . 208

Figure 3-12. Respiratory conditions (past year): Prevalence ratio (PR) adjusted for age, sex, race/ethnicity, and smoking status by agricultural group, U.S. residents age 17 and over, 1988-1994 _ . . . . . . . . 209

Figure 3-13. Respiratory conditions (ever): Prevalence ratio (PR) adjusted for age, sex, race/ethnicity, and smoking status by agricultural group, U.S. residents age 17 and over, $1988-1994 \ldots \ldots \ldots \ldots \ldots \ldots$

\section{Morbidity by Sex within Respiratory Condition and Agricultural Group-NHANES III}

Figure 3-14. Respiratory conditions (current), farm workers: Prevalence ratio (PR) adjusted for age, race/ ethnicity, and smoking status by sex, U.S. residents age 17 and over, $1988-1994 \ldots \ldots \ldots \ldots \ldots \ldots 211$

Figure 3-15. Respiratory conditions (current), farm managers: Prevalence ratio (PR) adjusted for age, race/

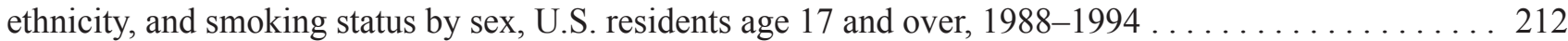

Figure 3-16. Respiratory conditions (current), other agricultural workers: Prevalence ratio (PR) adjusted for age, race/ethnicity, and smoking status by sex, U.S. residents age 17 and over, 1988-1994 . . . . . . 213

Figure 3-17. Respiratory conditions (past year), farm workers: Prevalence ratio (PR) adjusted for age, race/ ethnicity, and smoking status by sex, U.S. residents age 17 and over, 1988-1994 . . . . . . . . . . 214

Figure 3-18. Respiratory conditions (past year), farm managers: Prevalence ratio (PR) adjusted for age, race/ ethnicity, and smoking status by sex, U.S. residents age 17 and over, 1988-1994 . . . . . . . . . . . . 215 


\section{List of Tables and Figures}

Figure 3-19. Respiratory conditions (past year), other agricultural workers: Prevalence ratio (PR) adjusted for age, race/ethnicity, and smoking status by sex, U.S. residents age 17 and over, 1988-1994 . . . . . 216

Figure 3-20. Respiratory conditions (ever), farm workers: Prevalence ratio (PR) adjusted for age, race/

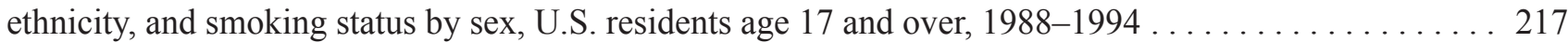

Figure 3-21. Respiratory conditions (ever), farm managers: Prevalence ratio (PR) adjusted for age, race/ ethnicity, and smoking status by sex, U.S. residents age 17 and over, $1988-1994 \ldots \ldots \ldots \ldots \ldots \ldots .218$

Figure 3-22. Respiratory conditions (ever), other agricultural workers: Prevalence ratio (PR) adjusted for age, race/ethnicity, and smoking status by sex, U.S. residents age 17 and over, 1988-1994 . . . . . . . 219

\section{Morbidity by Race/Ethnicity within Respiratory Condition and Agricultural Group-NHANES III}

Figure 3-23. Respiratory conditions (current), farm workers: Prevalence ratio (PR) adjusted for age, sex, and smoking status by race/ethnicity, U.S. residents age 17 and over, 1988-1994 . . . . . . . . . . . . . . . 220

Figure 3-24. Respiratory conditions (current), farm managers: Prevalence ratio (PR) adjusted for age, sex,

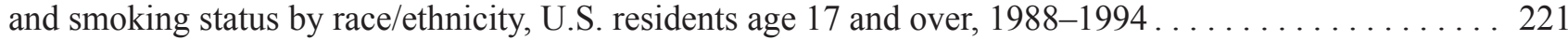

Figure 3-25. Respiratory conditions (current), other agricultural workers: Prevalence ratio (PR) adjusted for age, sex, and smoking status by race/ethnicity, U.S. residents age 17 and over, 1988-1994 . . . . . . 222

Figure 3-26. Respiratory conditions (past year), farm workers: Prevalence ratio (PR) adjusted for age, sex, and smoking status by race/ethnicity, U.S. residents age 17 and over, 1988-1994 . . . . . . . . . . . 223

Figure 3-27. Respiratory conditions (past year), farm managers: Prevalence ratio (PR) adjusted for age, sex, and smoking status by race/ethnicity, U.S. residents age 17 and over, 1988-1994 . . . . . . . . . . . 224

Figure 3-28. Respiratory conditions (past year), other agricultural workers: Prevalence ratio (PR) adjusted for age, sex, and smoking status by race/ethnicity, U.S. residents age 17 and over, 1988-1994 . . . . . 225

Figure 3-29. Respiratory conditions (ever), farm workers: Prevalence ratio (PR) adjusted for age, sex, and smoking status by race/ethnicity, U.S. residents age 17 and over, $1988-1994 \ldots \ldots \ldots \ldots \ldots \ldots \ldots$

Figure 3-30. Respiratory conditions (ever), farm managers: Prevalence ratio (PR) adjusted for age, sex, and

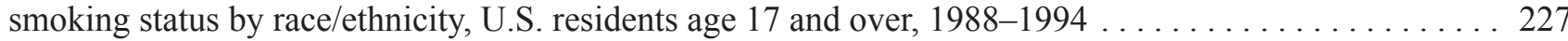

Figure 3-31. Respiratory conditions (ever), other agricultural workers: Prevalence ratio (PR) adjusted for age, sex, and smoking status by race/ethnicity, U.S. residents age 17 and over, 1988-1994 . . . . . . . 228

\section{Morbidity by Smoking Status within Respiratory Condition and Agricultural Group-NHANES III}

Figure 3-32. Respiratory conditions (current), farm workers: Prevalence ratio (PR) adjusted for age, sex, and race/ethnicity by smoking status, U.S. residents age 17 and over, 1988-1994 . . . . . . . . . . . . . . 229

Figure 3-33. Respiratory conditions (current), farm managers: Prevalence ratio (PR) adjusted for age, sex, and race/ethnicity by smoking status, U.S. residents age 17 and over, $1988-1994 \ldots \ldots \ldots \ldots \ldots \ldots$

Figure 3-34. Respiratory conditions (current), other agricultural workers: Prevalence ratio (PR) adjusted for age, sex, and race/ethnicity by smoking status, U.S. residents age 17 and over, 1988-1994 _ . . . . . 231

Figure 3-35. Respiratory conditions (past year), farm workers: Prevalence ratio (PR) adjusted for age, sex, and race/ethnicity by smoking status, U.S. residents age 17 and over, 1988-1994 . . . . . . . . . . . . . . . 232 
Figure 3-36. Respiratory conditions (past year), farm managers: Prevalence ratio (PR) adjusted for age, sex,

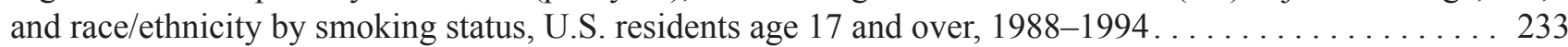

Figure 3-37. Respiratory conditions (past year), other agricultural workers: Prevalence ratio (PR) adjusted for age, sex, and race/ethnicity by smoking status, U.S. residents age 17 and over, 1988-1994 . . . . . 234

Figure 3-38. Respiratory conditions (ever), farm workers: Prevalence ratio (PR) adjusted for age, sex, and

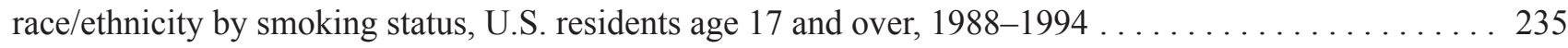

Figure 3-39. Respiratory conditions (ever), farm managers: Prevalence ratio (PR) adjusted for age, sex, and race/ethnicity by smoking status, U.S. residents age 17 and over, $1988-1994 \ldots \ldots \ldots \ldots \ldots \ldots \ldots$

Figure 3-40. Respiratory conditions (ever), other agricultural workers: Prevalence ratio (PR) adjusted for age, sex, and race/ethnicity by smoking status, U.S. residents age 17 and over, 1988-1994 _ . . . . . 237

\section{Morbidity by Agricultural Group within Spirometry Index: FEV 1 , FVC, PEF-NHANES III}

Table 3-20. Spirometry: Forced expiratory volume in one second $\left(\mathrm{FEV}_{1}\right)$, forced vital capacity (FVC), and peak expiratory flow (PEF) by agricultural group, U.S. residents age 17 and over, 1988-1994 . . . . . . 238

Table 3-21. Spirometry: Percent predicted forced expiratory volume in one second $\left(\mathrm{FEV}_{1}\right)$, forced vital capacity (FVC), and peak expiratory flow (PEF) by agricultural group, U.S. residents age 17 and over,

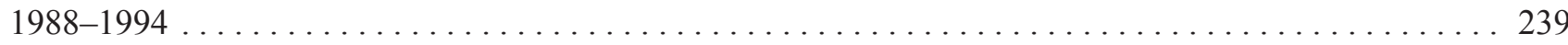

Table 3-22a. Obstructive abnormality: Estimated prevalence and prevalence ratio (PR) adjusted for age, sex, race/ethnicity, and smoking status by agricultural group, U.S. residents age 17 and over, 1988-1994 . . . 240

Table 3-22b. Restrictive abnormality: Estimated prevalence and prevalence ratio (PR) adjusted for age, sex, race/ethnicity, and smoking status by agricultural group, U.S. residents age 17 and over, 1988-1994 _. . 240

\section{Morbidity by Agricultural Group and Sex within Spirometry Index: FEV 1 , FVC, PEF-NHANES III}

Figure 3-41. Percent predicted forced expiratory volume in one second $\left(\mathrm{FEV}_{1}\right)$ by agricultural group and sex,

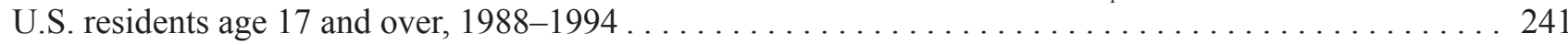

Figure 3-42. Percent predicted forced vital capacity (FVC) by agricultural group and sex, U.S. residents age

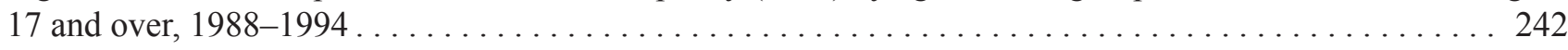

Figure 3-43. Percent predicted peak expiratory flow (PEF) by agricultural group and sex, U.S. residents age

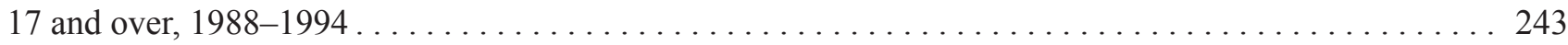

\section{Morbidity by Agricultural Group and Race/Ethnicity within Spirometry Index: FEV 1 , FVC, PEF-NHANES III}

Figure 3-44. Percent predicted forced expiratory volume in one second $\left(\mathrm{FEV}_{1}\right)$ by agricultural group and race/ethnicity, U.S. residents age 17 and over, $1988-1994 \ldots \ldots \ldots \ldots \ldots \ldots \ldots \ldots \ldots \ldots \ldots \ldots$

Figure 3-45. Percent predicted forced vital capacity (FVC) by agricultural group and race/ethnicity, U.S.

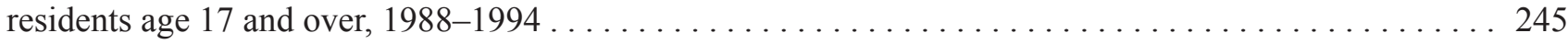

Figure 3-46. Percent predicted peak expiratory flow (PEF) by agricultural group and race/ethnicity, U.S.

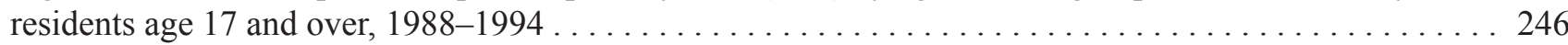


Morbidity by Agricultural Group and Smoking Status within Spirometry Index: FEV, FVC, PEF-NHANES III

Figure 3-47. Percent predicted forced expiratory volume in one second $\left(\mathrm{FEV}_{1}\right)$ by agricultural group and smoking status, U.S. residents age 17 and over, $1988-1994 \ldots \ldots \ldots \ldots \ldots \ldots \ldots \ldots \ldots \ldots \ldots$

Figure 3-48. Percent predicted forced vital capacity (FVC) by agricultural group and smoking status, U.S.

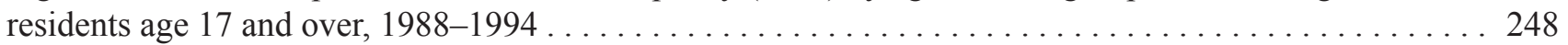

Figure 3-49. Percent predicted peak expiratory flow (PEF) by agricultural group and smoking status, U.S.

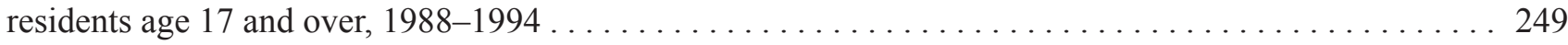

Morbidity by Agricultural Group and Sex within Spirometry Index: Obstructive and Restrictive Abnormality-NHANES III

Figure 3-50. Spirometry: Percent of workers with obstructive abnormality by agricultural group and sex,

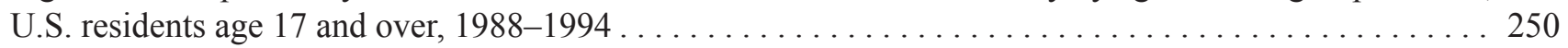

Figure 3-51. Spirometry: Percent of workers with restrictive abnormality by agricultural group and sex, U.S.

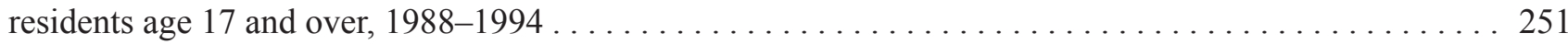

Morbidity by Agricultural Group and Race/Ethnicity within Spirometry Index: Obstructive and Restrictive Abnormality-NHANES III

Figure 3-52. Spirometry: Percent of workers with obstructive abnormality by agricultural group and race/

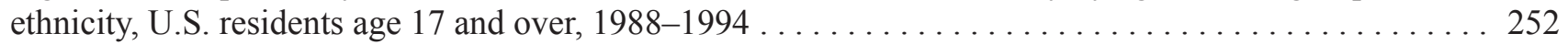

Figure 3-53. Spirometry: Percent of workers with restrictive abnormality by agricultural group and race/

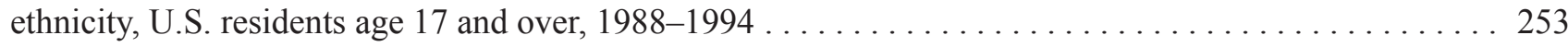

Morbidity by Agricultural Group and Smoking Status within Spirometry Index: Obstructive and Restrictive Abnormality-NHANES III

Figure 3-54. Spirometry: Percent of workers with obstructive abnormality by agricultural group and smoking status, U.S. residents age 17 and over, $1988-1994 \ldots \ldots \ldots \ldots \ldots \ldots \ldots \ldots \ldots \ldots \ldots \ldots \ldots \ldots \ldots$

Figure 3-55. Spirometry: Percent of workers with restrictive abnormality by agricultural group and smoking

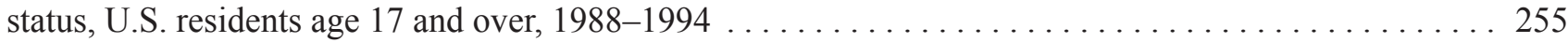

Morbidity by Agricultural Group within Dust Diseases of the Lung and Respiratory Conditions Due to Toxic Agents-SOII

Table 3-23. Dust diseases of the lung: Estimated incidence per 10,000 workers by agricultural group, 1995-

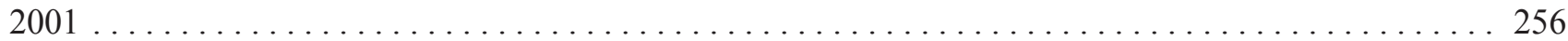

Table 3-24. Respiratory conditions due to toxic agents: Estimated incidence per 10,000 workers by

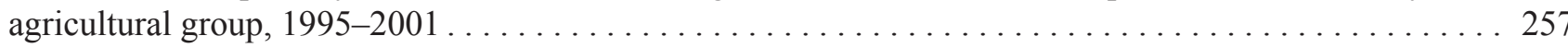




\section{Highlights and}

Limitations 



\section{Highlights}

These selected highlights summarize the major findings in the report, including a description of results that were statistically elevated. Mortality statistics were derived from deaths from 24 states for 1988-1998, while morbidity data came from two large population-based surveys of the U.S. undertaken in 1997-1999 and 1988-1994.

- Decedents whose death certificate indicated that they worked as crop workers had significantly elevated mortality for a number of respiratory conditions, including hypersensitivity pneumonitis (proportionate mortality more than 10 times higher than expected), asthma, bronchitis, histoplasmosis, tuberculosis, pneumonia, and influenza. (Tables $\mathrm{H}-1$ and $\mathrm{H}-2$ )

- Decedents whose death certificate indicated that they worked as livestock farm workers had significantly elevated mortality for several respiratory conditions, including hypersensitivity pneumonitis (proportionate mortality more than 50 times higher than expected), asthma, tuberculosis, and influenza. (Tables H-1 and H-2)

- Decedents whose death certificate indicated that they worked as landscape or horticultural workers had significantly elevated mortality for chronic obstructive pulmonary diseases (COPD), including chronic airways obstruction, and for abscesses of the lung and mediastinum. (Tables $\mathrm{H}-1$ and $\mathrm{H}-2)$
- Decedents whose death certificate indicated that they worked as forestry workers had significantly elevated mortality for tuberculosis, COPD, including chronic airways obstruction, and for pneumonia. (Tables $\mathrm{H}-1$ and $\mathrm{H}-2$ )

- Decedents whose death certificate indicated that they worked as fishery workers had significantly elevated mortality for COPD, including chronic airways obstruction. (Tables $\mathrm{H}-1$ and $\mathrm{H}-2$ )

- At least two of the agricultural groups studied in this report were noted to have significantly elevated mortality for several respiratory diseases, including tuberculosis, hypersensitivity pneumonitis, asthma, COPD, pneumonia, and influenza. Significantly elevated COPD mortality was noted in three agricultural groups (landscape and horticultural workers, forestry workers, and fishery workers). (Table H-2)

- Individuals who reported that their longest job held was farm worker had elevated prevalence of phlegm production compared to all non-agricultural workers. Prevalence of wheeze was elevated for female farm workers and shortness of breath was elevated for farm workers who had 'ever smoked.' (Table H-3)

- Farm workers had a prevalence ratio (PR) of 173 for obstructive abnormality. (Table 3-22a) 
Table H-1. Mortality: Significantly elevated proportionate mortality ratios (PMRs) by agricultural group

\begin{tabular}{|c|c|c|c|c|}
\hline \multirow[b]{2}{*}{ Disease (ICD-9 Code) } & \multirow{2}{*}{$\begin{array}{c}\text { Number of } \\
\text { Deaths }\end{array}$} & \multirow[b]{2}{*}{ PMR } & \multicolumn{2}{|c|}{ For more detail see: } \\
\hline & & & Table & Figure \\
\hline \multicolumn{5}{|l|}{ Crop Farm Workers } \\
\hline Hypersensitivity pneumonitis (495) & 23 & 1,228 & $2-73$ & $2-60$ \\
\hline Blastomycotic infection (116) & 14 & 245 & $2-43$ & $2-39$ \\
\hline Histoplasmosis (115) & 27 & 183 & $2-43$ & $2-38$ \\
\hline Bronchitis, not specified as acute or chronic (490) & 269 & 134 & $2-73$ & $2-55$ \\
\hline Abscess of lung and mediastinum (513) & 153 & 120 & $2-85$ & $2-71$ \\
\hline Pulmonary congestion \& hypostasis $(514)$ & 1,830 & 113 & $2-85$ & $2-72$ \\
\hline Asthma (493) & 813 & 111 & $2-73$ & $2-58$ \\
\hline Tuberculosis $(010-018)$ & 522 & 148 & $2-1$ & $2-1$ \\
\hline Miliary tuberculosis (018) & 35 & 196 & $2-37$ & $2-36$ \\
\hline Pulmonary tuberculosis $(011)$ & 437 & 152 & $2-37$ & $7-31$ \\
\hline Acute respiratory infections $(460-466)$ & 329 & 124 & $2-1$ & $2-5$ \\
\hline Acute upper respiratory infections of multiple or unspecified sites (465) & 87 & 160 & $2-55$ & $2-44$ \\
\hline Acute bronchitis and bronchiolitis (466) & 126 & 117 & $2-55$ & $2-45$ \\
\hline Pneumonia and influenza (480-487) & 26,114 & 109 & $2-1$ & $2-7$ \\
\hline Influenza (487) & 232 & 142 & $2-67$ & $2-54$ \\
\hline Other bacterial pneumonia (482) & 955 & 120 & $2-67$ & $2-50$ \\
\hline Pneumonia, organism unspecified (486) & 23,135 & 109 & $2-67$ & $2-53$ \\
\hline \multicolumn{5}{|l|}{ Livestock Farm Workers } \\
\hline Hypersensitivity pneumonitis (495) & 31 & 5,563 & $2-74$ & $2-60$ \\
\hline Other respiratory tuberculosis $(012)$ & 5 & 675 & $2-38$ & $2-32$ \\
\hline Tuberculosis of meninges and central nervous system (013) & 5 & 546 & $2-38$ & $2-33$ \\
\hline Asthma (493) & 276 & 150 & $2-74$ & $2-58$ \\
\hline Influenza (487) & 73 & 150 & $2-68$ & $2-54$ \\
\hline \multicolumn{5}{|l|}{ Landscape and Horticulture Workers } \\
\hline Abscess of lung and mediastinum (513) & 13 & 190 & $2-88$ & $2-71$ \\
\hline Chronic obstructive pulmonary disease and allied conditions (COPD) (490-496) & 799 & 109 & $2-4$ & $2-8$ \\
\hline Chronic airway obstruction, nec (496) & 624 & 111 & $2-76$ & $2-61$ \\
\hline \multicolumn{5}{|l|}{ Forestry Workers } \\
\hline Pulmonary tuberculosis $(011)$ & 41 & 143 & $2-41$ & $2-31$ \\
\hline Chronic obstructive pulmonary disease and allied conditions (COPD) (490-496) & 2,318 & 122 & $2-5$ & $2-8$ \\
\hline Chronic airway obstruction, nec (496) & 1,890 & 127 & $2-77$ & $2-61$ \\
\hline Pneumonia and influenza (480-487) & 1,771 & 116 & $2-5$ & $2-7$ \\
\hline Pneumonia, organism unspecified (486) & 1,564 & 117 & $2-71$ & $2-53$ \\
\hline \multicolumn{5}{|l|}{ Fishery Workers } \\
\hline Chronic obstructive pulmonary disease and allied conditions (COPD) (490-496) & 568 & 113 & $2-6$ & $2-8$ \\
\hline Chronic airway obstruction, nec (496) & 455 & 116 & $2-78$ & $2-61$ \\
\hline
\end{tabular}

nec - not elsewhere classified ICD - International Classification of Diseases

NOTE: PMRs are adjusted for age, sex, and race, U.S. residents age 15 and over, selected states (see Appendix D), 1988-1998. PMRs are significantly different from $100(\mathrm{p}<0.05)$.

SOURCE: National Center for Health Statistics multiple-cause-of-death data 
Table H-2. Mortality: Disease and disease categories with significantly elevated proportionate mortality ratios (PMRs) in two or more agricultural groups

\begin{tabular}{|c|c|c|c|c|c|}
\hline Disease (ICD-9 Code) & $\begin{array}{c}\text { Crop } \\
\text { Farm Workers }\end{array}$ & $\begin{array}{c}\text { Livestock } \\
\text { Farm Workers }\end{array}$ & $\begin{array}{c}\text { Landscape and } \\
\text { Horticulture Workers }\end{array}$ & $\begin{array}{l}\text { Forestry } \\
\text { Workers }\end{array}$ & $\begin{array}{l}\text { Fishery } \\
\text { Workers }\end{array}$ \\
\hline Pulmonary tuberculosis (011) & $\checkmark$ & & & $\checkmark$ & \\
\hline Abscess of lung and mediastinum (513) & $\checkmark$ & & $\checkmark$ & & \\
\hline Pneumonia/influenza (480-487) & $\checkmark$ & & & $\checkmark$ & \\
\hline Pneumonia, organism unspecified (486) & $\checkmark$ & & & $\checkmark$ & \\
\hline Influenza (487) & $\checkmark$ & $\checkmark$ & & & \\
\hline \multicolumn{3}{|c|}{ Chronic obstructive pulmonary disease (490-496) } & & $\checkmark$ & $\checkmark$ \\
\hline Asthma (493) & $\checkmark$ & $\checkmark$ & & & \\
\hline Hypersensitivity pneumonitis (495) & $\checkmark$ & $\checkmark$ & & & \\
\hline Chronic airway obstruction, nec (496) & & & $\checkmark$ & $\checkmark$ & $\checkmark$ \\
\hline
\end{tabular}

nec - not elsewhere classified

NOTE: Crop farm workers had 10, livestock farm workers had 2, and landscape and horticultural workers had 1 other respiratory diseases or disease categories with significantly elevated PMRs. See Table H-1. PMRs are adjusted for age, sex, and race, U.S. residents age 15 and over, selected states (see Appendix D), 1988-1998. PMRs are significantly different from $100(\mathrm{p}<0.05)$.

SOURCE: National Center for Health Statistics multiple-cause-of-death data

\section{Table H-3. Morbidity: Significantly elevated prevalence ratios (PRs) by agricultural group}

\begin{tabular}{llcc}
\hline \multicolumn{1}{c}{ Respiratory Condition } & PR & \multicolumn{2}{c}{ For more details see: } \\
\hline Farm Workers & & Table & Figure \\
\hline Phlegm (current) & 133 & $3-9$ & $3-11$ \\
$\quad$ Females & 226 & $3-14$ \\
$\quad$ Ever smoked & 156 & $3-32$ \\
Wheezing (apart from a cold), females & 155 & $3-20$ \\
Wheezing (past year), females & 146 & $3-17$ \\
Shortness of breath (current), ever smoked & 130 & $3-32$ \\
\hline
\end{tabular}

NOTE: PRs are adjusted for age, sex, race, and smoking (except for smoking-specific analyses), U.S. residents age 17 and over, 1988-1994. PRs are significantly different from $100(\mathrm{p}<0.05)$.

SOURCE: National Center for Health Statistics, Third National Health and Nutrition Examination Survey (NHANES III) 



\section{Limitations}

In addition to the following cautions, readers should see Appendix A for other limitations relating to specific sources of data presented in this report.

\section{General}

- In this report, the data are drawn from the major existing databases. However, other data may exist that would improve the completeness and reliability of the findings presented in this report. Readers who are aware of other data that should be considered for inclusion in future editions are encouraged to make their suggestions known (see Preface for contact information).

- Statistics in many tables and figures in this report are based on small numbers. Readers are cautioned that these can be unstable. Hence, inferences should be drawn with care, and should take the numerical basis into account.

- A decedent's or survey respondent's usual or current industry/occupation is not always indicative of the industry and occupation associated with the exposure that may be responsible for that individual's disease even when that disease is workrelated. Readers are therefore cautioned not to make definitive causative inferences about industries and occupations based solely on the various mortality and morbidity tables and figures presented in this report.

\section{Mortality Data}

- Data from only 24 states were used in the mortality analysis since reliable information on industry and occupation was not available for every state. These 24 states collectively account for 32 percent of the U.S. agricultural worker population (Table 1-2); they do not include the three states having the most agricultural employment (California, Texas, and Florida). Although the information presented is believed to be reasonably representative of health outcomes among all agricultural workers, it may not provide a fully accurate picture.
- Individuals affected by chronic diseases with long latency have much more time to change residences prior to death than individuals affected by acute diseases with short latency. Thus, state of residence at death does not necessarily represent the location of a decedent's occupational exposure, even for a death that results directly from occupational respiratory disease. However, unlike many other occupations, farmers often continue to work well beyond 65 years of age and $18 \%$ of the U.S. farm operators are over age $65^{1}$, indicating that farmers are less likely to change residences before death than other occupation.

- Work-related respiratory diseases are often chronic, may also have long latencies, but often may not be reported as the underlying cause of death. This led to a decision to consider both underlying and contributing causes of death in the mortality summary tables and figures in this report.

- Certifying physicians typically do not list all of a decedent's diseases on the death certificate. Therefore, even though contributing causes of death are considered, the mortality data presented in this report probably underestimate the occurrence of some or most respiratory diseases.

- As with any analysis based on death certificate data, there is undoubtedly some misclassification of cause of death. A treating physician may not correctly diagnose a particular disease during a patient's life or, as mentioned above, a certifying physician may fail to list a correctly diagnosed disease on the death certificate, particularly if another disease was directly responsible for the individual's death. In addition, the diagnoses listed on the death certificate sometimes are miscoded.

${ }^{1}$ U.S. Department of Commerce [1992]. Census of Agriculture. Washington, DC: U.S. Government Printing Office. 
- Data that depend, either directly or indirectly, on physician reporting or recording of occupational disease diagnoses can be influenced significantly by the physician's ability or willingness to suspect and evaluate a relationship between work and health. These, in turn, are influenced by evolving medical and scientific information, and by the legal, political and social environment. Some factors may lead to increased diagnosis and recording and reporting whereas others may reduce occupational disease recognition or reporting by physicians.

- The PMRs in this report have not been adjusted for smoking or any other confounding exposure because of lack of these data. Note that PMRs are vulnerable to difficulties in interpretation in that an elevated PMR may reflect an excess in a particular disease mortality or may simply arise from deficits in mortality from other diseases. The PMRs in this report are derived from data reported by only 24 states and omit data from some of the major agricultural states (e.g., California). They therefore may not be representative of the mortality patterns for the whole country. In addition, they may fail to indicate risks for some agricultural operations and situations not, or poorly, represented in the 24 states.

\section{Morbidity Data}

- Data from both the NHIS and NHANES surveys are restricted to a sample of householdbased respondents in the U.S. A typical round of NHIS or NHANES has about 30,000 respondents. Although weights reflecting the probability of selection for each survey respondent are provided (and were used in the analysis) to enable national estimates, the actual number of respondents is especially small when the data are disaggregated into groups (e.g., agricultural workers). For certain conditions such as emphysema and lung cancer, the numbers are especially small. Hence, the cautions given above for mortality data, against making broad inferences or generalizations from the data provided in this report, apply even more strongly here. In the case of the NHIS data, an attempt was made to compensate for small numbers by summing estimates from the most recent three years (1997-1999) for which survey data were available at the time of the analysis.

- Some of the conditions about which respondents were asked in these surveys relate to the individual's lifetime (e.g., has a doctor ever told you that you have asthma?), whereas others relate to a more recent period (e.g., during the past 12 months, have you had pneumonia?). Hence, the relationship between work and health may be conditional on the time frame of reference for the question, the individual's age, and whether the industry/occupation codes used in the analysis relate to the respondent's current or usual industry/ occupation. For the NHANES data, the industry/ occupation in which the respondent worked longest was used in the analysis, whereas for the NHIS data only the current industry/occupation was asked of the respondent.

- The questions asked about conditions in the NHANES and NHIS surveys are sensitive to the respondent's ability to recognize such conditions and to correctly answer the questions. Thus, there are potential reporting biases that may be associated, for example, with respondent age or socioeconomic status. The spirometric data from NHANES do not share this limitation, as they are objective measures of respiratory health derived from lung function tests.

- The method used to calculate confidence intervals for prevalence ratios assumes an underlying Poisson distribution and is strictly applicable to outcomes that are rare. Some of the outcomes reported in the survey (e.g., asthma) are not rare, and as a consequence the reported confidence intervals should be regarded as approximate. 
- Unlike the NHIS and NHANES data, publicuse data files were not available for the BLS injury and illness data. Only incidence rates summarized by industry for dust diseases of the lung and respiratory conditions due to toxic agents are publicly available, and it was not possible to adjust the survey results for factors such as age, sex, race/ethnicity, or smoking status. In the BLS data, work-related diseases are generally underrecognized and under-reported by employers. (Note: BLS confidential microdata for non-fatal injuries and illnesses is available for research, but users may access this data only at the BLS national office in Washington, D.C.)

- The agricultural occupation and industry coding systems for the source data employed in the presentation of the demographic, morbidity, and mortality data are broadly similar but differ in detail, preventing exact comparisons between them. See Appendices E, F, and G for descriptions of the industry and occupation codes relevant to this report. 



\section{Section 1}

Demographics 

Table 1-1. Demographic characteristics of employed U.S. agricultural workers by agricultural group and occupation, 2002

\begin{tabular}{lcrrr}
\hline & Number & \multicolumn{3}{c}{ Percent } \\
\cline { 3 - 6 } Occupation (Census Occupation Code) & (in thousands) & Female & Black & Hispanic \\
\hline Farm operators and managers & 1,168 & 24.5 & 1.2 & 4.2 \\
Farmers, except horticultural (473) & 898 & 25.5 & 0.7 & 2.5 \\
Horticultural specialty farmers (474) & 76 & 13.5 & 5.0 & 17.4 \\
Managers, farms, except horticultural (475) & 169 & 22.4 & 0.7 & 6.4 \\
Other agricultural and related occupations & 2,181 & 19.2 & 6.9 & 36.4 \\
Farm workers (479) & 716 & 21.0 & 4.7 & 45.4 \\
Supervisors, related agricultural occupations (485) & 188 & 7.7 & 5.5 & 19.1 \\
Groundskeepers and gardeners, except farm (486) & 973 & 7.8 & 10.0 & 35.4 \\
Animal caretakers, except farm (487) & 170 & 68.1 & 4.3 & 4.8 \\
Graders and sorters, agricultural products (488) & 68 & 67.7 & 2.8 & 71.1 \\
Timber cutting and logging (496) & 54 & 1.6 & 7.5 & 6.0 \\
\hline All Farming, Forestry, and Fishing Occupations & $\mathbf{3 , 4 8 0}$ & $\mathbf{2 0 . 6}$ & $\mathbf{4 . 9}$ & $\mathbf{2 4 . 4}$ \\
\hline
\end{tabular}

NOTE: Data for occupations with fewer than 50,000 employed are not published separately but are included in the total. See Appendices E, F, and G for occupations included in the analyses.

SOURCE: U.S. Bureau of Labor Statistics: Current Population Survey (ftp://ftp.bls.gov/pub/special.requests/lf/aa2002/aat11.txt) 


\section{Demographics}

Figure 1-1. Distribution of employed U.S. agricultural workers by sex, race, and ethnicity in comparison to all U.S. workers, 1997 and 2002

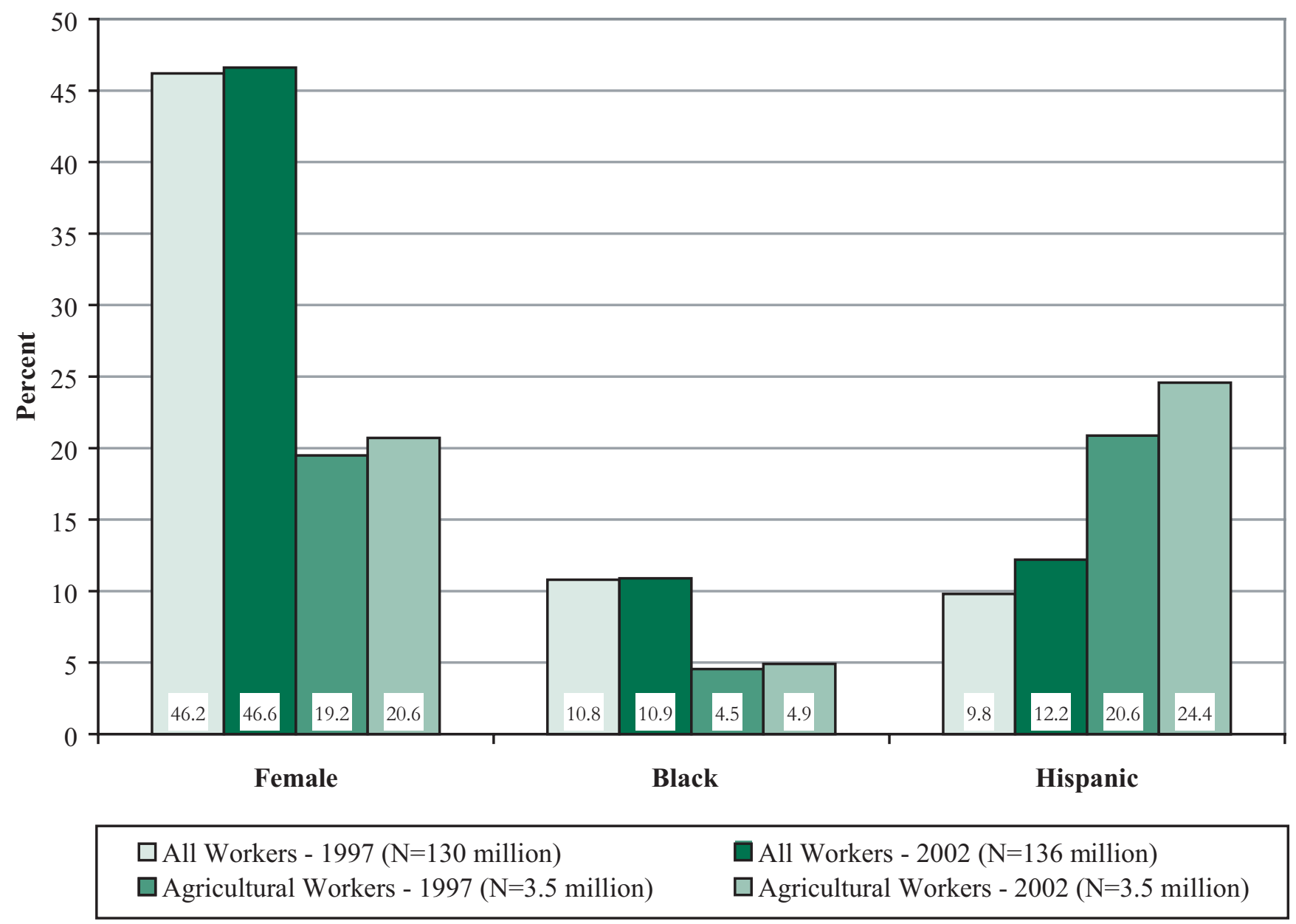

SOURCE: U.S. Bureau of Labor Statistics: Current Population Survey (ftp://ftp.bls.gov/pub/special.requests/lf/aa97/aat11.txt) (ftp://ftp.bls.gov/pub/ special.requests/lf/aa2002/aat11.txt) 
Figure 1-2. Distribution of employed U.S. agricultural groups by sex, race, and ethnicity, 1997

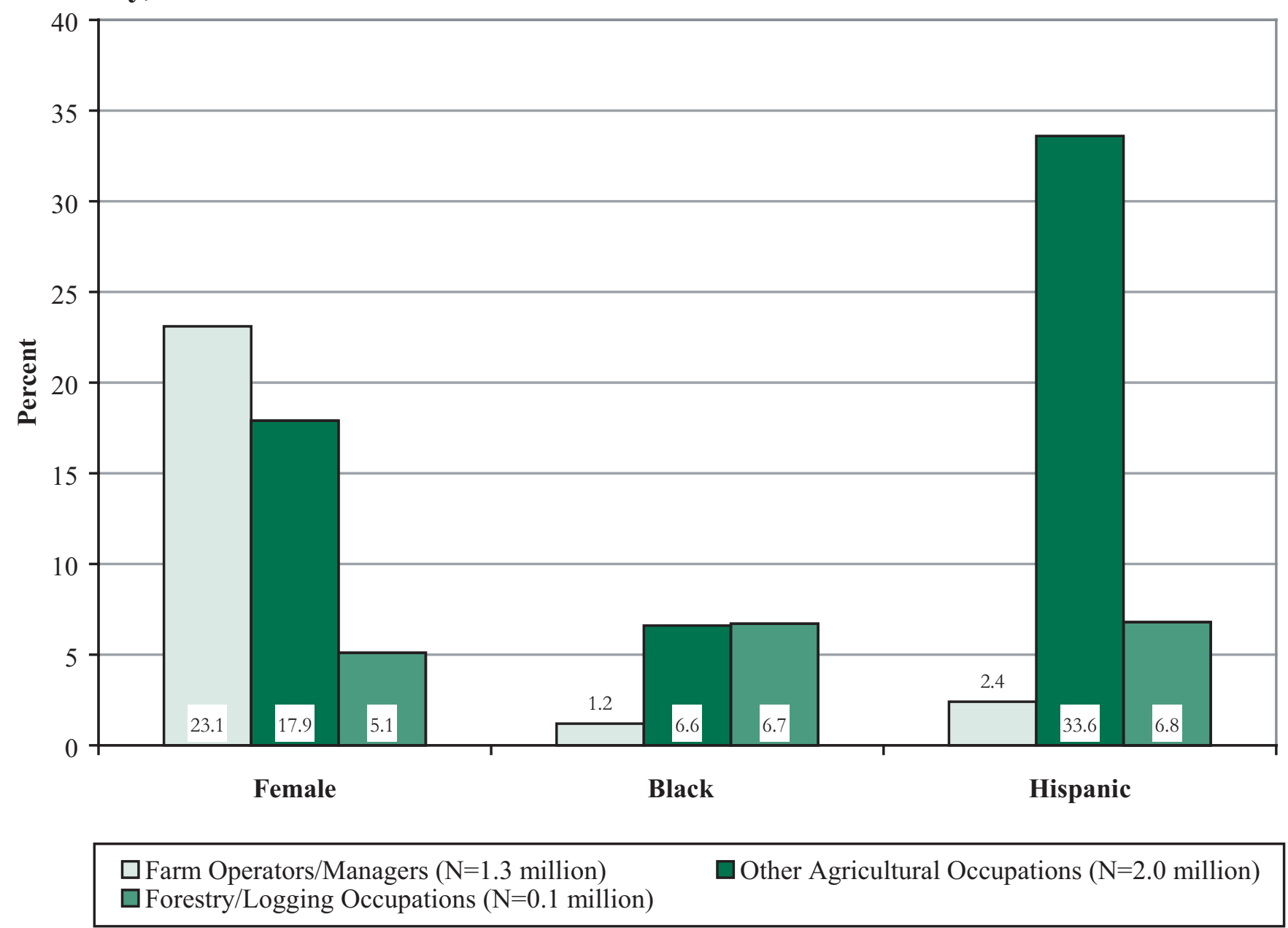

SOURCE: U.S. Bureau of Labor Statistics: Current Population Survey (ftp://ftp.bls.gov/pub/special.requests/lf/aa97/aat11.txt) 
Figure 1-3. Distribution of employed U.S. agricultural groups by sex, race, and ethnicity, 2002

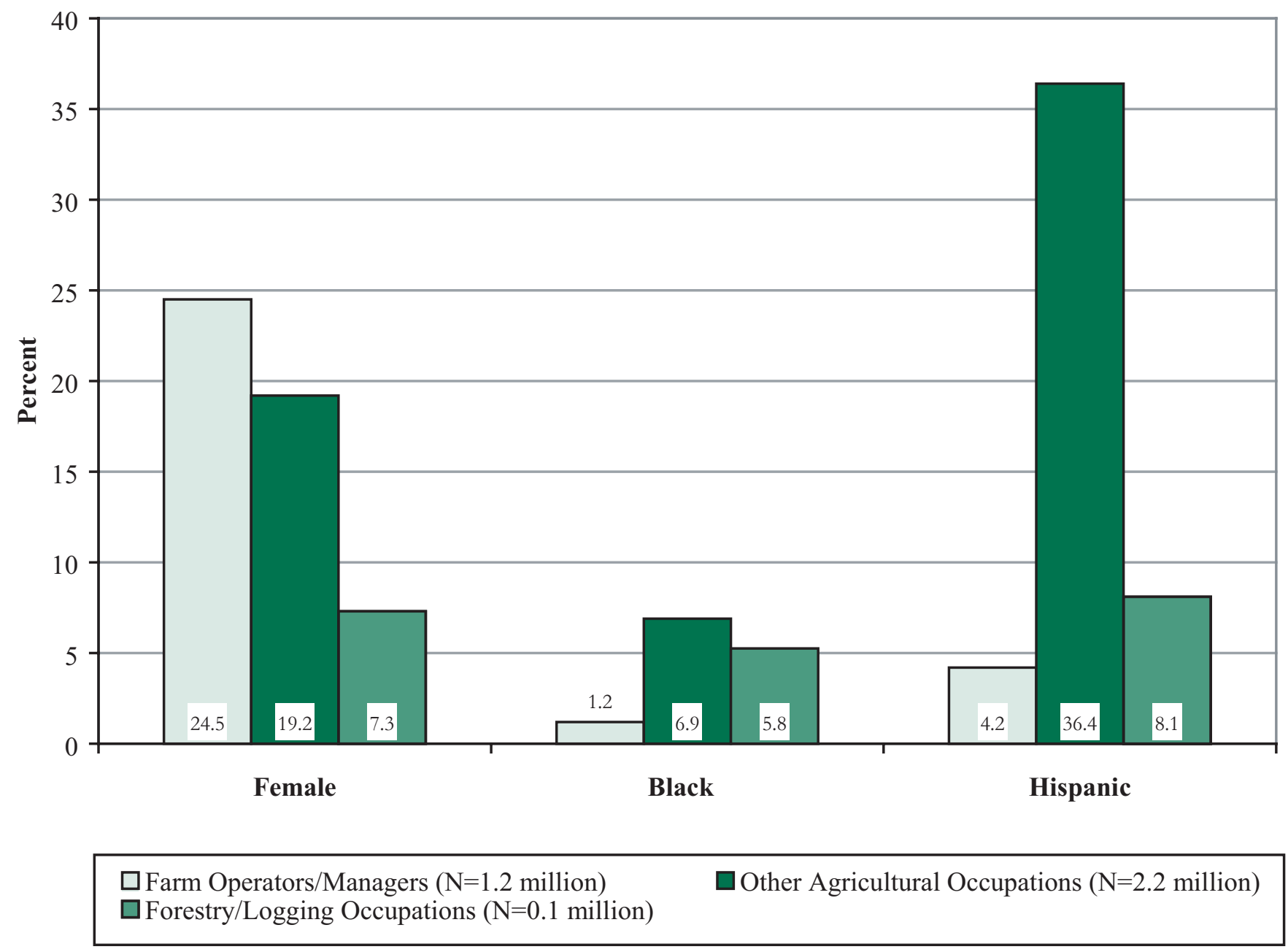

SOURCE: U.S. Bureau of Labor Statistics: Current Population Survey (ftp://ftp.bls.gov/pub/special.requests/lf/aa2002/aat11.txt) 
Table 1-2. Distribution of employed U.S. agricultural workers by state, 2002

\begin{tabular}{|c|c|c|c|c|c|}
\hline $\begin{array}{l}\text { States Used in the } \\
\text { Mortality Analysis } \\
\text { (Section 2) }\end{array}$ & $\begin{array}{c}\text { Number } \\
\text { Employed in } \\
\text { Agriculture } \\
\text { (in thousands) }\end{array}$ & $\begin{array}{c}\begin{array}{c}\text { Percent } \\
\text { of all }\end{array} \\
\text { Agricultural } \\
\text { Workers }\end{array}$ & $\begin{array}{r}\mathrm{N} \\
\text { Emr } \\
\text { Agr } \\
\text { (in th }\end{array}$ & $\begin{array}{l}\text { Number } \\
\text { nployed in } \\
\text { griculture } \\
\text { thousands) }\end{array}$ & $\begin{array}{c}\text { Percent } \\
\text { of all } \\
\text { Agricultural } \\
\text { Workers }\end{array}$ \\
\hline North Carolina & 107 & 3.1 & California & 513 & 14.7 \\
\hline Ohio & 95 & 2.7 & Texas & 297 & 8.5 \\
\hline Washington & 94 & 2.7 & Florida & 193 & 5.5 \\
\hline Wisconsin & 91 & 2.6 & New York & 122 & 3.5 \\
\hline Georgia & 89 & 2.5 & Illinois & 111 & 3.2 \\
\hline Indiana & 76 & 2.2 & Pennsylvania & 109 & 3.1 \\
\hline Tennessee & 67 & 1.9 & Minnesota & 102 & 2.9 \\
\hline Kansas & 56 & 1.6 & Michigan & 90 & 2.6 \\
\hline Kentucky & 56 & 1.6 & Missouri & 79 & 2.3 \\
\hline Colorado & 55 & 1.6 & Iowa & 71 & 2.0 \\
\hline Oklahoma & 53 & 1.5 & Oregon & 64 & 1.8 \\
\hline New Jersey & 46 & 1.3 & Virginia & 64 & 1.8 \\
\hline South Carolina & 40 & 1.1 & Arizona & 62 & 1.8 \\
\hline Idaho & 37 & 1.1 & Nebraska & 62 & 1.8 \\
\hline New Mexico & 27 & 0.8 & Maryland & 55 & 1.6 \\
\hline Utah & 26 & 0.7 & Arkansas & 53 & 1.5 \\
\hline Maine & 21 & 0.6 & Louisiana & 49 & 1.4 \\
\hline Hawaii & 20 & 0.6 & Mississippi & 47 & 1.3 \\
\hline Nevada & 18 & 0.5 & Massachusetts & 44 & 1.3 \\
\hline New Hampshire & 13 & 0.4 & Alabama & 38 & 1.1 \\
\hline Vermont & 13 & 0.4 & Montana & 35 & 1.0 \\
\hline West Virginia & 13 & 0.4 & South Dakota & 35 & 1.0 \\
\hline Alaska & 7 & 0.2 & North Dakota & 28 & 0.8 \\
\hline Rhode Island & 6 & 0.2 & Connecticut & 22 & 0.6 \\
\hline \multirow[t]{4}{*}{ Total } & \multirow[t]{4}{*}{1,126} & \multirow[t]{4}{*}{32.2} & Wyoming & 15 & 0.4 \\
\hline & & & Delaware & 7 & 0.2 \\
\hline & & & District of Columbia & ia 3 & 0.1 \\
\hline & & & Total, All States 3 & 3,496 & 100.0 \\
\hline
\end{tabular}

SOURCE: U.S. Bureau of Labor Statistics, Current Population Survey (http://www.bls.gov/opub/gp/pdf/gp02_14.pdf and http://www.bls.gov/opub/gp/pdf/ gp02_15.pdf) 



\section{Section 2}

Mortality 

Table 2-1. Crop farm workers: Proportionate mortality ratio (PMR) adjusted for age, sex, and race/ ethnicity by disease category, U.S. residents age 15 and over, selected states, 1988-1998

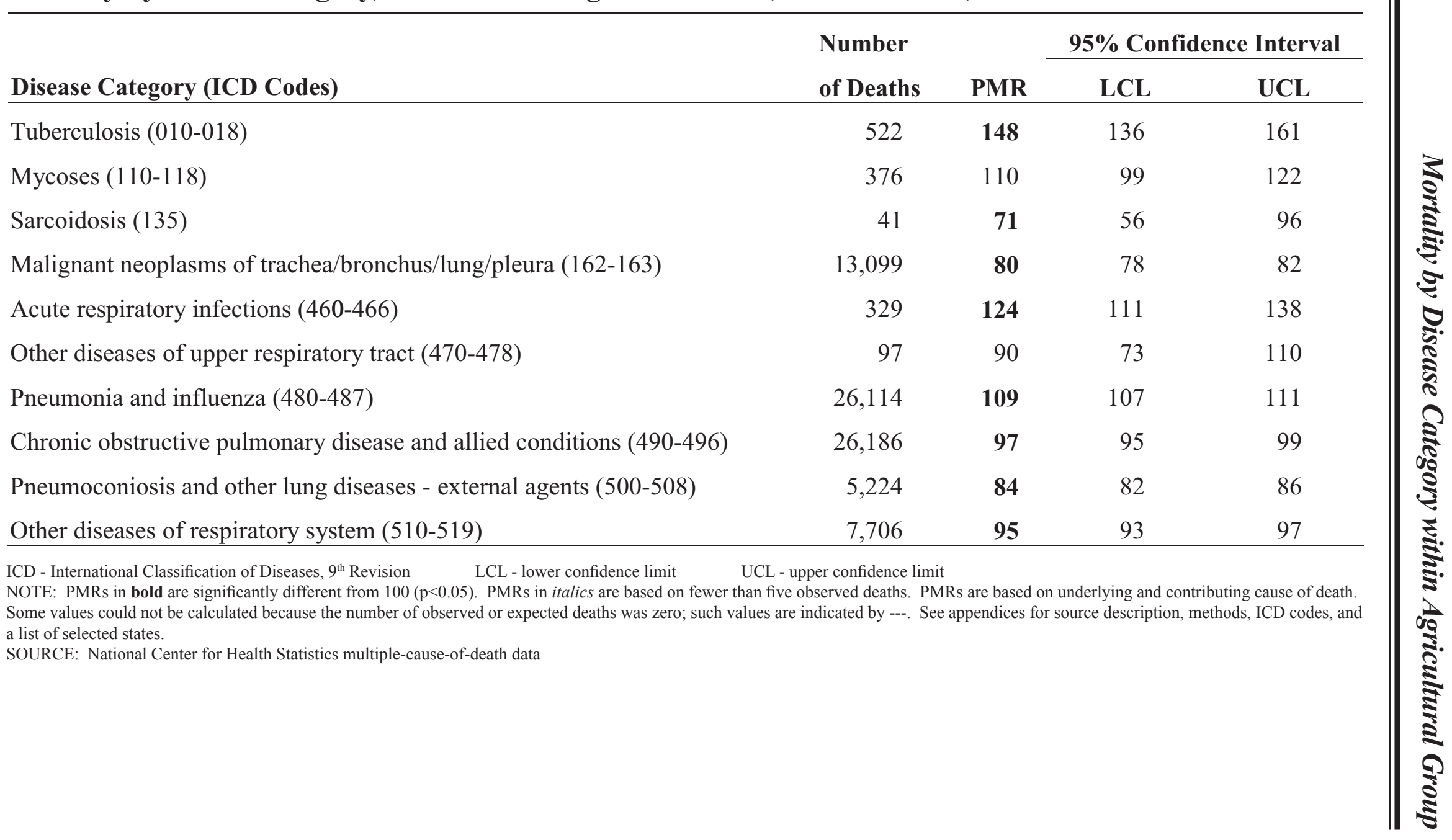


Table 2-2. Livestock farm workers: Proportionate mortality ratio (PMR) adjusted for age, sex, and race/ ethnicity by disease category, U.S. residents age 15 and over, selected states, 1988-1998

\begin{tabular}{|c|c|c|c|c|}
\hline \multirow[b]{2}{*}{ Disease Category (ICD Codes) } & \multirow{2}{*}{$\begin{array}{l}\text { Number } \\
\text { of Deaths }\end{array}$} & \multirow[b]{2}{*}{ PMR } & \multicolumn{2}{|c|}{ 95\% Confidence Interval } \\
\hline & & & LCL & $\mathbf{U C L}$ \\
\hline Tuberculosis (010-018) & 56 & 75 & 87 & 97 \\
\hline Mycoses (110-118) & 79 & 94 & 75 & 117 \\
\hline Sarcoidosis (135) & 9 & 103 & 47 & 195 \\
\hline Malignant neoplasms of trachea/bronchus/lung/pleura (162-163) & 2,960 & 68 & 66 & 70 \\
\hline Acute respiratory infections $(460-466)$ & 59 & 80 & 61 & 103 \\
\hline Other diseases of upper respiratory tract $(470-478)$ & 20 & 72 & 44 & 111 \\
\hline Pneumonia and influenza (480-487) & 6,391 & 99 & 97 & 101 \\
\hline Chronic obstructive pulmonary disease and allied conditions (490-496) & 6,956 & 93 & 91 & 95 \\
\hline Pneumoconiosis and other lung diseases - external agents (500-508) & 1,381 & 83 & 79 & 87 \\
\hline Other diseases of respiratory system (510-519) & 1,917 & 88 & 84 & 92 \\
\hline
\end{tabular}

ICD - International Classification of Diseases, $9^{\text {th }}$ Revision $\quad$ LCL - lower confidence limit

UCL - upper confidence limit

NOTE: PMRs in bold are significantly different from $100(\mathrm{p}<0.05)$. PMRs in italics are based on fewer than five observed deaths. PMRs are based on underlying and contributing cause of death. Some values could not be calculated because the number of observed or expected deaths was zero; such values are indicated by ---. See appendices for source description, methods. ICD codes, and a list of selected states.

SOURCE: National Center for Health Statistics multiple-cause-of-death data 
Table 2-3. Farm managers: Proportionate mortality ratio (PMR) adjusted for age, sex, and race/ ethnicity by disease category, U.S. residents age 15 and over, selected states, 1988-1998

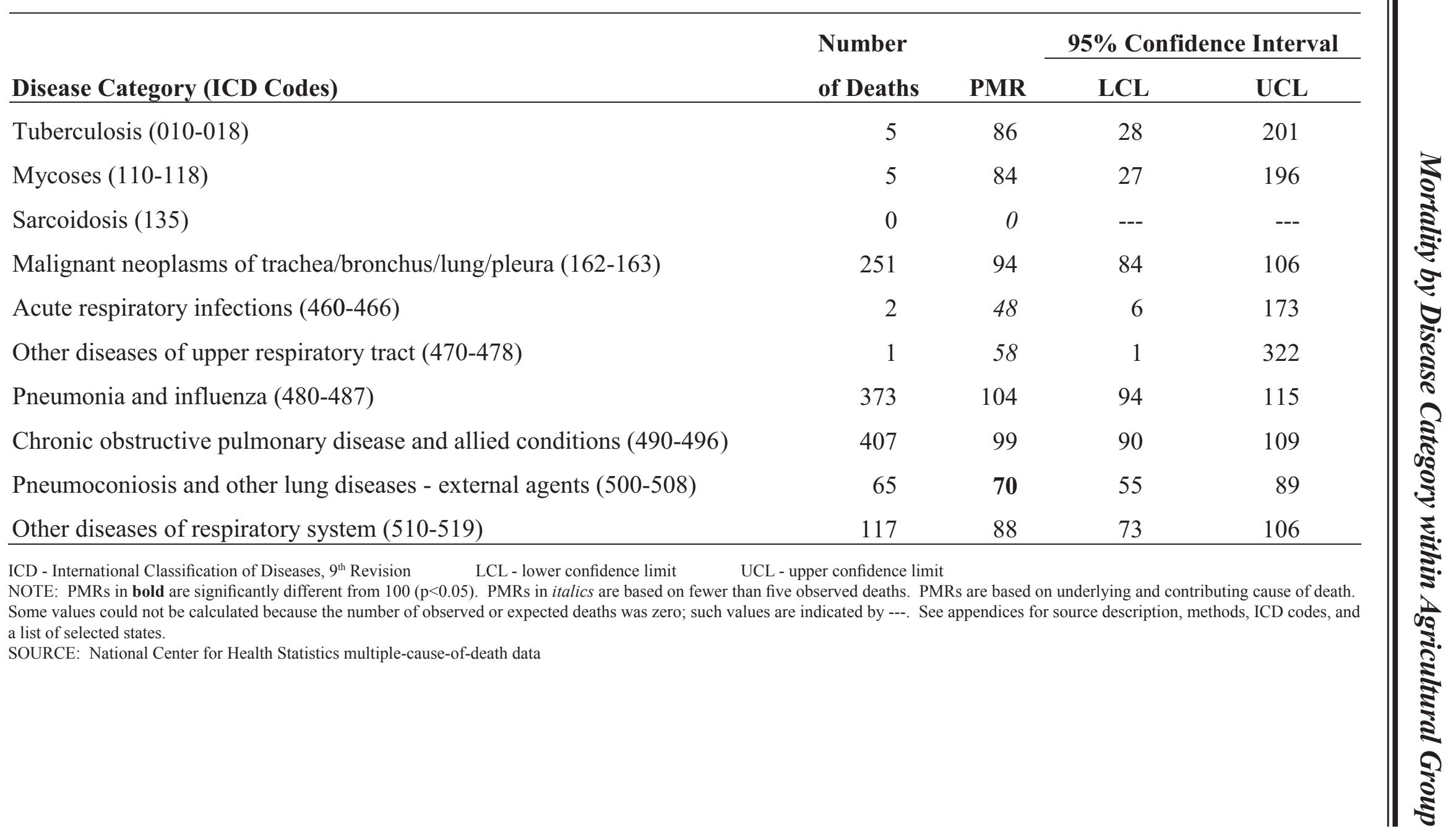


Table 2-4. Landscape and horticultural workers: Proportionate mortality ratio (PMR) adjusted for age, sex, and race/ethnicity by disease category, U.S. residents age 15 and over, selected states, 1988-1998

\begin{tabular}{|c|c|c|c|c|}
\hline \multirow[b]{2}{*}{ Disease Category (ICD Codes) } & \multirow{2}{*}{$\begin{array}{l}\text { Number } \\
\text { of Deaths }\end{array}$} & \multirow[b]{2}{*}{ PMR } & \multicolumn{2}{|c|}{ 95\% Confidence Interval } \\
\hline & & & $\mathbf{L C L}$ & $\mathbf{U C L}$ \\
\hline Tuberculosis $(010-018)$ & 16 & 80 & 46 & 130 \\
\hline Mycoses $(110-118)$ & 27 & 106 & 70 & 154 \\
\hline Sarcoidosis (135) & 2 & 34 & 4 & 123 \\
\hline Malignant neoplasms of trachea/bronchus/lung/pleura (162-163) & 647 & 97 & 90 & 105 \\
\hline Acute respiratory infections (460-466) & 9 & 108 & 50 & 205 \\
\hline Other diseases of upper respiratory tract (470-478) & 4 & 76 & 21 & 194 \\
\hline Pneumonia and influenza (480-487) & 607 & 94 & 87 & 102 \\
\hline Chronic obstructive pulmonary disease and allied conditions (490-496) & 799 & 109 & 102 & 117 \\
\hline Pneumoconiosis and other lung diseases - external agents (500-508) & 154 & 98 & 83 & 115 \\
\hline Other diseases of respiratory system (510-519) & 252 & 84 & 75 & 95 \\
\hline
\end{tabular}

ICD - International Classification of Diseases, $9^{\text {th }}$ Revision $\quad$ LCL - lower confidence limit $\quad$ UCL - upper confidence limit

NOTE: PMRs in bold are significantly different from $100(\mathrm{p}<0.05)$. PMRs in italics are based on fewer than five observed deaths. PMRs are based on underlying and contributing cause of death. Some values could not be calculated because the number of observed or expected deaths was zero; such values are indicated by ---. See appendices for source description, methods, ICD codes, and a list of selected states.

SOURCE: National Center for Health Statistics multiple-cause-of-death data 
Table 2-5. Forestry workers: Proportionate mortality ratio (PMR) adjusted for age, sex, and race/ ethnicity by disease category, U.S. residents age 15 and over, selected states, 1988-1998

\begin{tabular}{|c|c|c|c|c|}
\hline \multirow[b]{2}{*}{ Disease Category (ICD Codes) } & \multirow{2}{*}{$\begin{array}{l}\text { Number } \\
\text { of Deaths }\end{array}$} & \multirow[b]{2}{*}{ PMR } & \multicolumn{2}{|c|}{ 95\% Confidence Interval } \\
\hline & & & $\mathbf{L C L}$ & $\mathbf{U C L}$ \\
\hline Tuberculosis $(010-018)$ & 45 & 129 & 94 & 173 \\
\hline Mycoses (110-118) & 23 & 60 & 38 & 90 \\
\hline Sarcoidosis (135) & 4 & 49 & 13 & 125 \\
\hline Malignant neoplasms of trachea/bronchus/lung/pleura (162-163) & 1,553 & 102 & 97 & 107 \\
\hline Acute respiratory infections (460-466) & 15 & 83 & 46 & 137 \\
\hline Other diseases of upper respiratory tract (470-478) & 8 & 86 & 37 & 169 \\
\hline Pneumonia and influenza (480-487) & 1,771 & 116 & 111 & 122 \\
\hline Chronic obstructive pulmonary disease and allied conditions (490-496) & 2,318 & 122 & 117 & 127 \\
\hline Pneumoconiosis and other lung diseases - external agents (500-508) & 354 & 90 & 81 & 100 \\
\hline Other diseases of respiratory system (510-519) & 549 & 88 & 81 & 96 \\
\hline
\end{tabular}

ICD - International Classification of Diseases, $9^{\text {th }}$ Revision $\quad$ LCL - lower confidence limit $\quad$ UCL - upper confidence limit

NOTE: PMRs in bold are significantly different from $100(\mathrm{p}<0.05)$. PMRs in italics are based on fewer than five observed deaths. PMRs are based on underlying and contributing cause of death. Some values could not be calculated because the number of observed or expected deaths was zero; such values are indicated by ---. See appendices for source description, methods, ICD codes, and a list of selected states.

SOURCE: National Center for Health Statistics multiple-cause-of-death data 
Table 2-6. Fishery workers: Proportionate mortality ratio (PMR) adjusted for age, sex, and race/ ethnicity by disease category, U.S. residents age 15 and over, selected states, 1988-1998

\begin{tabular}{|c|c|c|c|c|}
\hline \multirow[b]{2}{*}{ Disease Category (ICD Codes) } & \multirow{2}{*}{$\begin{array}{l}\text { Number } \\
\text { of Deaths }\end{array}$} & \multirow[b]{2}{*}{ PMR } & \multicolumn{2}{|c|}{ 95\% Confidence Interval } \\
\hline & & & $\mathbf{L C L}$ & UCL \\
\hline Tuberculosis $(010-018)$ & 14 & 175 & 96 & 294 \\
\hline Mycoses (110-118) & 7 & 64 & 26 & 132 \\
\hline Sarcoidosis (135) & 4 & 256 & 70 & 655 \\
\hline Malignant neoplasms of trachea/bronchus/lung/pleura (162-163) & 426 & 108 & 98 & 119 \\
\hline Acute respiratory infections $(460-466)$ & 4 & 78 & 21 & 199 \\
\hline Other diseases of upper respiratory tract (470-478) & 5 & 193 & 62 & 451 \\
\hline Pneumonia and influenza (480-487) & 422 & 103 & 94 & 113 \\
\hline Chronic obstructive pulmonary disease and allied conditions (490-496) & 568 & 113 & 104 & 123 \\
\hline Pneumoconiosis and other lung diseases - external agents (500-508) & 98 & 94 & 76 & 115 \\
\hline Other diseases of respiratory system (510-519) & 150 & 88 & 75 & 103 \\
\hline
\end{tabular}

ICD - International Classification of Diseases, $9^{\text {th }}$ Revision $\quad$ LCL - lower confidence limit

UCL - upper confidence limit

NOTE: PMRs in bold are significantly different from $100(\mathrm{p}<0.05)$. PMRs in italics are based on fewer than five observed deaths. PMRs are based on underlying and contributing cause of death. Some values could not be calculated because the number of observed or expected deaths was zero; such values are indicated by ---. See appendices for source description, methods, ICD codes, and a list of selected states.

SOURCE: National Center for Health Statistics multiple-cause-of-death data 
Figure 2-1. Tuberculosis: Proportionate mortality ratio (PMR) adjusted for age, sex, and race/ethnicity by agricultural group, U.S. residents age 15 and over, selected states, 1988-1998

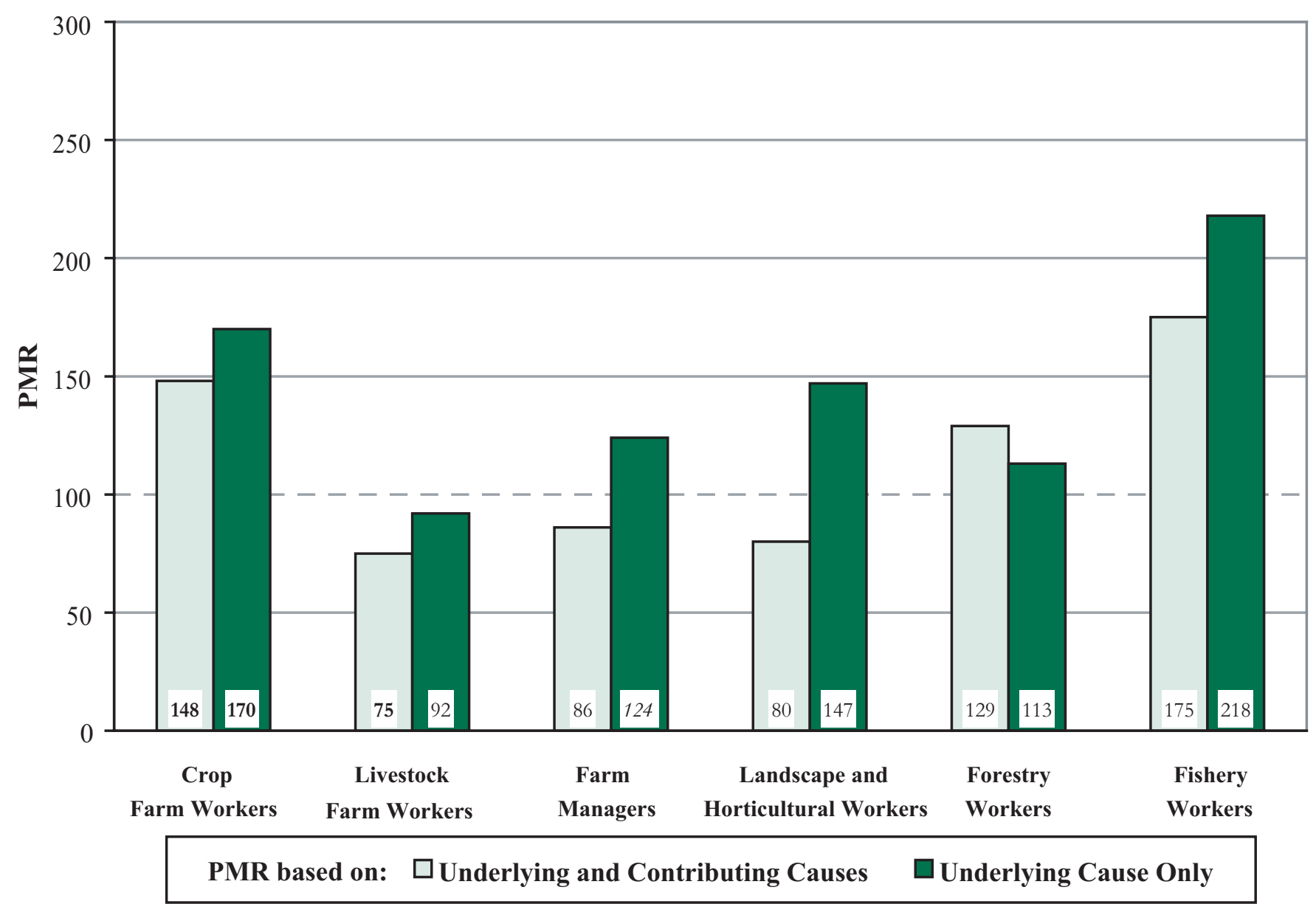

ICD - International Classification of Diseases, $9^{\text {th }}$ Revision

NOTE: Tuberculosis = ICD-9 codes 010-018. PMRs in bold are significantly different from $100(\mathrm{p}<0.05)$. PMRs in italics are based on fewer than five observed deaths. PMRs are based on underlying and contributing cause of death. See appendices for source description, methods, ICD codes, and a list of selected states.

SOURCE: National Center for Health Statistics multiple-cause-of-death data 
Figure 2-2. Mycoses: Proportionate mortality ratio (PMR) adjusted for age, sex, and race/ethnicity by agricultural group, U.S. residents age 15 and over, selected states, 1988-1998

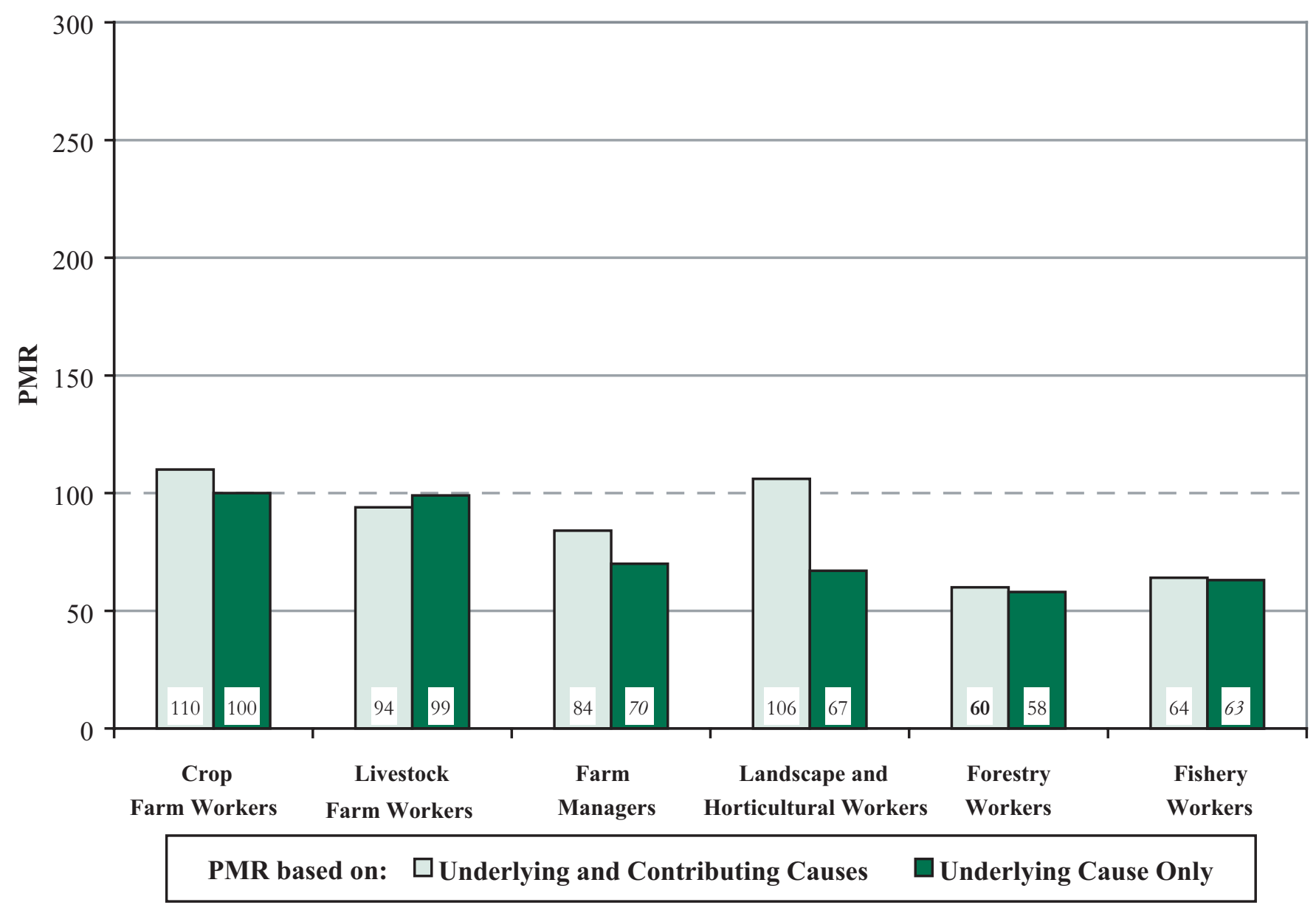

ICD - International Classification of Diseases, $9^{\text {th }}$ Revision

NOTE: Mycoses = ICD-9 codes 110-118. PMRs in bold are significantly different from $100(\mathrm{p}<0.05)$. PMRs in italics are based on fewer than five observed deaths. PMRs are based on underlying and contributing cause of death. See appendices for source description, methods, ICD codes, and a list of selected states.

SOURCE: National Center for Health Statistics multiple-cause-of-death data 
Figure 2-3. Sarcoidosis: Proportionate mortality ratio (PMR) adjusted for age, sex, and race/ethnicity by agricultural group, U.S. residents age 15 and over, selected states, 1988-1998

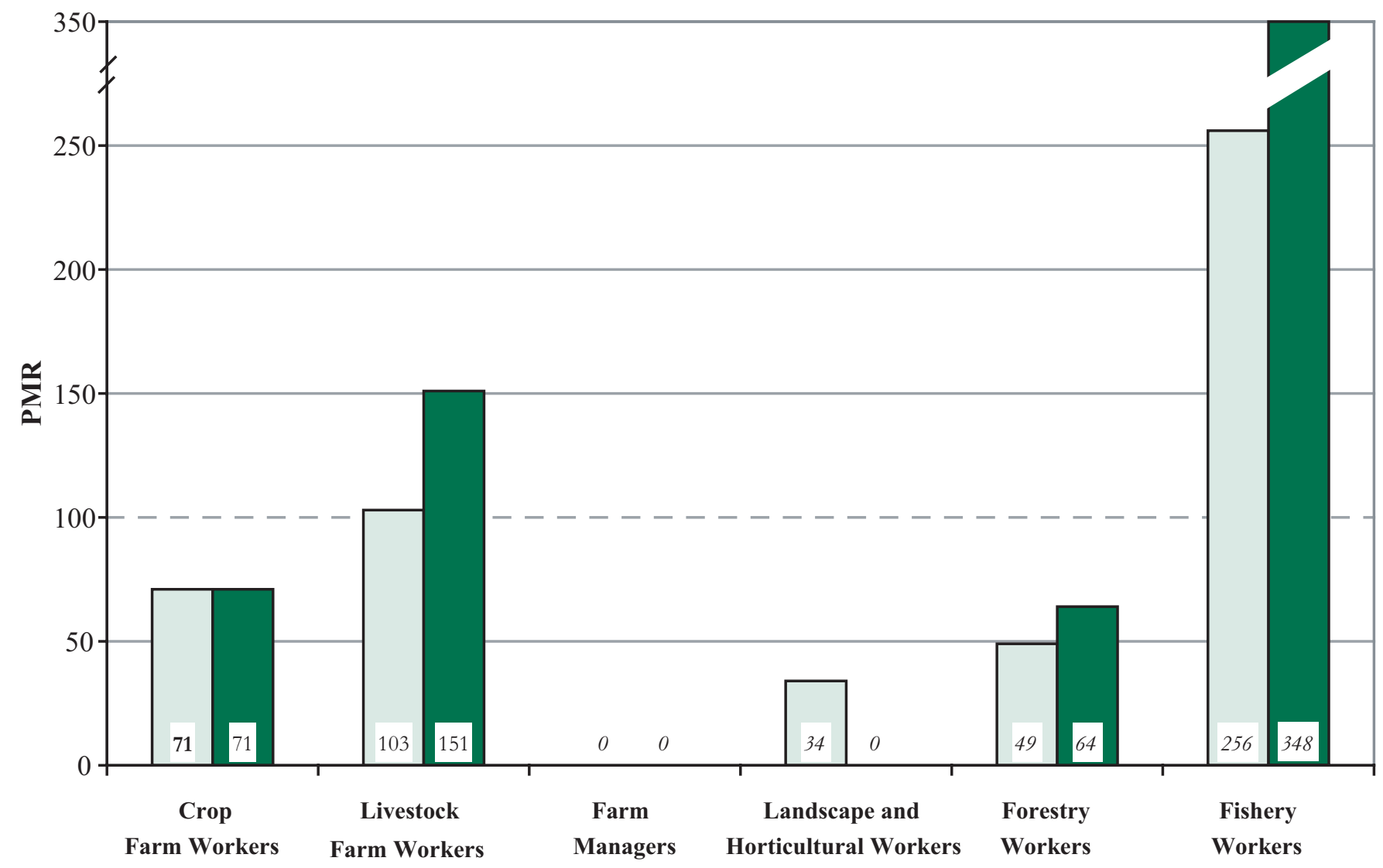

PMR based on: $\square$ Underlying and Contributing Causes $\quad \square$ Underlying Cause Only

ICD - International Classification of Diseases, $9^{\text {th }}$ Revision

NOTE: Sarcoidosis = ICD-9 code 135. PMRs in bold are significantly different from $100(\mathrm{p}<0.05)$. PMRs in italics are based on fewer than five observed deaths. PMRs are based on underlying and contributing cause of death. See appendices for source description, methods, ICD codes, and a list of selected states.

SOURCE: National Center for Health Statistics multiple-cause-of-death data 
Figure 2-4. Malignant neoplasms of trachea/bronchus/lung/pleura: Proportionate mortality ratio (PMR) adjusted for age, sex, and race/ethnicity by agricultural group, U.S. residents age 15 and over, selected states, 1988-1998

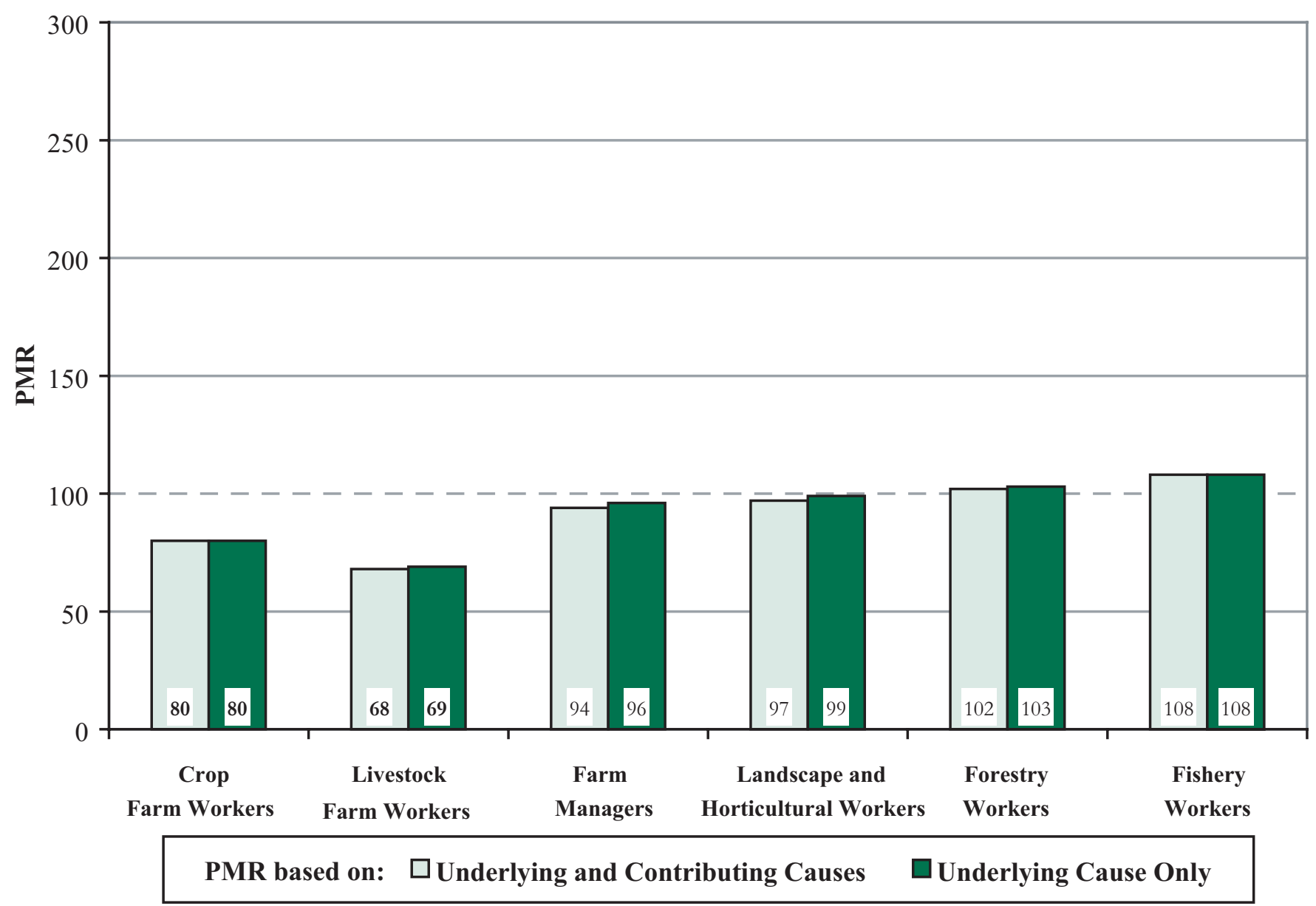

ICD - International Classification of Diseases, $9^{\text {th }}$ Revision

NOTE: Malignant neoplasms of trachea/bronchus/lung/pleura = ICD-9 codes 162-163. PMRs in bold are significantly different from 100 ( $<<0.05$ ). PMRs in italics are based on fewer than five observed deaths. PMRs are based on underlying and contributing cause of death. See appendices for source description, methods, ICD codes, and a list of selected states.

SOURCE: National Center for Health Statistics multiple-cause-of-death data 
Figure 2-5. Acute respiratory infections: Proportionate mortality ratio (PMR) adjusted for age, sex, and race/ethnicity by agricultural group, U.S. residents age 15 and over, selected states, 1988-1998

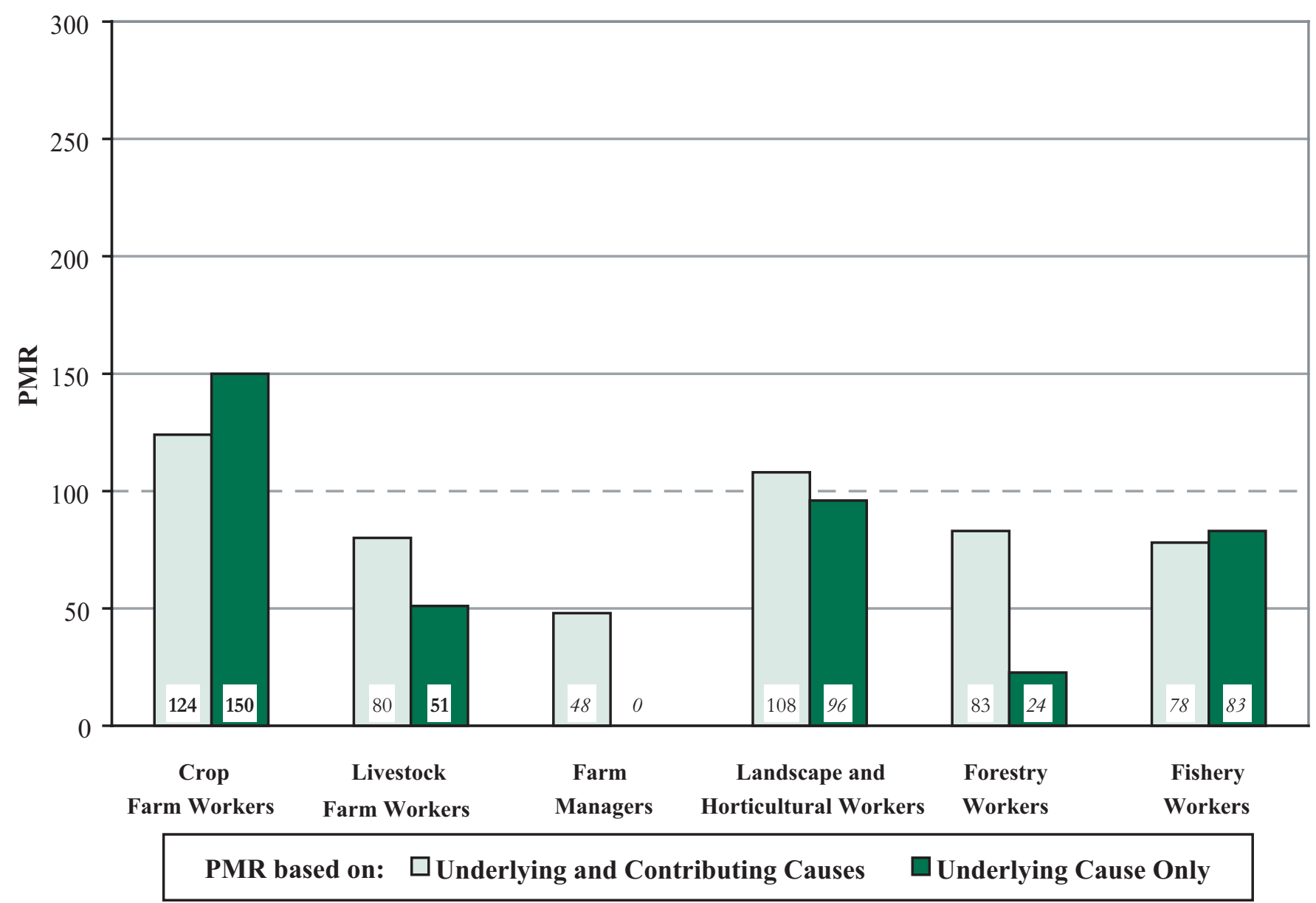

ICD - International Classification of Diseases, $9^{\text {th }}$ Revision

NOTE: Acute respiratory infections $=$ ICD-9 codes 460-466. PMRs in bold are significantly different from 100 ( $<<0.05)$. PMRs in italics are based on fewer than five observed deaths. PMRs are based on underlying and contributing cause of death. See appendices for source description, methods, ICD codes, and a list of selected states.

SOURCE: National Center for Health Statistics multiple-cause-of-death data 
Figure 2-6. Other diseases of upper respiratory tract: Proportionate mortality ratio (PMR) adjusted for age, sex, and race/ethnicity by agricultural group, U.S. residents age 15 and over, selected states, 1988-1998

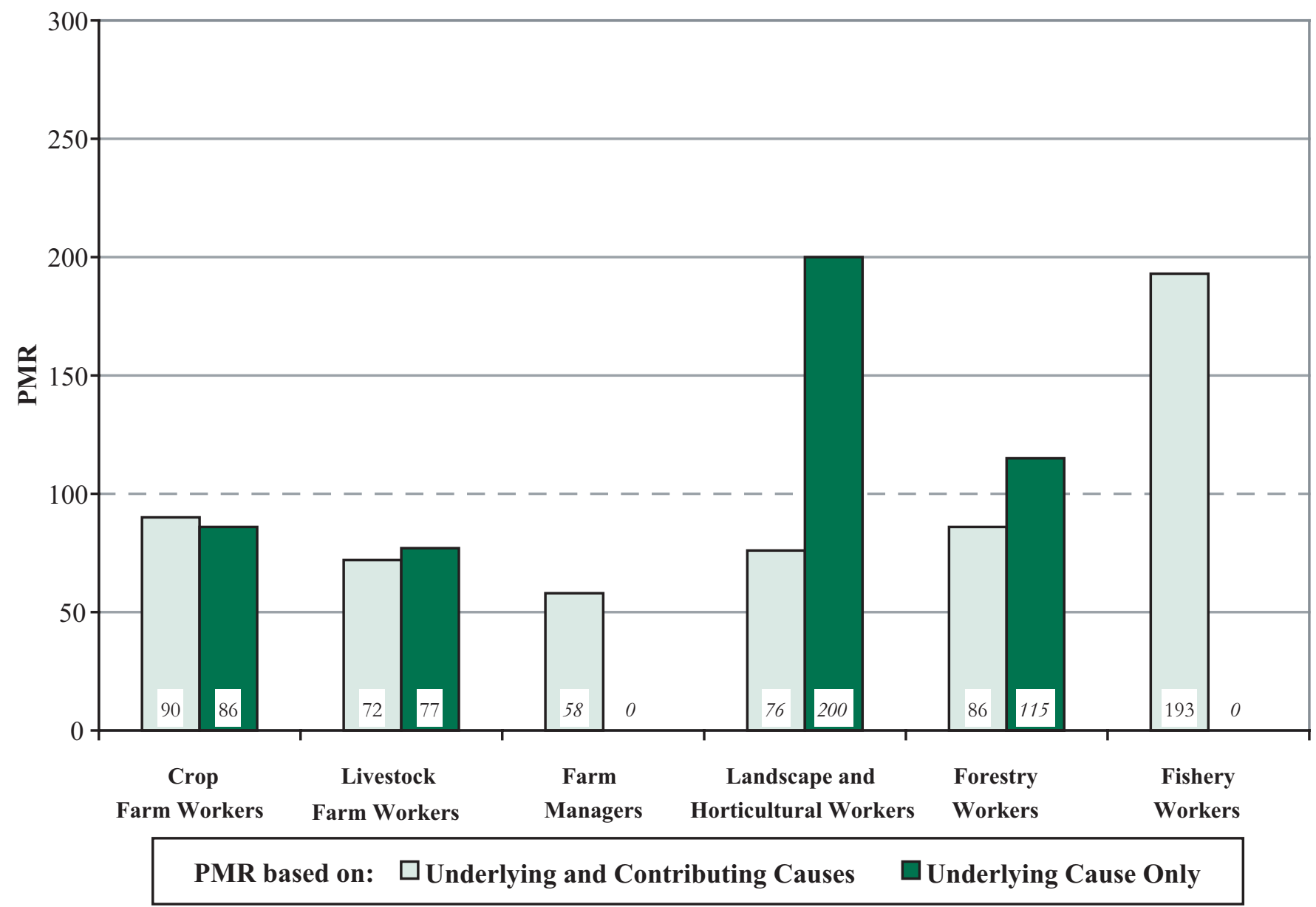

ICD - International Classification of Diseases, $9^{\text {th }}$ Revision

NOTE: Other diseases of upper respiratory tract $=$ ICD-9 codes 470-478. PMRs in bold are significantly different from 100 (p<0.05). PMRs in italics are based on fewer than five observed deaths. PMRs are based on underlying and contributing cause of death. See appendices for source description, methods, ICD codes, and a list of selected states.

SOURCE: National Center for Health Statistics multiple-cause-of-death data 
Figure 2-7. Pneumonia and influenza: Proportionate mortality ratio (PMR) adjusted for age, sex, and race/ethnicity by agricultural group, U.S. residents age 15 and over, selected states, 1988-1998

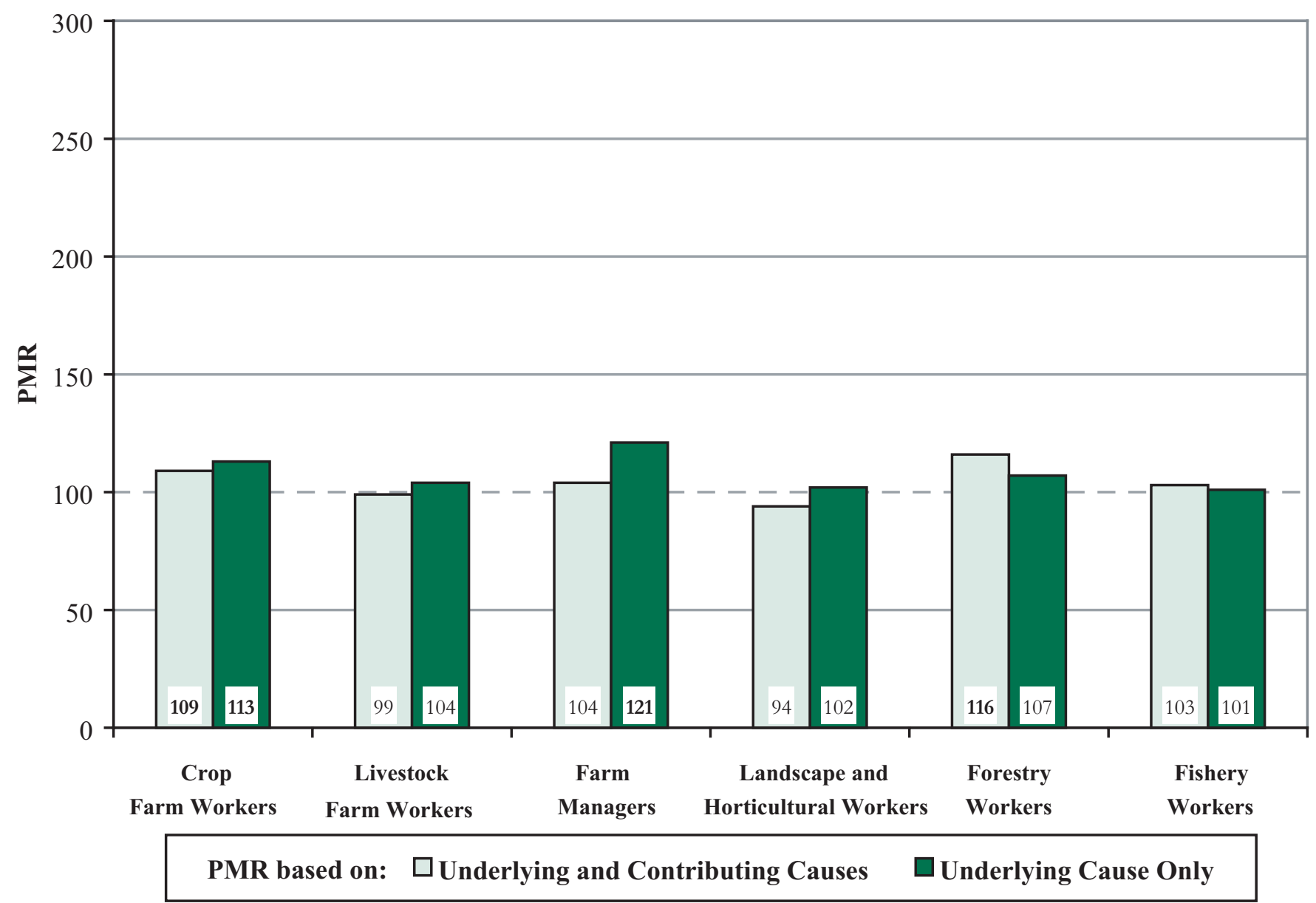

ICD - International Classification of Diseases, $9^{\text {th }}$ Revision

NOTE: Pneumonia and influenza = ICD-9 codes 480-487. PMRs in bold are significantly different from $100(\mathrm{p}<0.05)$. PMRs in italics are based on fewer than five observed deaths. PMRs are based on underlying and contributing cause of death. See appendices for source description, methods, ICD codes, and a list of selected states.

SOURCE: National Center for Health Statistics multiple-cause-of-death data 
Figure 2-8. Chronic obstructive pulmonary disease and allied conditions: Proportionate mortality ratio (PMR) adjusted for age, sex, and race/ethnicity by agricultural group, U.S. residents age 15 and over, selected states, 1988-1998

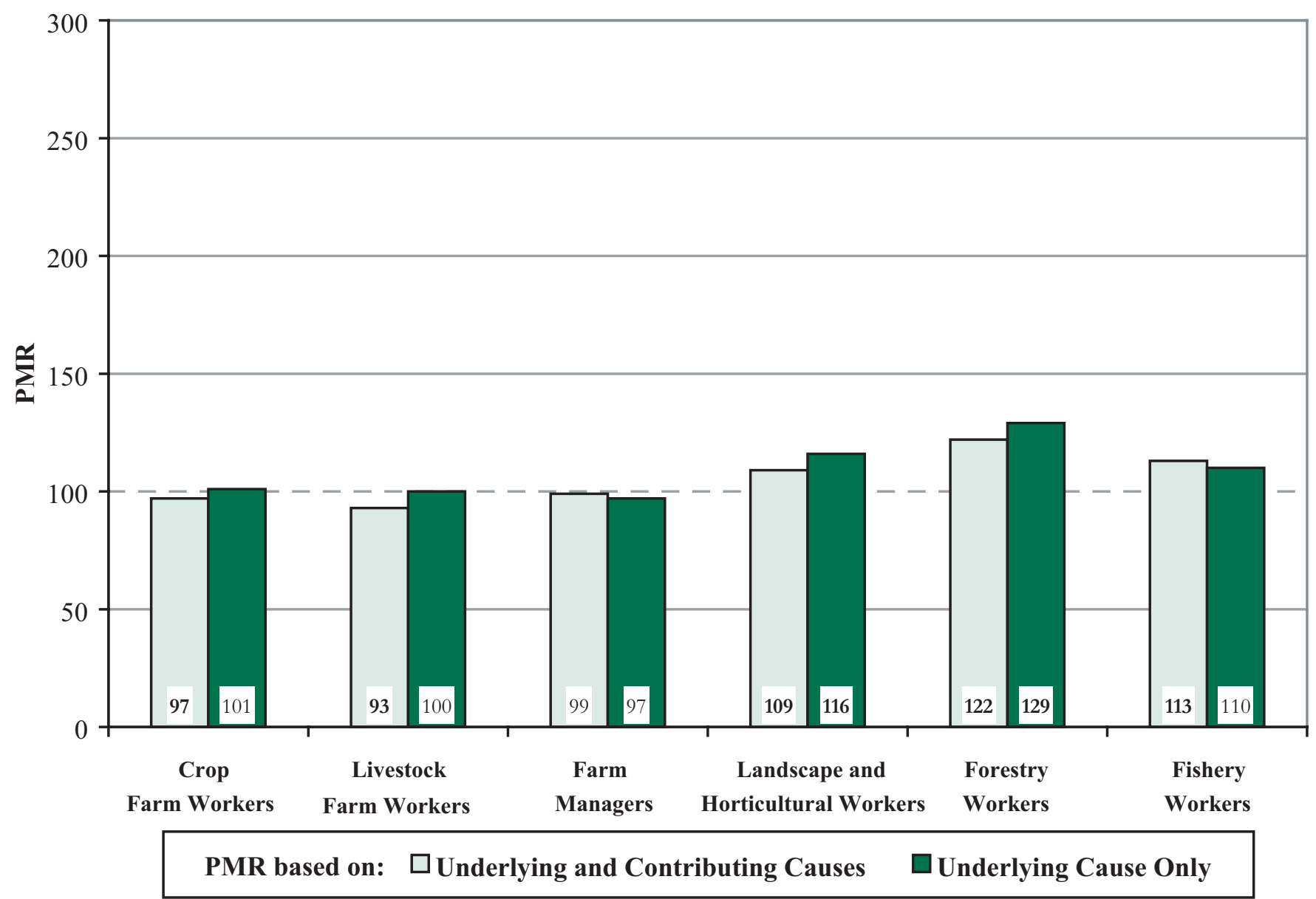

ICD - International Classification of Diseases, $9^{\text {th }}$ Revision

NOTE: Chronic obstructive pulmonary disease and allied conditions $=$ ICD-9 codes 490-496. PMRs in bold are significantly different from 100 ( $\mathrm{p}<0.05$ ). PMRs in italics are based on fewer than five observed deaths. PMRs are based on underlying and contributing cause of death. See appendices for source description, methods, ICD codes, and a list of selected states.

SOURCE: National Center for Health Statistics multiple-cause-of-death data 
Figure 2-9. Pneumoconioses and other lung diseases-external agents: Proportionate mortality ratio (PMR) adjusted for age, sex, and race/ethnicity by agricultural group, U.S. residents age 15 and over, selected states, 1988-1998

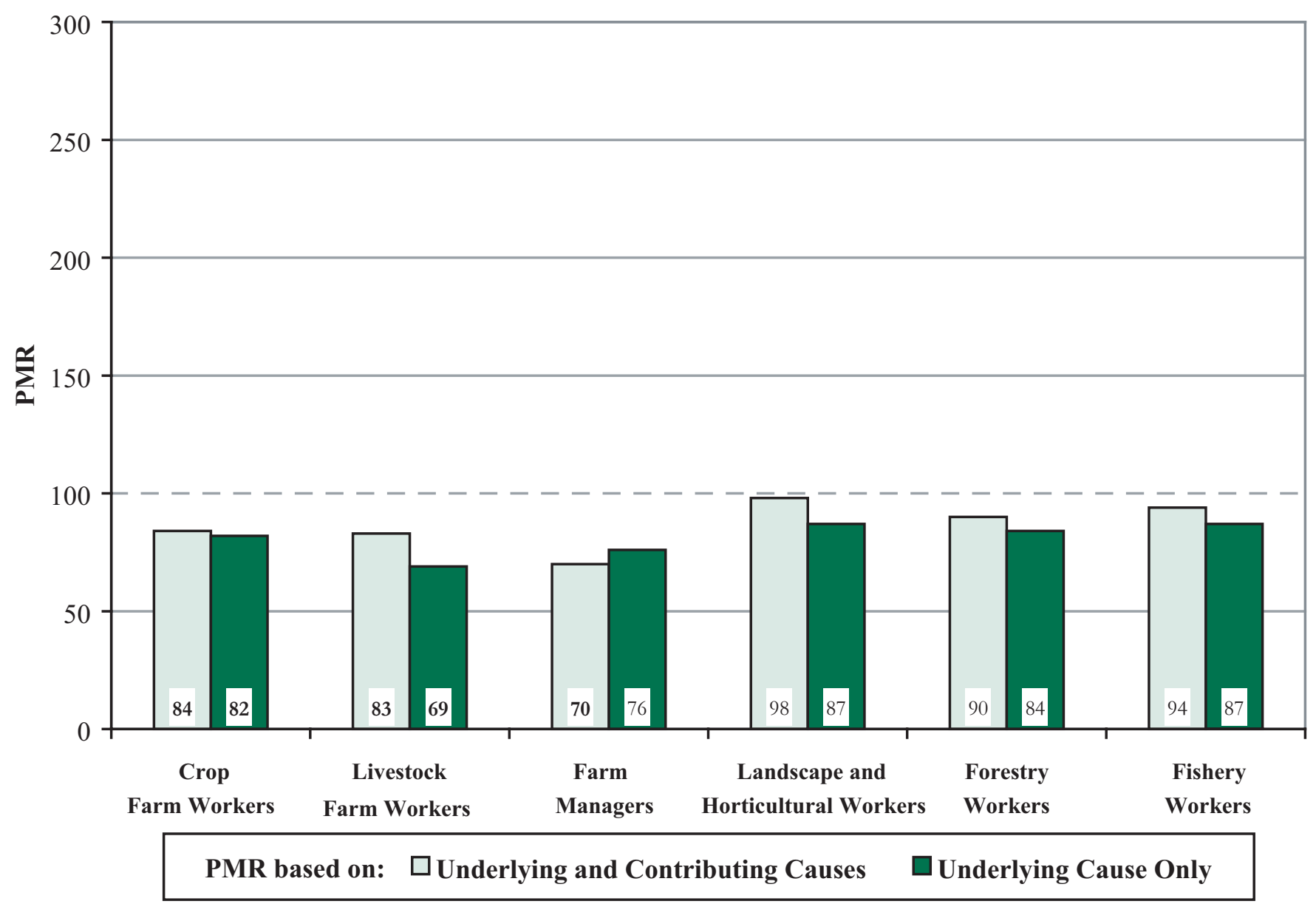

ICD - International Classification of Diseases, $9^{\text {th }}$ Revision

NOTE: Pneumoconioses and other lung diseases - external agents = ICD-9 codes 500-508. PMRs in bold are significantly different from 100 ( $p<0.05$ ). PMRs in italics are based on fewer than five observed deaths. PMRs are based on underlying and contributing cause of death. See appendices for source description, methods, ICD codes, and a list of selected states.

SOURCE: National Center for Health Statistics multiple-cause-of-death data 
Figure 2-10. Other diseases of respiratory system: Proportionate mortality ratio (PMR) adjusted for age, sex, and race/ethnicity by agricultural group, U.S. residents age 15 and over, selected states, 1988-1998

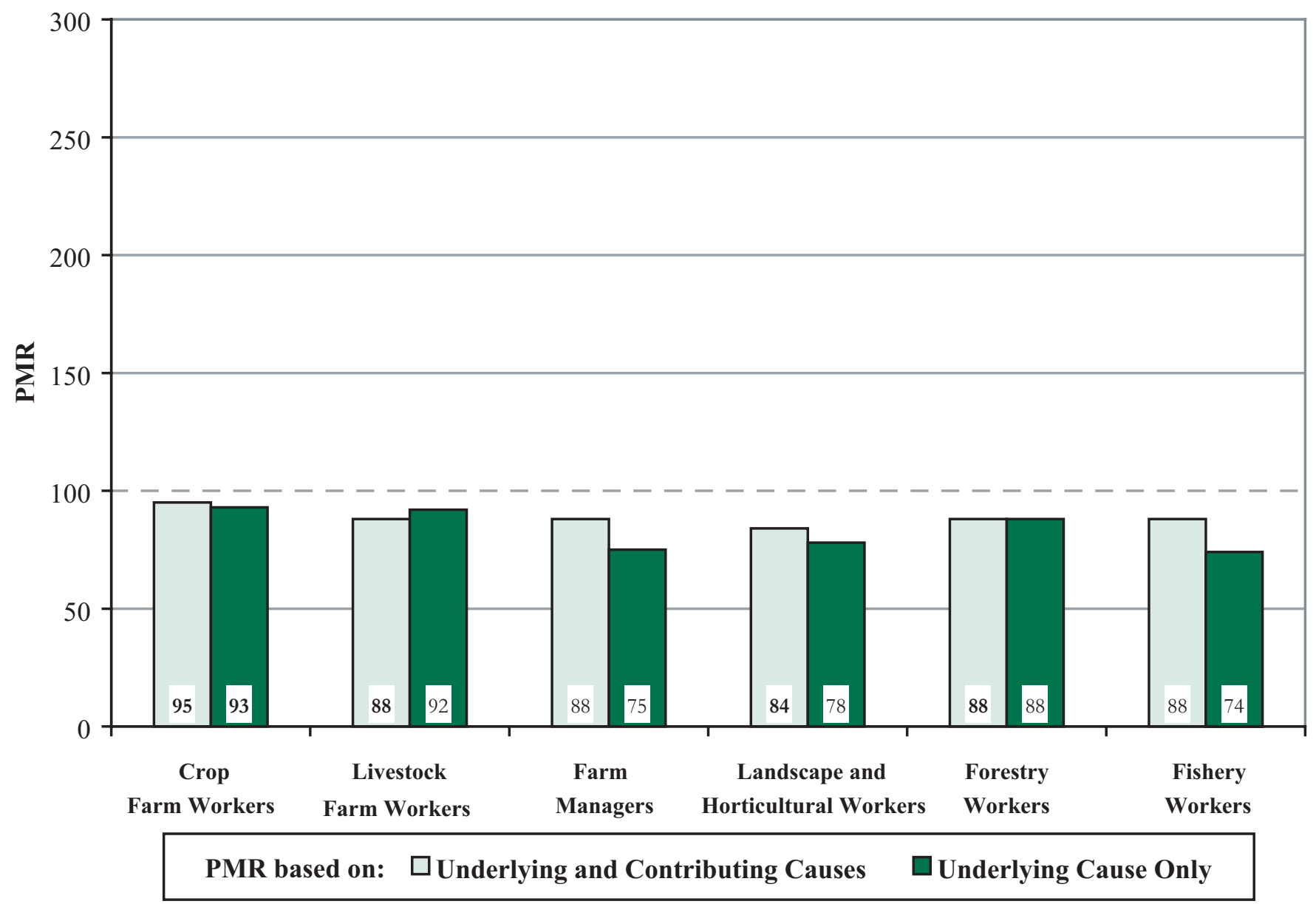

ICD - International Classification of Diseases, $9^{\text {th }}$ Revision

NOTE: Other diseases of respiratory system = ICD-9 codes 510-519. PMRs in bold are significantly different from 100 ( $<<0.05$ ). PMRs in italics are based on fewer than five observed deaths. PMRs are based on underlying and contributing cause of death. See appendices for source description, methods, ICD codes, and a list of selected states.

SOURCE: National Center for Health Statistics multiple-cause-of-death data 
Table 2-7. Crop farm workers, males: Proportionate mortality ratio (PMR) adjusted for age and race/ ethnicity by disease category, U.S. residents age 15 and over, selected states, 1988-1998

\begin{tabular}{|c|c|c|c|c|}
\hline Disease Category (ICD Codes) & $\begin{array}{l}\text { Number } \\
\text { of Deaths }\end{array}$ & PMR & \multicolumn{2}{|c|}{ 95\% Confidence Interval } \\
\hline Tuberculosis $(010-018)$ & 481 & 147 & 134 & 161 \\
\hline Mycoses (110-018) & 361 & 112 & 101 & 124 \\
\hline Sarcoidosis (135) & 34 & 76 & 52 & 106 \\
\hline Other diseases of upper respiratory tract (470-478) & 93 & 92 & 75 & 113 \\
\hline Pneumonia and influenza (480-487) & 24,848 & 110 & 108 & 112 \\
\hline Chronic obstructive pulmonary disease and allied conditions (490-496) & 25,521 & 97 & 95 & 99 \\
\hline
\end{tabular}


Table 2-8. Livestock farm workers, males: Proportionate mortality ratio (PMR) adjusted for age and race/ethnicity by disease category, U.S. residents age 15 and over, selected states, 1988-1998

\begin{tabular}{|c|c|c|c|c|}
\hline \multirow[b]{2}{*}{ Disease Category (ICD Codes) } & \multirow{2}{*}{$\begin{array}{l}\text { Number } \\
\text { of Deaths }\end{array}$} & \multirow[b]{2}{*}{ PMR } & \multicolumn{2}{|c|}{ 95\% Confidence Interval } \\
\hline & & & LCL & UCL \\
\hline Tuberculosis $(010-018)$ & 51 & 72 & 55 & 95 \\
\hline Mycoses (110-018) & 73 & 92 & 73 & 116 \\
\hline Sarcoidosis (135) & 9 & 119 & 55 & 226 \\
\hline Malignant neoplasms of trachea/bronchus/lung/pleura (162-163) & 2,835 & 68 & 66 & 71 \\
\hline Acute respiratory infections (460-466) & 55 & 80 & 61 & 104 \\
\hline Other diseases of upper respiratory tract (470-478) & 17 & 65 & 38 & 104 \\
\hline Pneumonia and influenza (480-487) & 6,060 & 99 & 97 & 102 \\
\hline Chronic obstructive pulmonary disease and allied conditions (490-496) & 6,712 & 93 & 91 & 95 \\
\hline Pneumoconiosis and other lung diseases - external agents (500-508) & 1,331 & 83 & 79 & 88 \\
\hline Other diseases of respiratory system (510-519) & 1,773 & 87 & 83 & 91 \\
\hline
\end{tabular}

ICD - International Classification of Diseases, $9^{\text {th }}$ Revision $\quad$ LCL - lower confidence limit $\quad$ UCL - upper confidence limit
NOTE: PMRs in bold are significantly different from $100(p<0.05)$. PMRs in italics are based on fewer than five observed deaths. PMRs are based on underlying and contributing cause of death. Some values could not be calculated because the number of observed or expected deaths was zero; such values are indicated by ---. See appendices for source description, methods, ICD codes, and a list of selected states.

SOURCE: National Center for Health Statistics multiple-cause-of-death data 
Table 2-9. Farm managers, males: Proportionate mortality ratio (PMR) adjusted for age and race/ ethnicity by disease category, U.S. residents age 15 and over, selected states, 1988-1998

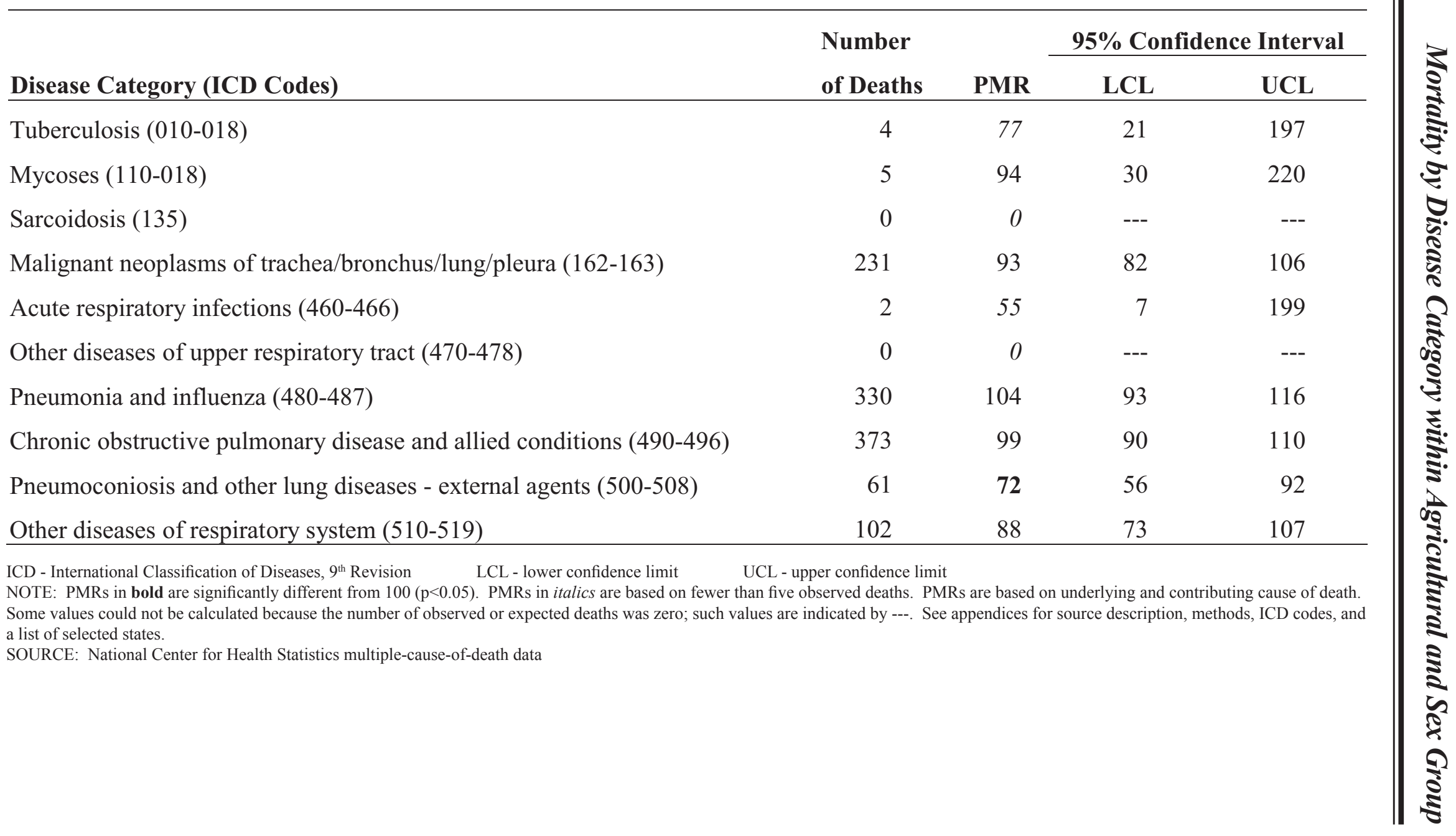


Table 2-10. Landscape and horticultural workers, males: Proportionate mortality ratio (PMR) adjusted for age and race/ethnicity by disease category, U.S. residents age 15 and over, selected states, 1988-1998

\begin{tabular}{lrrrr} 
& Number & & \multicolumn{2}{c}{$\mathbf{9 5 \%}$ Confidence Interval } \\
\cline { 3 - 5 } Disease Category (ICD Codes) & of Deaths & PMR & LCL & UCL \\
\hline Tuberculosis (010-018) & 16 & 84 & 48 & 136 \\
Mycoses (110-018) & 26 & 108 & 71 & 158 \\
Sarcoidosis (135) & 1 & 18 & 0 & 100 \\
Malignant neoplasms of trachea/bronchus/lung/pleura (162-163) & 615 & 98 & 91 & 106 \\
Acute respiratory infections (460-466) & 7 & 92 & 37 & 190 \\
Other diseases of upper respiratory tract (470-478) & 4 & 80 & 22 & 205 \\
Pneumonia and influenza (480-487) & 563 & 94 & 87 & 102 \\
Chronic obstructive pulmonary disease and allied conditions (490-496) & 741 & $\mathbf{1 0 8}$ & 101 \\
Pneumoconiosis and other lung diseases - external agents (500-508) & 149 & 100 & 85 & 116 \\
Other diseases of respiratory system (510-519) & 228 & $\mathbf{8 2}$ & 72 \\
\hline
\end{tabular}

ICD - International Classification of Diseases, $9^{\text {th }}$ Revision $\quad$ LCL - lower confidence limit $\quad$ UCL - upper confidence limit
NOTE: PMRs in bold are significantly different from $100(p<0.05)$. PMRs in italics are based on fewer than five observed deaths. PMRs are based on underlying and contributing cause of death. NOTE: PMRs in bold are significantly different from $100(\mathrm{p}<0.05)$. PMRs in italics are based on fewer than five observed deaths. PMRs are based on underlying and contributing cause of death.
Some values could not be calculated because the number of observed or expected deaths was zero; such values are indicated by ---. See appendices for source description, methods, ICD codes, and a list of selected states.

SOURCE: National Center for Health Statistics multiple-cause-of-death data 
Table 2-11. Forestry workers, males: Proportionate mortality ratio (PMR) adjusted for age and race/ ethnicity by disease category, U.S. residents age 15 and over, selected states, 1988-1998

\begin{tabular}{|c|c|c|c|c|}
\hline Disease Category (ICD Codes) & $\begin{array}{l}\text { Number } \\
\text { of Deaths }\end{array}$ & PMR & \multicolumn{2}{|c|}{ 95\% Confidence Interval } \\
\hline Tuberculosis (010-018) & 44 & 127 & 93 & 171 \\
\hline Mycoses (110-018) & 23 & 60 & 38 & 90 \\
\hline Sarcoidosis (135) & 4 & 50 & 14 & 128 \\
\hline Acute respiratory infections (460-466) & 15 & 84 & 47 & 139 \\
\hline Other diseases of upper respiratory tract (470-478) & 8 & 87 & 38 & 171 \\
\hline Pneumonia and influenza (480-487) & 1,758 & 116 & 111 & 122 \\
\hline Chronic obstructive pulmonary disease and allied conditions (490-496) & 2,308 & 123 & 118 & 128 \\
\hline
\end{tabular}


Table 2-12. Fishery workers, males: Proportionate mortality ratio (PMR) adjusted for age and race/ ethnicity by disease category, U.S. residents age 15 and over, selected states, 1988-1998

\begin{tabular}{|c|c|c|c|c|}
\hline \multirow[b]{2}{*}{ Disease Category (ICD Codes) } & \multirow{2}{*}{$\begin{array}{l}\text { Number } \\
\text { of Deaths }\end{array}$} & \multirow[b]{2}{*}{ PMR } & \multicolumn{2}{|c|}{ 95\% Confidence Interval } \\
\hline & & & $\mathbf{L C L}$ & UCL \\
\hline Tuberculosis (010-018) & 14 & 178 & 97 & 299 \\
\hline Mycoses (110-018) & 7 & 65 & 26 & 134 \\
\hline Sarcoidosis (135) & 4 & 265 & 72 & 678 \\
\hline Malignant neoplasms of trachea/bronchus/lung/pleura (162-163) & 415 & 107 & 97 & 118 \\
\hline Acute respiratory infections (460-466) & 4 & 80 & 22 & 205 \\
\hline Other diseases of upper respiratory tract (470-478) & 5 & 197 & 64 & 460 \\
\hline Pneumonia and influenza (480-487) & 416 & 104 & 95 & 114 \\
\hline Chronic obstructive pulmonary disease and allied conditions (490-496) & 556 & 112 & 103 & 122 \\
\hline Pneumoconiosis and other lung diseases - external agents (500-508) & 97 & 95 & 77 & 116 \\
\hline Other diseases of respiratory system $(510-519)$ & 148 & 89 & 76 & 105 \\
\hline
\end{tabular}

ICD - International Classification of Diseases, $9^{\text {th }}$ Revision $\quad$ LCL - lower confidence limit $\quad$ UCL - upper confidence limit

NOTE: PMRs in bold are significantly different from $100(\mathrm{p}<0.05)$. PMRs in italics are based on fewer than five observed deaths. PMRs are based on underlying and contributing cause of death. Some values could not be calculated because the number of observed or expected deaths was zero; such values are indicated by ---. See appendices for source description, methods, ICD codes, and a list of selected states.

SOURCE: National Center for Health Statistics multiple-cause-of-death data 
Table 2-13. Crop farm workers, females: Proportionate mortality ratio (PMR) adjusted for age and race/ethnicity by disease category, U.S. residents age 15 and over, selected states, 1988-1998

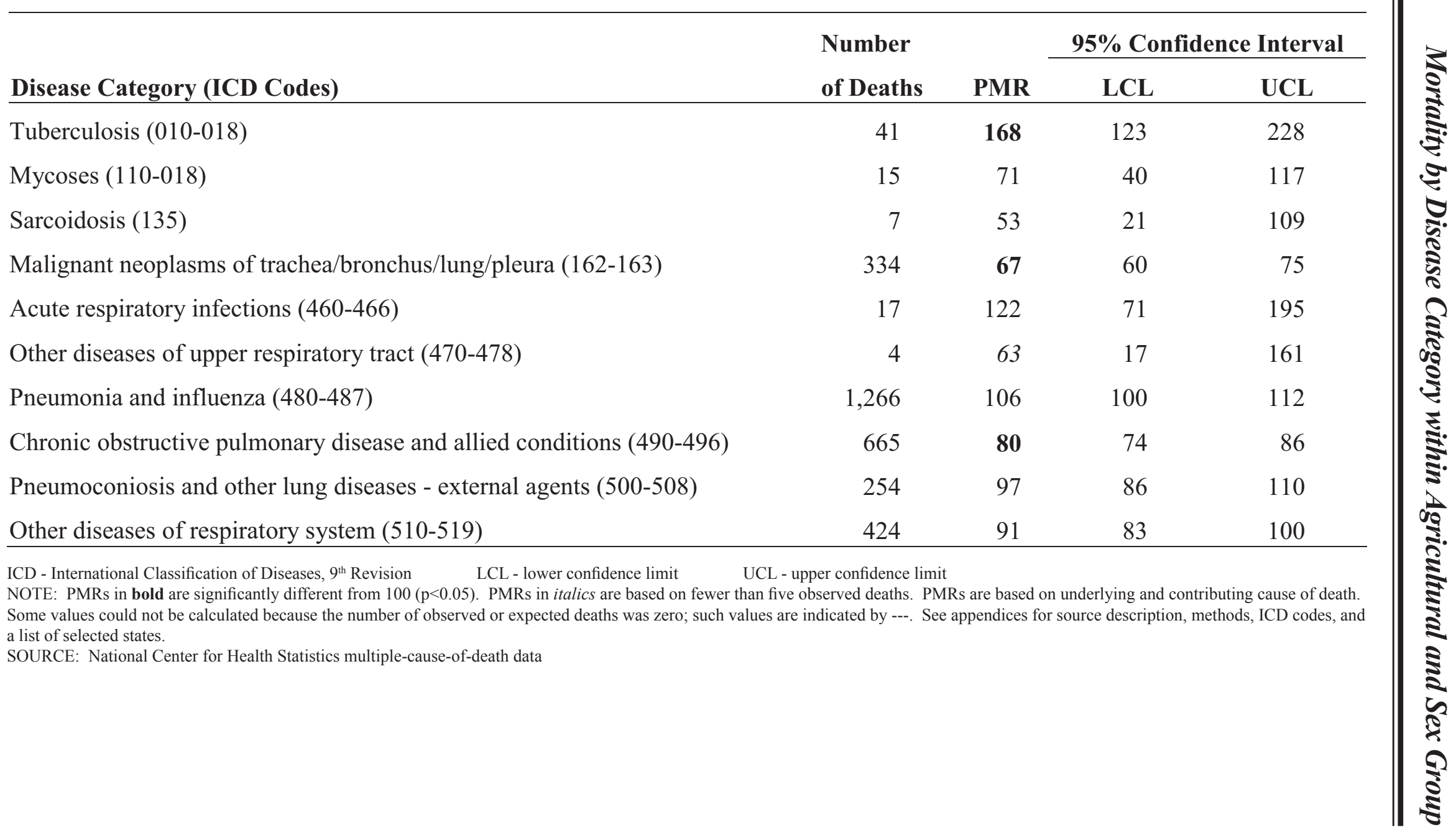


Table 2-14. Livestock farm workers, females: Proportionate mortality ratio (PMR) adjusted for age and race/ethnicity by disease category, U.S. residents age 15 and over, selected states, 1988-1998

\begin{tabular}{|c|c|c|c|c|}
\hline \multirow[b]{2}{*}{ Disease Category (ICD Codes) } & \multirow{2}{*}{$\begin{array}{l}\text { Number } \\
\text { of Deaths }\end{array}$} & \multirow[b]{2}{*}{ PMR } & \multicolumn{2}{|c|}{ 95\% Confidence Interval } \\
\hline & & & $\mathbf{L C L}$ & $\mathbf{U C L}$ \\
\hline Tuberculosis $(010-018)$ & 5 & 133 & 43 & 311 \\
\hline Mycoses (110-018) & 6 & 129 & 47 & 281 \\
\hline Sarcoidosis (135) & 0 & 0 & --- & --- \\
\hline Malignant neoplasms of trachea/bronchus/lung/pleura (162-163) & 125 & 78 & 66 & 93 \\
\hline Acute respiratory infections (460-466) & 4 & 79 & 22 & 202 \\
\hline Other diseases of upper respiratory tract $(470-478)$ & 3 & 191 & 39 & 558 \\
\hline Pneumonia and influenza (480-487) & 331 & 91 & 82 & 101 \\
\hline Chronic obstructive pulmonary disease and allied conditions (490-496) & 244 & 81 & 71 & 92 \\
\hline Pneumoconiosis and other lung diseases - external agents (500-508) & 50 & 71 & 53 & 94 \\
\hline Other diseases of respiratory system (510-519) & 144 & 108 & 92 & 127 \\
\hline
\end{tabular}

ICD - International Classification of Diseases, $9^{\text {th }}$ Revision $\quad$ LCL - lower confidence limit $\quad$ UCL - upper confidence limit

NOTE: PMRs in bold are significantly different from $100(\mathrm{p}<0.05)$. PMRs in italics are based on fewer than five observed deaths. PMRs are based on underlying and contributing cause of death.

Some values could not be calculated because the number of observed or expected deaths was zero; such values are indicated by ---. See appendices for source description, methods, ICD codes, and a list of selected states.

SOURCE: National Center for Health Statistics multiple-cause-of-death data 
Table 2-15. Farm managers, females: Proportionate mortality ratio (PMR) adjusted for age and race/ ethnicity by disease category, U.S. residents age 15 and over, selected states, 1988-1998

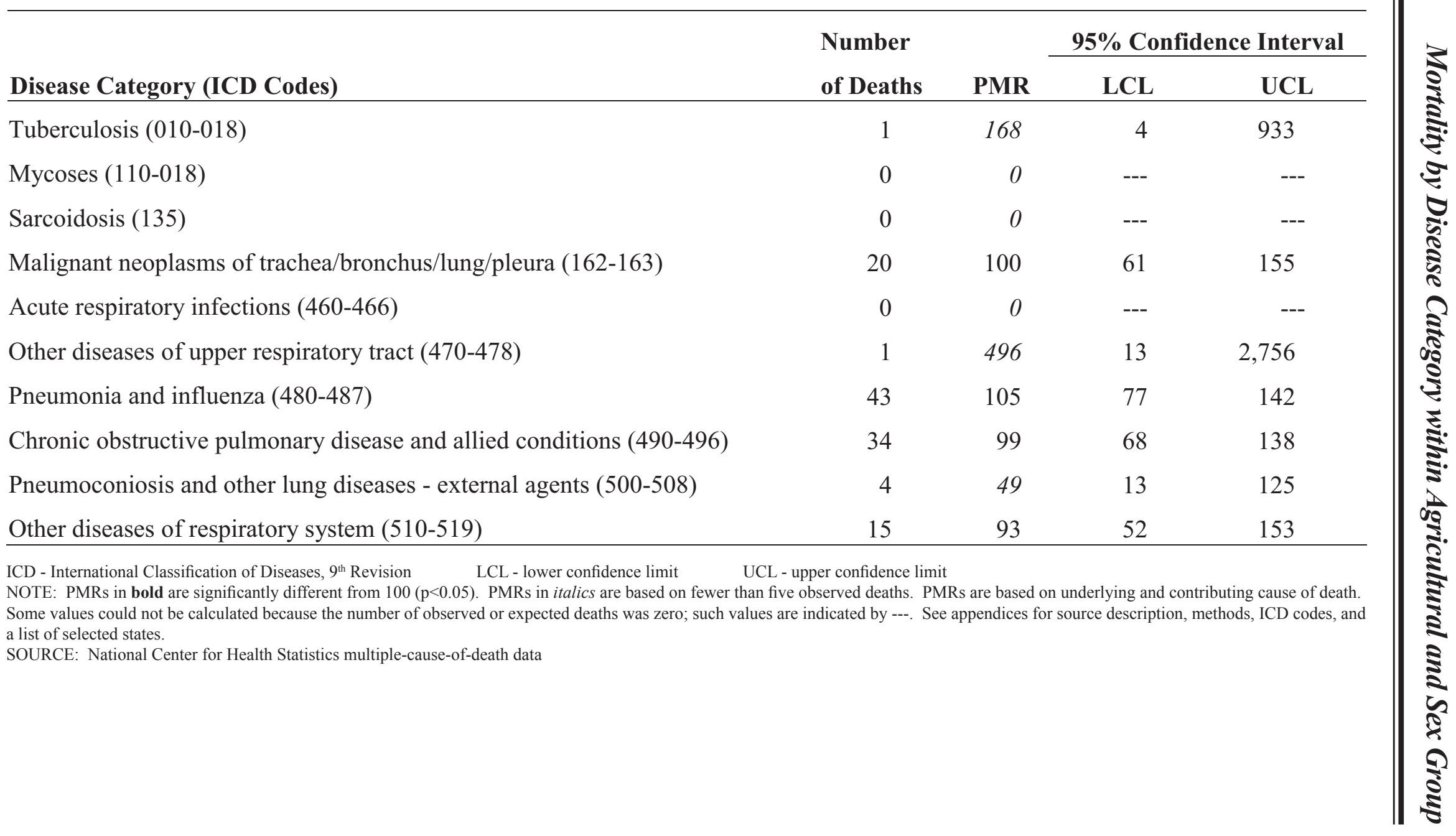


Table 2-16. Landscape and horticultural workers, females: Proportionate mortality ratio (PMR) adjusted for age and race/ethnicity by disease category, U.S. residents age 15 and over, selected states, 1988-1998

\begin{tabular}{|c|c|c|c|c|}
\hline \multirow[b]{2}{*}{ Disease Category (ICD Codes) } & \multirow{2}{*}{$\begin{array}{l}\text { Number } \\
\text { of Deaths }\end{array}$} & \multirow[b]{2}{*}{ PMR } & \multicolumn{2}{|c|}{ 95\% Confidence Interval } \\
\hline & & & LCL & $\mathbf{U C L}$ \\
\hline Tuberculosis $(010-018)$ & 0 & 0 & --- & --- \\
\hline Mycoses (110-018) & 1 & 77 & 2 & 428 \\
\hline Sarcoidosis (135) & 1 & 219 & 6 & 1,217 \\
\hline Malignant neoplasms of trachea/bronchus/lung/pleura (162-163) & 32 & 89 & 61 & 126 \\
\hline Acute respiratory infections (460-466) & 2 & 253 & 31 & 913 \\
\hline Other diseases of upper respiratory tract (470-478) & 0 & 0 & --- & --- \\
\hline Pneumonia and influenza (480-487) & 44 & 88 & 64 & 118 \\
\hline Chronic obstructive pulmonary disease and allied conditions (490-496) & 58 & 116 & 89 & 150 \\
\hline Pneumoconiosis and other lung diseases - external agents (500-508) & 5 & 53 & 17 & 124 \\
\hline Other diseases of respiratory system (510-519) & 24 & 101 & 65 & 150 \\
\hline
\end{tabular}

ICD - International Classification of Diseases, $9^{\text {th }}$ Revision $\quad$ LCL - lower confidence limit

UCL - upper confidence limit

NOTE: PMRs in bold are significantly different from $100(\mathrm{p}<0.05)$. PMRs in italics are based on fewer than five observed deaths. PMRs are based on underlying and contributing cause of death. Some values could not be calculated because the number of observed or expected deaths was zero; such values are indicated by ---. See appendices for source description, methods, ICD codes, and a list of selected states.

SOURCE: National Center for Health Statistics multiple-cause-of-death data 
Table 2-17. Forestry workers, females: Proportionate mortality ratio (PMR) adjusted for age and race/ ethnicity by disease category, U.S. residents age 15 and over, selected states, 1988-1998

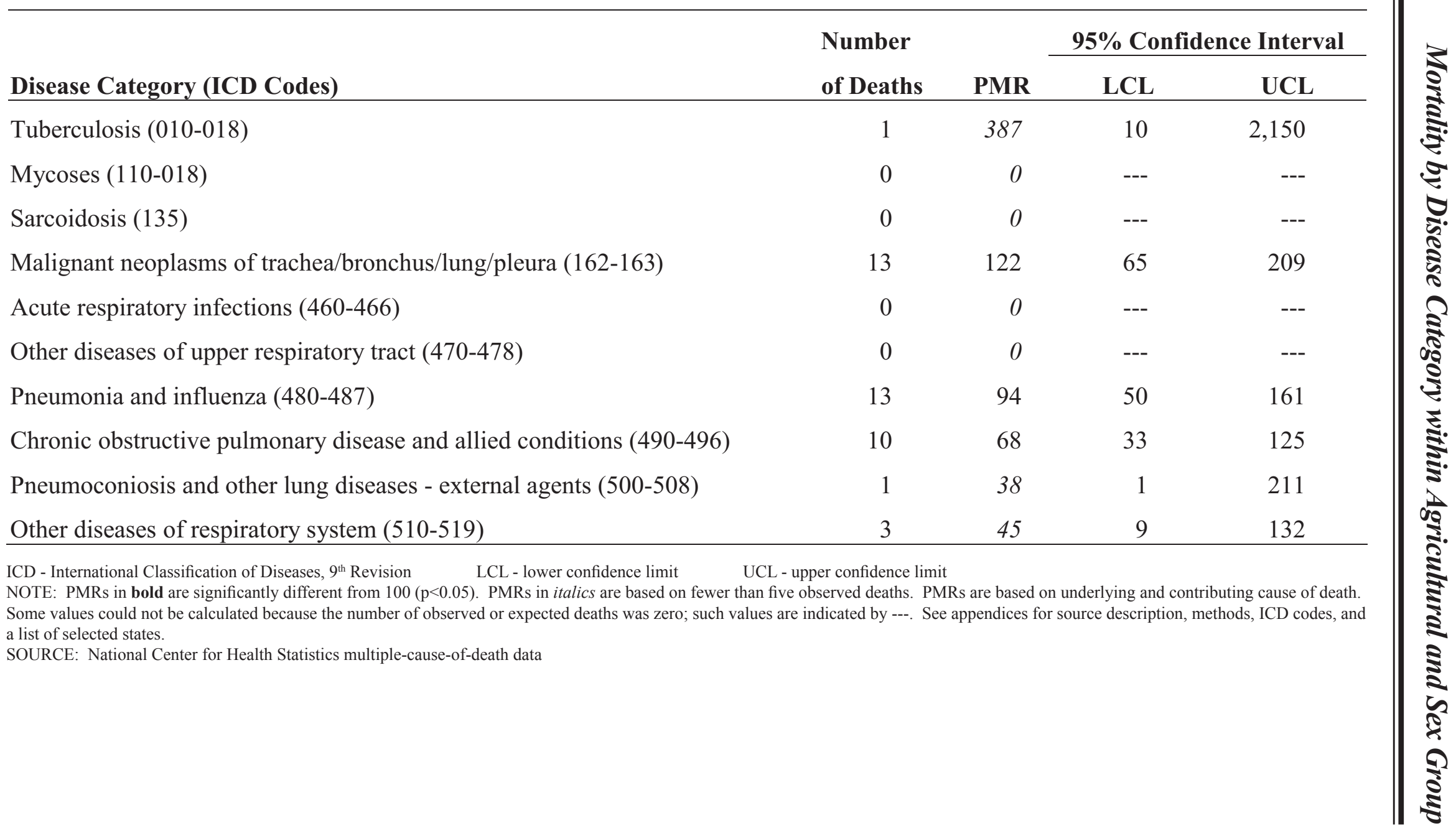


Table 2-18. Fishery workers, females: Proportionate mortality ratio (PMR) adjusted for age and race/ ethnicity by disease category, U.S. residents age 15 and over, selected states, 1988-1998

\begin{tabular}{|c|c|c|c|c|}
\hline \multirow[b]{2}{*}{ Disease Category (ICD Codes) } & \multirow{2}{*}{$\begin{array}{l}\text { Number } \\
\text { of Deaths }\end{array}$} & \multirow[b]{2}{*}{ PMR } & \multicolumn{2}{|c|}{ 95\% Confidence Interval } \\
\hline & & & $\mathbf{L C L}$ & $\mathbf{U C L}$ \\
\hline Tuberculosis $(010-018)$ & 0 & 0 & --- & --- \\
\hline Mycoses (110-018) & 0 & 0 & --- & --- \\
\hline Sarcoidosis (135) & 0 & 0 & --- & --- \\
\hline Malignant neoplasms of trachea/bronchus/lung/pleura (162-163) & 11 & 177 & 89 & 317 \\
\hline Acute respiratory infections (460-466) & 0 & 0 & --- & --- \\
\hline Other diseases of upper respiratory tract $(470-478)$ & 0 & 0 & --- & --- \\
\hline Pneumonia and influenza (480-487) & 6 & 72 & 26 & 157 \\
\hline Chronic obstructive pulmonary disease and allied conditions (490-496) & 12 & 136 & 70 & 237 \\
\hline Pneumoconiosis and other lung diseases - external agents (500-508) & 1 & 62 & 2 & 344 \\
\hline Other diseases of respiratory system (510-519) & 2 & 52 & 6 & 188 \\
\hline
\end{tabular}

ICD - International Classification of Diseases, $9^{\text {th }}$ Revision $\quad$ LCL - lower confidence limit $\quad$ UCL - upper confidence limit

NOTE: PMRs in bold are significantly different from $100(\mathrm{p}<0.05)$. PMRs in italics are based on fewer than five observed deaths. PMRs are based on underlying and contributing cause of death.

Some values could not be calculated because the number of observed or expected deaths was zero; such values are indicated by ---. See appendices for source description, methods, ICD codes, and a list of selected states.

SOURCE: National Center for Health Statistics multiple-cause-of-death data 
Figure 2-11. Tuberculosis: Proportionate mortality ratio (PMR) adjusted for age and race/ethnicity by agricultural group and sex, U.S. residents age 15 and over, selected states, 1988-1998

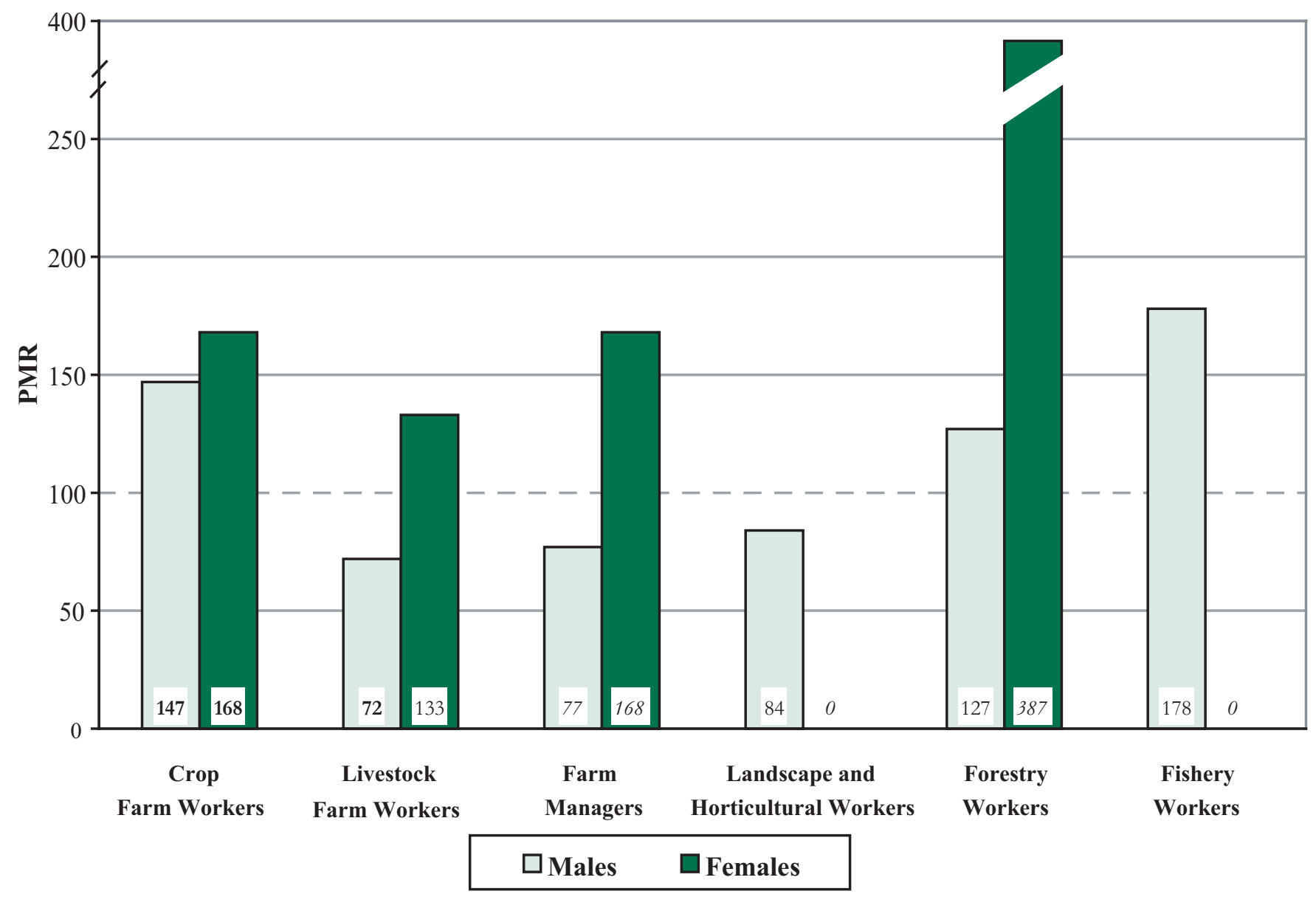

ICD - International Classification of Diseases, $9^{\text {th }}$ Revision

NOTE: Tuberculosis = ICD-9 codes 010-018. PMRs in bold are significantly different from $100(\mathrm{p}<0.05)$. PMRs in italics are based on fewer than five observed deaths. PMRs are based on underlying and contributing cause of death. See appendices for source description, methods, ICD codes, and a list of selected states.

SOURCE: National Center for Health Statistics multiple-cause-of-death data 
Figure 2-12. Mycoses: Proportionate mortality ratio (PMR) adjusted for age and race/ethnicity by agricultural group and sex, U.S. residents age 15 and over, selected states, 1988-1998

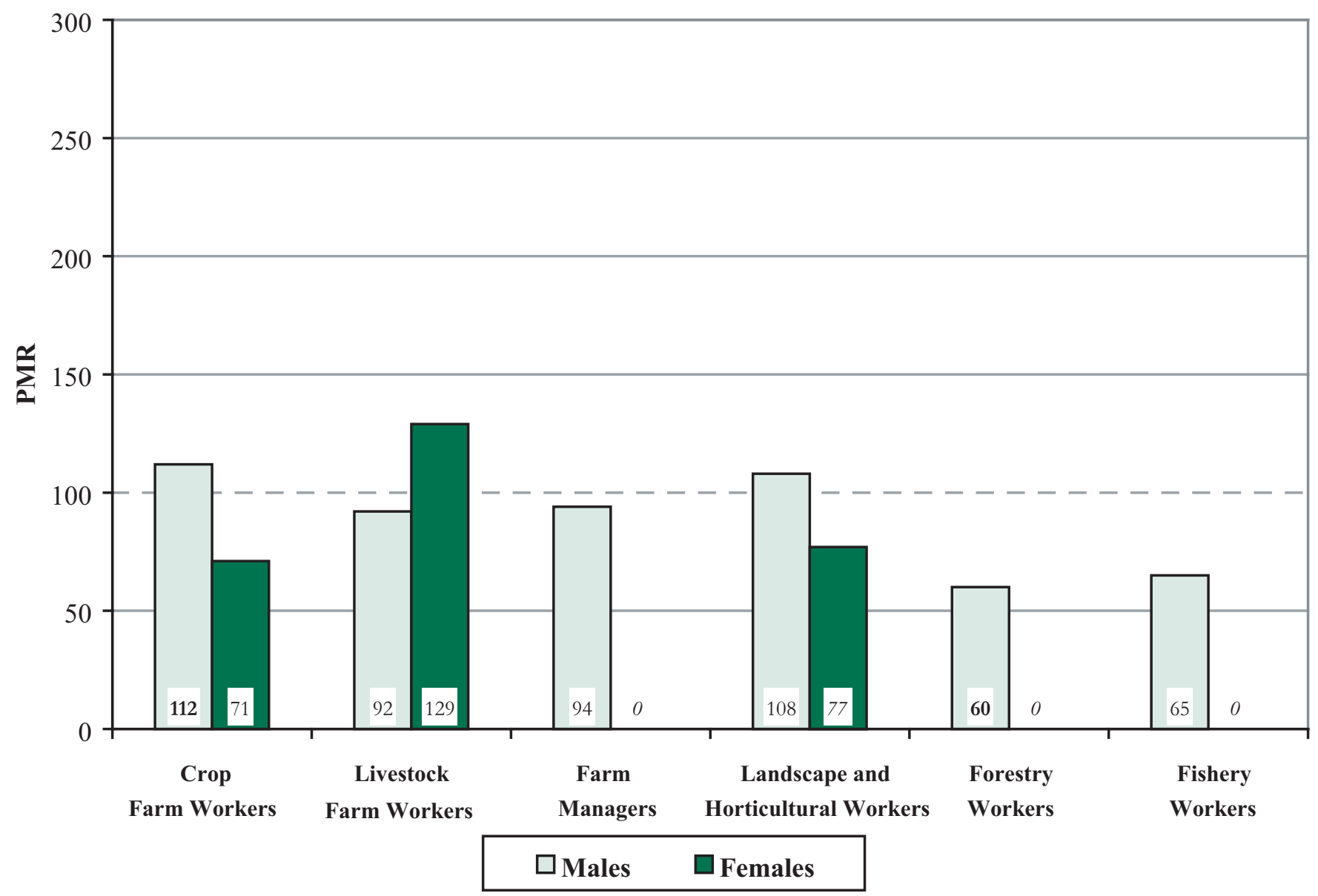

ICD - International Classification of Diseases, $9^{\text {th }}$ Revision

NOTE: Mycoses $=$ ICD-9 codes 110-118. PMRs in bold are significantly different from $100(\mathrm{p}<0.05)$. PMRs in italics are based on fewer than five observed deaths. PMRs are based on underlying and contributing cause of death. See appendices for source description, methods, ICD codes, and a list of selected states.

SOURCE: National Center for Health Statistics multiple-cause-of-death data 
Figure 2-13. Sarcoidosis: Proportionate mortality ratio (PMR) adjusted for age and race/ethnicity by agricultural group and sex, U.S. residents age 15 and over, selected states, 1988-1998

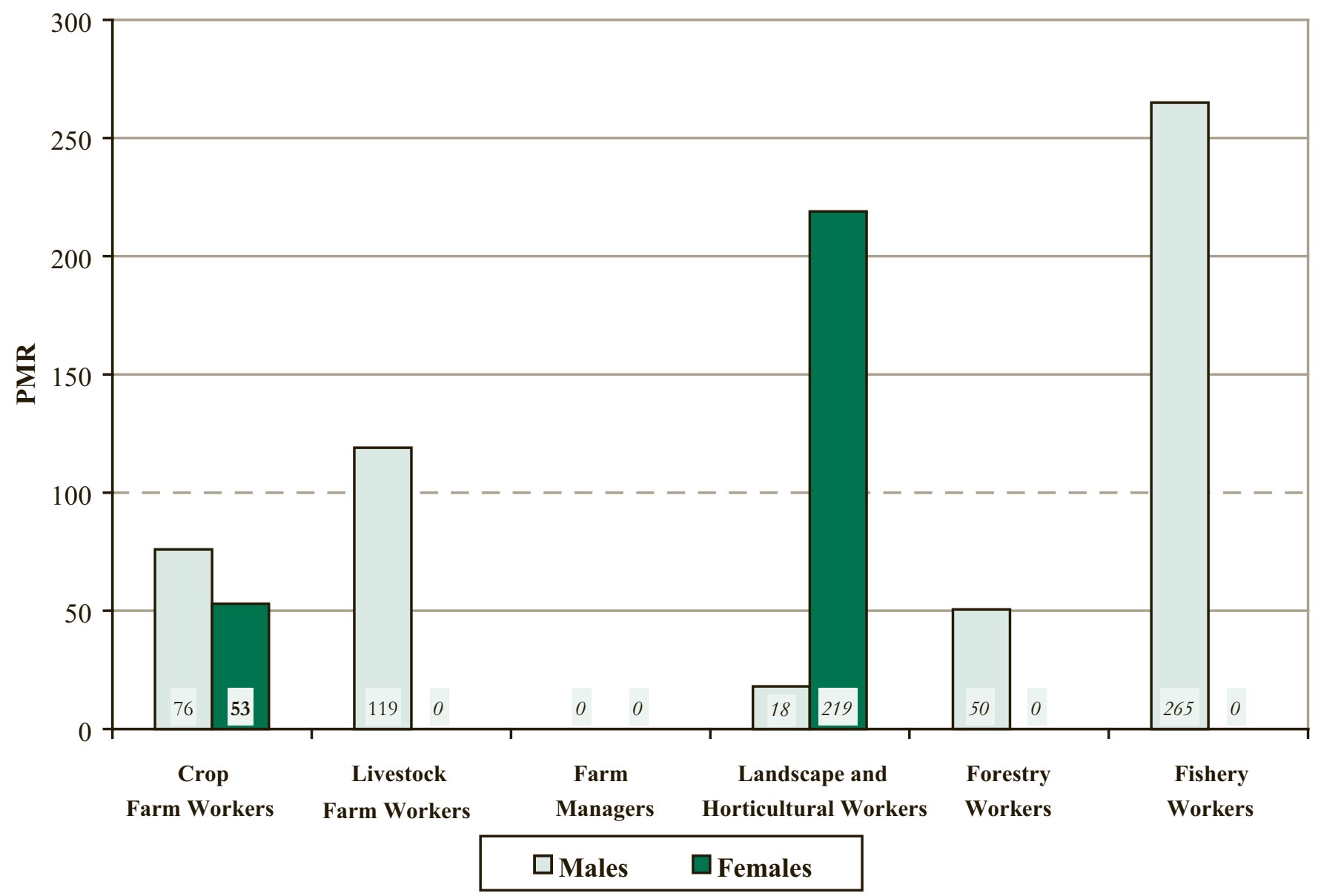

ICD - International Classification of Diseases, $9^{\text {th }}$ Revision

NOTE: Sarcoidosis $=$ ICD-9 code 135. PMRs in bold are significantly different from $100(\mathrm{p}<0.05)$. PMRs in italics are based on fewer than five observed deaths. PMRs are based on underlying and contributing cause of death. See appendices for source description, methods, ICD codes, and a list of selected states.

SOURCE: National Center for Health Statistics multiple-cause-of-death data 
Figure 2-14. Malignant neoplasms of trachea/bronchus/lung/pleura: Proportionate mortality ratio (PMR) adjusted for age and race/ethnicity by agricultural group and sex, U.S. residents age 15 and over, selected states, 1988-1998

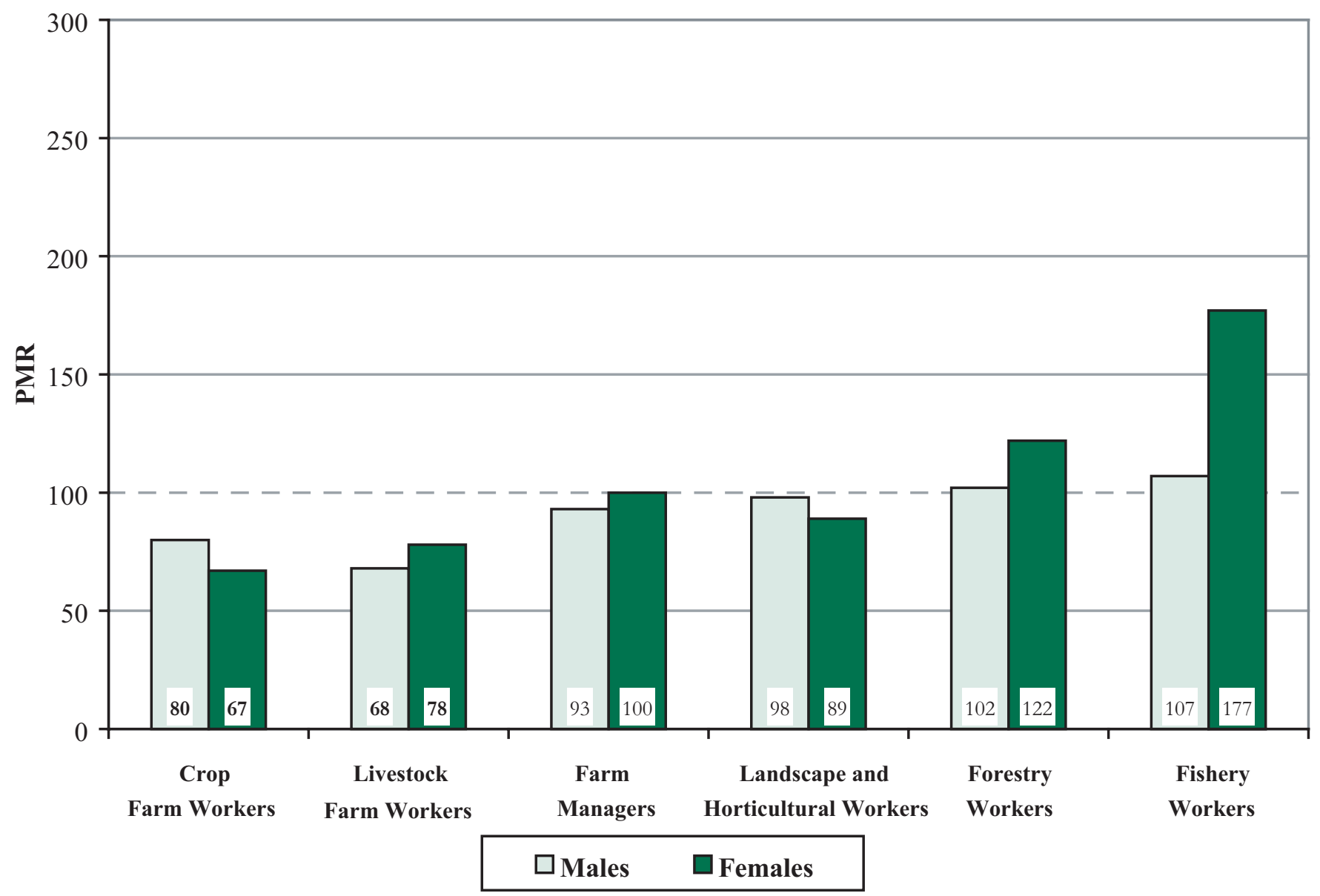

ICD - International Classification of Diseases, $9^{\text {th }}$ Revision

NOTE: Malignant neoplasms of trachea/bronchus/lung/pleura = ICD-9 codes 162-163. PMRs in bold are significantly different from 100 ( $\mathrm{p}<0.05$ ). PMRs in italics are based on fewer than five observed deaths. PMRs are based on underlying and contributing cause of death. See appendices for source description, methods, ICD codes, and a list of selected states.

SOURCE: National Center for Health Statistics multiple-cause-of-death data 
Figure 2-15. Acute respiratory infections: Proportionate mortality ratio (PMR) adjusted for age and race/ethnicity by agricultural group and sex, U.S. residents age 15 and over, selected states, 1988-1998

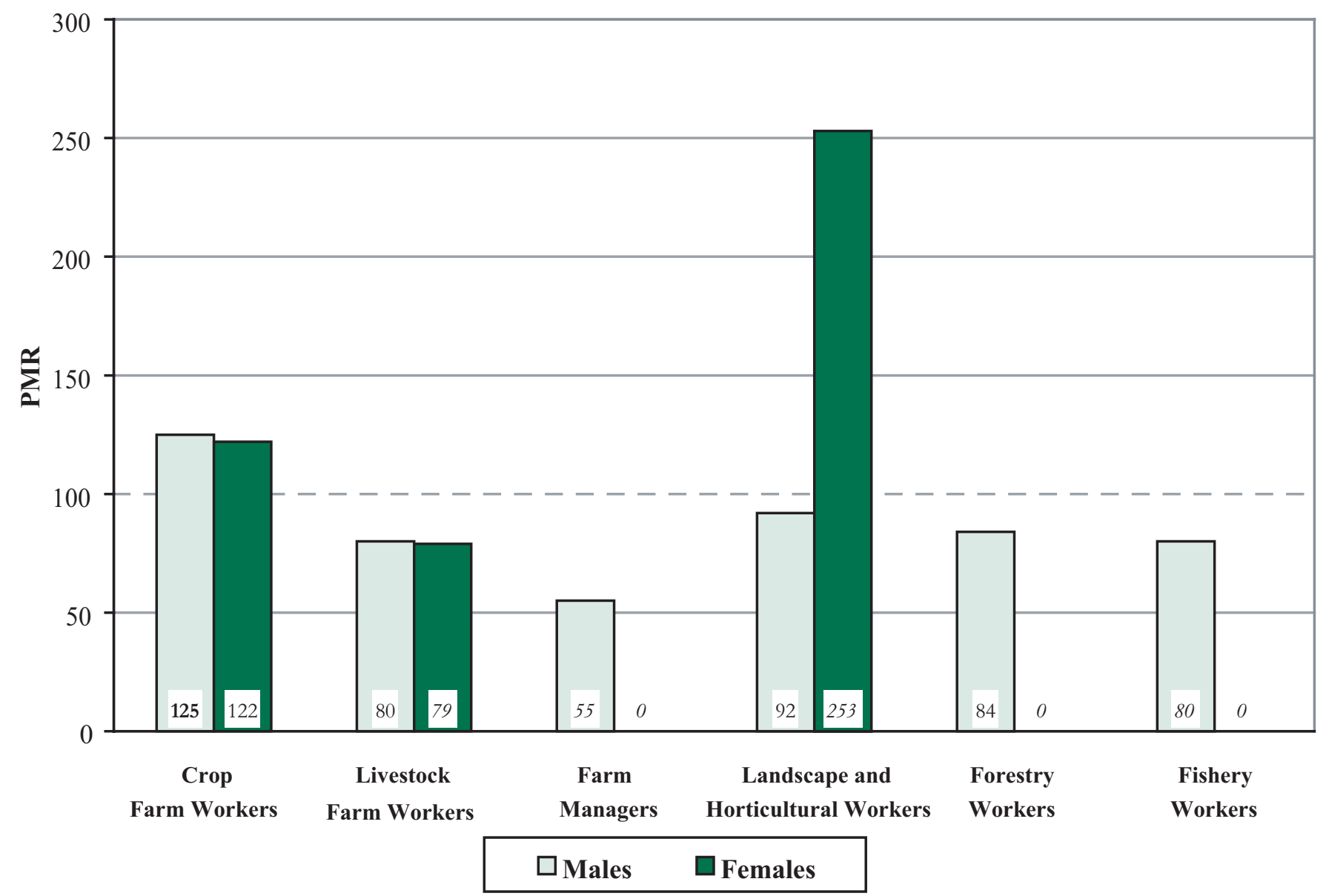

ICD - International Classification of Diseases, $9^{\text {th }}$ Revision

NOTE: Acute respiratory infections $=$ ICD-9 codes 460-466. PMRs in bold are significantly different from 100 ( $<<0.05$ ). PMRs in italics are based on fewer than five observed deaths. PMRs are based on underlying and contributing cause of death. See appendices for source description, methods, ICD codes, and a list of selected states.

SOURCE: National Center for Health Statistics multiple-cause-of-death data 
Figure 2-16. Other diseases of upper respiratory tract: Proportionate mortality ratio (PMR) adjusted for age and race/ethnicity by agricultural group and sex, U.S. residents age 15 and over, selected states, 1988-1998

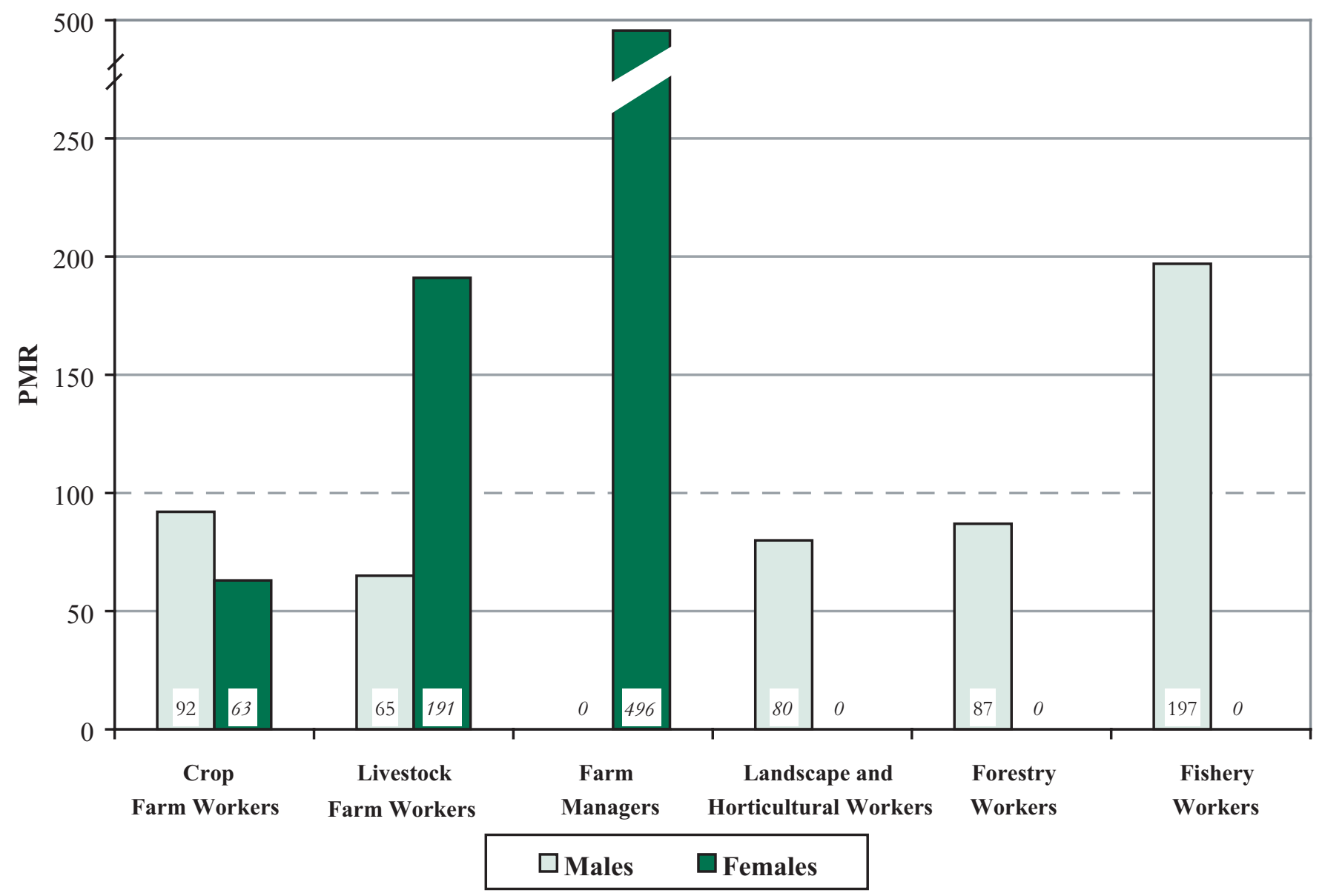

ICD - International Classification of Diseases, $9^{\text {th }}$ Revision

NOTE: Other diseases of upper respiratory tract $=$ ICD-9 codes 470-478. PMRs in bold are significantly different from 100 ( $<<0.05$ ). PMRs in italics are based on fewer than five observed deaths. PMRs are based on underlying and contributing cause of death. See appendices for source description, methods, ICD codes, and a list of selected states.

SOURCE: National Center for Health Statistics multiple-cause-of-death data 
Figure 2-17. Pneumonia and influenza: Proportionate mortality ratio (PMR) adjusted for age and race/ethnicity by agricultural group and sex, U.S. residents age 15 and over, selected states, 1988-1998

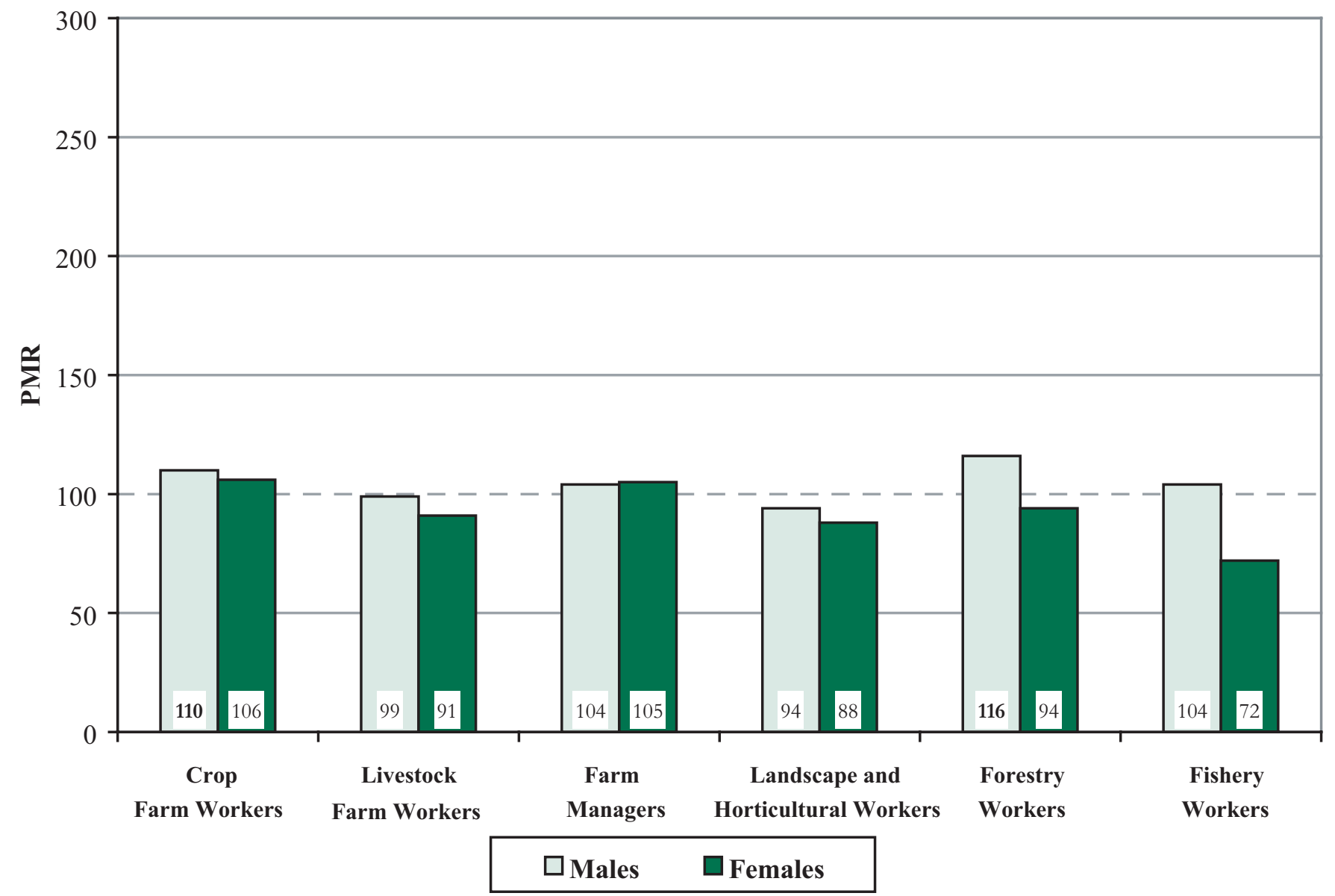

ICD - International Classification of Diseases, $9^{\text {th }}$ Revision

NOTE: Pneumonia and influenza = ICD-9 codes 480-487. PMRs in bold are significantly different from 100 ( $\mathrm{p}<0.05$ ). PMRs in italics are based on fewer than five observed deaths. PMRs are based on underlying and contributing cause of death. See appendices for source description, methods, ICD codes, and a list of selected states.

SOURCE: National Center for Health Statistics multiple-cause-of-death data 
Figure 2-18. Chronic obstructive pulmonary disease and allied conditions: Proportionate mortality ratio (PMR) adjusted for age and race/ethnicity by agricultural group and sex, U.S. residents age 15 and over, selected states, 1988-1998

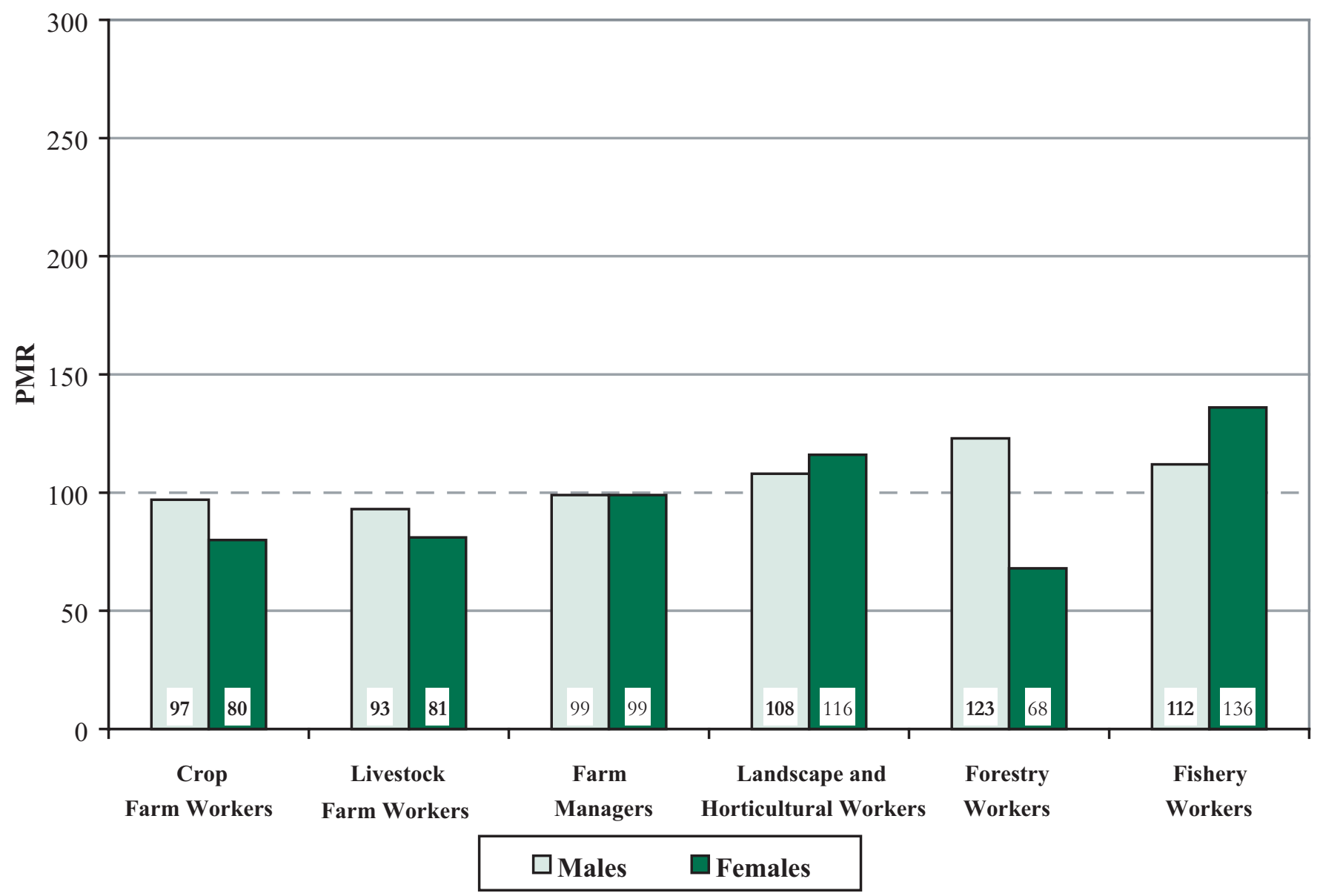

ICD - International Classification of Diseases, $9^{\text {th }}$ Revision

NOTE: Chronic obstructive pulmonary disease and allied conditions $=$ ICD-9 codes 490-496. PMRs in bold are significantly different from 100 ( $<<0.05$ ) PMRs in italics are based on fewer than five observed deaths. PMRs are based on underlying and contributing cause of death. See appendices for source description, methods, ICD codes, and a list of selected states.

SOURCE: National Center for Health Statistics multiple-cause-of-death data 
Figure 2-19. Pneumoconioses and other lung diseases-external agents: Proportionate mortality ratio (PMR) adjusted for age and race/ethnicity by agricultural group and sex, U.S. residents age 15 and over, selected states, 1988-1998

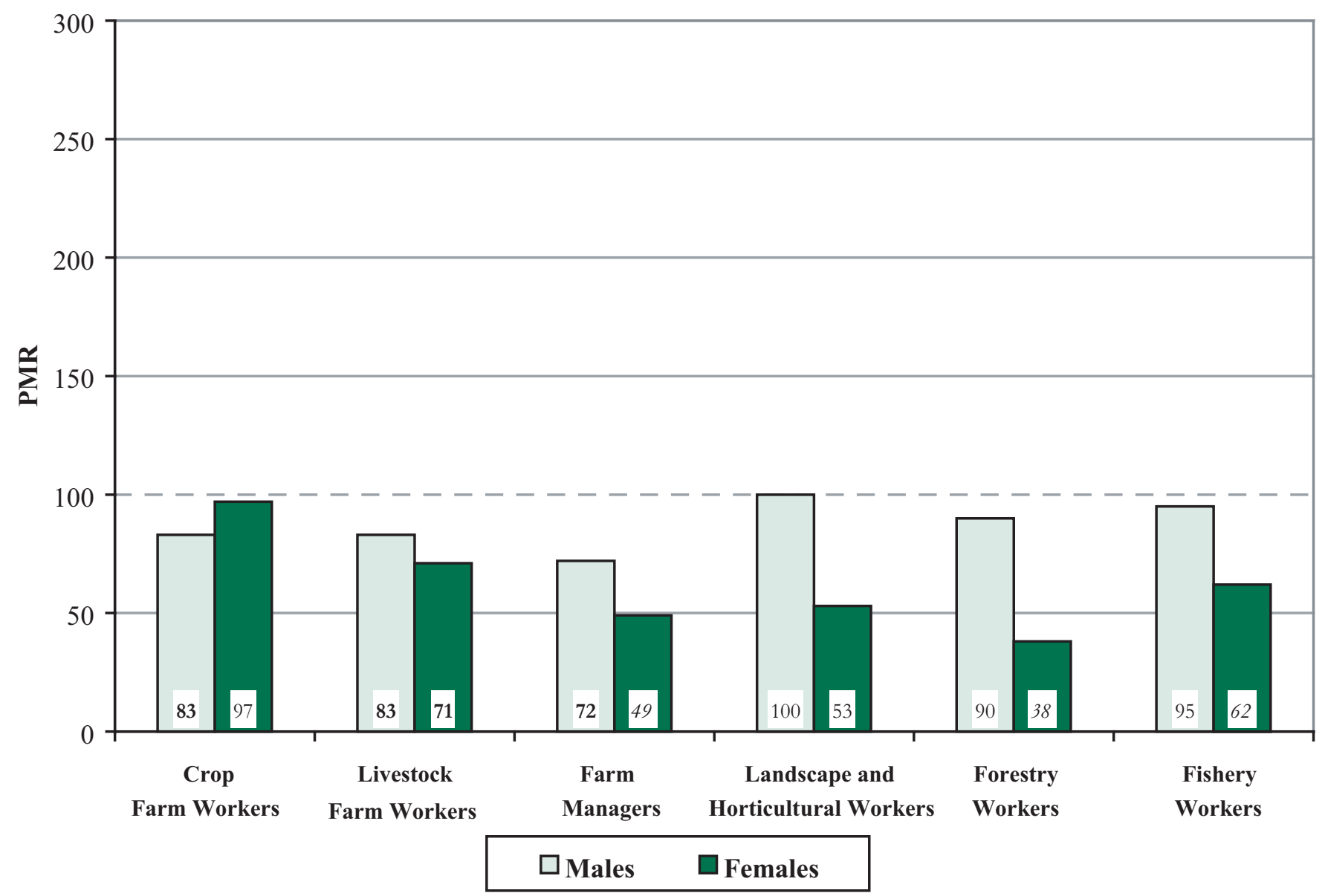

ICD - International Classification of Diseases, $9^{\text {th }}$ Revision

NOTE: Pneumoconioses and other lung diseases - external agents = ICD-9 codes 500-508. PMRs in bold are significantly different from 100 ( $<<0.05$ ). PMRs in italics are based on fewer than five observed deaths. PMRs are based on underlying and contributing cause of death. See appendices for source description, methods, ICD codes, and a list of selected states.

SOURCE: National Center for Health Statistics multiple-cause-of-death data 
Figure 2-20. Other diseases of respiratory system: Proportionate mortality ratio (PMR) adjusted for age and race/ethnicity by agricultural group and sex, U.S. residents age 15 and over, selected states, 1988-1998

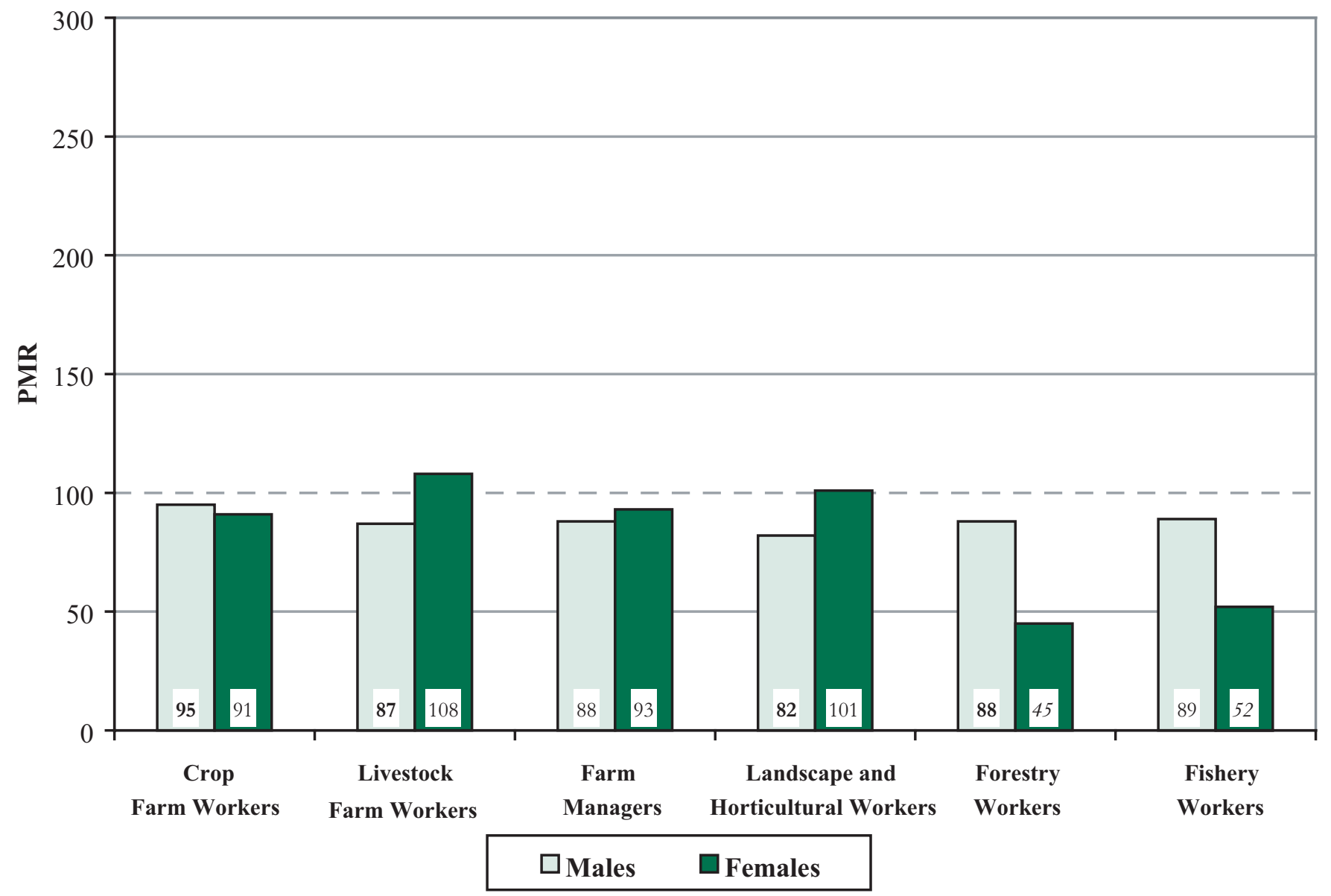

ICD - International Classification of Diseases, $9^{\text {th }}$ Revision

NOTE: Other diseases of respiratory system $=$ ICD-9 codes 510-519. PMRs in bold are significantly different from 100 ( $<<0.05$ ). PMRs in italics are based on fewer than five observed deaths. PMRs are based on underlying and contributing cause of death. See appendices for source description, methods, ICD codes, and a list of selected states.

SOURCE: National Center for Health Statistics multiple-cause-of-death data 
Table 2-19. Crop farm workers, white, non-Hispanic: Proportionate mortality ratio (PMR) adjusted for age and sex by disease category, U.S. residents age 15 and over, selected states, 1988-1998

\begin{tabular}{|c|c|c|c|c|}
\hline \multirow[b]{2}{*}{ Disease Category (ICD Codes) } & \multirow{2}{*}{$\begin{array}{c}\text { Number } \\
\text { of Deaths }\end{array}$} & \multirow[b]{2}{*}{ PMR } & \multicolumn{2}{|c|}{ 95\% Confidence Interval } \\
\hline & & & $\mathbf{L C L}$ & $\mathbf{U C L}$ \\
\hline Tuberculosis (010-018) & 223 & 134 & 118 & 153 \\
\hline Mycoses (110-018) & 268 & 118 & 105 & 133 \\
\hline Sarcoidosis (135) & 18 & 90 & 53 & 142 \\
\hline Malignant neoplasms of trachea/bronchus/lung/pleura (162-163) & 9,869 & 79 & 77 & 81 \\
\hline Acute respiratory infections (460-466) & 272 & 126 & 112 & 142 \\
\hline Other diseases of upper respiratory tract (470-478) & 69 & 89 & 70 & 113 \\
\hline Pneumonia and influenza (480-487) & 19,980 & 109 & 107 & 111 \\
\hline Chronic obstructive pulmonary disease and allied conditions (490-496) & 21,215 & 97 & 95 & 99 \\
\hline Pneumoconiosis and other lung diseases - external agents (500-508) & 3,944 & 82 & 79 & 85 \\
\hline Other diseases of respiratory system (510-519) & 5,832 & 95 & 93 & 97 \\
\hline \multicolumn{5}{|c|}{$\begin{array}{l}\text { ICD - International Classification of Diseases, } 9^{\text {th }} \text { Revision } \quad \text { LCL - lower confidence limit } \quad \text { UCL - upper confidence limit } \\
\text { NOTE: PMRs in bold are significantly different from } 100(\mathrm{p}<0.05) \text {. PMRs in italics are based on fewer than five observed deaths. PMRs are based on underlying and contributing cause of death. } \\
\text { Some values could not be calculated because the number of observed or expected deaths was zero; such values are indicated by ---. See appendices for source description, methods, ICD codes, and } \\
\text { a list of selected states. } \\
\text { SOURCE: National Center for Health Statistics multiple-cause-of-death data }\end{array}$} \\
\hline
\end{tabular}


Table 2-20. Livestock farm workers, white, non-Hispanic: Proportionate mortality ratio (PMR) adjusted for age and sex by disease category, U.S. residents age 15 and over, selected states, 1988-1998

\begin{tabular}{|c|c|c|c|c|}
\hline \multirow[b]{2}{*}{ Disease Category (ICD Codes) } & \multirow{2}{*}{$\begin{array}{l}\text { Number } \\
\text { of Deaths }\end{array}$} & \multirow[b]{2}{*}{ PMR } & \multicolumn{2}{|c|}{ 95\% Confidence Interval } \\
\hline & & & LCL & UCL \\
\hline Tuberculosis $(010-018)$ & 22 & 46 & 29 & 70 \\
\hline Mycoses (110-018) & 69 & 104 & 81 & 132 \\
\hline Sarcoidosis (135) & 8 & 134 & 58 & 264 \\
\hline Malignant neoplasms of trachea/bronchus/lung/pleura (162-163) & 2,324 & 65 & 63 & 68 \\
\hline Acute respiratory infections $(460-466)$ & 52 & 83 & 63 & 109 \\
\hline Other diseases of upper respiratory tract (470-478) & 17 & 75 & 44 & 120 \\
\hline Pneumonia and influenza (480-487) & 5,103 & 97 & 95 & 100 \\
\hline Chronic obstructive pulmonary disease and allied conditions (490-496) & 5,791 & 93 & 91 & 95 \\
\hline Pneumoconiosis and other lung diseases - external agents (500-508) & 1,129 & 82 & 77 & 87 \\
\hline Other diseases of respiratory system (510-519) & 1,532 & 86 & 82 & 90 \\
\hline
\end{tabular}

ICD - International Classification of Diseases, $9^{\text {th }}$ Revision

LCL - lower confidence limit

UCL - upper confidence limit

NOTE: PMRs in bold are significantly different from $100(\mathrm{p}<0.05)$. PMRs in italics are based on fewer than five observed deaths. PMRs are based on underlying and contributing cause of death. Some values could not be calculated because the number of observed or expected deaths was zero; such values are indicated by ---. See appendices for source description, methods, ICD codes, and a list of selected states.

SOURCE: National Center for Health Statistics multiple-cause-of-death data 
Table 2-21. Farm managers, white, non-Hispanic: Proportionate mortality ratio (PMR) adjusted for age and sex by disease category, U.S. residents age 15 and over, selected states, 1988-1998

\begin{tabular}{|c|c|c|c|c|}
\hline \multirow[b]{2}{*}{ Disease Category (ICD Codes) } & \multirow{2}{*}{$\begin{array}{l}\text { Number } \\
\text { of Deaths }\end{array}$} & \multirow[b]{2}{*}{ PMR } & \multicolumn{2}{|c|}{ 95\% Confidence Interval } \\
\hline & & & $\mathbf{L C L}$ & UCL \\
\hline Tuberculosis $(010-018)$ & 3 & 108 & 22 & 316 \\
\hline Mycoses $(110-018)$ & 4 & 91 & 25 & 233 \\
\hline Sarcoidosis (135) & 0 & 0 & --- & --- \\
\hline Malignant neoplasms of trachea/bronchus/lung/pleura (162-163) & 202 & 92 & 81 & 106 \\
\hline Acute respiratory infections (460-466) & 2 & 57 & 7 & 206 \\
\hline Other diseases of upper respiratory tract (470-478) & 0 & 0 & --- & --- \\
\hline Pneumonia and influenza (480-487) & 280 & 100 & 89 & 112 \\
\hline Chronic obstructive pulmonary disease and allied conditions (490-496) & 350 & 102 & 92 & 113 \\
\hline Pneumoconiosis and other lung diseases - external agents (500-508) & 49 & 69 & 51 & 91 \\
\hline Other diseases of respiratory system (510-519) & 92 & 90 & 73 & 110 \\
\hline
\end{tabular}

ICD - International Classification of Diseases, $9^{\text {th }}$ Revision

LCL - lower confidence limit

UCL - upper confidence limit

NOTE: PMRs in bold are significantly different from $100(\mathrm{p}<0.05)$. PMRs in italics are based on fewer than five observed deaths. PMRs are based on underlying and contributing cause of death. Some values could not be calculated because the number of observed or expected deaths was zero; such values are indicated by ---. See appendices for source description, methods, ICD codes, and a list of selected states.

SOURCE: National Center for Health Statistics multiple-cause-of-death data 
Table 2-22. Landscape and horticultural workers, white, non-Hispanic: Proportionate mortality ratio (PMR) adjusted for age and sex by disease category, U.S. residents age 15 and over, selected states, 1988-1998

\begin{tabular}{|c|c|c|c|c|}
\hline \multirow[b]{2}{*}{ Disease Category (ICD Codes) } & \multirow{2}{*}{$\begin{array}{l}\text { Number } \\
\text { of Deaths }\end{array}$} & \multirow[b]{2}{*}{ PMR } & \multicolumn{2}{|c|}{ 95\% Confidence Interval } \\
\hline & & & LCL & UCL \\
\hline Tuberculosis $(010-018)$ & 3 & 50 & 10 & 146 \\
\hline Mycoses (110-018) & 16 & 110 & 63 & 179 \\
\hline Sarcoidosis (135) & 0 & 0 & --- & --- \\
\hline Malignant neoplasms of trachea/bronchus/lung/pleura (162-163) & 444 & 98 & 89 & 108 \\
\hline Acute respiratory infections $(460-466)$ & 4 & 65 & 18 & 166 \\
\hline Other diseases of upper respiratory tract $(470-478)$ & 2 & 61 & 7 & 220 \\
\hline Pneumonia and influenza (480-487) & 385 & 92 & 83 & 102 \\
\hline Chronic obstructive pulmonary disease and allied conditions (490-496) & 604 & 113 & 105 & 122 \\
\hline Pneumoconiosis and other lung diseases - external agents (500-508) & 99 & 97 & 79 & 118 \\
\hline Other diseases of respiratory system (510-519) & 164 & 84 & 72 & 98 \\
\hline
\end{tabular}

ICD - International Classification of Diseases, $9^{\text {th }}$ Revision

LCL - lower confidence limit

UCL - upper confidence limit

NOTE: PMRs in bold are significantly different from $100(\mathrm{p}<0.05)$. PMRs in italics are based on fewer than five observed deaths. PMRs are based on underlying and contributing cause of death. Some values could not be calculated because the number of observed or expected deaths was zero; such values are indicated by ---. See appendices for source description, methods, ICD codes, and a list of selected states.

SOURCE: National Center for Health Statistics multiple-cause-of-death data 
Table 2-23. Forestry workers, white, non-Hispanic: Proportionate mortality ratio (PMR) adjusted for age and sex by disease category, U.S. residents age 15 and over, selected states, 1988-1998

\begin{tabular}{lrrrr}
\hline & Number & & \multicolumn{2}{c}{$\mathbf{9 5 \%}$ Confidence Interval } \\
\cline { 3 - 5 } Disease Category (ICD Codes) & of Deaths & PMR & LCL & UCL \\
\hline Tuberculosis (010-018) & 14 & 112 & 61 & 188 \\
Mycoses (110-018) & 16 & 70 & 40 & 114 \\
Sarcoidosis (135) & 1 & 48 & 1 & 95 \\
Malignant neoplasms of trachea/bronchus/lung/pleura (162-163) & 1,061 & 101 & 107 \\
Acute respiratory infections (460-466) & 12 & 87 & 45 & 152 \\
Other diseases of upper respiratory tract (470-478) & 4 & 65 & 18 & 169 \\
Pneumonia and influenza (480-487) & 1,231 & $\mathbf{1 1 5}$ & 109 \\
Chronic obstructive pulmonary disease and allied conditions (490-496) & 1,835 & $\mathbf{1 2 7}$ & 122 & 122 \\
Pneumoconiosis and other lung diseases - external agents (500-508) & 255 & 92 & 82 & 133 \\
Other diseases of respiratory system (510-519) & 362 & $\mathbf{8 4}$ & 76 \\
\hline
\end{tabular}

ICD - International Classification of Diseases, $9^{\text {th }}$ Revision

LCL - lower confidence limit

UCL - upper confidence limit

NOTE: PMRs in bold are significantly different from $100(\mathrm{p}<0.05)$. PMRs in italics are based on fewer than five observed deaths. PMRs are based on underlying and contributing cause of death. Some values could not be calculated because the number of observed or expected deaths was zero; such values are indicated by ---. See appendices for source description, methods, ICD codes, and a list of selected states.

SOURCE: National Center for Health Statistics multiple-cause-of-death data 
Table 2-24. Fishery workers, white, non-Hispanic: Proportionate mortality ratio (PMR) adjusted for age and sex by disease category, U.S. residents age 15 and over, selected states, 1988-1998

\begin{tabular}{lrrrr}
\hline & Number & & \multicolumn{2}{c}{$\mathbf{9 5 \%}$ Confidence Interval } \\
\cline { 2 - 5 } Disease Category (ICD Codes) & of Deaths & PMR & LCL & UCL \\
\hline Tuberculosis (010-018) & 6 & 154 & 56 & 336 \\
Mycoses (110-018) & 4 & 51 & 14 & 130 \\
Sarcoidosis (135) & 1 & 143 & 4 & 794 \\
Malignant neoplasms of trachea/bronchus/lung/pleura (162-163) & 333 & 107 & 96 & 119 \\
Acute respiratory infections (460-466) & 3 & 70 & 14 & 205 \\
Other diseases of upper respiratory tract (470-478) & 3 & 150 & 31 \\
Pneumonia and influenza (480-487) & 321 & 99 & 89 & 110 \\
Chronic obstructive pulmonary disease and allied conditions (490-496) & 502 & $\mathbf{1 1 9}$ & 110 & 130 \\
Pneumoconiosis and other lung diseases - external agents (500-508) & 78 & 94 & 75 & 117 \\
Other diseases of respiratory system (510-519) & 119 & 90 & 75 \\
\hline
\end{tabular}

ICD - International Classification of Diseases, $9^{\text {th }}$ Revision

LCL - lower confidence limit

UCL - upper confidence limit

NOTE: PMRs in bold are significantly different from $100(\mathrm{p}<0.05)$. PMRs in italics are based on fewer than five observed deaths. PMRs are based on underlying and contributing cause of death. Some values could not be calculated because the number of observed or expected deaths was zero; such values are indicated by ---. See appendices for source description, methods, ICD codes, and a list of selected states.

SOURCE: National Center for Health Statistics multiple-cause-of-death data 
Table 2-25. Crop farm workers, black, non-Hispanic: Proportionate mortality ratio (PMR) adjusted for age and sex by disease category, U.S. residents age 15 and over, selected states, 1988-1998

\begin{tabular}{|c|c|c|c|c|}
\hline \multirow[b]{2}{*}{ Disease Category (ICD Codes) } & \multirow{2}{*}{$\begin{array}{l}\text { Number } \\
\text { of Deaths }\end{array}$} & \multirow[b]{2}{*}{ PMR } & \multicolumn{2}{|c|}{ 95\% Confidence Interval } \\
\hline & & & $\mathbf{L C L}$ & $\mathbf{U C L}$ \\
\hline Tuberculosis $(010-018)$ & 231 & 188 & 165 & 214 \\
\hline Mycoses (110-018) & 69 & 94 & 73 & 119 \\
\hline Sarcoidosis (135) & 22 & 63 & 39 & 95 \\
\hline Malignant neoplasms of trachea/bronchus/lung/pleura (162-163) & 1,916 & 85 & 81 & 89 \\
\hline Acute respiratory infections (460-466) & 31 & 138 & 94 & 196 \\
\hline Other diseases of upper respiratory tract (470-478) & 20 & 115 & 70 & 178 \\
\hline Pneumonia and influenza (480-487) & 3,215 & 114 & 110 & 118 \\
\hline Chronic obstructive pulmonary disease and allied conditions (490-496) & 2,322 & 96 & 92 & 100 \\
\hline Pneumoconiosis and other lung diseases - external agents (500-508) & 734 & 95 & 89 & 102 \\
\hline Other diseases of respiratory system (510-519) & 1,011 & 94 & 89 & 100 \\
\hline \multicolumn{5}{|c|}{$\begin{array}{l}\text { ICD - International Classification of Diseases, } 9^{\text {th }} \text { Revision } \quad \text { LCL - lower confidence limit } \quad \text { UCL - upper confidence limit } \\
\text { NOTE: PMRs in bold are significantly different from } 100(\mathrm{p}<0.05) \text {. PMRs in italics are based on fewer than five observed deaths. PMRs are based on underlying and contributing cause of death. } \\
\text { Some values could not be calculated because the number of observed or expected deaths was zero; such values are indicated by ---. See appendices for source description, methods, ICD codes, and } \\
\text { a list of selected states. } \\
\text { SOURCE: National Center for Health Statistics multiple-cause-of-death data }\end{array}$} \\
\hline
\end{tabular}


Table 2-26. Livestock farm workers, black, non-Hispanic: Proportionate mortality ratio (PMR) adjusted for age and sex by disease category, U.S. residents age 15 and over, selected states, 1988-1998

\begin{tabular}{|c|c|c|c|c|}
\hline \multirow[b]{2}{*}{ Disease Category (ICD Codes) } & \multirow{2}{*}{$\begin{array}{l}\text { Number } \\
\text { of Deaths }\end{array}$} & \multirow[b]{2}{*}{ PMR } & \multicolumn{2}{|c|}{ 95\% Confidence Interval } \\
\hline & & & $\mathbf{L C L}$ & UCL \\
\hline Tuberculosis $(010-018)$ & 4 & 114 & 31 & 292 \\
\hline Mycoses (110-018) & 0 & 0 & --- & --- \\
\hline Sarcoidosis (135) & 0 & 0 & --- & --- \\
\hline Malignant neoplasms of trachea/bronchus/lung/pleura (162-163) & 57 & 103 & 79 & 134 \\
\hline Acute respiratory infections $(460-466)$ & 1 & 183 & 5 & 1,017 \\
\hline Other diseases of upper respiratory tract $(470-478)$ & 0 & 0 & --- & --- \\
\hline Pneumonia and influenza (480-487) & 69 & 111 & 87 & 141 \\
\hline Chronic obstructive pulmonary disease and allied conditions (490-496) & 49 & 92 & 68 & 122 \\
\hline Pneumoconiosis and other lung diseases - external agents (500-508) & 15 & 93 & 52 & 153 \\
\hline Other diseases of respiratory system (510-519) & 20 & 75 & 46 & 116 \\
\hline
\end{tabular}

ICD - International Classification of Diseases, $9^{\text {th }}$ Revision

LCL - lower confidence limit

UCL - upper confidence limit

NOTE: PMRs in bold are significantly different from $100(\mathrm{p}<0.05)$. PMRs in italics are based on fewer than five observed deaths. PMRs are based on underlying and contributing cause of death. Some values could not be calculated because the number of observed or expected deaths was zero; such values are indicated by ---. See appendices for source description, methods, ICD codes, and a list of selected states.

SOURCE: National Center for Health Statistics multiple-cause-of-death data 
Table 2-27. Farm managers, black, non-Hispanic: Proportionate mortality ratio (PMR) adjusted for age and sex by disease category, U.S. residents age 15 and over, selected states, 1988-1998

\begin{tabular}{|c|c|c|c|c|}
\hline \multirow[b]{2}{*}{ Disease Category (ICD Codes) } & \multirow{2}{*}{$\begin{array}{l}\text { Number } \\
\text { of Deaths }\end{array}$} & \multirow[b]{2}{*}{ PMR } & \multicolumn{2}{|c|}{ 95\% Confidence Interval } \\
\hline & & & $\mathbf{L C L}$ & UCL \\
\hline Tuberculosis $(010-018)$ & 0 & 0 & --- & --- \\
\hline Mycoses (110-018) & 0 & 0 & --- & --- \\
\hline Sarcoidosis (135) & 0 & 0 & --- & --- \\
\hline Malignant neoplasms of trachea/bronchus/lung/pleura (162-163) & 8 & 93 & 40 & 183 \\
\hline Acute respiratory infections (460-466) & 0 & 0 & --- & --- \\
\hline Other diseases of upper respiratory tract $(470-478)$ & 0 & 0 & --- & --- \\
\hline Pneumonia and influenza (480-487) & 9 & 92 & 42 & 175 \\
\hline Chronic obstructive pulmonary disease and allied conditions (490-496) & 4 & 47 & 13 & 120 \\
\hline Pneumoconiosis and other lung diseases - external agents (500-508) & 4 & 152 & 41 & 389 \\
\hline Other diseases of respiratory system (510-519) & 2 & 52 & 6 & 188 \\
\hline
\end{tabular}

ICD - International Classification of Diseases, $9^{\text {th }}$ Revision $\quad$ LCL - lower confidence limit $\quad$ UCL - upper confidence limit

NOTE: PMRs in bold are significantly different from $100(\mathrm{p}<0.05)$. PMRs in italics are based on fewer than five observed deaths. PMRs are based on underlying and contributing cause of death. Some values could not be calculated because the number of observed or expected deaths was zero; such values are indicated by ---. See appendices for source description, methods, ICD codes, and a list of selected states.

SOURCE: National Center for Health Statistics multiple-cause-of-death data 
Table 2-28. Landscape and horticultural workers, black, non-Hispanic: Proportionate mortality ratio (PMR) adjusted for age and sex by disease category, U.S. residents age 15 and over, selected states, 1988-1998

\begin{tabular}{|c|c|c|c|c|}
\hline \multirow[b]{2}{*}{ Disease Category (ICD Codes) } & \multirow{2}{*}{$\begin{array}{l}\text { Number } \\
\text { of Deaths }\end{array}$} & \multirow[b]{2}{*}{ PMR } & \multicolumn{2}{|c|}{ 95\% Confidence Interval } \\
\hline & & & $\mathbf{L C L}$ & $\mathbf{U C L}$ \\
\hline Tuberculosis $(010-018)$ & 9 & 86 & 39 & 163 \\
\hline Mycoses (110-018) & 8 & 102 & 44 & 201 \\
\hline Sarcoidosis (135) & 1 & 23 & 1 & 128 \\
\hline Malignant neoplasms of trachea/bronchus/lung/pleura (162-163) & 147 & 100 & 85 & 118 \\
\hline Acute respiratory infections (460-466) & 4 & 281 & 77 & 719 \\
\hline Other diseases of upper respiratory tract (470-478) & 2 & 149 & 18 & 538 \\
\hline Pneumonia and influenza (480-487) & 141 & 96 & 82 & 113 \\
\hline Chronic obstructive pulmonary disease and allied conditions (490-496) & 122 & 99 & 83 & 118 \\
\hline Pneumoconiosis and other lung diseases - external agents (500-508) & 32 & 89 & 61 & 126 \\
\hline Other diseases of respiratory system (510-519) & 61 & 88 & 69 & 113 \\
\hline
\end{tabular}

ICD - International Classification of Diseases, $9^{\text {th }}$ Revision

LCL - lower confidence limit

UCL - upper confidence limit

NOTE: PMRs in bold are significantly different from $100(\mathrm{p}<0.05)$. PMRs in italics are based on fewer than five observed deaths. PMRs are based on underlying and contributing cause of death. Some values could not be calculated because the number of observed or expected deaths was zero; such values are indicated by ---. See appendices for source description, methods, ICD codes, and a list of selected states.

SOURCE: National Center for Health Statistics multiple-cause-of-death data 
Table 2-29. Forestry workers, black, non-Hispanic: Proportionate mortality ratio (PMR) adjusted for age and sex by disease category, U.S. residents age 15 and over, selected states, 1988-1998

\begin{tabular}{|c|c|c|c|c|}
\hline \multirow[b]{2}{*}{ Disease Category (ICD Codes) } & \multirow{2}{*}{$\begin{array}{l}\text { Number } \\
\text { of Deaths }\end{array}$} & \multirow[b]{2}{*}{ PMR } & \multicolumn{2}{|c|}{ 95\% Confidence Interval } \\
\hline & & & LCL & UCL \\
\hline Tuberculosis (010-018) & 22 & 121 & 76 & 183 \\
\hline Mycoses (110-018) & 5 & 42 & 14 & 98 \\
\hline Sarcoidosis (135) & 3 & 52 & 11 & 152 \\
\hline Malignant neoplasms of trachea/bronchus/lung/pleura (162-163) & 357 & 101 & 91 & 112 \\
\hline Acute respiratory infections $(460-466)$ & 3 & 109 & 22 & 319 \\
\hline Other diseases of upper respiratory tract (470-478) & 4 & 174 & 47 & 445 \\
\hline Pneumonia and influenza (480-487) & 381 & 124 & 112 & 137 \\
\hline Chronic obstructive pulmonary disease and allied conditions (490-496) & 269 & 91 & 81 & 103 \\
\hline Pneumoconiosis and other lung diseases - external agents (500-508) & 77 & 95 & 75 & 119 \\
\hline Other diseases of respiratory system (510-519) & 138 & 101 & 85 & 119 \\
\hline
\end{tabular}

ICD - International Classification of Diseases, $9^{\text {th }}$ Revision $\quad$ LCL - lower confidence limit $\quad$ UCL - upper confidence limit

NOTE: PMRs in bold are significantly different from $100(\mathrm{p}<0.05)$. PMRs in italics are based on fewer than five observed deaths. PMRs are based on underlying and contributing cause of death. Some values could not be calculated because the number of observed or expected deaths was zero; such values are indicated by ---. See appendices for source description, methods, ICD codes, and a list of selected states.

SOURCE: National Center for Health Statistics multiple-cause-of-death data 
Table 2-30. Fishery workers, black, non-Hispanic: Proportionate mortality ratio (PMR) adjusted for age and sex by disease category, U.S. residents age 15 and over, selected states, 1988-1998

\begin{tabular}{|c|c|c|c|c|}
\hline \multirow[b]{2}{*}{ Disease Category (ICD Codes) } & \multirow{2}{*}{$\begin{array}{l}\text { Number } \\
\text { of Deaths }\end{array}$} & \multirow[b]{2}{*}{ PMR } & \multicolumn{2}{|c|}{ 95\% Confidence Interval } \\
\hline & & & $\mathbf{L C L}$ & $\mathbf{U C L}$ \\
\hline Tuberculosis $(010-018)$ & 2 & 191 & 23 & 690 \\
\hline Mycoses (110-018) & 1 & 71 & 2 & 394 \\
\hline Sarcoidosis (135) & 2 & 291 & 35 & 1,051 \\
\hline Malignant neoplasms of trachea/bronchus/lung/pleura (162-163) & 46 & 123 & 91 & 164 \\
\hline Acute respiratory infections $(460-466)$ & 1 & 317 & 8 & 1,761 \\
\hline Other diseases of upper respiratory tract (470-478) & 0 & 0 & --- & --- \\
\hline Pneumonia and influenza (480-487) & 46 & 130 & 96 & 174 \\
\hline Chronic obstructive pulmonary disease and allied conditions (490-496) & 21 & 64 & 40 & 98 \\
\hline Pneumoconiosis and other lung diseases - external agents (500-508) & 11 & 119 & 60 & 213 \\
\hline Other diseases of respiratory system (510-519) & 13 & 84 & 45 & 144 \\
\hline
\end{tabular}

ICD - International Classification of Diseases, $9^{\text {th }}$ Revision

LCL - lower confidence limit

UCL - upper confidence limit

NOTE: PMRs in bold are significantly different from $100(\mathrm{p}<0.05)$. PMRs in italics are based on fewer than five observed deaths. PMRs are based on underlying and contributing cause of death. Some values could not be calculated because the number of observed or expected deaths was zero; such values are indicated by ---. See appendices for source description, methods, ICD codes, and a list of selected states.

SOURCE: National Center for Health Statistics multiple-cause-of-death data 
Table 2-31. Crop farm workers, Hispanic: Proportionate mortality ratio (PMR) adjusted for age and sex by disease category, U.S. residents age 15 and over, selected states, 1988-1998

\begin{tabular}{|c|c|c|c|c|}
\hline \multirow[b]{2}{*}{ Disease Category (ICD Codes) } & \multirow{2}{*}{$\begin{array}{l}\text { Number } \\
\text { of Deaths }\end{array}$} & \multirow[b]{2}{*}{ PMR } & \multicolumn{2}{|c|}{ 95\% Confidence Interval } \\
\hline & & & LCL & UCL \\
\hline Tuberculosis $(010-018)$ & 12 & 76 & 39 & 133 \\
\hline Mycoses (110-018) & 8 & 67 & 29 & 132 \\
\hline Sarcoidosis (135) & 0 & 0 & --- & --- \\
\hline Malignant neoplasms of trachea/bronchus/lung/pleura (162-163) & 155 & 81 & 69 & 95 \\
\hline Acute respiratory infections $(460-466)$ & 9 & 349 & 160 & 662 \\
\hline Other diseases of upper respiratory tract (470-478) & 3 & 108 & 22 & 316 \\
\hline Pneumonia and influenza (480-487) & 447 & 109 & 99 & 120 \\
\hline Chronic obstructive pulmonary disease and allied conditions (490-496) & 385 & 109 & 99 & 120 \\
\hline Pneumoconiosis and other lung diseases - external agents (500-508) & 78 & 72 & 57 & 90 \\
\hline Other diseases of respiratory system (510-519) & 160 & 85 & 72 & 99 \\
\hline
\end{tabular}

ICD - International Classification of Diseases, $9^{\text {th }}$ Revision

LCL - lower confidence limit

UCL - upper confidence limit

NOTE: PMRs in bold are significantly different from $100(\mathrm{p}<0.05)$. PMRs in italics are based on fewer than five observed deaths. PMRs are based on underlying and contributing cause of death. Some values could not be calculated because the number of observed or expected deaths was zero; such values are indicated by ---. See appendices for source description, methods, ICD codes, and a list of selected states.

SOURCE: National Center for Health Statistics multiple-cause-of-death data 
Table 2-32. Livestock farm workers, Hispanic: Proportionate mortality ratio (PMR) adjusted for age and sex by disease category, U.S. residents age 15 and over, selected states, 1988-1998

\begin{tabular}{|c|c|c|c|c|}
\hline \multirow[b]{2}{*}{ Disease Category (ICD Codes) } & \multirow{2}{*}{$\begin{array}{l}\text { Number } \\
\text { of Deaths }\end{array}$} & \multirow[b]{2}{*}{ PMR } & \multicolumn{2}{|c|}{ 95\% Confidence Interval } \\
\hline & & & LCL & UCL \\
\hline Tuberculosis $(010-018)$ & 9 & 165 & 76 & 313 \\
\hline Mycoses (110-018) & 1 & 36 & 1 & 200 \\
\hline Sarcoidosis (135) & 0 & 0 & --- & --- \\
\hline Malignant neoplasms of trachea/bronchus/lung/pleura (162-163) & 73 & 91 & 72 & 115 \\
\hline Acute respiratory infections (460-466) & 0 & 0 & --- & --- \\
\hline Other diseases of upper respiratory tract (470-478) & 1 & 131 & 3 & 728 \\
\hline Pneumonia and influenza (480-487) & 223 & 114 & 100 & 130 \\
\hline Chronic obstructive pulmonary disease and allied conditions (490-496) & 194 & 110 & 95 & 127 \\
\hline Pneumoconiosis and other lung diseases - external agents (500-508) & 55 & 100 & 76 & 130 \\
\hline Other diseases of respiratory system $(510-519)$ & 53 & 69 & 53 & 90 \\
\hline
\end{tabular}

ICD - International Classification of Diseases, $9^{\text {th }}$ Revision

LCL - lower confidence limit

UCL - upper confidence limit

NOTE: PMRs in bold are significantly different from $100(\mathrm{p}<0.05)$. PMRs in italics are based on fewer than five observed deaths. PMRs are based on underlying and contributing cause of death. Some values could not be calculated because the number of observed or expected deaths was zero; such values are indicated by ---. See appendices for source description, methods, ICD codes, and a list of selected states.

SOURCE: National Center for Health Statistics multiple-cause-of-death data 
Table 2-33. Farm managers, Hispanic: Proportionate mortality ratio (PMR) adjusted for age and sex by disease category, U.S. residents age 15 and over, selected states, 1988-1998

\begin{tabular}{|c|c|c|c|c|}
\hline \multirow[b]{2}{*}{ Disease Category (ICD Codes) } & \multirow{2}{*}{$\begin{array}{l}\text { Number } \\
\text { of Deaths }\end{array}$} & \multirow[b]{2}{*}{ PMR } & \multicolumn{2}{|c|}{ 95\% Confidence Interval } \\
\hline & & & LCL & UCL \\
\hline Tuberculosis $(010-018)$ & 1 & 165 & 4 & 917 \\
\hline Mycoses (110-018) & 1 & 264 & 7 & 1,467 \\
\hline Sarcoidosis (135) & 0 & 0 & --- & --- \\
\hline Malignant neoplasms of trachea/bronchus/lung/pleura (162-163) & 4 & 50 & 14 & 128 \\
\hline Acute respiratory infections $(460-466)$ & 0 & 0 & --- & --- \\
\hline Other diseases of upper respiratory tract $(470-478)$ & 0 & 0 & --- & --- \\
\hline Pneumonia and influenza (480-487) & 22 & 118 & 74 & 179 \\
\hline Chronic obstructive pulmonary disease and allied conditions (490-496) & 19 & 116 & 70 & 181 \\
\hline Pneumoconiosis and other lung diseases - external agents (500-508) & 1 & 20 & 1 & 111 \\
\hline Other diseases of respiratory system (510-519) & 8 & 102 & 44 & 201 \\
\hline
\end{tabular}

ICD - International Classification of Diseases, $9^{\text {th }}$ Revision $\quad$ LCL - lower confidence limit $\quad$ UCL - upper confidence limit

NOTE: PMRs in bold are significantly different from $100(\mathrm{p}<0.05)$. PMRs in italics are based on fewer than five observed deaths. PMRs are based on underlying and contributing cause of death. Some values could not be calculated because the number of observed or expected deaths was zero; such values are indicated by ---. See appendices for source description, methods, ICD codes, and a list of selected states.

SOURCE: National Center for Health Statistics multiple-cause-of-death data 
Table 2-34. Landscape and horticultural workers, Hispanic: Proportionate mortality ratio (PMR) adjusted for age and sex by disease category, U.S. residents age 15 and over, selected states, 1988-1998

\begin{tabular}{|c|c|c|c|c|}
\hline \multirow[b]{2}{*}{ Disease Category (ICD Codes) } & \multirow{2}{*}{$\begin{array}{l}\text { Number } \\
\text { of Deaths }\end{array}$} & \multirow[b]{2}{*}{ PMR } & \multicolumn{2}{|c|}{ 95\% Confidence Interval } \\
\hline & & & LCL & UCL \\
\hline Tuberculosis (010-018) & 2 & 124 & 15 & 448 \\
\hline Mycoses (110-018) & 1 & 62 & 2 & 344 \\
\hline Sarcoidosis (135) & 0 & 0 & --- & --- \\
\hline Malignant neoplasms of trachea/bronchus/lung/pleura (162-163) & 10 & 71 & 34 & 131 \\
\hline Acute respiratory infections (460-466) & 0 & 0 & --- & --- \\
\hline Other diseases of upper respiratory tract (470-478) & 0 & 0 & --- & --- \\
\hline Pneumonia and influenza (480-487) & 23 & 87 & 55 & 131 \\
\hline Chronic obstructive pulmonary disease and allied conditions (490-496) & 17 & 88 & 51 & 141 \\
\hline Pneumoconiosis and other lung diseases - external agents (500-508) & 6 & 98 & 36 & 214 \\
\hline Other diseases of respiratory system (510-519) & 13 & 83 & 44 & 142 \\
\hline
\end{tabular}

ICD - International Classification of Diseases, $9^{\text {th }}$ Revision

LCL - lower confidence limit

UCL - upper confidence limit

NOTE: PMRs in bold are significantly different from $100(\mathrm{p}<0.05)$. PMRs in italics are based on fewer than five observed deaths. PMRs are based on underlying and contributing cause of death. Some values could not be calculated because the number of observed or expected deaths was zero; such values are indicated by ---. See appendices for source description, methods, ICD codes, and a list of selected states.

SOURCE: National Center for Health Statistics multiple-cause-of-death data 
Table 2-35. Forestry workers, Hispanic: Proportionate mortality ratio (PMR) adjusted for age and sex by disease category, U.S. residents age 15 and over, selected states, 1988-1998

\begin{tabular}{|c|c|c|c|c|}
\hline \multirow[b]{2}{*}{ Disease Category (ICD Codes) } & \multirow{2}{*}{$\begin{array}{l}\text { Number } \\
\text { of Deaths }\end{array}$} & \multirow[b]{2}{*}{ PMR } & \multicolumn{2}{|c|}{ 95\% Confidence Interval } \\
\hline & & & $\mathbf{L C L}$ & UCL \\
\hline Tuberculosis (010-018) & 1 & 142 & 4 & 789 \\
\hline Mycoses (110-018) & 0 & 0 & --- & --- \\
\hline Sarcoidosis (135) & 0 & 0 & --- & --- \\
\hline Malignant neoplasms of trachea/bronchus/lung/pleura (162-163) & 8 & 83 & 36 & 163 \\
\hline Acute respiratory infections (460-466) & 0 & 0 & --- & --- \\
\hline Other diseases of upper respiratory tract (470-478) & 0 & 0 & --- & --- \\
\hline Pneumonia and influenza (480-487) & 18 & 85 & 50 & 134 \\
\hline Chronic obstructive pulmonary disease and allied conditions (490-496) & 22 & 116 & 73 & 176 \\
\hline Pneumoconiosis and other lung diseases - external agents (500-508) & 3 & 52 & 11 & 152 \\
\hline Other diseases of respiratory system (510-519) & 7 & 77 & 31 & 159 \\
\hline
\end{tabular}

ICD - International Classification of Diseases, $9^{\text {th }}$ Revision

LCL - lower confidence limit

UCL - upper confidence limit

NOTE: PMRs in bold are significantly different from $100(\mathrm{p}<0.05)$. PMRs in italics are based on fewer than five observed deaths. PMRs are based on underlying and contributing cause of death. Some values could not be calculated because the number of observed or expected deaths was zero; such values are indicated by ---. See appendices for source description, methods, ICD codes, and a list of selected states.

SOURCE: National Center for Health Statistics multiple-cause-of-death data 
Table 2-36. Fishery workers, Hispanic: Proportionate mortality ratio (PMR) adjusted for age and sex by disease category, U.S. residents age 15 and over, selected states, 1988-1998

\begin{tabular}{|c|c|c|c|c|}
\hline \multirow[b]{2}{*}{ Disease Category (ICD Codes) } & \multirow{2}{*}{$\begin{array}{l}\text { Number } \\
\text { of Deaths }\end{array}$} & \multirow[b]{2}{*}{ PMR } & \multicolumn{2}{|c|}{ 95\% Confidence Interval } \\
\hline & & & LCL & $\mathbf{U C L}$ \\
\hline Tuberculosis (010-018) & 1 & 888 & 22 & 4,933 \\
\hline Mycoses (110-018) & 0 & 0 & --- & --- \\
\hline Sarcoidosis (135) & 0 & 0 & --- & --- \\
\hline Malignant neoplasms of trachea/bronchus/lung/pleura (162-163) & 0 & 0 & --- & --- \\
\hline Acute respiratory infections (460-466) & 0 & 0 & --- & --- \\
\hline Other diseases of upper respiratory tract (470-478) & 0 & 0 & --- & --- \\
\hline Pneumonia and influenza (480-487) & 2 & 81 & 10 & 292 \\
\hline Chronic obstructive pulmonary disease and allied conditions (490-496) & 3 & 149 & 31 & 436 \\
\hline Pneumoconiosis and other lung diseases - external agents (500-508) & 0 & 0 & --- & --- \\
\hline Other diseases of respiratory system (510-519) & 0 & 0 & --- & --- \\
\hline
\end{tabular}

ICD - International Classification of Diseases, $9^{\text {th }}$ Revision

LCL - lower confidence limit

UCL - upper confidence limit

NOTE: PMRs in bold are significantly different from $100(\mathrm{p}<0.05)$. PMRs in italics are based on fewer than five observed deaths. PMRs are based on underlying and contributing cause of death. Some values could not be calculated because the number of observed or expected deaths was zero; such values are indicated by ---. See appendices for source description, methods, ICD codes, and a list of selected states.

SOURCE: National Center for Health Statistics multiple-cause-of-death data 
Figure 2-21. Tuberculosis: Proportionate mortality ratio (PMR) adjusted for age and sex by agricultural group and race/ethnicity, U.S. residents age 15 and over, selected states, 1988-1998

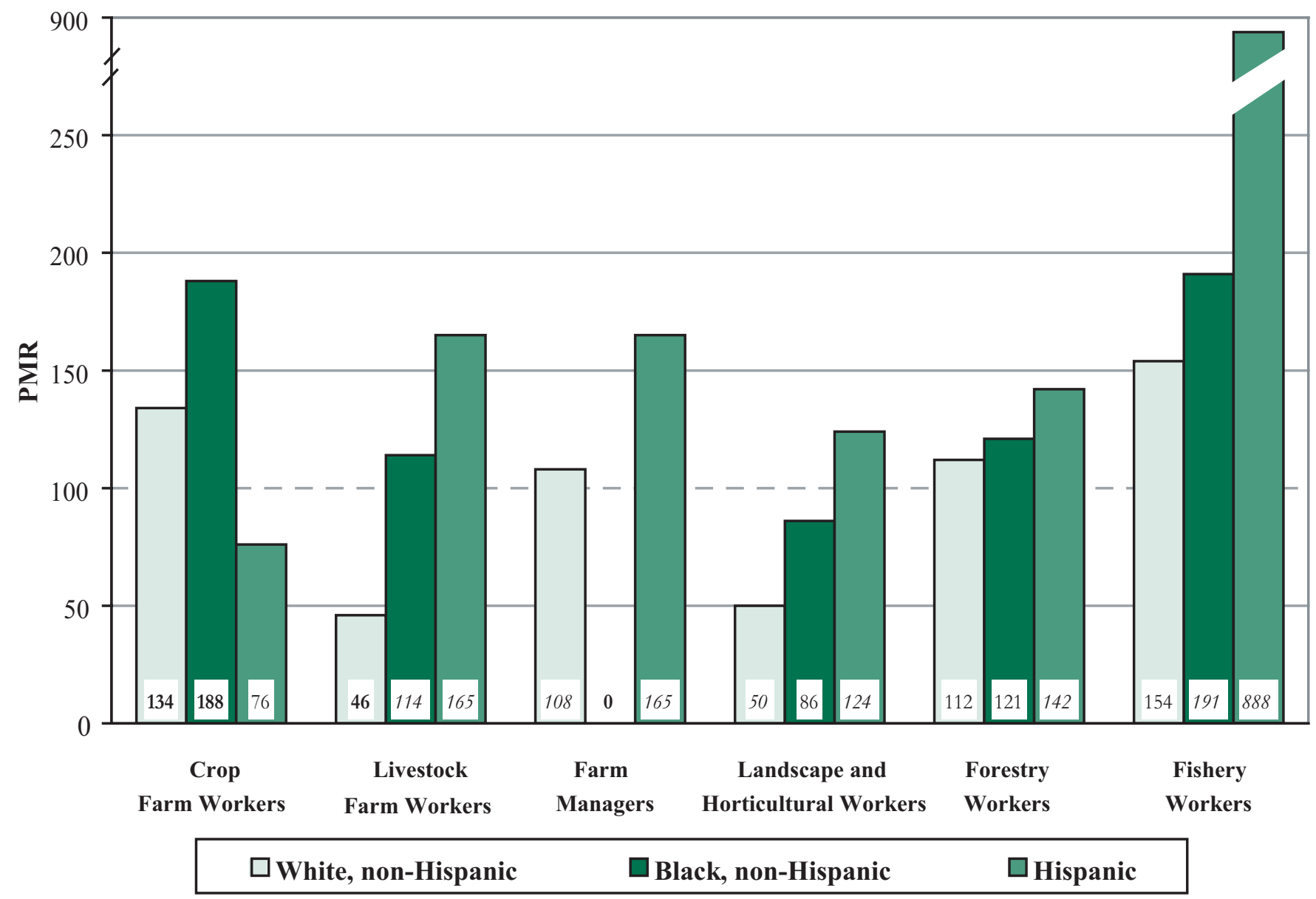

ICD - International Classification of Diseases, $9^{\text {th }}$ Revision

NOTE: Tuberculosis = ICD-9 codes 010-018. PMRs in bold are significantly different from $100(\mathrm{p}<0.05)$. PMRs in italics are based on fewer than five observed deaths. PMRs are based on underlying and contributing cause of death. See appendices for source description, methods, ICD codes, and a list of selected states.

SOURCE: National Center for Health Statistics multiple-cause-of-death data 
Figure 2-22. Mycoses: Proportionate mortality ratio (PMR) adjusted for age and sex by agricultural group and race/ethnicity, U.S. residents age 15 and over, selected states, 1988-1998

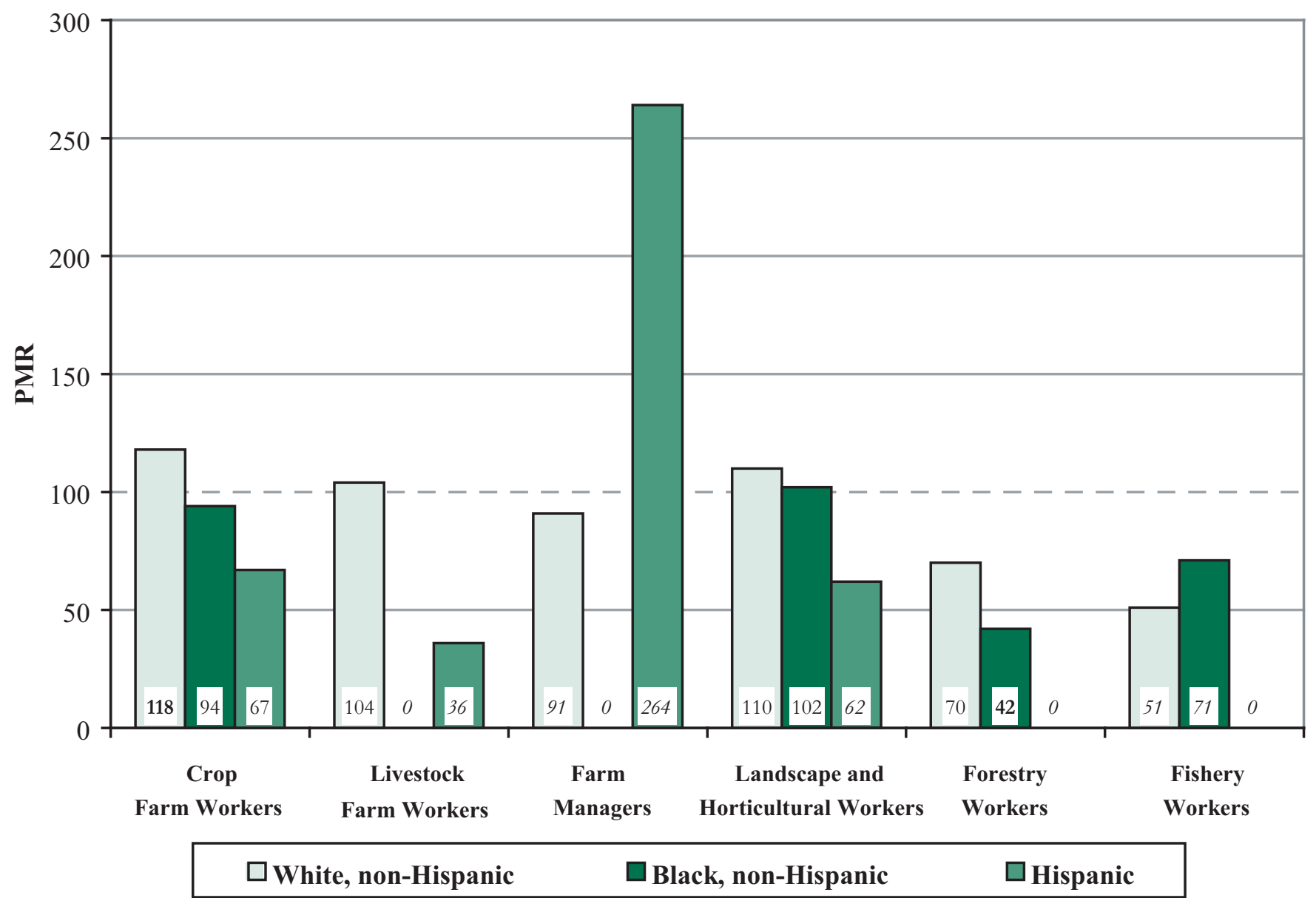

ICD - International Classification of Diseases, $9^{\text {th }}$ Revision

NOTE: Mycoses = ICD-9 codes 110-118. PMRs in bold are significantly different from $100(\mathrm{p}<0.05)$. PMRs in italics are based on fewer than five

observed deaths. PMRs are based on underlying and contributing cause of death. See appendices for source description, methods, ICD codes, and a list of selected states.

SOURCE: National Center for Health Statistics multiple-cause-of-death data 
Figure 2-23. Sarcoidosis: Proportionate mortality ratio (PMR) adjusted for age and sex by agricultural group and race/ethnicity, U.S. residents age 15 and over, selected states, 1988-1998

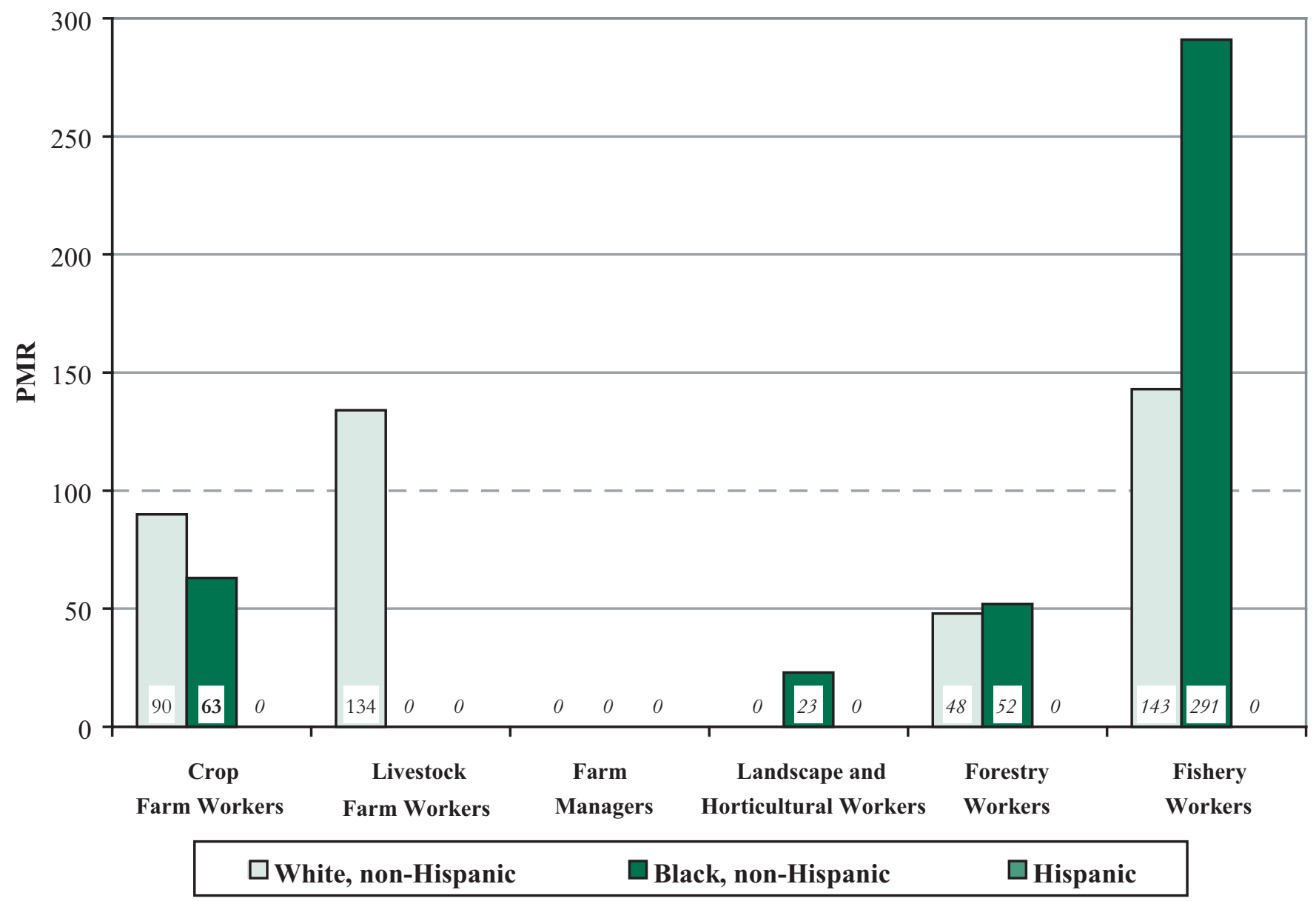

ICD - International Classification of Diseases, $9^{\text {th }}$ Revision

NOTE: Sarcoidosis = ICD-9 code 135. PMRs in bold are significantly different from $100(\mathrm{p}<0.05)$. PMRs in italics are based on fewer than five observed deaths. PMRs are based on underlying and contributing cause of death. See appendices for source description, methods, ICD codes, and a list of selected states.

SOURCE: National Center for Health Statistics multiple-cause-of-death data 
Figure 2-24. Malignant neoplasms of trachea/bronchus/lung/pleura: Proportionate mortality ratio (PMR) adjusted for age and sex by agricultural group and race/ ethnicity, U.S. residents age 15 and over, selected states, 1988-1998

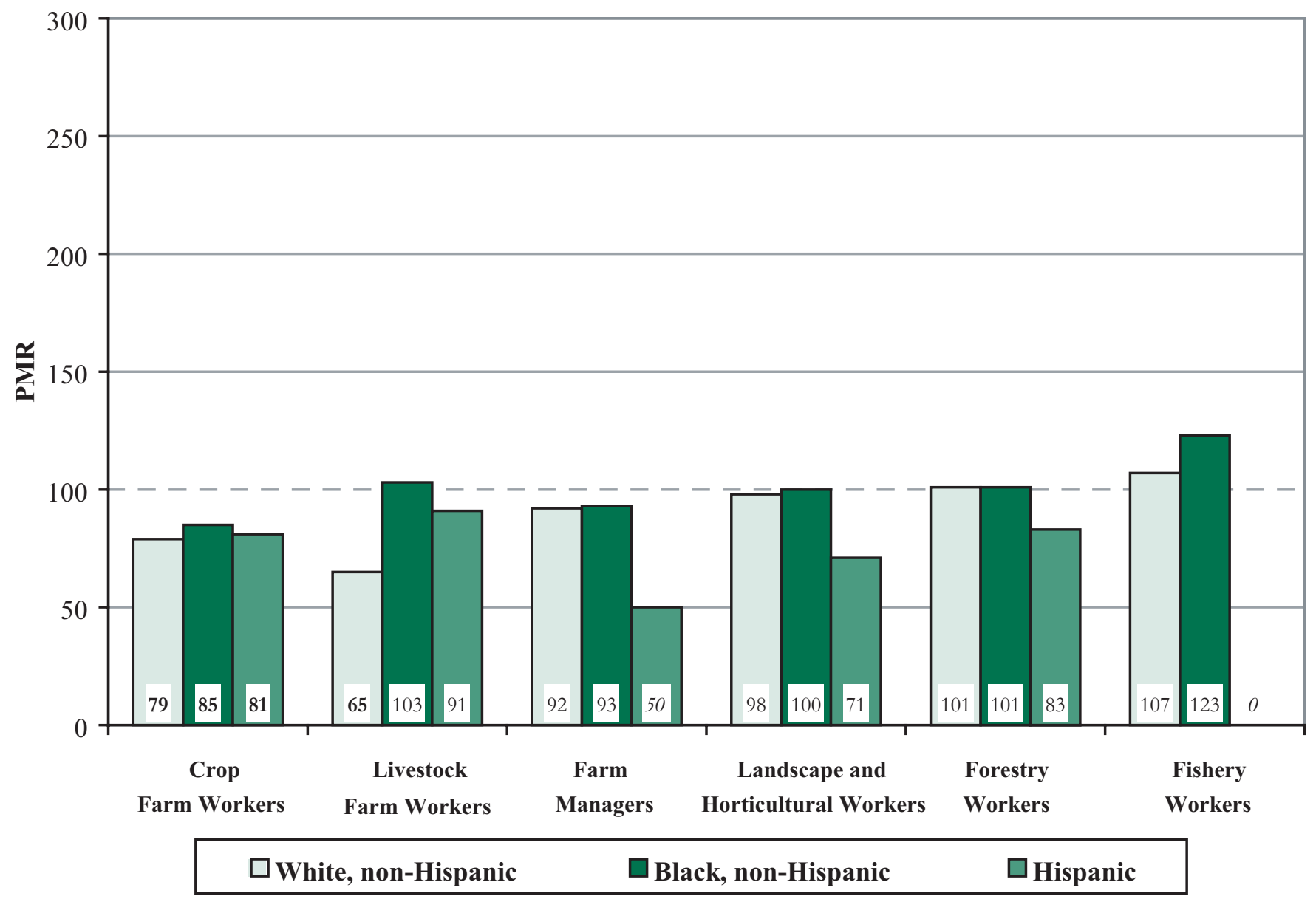

ICD - International Classification of Diseases, $9^{\text {th }}$ Revision

NOTE: Malignant neoplasms of trachea/bronchus/lung/pleura = ICD-9 codes 162-163. PMRs in bold are significantly different from 100 ( $<<0.05$ ).

PMRs in italics are based on fewer than five observed deaths. PMRs are based on underlying and contributing cause of death. See appendices for source description, methods, ICD codes, and a list of selected states.

SOURCE: National Center for Health Statistics multiple-cause-of-death data 
Figure 2-25. Acute respiratory infections: Proportionate mortality ratio (PMR) adjusted for age and sex by agricultural group and race/ethnicity, U.S. residents age 15 and over, selected states, 1988-1998

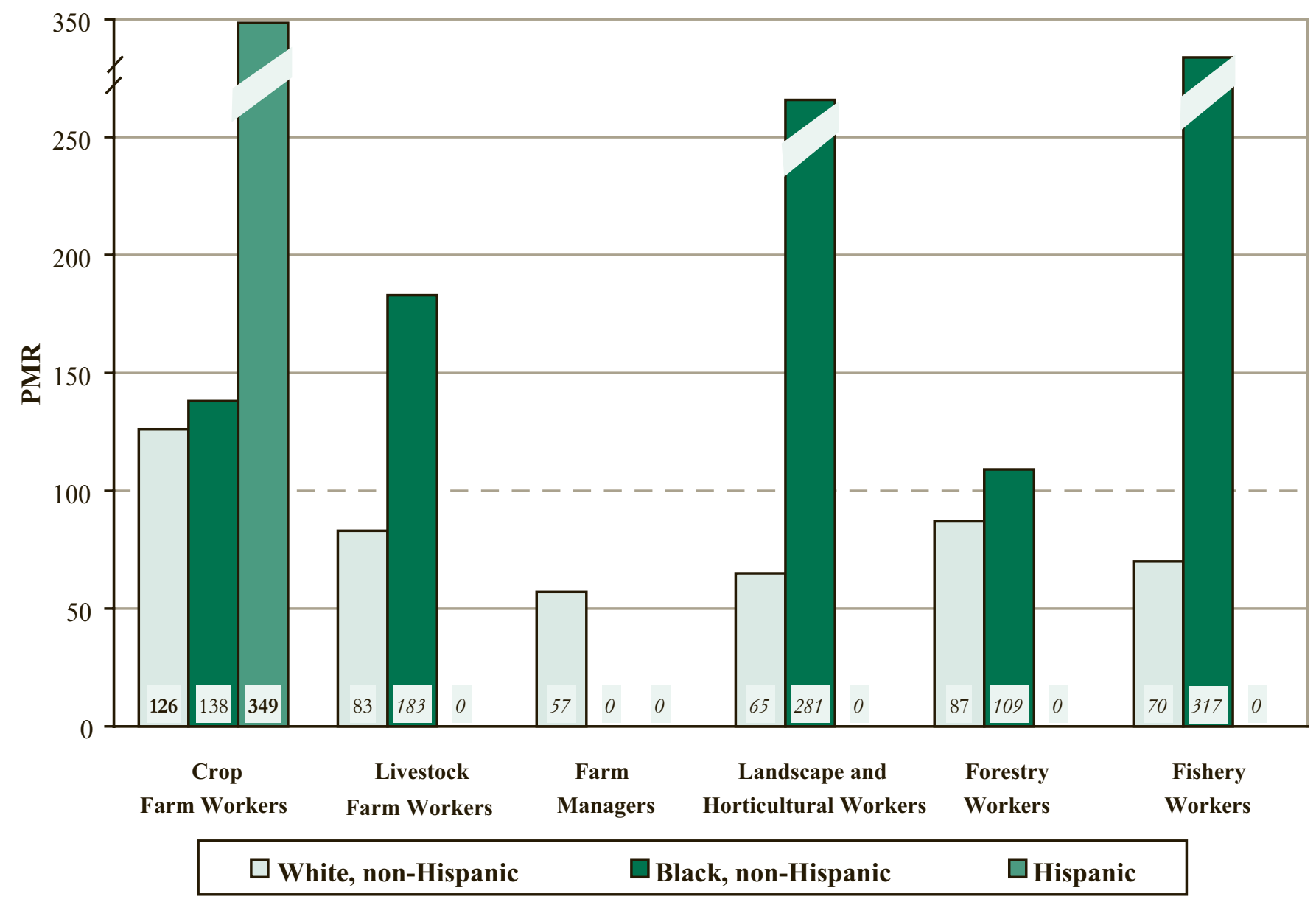

ICD - International Classification of Diseases, $9^{\text {th }}$ Revision

NOTE: Acute respiratory infections $=$ ICD-9 codes 460-466. PMRs in bold are significantly different from 100 ( $<<0.05$ ). PMRs in italics are based on fewer than five observed deaths. PMRs are based on underlying and contributing cause of death. See appendices for source description, methods, ICD codes, and a list of selected states.

SOURCE: National Center for Health Statistics multiple-cause-of-death data 
Figure 2-26. Other diseases of upper respiratory tract: Proportionate mortality ratio (PMR) adjusted for age and sex by agricultural group and race/ethnicity, U.S. residents age 15 and over, selected states, 1988-1998

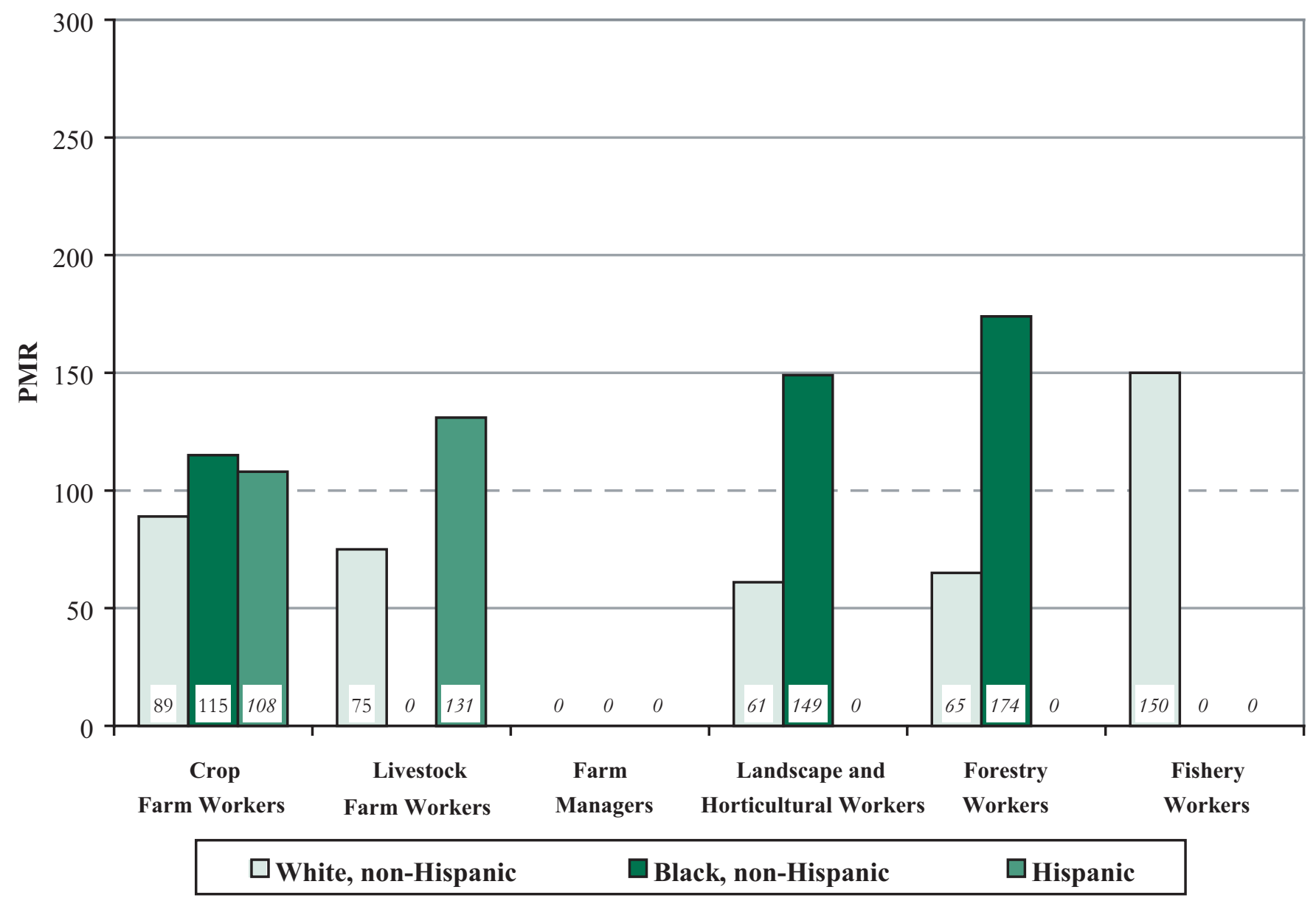

ICD - International Classification of Diseases, $9^{\text {th }}$ Revision

NOTE: Other diseases of upper respiratory tract $=$ ICD-9 codes 470-478. PMRs in bold are significantly different from 100 ( $<<0.05$ ). PMRs in italics are based on fewer than five observed deaths. PMRs are based on underlying and contributing cause of death. See appendices for source description, methods, ICD codes, and a list of selected states.

SOURCE: National Center for Health Statistics multiple-cause-of-death data 
Figure 2-27. Pneumonia and influenza: Proportionate mortality ratio (PMR) adjusted for age and sex by agricultural group and race/ethnicity, U.S. residents age 15 and over, selected states, 1988-1998

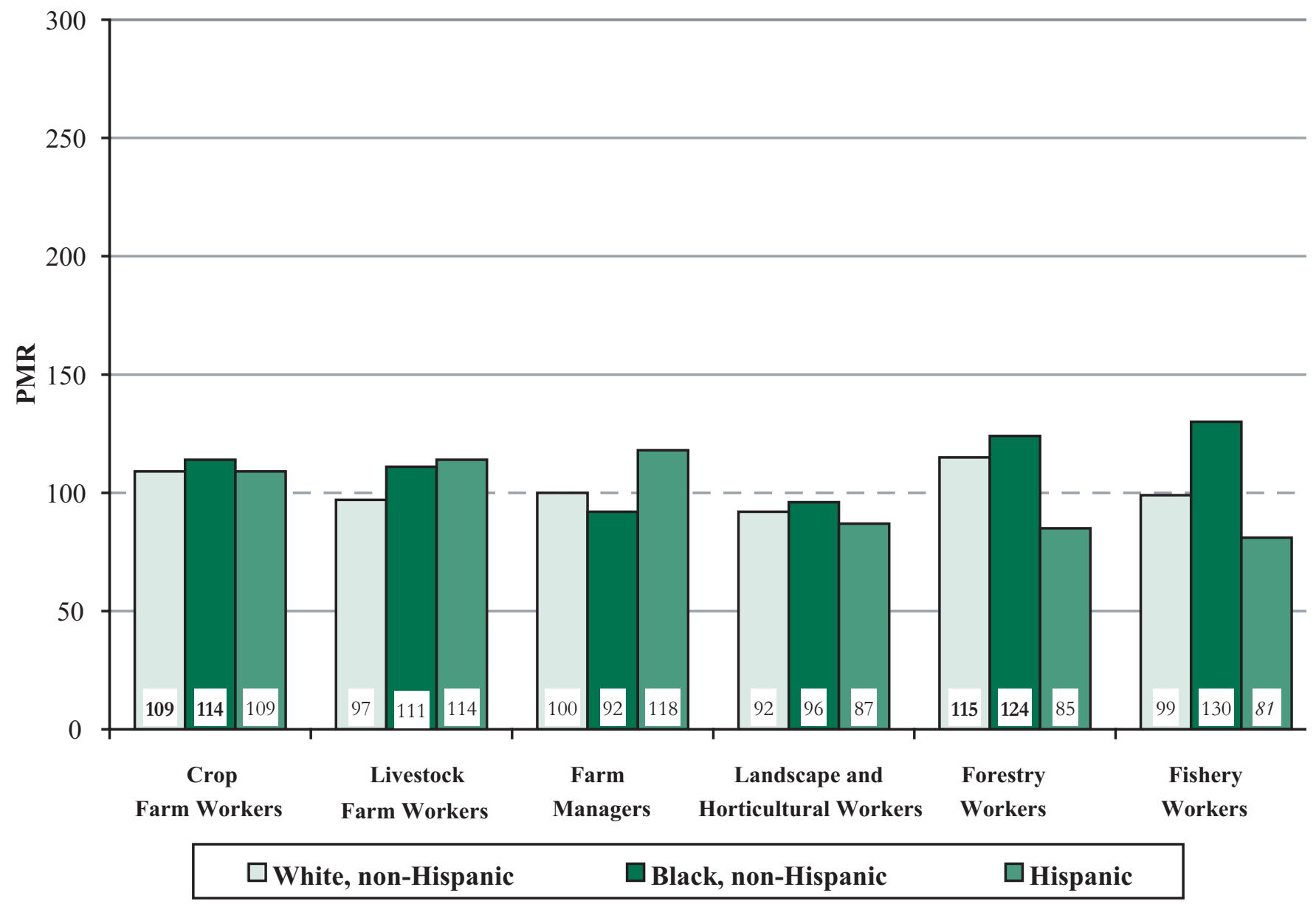

ICD - International Classification of Diseases, $9^{\text {th }}$ Revision

NOTE: Pneumonia and influenza = ICD-9 codes 480-487. PMRs in bold are significantly different from 100 ( $\mathrm{p}<0.05$ ). PMRs in italics are based on fewer than five observed deaths. PMRs are based on underlying and contributing cause of death. See appendices for source description, methods, ICD codes, and a list of selected states.

SOURCE: National Center for Health Statistics multiple-cause-of-death data 
Figure 2-28. Chronic obstructive pulmonary disease and allied conditions: Proportionate mortality ratio (PMR) adjusted for age and sex by agricultural group and race/ethnicity, U.S. residents age 15 and over, selected states, 1988-1998

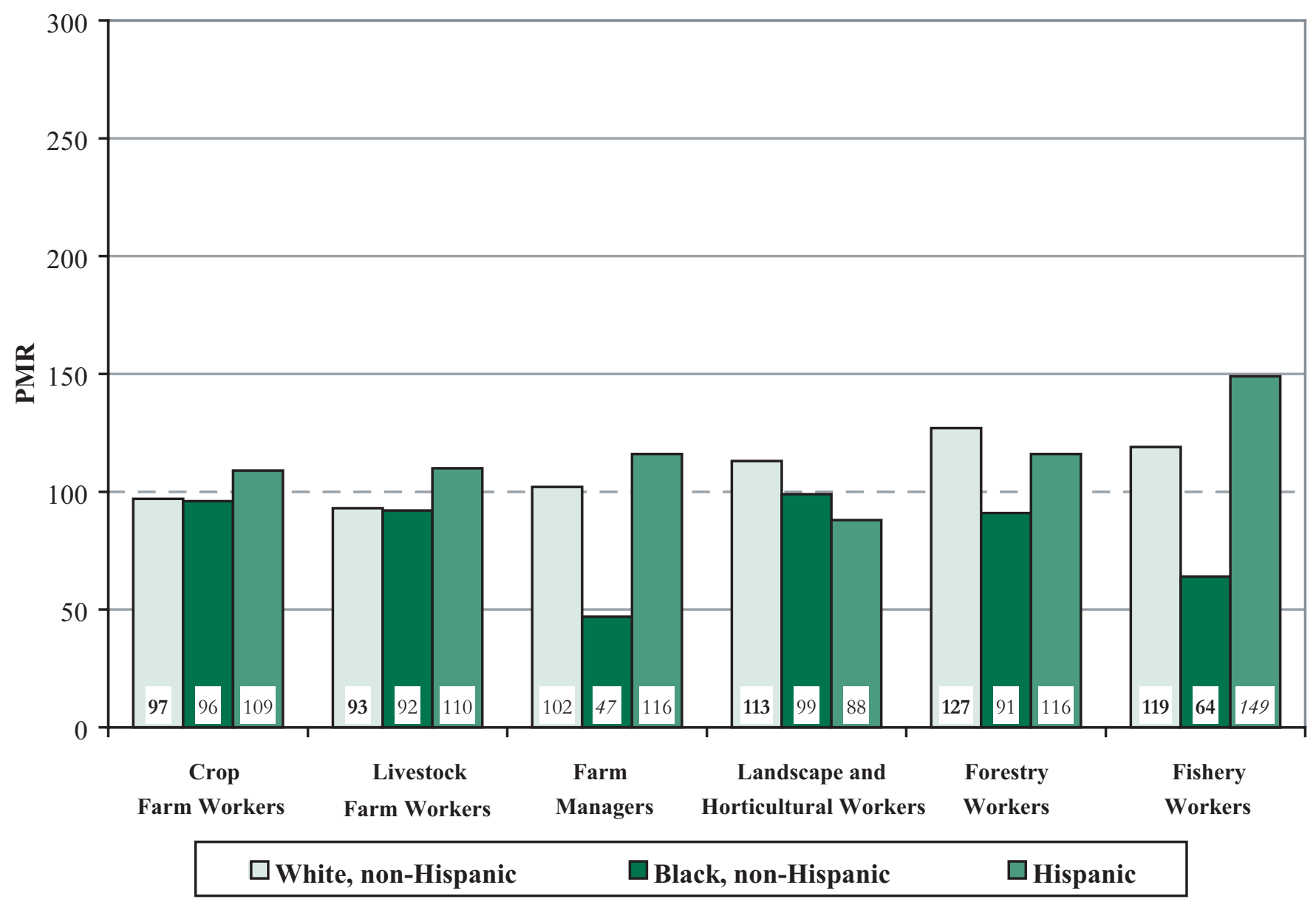

ICD - International Classification of Diseases, $9^{\text {th }}$ Revision

NOTE: Chronic obstructive pulmonary disease and allied conditions = ICD-9 codes 490-496. PMRs in bold are significantly different from 100 ( $\mathrm{p}<0.05$ ).

PMRs in italics are based on fewer than five observed deaths. PMRs are based on underlying and contributing cause of death. See appendices for source description, methods, ICD codes, and a list of selected states.

SOURCE: National Center for Health Statistics multiple-cause-of-death data 
Figure 2-29. Pneumoconioses and other lung diseases-external agents: Proportionate mortality ratio (PMR) adjusted for age and sex by agricultural group and race/ethnicity, U.S. residents age 15 and over, selected states, 1988-1998

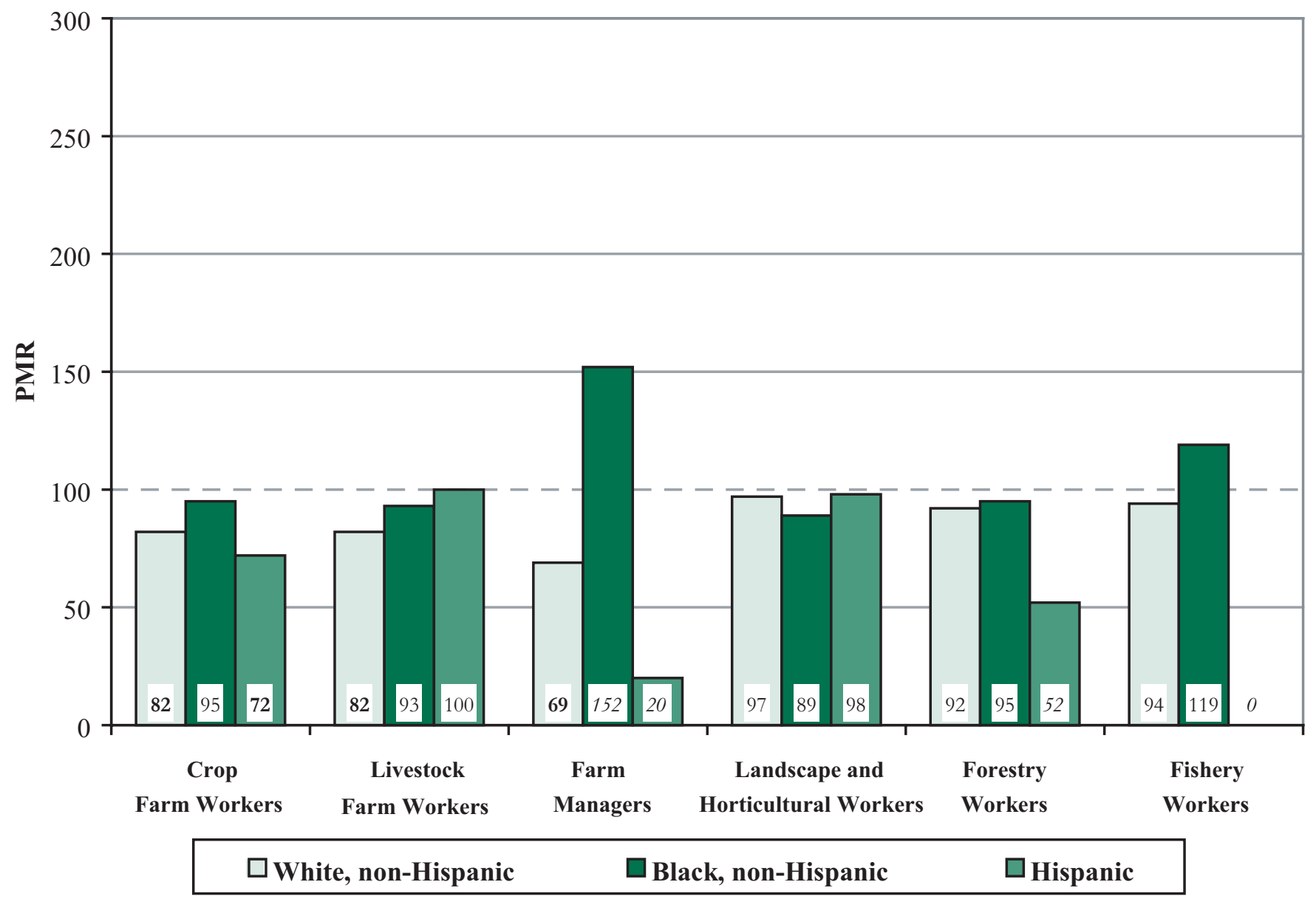

ICD - International Classification of Diseases, $9^{\text {th }}$ Revision

NOTE: Pneumoconioses and other lung diseases - external agents = ICD-9 codes 500-508. PMRs in bold are significantly different from 100 ( $<<0.05$ ). PMRs in italics are based on fewer than five observed deaths. PMRs are based on underlying and contributing cause of death. See appendices for source description, methods, ICD codes, and a list of selected states.

SOURCE: National Center for Health Statistics multiple-cause-of-death data 
Figure 2-30. Other diseases of respiratory system: Proportionate mortality ratio (PMR) adjusted for age and sex by agricultural group and race/ethnicity, U.S. residents age 15 and over, selected states, 1988-1998

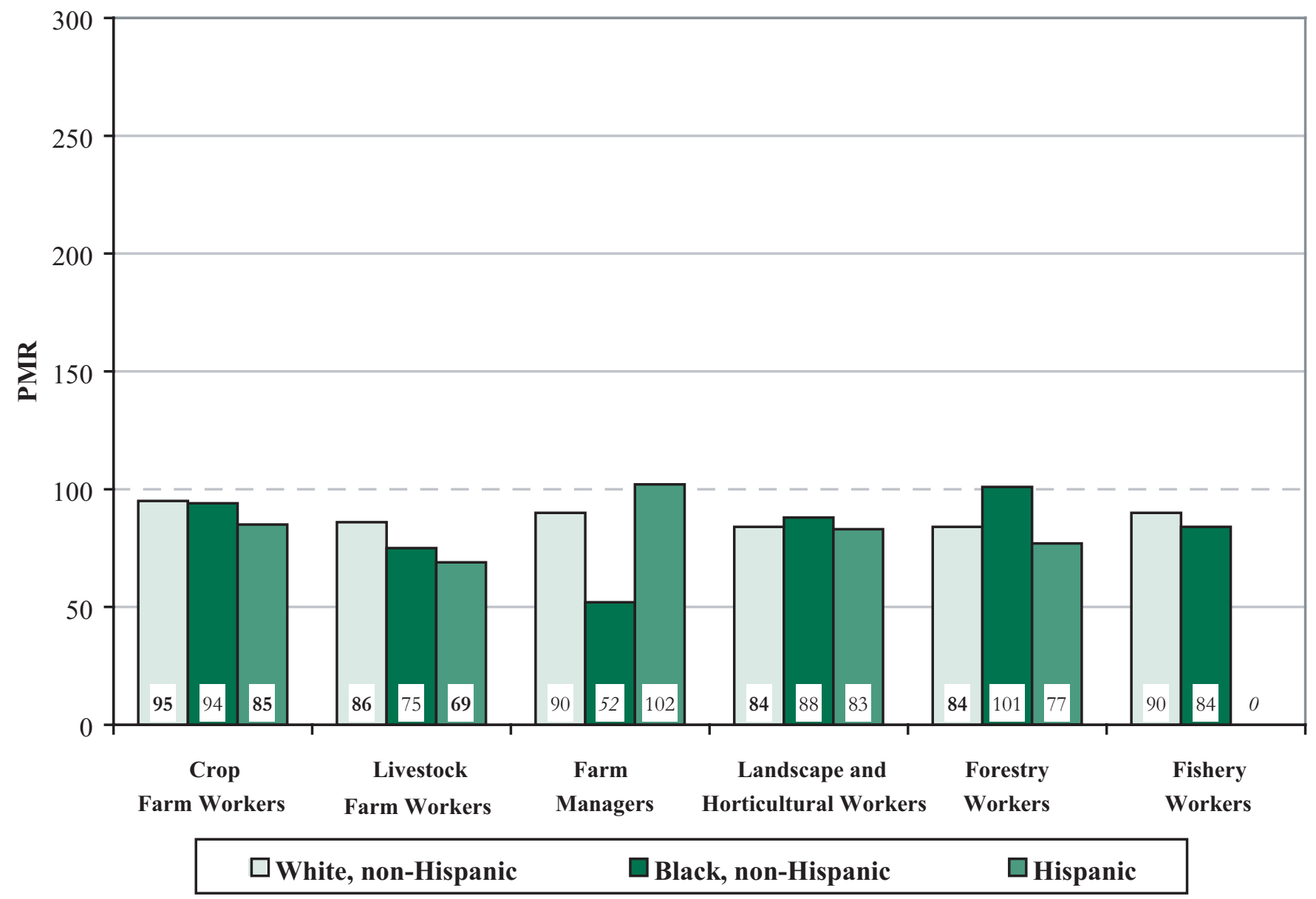

ICD - International Classification of Diseases, $9^{\text {th }}$ Revision

NOTE: Other diseases of respiratory system $=$ ICD-9 codes 510-519. PMRs in bold are significantly different from 100 ( $<<0.05$ ). PMRs in italics are based on fewer than five observed deaths. PMRs are based on underlying and contributing cause of death. See appendices for source description, methods, ICD codes, and a list of selected states.

SOURCE: National Center for Health Statistics multiple-cause-of-death data 
Table 2-37. Crop farm workers: Proportionate mortality ratio (PMR) adjusted for age, sex, and race/ ethnicity for tuberculosis, U.S. residents age 15 and over, selected states, 1988-1998

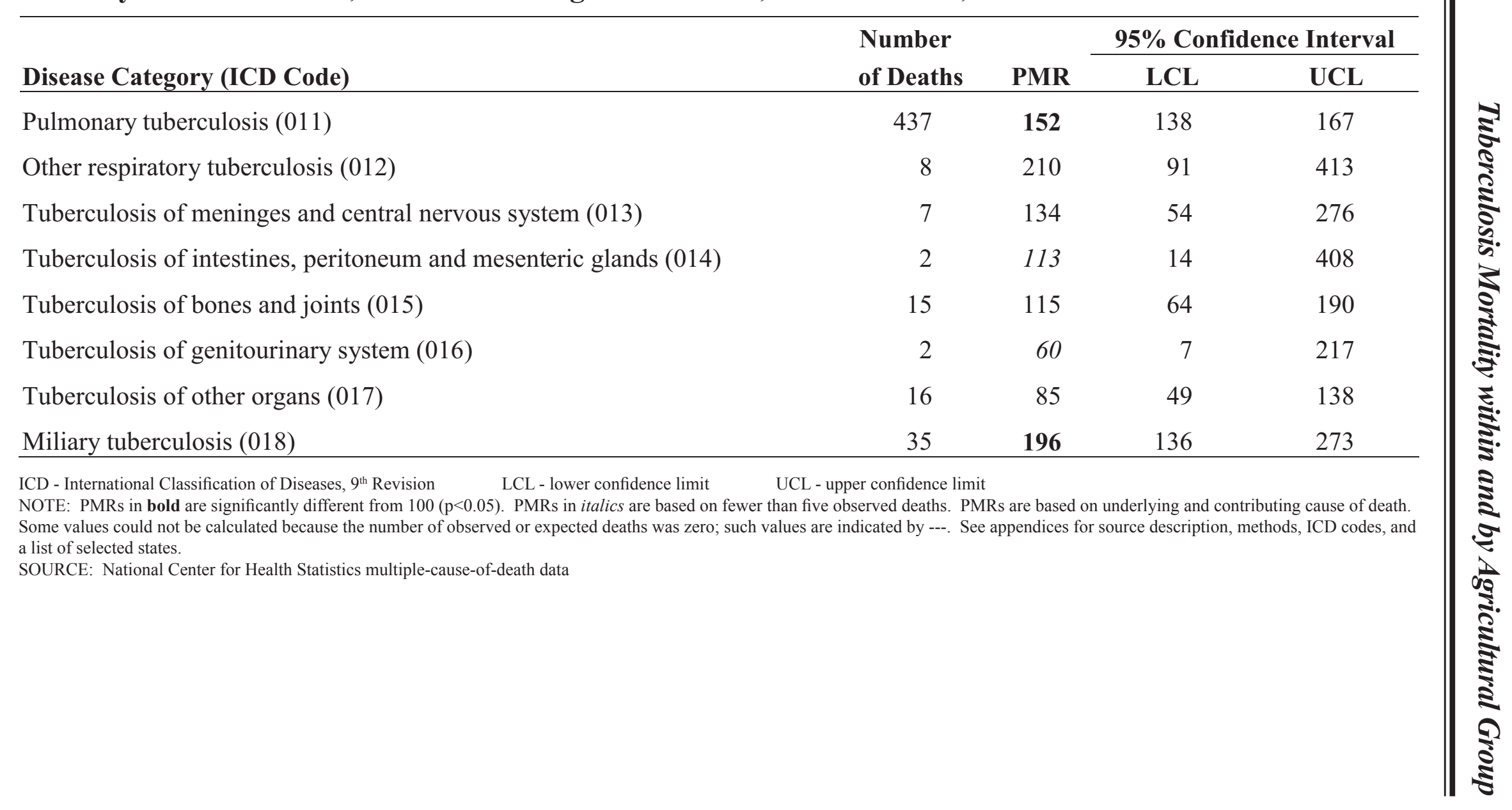


Table 2-38. Livestock farm workers: Proportionate mortality ratio (PMR) adjusted for age, sex, and race/ethnicity for tuberculosis, U.S. residents age 15 and over, selected states, 1988-1998

\begin{tabular}{|c|c|c|c|c|}
\hline \multirow[b]{2}{*}{ Disease Category (ICD Code) } & \multirow{2}{*}{$\begin{array}{l}\text { Number } \\
\text { of Deaths }\end{array}$} & \multirow[b]{2}{*}{ PMR } & \multicolumn{2}{|c|}{ 95\% Confidence Interval } \\
\hline & & & $\mathbf{L C L}$ & UCL \\
\hline Pulmonary tuberculosis $(011)$ & 37 & 60 & 43 & 83 \\
\hline Other respiratory tuberculosis $(012)$ & 5 & 675 & 218 & 1,577 \\
\hline Tuberculosis of meninges and central nervous system (013) & 5 & 546 & 177 & 1,246 \\
\hline Tuberculosis of intestines, peritoneum and mesenteric glands (014) & 0 & 0 & --- & --- \\
\hline Tuberculosis of bones and joints $(015)$ & 2 & 65 & 8 & 235 \\
\hline Tuberculosis of genitourinary system $(016)$ & 1 & 130 & 3 & 722 \\
\hline Tuberculosis of other organs (017) & 2 & 44 & 5 & 159 \\
\hline Miliary tuberculosis (018) & 4 & 143 & 39 & 366 \\
\hline
\end{tabular}


Table 2-39. Farm managers: Proportionate mortality ratio (PMR) adjusted for age, sex, and race/ ethnicity for tuberculosis, U.S. residents age 15 and over, selected states, 1988-1998

\begin{tabular}{|c|c|c|c|c|}
\hline Disease Category (ICD Code) & $\begin{array}{l}\text { Number } \\
\text { of Deaths }\end{array}$ & PMR & \multicolumn{2}{|c|}{ 95\% Confidence Interval } \\
\hline Pulmonary tuberculosis (011) & 4 & 84 & 23 & 215 \\
\hline Tuberculosis of meninges and central nervous system (013) & 0 & 0 & --- & --- \\
\hline Tuberculosis of intestines, peritoneum and mesenteric glands (014) & 0 & 0 & --- & --- \\
\hline Tuberculosis of genitourinary system (016) & 1 & 2,053 & 52 & 11,406 \\
\hline Tuberculosis of other organs (017) & 0 & 0 & --- & --- \\
\hline Miliary tuberculosis $(018)$ & 0 & 0 & --- & --- \\
\hline \multicolumn{5}{|c|}{$\begin{array}{l}\text { ICD - International Classification of Diseases, } 9^{\text {th }} \text { Revision } \quad \text { LCL - lower confidence limit } \quad \text { UCL - upper confidence limit } \\
\text { NOTE: PMRRs in bold are significantly different from } 100 \text { ( }<<0.05) \text {. PMRs in italics are based on fewer than five observed deaths. PMRs are based on underlying and contributing cause of death. } \\
\text { Some values could not be calculated because the number of observed or expected deaths was zero; such values are indicated by ---. See appendices for source description, methods, ICD codes, and } \\
\text { a list of selected states. } \\
\text { SOURCE: National Center for Health Statistics multiple-cause-of-death data }\end{array}$} \\
\hline
\end{tabular}


Table 2-40. Landscape and horticultural workers: Proportionate mortality ratio (PMR) adjusted for age, sex, and race/ethnicity for tuberculosis, U.S. residents age 15 and over, selected states, 1988-1998

\begin{tabular}{|c|c|c|c|c|}
\hline \multirow[b]{2}{*}{ Disease Category (ICD Code) } & \multirow{2}{*}{$\begin{array}{l}\text { Number } \\
\text { of Deaths }\end{array}$} & \multirow[b]{2}{*}{ PMR } & \multicolumn{2}{|c|}{ 95\% Confidence Interval } \\
\hline & & & LCL & UCL \\
\hline Pulmonary tuberculosis $(011)$ & 14 & 88 & 48 & 148 \\
\hline Other respiratory tuberculosis $(012)$ & 0 & 0 & --- & --- \\
\hline Tuberculosis of meninges and central nervous system (013) & 1 & 209 & 5 & 1,161 \\
\hline Tuberculosis of intestines, peritoneum and mesenteric glands (014) & 0 & 0 & --- & --- \\
\hline Tuberculosis of bones and joints $(015)$ & 0 & 0 & --- & --- \\
\hline Tuberculosis of genitourinary system (016) & 0 & 0 & --- & --- \\
\hline Tuberculosis of other organs $(017)$ & 1 & 88 & 2 & 489 \\
\hline Miliary tuberculosis (018) & 0 & 0 & --- & --- \\
\hline
\end{tabular}

ICD - International Classification of Diseases, $9^{\text {th }}$ Revision

LCL - lower confidence limit

UCL - upper confidence limit

NOTE: PMRs in bold are significantly different from $100(\mathrm{p}<0.05)$. PMRs in italics are based on fewer than five observed deaths. PMRs are based on underlying and contributing cause of death. Some values could not be calculated because the number of observed or expected deaths was zero; such values are indicated by ---. See appendices for source description, methods, ICD codes, and a list of selected states.

SOURCE: National Center for Health Statistics multiple-cause-of-death data 
Table 2-41. Forestry workers: Proportionate mortality ratio (PMR) adjusted for age, sex, and race/ ethnicity for tuberculosis, U.S. residents age 15 and over, selected states, 1988-1998

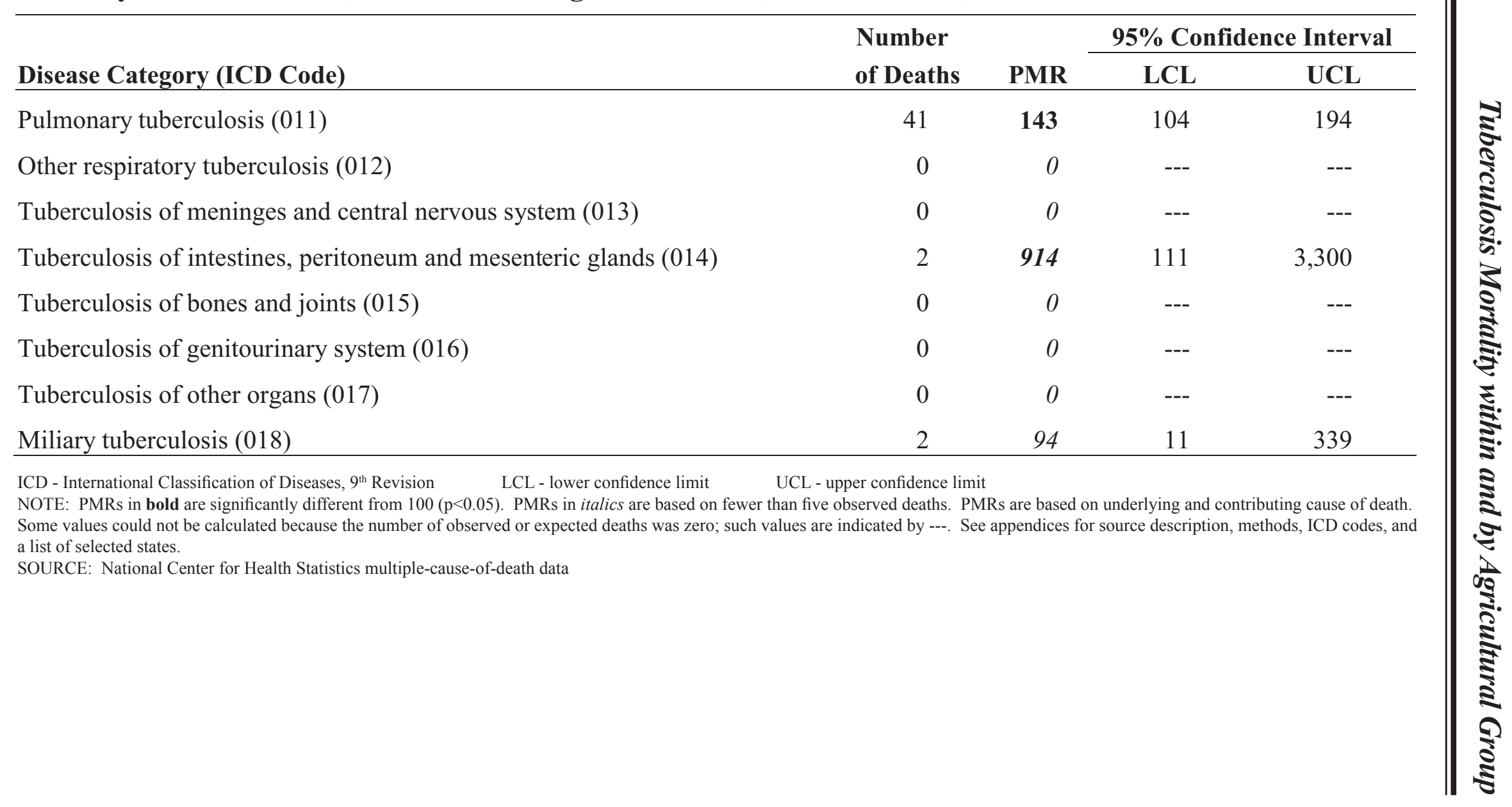


Table 2-42. Fishery workers: Proportionate mortality ratio (PMR) adjusted for age, sex, and race/ ethnicity for tuberculosis, U.S. residents age 15 and over, selected states, 1988-1998

\begin{tabular}{|c|c|c|c|c|}
\hline \multirow[b]{2}{*}{ Disease Category (ICD Code) } & \multirow{2}{*}{$\begin{array}{l}\text { Number } \\
\text { of Deaths }\end{array}$} & \multirow[b]{2}{*}{ PMR } & \multicolumn{2}{|c|}{ 95\% Confidence Interval } \\
\hline & & & $\mathbf{L C L}$ & $\mathbf{U C L}$ \\
\hline Pulmonary tuberculosis (011) & 10 & 156 & 75 & 287 \\
\hline Other respiratory tuberculosis $(012)$ & 0 & 0 & --- & --- \\
\hline Tuberculosis of meninges and central nervous system (013) & 0 & 0 & --- & --- \\
\hline Tuberculosis of intestines, peritoneum and mesenteric glands (014) & 0 & 0 & --- & --- \\
\hline Tuberculosis of bones and joints (015) & 0 & 0 & --- & --- \\
\hline Tuberculosis of genitourinary system (016) & 1 & 1,771 & 45 & 9,839 \\
\hline Tuberculosis of other organs $(017)$ & 1 & 218 & 6 & 1,211 \\
\hline Miliary tuberculosis (018) & 2 & 411 & 50 & 1,484 \\
\hline
\end{tabular}

ICD - International Classification of Diseases, $9^{\text {th }}$ Revision

LCL - lower confidence limit

UCL - upper confidence limit

NOTE: PMRs in bold are significantly different from $100(\mathrm{p}<0.05)$. PMRs in italics are based on fewer than five observed deaths. PMRs are based on underlying and contributing cause of death. Some values could not be calculated because the number of observed or expected deaths was zero; such values are indicated by ---. See appendices for source description, methods, ICD codes, and a list of selected states.

SOURCE: National Center for Health Statistics multiple-cause-of-death data 
Figure 2-31. Pulmonary tuberculosis: Proportionate mortality ratio (PMR) adjusted for age, sex, and race/ethnicity by agricultural group, U.S. residents age 15 and over, selected states, 1988-1998

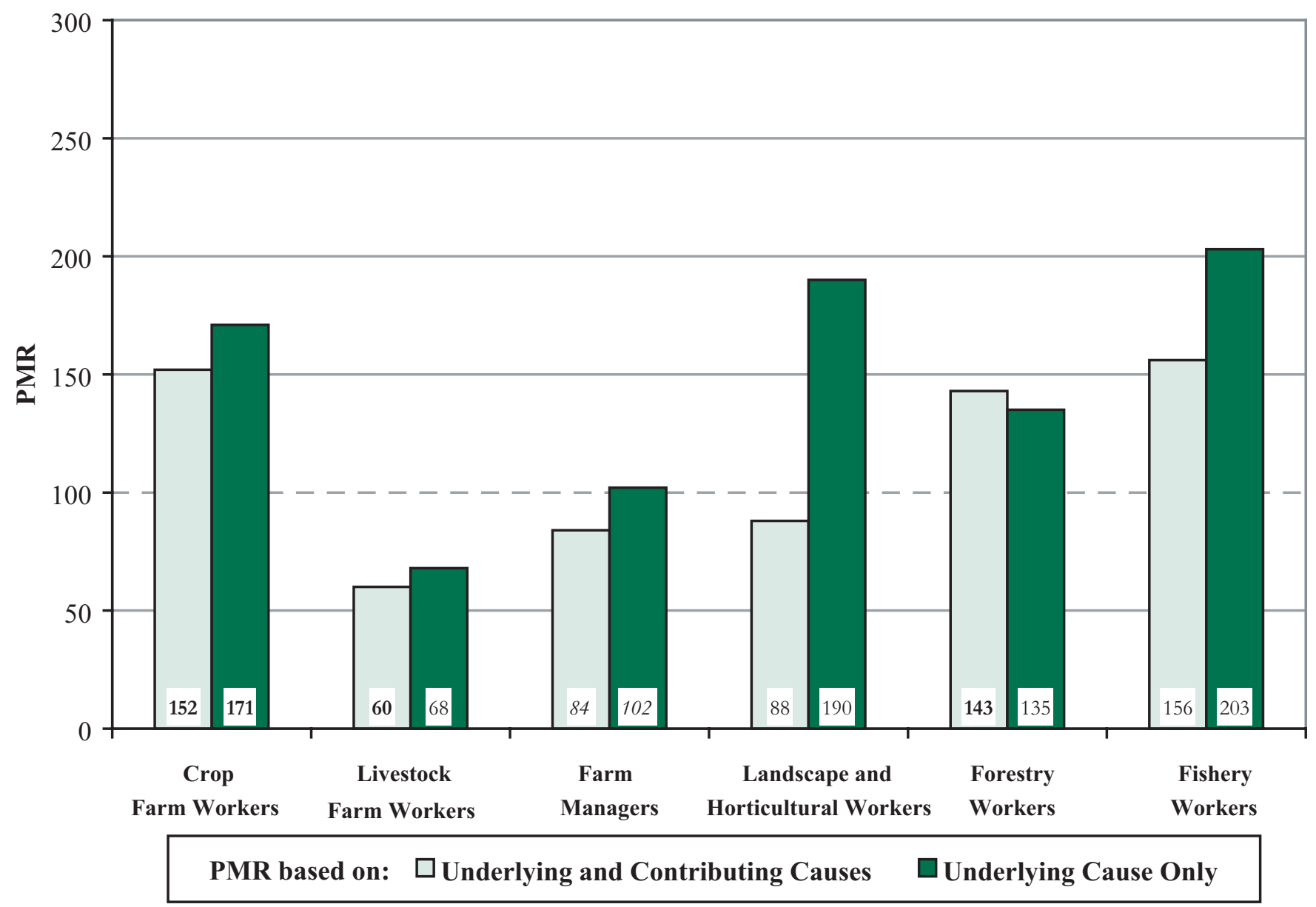

ICD - International Classification of Diseases, $9^{\text {th }}$ Revision

NOTE: Pulmonary tuberculosis = ICD-9 code 011. PMRs in bold are significantly different from $100(\mathrm{p}<0.05)$. PMRs in italics are based on fewer than five observed deaths. PMRs are based on underlying and contributing cause of death. See appendices for source description, methods, ICD codes, and a list of selected states.

SOURCE: National Center for Health Statistics multiple-cause-of-death data 
Figure 2-32. Other respiratory tuberculosis: Proportionate mortality ratio (PMR) adjusted for age, sex, and race/ethnicity by agricultural group, U.S. residents age 15 and over, selected states, 1988-1998

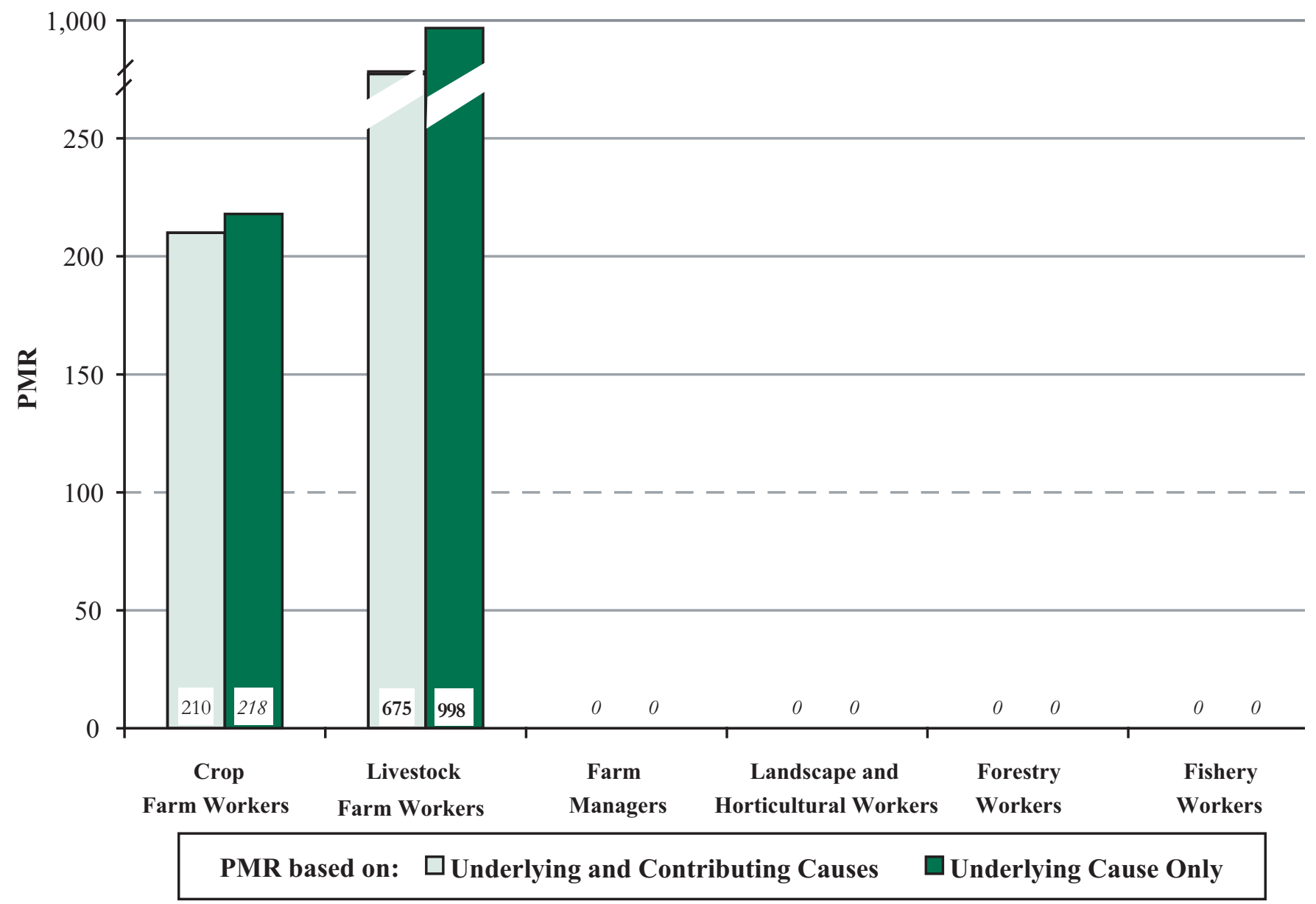

ICD - International Classification of Diseases, $9^{\text {th }}$ Revision

NOTE: Other respiratory tuberculosis = ICD-9 code 012. PMRs in bold are significantly different from 100 ( $<<0.05$ ). PMRs in italics are based on fewer than five observed deaths. PMRs are based on underlying and contributing cause of death. See appendices for source description, methods, ICD codes, and a list of selected states.

SOURCE: National Center for Health Statistics multiple-cause-of-death data 
Figure 2-33. Tuberculosis of meninges and central nervous system: Proportionate mortality ratio (PMR) adjusted for age, sex, and race/ethnicity by agricultural group, U.S. residents age 15 and over, selected states, 1988-1998

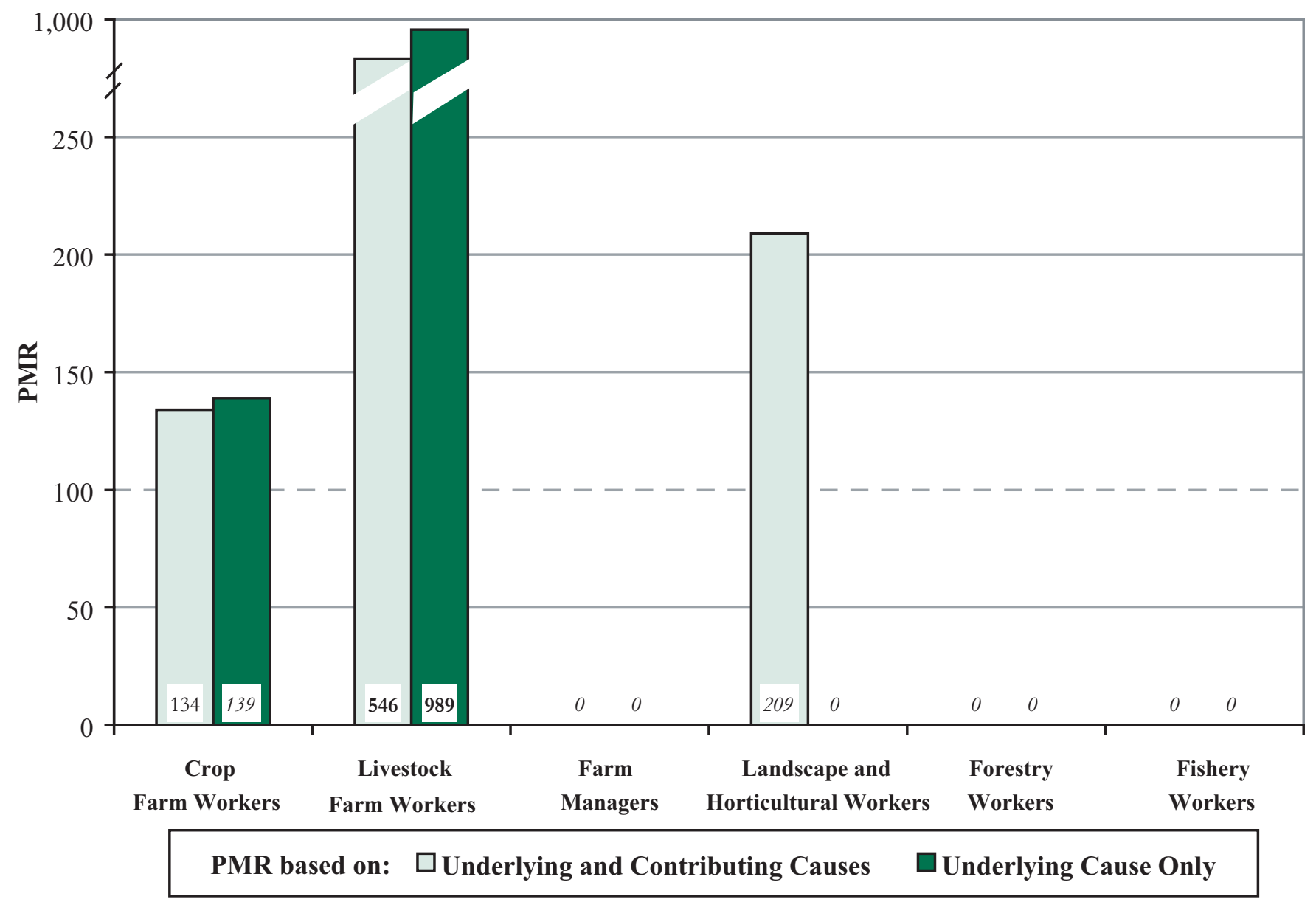

ICD - International Classification of Diseases, $9^{\text {th }}$ Revision

NOTE: Tuberculosis of meninges and central nervous system = ICD-9 code 013. PMRs in bold are significantly different from 100 ( $<<0.05$ ). PMRs in italics are based on fewer than five observed deaths. PMRs are based on underlying and contributing cause of death. See appendices for source description, methods, ICD codes, and a list of selected states.

SOURCE: National Center for Health Statistics multiple-cause-of-death data 
Figure 2-34. Tuberculosis of bones and joints: Proportionate mortality ratio (PMR) adjusted for age, sex, and race/ethnicity by agricultural group, U.S. residents age 15 and over, selected states, 1988-1998

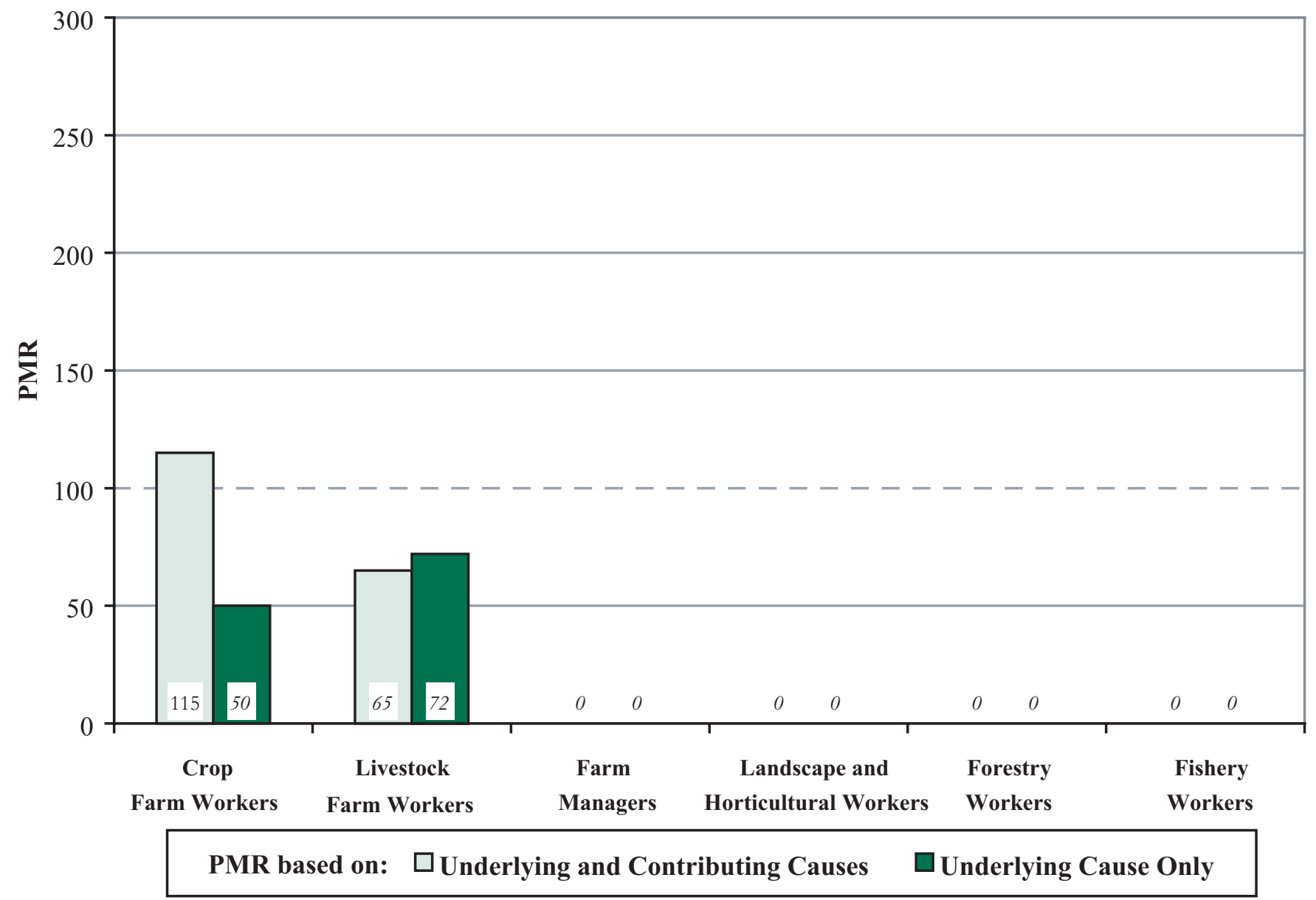

ICD - International Classification of Diseases, $9^{\text {th }}$ Revision

NOTE: Tuberculosis of bones and joints = ICD-9 code 015 . PMRs in bold are significantly different from 100 ( $\mathrm{p}<0.05)$. PMRs in italics are based on fewer than five observed deaths. PMRs are based on underlying and contributing cause of death. See appendices for source description, methods, ICD codes, and a list of selected states.

SOURCE: National Center for Health Statistics multiple-cause-of-death data 
Figure 2-35. Tuberculosis of other organs: Proportionate mortality ratio (PMR) adjusted for age, sex, and race/ethnicity by agricultural group, U.S. residents age 15 and over, selected states, 1988-1998

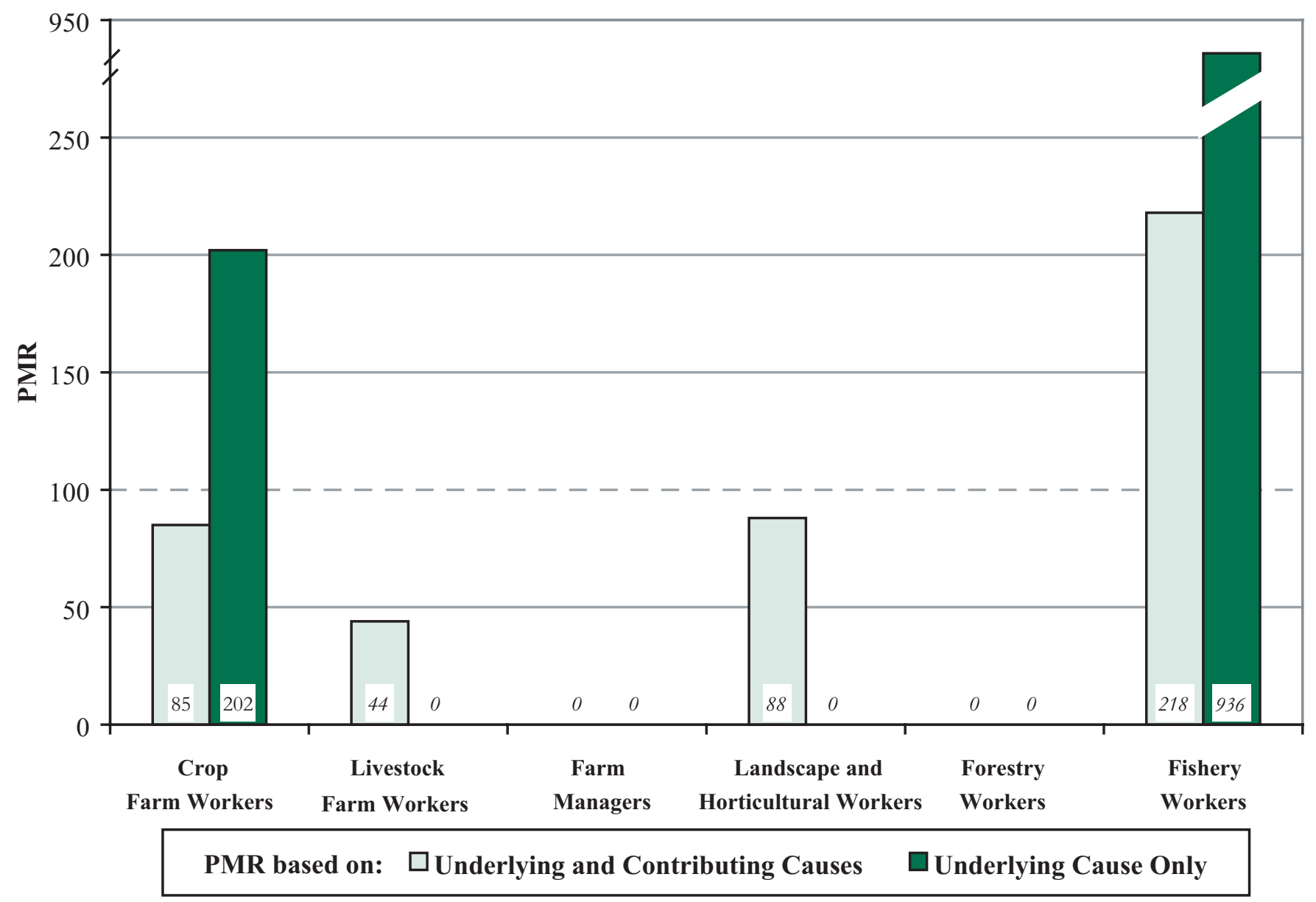

ICD - International Classification of Diseases, $9^{\text {th }}$ Revision

NOTE: Tuberculosis of other organs $=$ ICD-9 code 017 . PMRs in bold are significantly different from 100 ( $<<0.05)$. PMRs in italics are based on fewer than five observed deaths. PMRs are based on underlying and contributing cause of death. See appendices for source description, methods, ICD codes, and a list of selected states.

SOURCE: National Center for Health Statistics multiple-cause-of-death data 
Figure 2-36. Miliary tuberculosis: Proportionate mortality ratio (PMR) adjusted for age, sex, and race/ethnicity by agricultural group, U.S. residents age 15 and over, selected states, 1988-1998

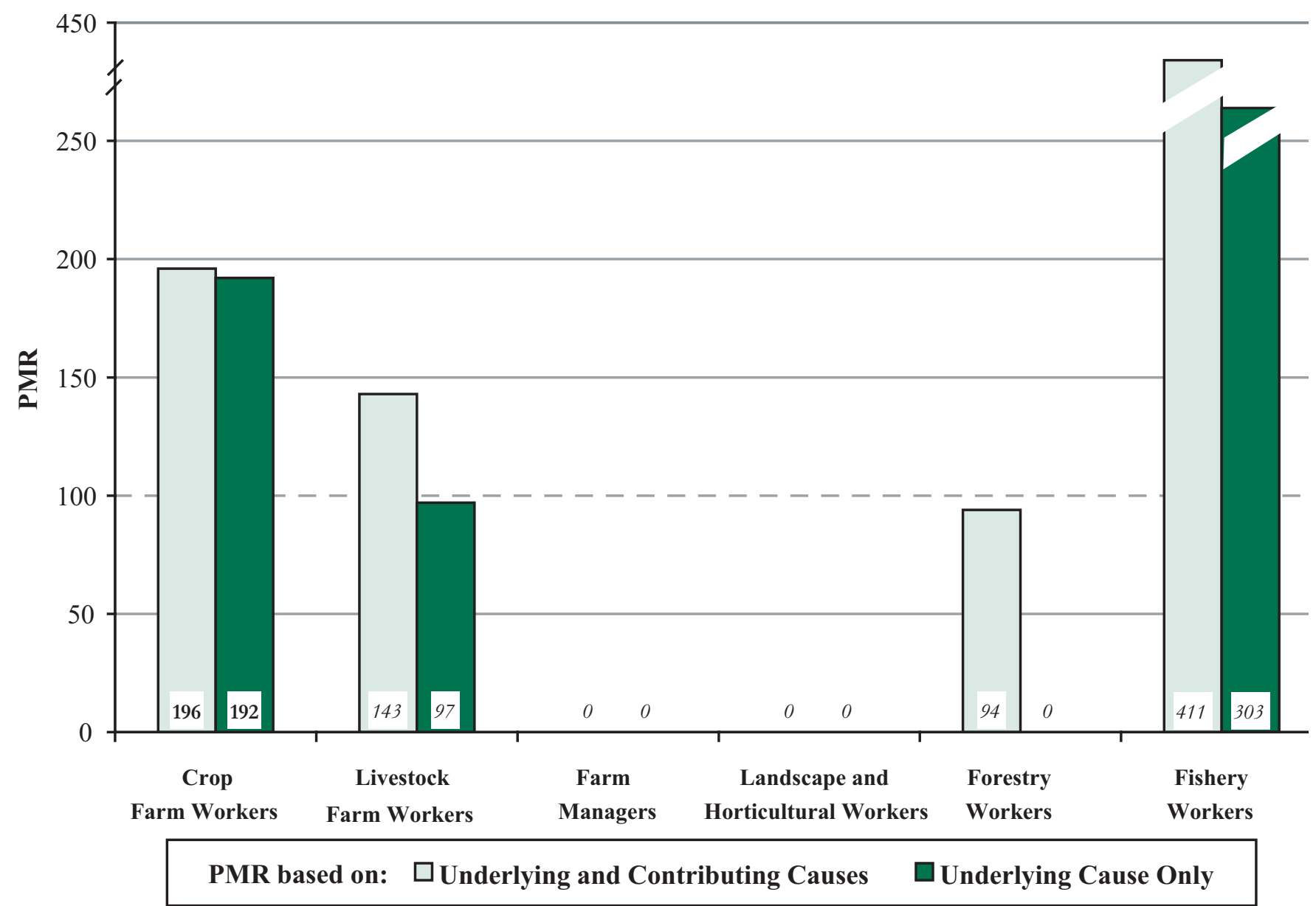

ICD - International Classification of Diseases, $9^{\text {th }}$ Revision

NOTE: Miliary tuberculosis = ICD-9 code 018. PMRs in bold are significantly different from $100(\mathrm{p}<0.05)$. PMRs in italics are based on fewer than five observed deaths. PMRs are based on underlying and contributing cause of death. See appendices for source description, methods, ICD codes, and a list of selected states.

SOURCE: National Center for Health Statistics multiple-cause-of-death data 
Table 2-43. Crop farm workers: Proportionate mortality ratio (PMR) adjusted for age, sex, and race/ ethnicity for mycoses, U.S. residents age 15 and over, selected states, 1988-1998

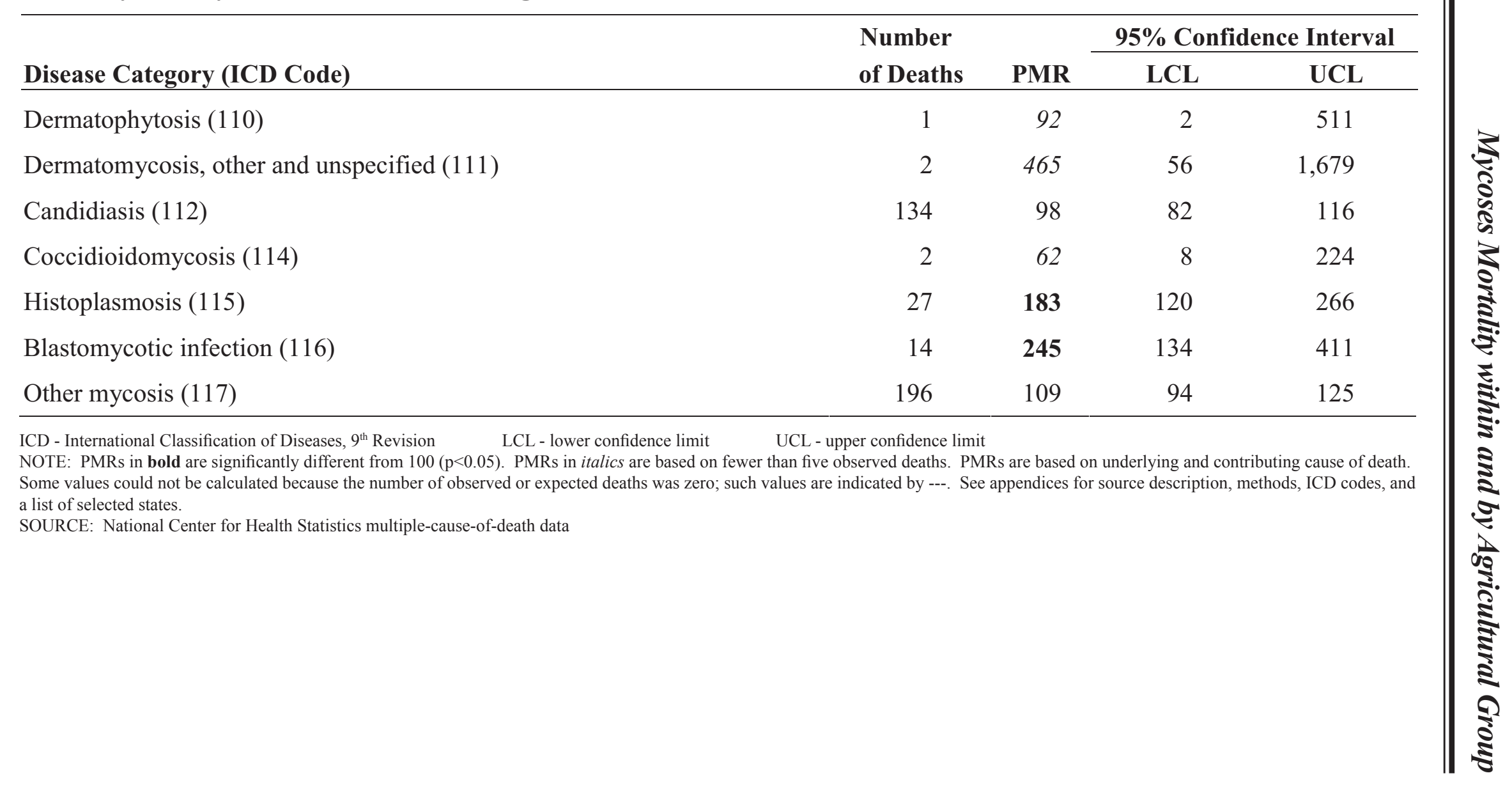


Table 2-44. Livestock farm workers: Proportionate mortality ratio (PMR) adjusted for age, sex, and race/ethnicity for mycoses, U.S. residents age 15 and over, selected states, 1988-1998

\begin{tabular}{|c|c|c|c|c|}
\hline \multirow[b]{2}{*}{ Disease Category (ICD Code) } & \multirow{2}{*}{$\begin{array}{l}\text { Number } \\
\text { of Deaths }\end{array}$} & \multirow[b]{2}{*}{ PMR } & \multicolumn{2}{|c|}{ 95\% Confidence Interval } \\
\hline & & & $\mathbf{L C L}$ & UCL \\
\hline Dermatophytosis (110) & 0 & 0 & --- & --- \\
\hline Dermatomycosis, other and unspecified (111) & 0 & 0 & --- & --- \\
\hline Candidiasis (112) & 32 & 95 & 65 & 134 \\
\hline Coccidioidomycosis (114) & 2 & 240 & 29 & 866 \\
\hline Histoplasmosis (115) & 2 & 49 & 6 & 177 \\
\hline Blastomycotic infection (116) & 2 & 132 & 16 & 477 \\
\hline Other mycosis (117) & 41 & 94 & 69 & 128 \\
\hline
\end{tabular}


Table 2-45. Farm managers: Proportionate mortality ratio (PMR) adjusted for age, sex, and race/ ethnicity for mycoses, U.S. residents age 15 and over, selected states, 1988-1998

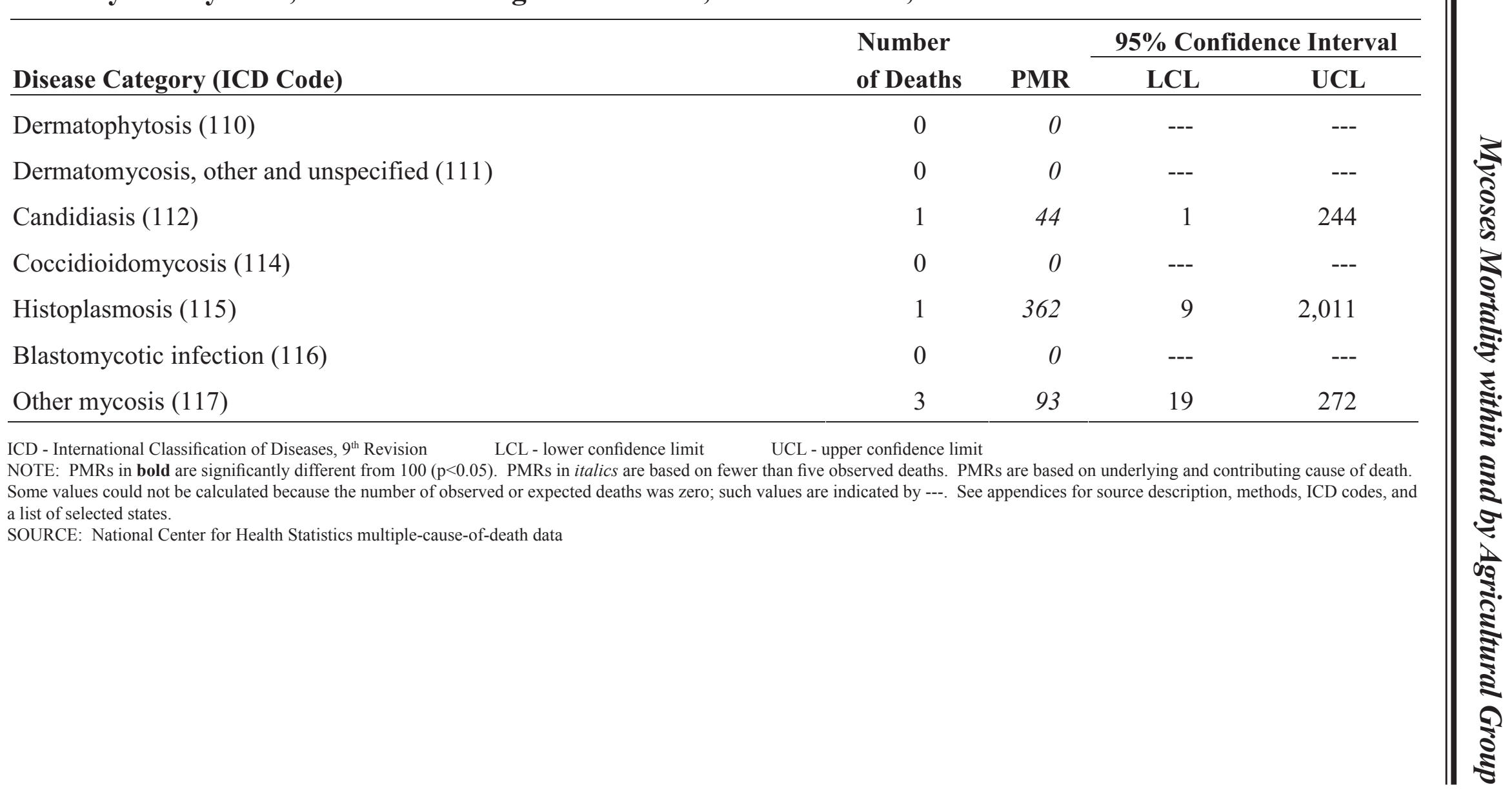


Table 2-46. Landscape and horticultural workers: Proportionate mortality ratio (PMR) adjusted for age, sex, and race/ethnicity for mycoses, U.S. residents age 15 and over, selected states, 1988-1998

\begin{tabular}{|c|c|c|c|c|}
\hline \multirow[b]{2}{*}{ Disease Category (ICD Code) } & \multirow{2}{*}{$\begin{array}{l}\text { Number } \\
\text { of Deaths }\end{array}$} & \multirow[b]{2}{*}{ PMR } & \multicolumn{2}{|c|}{ 95\% Confidence Interval } \\
\hline & & & $\mathbf{L C L}$ & UCL \\
\hline Dermatophytosis (110) & 1 & 767 & 19 & 4,261 \\
\hline Dermatomycosis, other and unspecified (111) & 0 & 0 & --- & --- \\
\hline Candidiasis (112) & 10 & 110 & 53 & 202 \\
\hline Coccidioidomycosis (114) & 0 & 0 & --- & --- \\
\hline Histoplasmosis (115) & 2 & 130 & 16 & 469 \\
\hline Blastomycotic infection (116) & 0 & 0 & --- & --- \\
\hline Other mycosis (117) & 14 & 100 & 55 & 168 \\
\hline
\end{tabular}


Table 2-47. Forestry workers: Proportionate mortality ratio (PMR) adjusted for age, sex, and race/ ethnicity for mycoses, U.S. residents age 15 and over, selected states, 1988-1998

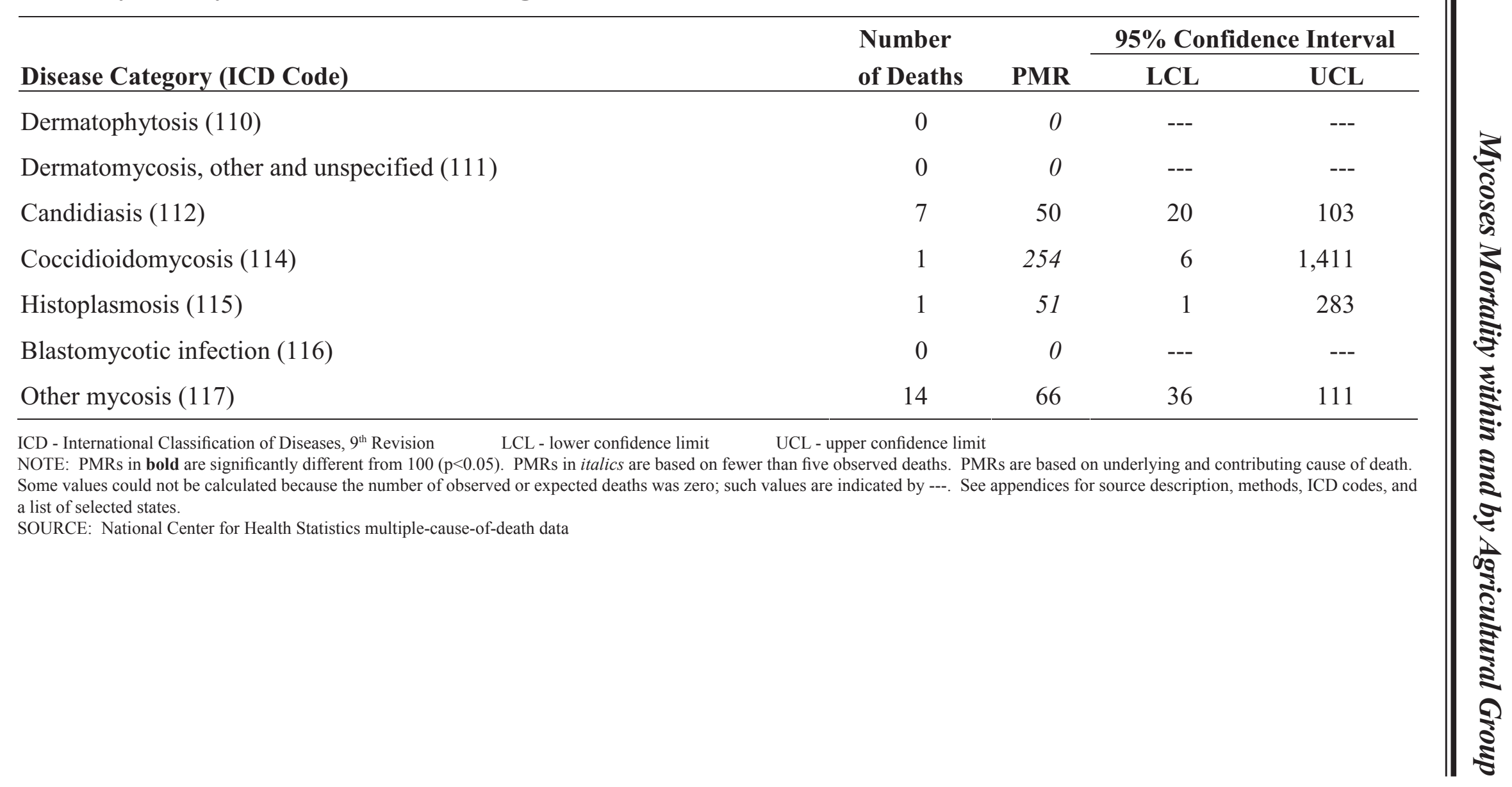


Table 2-48. Fishery workers: Proportionate mortality ratio (PMR) adjusted for age, sex, and race/ ethnicity for mycoses, U.S. residents age 15 and over, selected states, 1988-1998

\begin{tabular}{|c|c|c|c|c|}
\hline Disease Category (ICD Code) & $\begin{array}{l}\text { Number } \\
\text { of Deaths }\end{array}$ & PMR & \multicolumn{2}{|c|}{ 95\% Confidence Interval } \\
\hline Dermatophytosis (110) & 0 & 0 & --- & --- \\
\hline Candidiasis (112) & 1 & 26 & 1 & 144 \\
\hline Coccidioidomycosis (114) & 0 & 0 & --- & --- \\
\hline Blastomycotic infection (116) & 0 & 0 & --- & --- \\
\hline Other mycosis (117) & 5 & 82 & 27 & 192 \\
\hline \multicolumn{5}{|c|}{$\begin{array}{l}\text { ICD - International Classification of Diseases, } 9^{\text {th }} \text { Revision } \quad \text { LCL - lower confidence limit } \quad \text { UCL - upper confidence limit } \\
\text { NOTE: PMRs in bold are significantly different from } 100(\mathrm{p}<0.05) \text {. PMRs in italics are based on fewer than five observed deaths. PMRs are based on underlying and contributing cause of death. } \\
\text { Some values could not be calculated because the number of observed or expected deaths was zero; such values are indicated by ---. See appendices for source description, methods, ICD codes, and } \\
\text { a list of selected states. } \\
\text { SOURCE: National Center for Health Statistics multiple-cause-of-death data }\end{array}$} \\
\hline
\end{tabular}


Figure 2-37. Candidiasis: Proportionate mortality ratio (PMR) adjusted for age, sex, and race/ethnicity by agricultural group, U.S. residents age 15 and over, selected states, 1988-1998

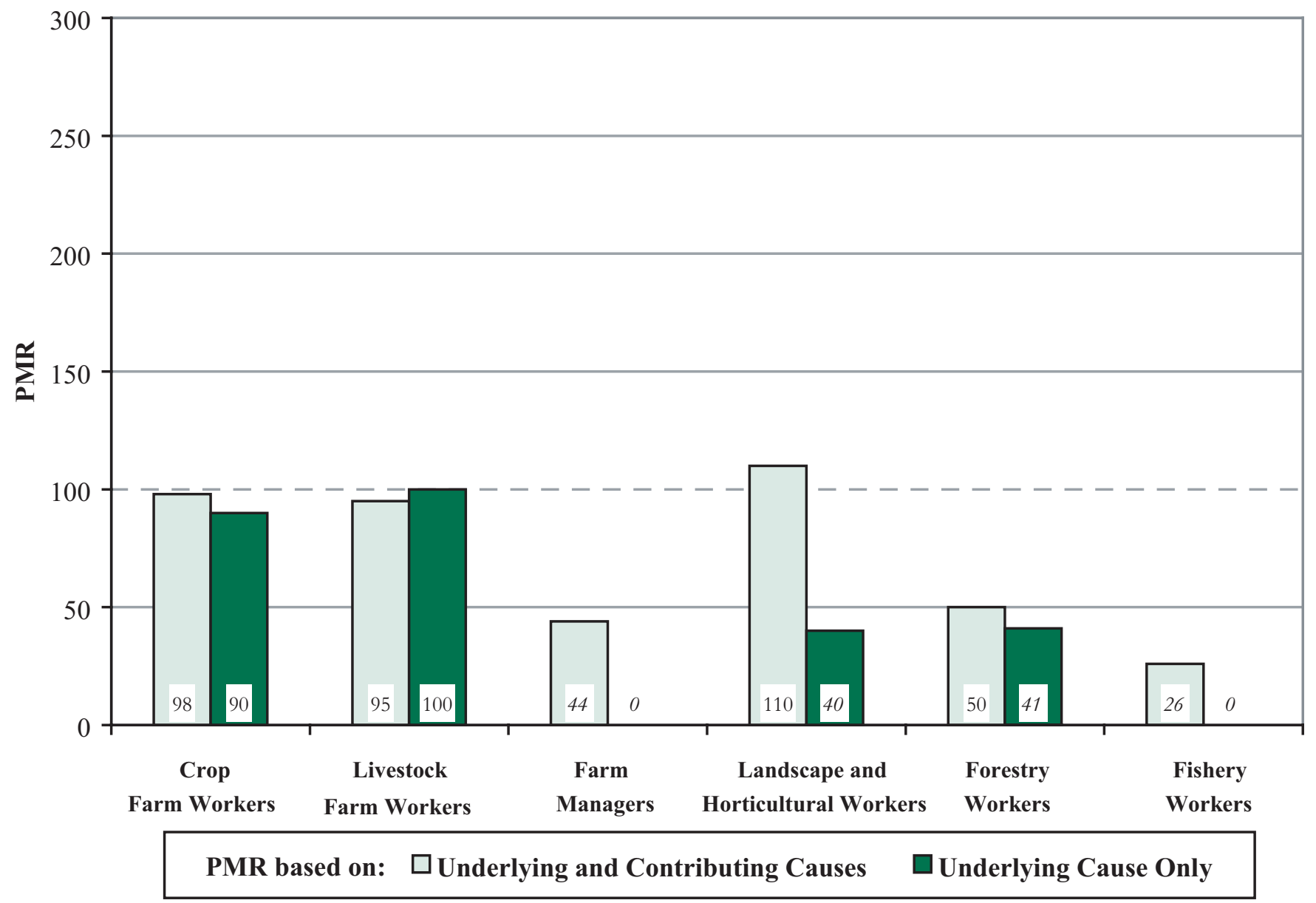

ICD - International Classification of Diseases, $9^{\text {th }}$ Revision

NOTE: Candidiasis = ICD-9 code 112. PMRs in bold are significantly different from $100(\mathrm{p}<0.05)$. PMRs in italics are based on fewer than five observed deaths. PMRs are based on underlying and contributing cause of death. See appendices for source description, methods, ICD codes, and a list of selected states.

SOURCE: National Center for Health Statistics multiple-cause-of-death data 
Figure 2-38. Histoplasmosis: Proportionate mortality ratio (PMR) adjusted for age, sex, and race/ethnicity by agricultural group, U.S. residents age 15 and over, selected states, 1988-1998

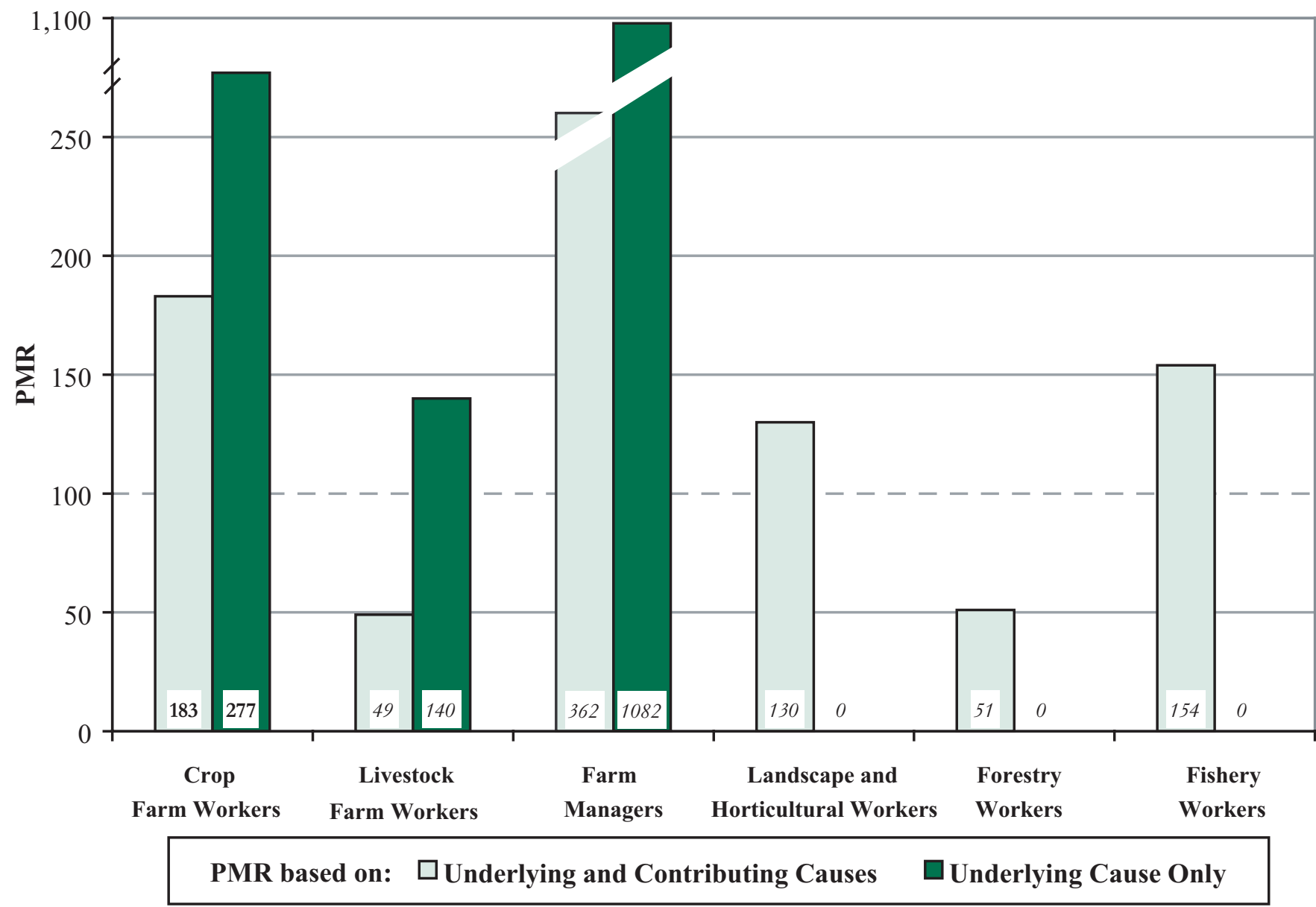

ICD - International Classification of Diseases, $9^{\text {th }}$ Revision

NOTE: Histoplasmosis $=$ ICD-9 code 115. PMRs in bold are significantly different from $100(\mathrm{p}<0.05)$. PMRs in italics are based on fewer than five observed deaths. PMRs are based on underlying and contributing cause of death. See appendices for source description, methods, ICD codes, and a list of selected states.

SOURCE: National Center for Health Statistics multiple-cause-of-death data 
Figure 2-39. Blastomycotic infection: Proportionate mortality ratio (PMR) adjusted for age, sex, and race/ethnicity by agricultural group, U.S. residents age 15 and over, selected states, 1988-1998

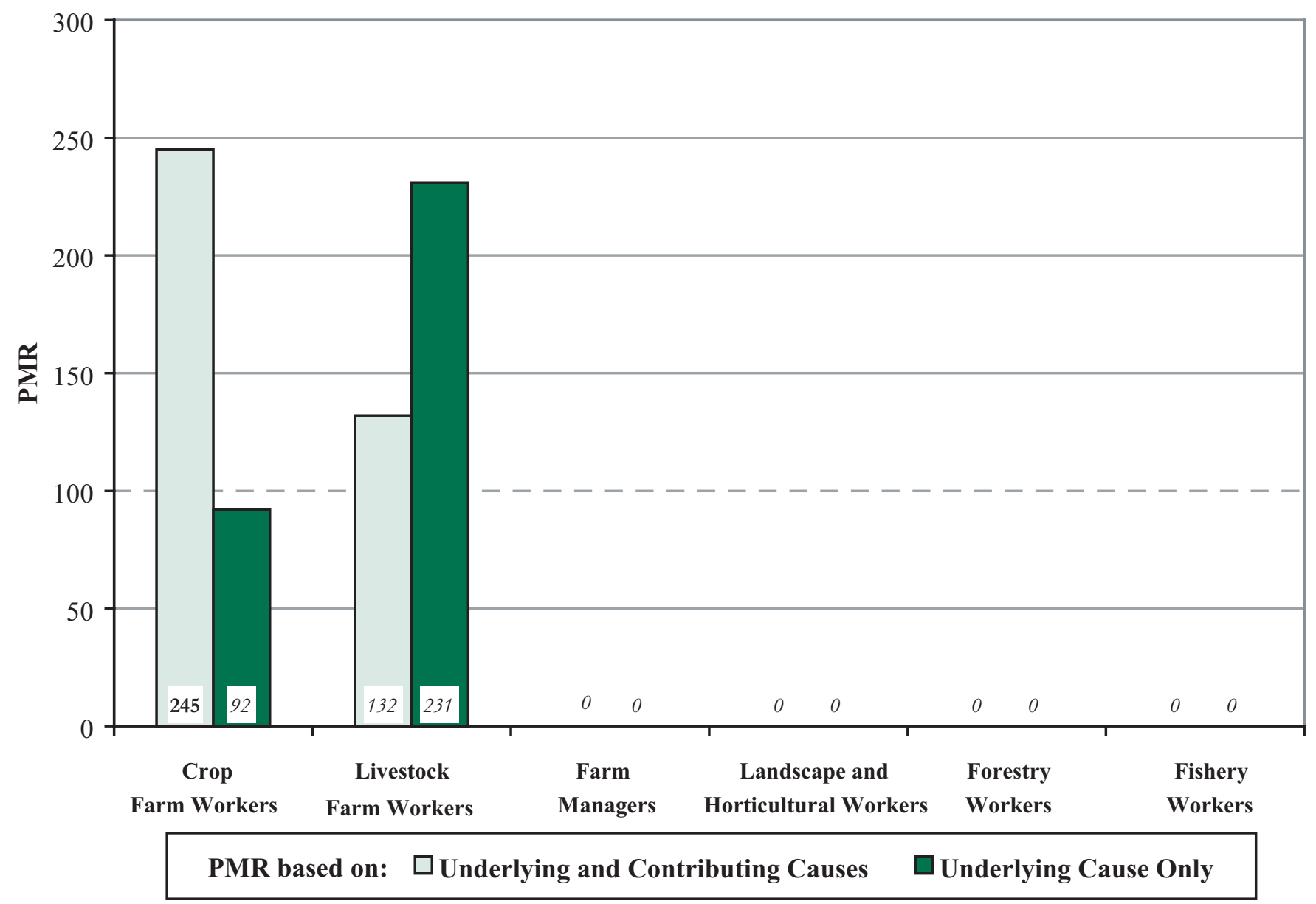

ICD - International Classification of Diseases, $9^{\text {th }}$ Revision

NOTE: Blastomycotic infection = ICD-9 code 116. PMRs in bold are significantly different from $100(\mathrm{p}<0.05)$. PMRs in italics are based on fewer than five observed deaths. PMRs are based on underlying and contributing cause of death. See appendices for source description, methods, ICD codes, and a list of selected states.

SOURCE: National Center for Health Statistics multiple-cause-of-death data 
Figure 2-40. Other mycoses: Proportionate mortality ratio (PMR) adjusted for age, sex, and race/ethnicity by agricultural group, U.S. residents age 15 and over, selected states, 1988-1998

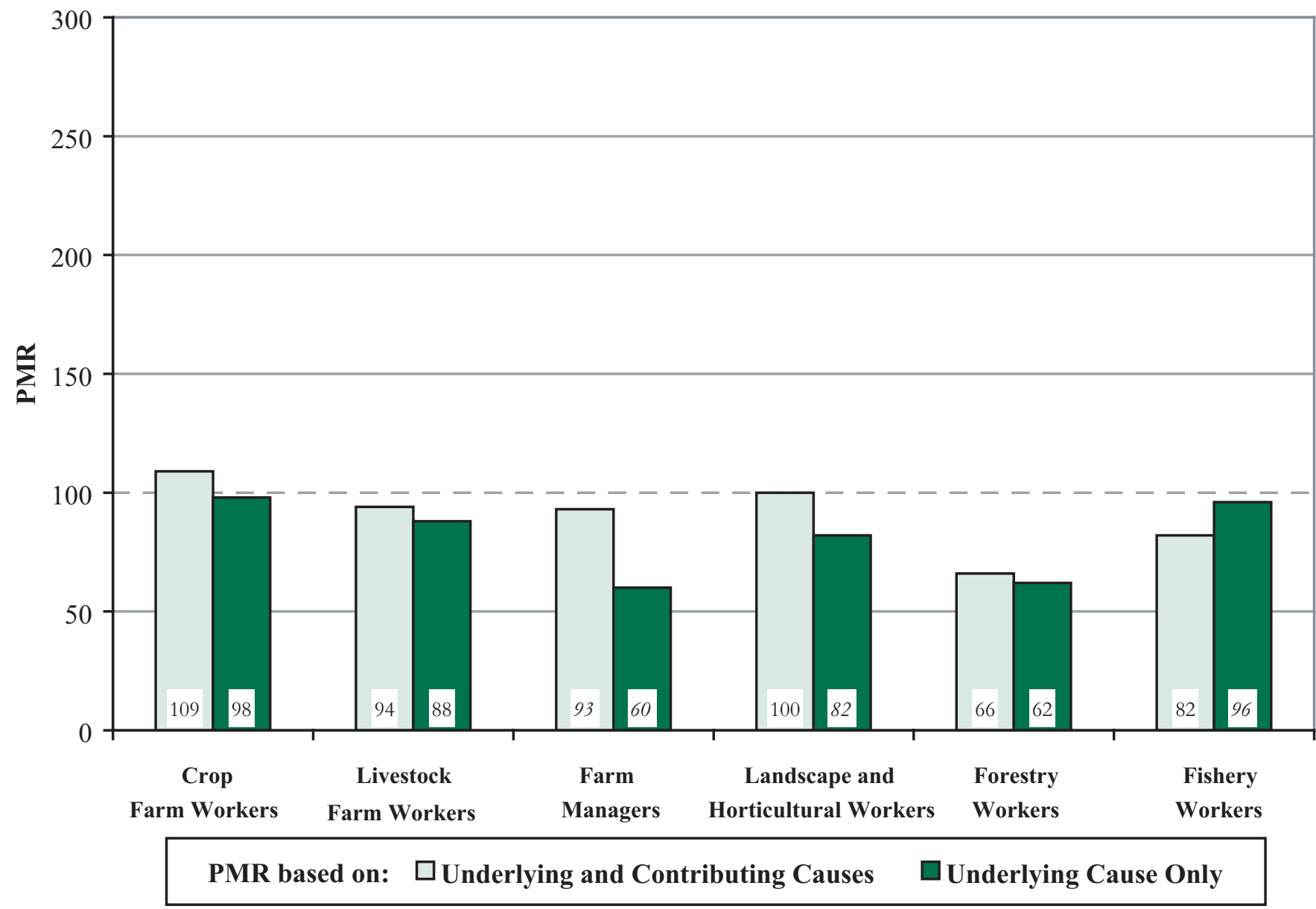

ICD - International Classification of Diseases, $9^{\text {th }}$ Revision

NOTE: Other mycoses = ICD-9 code 117. PMRs in bold are significantly different from 100 ( $\mathrm{p}<0.05)$. PMRs in italics are based on fewer than five observed deaths. PMRs are based on underlying and contributing cause of death. See appendices for source description, methods, ICD codes, and a list of selected states.

SOURCE: National Center for Health Statistics multiple-cause-of-death data 
Table 2-49. Crop farm workers: Proportionate mortality ratio (PMR) adjusted for age, sex, and race/ ethnicity for malignant neoplasms of trachea/bronchus/lung/pleura, U.S. residents age 15 and over, selected states, 1988-1998

\begin{tabular}{lrrrr}
\hline & Number & & \multicolumn{2}{c}{ 95\% Confidence Interval } \\
\cline { 3 - 5 } Disease Category (ICD Code) & of Deaths & PMR & LCL & UCL \\
\hline Malignant neoplasm of trachea, bronchus, and lung (162) & 13,080 & $\mathbf{8 0}$ & 78 & 82 \\
Malignant neoplasm of pleura (163) & 19 & $\mathbf{3 0}$ & 18 & 47 \\
\hline
\end{tabular}

ICD - International Classification of Diseases, $9^{\text {th }}$ Revision

LCL - lower confidence limit

UCL - upper confidence limit

NOTE: PMRs in bold are significantly different from $100(\mathrm{p}<0.05)$. PMRs in italics are based on fewer than five observed deaths. PMRs are based on underlying and contributing cause of death. Some values could not be calculated because the number of observed or expected deaths was zero; such values are indicated by ---. See appendices for source description, methods, ICD codes, and a list of selected states.

SOURCE: National Center for Health Statistics multiple-cause-of-death data 
Table 2-50. Livestock farm workers: Proportionate mortality ratio (PMR) adjusted for age, sex, and race/ethnicity for malignant neoplasms of trachea/bronchus/lung/pleura, U.S. residents age 15 and over, selected states, 1988-1998

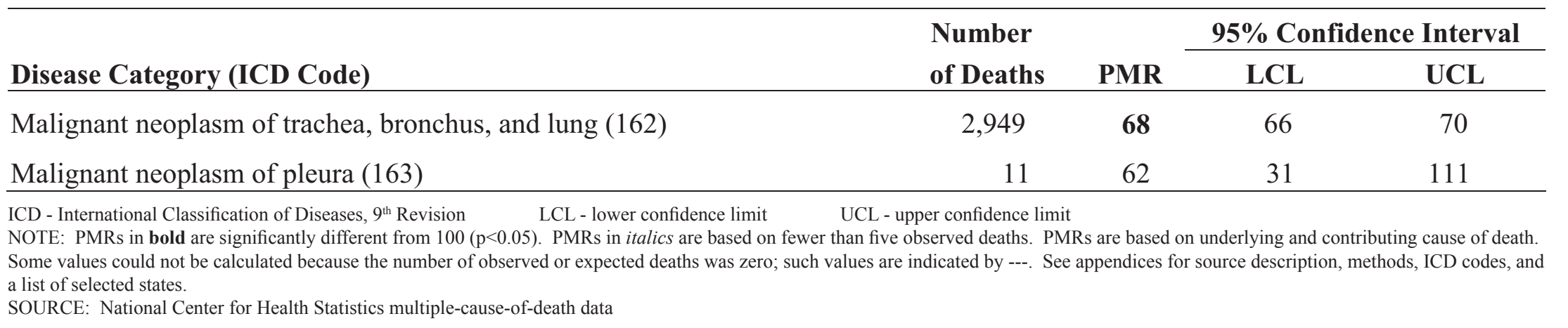


Table 2-51. Farm managers: Proportionate mortality ratio (PMR) adjusted for age, sex, and race/ ethnicity for malignant neoplasms of trachea/bronchus/lung/pleura, U.S. residents age 15 and over, selected states, 1988-1998

\begin{tabular}{|c|c|c|c|c|}
\hline \multirow[b]{2}{*}{ Disease Category (ICD Code) } & \multirow{2}{*}{$\begin{array}{l}\text { Number } \\
\text { of Deaths }\end{array}$} & \multirow[b]{2}{*}{ PMR } & \multicolumn{2}{|c|}{ 95\% Confidence Interval } \\
\hline & & & $\mathbf{L C L}$ & $\mathbf{U C L}$ \\
\hline Malignant neoplasm of trachea, bronchus, and lung (162) & 250 & 94 & 83 & 106 \\
\hline Malignant neoplasm of pleura (163) & 1 & 98 & 2 & 544 \\
\hline \multicolumn{5}{|c|}{$\begin{array}{l}\text { ICD - International Classification of Diseases, } 9^{\text {th }} \text { Revision } \quad \text { LCL - lower confidence limit } \quad \text { UCL - upper confidence limit } \\
\text { NOTE: PMRs in bold are significantly different from } 100(p<0.05) \text {. PMRs in italics are based on fewer than five observed deaths. PMRs are based on underlying and contributing cause of death. } \\
\text { Some values could not be calculated because the number of observed or expected deaths was zero; such values are indicated by ---. See appendices for source description, methods, ICD codes, and } \\
\text { a list of selected states. } \\
\text { SOURCE: National Center for Health Statistics multiple-cause-of-death data }\end{array}$} \\
\hline
\end{tabular}


Table 2-52. Landscape and horticultural workers: Proportionate mortality ratio (PMR) adjusted for age, sex, and race/ethnicity for malignant neoplasms of trachea/bronchus/lung/pleura, U.S. residents age 15 and over, selected states, 1988-1998

\begin{tabular}{|c|c|c|c|c|}
\hline \multirow[b]{2}{*}{ Disease Category (ICD Code) } & \multirow{2}{*}{$\begin{array}{l}\text { Number } \\
\text { of Deaths }\end{array}$} & \multirow[b]{2}{*}{ PMR } & \multicolumn{2}{|c|}{ 95\% Confidence Interval } \\
\hline & & & $\mathbf{L C L}$ & $\mathbf{U C L}$ \\
\hline Malignant neoplasm of trachea, bronchus, and lung (162) & 642 & 97 & 90 & 105 \\
\hline Malignant neoplasm of pleura (163) & 5 & 235 & 76 & 235 \\
\hline
\end{tabular}

SOURCE: National Center for Health Statistics multiple-cause-of-death data 
Table 2-53. Forestry workers: Proportionate mortality ratio (PMR) adjusted for age, sex, and race/ ethnicity for malignant neoplasms of trachea/bronchus/lung/pleura, U.S. residents age 15 and over, selected states, 1988-1998

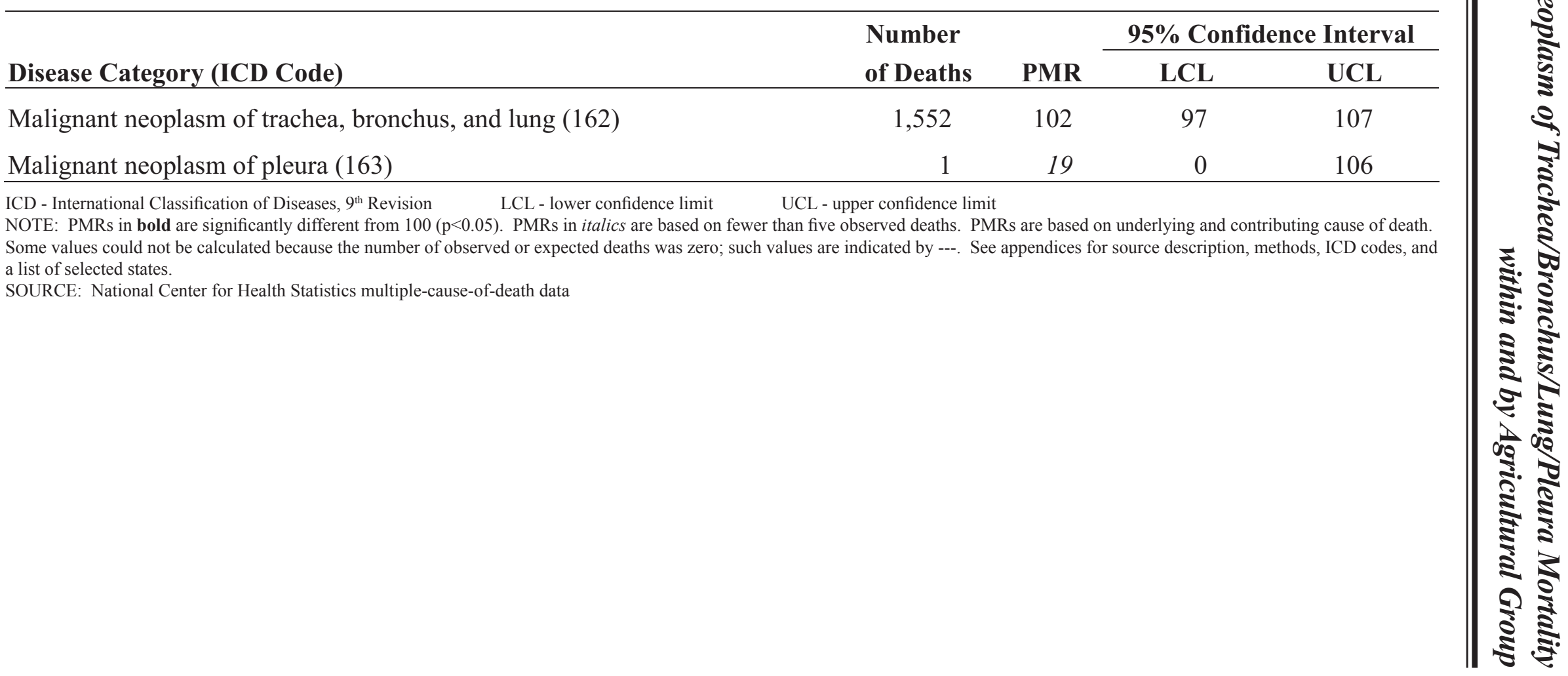


Table 2-54. Fishery workers: Proportionate mortality ratio (PMR) adjusted for age, sex, and race/ ethnicity for malignant neoplasms of trachea/bronchus/lung/pleura, U.S. residents age 15 and over, selected states, 1988-1998

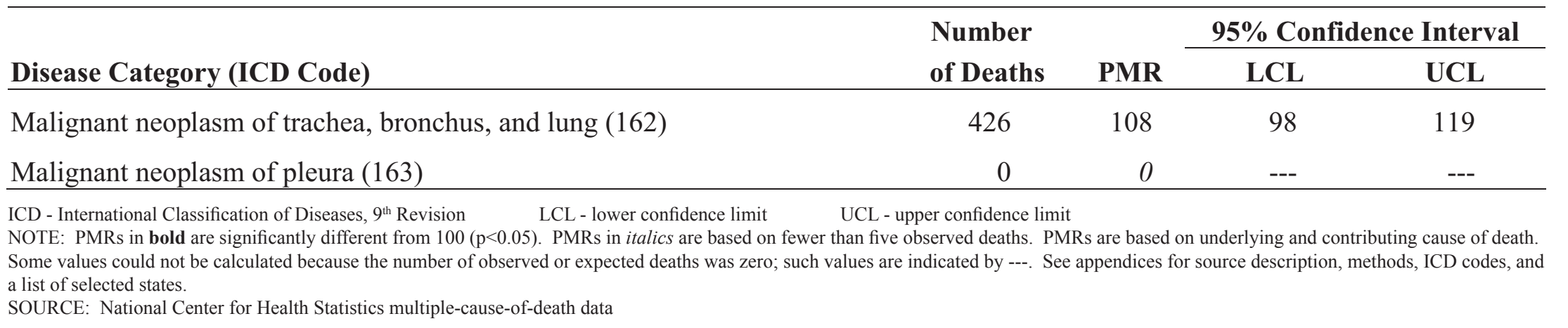


Figure 2-41. Malignant neoplasm of trachea, bronchus, and lung: Proportionate mortality ratio (PMR) adjusted for age, sex, and race/ethnicity by agricultural group, U.S. residents age 15 and over, selected states, 1988-1998

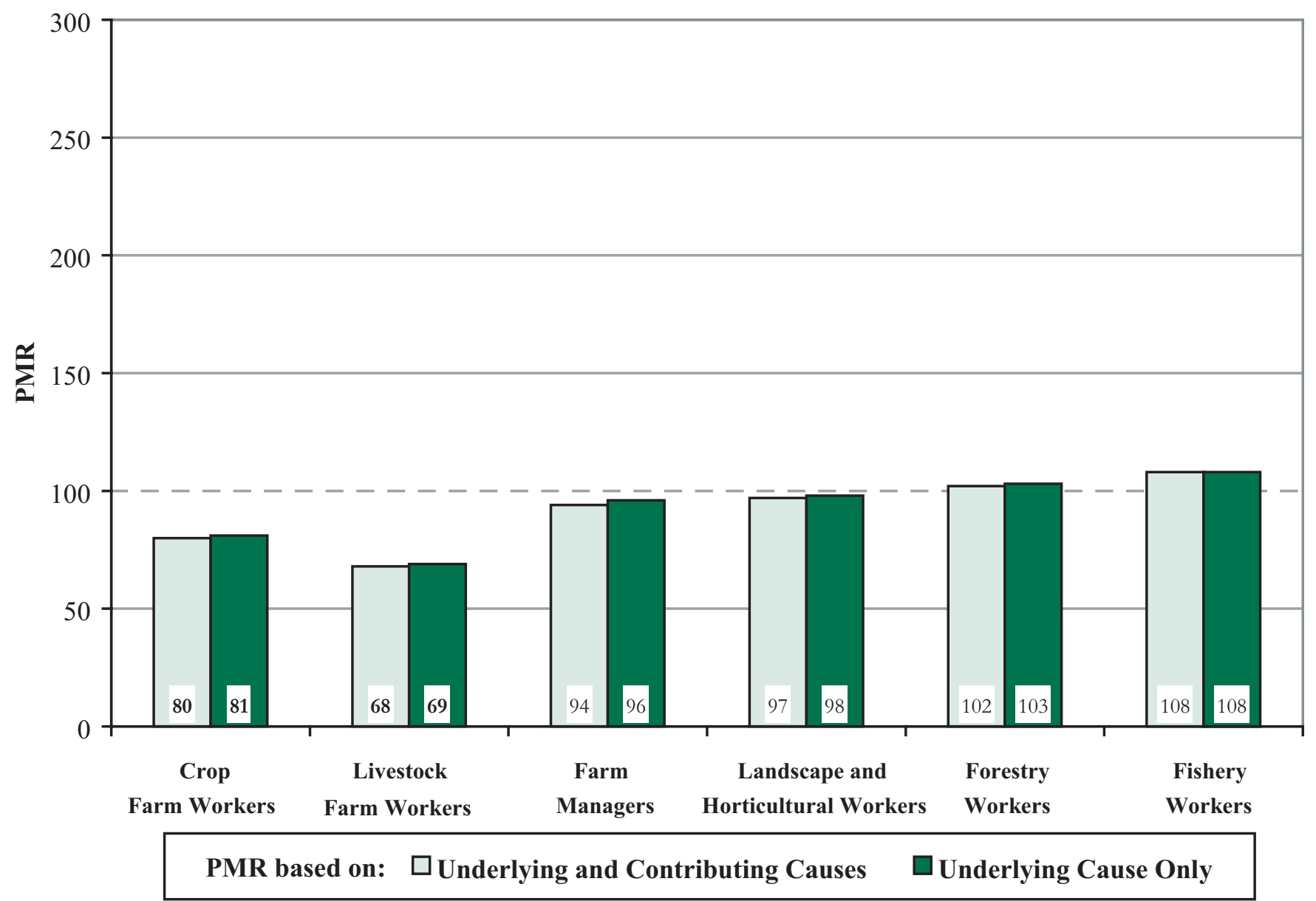

ICD - International Classification of Diseases, $9^{\text {th }}$ Revision

NOTE: Malignant neoplasm of trachea, bronchus, and lung = ICD-9 code 162 . PMRs in bold are significantly different from 100 ( $<<0.05$ ). PMRs in italics are based on fewer than five observed deaths. PMRs are based on underlying and contributing cause of death. See appendices for source description, methods, ICD codes, and a list of selected states.

SOURCE: National Center for Health Statistics multiple-cause-of-death data 
Figure 2-42. Malignant neoplasm of pleura: Proportionate mortality ratio (PMR) adjusted for age, sex, and race/ethnicity by agricultural group, U.S. residents age 15 and over, selected states, 1988-1998

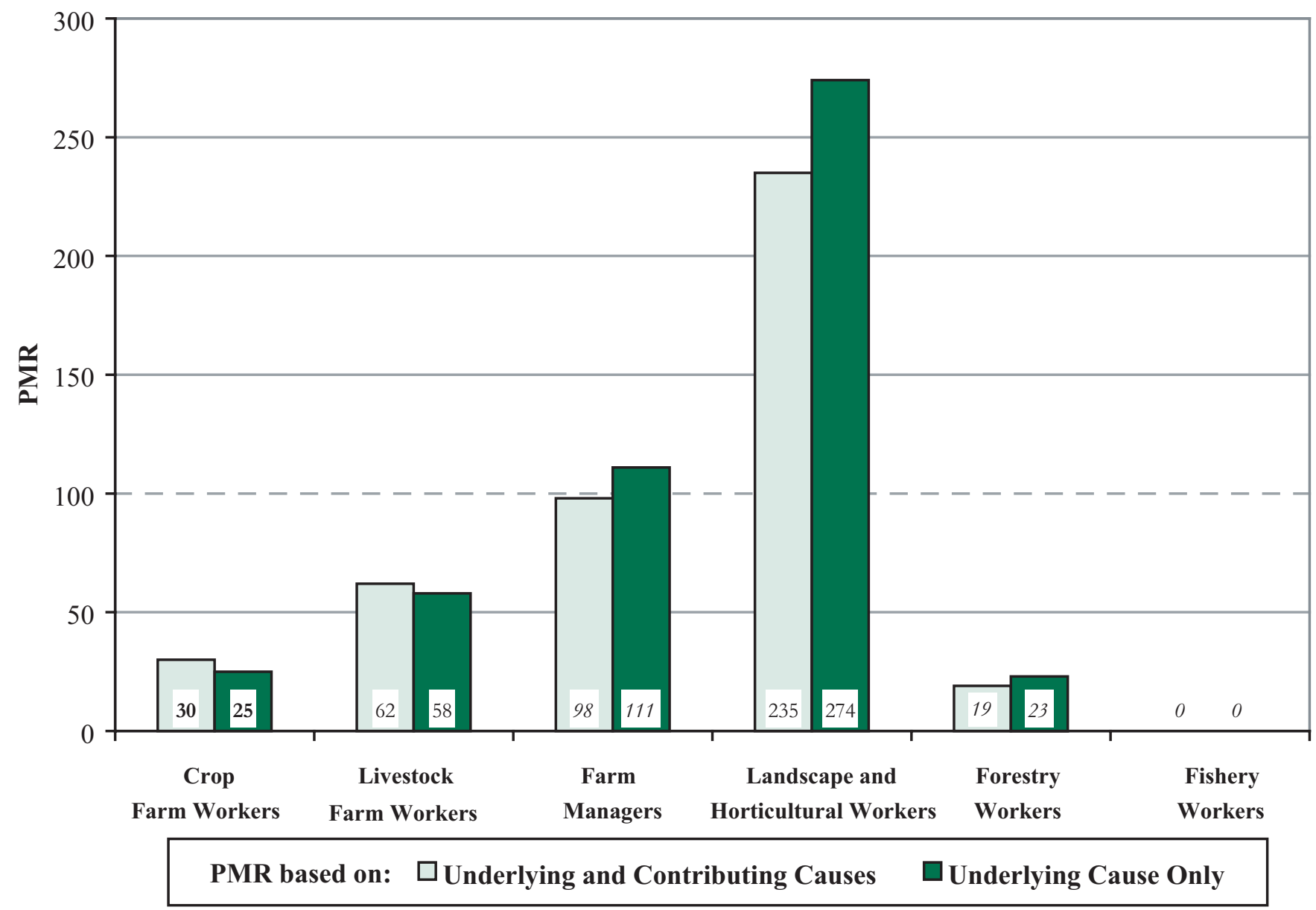

ICD - International Classification of Diseases, $9^{\text {th }}$ Revision

NOTE: Malignant neoplasm of pleura = ICD-9 code 163. PMRs in bold are significantly different from $100(\mathrm{p}<0.05)$. PMRs in italics are based on fewer than five observed deaths. PMRs are based on underlying and contributing cause of death. See appendices for source description, methods, ICD codes, and a list of selected states.

SOURCE: National Center for Health Statistics multiple-cause-of-death data 
Table 2-55. Crop farm workers: Proportionate mortality ratio (PMR) adjusted for age, sex, and race/ ethnicity for acute respiratory infections, U.S. residents age 15 and over, selected states, 1988-1998

\begin{tabular}{lrrrr}
\hline & Number & & \multicolumn{2}{c}{ 95\% Confidence Interval } \\
\cline { 2 - 5 } Disease Category (ICD Code) & of Deaths & PMR & LCL & UCL \\
\hline Acute nasopharyngitis (460) & 3 & 143 & 29 & 418 \\
Acute pharyngitis (462) & 5 & 99 & 32 & 231 \\
Acute tonsillitis (463) & 1 & 205 & 5 & 1,139 \\
Acute laryngitis and tracheitis (464) & 7 & 92 & 37 & 129 \\
Acute upper respiratory infections of multiple or unspecified sites (465) & 87 & $\mathbf{1 6 0}$ & 197 \\
Acute bronchitis and bronchiolitis (466) & 226 & $\mathbf{1 1 7}$ & 103 & 133 \\
\hline
\end{tabular}

ICD - International Classification of Diseases, $9^{\text {th }}$ Revision

LCL - lower confidence limit

UCL - upper confidence limit

NOTE: PMRs in bold are significantly different from $100(\mathrm{p}<0.05)$. PMRs in italics are based on fewer than five observed deaths. PMRs are based on underlying and contributing cause of death.

Some values could not be calculated because the number of observed or expected deaths was zero; such values are indicated by ---. See appendices for source description, methods, ICD codes, and

a list of selected states.

SOURCE: National Center for Health Statistics multiple-cause-of-death data 
Table 2-56. Livestock farm workers: Proportionate mortality ratio (PMR) adjusted for age, sex, and race/ethnicity for acute respiratory infections, U.S. residents age 15 and over, selected states, 1988-1998

\begin{tabular}{lrrrr} 
& Number & & \multicolumn{2}{c}{ 95\% Confidence Interval } \\
\cline { 3 - 5 } Disease Category (ICD Code) & of Deaths & PMR & LCL & UCL \\
\hline Acute nasopharyngitis (460) & 2 & 348 & 42 & 1,256 \\
Acute pharyngitis (462) & 1 & 76 & 2 & -122 \\
Acute tonsillitis (463) & 0 & 0 & --- \\
Acute laryngitis and tracheitis (464) & 1 & 52 & 1 & 79 \\
Acute upper respiratory infections of multiple or unspecified sites (465) & 20 & 129 & 199 \\
Acute bronchitis and bronchiolitis (466) & 35 & $\mathbf{6 5}$ & 45 & 90 \\
\hline
\end{tabular}

ICD - International Classification of Diseases, $9^{\text {th }}$ Revision

LCL - lower confidence limit UCL - upper confidence limit

NOTE: PMRs in bold are significantly different from $100(\mathrm{p}<0.05)$. PMRs in italics are based on fewer than five observed deaths. PMRs are based on underlying and contributing cause of death. Some values could not be calculated because the number of observed or expected deaths was zero; such values are indicated by ---. See appendices for source description, methods, ICD codes, and a list of selected states.

SOURCE: National Center for Health Statistics multiple-cause-of-death data 
Table 2-57. Farm managers: Proportionate mortality ratio (PMR) adjusted for age, sex, and race/ ethnicity for acute respiratory infections, U.S. residents age 15 and over, selected states, 1988-1998

\begin{tabular}{|c|c|c|c|c|}
\hline \multirow[b]{2}{*}{ Disease Category (ICD Code) } & \multirow{2}{*}{$\begin{array}{l}\text { Number } \\
\text { of Deaths }\end{array}$} & \multirow[b]{2}{*}{ PMR } & \multicolumn{2}{|c|}{ 95\% Confidence Interval } \\
\hline & & & $\mathbf{L C L}$ & UCL \\
\hline Acute nasopharyngitis (460) & 0 & 0 & --- & --- \\
\hline Acute pharyngitis (462) & 0 & 0 & --- & --- \\
\hline Acute tonsillitis (463) & 0 & 0 & --- & --- \\
\hline Acute laryngitis and tracheitis (464) & 0 & 0 & --- & --- \\
\hline Acute upper respiratory infections of multiple or unspecified sites (465) & 0 & 0 & --- & --- \\
\hline Acute bronchitis and bronchiolitis (466) & 2 & 66 & 8 & 238 \\
\hline
\end{tabular}

\section{ICD - International Classification of Diseases, $9^{\text {th }}$ Revision $\quad$ LCL - lower confidence limit $\quad$ UCL - upper confidence limit}

NOTE: PMRs in bold are significantly different from $100(\mathrm{p}<0.05)$. PMRs in italics are based on fewer than five observed deaths. PMRs are based on underlying and contributing cause of death. Some values could not be calculated because the number of observed or expected deaths was zero; such values are indicated by ---. See appendices for source description, methods, ICD codes, and a list of selected states.

SOURCE: National Center for Health Statistics multiple-cause-of-death data 
Table 2-58. Landscape and horticultural workers: Proportionate mortality ratio (PMR) adjusted for age, sex, and race/ethnicity for acute respiratory infections, U.S. residents age 15 and over, selected states, 1988-1998

\begin{tabular}{|c|c|c|c|c|}
\hline \multirow[b]{2}{*}{ Disease Category (ICD Code) } & \multirow{2}{*}{$\begin{array}{l}\text { Number } \\
\text { of Deaths }\end{array}$} & \multirow[b]{2}{*}{ PMR } & \multicolumn{2}{|c|}{ 95\% Confidence Interval } \\
\hline & & & $\mathbf{L C L}$ & UCL \\
\hline Acute nasopharyngitis (460) & 0 & 0 & --- & --- \\
\hline Acute pharyngitis (462) & 1 & 425 & 11 & 2,361 \\
\hline Acute tonsillitis (463) & 0 & 0 & --- & --- \\
\hline Acute laryngitis and tracheitis (464) & 1 & 179 & 5 & 994 \\
\hline Acute upper respiratory infections of multiple or unspecified sites (465) & 3 & 186 & 38 & 544 \\
\hline Acute bronchitis and bronchiolitis (466) & 4 & 70 & 19 & 179 \\
\hline
\end{tabular}

ICD - International Classification of Diseases, $9^{\text {th }}$ Revision

LCL - lower confidence limit

UCL - upper confidence limit

NOTE: PMRs in bold are significantly different from $100(\mathrm{p}<0.05)$. PMRs in italics are based on fewer than five observed deaths. PMRs are based on underlying and contributing cause of death. Some values could not be calculated because the number of observed or expected deaths was zero; such values are indicated by ---. See appendices for source description, methods, ICD codes, and a list of selected states.

SOURCE: National Center for Health Statistics multiple-cause-of-death data 
Table 2-59. Forestry workers: Proportionate mortality ratio (PMR) adjusted for age, sex, and race/ ethnicity for acute respiratory infections, U.S. residents age 15 and over, selected states, 1988-1998

\begin{tabular}{lcccc}
\hline & \multicolumn{2}{c}{$\begin{array}{c}\text { Number } \\
\text { Disease Category (ICD Code) }\end{array}$} & \multicolumn{2}{c}{ 95\% Confidence Interval } \\
\cline { 3 - 5 } Acute nasopharyngitis (460) & 0 & 0 & LCL & UCL \\
Acute pharyngitis (462) & 0 & 0 & --- \\
Acute tonsillitis (463) & 0 & 0 & --- \\
Acute laryngitis and tracheitis (464) & 0 & 0 & --- & -- \\
Acute upper respiratory infections of multiple or unspecified sites (465) & 3 & 89 & 18 & 260 \\
Acute bronchitis and bronchiolitis (466) & 12 & 91 & 47 & 159 \\
\hline
\end{tabular}

ICD - International Classification of Diseases, $9^{\text {th }}$ Revision

LCL - lower confidence limit

UCL - upper confidence limit

NOTE: PMRs in bold are significantly different from $100(\mathrm{p}<0.05)$. PMRs in italics are based on fewer than five observed deaths. PMRs are based on underlying and contributing cause of death. Some values could not be calculated because the number of observed or expected deaths was zero; such values are indicated by ---. See appendices for source description, methods, ICD codes, and a list of selected states.

SOURCE: National Center for Health Statistics multiple-cause-of-death data 
Table 2-60. Fishery workers: Proportionate mortality ratio (PMR) adjusted for age, sex, and race/ ethnicity for acute respiratory infections, U.S. residents age 15 and over, selected states, 1988-1998

\begin{tabular}{lcrcr}
\hline & Number & & \multicolumn{2}{c}{ 95\% Confidence Interval } \\
\cline { 2 - 5 } Disease Category (ICD Code) & of Deaths & PMR & LCL & UCL \\
\hline Acute nasopharyngitis (460) & 0 & 0 & --- \\
Acute pharyngitis (462) & 0 & 0 & --- & --- \\
Acute tonsillitis (463) & 0 & 0 & --- & --- \\
Acute laryngitis and tracheitis (464) & 0 & 0 & --- \\
Acute upper respiratory infections of multiple or unspecified sites (465) & 1 & 102 & 367 \\
Acute bronchitis and bronchiolitis (466) & 3 & 81 & 17 & 237 \\
\hline
\end{tabular}

NOTE: PMRs in bold are significantly different from $100(\mathrm{p}<0.05)$. PMRs in italics are based on fewer than five observed deaths. PMRs are based on underlying and contributing cause of death. Some values could not be calculated because the number of observed or expected deaths was zero; such values are indicated by ---. See appendices for source description, methods, ICD codes, and a list of selected states.

SOURCE: National Center for Health Statistics multiple-cause-of-death data 
Figure 2-43. Acute laryngitis and tracheitis: Proportionate mortality ratio (PMR) adjusted for age, sex, and race/ethnicity by agricultural group, U.S. residents age 15 and over, selected states, 1988-1998

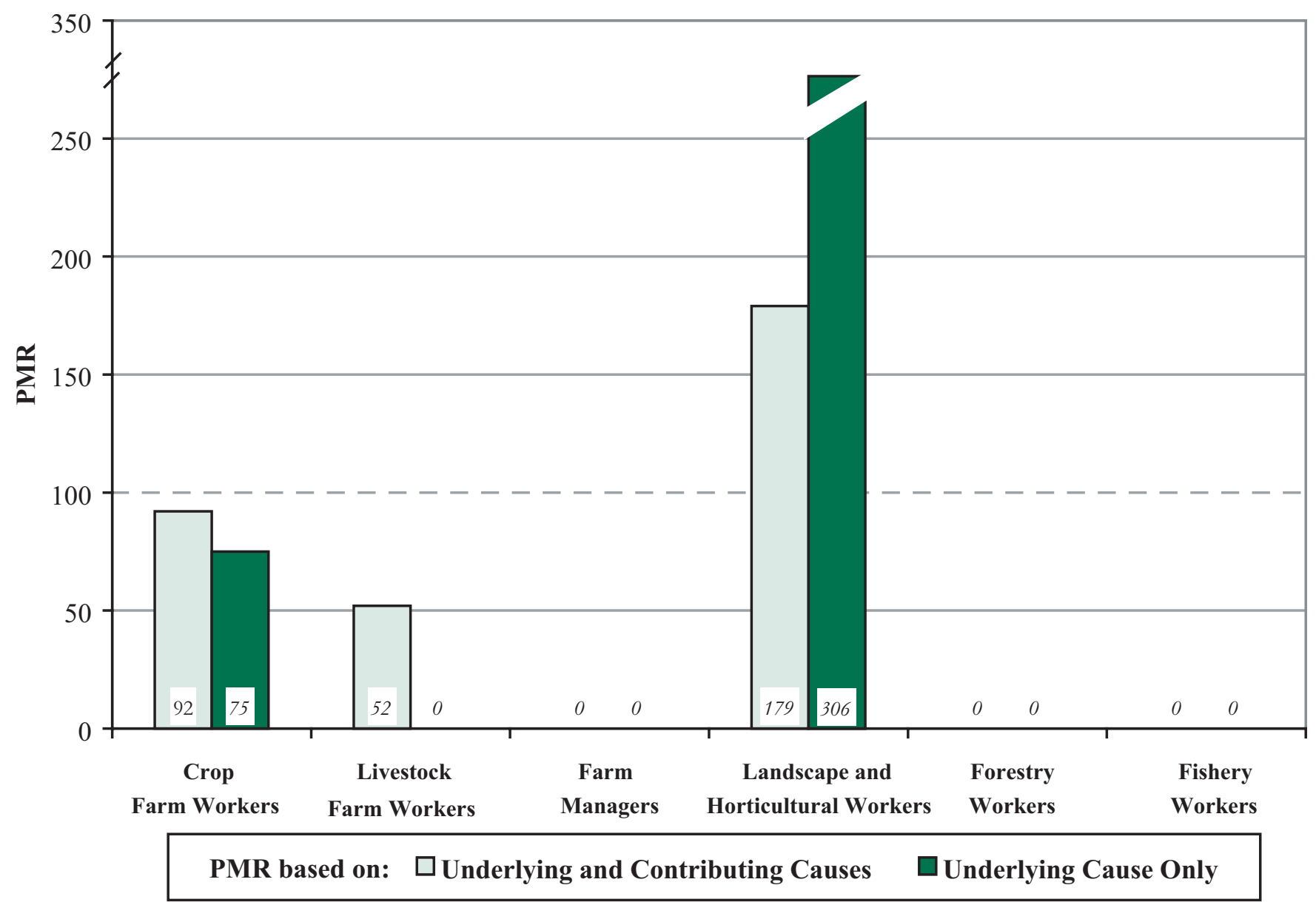

ICD - International Classification of Diseases, $9^{\text {th }}$ Revision

NOTE: Acute laryngitis and tracheitis = ICD-9 code 464. PMRs in bold are significantly different from 100 ( $<<0.05)$. PMRs in italics are based on fewer

than five observed deaths. PMRs are based on underlying and contributing cause of death. See appendices for source description, methods, ICD codes, and

a list of selected states.

SOURCE: National Center for Health Statistics multiple-cause-of-death data 
Figure 2-44. Acute upper respiratory infections of multiple or unspecified sites: Proportionate mortality ratio (PMR) adjusted for age, sex, and race/ethnicity by agricultural group, U.S. residents age 15 and over, selected states, 1988-1998

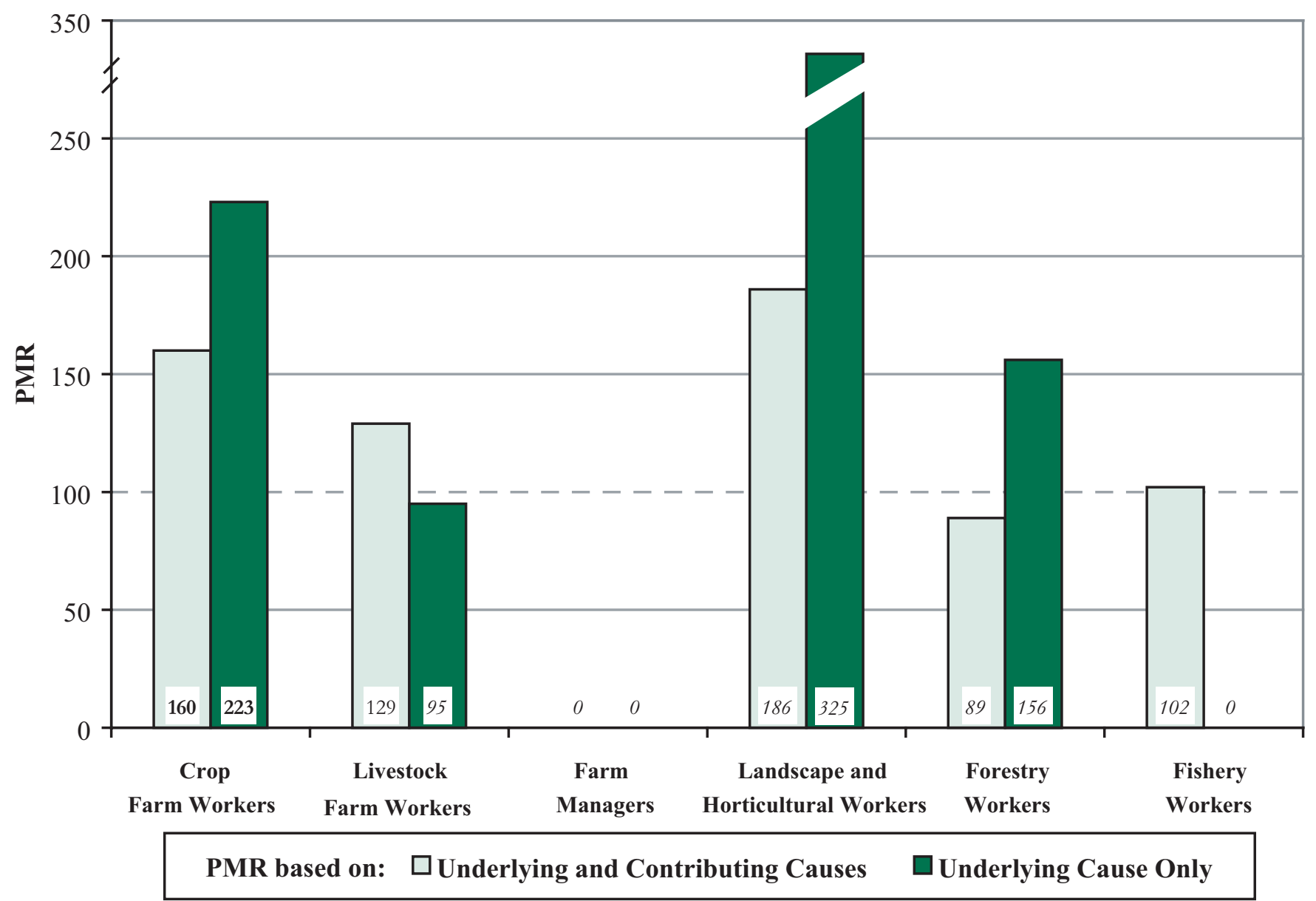

ICD - International Classification of Diseases, $9^{\text {th }}$ Revision

NOTE: Acute upper respiratory infections of multiple or unspecified sites $=$ ICD-9 code 465 . PMRs in bold are significantly different from 100 ( $p<0.05$ ).

PMRs in italics are based on fewer than five observed deaths. PMRs are based on underlying and contributing cause of death. See appendices for source description, methods, ICD codes, and a list of selected states.

SOURCE: National Center for Health Statistics multiple-cause-of-death data 
Figure 2-45. Acute bronchitis and bronchiolitis: Proportionate mortality ratio (PMR) adjusted for age, sex, and race/ethnicity by agricultural group, U.S. residents age 15 and over, selected states, 1988-1998

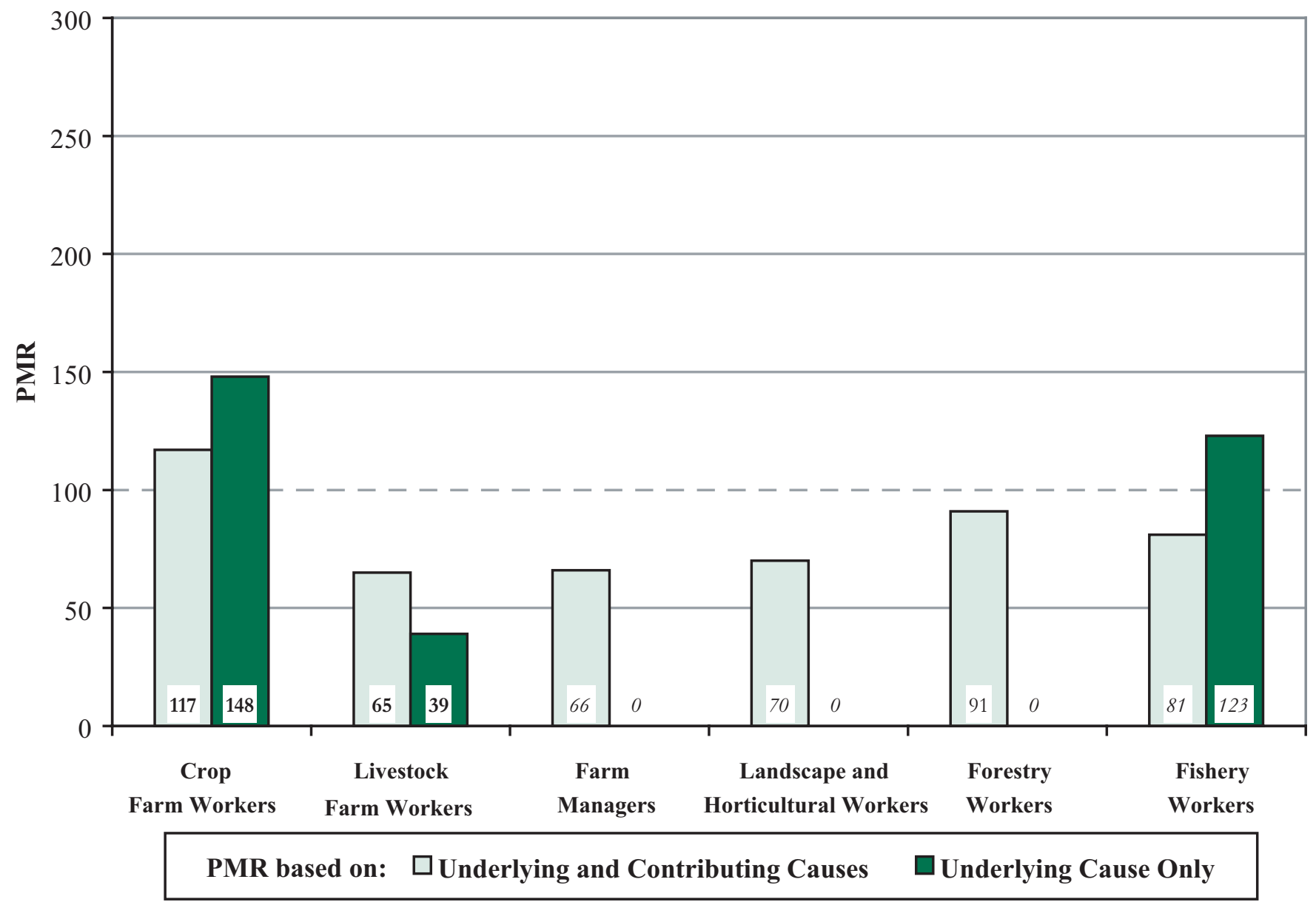

ICD - International Classification of Diseases, $9^{\text {th }}$ Revision

NOTE: Acute bronchitis and bronchiolitis $=$ ICD-9 code 466. PMRs in bold are significantly different from 100 ( $<<0.05)$. PMRs in italics are based on

fewer than five observed deaths. PMRs are based on underlying and contributing cause of death. See appendices for source description, methods, ICD codes, and a list of selected states.

SOURCE: National Center for Health Statistics multiple-cause-of-death data 
Table 2-61. Crop farm workers: Proportionate mortality ratio (PMR) adjusted for age, sex, and race/ ethnicity for other diseases of upper respiratory tract, U.S. residents age 15 and over, selected states, 1988-1998

\begin{tabular}{|c|c|c|c|c|}
\hline \multirow[b]{2}{*}{ Disease Category (ICD Code) } & \multirow{2}{*}{$\begin{array}{l}\text { Number } \\
\text { of Deaths }\end{array}$} & \multirow[b]{2}{*}{ PMR } & \multicolumn{2}{|c|}{ 95\% Confidence Interval } \\
\hline & & & LCL & UCL \\
\hline Nasal polyps (471) & 1 & 91 & 2 & 506 \\
\hline Chronic pharyngitis and nasopharyngitis (472) & 1 & 68 & 2 & 378 \\
\hline Chronic sinusitis (473) & 19 & 72 & 43 & 113 \\
\hline Chronic disease of tonsils and adenoids (474) & 3 & 161 & 33 & 471 \\
\hline Peritonsillar abscess (475) & 2 & 150 & 18 & 542 \\
\hline Allergic rhinitis (477) & 6 & 214 & 78 & 466 \\
\hline Other diseases of upper respiratory tract (478) & 65 & 90 & 70 & 115 \\
\hline
\end{tabular}

ICD - International Classification of Diseases, $9^{\text {th }}$ Revision $\quad$ LCL - lower confidence limit $\quad$ UCL - upper confidence limit
NOTE: PMRs in bold are significantly different from $100(p<0.05)$. PMRs in italics are based on fewer than five observed deaths. PMRs are based on underlying and contributing cause of death. Some values could not be calculated because the number of observed or expected deaths was zero; such values are indicated by ---. See appendices for source description, methods, ICD codes, and a list of selected states.

SOURCE: National Center for Health Statistics multiple-cause-of-death data 
Table 2-62. Livestock farm workers: Proportionate mortality ratio (PMR) adjusted for age, sex, and race/ethnicity for other diseases of upper respiratory tract, U.S. residents age 15 and over, selected states, 1988-1998

\begin{tabular}{|c|c|c|c|c|}
\hline \multirow[b]{2}{*}{ Disease Category (ICD Code) } & \multirow{2}{*}{$\begin{array}{l}\text { Number } \\
\text { of Deaths }\end{array}$} & \multirow[b]{2}{*}{ PMR } & \multicolumn{2}{|c|}{ 95\% Confidence Interval } \\
\hline & & & $\mathbf{L C L}$ & UCL \\
\hline Nasal polyps (471) & 0 & 0 & --- & --- \\
\hline Chronic pharyngitis and nasopharyngitis (472) & 0 & 0 & --- & --- \\
\hline Chronic sinusitis (473) & 8 & 117 & 50 & 230 \\
\hline Chronic disease of tonsils and adenoids (474) & 1 & 207 & 5 & 1,150 \\
\hline Peritonsillar abscess $(475)$ & 1 & 283 & 7 & 1,572 \\
\hline Allergic rhinitis (477) & 1 & 117 & 3 & 650 \\
\hline Other diseases of upper respiratory tract (478) & 9 & 49 & 22 & 93 \\
\hline
\end{tabular}

ICD - International Classification of Diseases, $9^{\text {th }}$ Revision $\quad$ LCL - lower confidence limit $\quad$ UCL - upper confidence limit
NOTE: PMRs in bold are significantly different from $100(p<0.05)$. PMRs in italics are based on fewer than five observed deaths. PMRs are based on underlying and contributing cause of death. Some values could not be calculated because the number of observed or expected deaths was zero; such values are indicated by ---. See appendices for source description, methods, ICD codes, and a list of selected states.

SOURCE: National Center for Health Statistics multiple-cause-of-death data 
Table 2-63. Farm managers: Proportionate mortality ratio (PMR) adjusted for age, sex, and race/ ethnicity for other diseases of upper respiratory tract, U.S. residents age 15 and over, selected states, 1988-1998

\begin{tabular}{|c|c|c|c|c|}
\hline \multirow[b]{2}{*}{ Disease Category (ICD Code) } & \multirow{2}{*}{$\begin{array}{l}\text { Number } \\
\text { of Deaths }\end{array}$} & \multirow[b]{2}{*}{ PMR } & \multicolumn{2}{|c|}{ 95\% Confidence Interval } \\
\hline & & & LCL & UCL \\
\hline Nasal polyps (471) & 0 & 0 & --- & --- \\
\hline Chronic pharyngitis and nasopharyngitis (472) & 0 & 0 & --- & --- \\
\hline Chronic sinusitis (473) & 1 & 205 & 5 & 1,139 \\
\hline Chronic disease of tonsils and adenoids (474) & 0 & 0 & --- & --- \\
\hline Peritonsillar abscess $(475)$ & 0 & 0 & --- & --- \\
\hline Allergic rhinitis (477) & 0 & 0 & --- & --- \\
\hline Other diseases of upper respiratory tract (478) & 0 & 0 & --- & --- \\
\hline
\end{tabular}

\section{ICD - International Classification of Diseases, $9^{\text {th }}$ Revision $\quad$ LCL - lower confidence limit $\quad$ UCL - upper confidence limit}

NOTE: PMRs in bold are significantly different from $100(\mathrm{p}<0.05)$. PMRs in italics are based on fewer than five observed deaths. PMRs are based on underlying and contributing cause of death. Some values could not be calculated because the number of observed or expected deaths was zero; such values are indicated by ---. See appendices for source description, methods, ICD codes, and a list of selected states.

SOURCE: National Center for Health Statistics multiple-cause-of-death data 
Table 2-64. Landscape and horticultural workers: Proportionate mortality ratio (PMR) adjusted for age, sex, and race/ethnicity for other diseases of upper respiratory tract, U.S. residents age 15 and over, selected states, 1988-1998

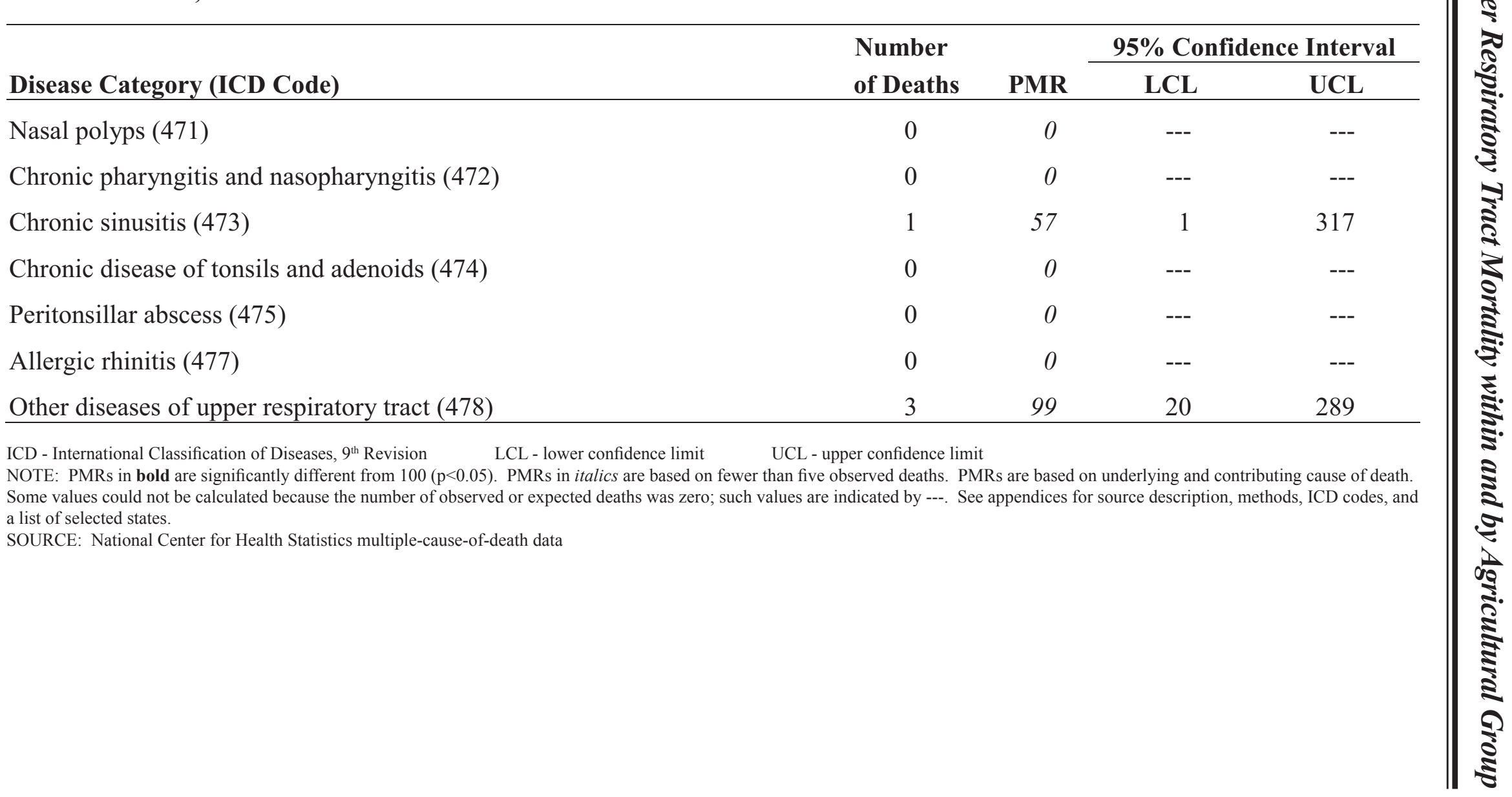


Table 2-65. Forestry workers: Proportionate mortality ratio (PMR) adjusted for age, sex, and race/ ethnicity for other diseases of upper respiratory tract, U.S. residents age 15 and over, selected states, 1988-1998

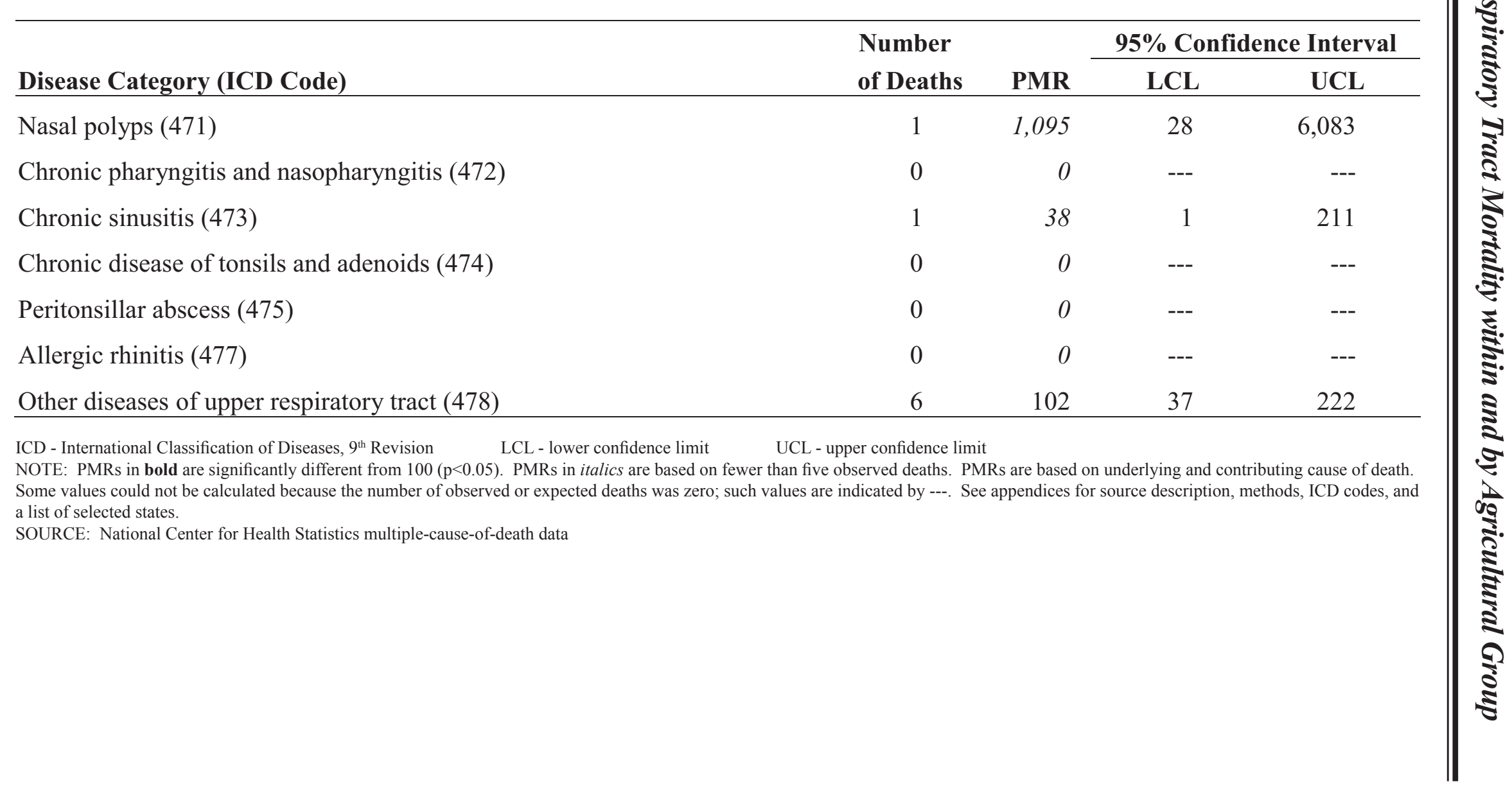


Table 2-66. Fishery workers: Proportionate mortality ratio (PMR) adjusted for age, sex, and race/ ethnicity for other diseases of upper respiratory tract, U.S. residents age 15 and over, selected states, 1988-1998

\begin{tabular}{|c|c|c|c|c|}
\hline \multirow[b]{2}{*}{ Disease Category (ICD Code) } & \multirow{2}{*}{$\begin{array}{l}\text { Number } \\
\text { of Deaths }\end{array}$} & \multirow[b]{2}{*}{ PMR } & \multicolumn{2}{|c|}{ 95\% Confidence Interval } \\
\hline & & & $\mathbf{L C L}$ & UCL \\
\hline Nasal polyps (471) & 0 & 0 & --- & --- \\
\hline Chronic pharyngitis and nasopharyngitis (472) & 0 & 0 & --- & --- \\
\hline Chronic sinusitis (473) & 0 & 0 & --- & --- \\
\hline Chronic disease of tonsils and adenoids (474) & 1 & 1,376 & 35 & 7,644 \\
\hline Peritonsillar abscess $(475)$ & 0 & 0 & --- & --- \\
\hline Allergic rhinitis (477) & 1 & 1,619 & 41 & 8,994 \\
\hline Other diseases of upper respiratory tract (478) & 3 & 191 & 39 & 558 \\
\hline
\end{tabular}

ICD - International Classification of Diseases, $9^{\text {th }}$ Revision $\quad$ LCL - lower confidence limit $\quad$ UCL - upper confidence limit
NOTE: PMRs in bold are significantly different from $100(p<0.05)$. PMRs in italics are based on fewer than five observed deaths. PMRs are based on underlying and contributing cause of death. Some values could not be calculated because the number of observed or expected deaths was zero; such values are indicated by ---. See appendices for source description, methods, ICD codes, and a list of selected states.

SOURCE: National Center for Health Statistics multiple-cause-of-death data 
Figure 2-46. Chronic sinusitis: Proportionate mortality ratio (PMR) adjusted for age, sex, and race/ethnicity by agricultural group, U.S. residents age 15 and over, selected states, 1988-1998

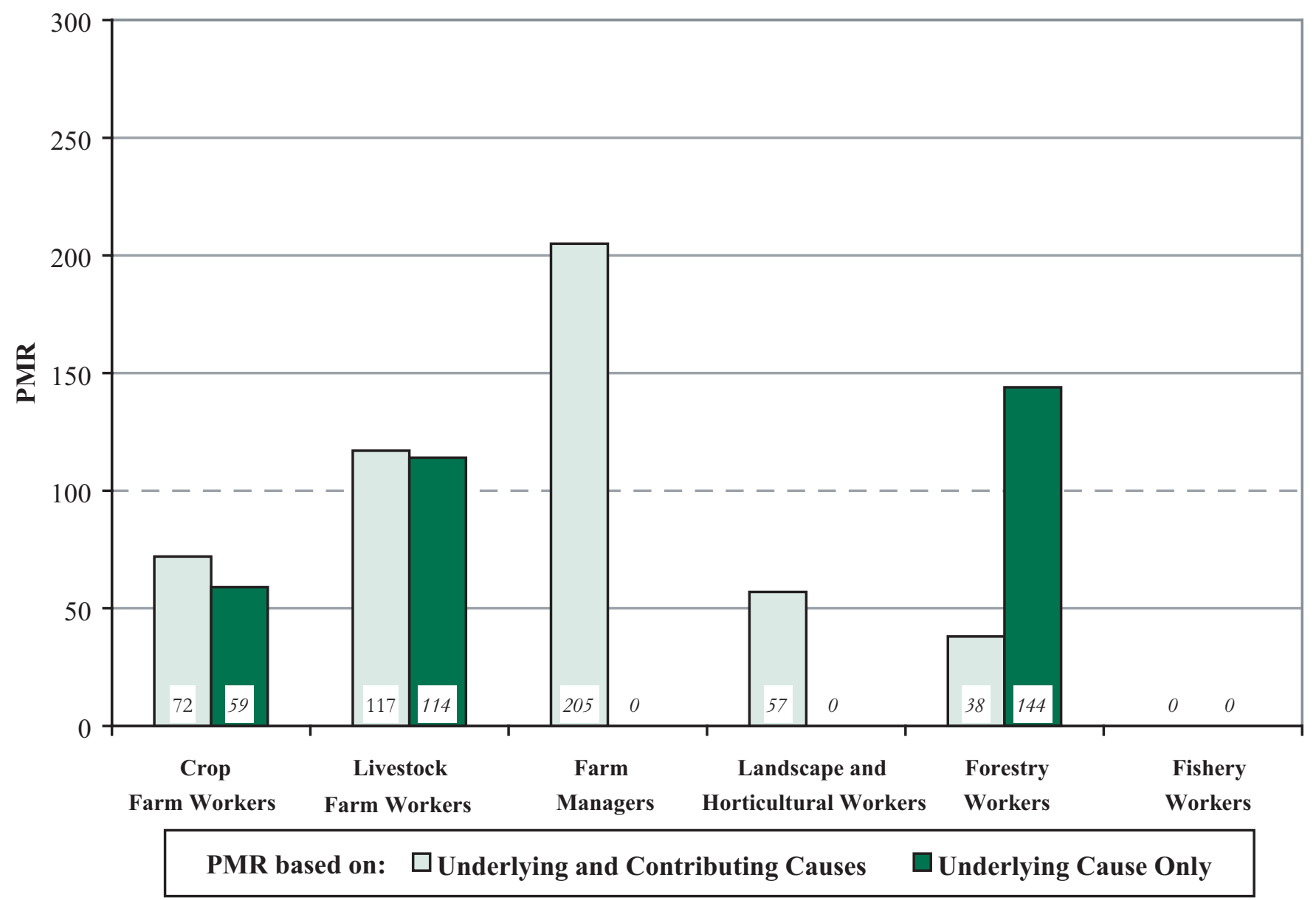

ICD - International Classification of Diseases, $9^{\text {th }}$ Revision

NOTE: Chronic sinusitis = ICD-9 code 473. PMRs in bold are significantly different from $100(\mathrm{p}<0.05)$. PMRs in italics are based on fewer than five observed deaths. PMRs are based on underlying and contributing cause of death. See appendices for source description, methods, ICD codes, and a list of selected states.

SOURCE: National Center for Health Statistics multiple-cause-of-death data 
Figure 2-47. Other diseases of upper respiratory tract: Proportionate mortality ratio (PMR) adjusted for age, sex, and race/ethnicity by agricultural group, U.S. residents age 15 and over, selected states, 1988-1998

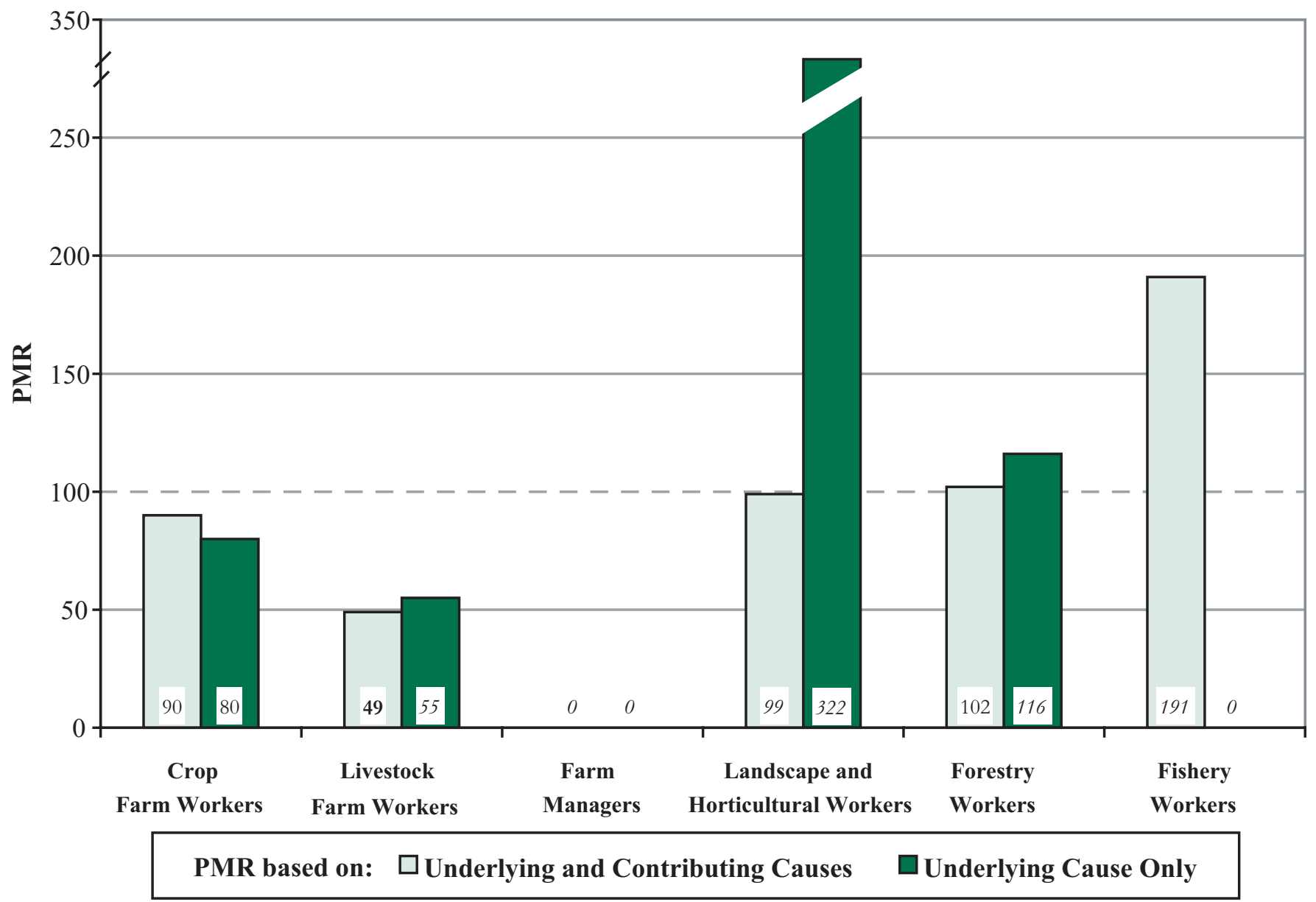

ICD - International Classification of Diseases, $9^{\text {th }}$ Revision

NOTE: Other diseases of upper respiratory tract $=$ ICD-9 code 478 . PMRs in bold are significantly different from 100 ( $<<0.05$ ). PMRs in italics are based on fewer than five observed deaths. PMRs are based on underlying and contributing cause of death. See appendices for source description, methods, ICD codes, and a list of selected states.

SOURCE: National Center for Health Statistics multiple-cause-of-death data 
Table 2-67. Crop farm workers: Proportionate mortality ratio (PMR) adjusted for age, sex, and race/ ethnicity for pneumonia and influenza, U.S. residents age 15 and over, selected states, 1988-1998

\begin{tabular}{|c|c|c|c|c|}
\hline \multirow[b]{2}{*}{ Disease Category (ICD Code) } & \multirow{2}{*}{$\begin{array}{l}\text { Number } \\
\text { of Deaths }\end{array}$} & \multirow[b]{2}{*}{ PMR } & \multicolumn{2}{|c|}{ 95\% Confidence Interval } \\
\hline & & & $\mathbf{L C L}$ & $\mathbf{U C L}$ \\
\hline Viral pneumonia (480) & 60 & 110 & 84 & 142 \\
\hline Pneumococcal pneumonia [Streptococcus pneumoniae pneumonia] (481) & 347 & 98 & 88 & 109 \\
\hline Other bacterial pneumonia (482) & 955 & 120 & 113 & 128 \\
\hline Pneumonia due to other specified organism (483) & 20 & 113 & 69 & 175 \\
\hline Bronchopneumonia, organism unspecified (485) & 1,365 & 106 & 100 & 112 \\
\hline Pneumonia, organism unspecified (486) & 23,135 & 109 & 107 & 111 \\
\hline Influenza (487) & 232 & 142 & 125 & 162 \\
\hline
\end{tabular}


Table 2-68. Livestock farm workers: Proportionate mortality ratio (PMR) adjusted for age, sex, and race/ethnicity for pneumonia and influenza, U.S. residents age 15 and over, selected states, 1988-1998

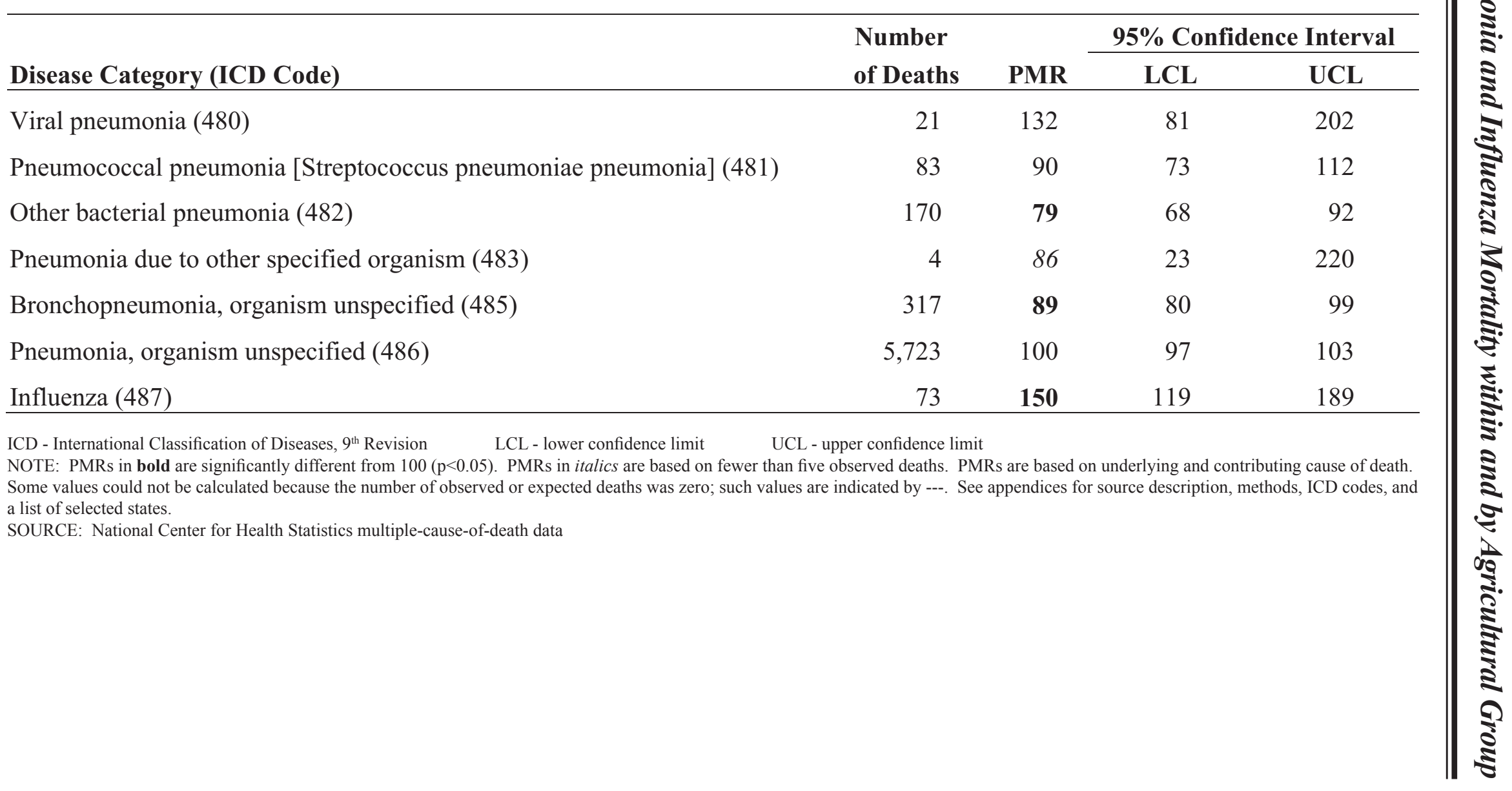


Table 2-69. Farm managers: Proportionate mortality ratio (PMR) adjusted for age, sex, and race/ ethnicity for pneumonia and influenza, U.S. residents age 15 and over, selected states, 1988-1998

\begin{tabular}{|c|c|c|c|c|}
\hline \multirow[b]{2}{*}{ Disease Category (ICD Code) } & \multirow{2}{*}{$\begin{array}{l}\text { Number } \\
\text { of Deaths }\end{array}$} & \multirow[b]{2}{*}{ PMR } & \multicolumn{2}{|c|}{ 95\% Confidence Interval } \\
\hline & & & LCL & UCL \\
\hline Viral pneumonia (480) & 2 & 208 & 13 & 390 \\
\hline Pneumococcal pneumonia [Streptococcus pneumoniae pneumonia\} (481) & 6 & 109 & 40 & 237 \\
\hline Other bacterial pneumonia (482) & 10 & 83 & 40 & 153 \\
\hline Pneumonia due to other specified organism (483) & 0 & 0 & --- & --- \\
\hline Bronchopneumonia, organism unspecified (485) & 14 & 72 & 39 & 121 \\
\hline Pneumonia, organism unspecified (486) & 337 & 106 & 95 & 118 \\
\hline Influenza (487) & 4 & 155 & 42 & 396 \\
\hline
\end{tabular}


Table 2-70. Landscape and horticultural workers: Proportionate mortality ratio (PMR) adjusted for age, sex, and race/ethnicity for pneumonia and influenza, U.S. residents age 15 and over, selected states, 1988-1998

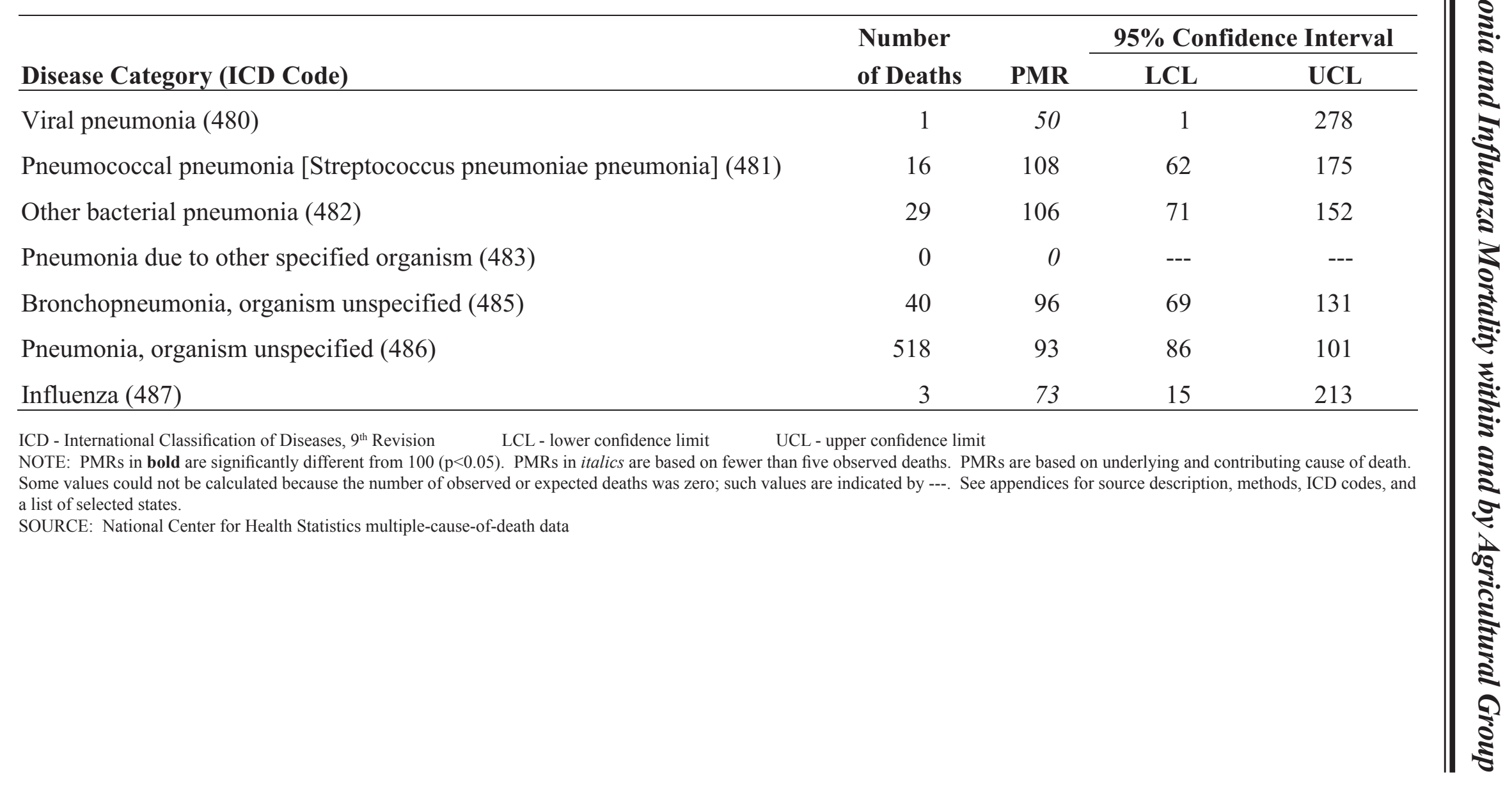


Table 2-71. Forestry workers: Proportionate mortality ratio (PMR) adjusted for age, sex, and race/ ethnicity for pneumonia and influenza, U.S. residents age 15 and over, selected states, 1988-1998

\begin{tabular}{|c|c|c|c|c|}
\hline \multirow[b]{2}{*}{ Disease Category (ICD Code) } & \multirow{2}{*}{$\begin{array}{l}\text { Number } \\
\text { of Deaths }\end{array}$} & \multirow[b]{2}{*}{ PMR } & \multicolumn{2}{|c|}{ 95\% Confidence Interval } \\
\hline & & & LCL & UCL \\
\hline Viral pneumonia (480) & 3 & 78 & 16 & 228 \\
\hline Pneumococcal pneumonia [Streptococcus pneumoniae pneumonia] (481) & 28 & 102 & 68 & 147 \\
\hline Other bacterial pneumonia (482) & 65 & 112 & 88 & 143 \\
\hline Pneumonia due to other specified organism (483) & 2 & 133 & 16 & 480 \\
\hline Bronchopneumonia, organism unspecified (485) & 93 & 104 & 85 & 127 \\
\hline Pneumonia, organism unspecified (486) & 1,564 & 117 & 111 & 123 \\
\hline Influenza (487) & 16 & 169 & 97 & 274 \\
\hline
\end{tabular}


Table 2-72. Fishery workers: Proportionate mortality ratio (PMR) adjusted for age, sex, and race/ ethnicity for pneumonia and influenza, U.S. residents age 15 and over, selected states, 1988-1998

\begin{tabular}{|c|c|c|c|c|}
\hline \multirow[b]{2}{*}{ Disease Category (ICD Code) } & \multirow{2}{*}{$\begin{array}{l}\text { Number } \\
\text { of Deaths }\end{array}$} & \multirow[b]{2}{*}{ PMR } & \multicolumn{2}{|c|}{ 95\% Confidence Interval } \\
\hline & & & LCL & UCL \\
\hline Viral pneumonia (480) & 1 & 86 & 2 & 478 \\
\hline Other bacterial pneumonia (482) & 12 & 77 & 40 & 134 \\
\hline Pneumonia due to other specified organism (483) & 0 & 0 & --- & --- \\
\hline Pneumonia, organism unspecified (486) & 370 & 104 & 94 & 115 \\
\hline Influenza (487) & 2 & 73 & 9 & 264 \\
\hline \multicolumn{5}{|c|}{$\begin{array}{l}\text { ICD - International Classification of Diseases, } 9^{\text {th }} \text { Revision } \quad \text { LCL - lower confidence limit } \quad \text { UCL - upper confidence limit } \\
\text { NOTE: PMRs in bold are significantly different from } 100(p<0.05) \text {. PMRs in italics are based on fewer than five observed deaths. PMRs are based on underlying and contributing cause of death. } \\
\text { Some values could not be calculated because the number of observed or expected deaths was zero; such values are indicated by --.. See appendices for source description, methods, ICD codes, and } \\
\text { a list of selected states. } \\
\text { SOURCE: National Center for Health Statistics multiple-cause-of-death data }\end{array}$} \\
\hline
\end{tabular}


Figure 2-48. Viral pneumonia: Proportionate mortality ratio (PMR) adjusted for age, sex, and race/ethnicity by agricultural group, U.S. residents age 15 and over, selected states, 1988-1998

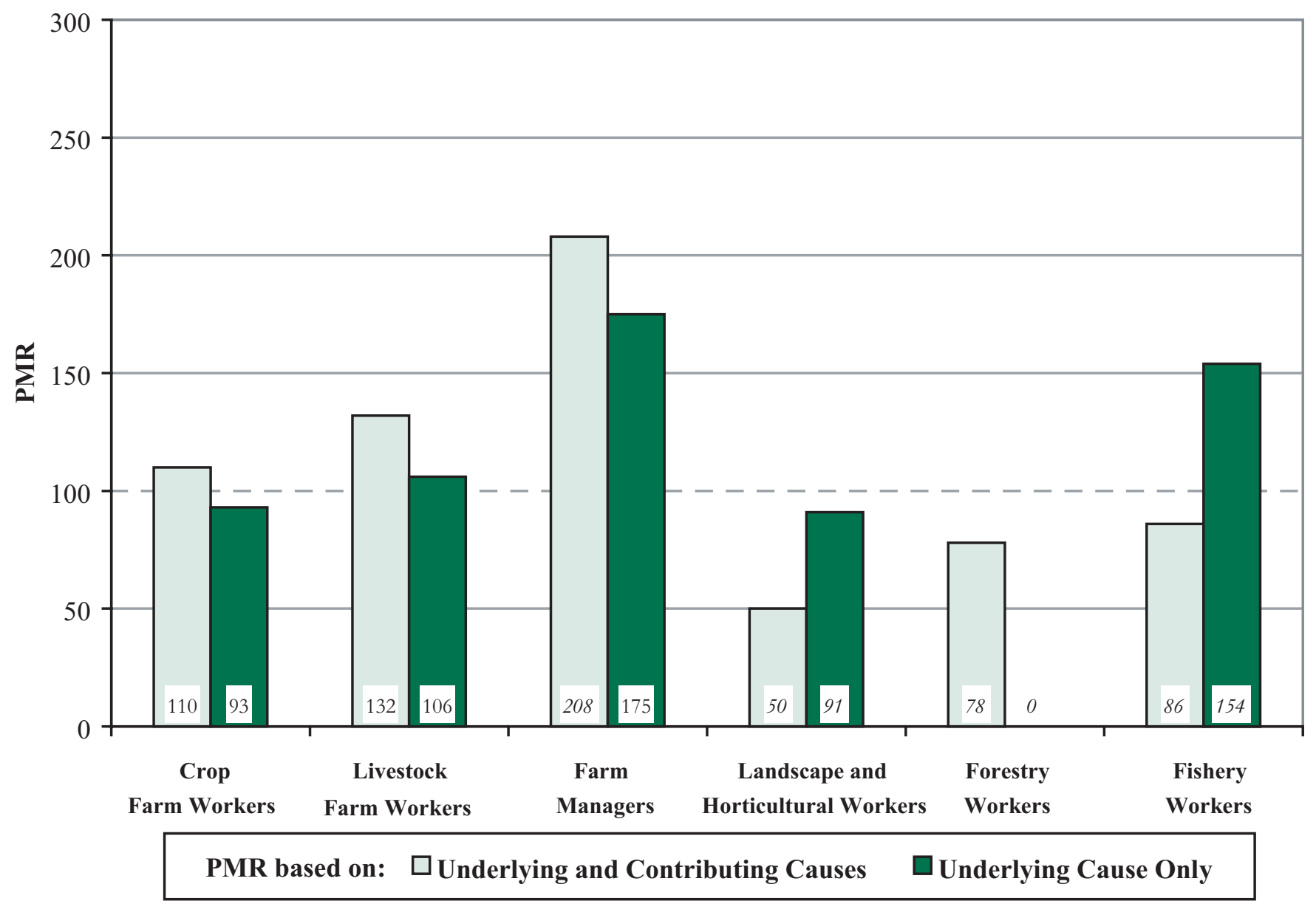

ICD - International Classification of Diseases, $9^{\text {th }}$ Revision

NOTE: Viral pneumonia = ICD-9 code 480. PMRs in bold are significantly different from $100(\mathrm{p}<0.05)$. PMRs in italics are based on fewer than five observed deaths. PMRs are based on underlying and contributing cause of death. See appendices for source description, methods, ICD codes, and a list of selected states.

SOURCE: National Center for Health Statistics multiple-cause-of-death data 
Figure 2-49. Pneumococcal pneumonia: Proportionate mortality ratio (PMR) adjusted for age, sex, and race/ethnicity by agricultural group, U.S. residents age 15 and over, selected states, 1988-1998

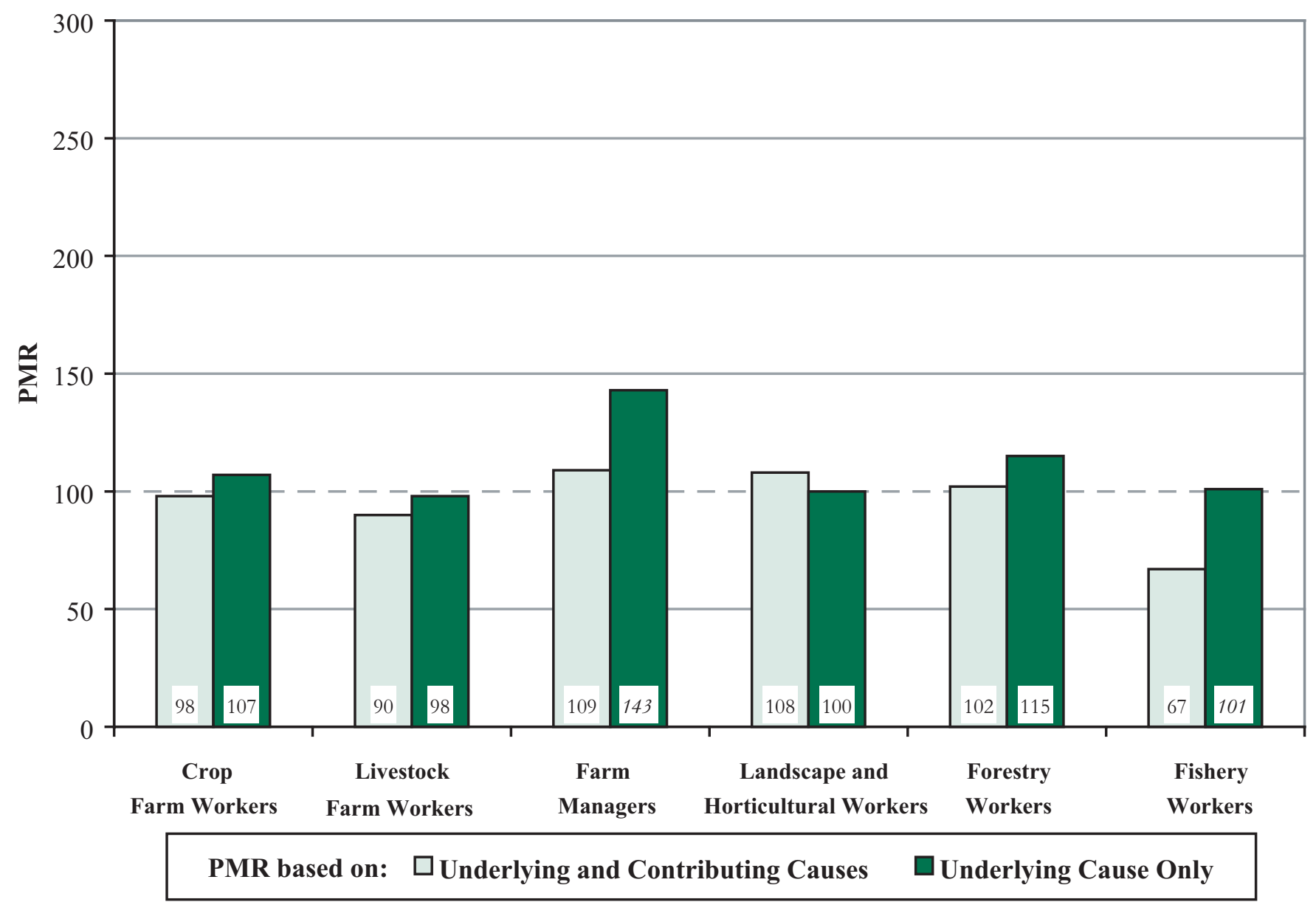

ICD - International Classification of Diseases, $9^{\text {th }}$ Revision

NOTE: Pneumococcal pneumonia = ICD-9 code 481. PMRs in bold are significantly different from 100 ( $<<0.05$ ). PMRs in italics are based on fewer than five observed deaths. PMRs are based on underlying and contributing cause of death. See appendices for source description, methods, ICD codes, and a list of selected states.

SOURCE: National Center for Health Statistics multiple-cause-of-death data 
Figure 2-50. Other bacterial pneumonia: Proportionate mortality ratio (PMR) adjusted for age, sex, and race/ethnicity by agricultural group, U.S. residents age 15 and over, selected states, 1988-1998

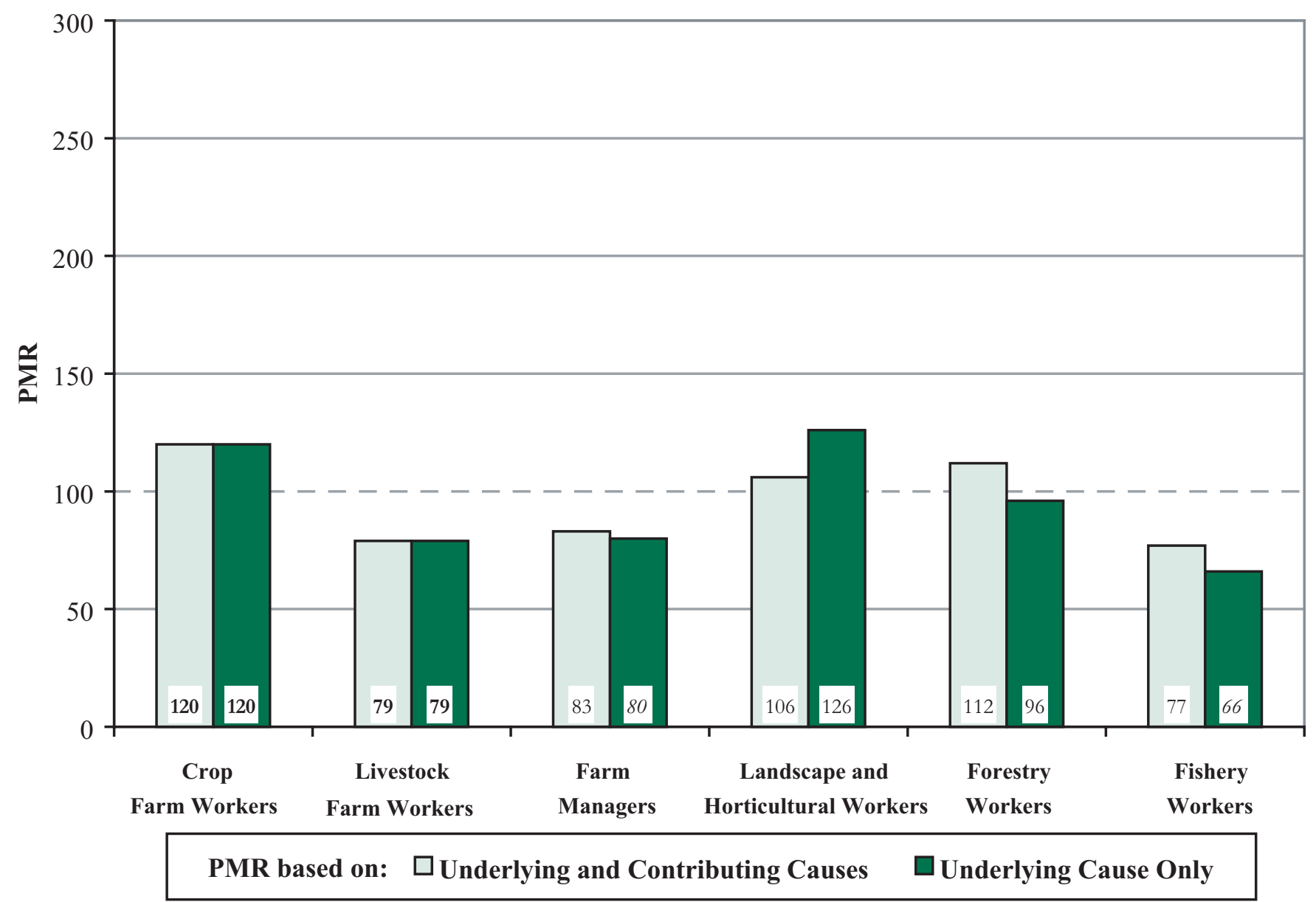

ICD - International Classification of Diseases, $9^{\text {th }}$ Revision

NOTE: Other bacterial pneumonia $=$ ICD-9 code 482. PMRs in bold are significantly different from 100 ( $\mathrm{p}<0.05)$. PMRs in italics are based on fewer than five observed deaths. PMRs are based on underlying and contributing cause of death. See appendices for source description, methods, ICD codes, and a list of selected states.

SOURCE: National Center for Health Statistics multiple-cause-of-death data 
Figure 2-51. Pneumonia due to other specified organism: Proportionate mortality ratio (PMR) adjusted for age, sex, and race/ethnicity by agricultural group, U.S. residents age 15 and over, selected states, 1988-1998

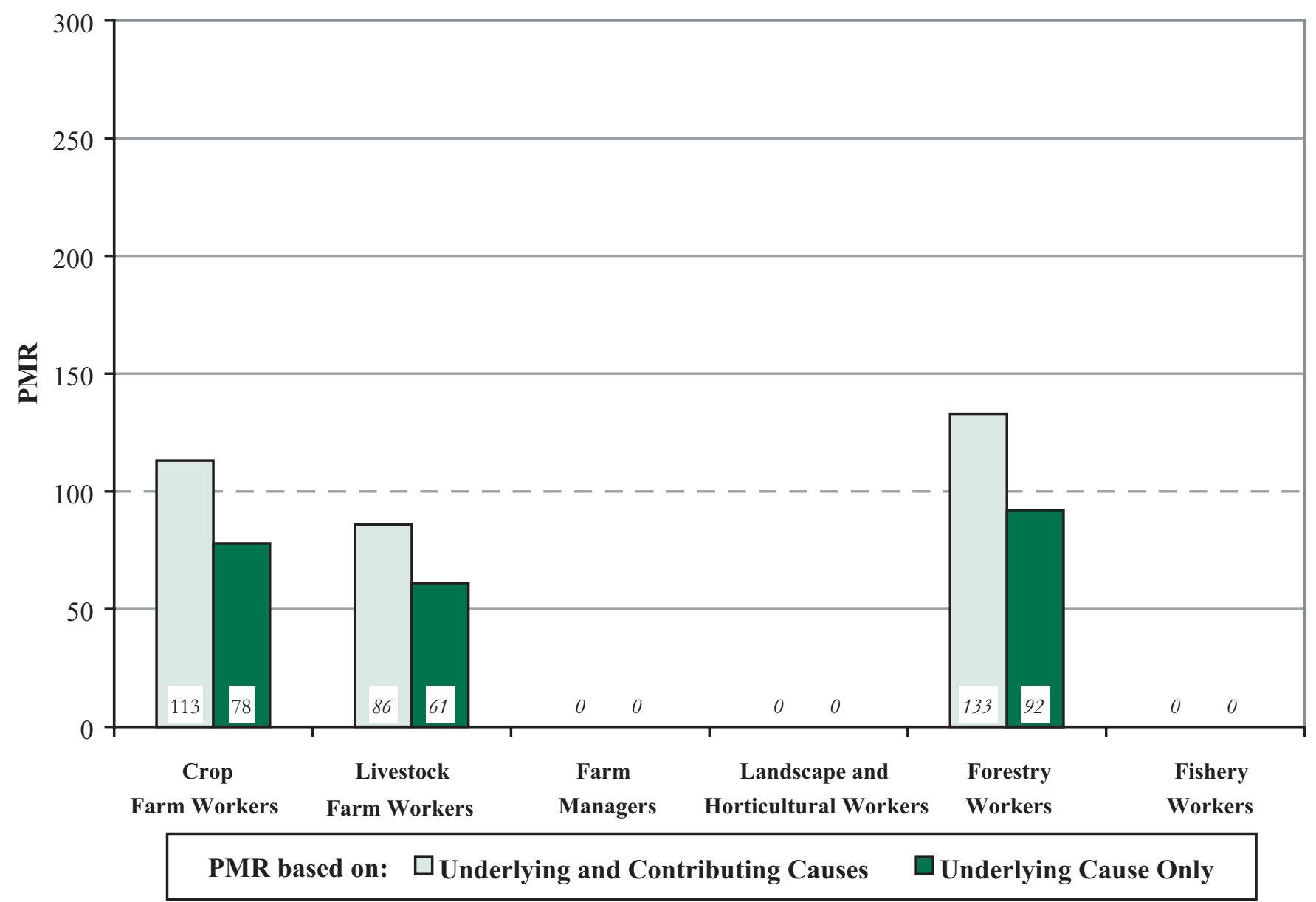

ICD - International Classification of Diseases, $9^{\text {th }}$ Revision

NOTE: Other bacterial pneumonia = ICD-9 code 482. PMRs in bold are significantly different from 100 ( $<<0.05)$. PMRs in italics are based on fewer than five observed deaths. PMRs are based on underlying and contributing cause of death. See appendices for source description, methods, ICD codes, and a list of selected states.

SOURCE: National Center for Health Statistics multiple-cause-of-death data 
Figure 2-52. Bronchopneumonia, organism unspecified: Proportionate mortality ratio (PMR) adjusted for age, sex, and race/ethnicity by agricultural group, U.S. residents age 15 and over, selected states, 1988-1998

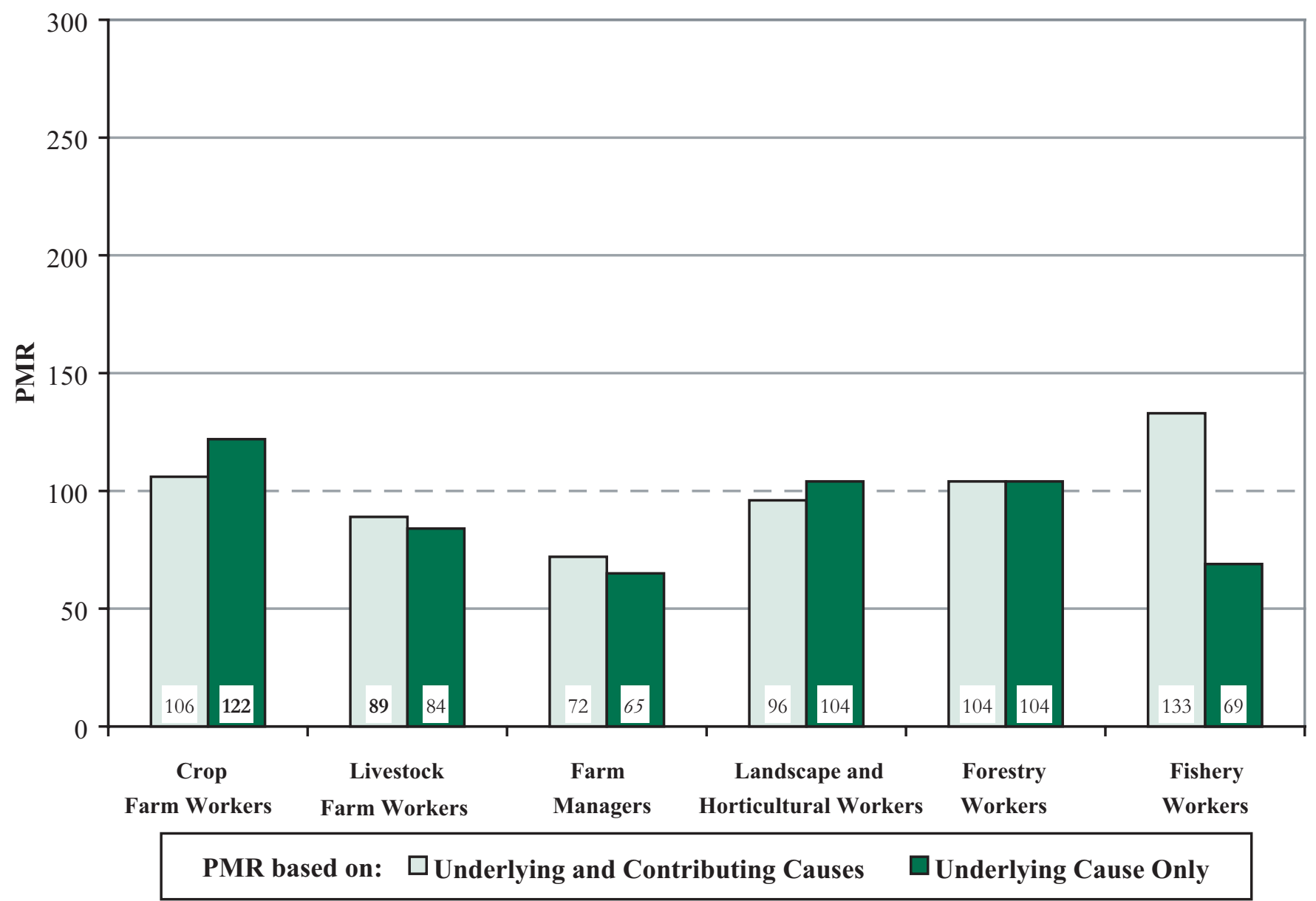

ICD - International Classification of Diseases, $9^{\text {th }}$ Revision

NOTE: Bronchopneumonia, organism unspecified = ICD-9 code 485 . PMRs in bold are significantly different from 100 ( $\mathrm{p}<0.05$ ). PMRs in italics are based on fewer than five observed deaths. PMRs are based on underlying and contributing cause of death. See appendices for source description, methods, ICD codes, and a list of selected states.

SOURCE: National Center for Health Statistics multiple-cause-of-death data 
Figure 2-53. Pneumonia, organism unspecified: Proportionate mortality ratio (PMR) adjusted for age, sex, and race/ethnicity by agricultural group, U.S. residents age 15 and over, selected states, 1988-1998

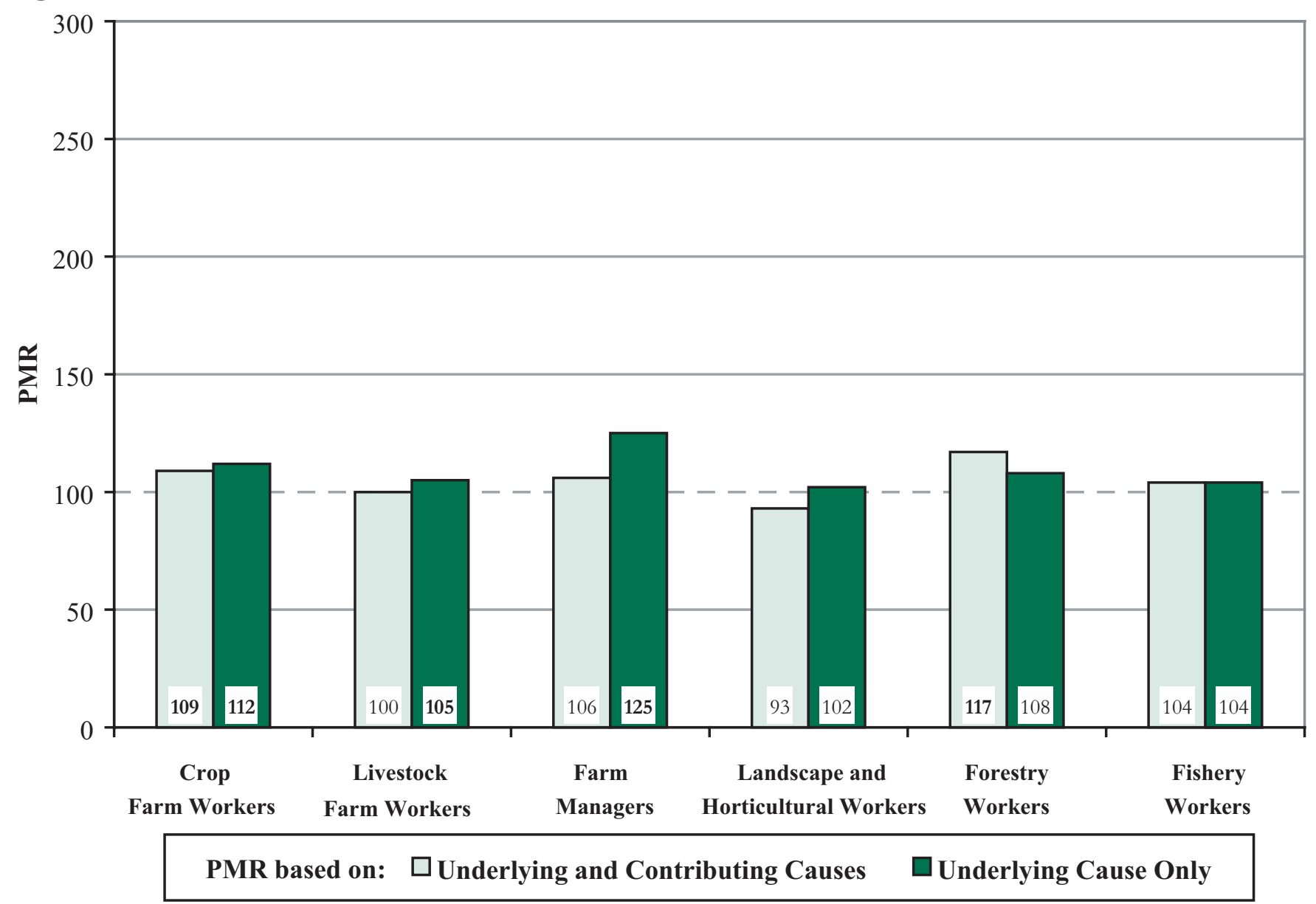

ICD - International Classification of Diseases, $9^{\text {th }}$ Revision

NOTE: Pneumonia, organism unspecified = ICD-9 code 486. PMRs in bold are significantly different from 100 ( $<<0.05$ ). PMRs in italics are based on fewer than five observed deaths. PMRs are based on underlying and contributing cause of death. See appendices for source description, methods, ICD codes, and a list of selected states.

SOURCE: National Center for Health Statistics multiple-cause-of-death data 
Figure 2-54. Influenza: Proportionate mortality ratio (PMR) adjusted for age, sex, and race/ethnicity by agricultural group, U.S. residents age 15 and over, selected states, 1988-1998

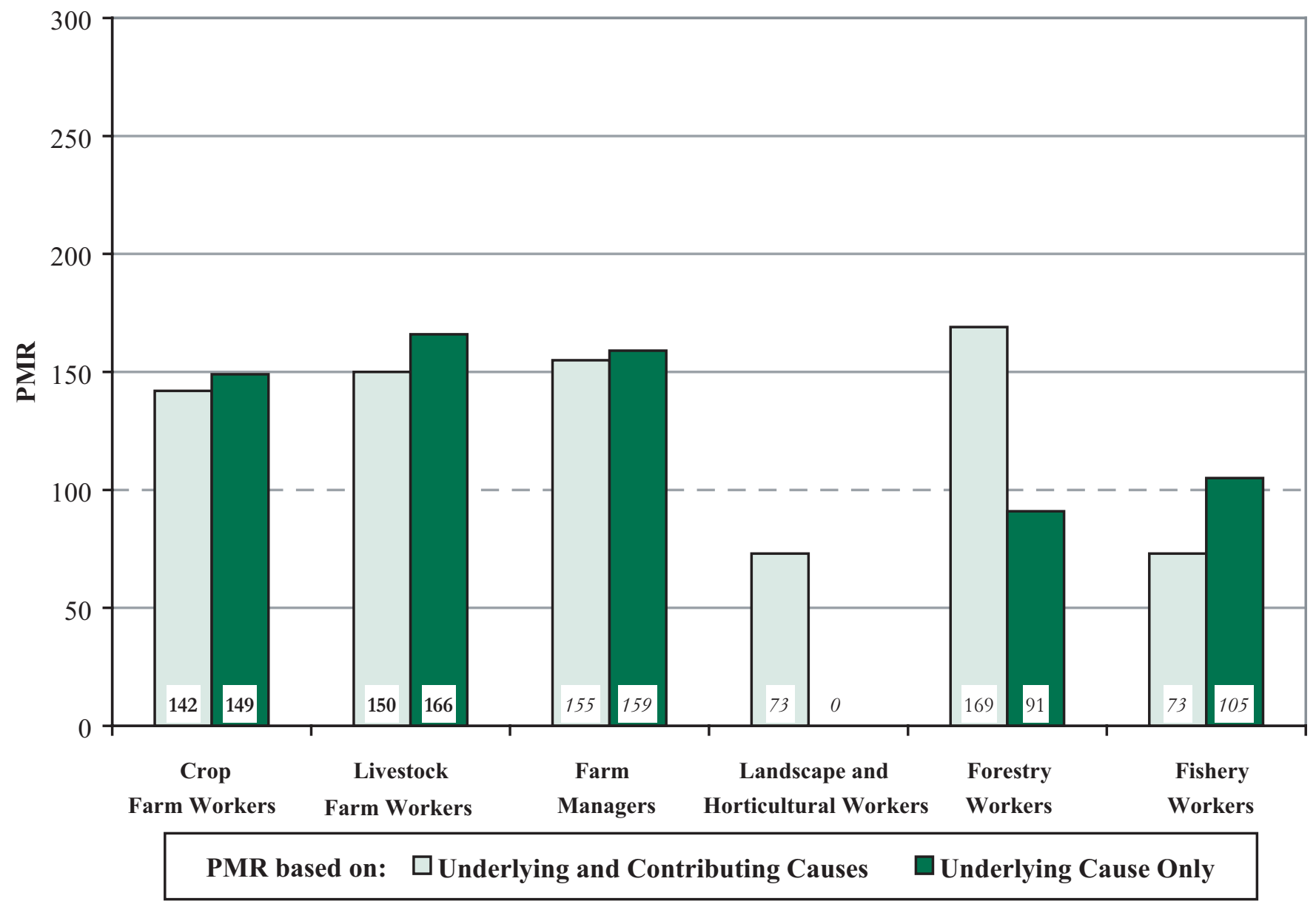

ICD - International Classification of Diseases, $9^{\text {th }}$ Revision

NOTE: Influenza = ICD-9 code 487. PMRs in bold are significantly different from $100(\mathrm{p}<0.05)$. PMRs in italics are based on fewer than five observed deaths. PMRs are based on underlying and contributing cause of death. See appendices for source description, methods, ICD codes, and a list of selected states.

SOURCE: National Center for Health Statistics multiple-cause-of-death data 
Table 2-73. Crop farm workers: Proportionate mortality ratio (PMR) adjusted for age, sex, and race/ ethnicity for chronic obstructive pulmonary disease and allied conditions, U.S. residents age 15 and over, selected states, 1988-1998

\begin{tabular}{lrrrr}
\hline & \multicolumn{2}{c}{ Number } & & \multicolumn{2}{c}{$\mathbf{9 5 \%}$ Confidence Interval } \\
\cline { 3 - 5 } Disease Category (ICD Code) & of Deaths & PMR & LCL & UCL \\
\hline Bronchitis, not specified as acute or chronic (490) & 269 & $\mathbf{1 3 4}$ & 119 & 151 \\
Chronic bronchitis (491) & 679 & 103 & 96 & 111 \\
Emphysema (492) & 3,265 & $\mathbf{8 6}$ & 83 & 104 \\
Asthma (493) & 813 & $\mathbf{1 1 1}$ & 119 \\
Bronchiectasis (494) & 139 & 90 & 76 & 106 \\
Hypersensitivity pneumonitis (495) & 23 & $\mathbf{1 , 2 2 8}$ & 777 & 1,844 \\
Chronic airway obstruction, not elsewhere classified (496) & 20,998 & $\mathbf{9 7}$ & 95 & 99 \\
\hline
\end{tabular}

ICD - International Classification of Diseases, $9^{\text {th }}$ Revision $\quad$ LCL - lower confidence limit $\quad$ UCL - upper confidence limit

NOTE: PMRs in bold are significantly different from $100(\mathrm{p}<0.05)$. PMRs in italics are based on fewer than five observed deaths. PMRs are based on underlying and contributing cause of death.

Some values could not be calculated because the number of observed or expected deaths was zero; such values are indicated by ---. See appendices for source description, methods, ICD codes, and a list of selected states.

SOURCE: National Center for Health Statistics multiple-cause-of-death data 
Table 2-74. Livestock farm workers: Proportionate mortality ratio (PMR) adjusted for age, sex, and race/ethnicity for chronic obstructive pulmonary disease and allied conditions, U.S. residents age 15 and over, selected states, 1988-1998

\begin{tabular}{lrrrr} 
& & Number & & \multicolumn{2}{c}{$\mathbf{9 5 \%}$ Confidence Interval } \\
\cline { 3 - 5 } Disease Category (ICD Code) & of Deaths & PMR & LCL & UCL \\
\hline Bronchitis, not specified as acute or chronic (490) & 58 & 103 & 79 & 133 \\
Chronic bronchitis (491) & 161 & 88 & 76 & 103 \\
Emphysema (492) & 956 & $\mathbf{9 1}$ & 85 & 133 \\
Asthma (493) & 276 & $\mathbf{1 5 0}$ & 57 & 169 \\
Bronchiectasis (494) & 35 & 79 & 55 & 110 \\
Hypersensitivity pneumonitis (495) & 31 & $\mathbf{5 , 5 6 3}$ & 3,779 & 7,904 \\
Chronic airway obstruction, not elsewhere classified (496) & 5,439 & $\mathbf{9 1}$ & 89 & 93 \\
\hline
\end{tabular}

ICD - International Classification of Diseases, $9^{\text {th }}$ Revision $\quad$ LCL - lower confidence limit $\quad$ UCL - upper confidence limit

NOTE: PMRs in bold are significantly different from $100(\mathrm{p}<0.05)$. PMRs in italics are based on fewer than five observed deaths. PMRs are based on underlying and contributing cause of death. Some values could not be calculated because the number of observed or expected deaths was zero; such values are indicated by ---. See appendices for source description, methods, ICD codes, and a list of selected states.

SOURCE: National Center for Health Statistics multiple-cause-of-death data 
Table 2-75. Farm managers: Proportionate mortality ratio (PMR) adjusted for age, sex, and race/ ethnicity for chronic obstructive pulmonary disease and allied conditions, U.S. residents age 15 and over, selected states, 1988-1998

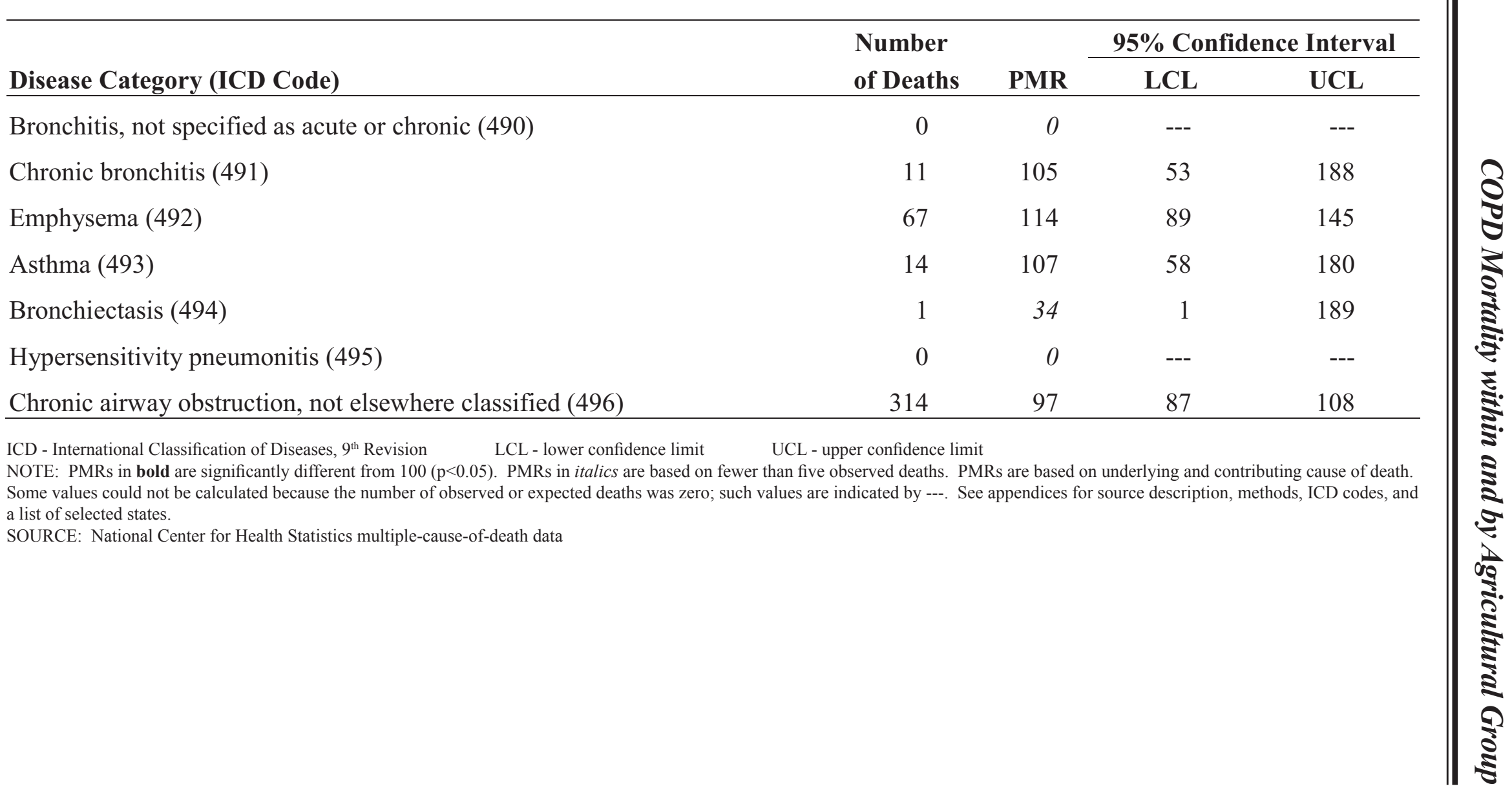


Table 2-76. Landscape and horticultural workers: Proportionate mortality ratio (PMR) adjusted for age, sex, and race/ethnicity for chronic obstructive pulmonary disease and allied conditions, U.S. residents age 15 and over, selected states, 1988-1998

\begin{tabular}{|c|c|c|c|c|}
\hline \multirow[b]{2}{*}{ Disease Category (ICD Code) } & \multirow{2}{*}{$\begin{array}{l}\text { Number } \\
\text { of Deaths }\end{array}$} & \multirow[b]{2}{*}{ PMR } & \multicolumn{2}{|c|}{ 95\% Confidence Interval } \\
\hline & & & $\mathbf{L C L}$ & UCL \\
\hline Bronchitis, not specified as acute or chronic (490) & 5 & 85 & 28 & 199 \\
\hline Chronic bronchitis (491) & 23 & 124 & 78 & 186 \\
\hline Emphysema (492) & 114 & 105 & 87 & 126 \\
\hline Asthma (493) & 33 & 92 & 63 & 129 \\
\hline Bronchiectasis (494) & 0 & 0 & --- & --- \\
\hline Hypersensitivity pneumonitis (495) & 0 & 0 & --- & --- \\
\hline Chronic airway obstruction, not elsewhere classified (496) & 624 & 111 & 103 & 120 \\
\hline
\end{tabular}

ICD - International Classification of Diseases, $9^{\text {th }}$ Revision $\quad$ LCL - lower confidence limit $\quad$ UCL - upper confidence limit

NOTE: PMRs in bold are significantly different from $100(\mathrm{p}<0.05)$. PMRs in italics are based on fewer than five observed deaths. PMRs are based on underlying and contributing cause of death. Some values could not be calculated because the number of observed or expected deaths was zero; such values are indicated by ---. See appendices for source description, methods, ICD codes, and a list of selected states.

SOURCE: National Center for Health Statistics multiple-cause-of-death data 
Table 2-77. Forestry workers: Proportionate mortality ratio (PMR) adjusted for age, sex, and race/ ethnicity for chronic obstructive pulmonary disease and allied conditions, U.S. residents age 15 and over, selected states, 1988-1998

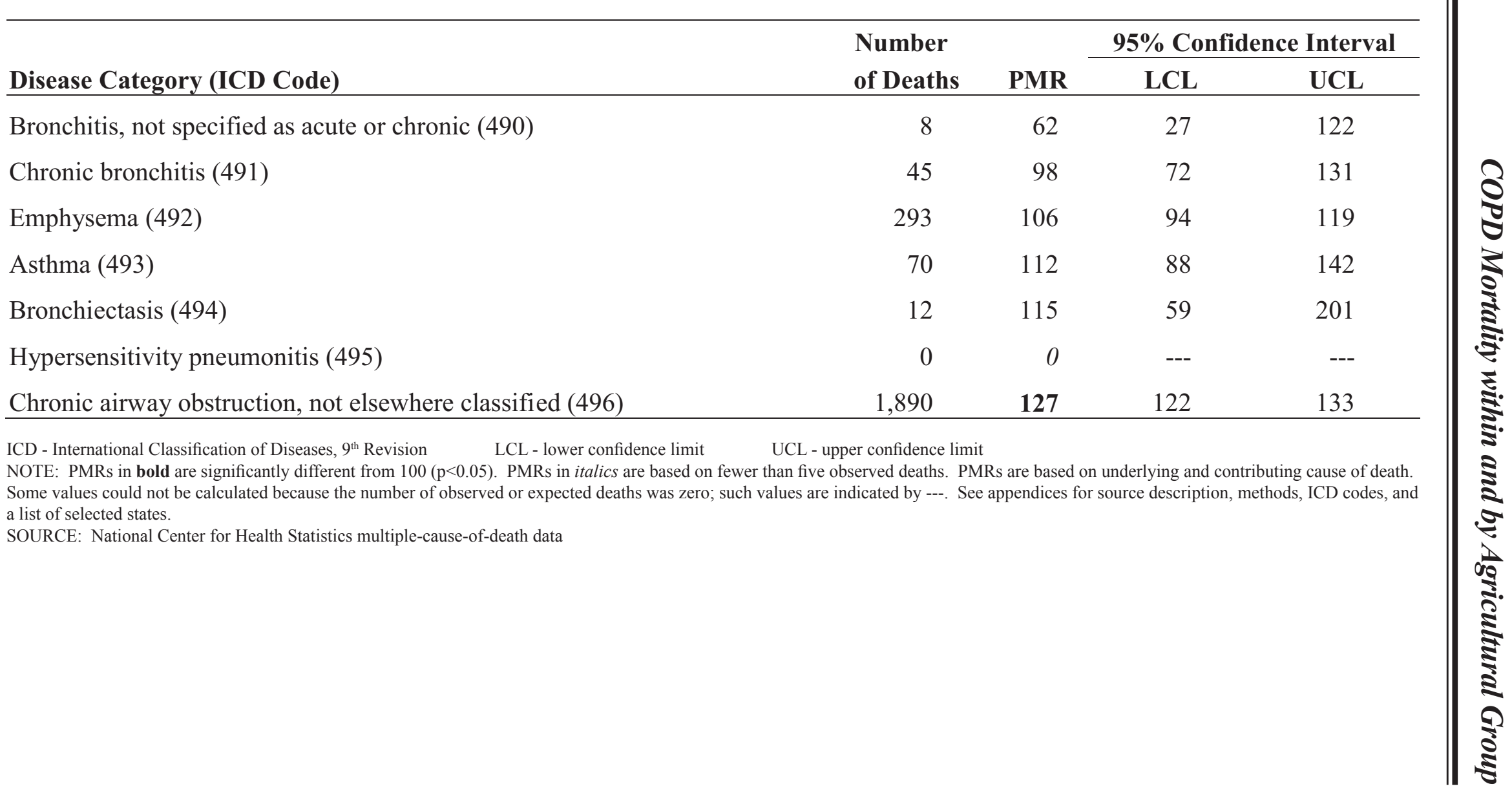


Table 2-78. Fishery workers: Proportionate mortality ratio (PMR) adjusted for age, sex, and race/ ethnicity for chronic obstructive pulmonary disease and allied conditions, U.S. residents age 15 and over, selected states, 1988-1998

\begin{tabular}{|c|c|c|c|c|}
\hline \multirow[b]{2}{*}{ Disease Category (ICD Code) } & \multirow{2}{*}{$\begin{array}{l}\text { Number } \\
\text { of Deaths }\end{array}$} & \multirow[b]{2}{*}{ PMR } & \multicolumn{2}{|c|}{ 95\% Confidence Interval } \\
\hline & & & LCL & UCL \\
\hline Bronchitis, not specified as acute or chronic (490) & 4 & 111 & 30 & 284 \\
\hline Chronic bronchitis (491) & 13 & 103 & 55 & 176 \\
\hline Emphysema (492) & 87 & 117 & 94 & 144 \\
\hline Asthma (493) & 9 & 53 & 24 & 101 \\
\hline Bronchiectasis (494) & 0 & 0 & --- & --- \\
\hline Hypersensitivity pneumonitis (495) & 0 & 0 & --- & --- \\
\hline Chronic airway obstruction, not elsewhere classified (496) & 455 & 116 & 106 & 127 \\
\hline
\end{tabular}

ICD - International Classification of Diseases, $9^{\text {th }}$ Revision $\quad$ LCL - lower confidence limit $\quad$ UCL - upper confidence limit

NOTE: PMRs in bold are significantly different from $100(\mathrm{p}<0.05)$. PMRs in italics are based on fewer than five observed deaths. PMRs are based on underlying and contributing cause of death. Some values could not be calculated because the number of observed or expected deaths was zero; such values are indicated by ---. See appendices for source description, methods, ICD codes, and a list of selected states.

SOURCE: National Center for Health Statistics multiple-cause-of-death data 
Figure 2-55. Bronchitis, not specified as acute or chronic: Proportionate mortality ratio (PMR) adjusted for age, sex, and race/ethnicity by agricultural group, U.S. residents age 15 and over, selected states, 1988-1998

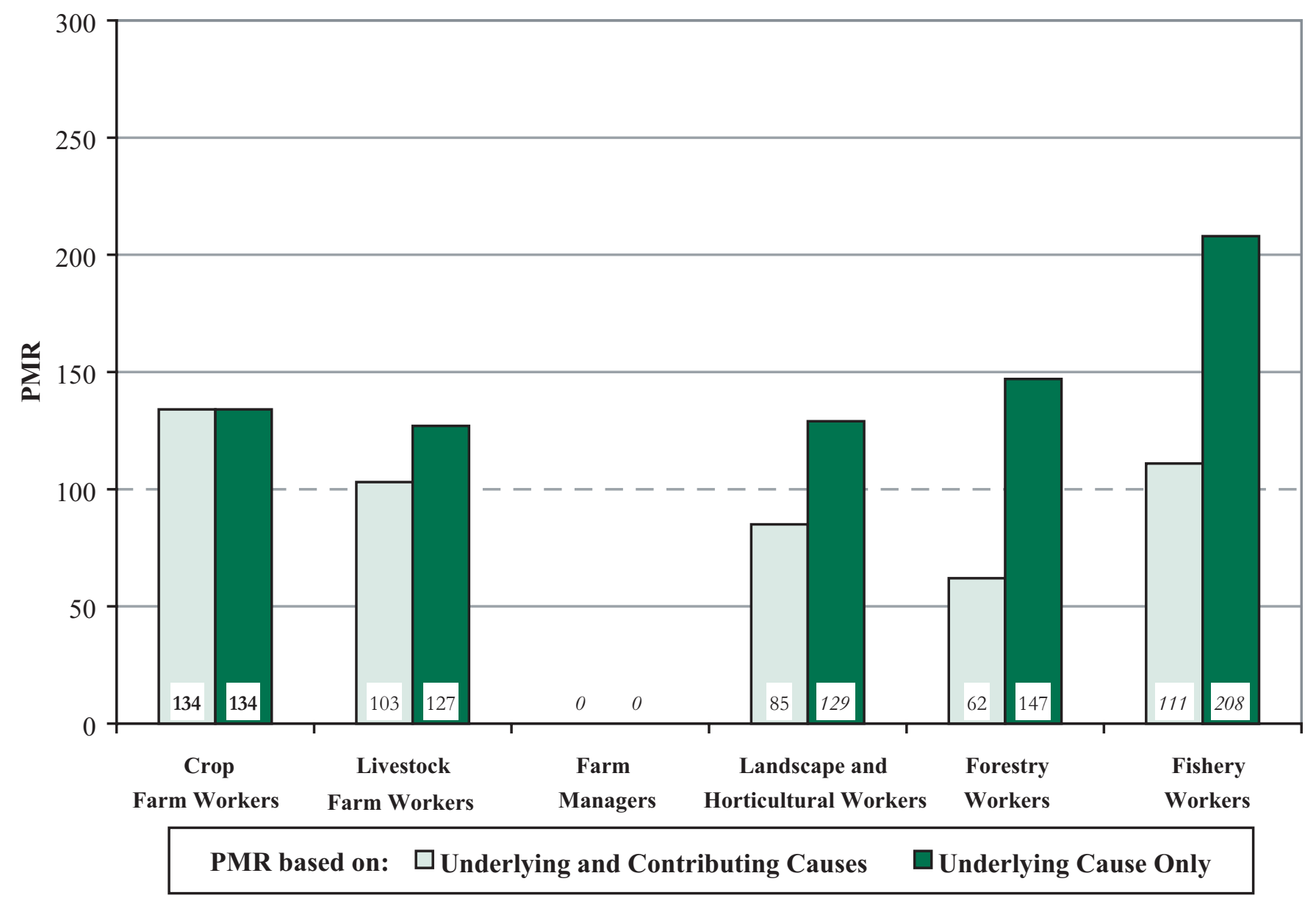

ICD - International Classification of Diseases, $9^{\text {th }}$ Revision

NOTE: Bronchitis, not specified as acute or chronic $=$ ICD-9 code 490. PMRs in bold are significantly different from 100 (p $<0.05$ ). PMRs in italics are based on fewer than five observed deaths. PMRs are based on underlying and contributing cause of death. See appendices for source description, methods, ICD codes, and a list of selected states.

SOURCE: National Center for Health Statistics multiple-cause-of-death data 
Figure 2-56. Chronic bronchitis: Proportionate mortality ratio (PMR) adjusted for age, sex, and race/ethnicity by agricultural group, U.S. residents age 15 and over, selected states, 1988-1998

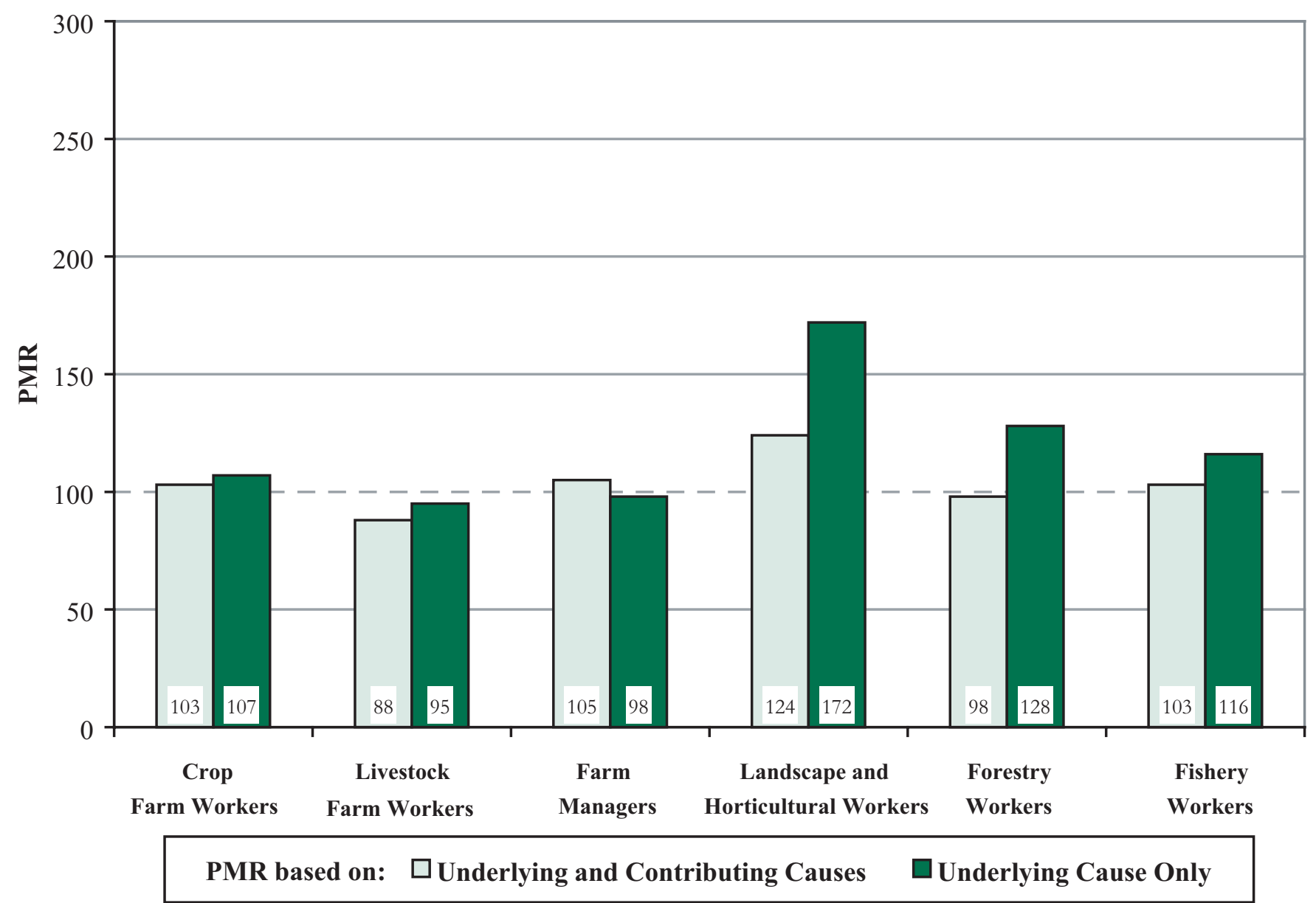

ICD - International Classification of Diseases, $9^{\text {th }}$ Revision

NOTE: Chronic bronchitis = ICD-9 code 491. PMRs in bold are significantly different from $100(\mathrm{p}<0.05)$. PMRs in italics are based on fewer than five observed deaths. PMRs are based on underlying and contributing cause of death. See appendices for source description, methods, ICD codes, and a list of selected states.

SOURCE: National Center for Health Statistics multiple-cause-of-death data 
Figure 2-57. Emphysema: Proportionate mortality ratio (PMR) adjusted for age, sex, and race/ethnicity by agricultural group, U.S. residents age 15 and over, selected states, 1988-1998

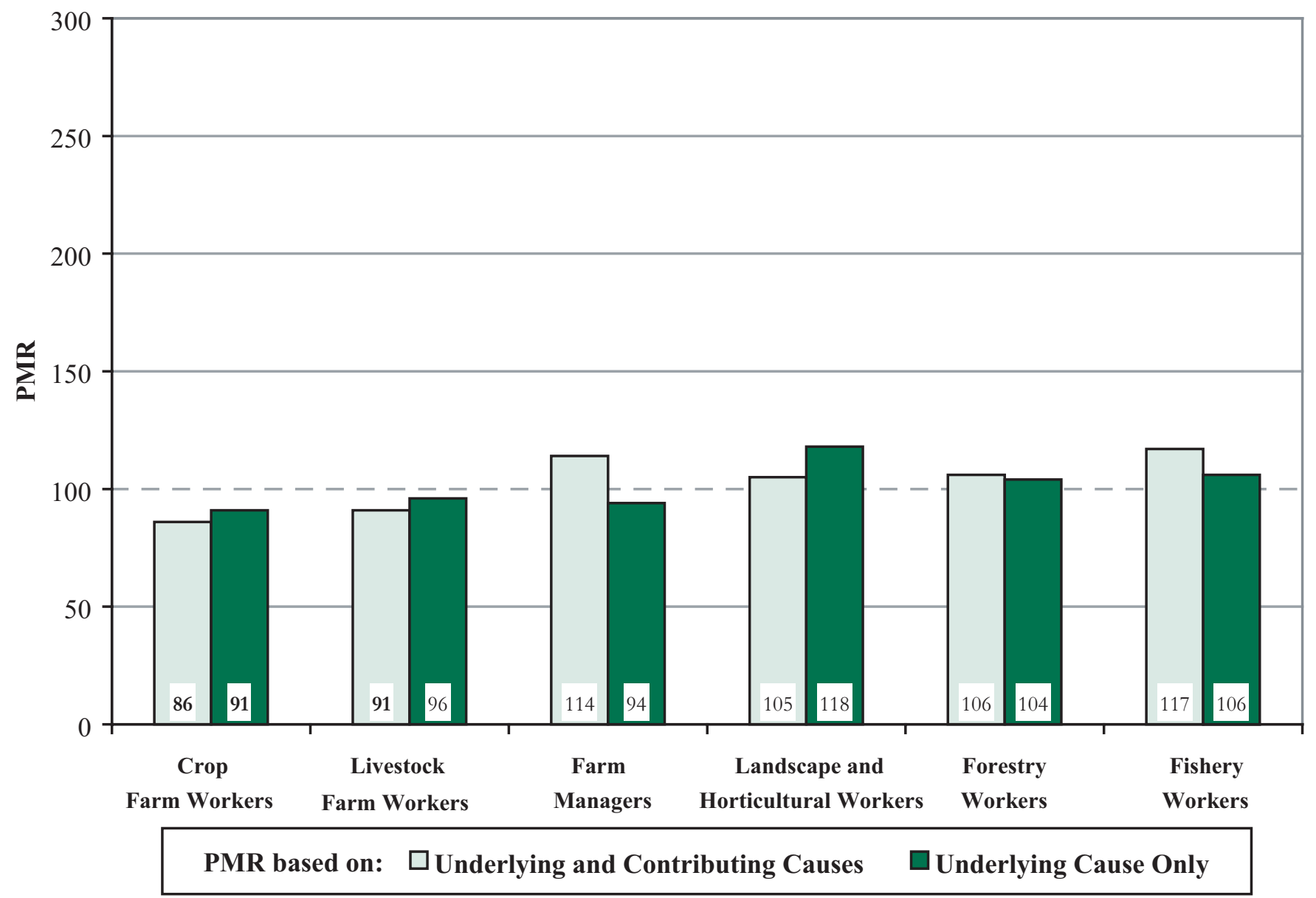

ICD - International Classification of Diseases, $9^{\text {th }}$ Revision

NOTE: Emphysema = ICD-9 code 492. PMRs in bold are significantly different from $100(\mathrm{p}<0.05)$. PMRs in italics are based on fewer than five observed deaths. PMRs are based on underlying and contributing cause of death. See appendices for source description, methods, ICD codes, and a list of selected states.

SOURCE: National Center for Health Statistics multiple-cause-of-death data 
Figure 2-58. Asthma: Proportionate mortality ratio (PMR) adjusted for age, sex, and race/ethnicity by agricultural group, U.S. residents age 15 and over, selected states, 1988-1998

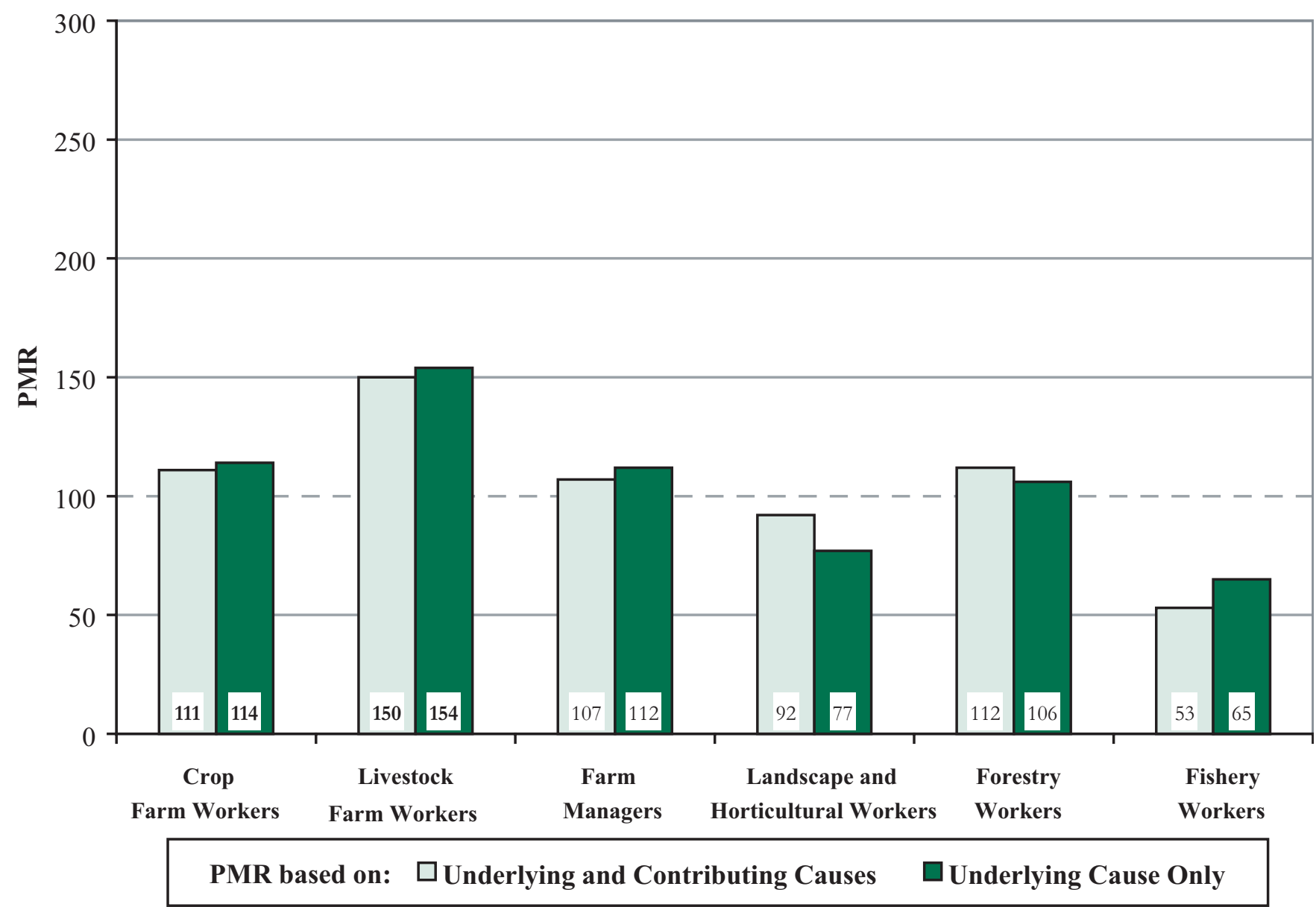

ICD - International Classification of Diseases, $9^{\text {th }}$ Revision

NOTE: Asthma $=$ ICD-9 code 493. PMRs in bold are significantly different from $100(\mathrm{p}<0.05)$. PMRs in italics are based on fewer than five observed deaths. PMRs are based on underlying and contributing cause of death. See appendices for source description, methods, ICD codes, and a list of selected states.

SOURCE: National Center for Health Statistics multiple-cause-of-death data 
Figure 2-59. Bronchiectasis: Proportionate mortality ratio (PMR) adjusted for age, sex, and race/ethnicity by agricultural group, U.S. residents age 15 and over, selected states, 1988-1998

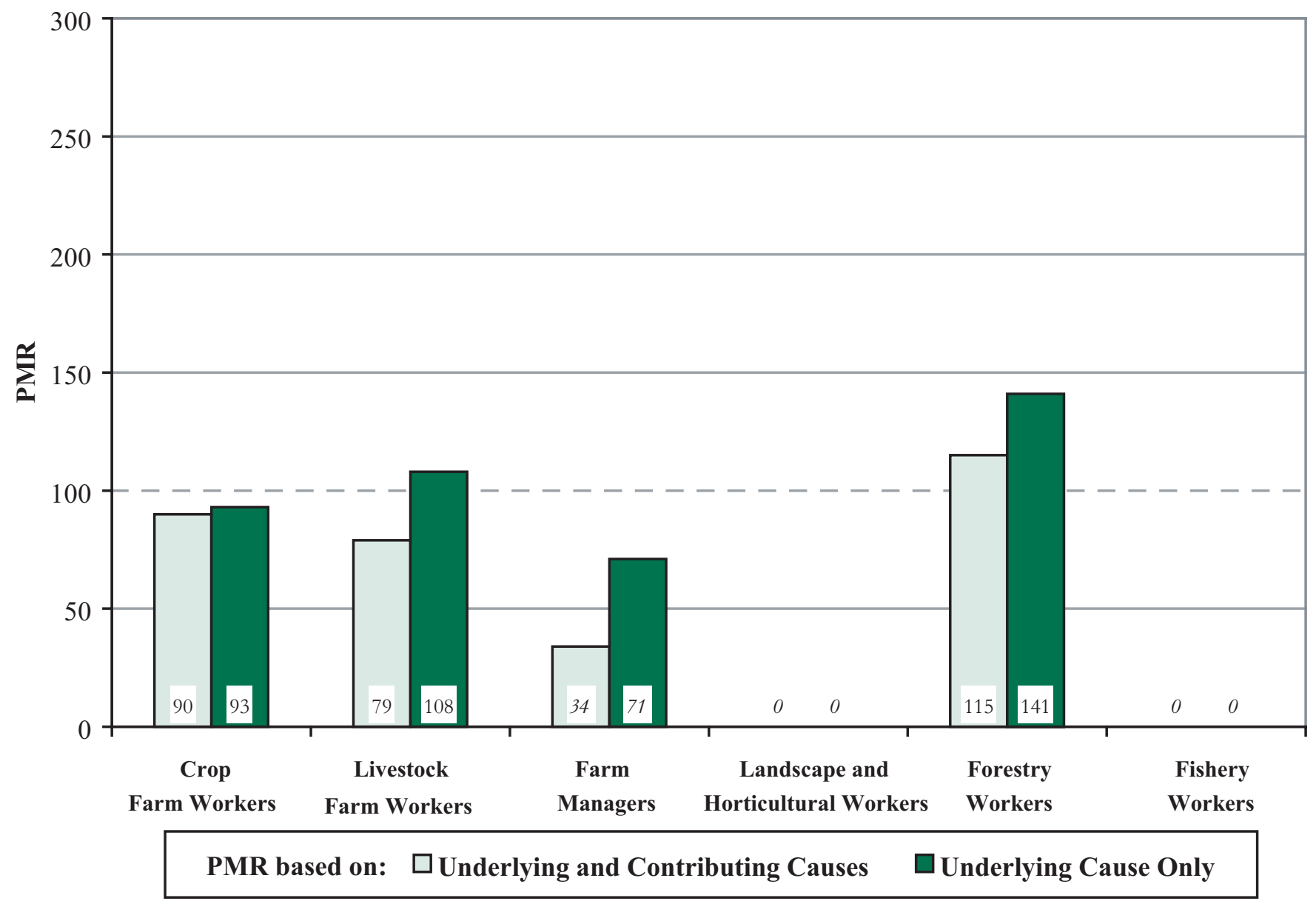

ICD - International Classification of Diseases, $9^{\text {th }}$ Revision

NOTE: Bronchiectasis = ICD-9 code 494. PMRs in bold are significantly different from $100(\mathrm{p}<0.05)$. PMRs in italics are based on fewer than five observed deaths. PMRs are based on underlying and contributing cause of death. See appendices for source description, methods, ICD codes, and a list of selected states.

SOURCE: National Center for Health Statistics multiple-cause-of-death data 
Figure 2-60. Hypersensitivity pneumonitis: Proportionate mortality ratio (PMR) adjusted for age, sex, and race/ethnicity by agricultural group, U.S. residents age 15 and over, selected states, 1988-1998

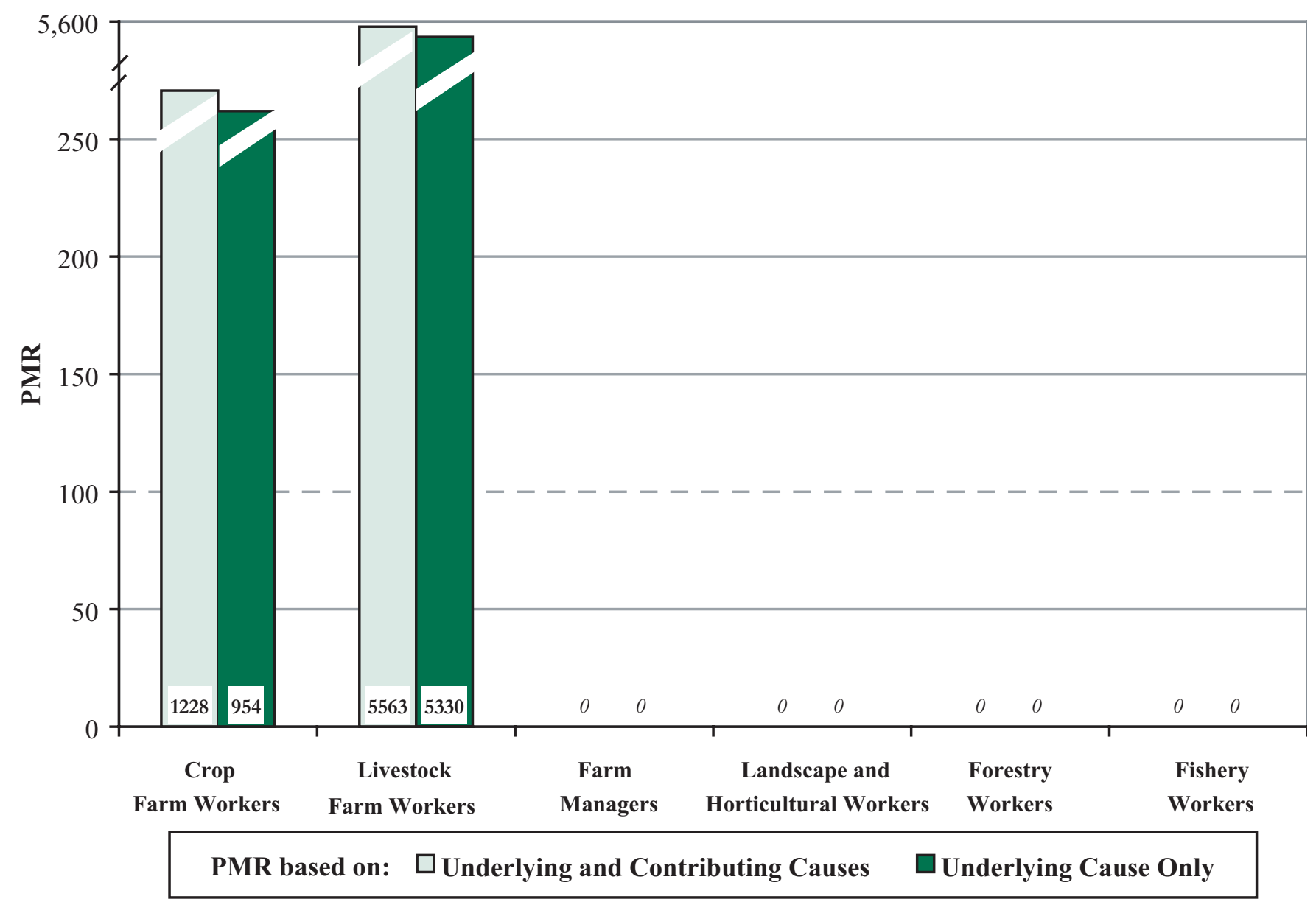

ICD - International Classification of Diseases, $9^{\text {th }}$ Revision

NOTE: Hypersensitivity pneumonitis = ICD-9 code 495. PMRs in bold are significantly different from $100(\mathrm{p}<0.05)$. PMRs in italics are based on fewer

than five observed deaths. PMRs are based on underlying and contributing cause of death. See appendices for source description, methods, ICD codes, and a list of selected states.

SOURCE: National Center for Health Statistics multiple-cause-of-death data 
Figure 2-61. Chronic airway obstruction, not elsewhere classified: Proportionate mortality ratio (PMR) adjusted for age, sex, and race/ethnicity by agricultural group, U.S. residents age 15 and over, selected states, 1988-1998

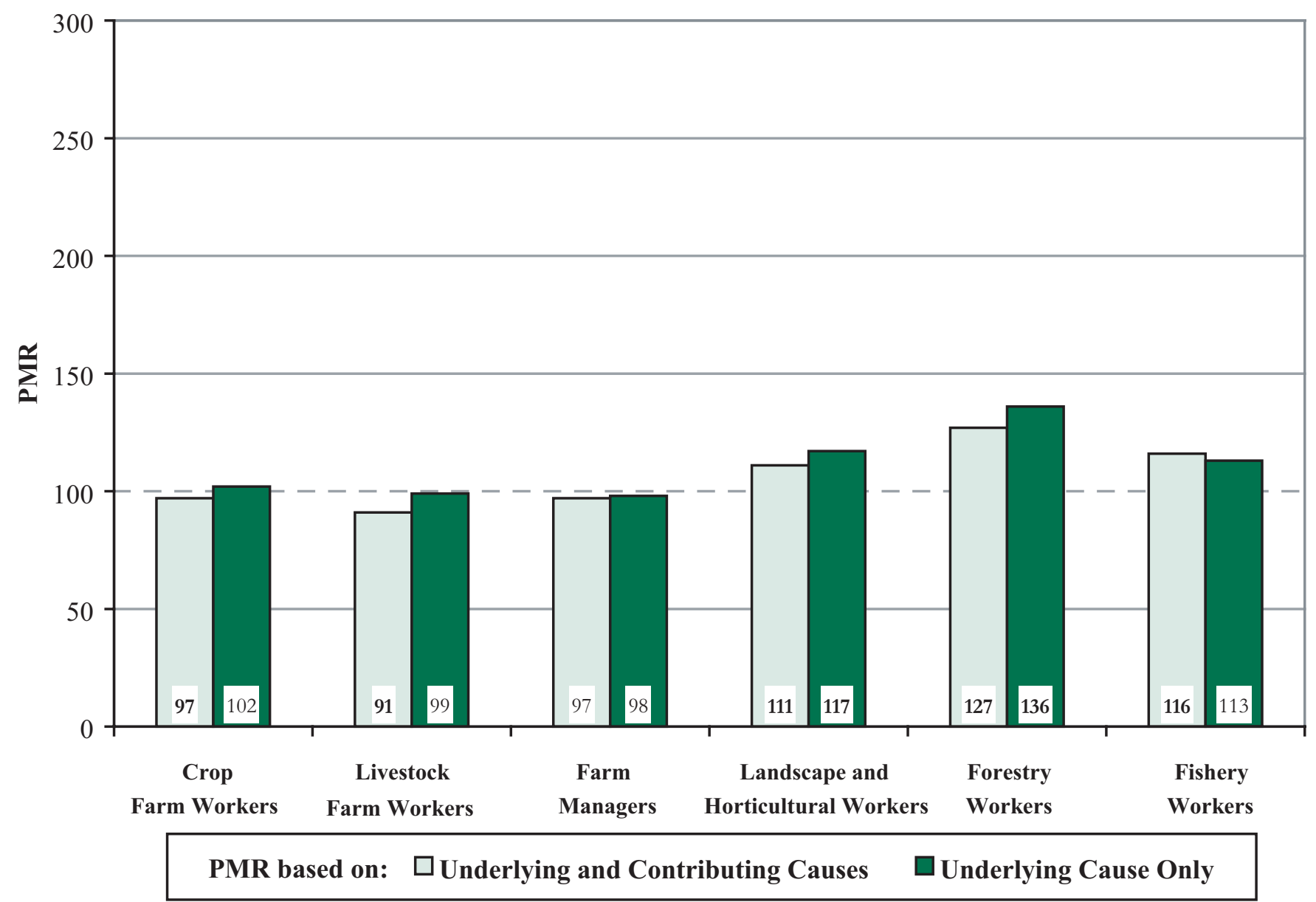

ICD - International Classification of Diseases, $9^{\text {th }}$ Revision

NOTE: Chronic airway obstruction, not elsewhere classified = ICD-9 code 496. PMRs in bold are significantly different from 100 ( $<<0.05$ ). PMRs in italics are based on fewer than five observed deaths. PMRs are based on underlying and contributing cause of death. See appendices for source description, methods, ICD codes, and a list of selected states.

SOURCE: National Center for Health Statistics multiple-cause-of-death data 
Table 2-79. Crop farm workers: Proportionate mortality ratio (PMR) adjusted for age, sex, and race/ ethnicity for pneumoconiosis and other lung diseases-external agents, U.S. residents age 15 and over, selected states, 1988-1998

\begin{tabular}{|c|c|c|c|c|}
\hline \multirow[b]{2}{*}{ Disease Category (ICD Code) } & \multirow{2}{*}{$\begin{array}{l}\text { Number } \\
\text { of Deaths }\end{array}$} & \multirow[b]{2}{*}{ PMR } & \multicolumn{2}{|c|}{$\mathbf{9 5 \%}$ Confidence Interva } \\
\hline & & & $\mathbf{L C L}$ & UCL \\
\hline Coal workers' pneumoconiosis (500) & 41 & 9 & 7 & 12 \\
\hline Asbestosis (501) & 22 & 11 & 7 & 17 \\
\hline Pneumoconiosis due to other silica or silicates (502) & 17 & 22 & 13 & 35 \\
\hline Pneumoconiosis due to other inorganic dust (503) & 1 & 63 & 2 & 350 \\
\hline Pneumoconiosis due to inhalation of other dust (504) & 1 & 28 & 1 & 156 \\
\hline Pneumoconiosis, unspecified (505) & 19 & 17 & 10 & 27 \\
\hline Respiratory conditions due to chemical fumes and vapors (506) & 0 & 0 & --- & --- \\
\hline Pneumonitis due to solids and liquids (507) & 5,094 & 95 & 93 & 98 \\
\hline Respiratory conditions due to other and unspecified external agents (508) & 29 & 66 & 44 & 95 \\
\hline
\end{tabular}

ICD - International Classification of Diseases, $9^{\text {th }}$ Revision $\quad$ LCL - lower confidence limit $\quad$ UCL - upper confidence limit

NOTE: PMRs in bold are significantly different from $100(\mathrm{p}<0.05)$. PMRs in italics are based on fewer than five observed deaths. PMRs are based on underlying and contributing cause of death. Some values could not be calculated because the number of observed or expected deaths was zero; such values are indicated by ---. See appendices for source description, methods, ICD codes, and a list of selected states.

SOURCE: National Center for Health Statistics multiple-cause-of-death data 
Table 2-80. Livestock farm workers: Proportionate mortality ratio (PMR) adjusted for age, sex, and race/ethnicity for pneumoconiosis and other lung diseases-external agents, U.S. residents age 15 and over, selected states, 1988-1998

\begin{tabular}{|c|c|c|c|c|}
\hline \multirow[b]{2}{*}{ Disease Category (ICD Code) } & \multirow{2}{*}{$\begin{array}{l}\text { Number } \\
\text { of Deaths }\end{array}$} & \multirow[b]{2}{*}{ PMR } & \multicolumn{2}{|c|}{ 95\% Confidence Interval } \\
\hline & & & $\mathbf{L C L}$ & $\mathbf{U C L}$ \\
\hline Coal workers' pneumoconiosis (500) & 7 & 6 & 2 & 12 \\
\hline Asbestosis (501) & 9 & 16 & 7 & 30 \\
\hline Pneumoconiosis due to other silica or silicates (502) & 6 & 30 & 11 & 65 \\
\hline Pneumoconiosis due to other inorganic dust (503) & 0 & 0 & --- & -- \\
\hline Pneumoconiosis due to inhalation of other dust (504) & 0 & 0 & --- & --- \\
\hline Pneumoconiosis, unspecified (505) & 6 & 20 & 7 & 44 \\
\hline Respiratory conditions due to chemical fumes and vapors (506) & 3 & 364 & 75 & 1,064 \\
\hline Pneumonitis due to solids and liquids (507) & 1,346 & 95 & 90 & 100 \\
\hline Respiratory conditions due to other and unspecified external agents (508) & 4 & 33 & 9 & 84 \\
\hline
\end{tabular}

ICD - International Classification of Diseases, $9^{\text {th }}$ Revision $\quad$ LCL - lower confidence limit $\quad$ UCL - upper confidence limit

NOTE: PMRs in bold are significantly different from $100(\mathrm{p}<0.05)$. PMRs in italics are based on fewer than five observed deaths. PMRs are based on underlying and contributing cause of death. Some values could not be calculated because the number of observed or expected deaths was zero; such values are indicated by ---. See appendices for source description, methods, ICD codes, and a list of selected states.

SOURCE: National Center for Health Statistics multiple-cause-of-death data 
Table 2-81. Farm managers: Proportionate mortality ratio (PMR) adjusted for age, sex, and race/ ethnicity for pneumoconiosis and other lung diseases-external agents, U.S. residents age 15 and over, selected states, 1988-1998

\begin{tabular}{|c|c|c|c|c|}
\hline \multirow[b]{2}{*}{ Disease Category (ICD Code) } & \multirow{2}{*}{$\begin{array}{l}\text { Number } \\
\text { of Deaths }\end{array}$} & \multirow[b]{2}{*}{ PMR } & \multicolumn{2}{|c|}{ 95\% Confidence Interval } \\
\hline & & & $\mathbf{L C L}$ & $\mathbf{U C L}$ \\
\hline Coal workers' pneumoconiosis (500) & 0 & 0 & --- & --- \\
\hline Asbestosis (501) & 1 & 33 & 1 & 183 \\
\hline Pneumoconiosis due to other silica or silicates (502) & 1 & 84 & 2 & 467 \\
\hline Pneumoconiosis due to other inorganic dust (503) & 0 & 0 & --- & --- \\
\hline Pneumoconiosis due to inhalation of other dust (504) & 1 & 1,969 & 50 & 10,939 \\
\hline Pneumoconiosis, unspecified (505) & 0 & 0 & --- & --- \\
\hline Respiratory conditions due to chemical fumes and vapors (506) & 0 & 0 & --- & --- \\
\hline Pneumonitis due to solids and liquids (507) & 61 & 76 & 59 & 98 \\
\hline Respiratory conditions due to other and unspecified external agents (508) & 1 & 134 & 3 & 744 \\
\hline
\end{tabular}

ICD - International Classification of Diseases, $9^{\text {th }}$ Revision $\quad$ LCL - lower confidence limit $\quad$ UCL - upper confidence limit

NOTE: PMRs in bold are significantly different from $100(\mathrm{p}<0.05)$. PMRs in italics are based on fewer than five observed deaths. PMRs are based on underlying and contributing cause of death. Some values could not be calculated because the number of observed or expected deaths was zero; such values are indicated by ---. See appendices for source description, methods, ICD codes, and a list of selected states.

SOURCE: National Center for Health Statistics multiple-cause-of-death data 
Table 2-82. Landscape and horticultural workers: Proportionate mortality ratio (PMR) adjusted for age, sex, and race/ethnicity for pneumoconiosis and other lung diseases-external agents, U.S. residents age 15 and over, selected states, 1988-1998

\begin{tabular}{|c|c|c|c|c|}
\hline \multirow[b]{2}{*}{ Disease Category (ICD Code) } & \multirow{2}{*}{$\begin{array}{l}\text { Number } \\
\text { of Deaths }\end{array}$} & \multirow[b]{2}{*}{ PMR } & \multicolumn{2}{|c|}{ 95\% Confidence Interval } \\
\hline & & & $\mathbf{L C L}$ & $\mathbf{U C L}$ \\
\hline Coal workers' pneumoconiosis (500) & 2 & 20 & 2 & 72 \\
\hline Asbestosis (501) & 4 & 79 & 22 & 202 \\
\hline Pneumoconiosis due to other silica or silicates (502) & 0 & 0 & --- & --- \\
\hline Pneumoconiosis due to other inorganic dust (503) & 0 & 0 & --- & --- \\
\hline Pneumoconiosis due to inhalation of other dust (504) & 0 & 0 & --- & --- \\
\hline Pneumoconiosis, unspecified (505) & 1 & 38 & 1 & 211 \\
\hline Respiratory conditions due to chemical fumes and vapors (506) & 0 & 0 & --- & --- \\
\hline Pneumonitis due to solids and liquids (507) & 146 & 108 & 92 & 127 \\
\hline Respiratory conditions due to other and unspecified external agents (508) & 1 & 58 & 1 & 322 \\
\hline \multicolumn{5}{|c|}{$\begin{array}{l}\text { ICD - International Classification of Diseases, } 9^{\text {th }} \text { Revision } \quad \text { LCL - lower confidence limit } \quad \text { UCL - upper confidence limit } \\
\text { NOTE: PMRs in bold are significantly different from } 100(\mathrm{p}<0.05) \text {. PMRs in italics are based on fewer than five observed deaths. PMRs are based on underlying and contributing cause of death } \\
\text { Some values could not be calculated because the number of observed or expected deaths was zero; such values are indicated by ---. See appendices for source description, methods, ICD codes, ar } \\
\text { a list of selected states. } \\
\text { SOURCE: National Center for Health Statistics multiple-cause-of-death data }\end{array}$} \\
\hline
\end{tabular}


Table 2-83. Forestry workers: Proportionate mortality ratio (PMR) adjusted for age, sex, and race/ ethnicity for pneumoconiosis and other lung diseases-external agents, U.S. residents age 15 and over, selected states, 1988-1998

\begin{tabular}{|c|c|c|c|c|}
\hline \multirow[b]{2}{*}{ Disease Category (ICD Code) } & \multirow{2}{*}{$\begin{array}{l}\text { Number } \\
\text { of Deaths }\end{array}$} & \multirow[b]{2}{*}{ PMR } & \multicolumn{2}{|c|}{ 95\% Confidence Interval } \\
\hline & & & $\mathbf{L C L}$ & UCL \\
\hline Coal workers' pneumoconiosis (500) & 10 & 34 & 16 & 63 \\
\hline Asbestosis (501) & 6 & 42 & 15 & 92 \\
\hline Pneumoconiosis due to other silica or silicates (502) & 1 & 17 & 0 & 94 \\
\hline Pneumoconiosis due to other inorganic dust (503) & 0 & 0 & --- & --- \\
\hline Pneumoconiosis due to inhalation of other dust (504) & 0 & 0 & --- & --- \\
\hline Pneumoconiosis, unspecified (505) & 6 & 81 & 30 & 176 \\
\hline Respiratory conditions due to chemical fumes and vapors (506) & 0 & 0 & --- & --- \\
\hline Pneumonitis due to solids and liquids (507) & 330 & 99 & 89 & 110 \\
\hline Respiratory conditions due to other and unspecified external agents (508) & 1 & 25 & 1 & 139 \\
\hline
\end{tabular}

ICD - International Classification of Diseases, $9^{\text {th }}$ Revision $\quad$ LCL - lower confidence limit $\quad$ UCL - upper confidence limit

NOTE: PMRs in bold are significantly different from $100(\mathrm{p}<0.05)$. PMRs in italics are based on fewer than five observed deaths. PMRs are based on underlying and contributing cause of death. Some values could not be calculated because the number of observed or expected deaths was zero; such values are indicated by ---. See appendices for source description, methods, ICD codes, and a list of selected states.

SOURCE: National Center for Health Statistics multiple-cause-of-death data 
Table 2-84. Fishery workers: Proportionate mortality ratio (PMR) adjusted for age, sex, and race/ ethnicity for pneumoconiosis and other lung diseases-external agents, U.S. residents age 15 and over, selected states, 1988-1998

\begin{tabular}{|c|c|c|c|c|}
\hline \multirow[b]{2}{*}{ Disease Category (ICD Code) } & \multirow{2}{*}{$\begin{array}{l}\text { Number } \\
\text { of Deaths }\end{array}$} & \multirow[b]{2}{*}{ PMR } & \multicolumn{2}{|c|}{$\mathbf{9 5 \%}$ Confidence Interva } \\
\hline & & & $\mathbf{L C L}$ & UCL \\
\hline Coal workers' pneumoconiosis (500) & 0 & 0 & --- & --- \\
\hline Asbestosis (501) & 4 & 104 & 28 & 266 \\
\hline Pneumoconiosis due to other silica or silicates (502) & 0 & 0 & --- & --- \\
\hline Pneumoconiosis due to other inorganic dust (503) & 0 & 0 & --- & --- \\
\hline Pneumoconiosis due to inhalation of other dust (504) & 0 & 0 & --- & --- \\
\hline Pneumoconiosis, unspecified (505) & 0 & 0 & --- & --- \\
\hline Respiratory conditions due to chemical fumes and vapors (506) & 0 & 0 & --- & --- \\
\hline Pneumonitis due to solids and liquids (507) & 92 & 104 & 85 & 128 \\
\hline Respiratory conditions due to other and unspecified external agents (508) & 2 & 188 & 23 & 679 \\
\hline
\end{tabular}

ICD - International Classification of Diseases, $9^{\text {th }}$ Revision $\quad$ LCL - lower confidence limit $\quad$ UCL - upper confidence limit

NOTE: PMRs in bold are significantly different from $100(\mathrm{p}<0.05)$. PMRs in italics are based on fewer than five observed deaths. PMRs are based on underlying and contributing cause of death. Some values could not be calculated because the number of observed or expected deaths was zero; such values are indicated by ---. See appendices for source description, methods, ICD codes, and a list of selected states.

SOURCE: National Center for Health Statistics multiple-cause-of-death data 
Figure 2-62. Coal workers' pneumoconiosis: Proportionate mortality ratio (PMR) adjusted for age, sex, and race/ethnicity by agricultural group, U.S. residents age 15 and over, selected states, 1988-1998

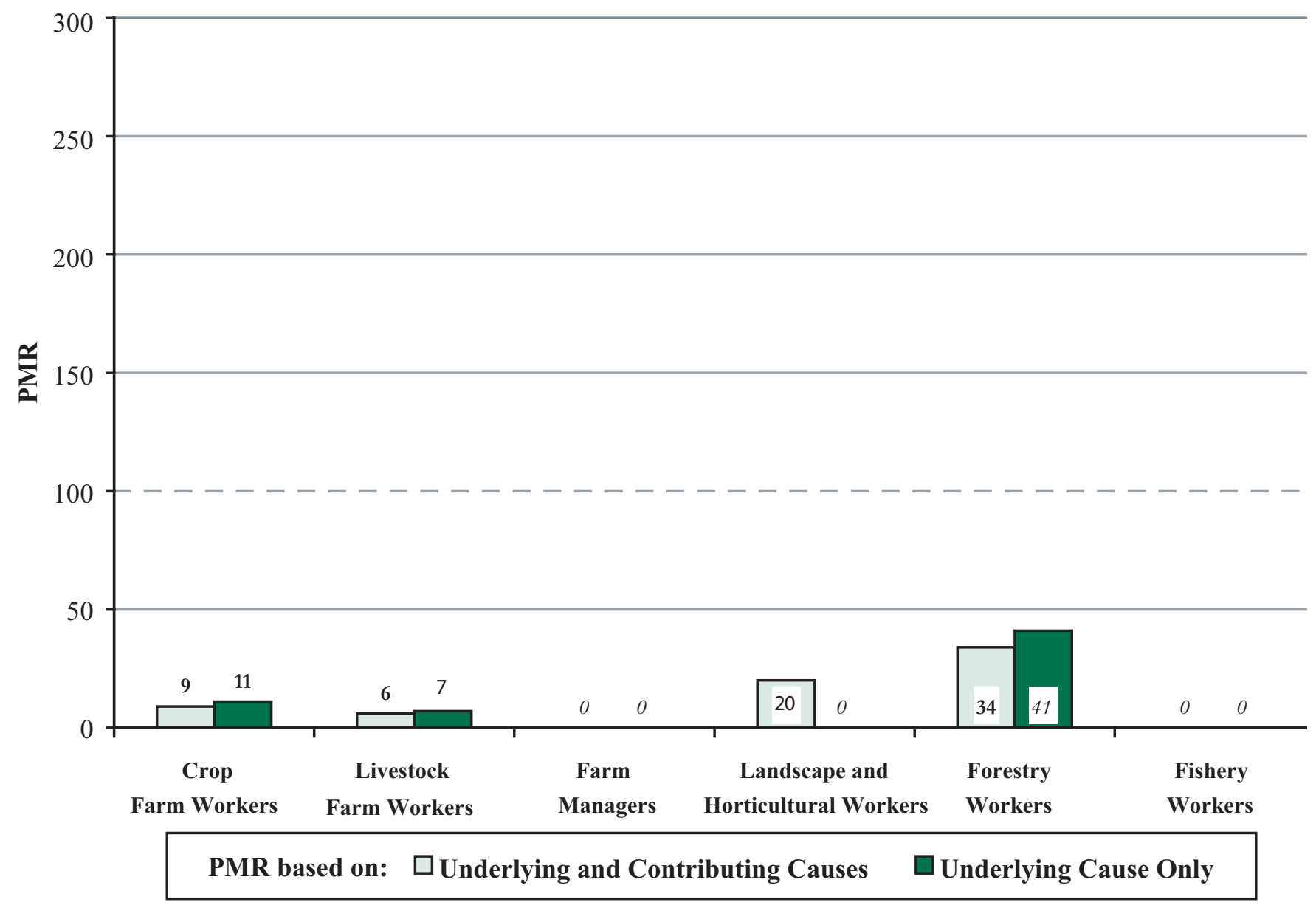

ICD - International Classification of Diseases, $9^{\text {th }}$ Revision

NOTE: Coal workers' pneumoconiosis = ICD-9 code 500. PMRs in bold are significantly different from 100 ( $\mathrm{p}<0.05$ ). PMRs in italics are based on fewer than five observed deaths. PMRs are based on underlying and contributing cause of death. See appendices for source description, methods, ICD codes, and a list of selected states.

SOURCE: National Center for Health Statistics multiple-cause-of-death data 
Figure 2-63. Asbestosis: Proportionate mortality ratio (PMR) adjusted for age, sex, and race/ethnicity by agricultural group, U.S. residents age 15 and over, selected states, 1988-1998

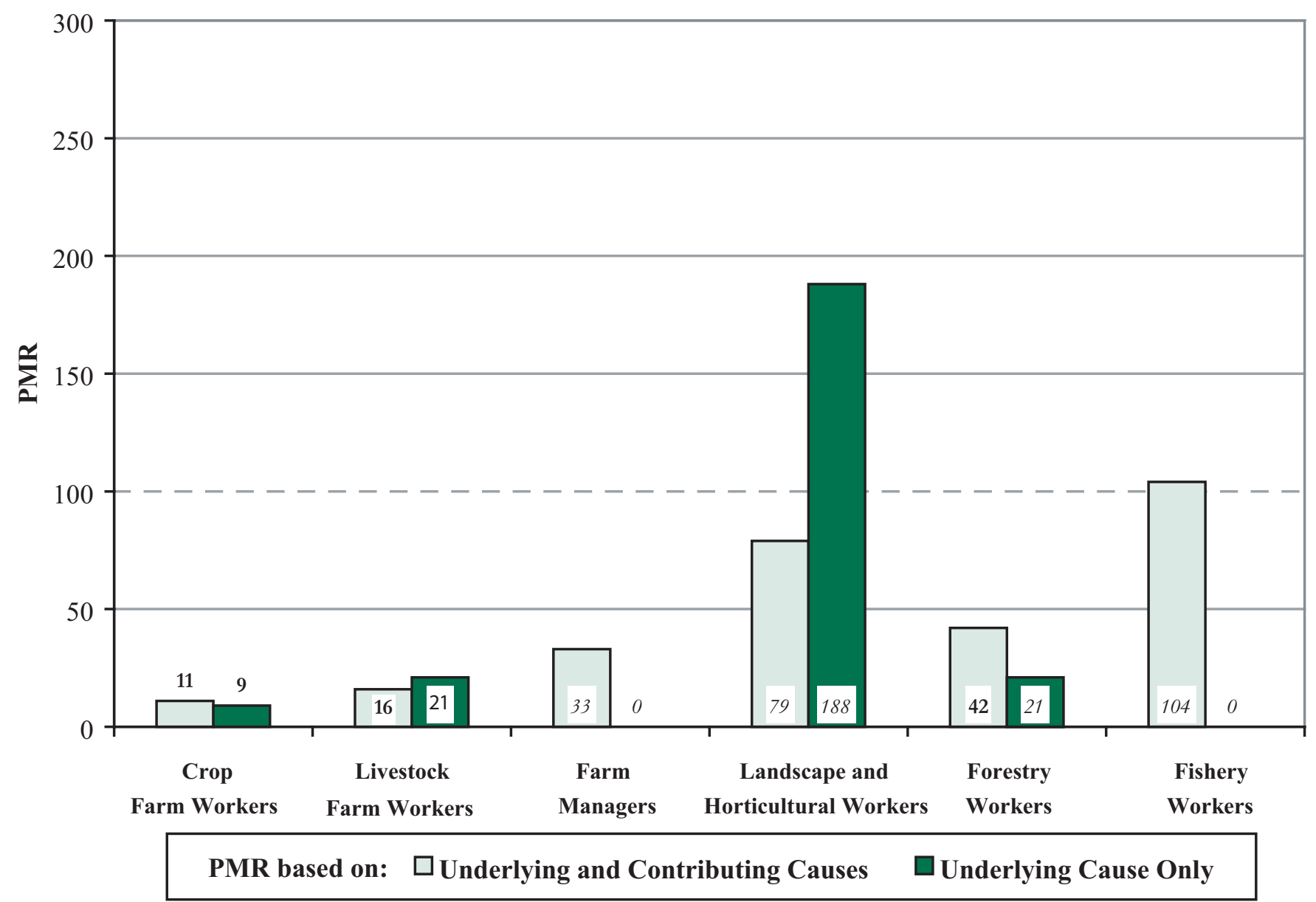

ICD - International Classification of Diseases, $9^{\text {th }}$ Revision

NOTE: Asbestosis = ICD-9 code 501. PMRs in bold are significantly different from $100(\mathrm{p}<0.05)$. PMRs in italics are based on fewer than five observed deaths. PMRs are based on underlying and contributing cause of death. See appendices for source description, methods, ICD codes, and a list of selected states.

SOURCE: National Center for Health Statistics multiple-cause-of-death data 
Figure 2-64. Pneumoconiosis due to other silica or silicates: Proportionate mortality ratio (PMR) adjusted for age, sex, and race/ethnicity by agricultural group, U.S. residents age 15 and over, selected states, 1988-1998

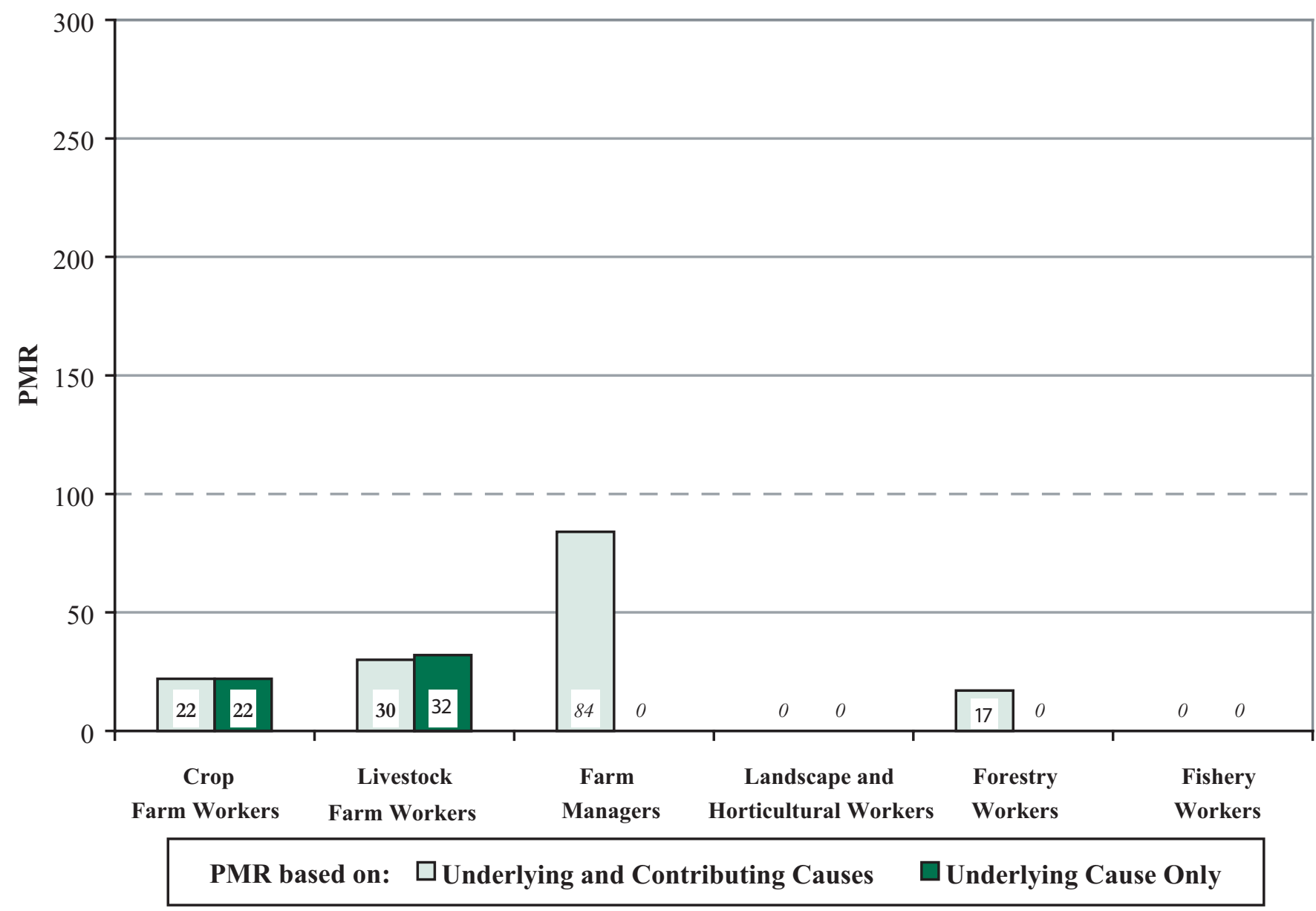

ICD - International Classification of Diseases, $9^{\text {th }}$ Revision

NOTE: Pneumoconiosis due to other silica or silicates $=$ ICD-9 code 502. PMRs in bold are significantly different from 100 ( $\mathrm{p}<0.05$ ). PMRs in italics are based on fewer than five observed deaths. PMRs are based on underlying and contributing cause of death. See appendices for source description, methods, ICD codes, and a list of selected states.

SOURCE: National Center for Health Statistics multiple-cause-of-death data 
Figure 2-65. Pneumoconiosis, unspecified: Proportionate mortality ratio (PMR) adjusted for age, sex, and race/ethnicity by agricultural group, U.S. residents age 15 and over, selected states, 1988-1998

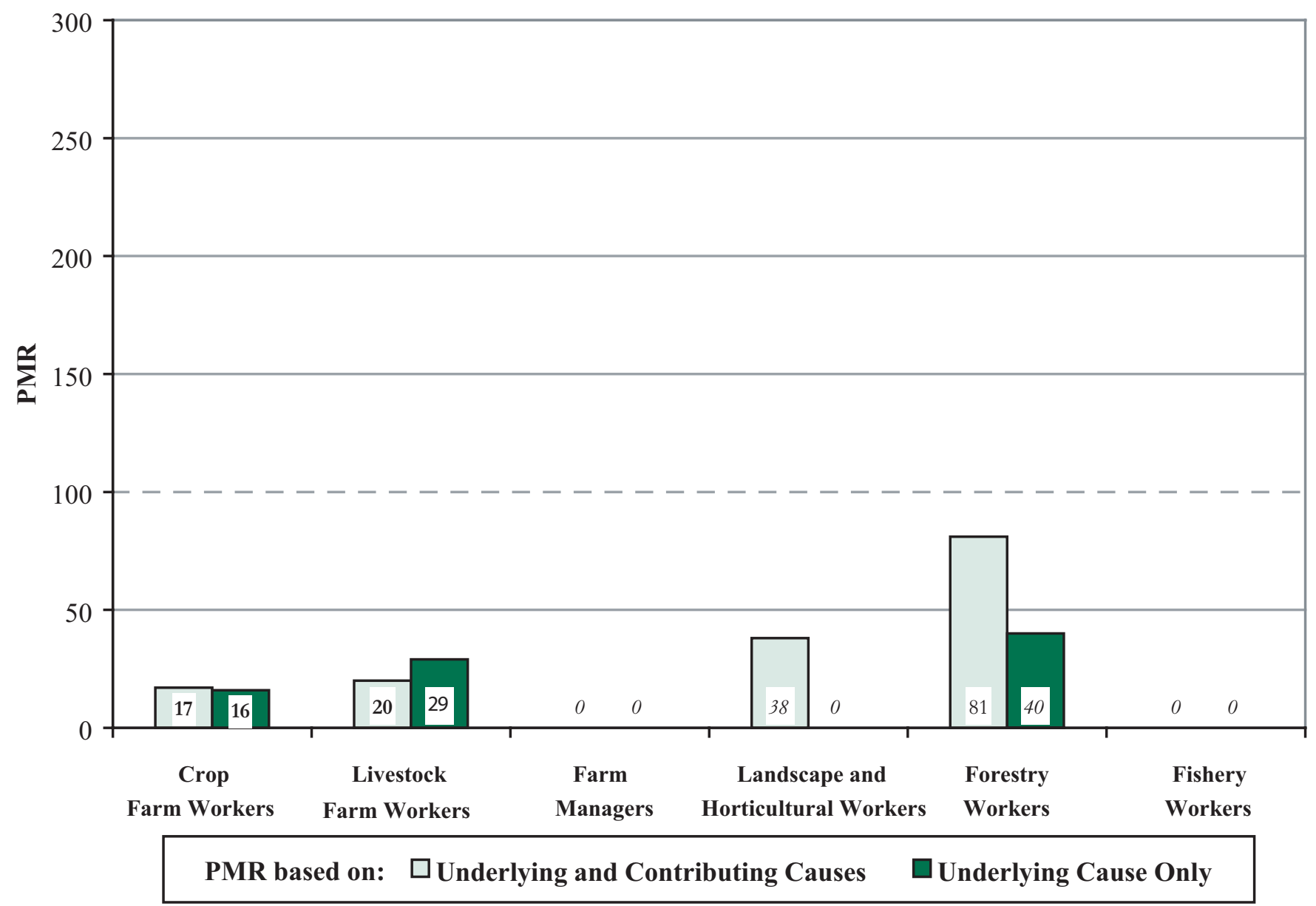

ICD - International Classification of Diseases, $9^{\text {th }}$ Revision

NOTE: Pneumoconiosis, unspecified $=$ ICD-9 code 505. PMRs in bold are significantly different from $100(\mathrm{p}<0.05)$. PMRs in italics are based on fewer than five observed deaths. PMRs are based on underlying and contributing cause of death. See appendices for source description, methods, ICD codes, and a list of selected states.

SOURCE: National Center for Health Statistics multiple-cause-of-death data 
Figure 2-66. Pneumonitis due to solids and liquids: Proportionate mortality ratio (PMR) adjusted for age, sex, and race/ethnicity by agricultural group, U.S. residents age 15 and over, selected states, 1988-1998

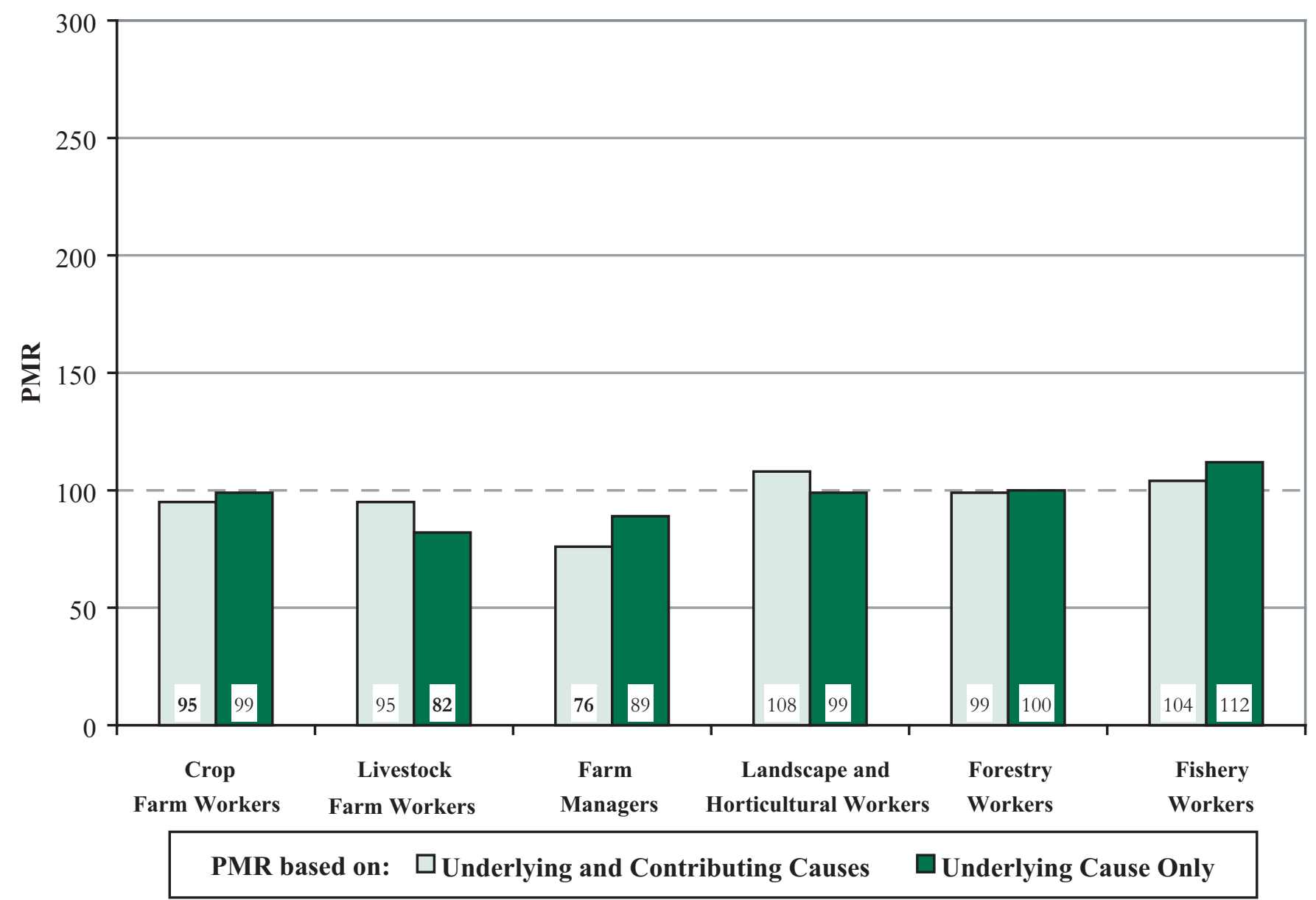

ICD - International Classification of Diseases, $9^{\text {th }}$ Revision

NOTE: Pneumonitis due to solids and liquids = ICD-9 code 507. PMRs in bold are significantly different from 100 ( $\mathrm{p}<0.05$ ). PMRs in italics are based

on fewer than five observed deaths. PMRs are based on underlying and contributing cause of death. See appendices for source description, methods, ICD codes, and a list of selected states.

SOURCE: National Center for Health Statistics multiple-cause-of-death data 
Figure 2-67. Respiratory conditions due to other and unspecified external agents: Proportionate mortality ratio (PMR) adjusted for age, sex, and race/ethnicity by agricultural group, U.S. residents age 15 and over, selected states, 1988-1998

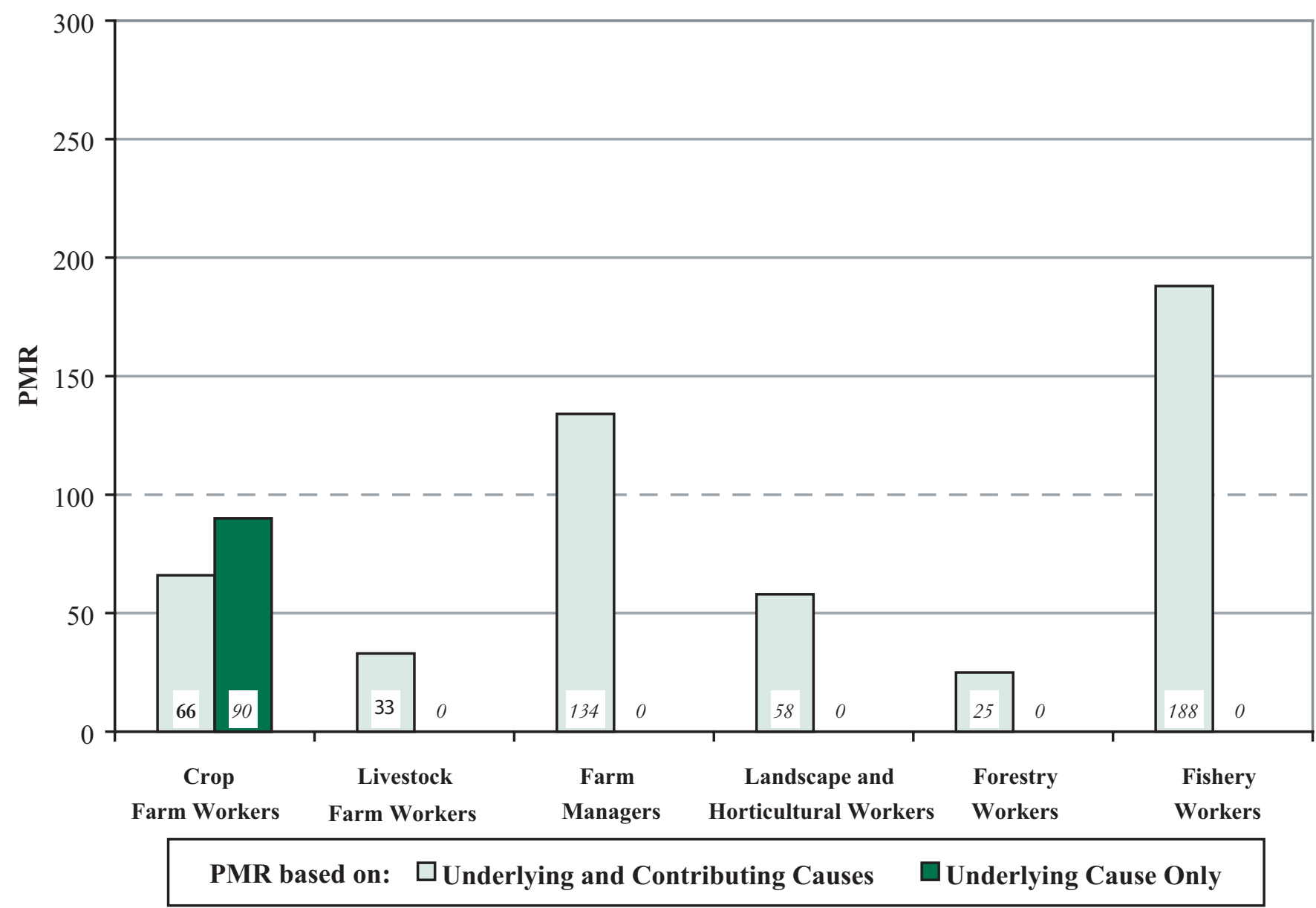

ICD - International Classification of Diseases, $9^{\text {th }}$ Revision

NOTE: Respiratory conditions due to other and unspecified external agents $=$ ICD-9 code 508 . PMRs in bold are significantly different from 100 ( $\mathrm{p}<0.05$ ). PMRs in italics are based on fewer than five observed deaths. PMRs are based on underlying and contributing cause of death. See appendices for source description, methods, ICD codes, and a list of selected states.

SOURCE: National Center for Health Statistics multiple-cause-of-death data 
Table 2-85. Crop farm workers: Proportionate mortality ratio (PMR) adjusted for age, sex, and race/ ethnicity for other diseases of respiratory system, U.S. residents age 15 and over, selected states, 1988-1998

\begin{tabular}{lrrrr}
\hline & Number & & \multicolumn{2}{c}{$\mathbf{9 5 \%}$ Confidence Interval } \\
\cline { 3 - 6 } Disease Category (ICD Code) & of Deaths & PMR & LCL & UCL \\
\hline Empyema (510) & 134 & 90 & 76 & 107 \\
Pleurisy (511) & 952 & $\mathbf{8 9}$ & 84 & 95 \\
Pneumothorax (512) & 301 & 95 & 85 & 106 \\
Abscess of lung and mediastinum (513) & 153 & $\mathbf{1 2 0}$ & 102 & 141 \\
Pulmonary congestion and hypostasis (514) & 1,830 & $\mathbf{1 1 3}$ & 108 & 118 \\
Postinflammatory pulmonary fibrosis (515) & 1,165 & $\mathbf{8 7}$ & 82 & 92 \\
Other alveolar and parietoalveolar pneumonopathy (516) & 74 & 80 & 63 & 101 \\
Other diseases of the lung (518) & 2,701 & $\mathbf{8 8}$ & 85 & 91 \\
Other diseases of respiratory system (519) & 396 & 104 & 94 & 115 \\
\hline
\end{tabular}

ICD - International Classification of Diseases, $9^{\text {th }}$ Revision

LCL - lower confidence limit

UCL - upper confidence limit

NOTE: PMRs in bold are significantly different from $100(\mathrm{p}<0.05)$. PMRs in italics are based on fewer than five observed deaths. PMRs are based on underlying and contributing cause of death. Some values could not be calculated because the number of observed or expected deaths was zero; such values are indicated by ---. See appendices for source description, methods, ICD codes, and a list of selected states.

SOURCE: National Center for Health Statistics multiple-cause-of-death data 
Table 2-86. Livestock farm workers: Proportionate mortality ratio (PMR) adjusted for age, sex, and race/ ethnicity for other diseases of respiratory system, U.S. residents age 15 and over, selected states, 1988-1998

\begin{tabular}{|c|c|c|c|c|}
\hline \multirow[b]{2}{*}{ Disease Category (ICD Code) } & \multirow{2}{*}{$\begin{array}{l}\text { Number } \\
\text { of Deaths }\end{array}$} & \multirow[b]{2}{*}{ PMR } & \multicolumn{2}{|c|}{ 95\% Confidence Interval } \\
\hline & & & $\mathbf{L C L}$ & $\mathbf{U C L}$ \\
\hline Empyema (510) & 47 & 119 & 88 & 158 \\
\hline Pneumothorax (512) & 47 & 58 & 43 & 77 \\
\hline Abscess of lung and mediastinum (513) & 29 & 93 & 62 & 134 \\
\hline Postinflammatory pulmonary fibrosis (515) & 322 & 87 & 78 & 97 \\
\hline Other alveolar and parietoalveolar pneumonopathy (516) & 19 & 75 & 45 & 117 \\
\hline Other diseases of the lung (518) & 676 & 83 & 77 & 89 \\
\hline Other diseases of respiratory system (519) & 105 & 104 & 86 & 126 \\
\hline
\end{tabular}


Table 2-87. Farm managers: Proportionate mortality ratio (PMR) adjusted for age, sex, and race/ethnicity for other diseases of respiratory system, U.S. residents age 15 and over, selected states, 1988-1998

\begin{tabular}{lrrrr}
\hline & Number & & \multicolumn{2}{c}{ 95\% Confidence Interval } \\
\cline { 3 - 5 } Disease Category (ICD Code) & of Deaths & PMR & LCL & UCL \\
\hline Empyema (510) & 2 & 80 & 10 & 289 \\
Pleurisy (511) & 15 & 90 & 50 & 149 \\
Pneumothorax (512) & 8 & 155 & 67 & 305 \\
Abscess of lung and mediastinum (513) & 1 & 50 & 49 & 124 \\
Pulmonary congestion and hypostasis (514) & 20 & 80 & 49 \\
Postinflammatory pulmonary fibrosis (515) & 22 & 97 & 61 \\
Other alveolar and parietoalveolar pneumonopathy (516) & 1 & 62 & 2 & 344 \\
Other diseases of the lung (518) & 46 & 91 & 67 & 121 \\
Other diseases of respiratory system (519) & 2 & 32 & 4 & 116 \\
\hline
\end{tabular}

ICD - International Classification of Diseases, $9^{\text {th }}$ Revision

LCL - lower confidence limit

UCL - upper confidence limit

NOTE: PMRs in bold are significantly different from $100(\mathrm{p}<0.05)$. PMRs in italics are based on fewer than five observed deaths. PMRs are based on underlying and contributing cause of death. Some values could not be calculated because the number of observed or expected deaths was zero; such values are indicated by ---. See appendices for source description, methods, ICD codes, and a list of selected states.

SOURCE: National Center for Health Statistics multiple-cause-of-death data 
Table 2-88. Landscape and horticultural workers: Proportionate mortality ratio (PMR) adjusted for age, sex, and race/ethnicity for other diseases of respiratory system, U.S. residents age 15 and over, selected states, 1988-1998

\begin{tabular}{|c|c|c|c|c|}
\hline \multirow[b]{2}{*}{ Disease Category (ICD Code) } & \multirow{2}{*}{$\begin{array}{l}\text { Number } \\
\text { of Deaths }\end{array}$} & \multirow[b]{2}{*}{ PMR } & \multicolumn{2}{|c|}{ 95\% Confidence Interval } \\
\hline & & & $\mathbf{L C L}$ & $\mathbf{U C L}$ \\
\hline Empyema (510) & 9 & 126 & 58 & 239 \\
\hline Pneumothorax (512) & 10 & 72 & 35 & 132 \\
\hline Abscess of lung and mediastinum (513) & 13 & 190 & 101 & 325 \\
\hline Postinflammatory pulmonary fibrosis (515) & 31 & 79 & 54 & 112 \\
\hline Other alveolar and parietoalveolar pneumonopathy (516) & 2 & 43 & 5 & 155 \\
\hline Other diseases of the lung (518) & 98 & 80 & 65 & 98 \\
\hline Other diseases of respiratory system (519) & 11 & 71 & 36 & 127 \\
\hline
\end{tabular}


Table 2-89. Forestry workers: Proportionate mortality ratio (PMR) adjusted for age, sex, and race/ ethnicity for other diseases of respiratory system, U.S. residents age 15 and over, selected states, 1988-1998

\begin{tabular}{|c|c|c|c|c|}
\hline \multirow[b]{2}{*}{ Disease Category (ICD Code) } & \multirow{2}{*}{$\begin{array}{l}\text { Number } \\
\text { of Deaths }\end{array}$} & \multirow[b]{2}{*}{ PMR } & \multicolumn{2}{|c|}{ 95\% Confidence Interval } \\
\hline & & & LCL & UCL \\
\hline Empyema (510) & 9 & 65 & 30 & 123 \\
\hline Pneumothorax (512) & 24 & 89 & 57 & 132 \\
\hline Abscess of lung and mediastinum (513) & 13 & 99 & 53 & 169 \\
\hline Postinflammatory pulmonary fibrosis (515) & 64 & 69 & 54 & 88 \\
\hline Other alveolar and parietoalveolar pneumonopathy (516) & 5 & 62 & 20 & 145 \\
\hline Other diseases of the lung (518) & 223 & 91 & 80 & 104 \\
\hline Other diseases of respiratory system (519) & 36 & 117 & 84 & 162 \\
\hline
\end{tabular}


Table 2-90. Fishery workers: Proportionate mortality ratio (PMR) adjusted for age, sex, and race/ethnicity for other diseases of respiratory system, U.S. residents age 15 and over, selected states, 1988-1998

\begin{tabular}{|c|c|c|c|c|}
\hline \multirow[b]{2}{*}{ Disease Category (ICD Code) } & \multirow{2}{*}{$\begin{array}{l}\text { Number } \\
\text { of Deaths }\end{array}$} & \multirow[b]{2}{*}{ PMR } & \multicolumn{2}{|c|}{ 95\% Confidence Interval } \\
\hline & & & $\mathbf{L C L}$ & UCL \\
\hline Empyema (510) & 3 & 79 & 16 & 231 \\
\hline Pleurisy (511) & 13 & 64 & 34 & 109 \\
\hline Pneumothorax (512) & 6 & 84 & 31 & 183 \\
\hline Abscess of lung and mediastinum (513) & 6 & 183 & 67 & 399 \\
\hline Pulmonary congestion and hypostasis (514) & 32 & 101 & 69 & 103 \\
\hline Postinflammatory pulmonary fibrosis (515) & 24 & 94 & 60 & 140 \\
\hline Other alveolar and parietoalveolar pneumonopathy (516) & 2 & 87 & 11 & 314 \\
\hline Other diseases of the lung (518) & 60 & 89 & 68 & 115 \\
\hline Other diseases of respiratory system (519) & 4 & 48 & 13 & 123 \\
\hline
\end{tabular}

ICD - International Classification of Diseases, $9^{\text {th }}$ Revision

LCL - lower confidence limit

UCL - upper confidence limit

NOTE: PMRs in bold are significantly different from $100(\mathrm{p}<0.05)$. PMRs in italics are based on fewer than five observed deaths. PMRs are based on underlying and contributing cause of death. Some values could not be calculated because the number of observed or expected deaths was zero; such values are indicated by ---. See appendices for source description, methods, ICD codes, and a list of selected states.

SOURCE: National Center for Health Statistics multiple-cause-of-death data 
Figure 2-68. Empyema: Proportionate mortality ratio (PMR) adjusted for age, sex, and race/ethnicity by agricultural group, U.S. residents age 15 and over, selected states, 1988-1998

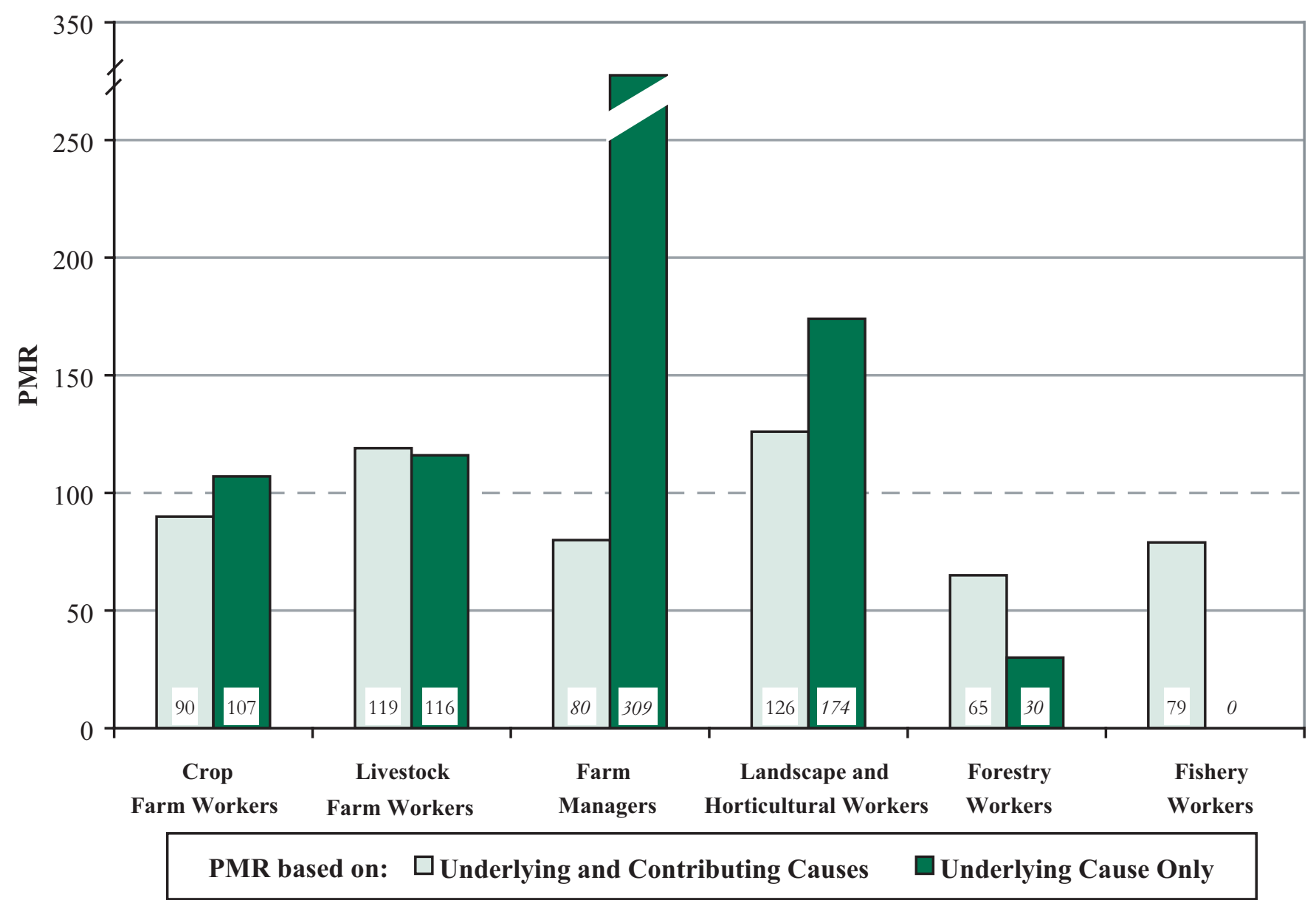

ICD - International Classification of Diseases, $9^{\text {th }}$ Revision

NOTE: Empyema = ICD-9 code 510. PMRs in bold are significantly different from $100(\mathrm{p}<0.05)$. PMRs in italics are based on fewer than five observed deaths. PMRs are based on underlying and contributing cause of death. See appendices for source description, methods, ICD codes, and a list of selected states.

SOURCE: National Center for Health Statistics multiple-cause-of-death data 
Figure 2-69. Pleurisy: Proportionate mortality ratio (PMR) adjusted for age, sex, and race/ethnicity by agricultural group, U.S. residents age 15 and over, selected states, 1988-1998

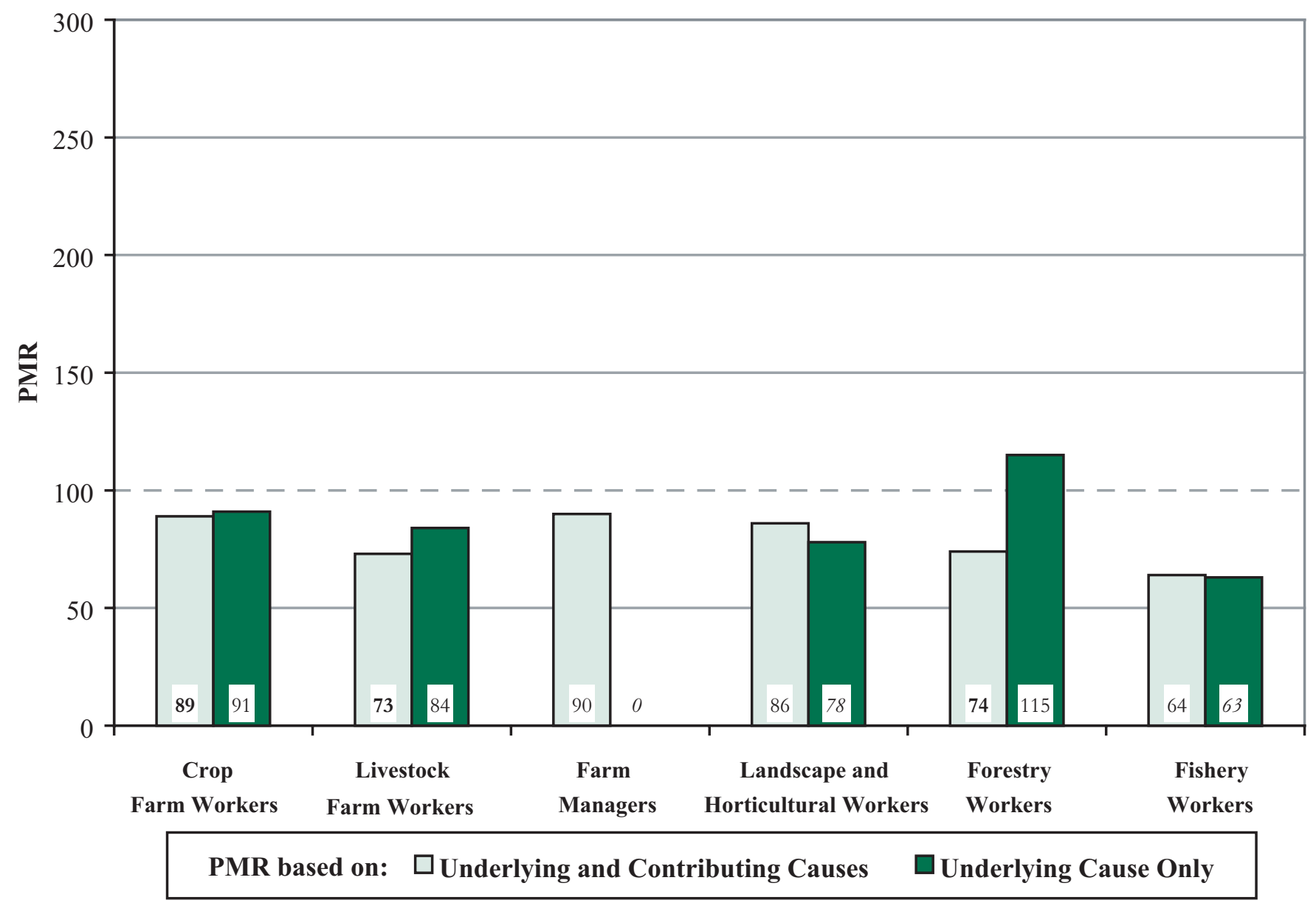

ICD - International Classification of Diseases, $9^{\text {th }}$ Revision

NOTE: Pleurisy = ICD-9 code 511. PMRs in bold are significantly different from $100(\mathrm{p}<0.05)$. PMRs in italics are based on fewer than five observed

deaths. PMRs are based on underlying and contributing cause of death. See appendices for source description, methods, ICD codes, and a list of selected states.

SOURCE: National Center for Health Statistics multiple-cause-of-death data 
Figure 2-70. Pneumothorax: Proportionate mortality ratio (PMR) adjusted for age, sex, and race/ethnicity by agricultural group, U.S. residents age 15 and over, selected states, 1988-1998

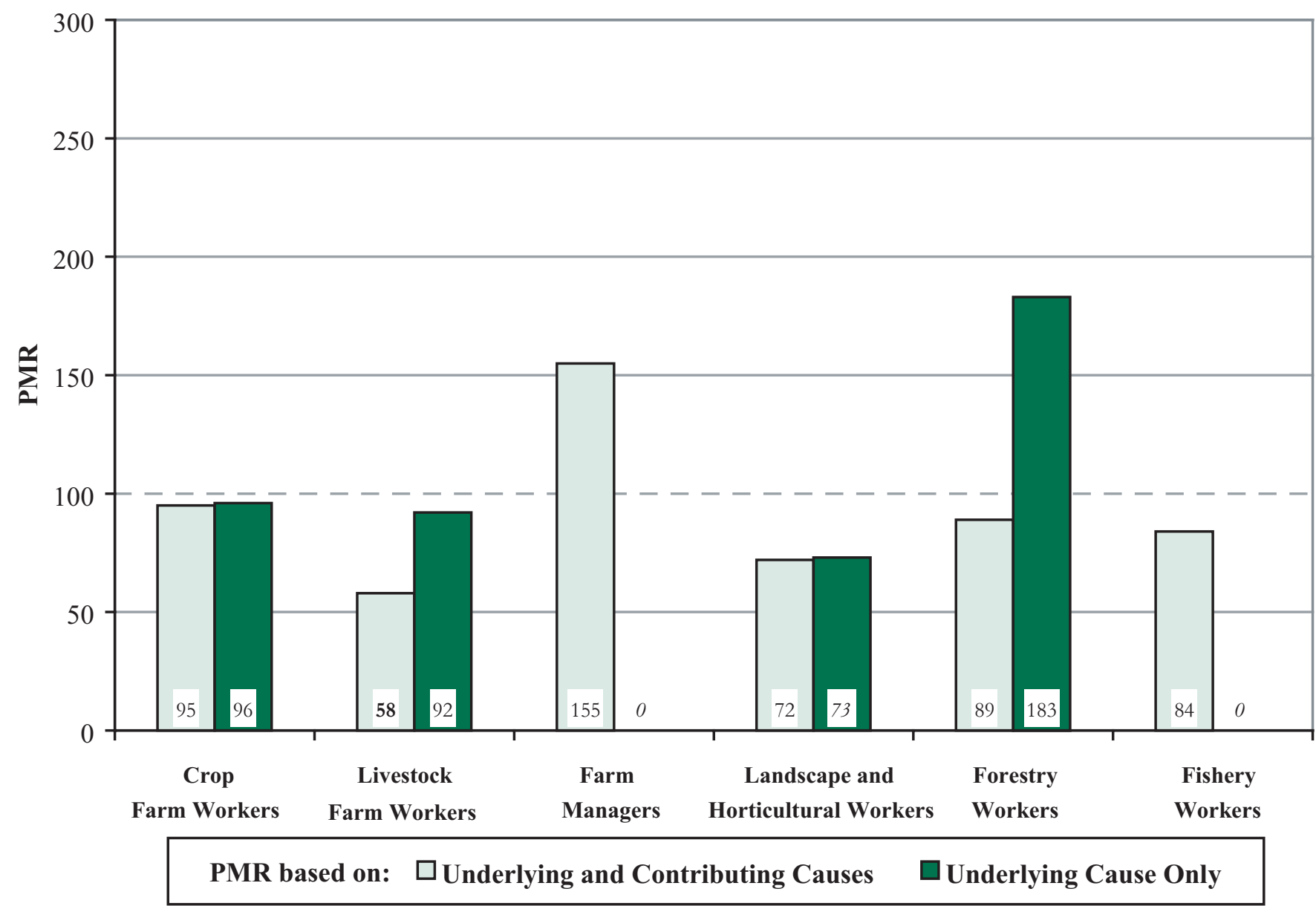

ICD - International Classification of Diseases, $9^{\text {th }}$ Revision

NOTE: Pneumothorax = ICD-9 code 512. PMRs in bold are significantly different from $100(\mathrm{p}<0.05)$. PMRs in italics are based on fewer than five observed deaths. PMRs are based on underlying and contributing cause of death. See appendices for source description, methods, ICD codes, and a list of selected states.

SOURCE: National Center for Health Statistics multiple-cause-of-death data 
Figure 2-71. Abscess of lung and mediastinum: Proportionate mortality ratio (PMR) adjusted for age, sex, and race/ethnicity by agricultural group, U.S. residents age 15 and over, selected states, 1988-1998

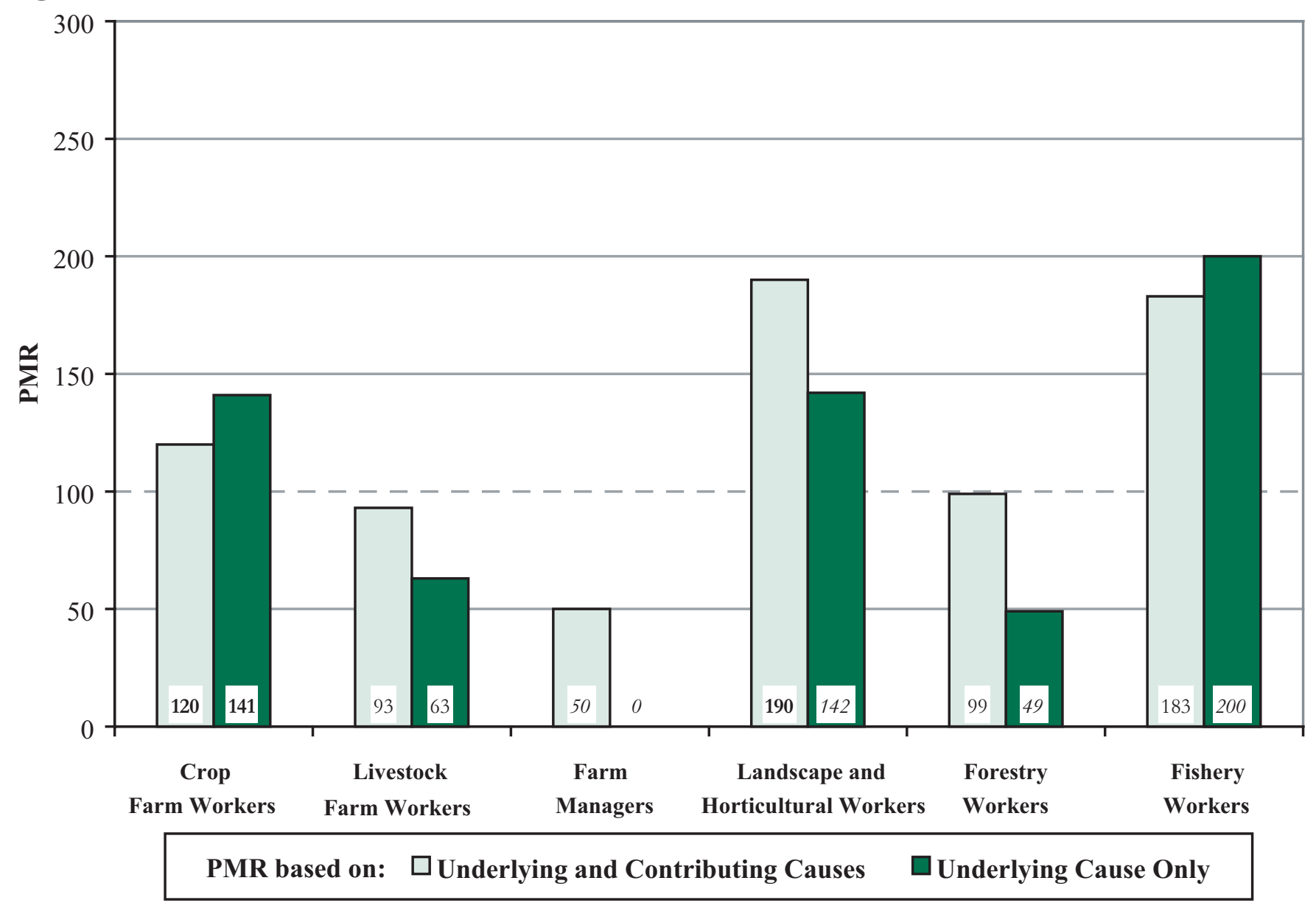

ICD - International Classification of Diseases, $9^{\text {th }}$ Revision

NOTE: Abscess of lung and mediastinum = ICD-9 code 513. PMRs in bold are significantly different from 100 (p<0.05). PMRs in italics are based on fewer than five observed deaths. PMRs are based on underlying and contributing cause of death. See appendices for source description, methods, ICD codes, and a list of selected states.

SOURCE: National Center for Health Statistics multiple-cause-of-death data 
Figure 2-72. Pulmonary congestion and hypostasis: Proportionate mortality ratio (PMR) adjusted for age, sex, and race/ethnicity by agricultural group, U.S. residents age 15 and over, selected states, 1988-1998

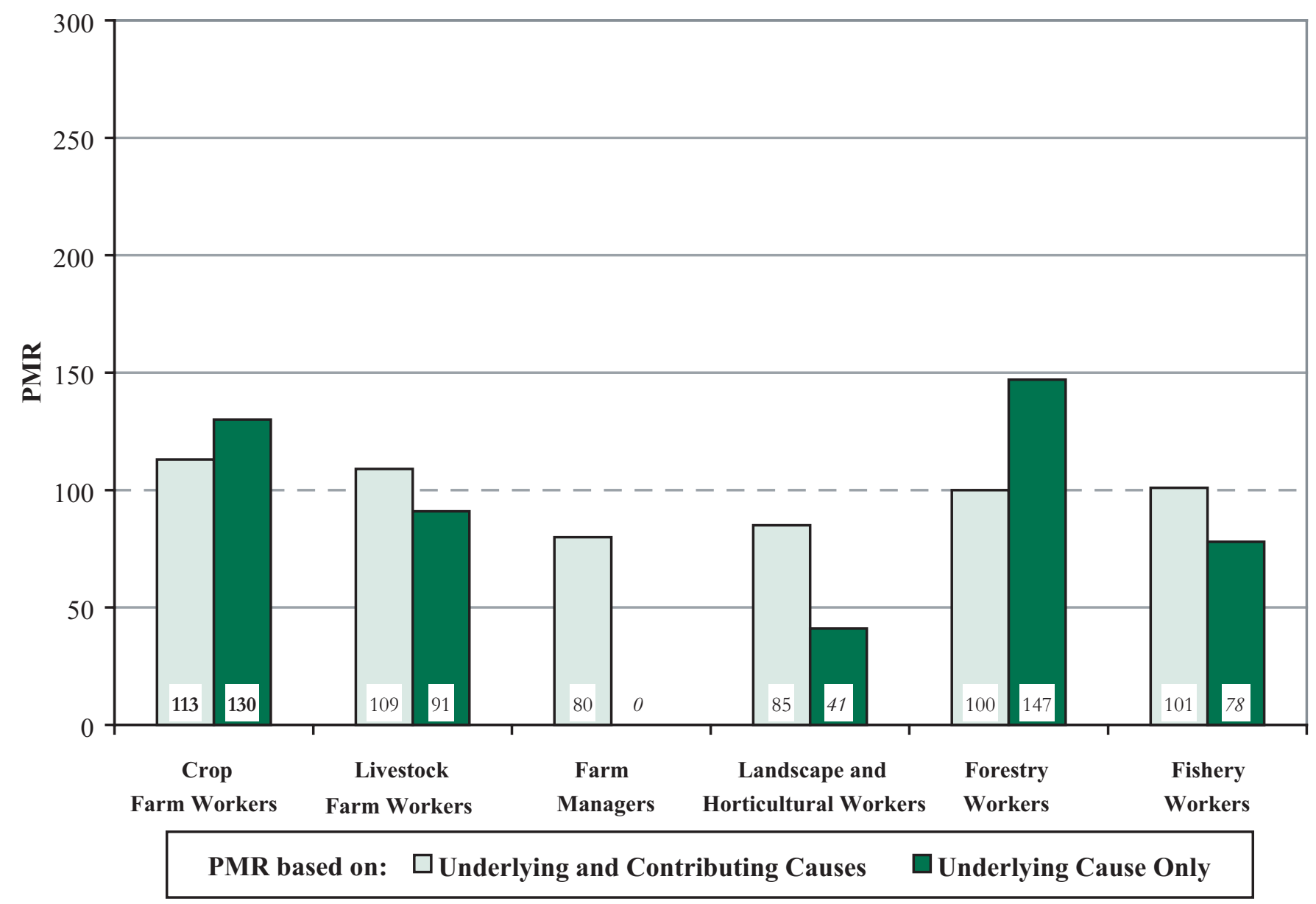

ICD - International Classification of Diseases, $9^{\text {th }}$ Revision

NOTE: Pulmonary congestion and hypostasis = ICD-9 code 514. PMRs in bold are significantly different from 100 (p<0.05). PMRs in italics are based on fewer than five observed deaths. PMRs are based on underlying and contributing cause of death. See appendices for source description, methods, ICD codes, and a list of selected states.

SOURCE: National Center for Health Statistics multiple-cause-of-death data 
Figure 2-73. Postinflammatory pulmonary fibrosis: Proportionate mortality ratio (PMR) adjusted for age, sex, and race/ethnicity by agricultural group, U.S. residents age 15 and over, selected states, 1988-1998

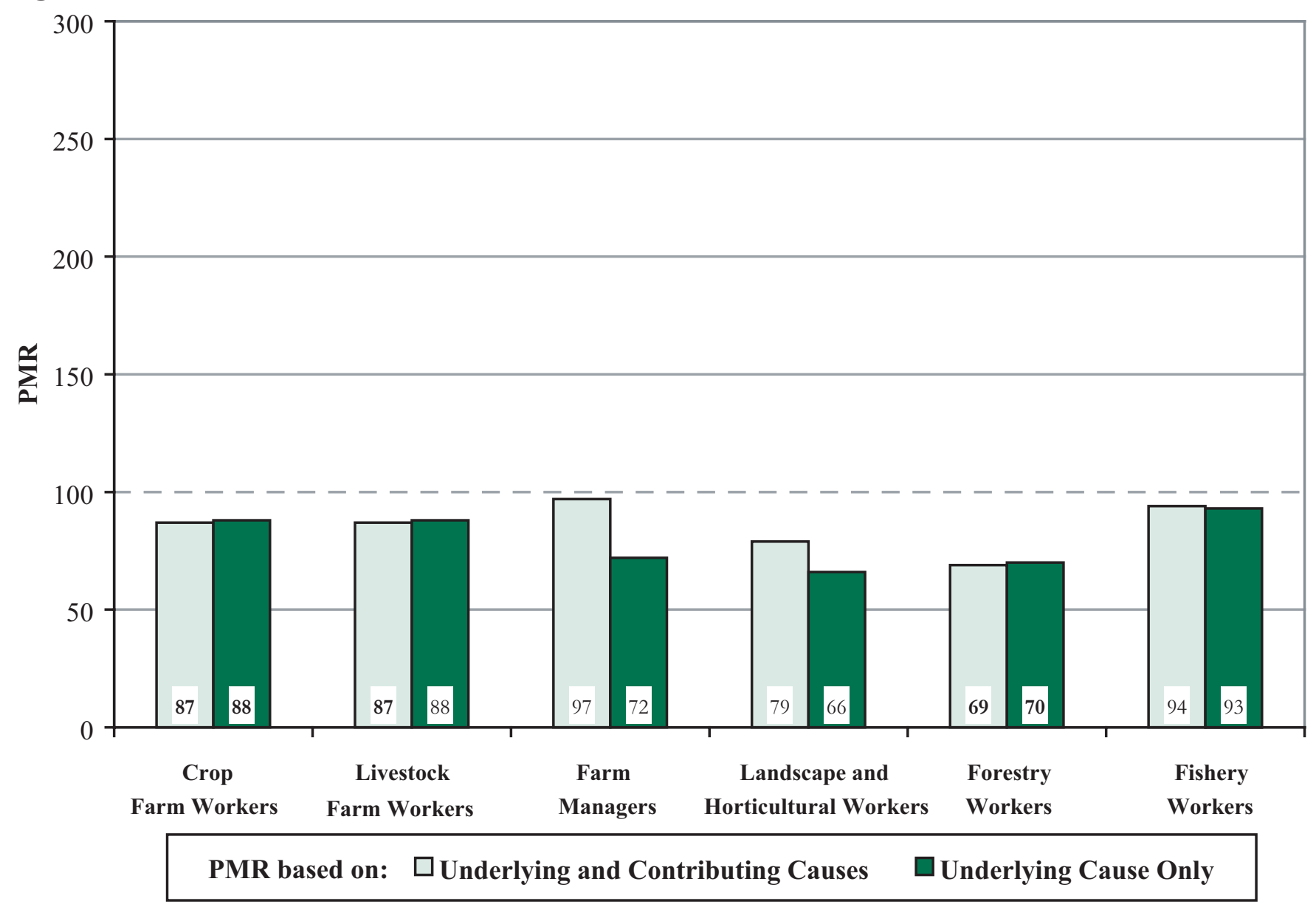

ICD - International Classification of Diseases, $9^{\text {th }}$ Revision

NOTE: Postinflammatory pulmonary fibrosis = ICD-9 code 515. PMRs in bold are significantly different from 100 ( $<<0.05)$. PMRs in italics are based on fewer than five observed deaths. PMRs are based on underlying and contributing cause of death. See appendices for source description, methods, ICD codes, and a list of selected states.

SOURCE: National Center for Health Statistics multiple-cause-of-death data 
Figure 2-74. Other alveolar and parietoalveolar pneumonopathy: Proportionate mortality ratio (PMR) adjusted for age, sex, and race/ethnicity by agricultural group, U.S. residents age 15 and over, selected states, 1988-1998

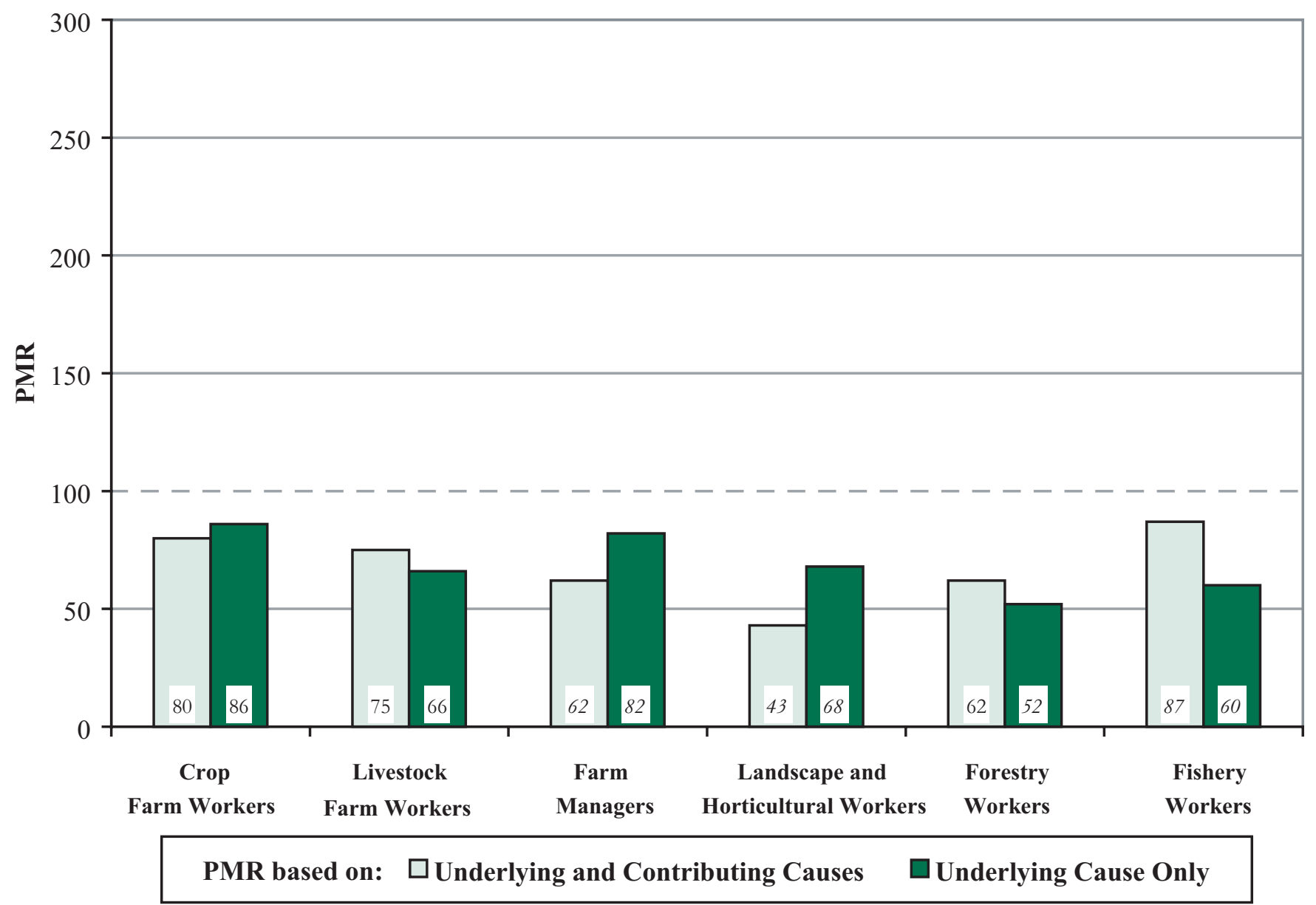

ICD - International Classification of Diseases, $9^{\text {th }}$ Revision

NOTE: Other alveolar and parietoalveolar pneumonopathy = ICD-9 code 516. PMRs in bold are significantly different from 100 ( $\mathrm{p}<0.05$ ). PMRs in italics are based on fewer than five observed deaths. PMRs are based on underlying and contributing cause of death. See appendices for source description, methods, ICD codes, and a list of selected states.

SOURCE: National Center for Health Statistics multiple-cause-of-death data 
Figure 2-75. Other diseases of the lung: Proportionate mortality ratio (PMR) adjusted for age, sex, and race/ethnicity by agricultural group, U.S. residents age 15 and over, selected states, 1988-1998

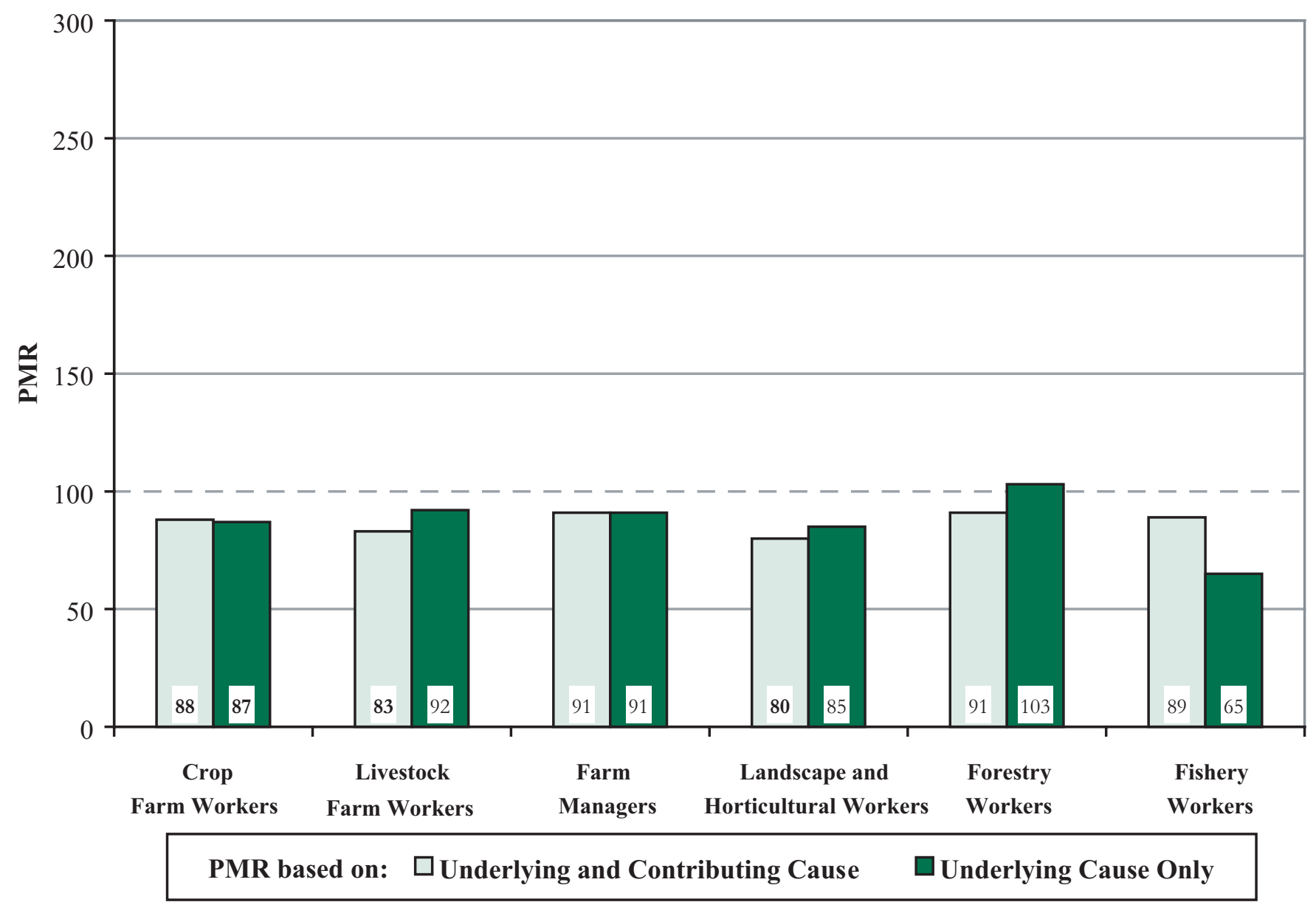

ICD - International Classification of Diseases, $9^{\text {th }}$ Revision

NOTE: Other diseases of the lung = ICD-9 code 518. PMRs in bold are significantly different from $100(\mathrm{p}<0.05)$. PMRs in italics are based on fewer than five observed deaths. PMRs are based on underlying and contributing cause of death. See appendices for source description, methods, ICD codes, and a list of selected states.

SOURCE: National Center for Health Statistics multiple-cause-of-death data 
Figure 2-76. Other diseases of respiratory system: Proportionate mortality ratio (PMR) adjusted for age, sex, and race/ethnicity by agricultural group, U.S. residents age 15 and over, selected states, 1988-1998

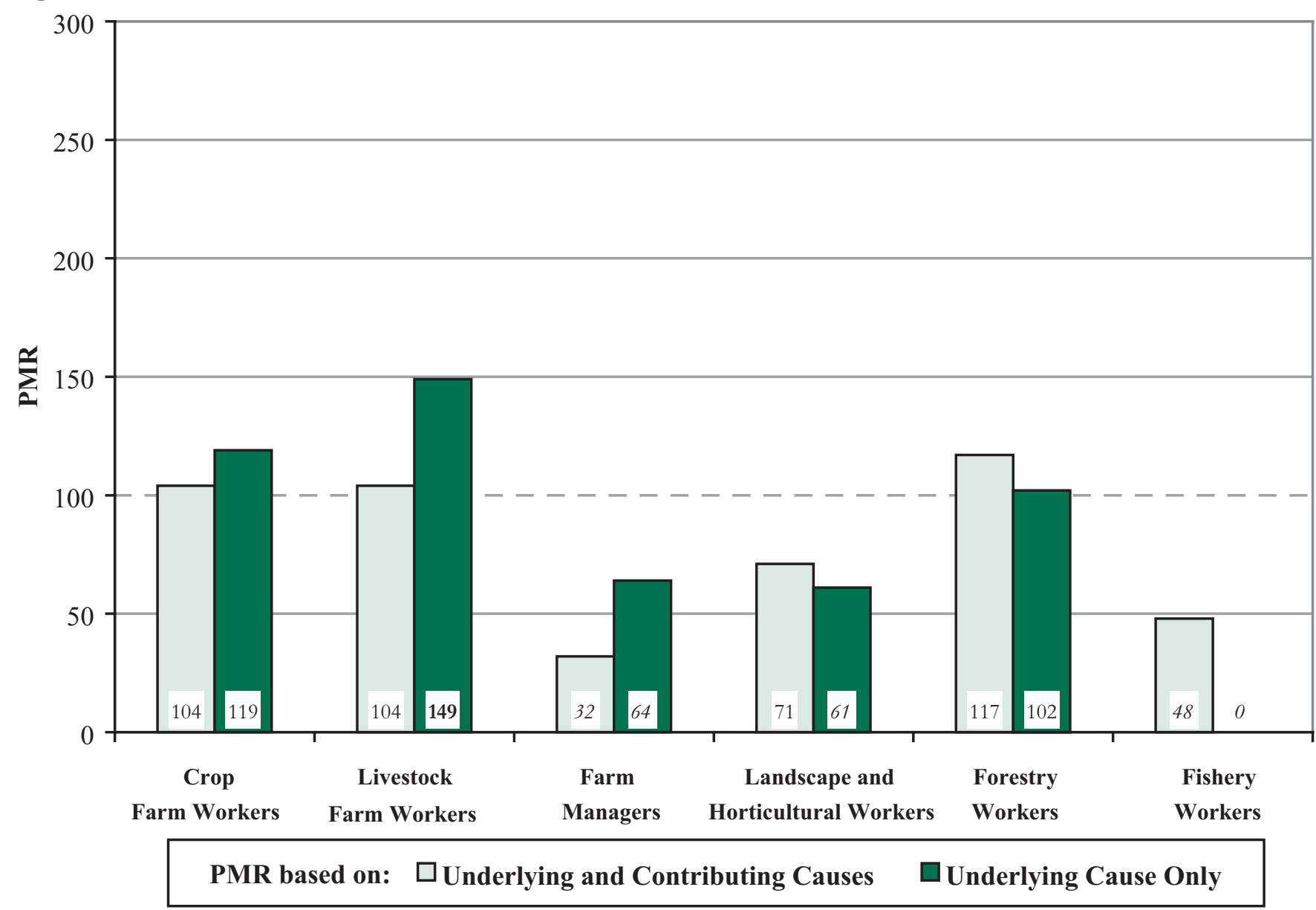

ICD - International Classification of Diseases, $9^{\text {th }}$ Revision

NOTE: Other diseases of respiratory system $=$ ICD-9 code 519 . PMRs in bold are significantly different from $100(\mathrm{p}<0.05)$. PMRs in italics are based on fewer than five observed deaths. PMRs are based on underlying and contributing cause of death. See appendices for source description, methods, ICD codes, and a list of selected states.

SOURCE: National Center for Health Statistics multiple-cause-of-death data 


\section{Section 3}

Morbidity 

Table 3-1. Hayfever (past year): Estimated prevalence and prevalence ratio (PR) adjusted for age, sex, race/ethnicity, and smoking status by agricultural group and survey year, U.S. residents age 18 and over, 1997-1999

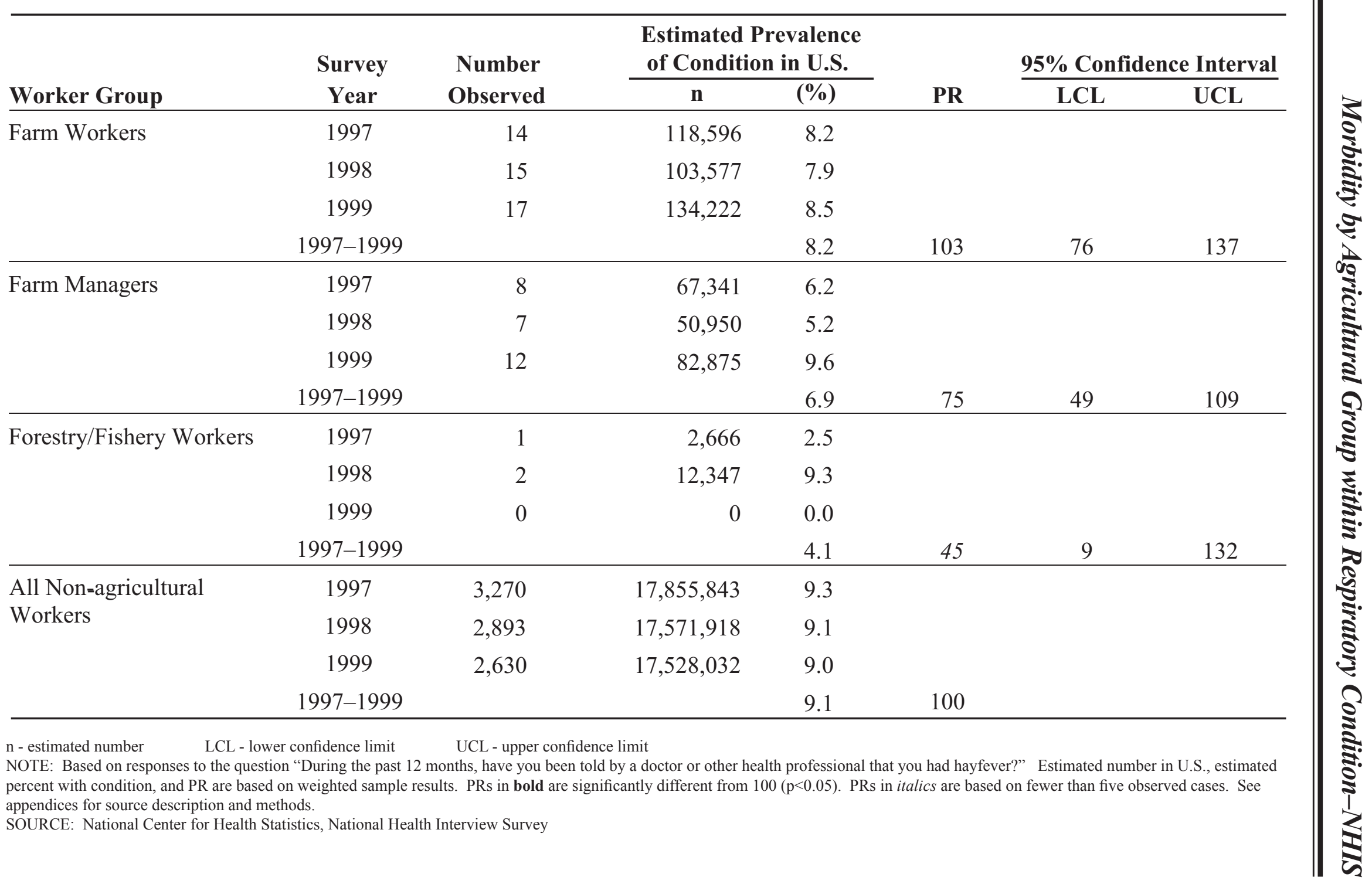


Table 3-2. Sinusitis (past year): Estimated prevalence and prevalence ratio (PR) adjusted for age, sex, race/ethnicity, and smoking status by agricultural group and survey year, U.S. residents age 18 and over, 1997-1999

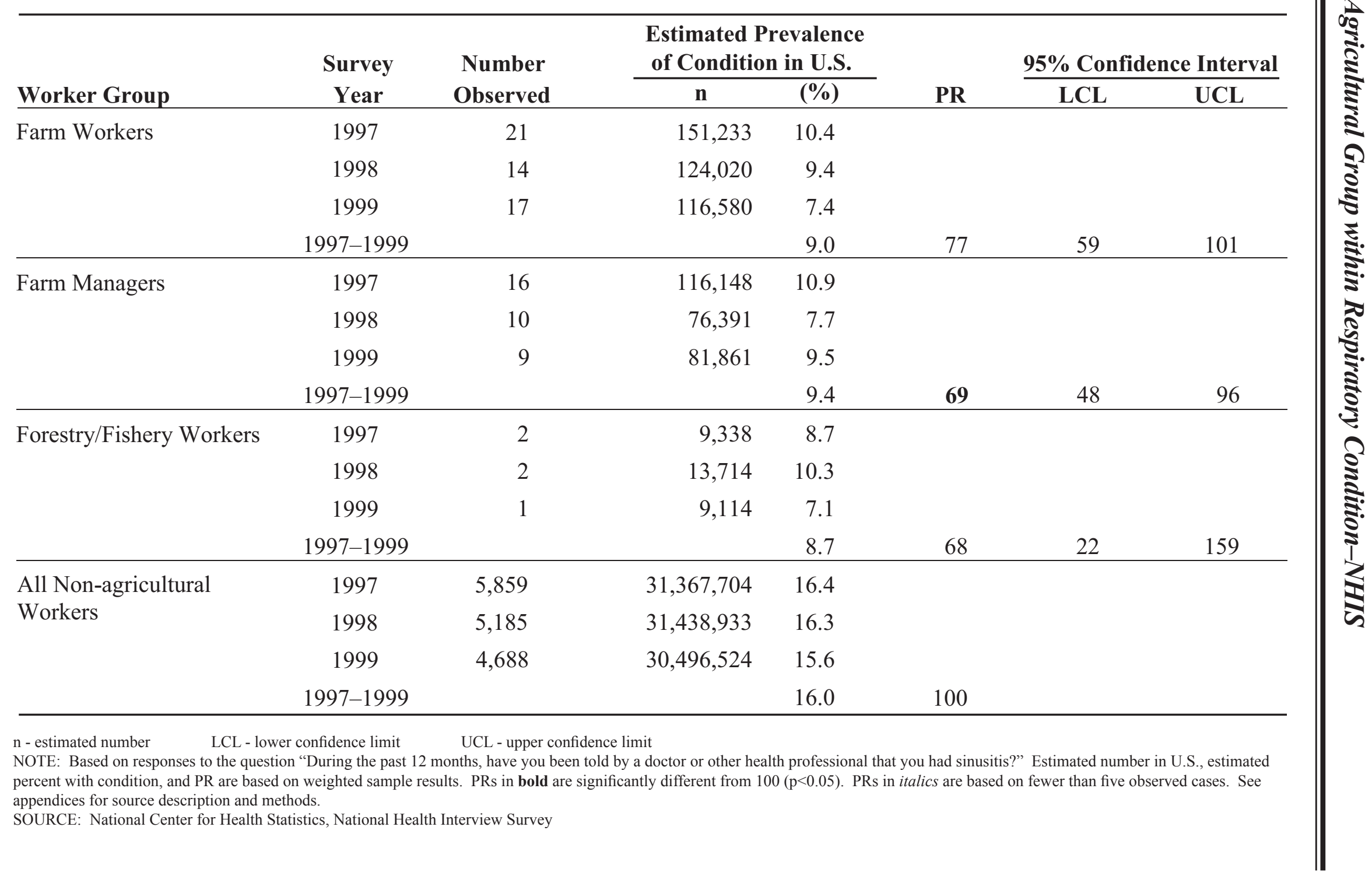


Table 3-3. Chronic bronchitis (past year): Estimated prevalence and prevalence ratio (PR) adjusted for age, sex, race/ethnicity, and smoking status by agricultural group and survey year, U.S. residents age 18 and over, 1997-1999

\begin{tabular}{|c|c|c|c|c|c|c|c|}
\hline \multirow[b]{2}{*}{ Worker Group } & \multirow{2}{*}{$\begin{array}{c}\text { Survey } \\
\text { Year }\end{array}$} & \multirow{2}{*}{$\begin{array}{c}\text { Number } \\
\text { Observed }\end{array}$} & \multicolumn{2}{|c|}{$\begin{array}{l}\text { Estimated Prevalence } \\
\text { of Condition in U.S. }\end{array}$} & \multirow[b]{2}{*}{ PR } & \multicolumn{2}{|c|}{ 95\% Confidence Interval } \\
\hline & & & $\mathbf{n}$ & $(\%)$ & & LCL & UCL \\
\hline \multirow{2}{*}{ Farm Workers } & 1998 & 8 & 69,040 & 5.2 & & & \\
\hline & 1999 & 7 & 40,345 & 2.6 & & & \\
\hline \multirow{3}{*}{ Farm Managers } & 1998 & 1 & 6,507 & 0.7 & & & \\
\hline & 1999 & 3 & 20,890 & 2.4 & & & \\
\hline & 1997-1999 & & & 1.8 & 52 & 22 & 102 \\
\hline Forestry/Fishery Workers & 1997 & 0 & 0 & 0.0 & & & \\
\hline \multirow{4}{*}{$\begin{array}{l}\text { All Non-agricultural } \\
\text { Workers }\end{array}$} & 1997 & 1,857 & $9,593,307$ & 5.0 & & & \\
\hline & 1998 & 1,554 & $8,863,177$ & 4.6 & & & \\
\hline & 1999 & 1,409 & $8,751,682$ & 4.5 & & & \\
\hline & 1997-1999 & & & 4.7 & 100 & & \\
\hline $\begin{array}{l}\mathrm{n} \text { - estimated number } \quad \text { LCL - lo } \\
\text { NOTE: Based on responses to the ques } \\
\text { estimated percent with condition, and P } \\
\text { cases. See appendices for source descr } \\
\text { SOURCE: National Center for Health }\end{array}$ & $\begin{array}{l}\text { confidence limit } \\
\text { "During the past } 1 \\
\text { re based on weight } \\
\text { on and methods. } \\
\text { istics, National Hea }\end{array}$ & $\begin{array}{l}\text { UCL - upper c } \\
\text { nonths, have you b } \\
\text { ample results. PR } \\
\text { Interview Survey }\end{array}$ & $\begin{array}{l}\text { mit } \\
\text { a doctor or other } \\
\text { e significantly dif }\end{array}$ & $\begin{array}{l}\text { professional } \\
\text { rom } 100(\mathrm{p} \text { - }\end{array}$ & $\begin{array}{l}\text { u had cht } \\
\text { PRs in it }\end{array}$ & $\begin{array}{l}\text { bronchitis?" Es } \\
\text { are based on fey }\end{array}$ & $\begin{array}{l}\text { mber in U.S., } \\
\text { e observed }\end{array}$ \\
\hline
\end{tabular}


Table 3-4. Emphysema (ever): Estimated prevalence and prevalence ratio (PR) adjusted for age, sex, race/ethnicity, and smoking status by agricultural group and survey year, U.S. residents age 18 and over, 1997-1999

\begin{tabular}{|c|c|c|c|c|c|c|c|}
\hline \multirow[b]{2}{*}{ Worker Group } & \multirow{2}{*}{$\begin{array}{c}\text { Survey } \\
\text { Year }\end{array}$} & \multirow{2}{*}{$\begin{array}{c}\text { Number } \\
\text { Observed }\end{array}$} & \multicolumn{2}{|c|}{$\begin{array}{l}\text { Estimated Prevalence } \\
\text { of Condition in U.S. }\end{array}$} & \multirow[b]{2}{*}{ PR } & \multicolumn{2}{|c|}{ 95\% Confidence Interval } \\
\hline & & & $\mathbf{n}$ & $(\%)$ & & $\mathbf{L C L}$ & UCL \\
\hline \multirow{2}{*}{ Farm Workers } & 1998 & 3 & 20,760 & 1.6 & & & \\
\hline & 1999 & 1 & 1,969 & 0.1 & & & \\
\hline & 1998 & 0 & 0 & 0.0 & & & \\
\hline & 1999 & 0 & 0 & 0.0 & & & \\
\hline & 1997-1999 & & & 0.4 & 20 & 1 & 111 \\
\hline Forestry/Fishery Workers & 1997 & 0 & 0 & 0.0 & & & \\
\hline \multirow{4}{*}{$\begin{array}{l}\text { All Non-agricultural } \\
\text { Workers }\end{array}$} & 1997 & 612 & $3,205,415$ & 1.7 & & & \\
\hline & 1998 & 542 & $2,934,923$ & 1.5 & & & \\
\hline & 1999 & 460 & $2,783,974$ & 1.4 & & & \\
\hline & 1997-1999 & & & 1.5 & 100 & & \\
\hline $\begin{array}{l}\mathrm{n} \text { - estimated number } \quad \text { LCL - lo } \\
\text { NOTE: Based on responses to the ques } \\
\text { condition, and PR are based on weight } \\
\text { source description and methods. } \\
\text { SOURCE: National Center for Health }\end{array}$ & $\begin{array}{l}\text { confidence limit } \\
\text { "Have you EVER } \\
\text { mple results. PRs } \\
\text { istics, National Hea }\end{array}$ & $\begin{array}{l}\text { UCL - upper ce } \\
\text { t told by a doctor } \\
\text { old are significant } \\
\text { nterview Survey }\end{array}$ & $\begin{array}{l}\text { nit } \\
\text { lth professional t1 } \\
\text { from } 100(p<0.05\end{array}$ & $\begin{array}{l}\text { had emph } \\
\text { in italics a }\end{array}$ & $\begin{array}{l}\text { Estima } \\
\text { on fewc }\end{array}$ & $\begin{array}{l}\text { nber in U.S., es } \\
\text { five observed c }\end{array}$ & $\begin{array}{l}\text { rcent with } \\
\text { appendices for }\end{array}$ \\
\hline
\end{tabular}


Table 3-5. Asthma (ever): Estimated prevalence and prevalence ratio (PR) adjusted for age, sex, race/ ethnicity, and smoking status by agricultural group and survey year, U.S. residents age 18 and over, 1997-1999

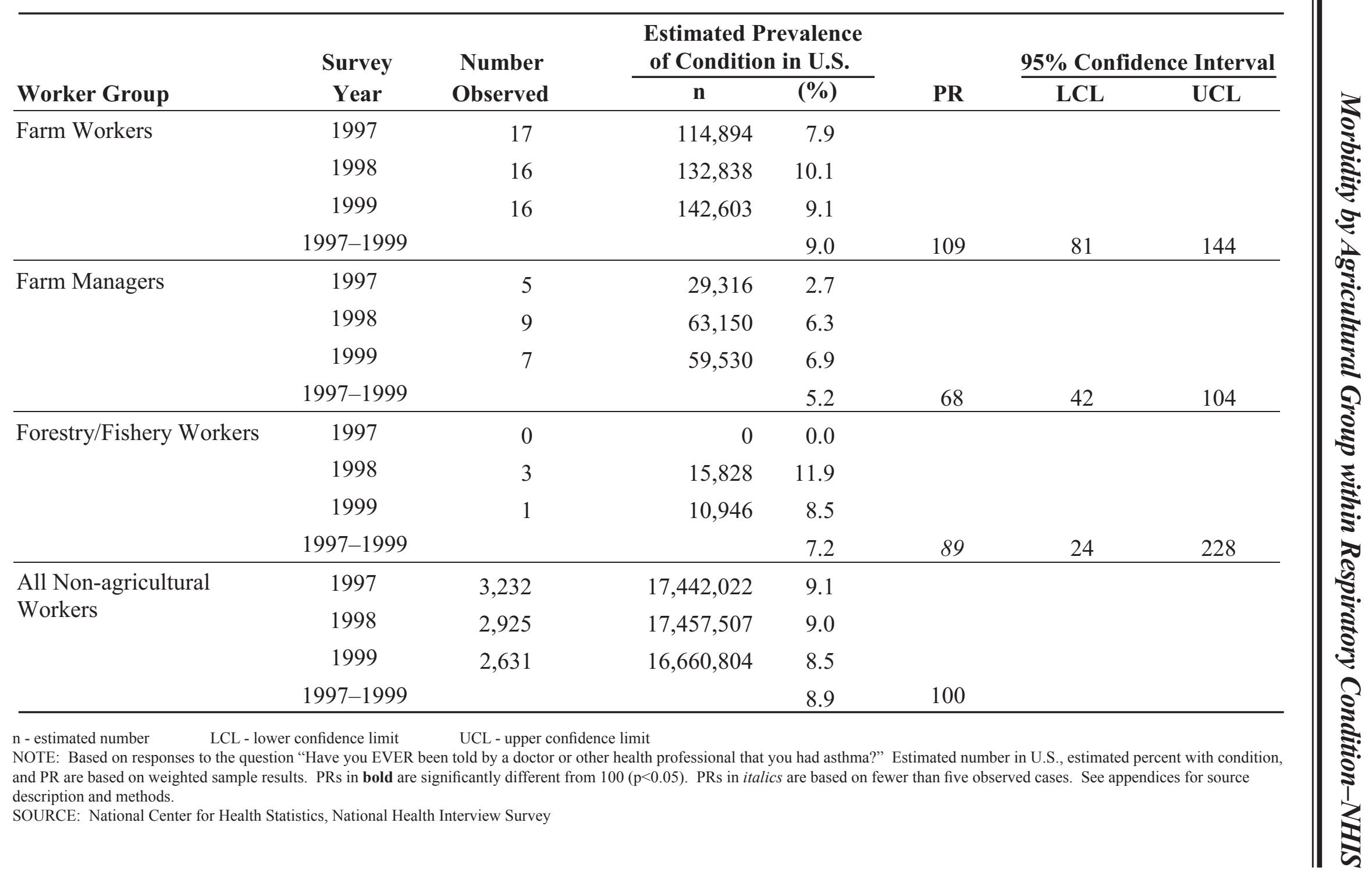


Table 3-6. Lung cancer (ever): Estimated prevalence and prevalence ratio (PR) adjusted for age, sex, race/ethnicity, and smoking status by agricultural group and survey year, U.S. residents age 18 and over, 1997-1999

\begin{tabular}{|c|c|c|c|c|c|c|c|}
\hline \multirow[b]{2}{*}{ Worker Group } & \multirow{2}{*}{$\begin{array}{l}\text { Survey } \\
\text { Year }\end{array}$} & \multirow{2}{*}{$\begin{array}{l}\text { Number } \\
\text { Observed }\end{array}$} & \multicolumn{2}{|c|}{$\begin{array}{l}\text { Estimated Prevalence } \\
\text { of Condition in U.S. }\end{array}$} & \multirow[b]{2}{*}{ PR } & \multicolumn{2}{|c|}{$\mathbf{9 5 \%}$ Confidence Interval } \\
\hline & & & $\mathbf{n}$ & $(\%)$ & & LCL & $\mathbf{U C L}$ \\
\hline \multirow{2}{*}{ Farm Workers } & 1998 & 0 & 0 & 0.0 & & & \\
\hline & 1999 & 0 & 0 & 0.0 & & & \\
\hline \multirow{3}{*}{ Farm Managers } & 1998 & 0 & 0 & 0.0 & & & \\
\hline & 1999 & 1 & 13,927 & 1.6 & & & \\
\hline & 1997-1999 & & & 0.5 & 223 & 6 & 1,239 \\
\hline Forestry/Fishery Workers & 1997 & 0 & 0 & 0.0 & & & \\
\hline \multirow{4}{*}{$\begin{array}{l}\text { All Non-agricultural } \\
\text { Workers }\end{array}$} & 1997 & 78 & 388,060 & 0.2 & & & \\
\hline & 1998 & 54 & 339,485 & 0.2 & & & \\
\hline & 1999 & 70 & 439,496 & 0.2 & & & \\
\hline & 1997-1999 & & & 0.2 & 100 & & \\
\hline $\begin{array}{l}\mathrm{n} \text { - estimated number } \\
\text { NOTE: Based on responses to the ques } \\
\text { was it? ... lung?" Estimated number in } \\
\text { PRs in italics are based on fewer than fi } \\
\text { SOURCE: National Center for Health }\end{array}$ & $\begin{array}{l}\text { confidence limit } \\
\text { "Have you EVER } \\
\text {, estimated percent } \\
\text { observed cases. See } \\
\text { istics, National Hea }\end{array}$ & $\begin{array}{l}\text { UCL - upper co } \\
\text { n told by a doctor } \\
\text { h condition, and } \mathrm{P} \\
\text { oendices for sourc } \\
\text { Interview Survey }\end{array}$ & $\begin{array}{l}\text { mit } \\
\text { alth professional } t \\
\text { d on weighted san } \\
\text { on and methods. }\end{array}$ & $\begin{array}{l}\text { had cancer } \\
\text { ults. PRs in }\end{array}$ & $\begin{array}{l}\text { lignancy } \\
\text { re signifi }\end{array}$ & $\begin{array}{l}\text { yy kind? What } \mathrm{k} \\
\text { different from }\end{array}$ & \\
\hline
\end{tabular}


Figure 3-1. Respiratory conditions: Prevalence ratio (PR) adjusted for age, sex, race/ethnicity, and smoking status by agricultural group, U.S. residents age 18 and over, 1997-1999

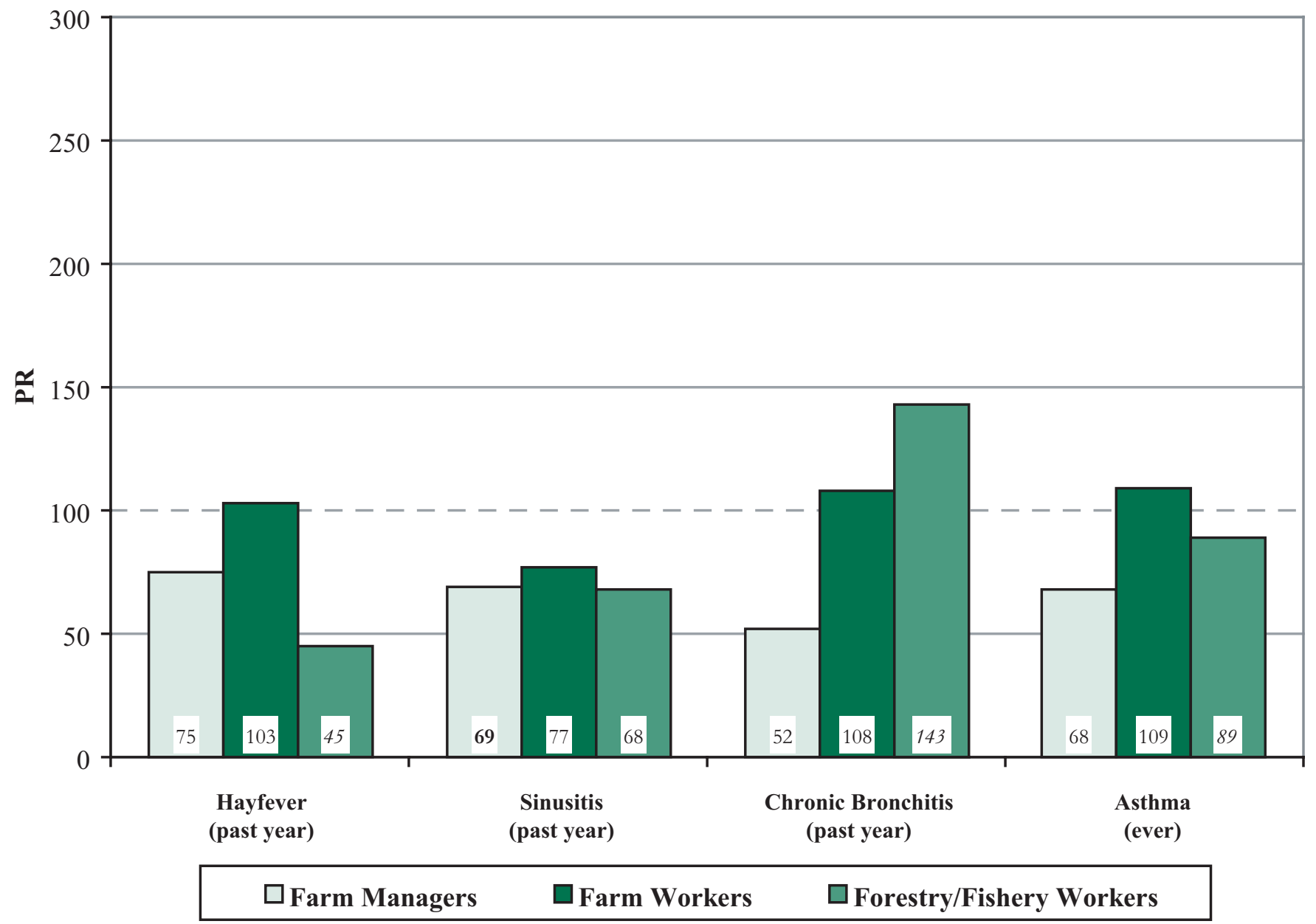

NOTE: Based on responses to the following questions:

"During the past 12 months, have you been told by a doctor or other health professional that you had hayfever?"

"During the past 12 months, have you been told by a doctor or other health professional that you had sinusitis?"

"During the past 12 months, have you been told by a doctor or other health professional that you had chronic bronchitis?"

"Have you EVER been told by a doctor or other health professional that you had asthma?"

PRs in bold are significantly different from $100(\mathrm{p}<0.05)$. PRs in italics are based on fewer than five observed cases. See appendices for source description and methods.

SOURCE: National Center for Health Statistics, National Health Interview Survey 
Figure 3-2. Farm workers: Prevalence ratio (PR) adjusted for age, race/ethnicity, and smoking status by respiratory condition and sex, U.S. residents age 18 and over, 1997-1999

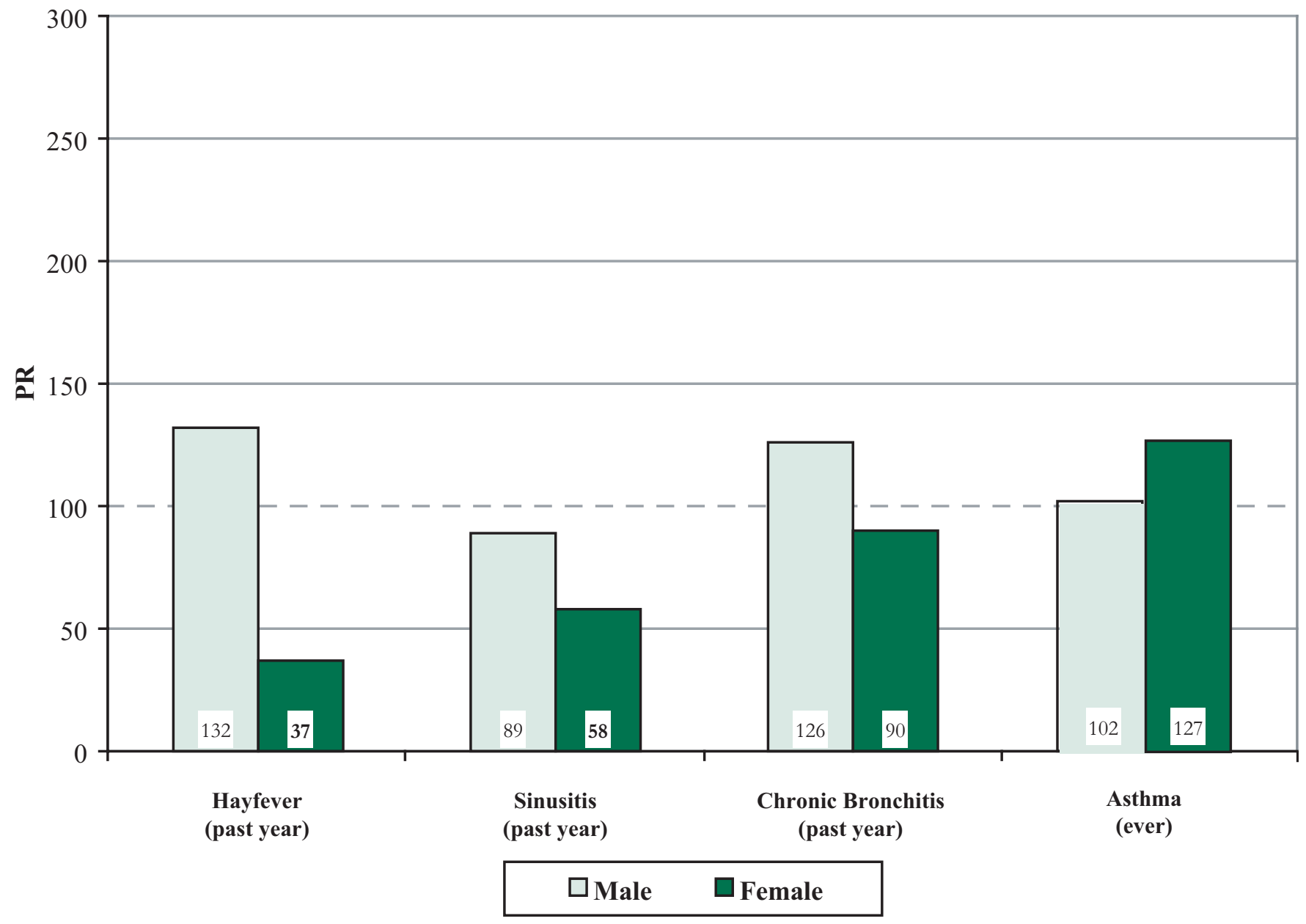

NOTE: Based on responses to the following questions:

"During the past 12 months, have you been told by a doctor or other health professional that you had hayfever?"

"During the past 12 months, have you been told by a doctor or other health professional that you had sinusitis?"

"During the past 12 months, have you been told by a doctor or other health professional that you had chronic bronchitis?"

"Have you EVER been told by a doctor or other health professional that you had asthma?"

PRs in bold are significantly different from $100(\mathrm{p}<0.05)$. PRs in italics are based on fewer than five observed cases. See appendices for source description and methods.

SOURCE: National Center for Health Statistics, National Health Interview Survey 
Figure 3-3. Farm managers: Prevalence ratio (PR) adjusted for age, race/ethnicity, and smoking status by respiratory condition and sex, U.S. residents age 18 and over, 1997-1999

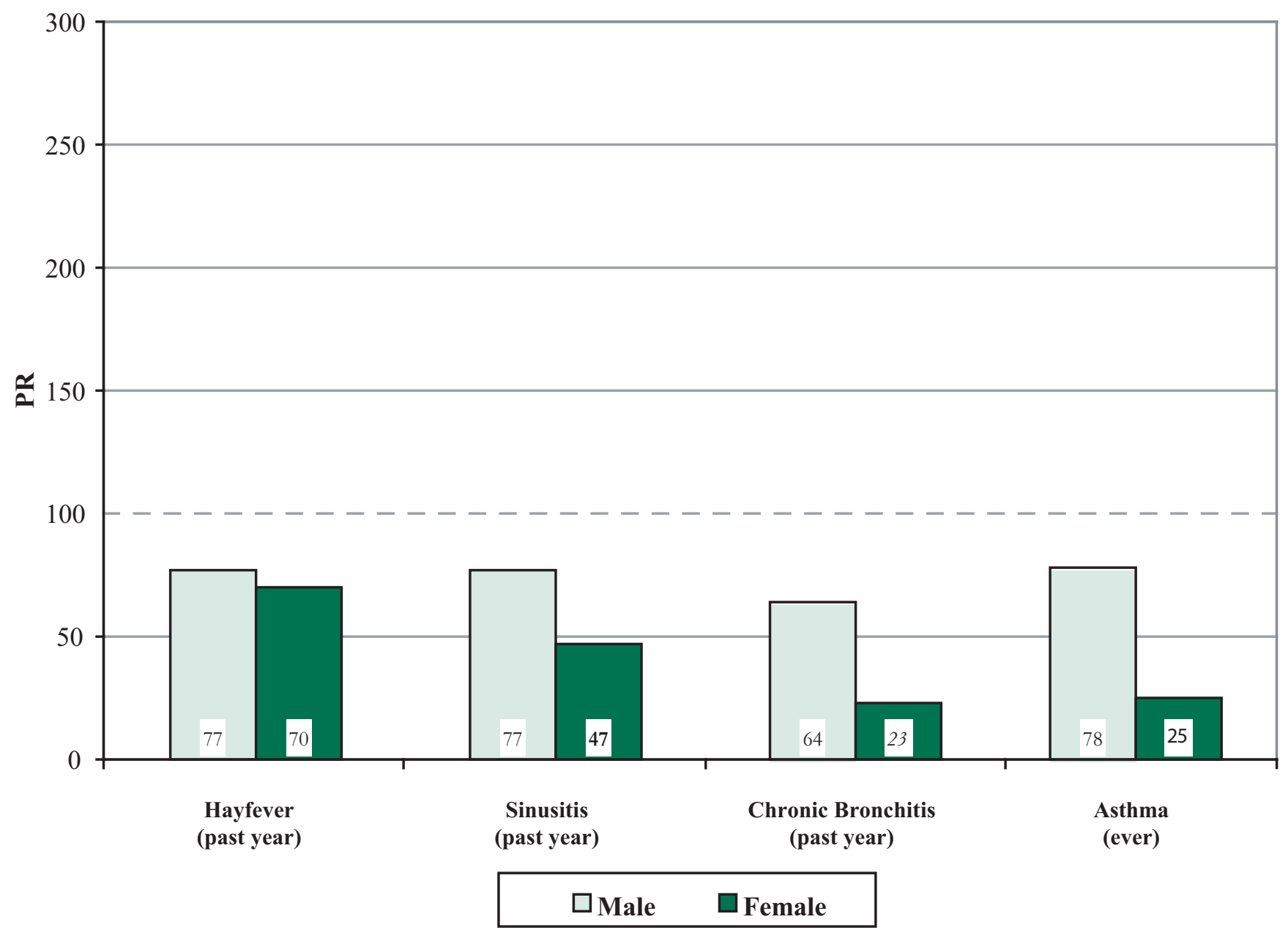

NOTE: Based on responses to the following questions:

"During the past 12 months, have you been told by a doctor or other health professional that you had hayfever?"

"During the past 12 months, have you been told by a doctor or other health professional that you had sinusitis?"

"During the past 12 months, have you been told by a doctor or other health professional that you had chronic bronchitis?"

"Have you EVER been told by a doctor or other health professional that you had asthma?"

PRs in bold are significantly different from $100(\mathrm{p}<0.05)$. PRs in italics are based on fewer than five observed cases. See appendices for source description and methods.

SOURCE: National Center for Health Statistics, National Health Interview Survey 
Figure 3-4. Forestry/fishery workers: Prevalence ratio (PR) adjusted for age, race/ ethnicity, and smoking status by respiratory condition and sex, U.S. residents age 18 and over, 1997-1999

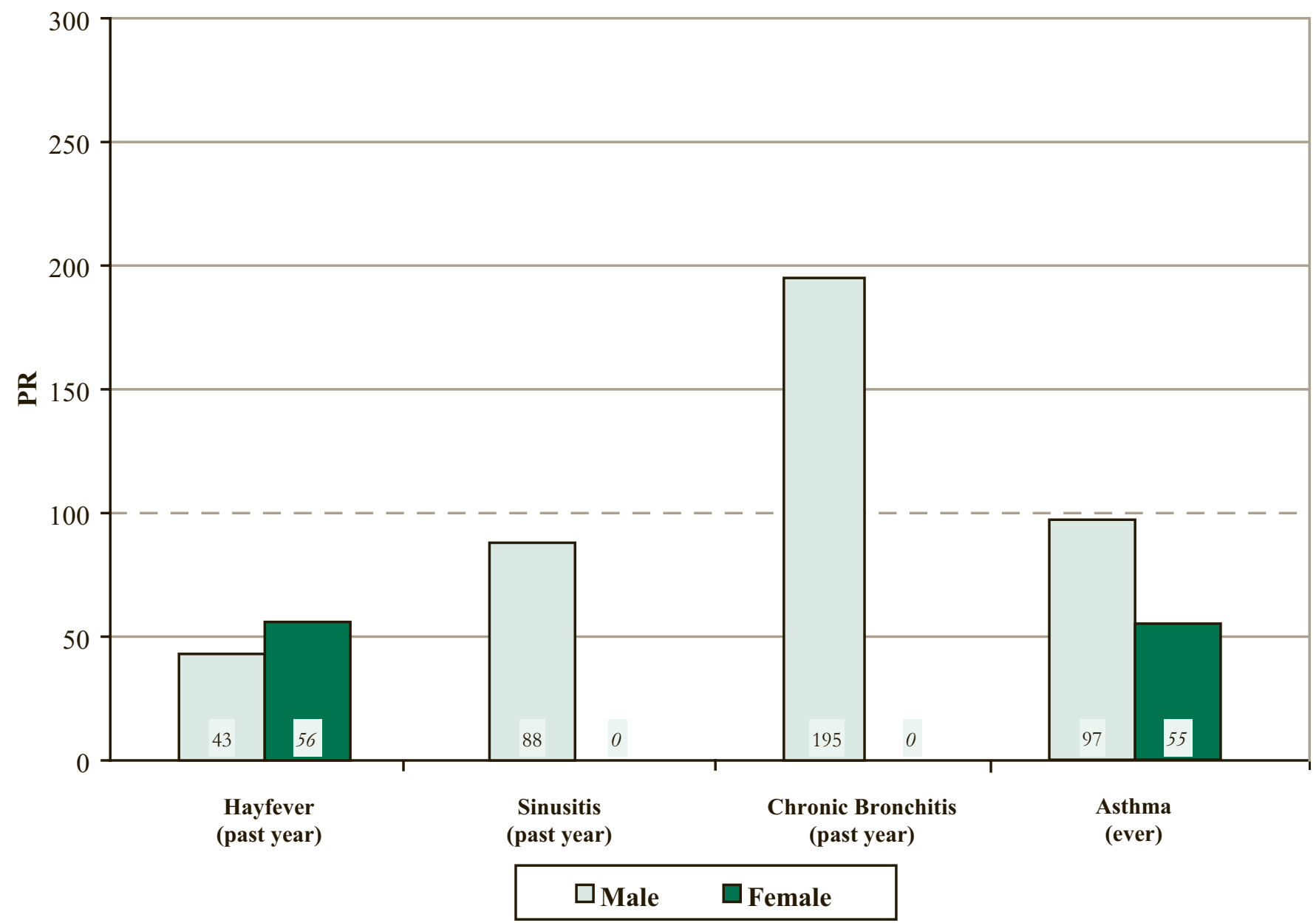

NOTE: Based on responses to the following questions:

"During the past 12 months, have you been told by a doctor or other health professional that you had hayfever?"

"During the past 12 months, have you been told by a doctor or other health professional that you had sinusitis?"

"During the past 12 months, have you been told by a doctor or other health professional that you had chronic bronchitis?"

"Have you EVER been told by a doctor or other health professional that you had asthma?"

PRs in bold are significantly different from $100(\mathrm{p}<0.05)$. PRs in italics are based on fewer than five observed cases. See appendices for source description and methods.

SOURCE: National Center for Health Statistics, National Health Interview Survey 
Figure 3-5. Farm workers: Prevalence ratio (PR) adjusted for age, sex, and smoking status by respiratory condition and race/ethnicity, U.S. residents age 18 and over, 1997-1999

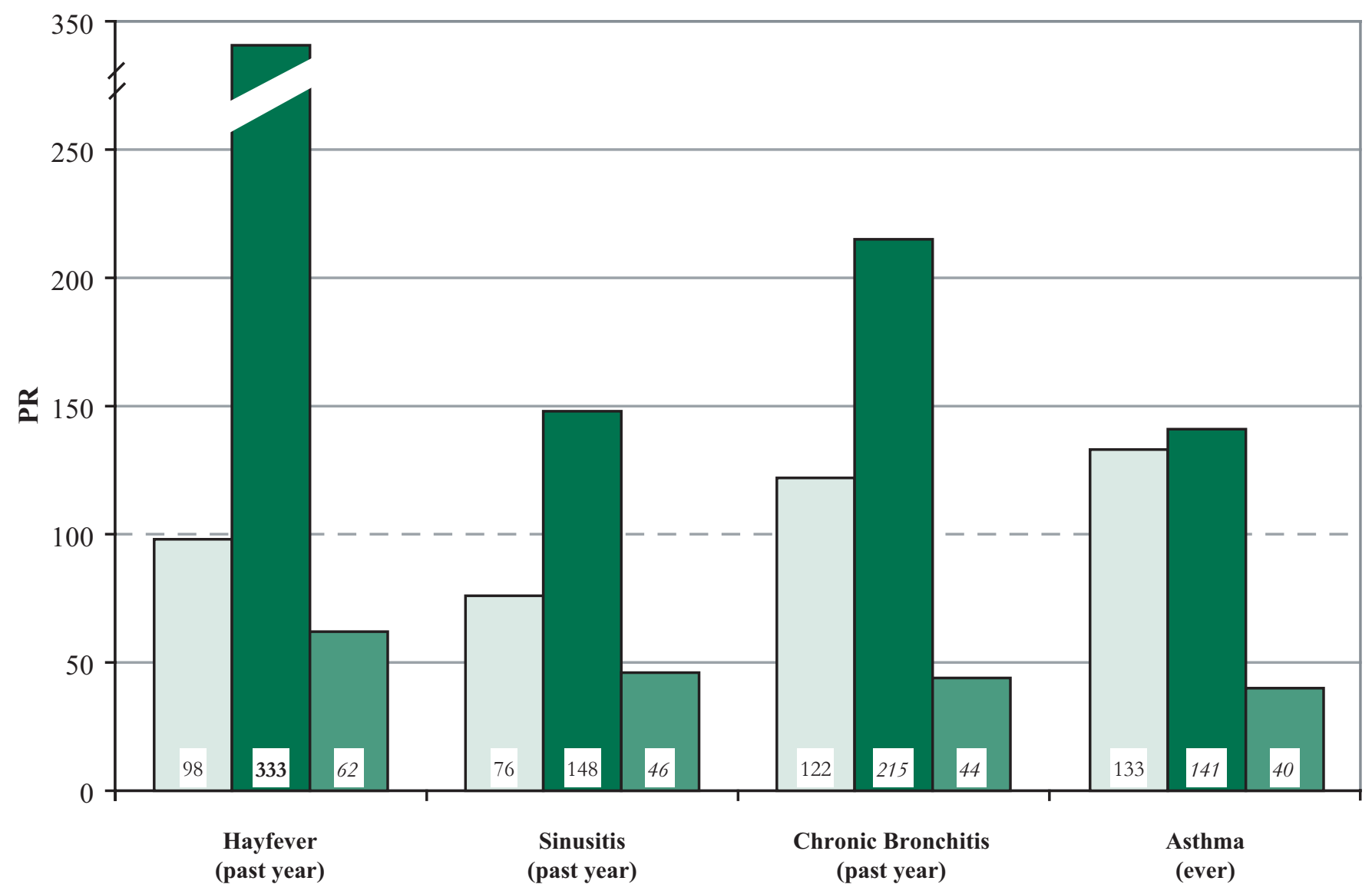

White, non-Hispanic

Black, non-Hispanic

$\square$ Hispanic

NOTE: Based on responses to the following questions:

"During the past 12 months, have you been told by a doctor or other health professional that you had hayfever?"

"During the past 12 months, have you been told by a doctor or other health professional that you had sinusitis?"

"During the past 12 months, have you been told by a doctor or other health professional that you had chronic bronchitis?"

"Have you EVER been told by a doctor or other health professional that you had asthma?"

PRs in bold are significantly different from $100(\mathrm{p}<0.05)$. PRs in italics are based on fewer than five observed cases. See appendices for source description and methods.

SOURCE: National Center for Health Statistics, National Health Interview Survey 
Figure 3-6. Farm managers: Prevalence ratio (PR) adjusted for age, sex, and smoking status by respiratory condition and race/ethnicity, U.S. residents age 18 and over, 1997-1999

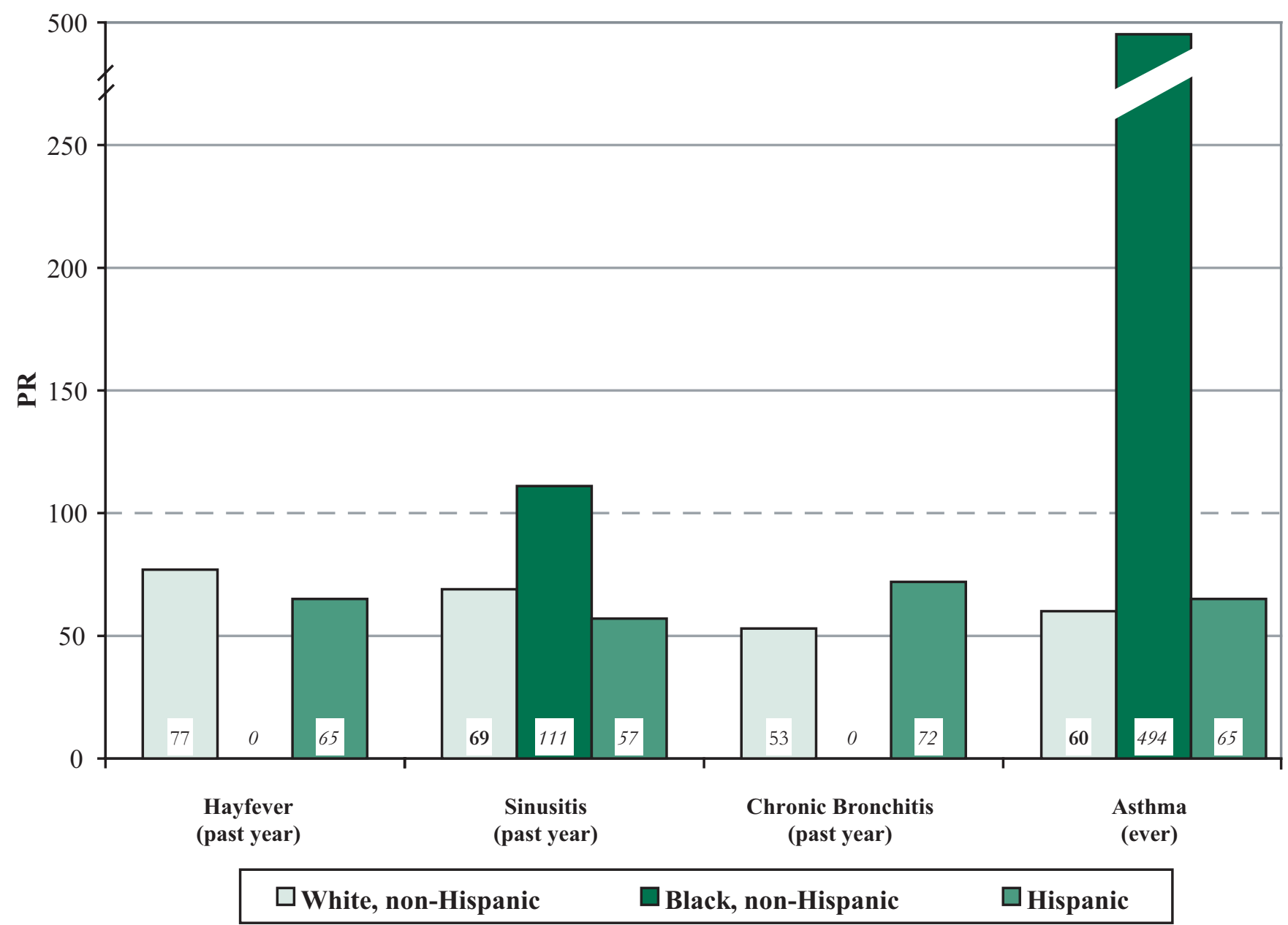

NOTE: Based on responses to the following questions:

"During the past 12 months, have you been told by a doctor or other health professional that you had hayfever?"

"During the past 12 months, have you been told by a doctor or other health professional that you had sinusitis?"

"During the past 12 months, have you been told by a doctor or other health professional that you had chronic bronchitis?"

"Have you EVER been told by a doctor or other health professional that you had asthma?"

PRs in bold are significantly different from $100(\mathrm{p}<0.05)$. PRs in italics are based on fewer than five observed cases. See appendices for source description and methods.

SOURCE: National Center for Health Statistics, National Health Interview Survey 
Figure 3-7. Forestry/fishery workers: Prevalence ratio (PR) adjusted for age, sex, and smoking status by respiratory condition and race/ethnicity, U.S. residents age 18 and over, 1997-1999

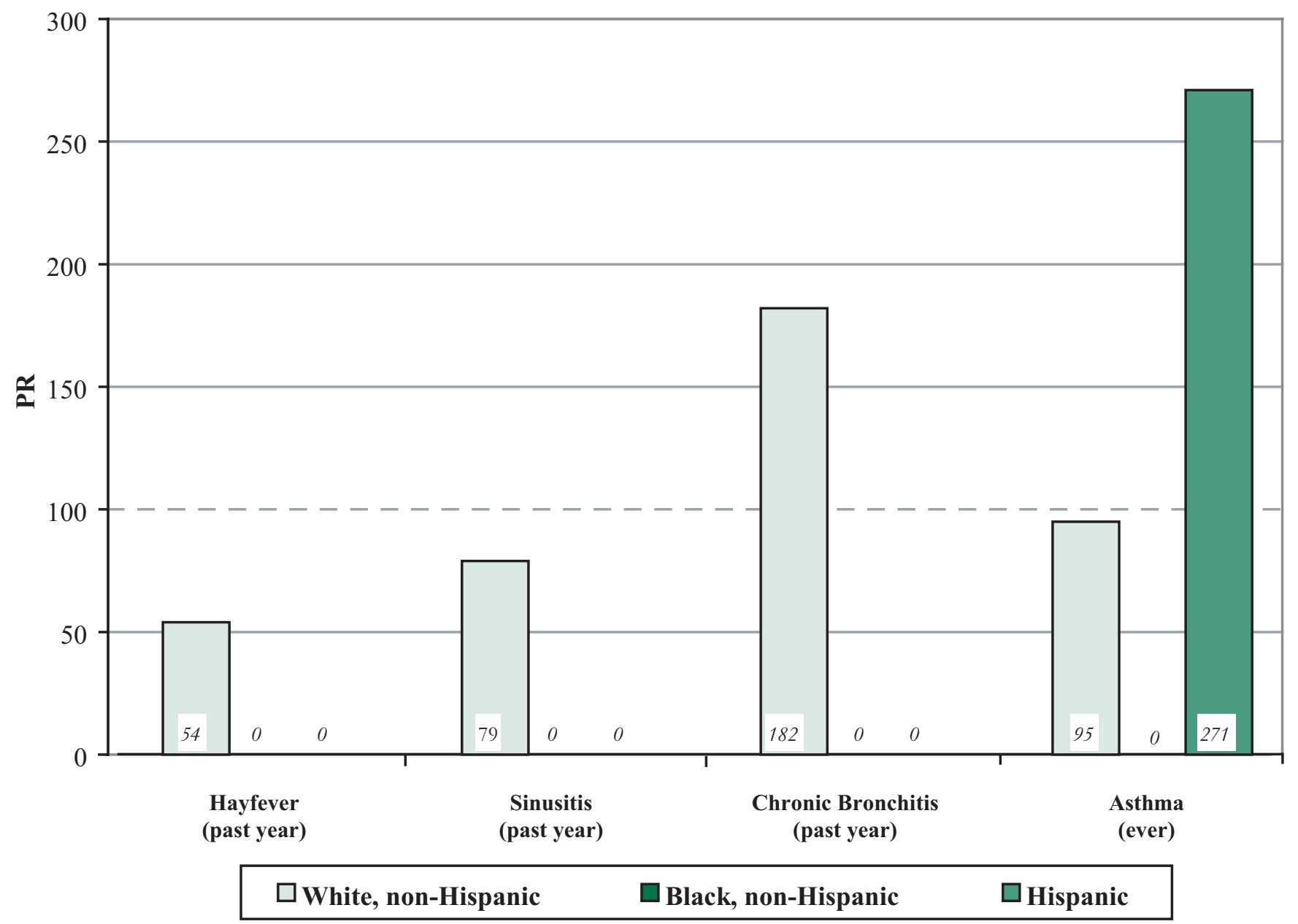

NOTE: Based on responses to the following questions:

"During the past 12 months, have you been told by a doctor or other health professional that you had hayfever?"

"During the past 12 months, have you been told by a doctor or other health professional that you had sinusitis?"

"During the past 12 months, have you been told by a doctor or other health professional that you had chronic bronchitis?"

"Have you EVER been told by a doctor or other health professional that you had asthma?"

PRs in bold are significantly different from $100(\mathrm{p}<0.05)$. PRs in italics are based on fewer than five observed cases. See appendices for source description and methods.

SOURCE: National Center for Health Statistics, National Health Interview Survey 
Figure 3-8. Farm workers: Prevalence ratio (PR) adjusted for age, sex, and race/ ethnicity by respiratory condition and smoking status, U.S. residents age 18 and over, 1997-1999

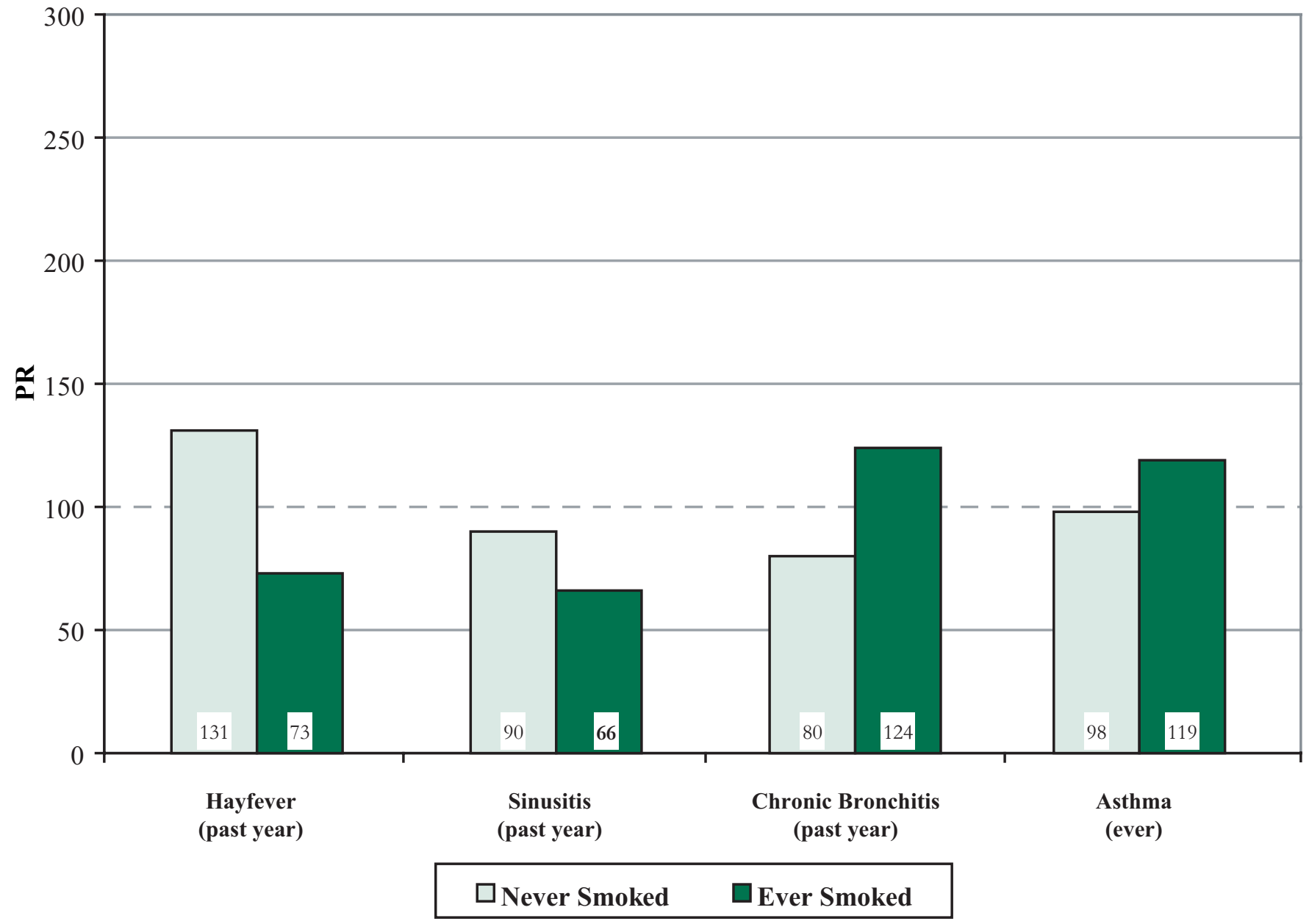

NOTE: Based on responses to the following questions:

"During the past 12 months, have you been told by a doctor or other health professional that you had hayfever?"

"During the past 12 months, have you been told by a doctor or other health professional that you had sinusitis?"

"During the past 12 months, have you been told by a doctor or other health professional that you had chronic bronchitis?"

"Have you EVER been told by a doctor or other health professional that you had asthma?"

PRs in bold are significantly different from $100(\mathrm{p}<0.05)$. PRs in italics are based on fewer than five observed cases. See appendices for source description and methods.

SOURCE: National Center for Health Statistics, National Health Interview Survey 
Figure 3-9. Farm managers: Prevalence ratio (PR) adjusted for age, sex, and race/ ethnicity by respiratory condition and smoking status, U.S. residents age 18 and over, 1997-1999

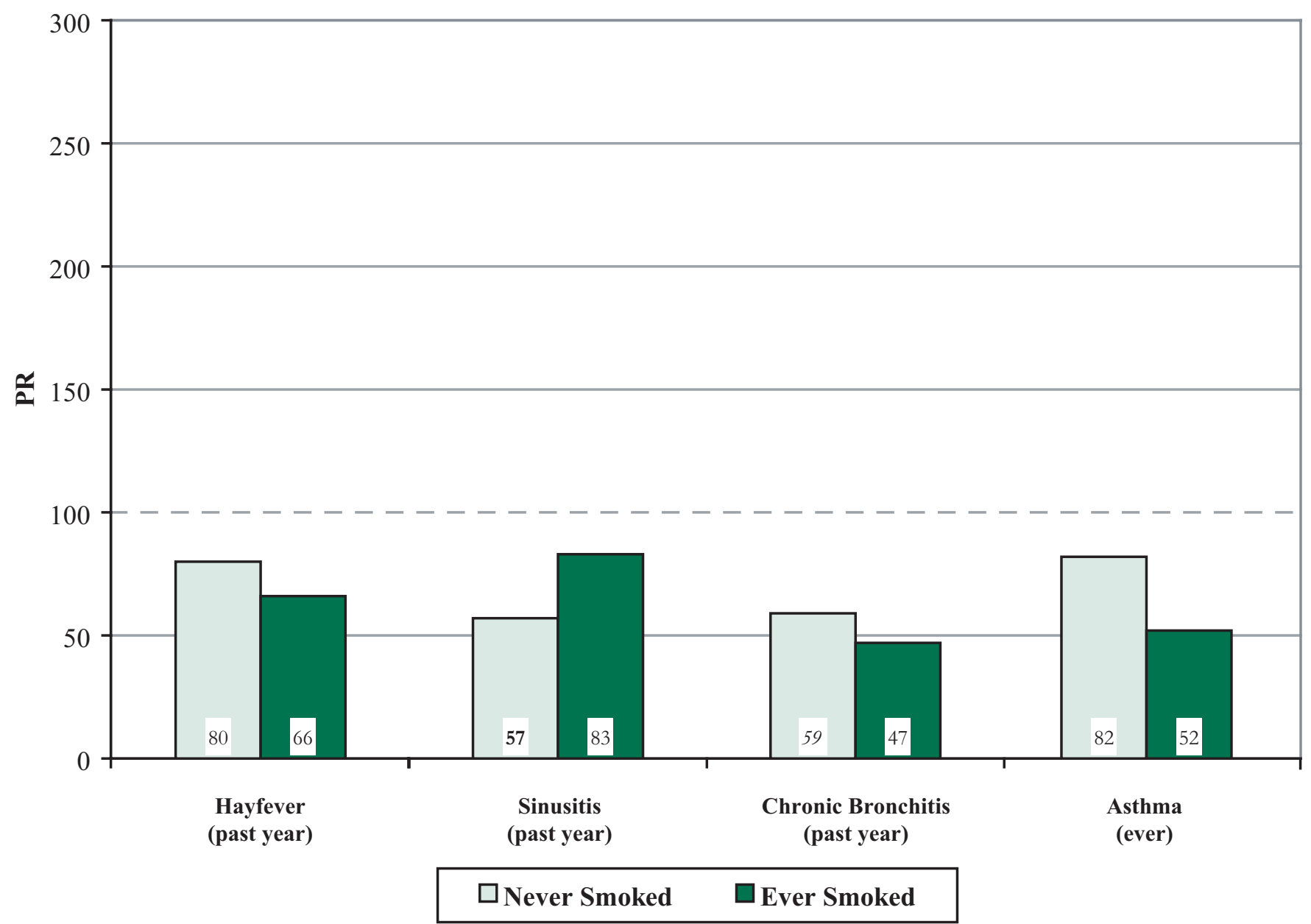

NOTE: Based on responses to the following questions:

"During the past 12 months, have you been told by a doctor or other health professional that you had hayfever?"

"During the past 12 months, have you been told by a doctor or other health professional that you had sinusitis?"

"During the past 12 months, have you been told by a doctor or other health professional that you had chronic bronchitis?"

"Have you EVER been told by a doctor or other health professional that you had asthma?"

PRs in bold are significantly different from $100(\mathrm{p}<0.05)$. PRs in italics are based on fewer than five observed cases. See appendices for source description and methods.

SOURCE: National Center for Health Statistics, National Health Interview Survey 
Figure 3-10. Forestry/fishery workers: Prevalence ratio (PR) adjusted for age, sex, and race/ethnicity by respiratory condition and smoking status, U.S. residents age 18 and over, 1997-1999

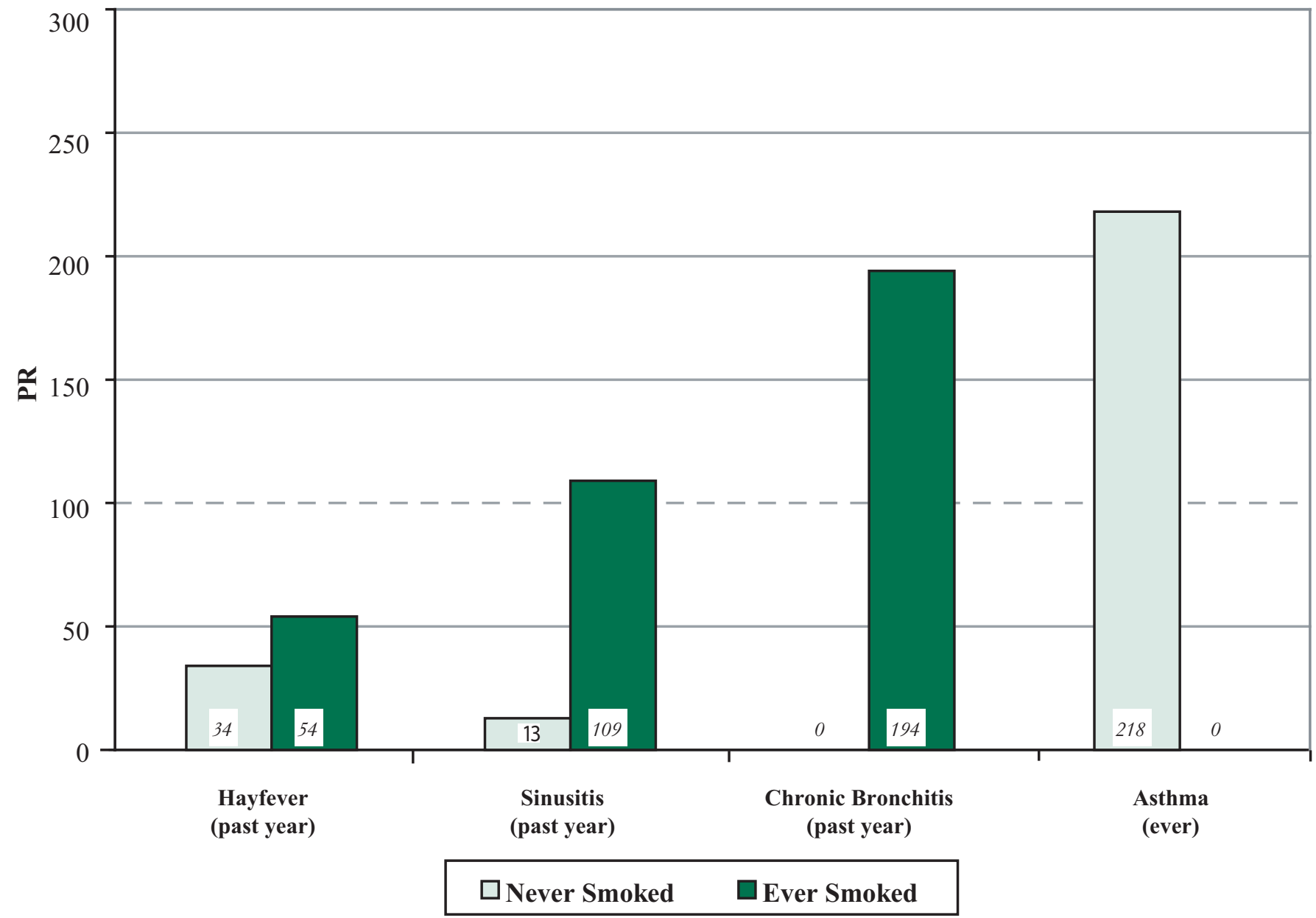

NOTE: Based on responses to the following questions:

"During the past 12 months, have you been told by a doctor or other health professional that you had hayfever?"

"During the past 12 months, have you been told by a doctor or other health professional that you had sinusitis?"

"During the past 12 months, have you been told by a doctor or other health professional that you had chronic bronchitis?"

"Have you EVER been told by a doctor or other health professional that you had asthma?"

PRs in bold are significantly different from $100(\mathrm{p}<0.05)$. PRs in italics are based on fewer than five observed cases. See appendices for source description and methods.

SOURCE: National Center for Health Statistics, National Health Interview Survey 
Table 3-7. Wheezing, apart from a cold (current): Estimated prevalence and prevalence ratio (PR) adjusted for age, sex, race/ethnicity, and smoking status by agricultural group, U.S. residents age 17 and over, 1988-1994

\begin{tabular}{|c|c|c|c|c|c|c|}
\hline \multirow[b]{2}{*}{ Worker Group } & \multirow{2}{*}{$\begin{array}{l}\text { Number } \\
\text { Observed }\end{array}$} & \multicolumn{2}{|c|}{$\begin{array}{l}\text { Estimated Prevalence } \\
\text { of Condition in U.S. }\end{array}$} & \multirow[b]{2}{*}{ PR } & \multicolumn{2}{|c|}{ 95\% Confidence Interval } \\
\hline & & $\mathbf{n}$ & $(\%)$ & & $\mathrm{LCL}$ & $\overline{\mathbf{U C L}}$ \\
\hline Farm Workers & 95 & 436,107 & 12.0 & 111 & 90 & 136 \\
\hline Farm Managers & 53 & 370,558 & 14.1 & 115 & 88 & 151 \\
\hline Other Agricultural Workers & 19 & 138,713 & 10.7 & 100 & 60 & 156 \\
\hline All Non-agricultural Workers & 1,905 & $21,380,987$ & 12.0 & 100 & & \\
\hline
\end{tabular}

n - estimated number

LCL - lower confidence limit

UCL - upper confidence limit

NOTE: Based on responses to the question "Apart from when you have a cold, does your chest ever sound wheezy or whistling?" Estimated number in U.S., estimated percent with condition, and PR are based on weighted sample results. PRs in bold are significantly different from $100(\mathrm{p}<0.05)$. PRs in italics are based on fewer than five observed cases. See appendices for source description and methods.

SOURCE: National Center for Health Statistics, Third National Health and Nutrition Examination Survey (NHANES III) 
Table 3-8. Cough (current): Estimated prevalence and prevalence ratio (PR) adjusted for age, sex, race/ ethnicity, and smoking status by agricultural group, U.S. residents age 17 and over, 1988-1994

\begin{tabular}{|c|c|c|c|c|c|c|}
\hline \multirow[b]{2}{*}{ Worker Group } & \multirow{2}{*}{$\begin{array}{l}\text { Number } \\
\text { Observed }\end{array}$} & \multicolumn{2}{|c|}{$\begin{array}{l}\text { Estimated Prevalence } \\
\text { of Condition in U.S. }\end{array}$} & \multirow[b]{2}{*}{ PR } & \multicolumn{2}{|c|}{ 95\% Confidence Interval } \\
\hline & & $\mathbf{n}$ & $(\%)$ & & $\mathrm{LCL}$ & UCL \\
\hline Farm Workers & 56 & 330,763 & 9.1 & 108 & 82 & 140 \\
\hline Farm Managers & 48 & 231,149 & 8.8 & 90 & 67 & 119 \\
\hline Other Agricultural Workers & 15 & 84,140 & 6.5 & 74 & 41 & 122 \\
\hline All Non-agricultural Workers & 1,493 & $16,332,052$ & 9.2 & 100 & & \\
\hline
\end{tabular}

$\mathrm{n}$ - estimated number

LCL - lower confidence limit

UCL - upper confidence limit

NOTE: Based on responses to the question "Do you usually cough on most days for 3 consecutive months or more during the year?" Estimated number in U.S., estimated percent with condition, and PR are based on weighted sample results. PRs in bold are significantly different from $100(\mathrm{p}<0.05)$. PRs in italics are based on fewer than five observed cases. See appendices for source

description and methods.

SOURCE: National Center for Health Statistics, Third National Health and Nutrition Examination Survey (NHANES III) 
Table 3-9. Phlegm (current): Estimated prevalence and prevalence ratio (PR) adjusted for age, sex, race/ ethnicity, and smoking status by agricultural group, U.S. residents age 17 and over, 1988-1994

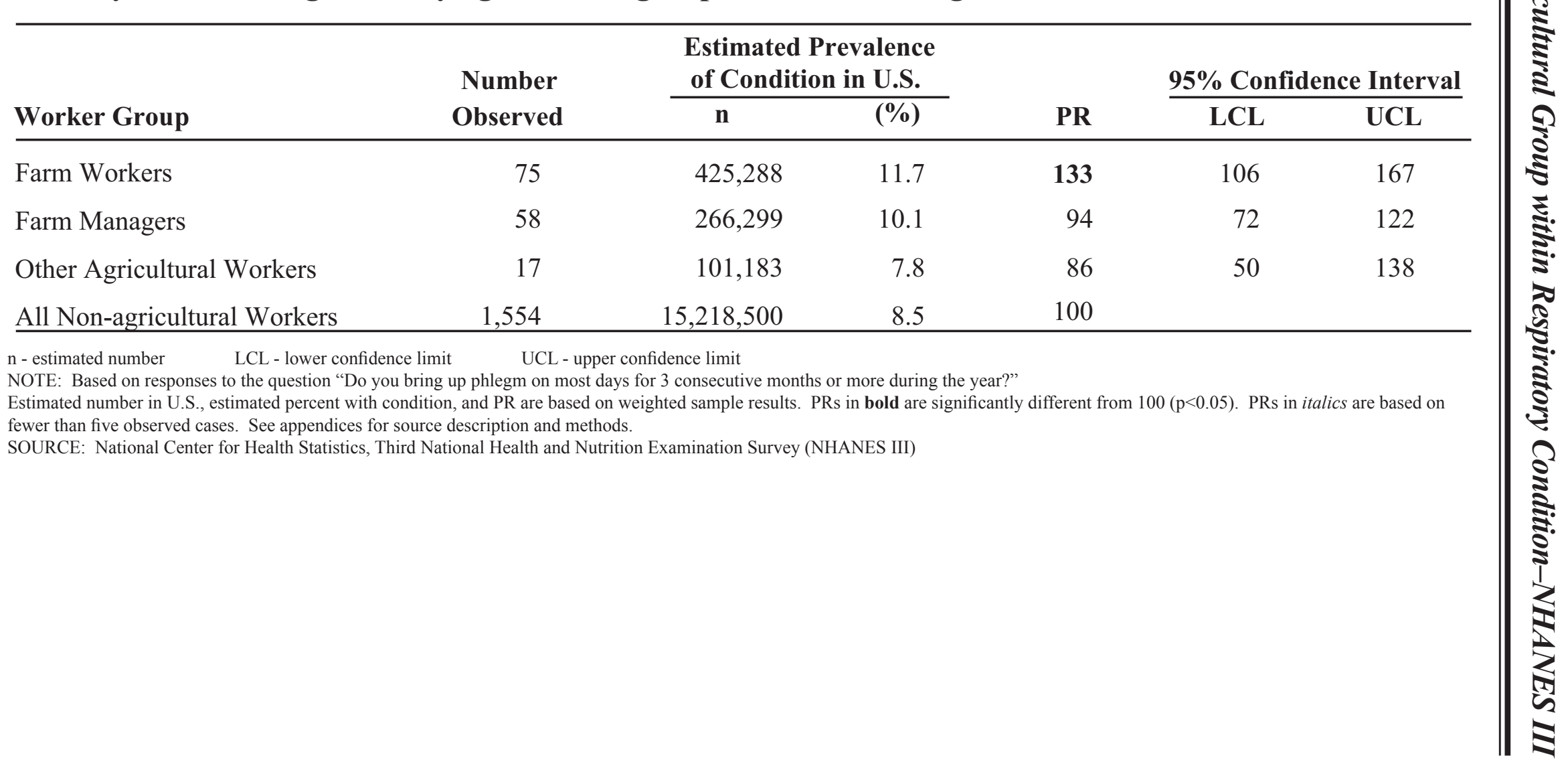


Table 3-10. Shortness of breath (current): Estimated prevalence and prevalence ratio (PR) adjusted for age, sex, race/ethnicity, and smoking status by agricultural group, U.S. residents age 17 and over, 19881994

\begin{tabular}{|c|c|c|c|c|c|c|}
\hline \multirow[b]{2}{*}{ Worker Group } & \multirow{2}{*}{$\begin{array}{l}\text { Number } \\
\text { Observed }\end{array}$} & \multicolumn{2}{|c|}{$\begin{array}{l}\text { Estimated Prevalence } \\
\text { of Condition in U.S. }\end{array}$} & \multirow[b]{2}{*}{ PR } & \multicolumn{2}{|c|}{ 95\% Confidence Interval } \\
\hline & & $\mathbf{n}$ & $(\%)$ & & LCL & UCL \\
\hline Farm Workers & 218 & 915,335 & 25.2 & 110 & 97 & 126 \\
\hline Farm Managers & 125 & 615,034 & 23.5 & 94 & 79 & 112 \\
\hline Other Agricultural Workers & 41 & 249,033 & 19.2 & 101 & 74 & 137 \\
\hline All Non-agricultural Workers & 4,642 & $41,389,383$ & 23.3 & 100 & & \\
\hline
\end{tabular}

$\mathrm{n}$ - estimated number

LCL - lower confidence limit

UCL - upper confidence limit

NOTE: Based on responses to the question "Are you troubled by shortness of breath when hurrying on level ground or walking up a slight hill?"

Estimated number in U.S., estimated percent with condition, and PR are based on weighted sample results. PRs in bold are significantly different from 100 (p<0.05). PRs in italics are based on fewer than five observed cases. See appendices for source description and methods.

SOURCE: National Center for Health Statistics, Third National Health and Nutrition Examination Survey (NHANES III) 
Table 3-11. Stuffy, itchy, runny nose (past year): Estimated prevalence and prevalence ratio (PR) adjusted for age, sex, race/ethnicity, and smoking status by agricultural group, U.S. residents age 17 and over, 1988-1994

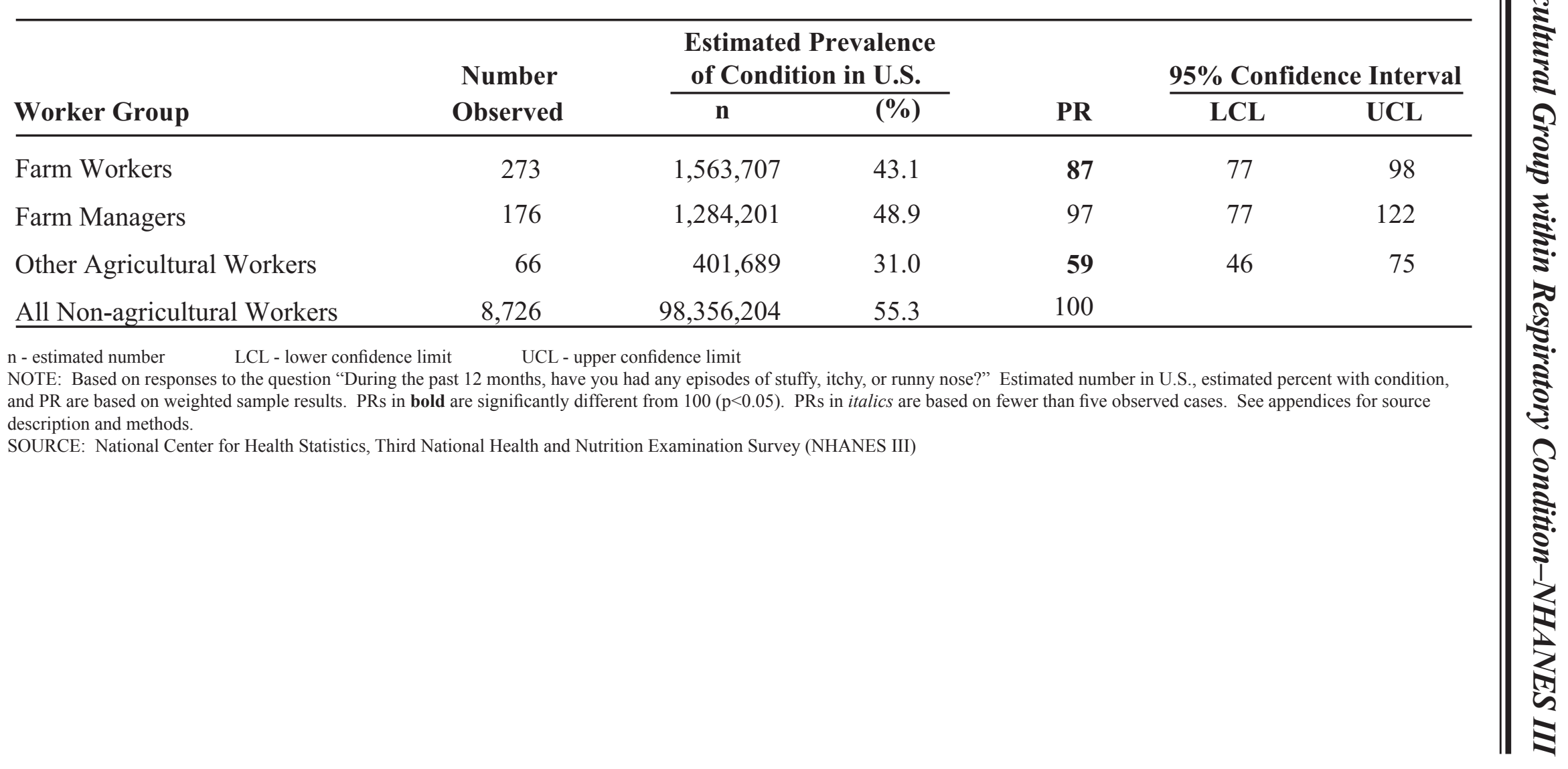


Table 3-12. Cold or flu (past year): Estimated prevalence and prevalence ratio (PR) adjusted for age, sex, race/ethnicity, and smoking status by agricultural group, U.S. residents age 17 and over, 1988-1994

\begin{tabular}{|c|c|c|c|c|c|c|}
\hline \multirow[b]{2}{*}{ Worker Group } & \multirow{2}{*}{$\begin{array}{c}\text { Number } \\
\text { Observed }\end{array}$} & \multicolumn{2}{|c|}{$\begin{array}{l}\text { Estimated Prevalence } \\
\text { of Condition in U.S. }\end{array}$} & \multirow[b]{2}{*}{ PR } & \multicolumn{2}{|c|}{ 95\% Confidence Interval } \\
\hline & & $\mathbf{n}$ & $(\%)$ & & $\mathrm{LCL}$ & UCL \\
\hline Farm Workers & 502 & $2,373,949$ & 65.4 & 96 & 88 & 105 \\
\hline Farm Managers & 203 & $1,767,314$ & 67.2 & 108 & 95 & 124 \\
\hline Other Agricultural Workers & 117 & 770,838 & 59.4 & 83 & 69 & 100 \\
\hline All Non-agricultural Workers & 12,029 & $123,171,528$ & 69.2 & 100 & & \\
\hline
\end{tabular}

$\mathrm{n}$ - estimated number

LCL - lower confidence limit

UCL - upper confidence limit

NOTE: Based on responses to the question "During the past 12 months, have you had a cold or the flu?" Estimated number in U.S., estimated percent with condition, and PR are based on weighted sample results. PRs in bold are significantly different from $100(\mathrm{p}<0.05)$. PRs in italics are based on fewer than five observed cases. See appendices for source description and methods. SOURCE: National Center for Health Statistics, Third National Health and Nutrition Examination Survey (NHANES III) 
Table 3-13. Sinusitis (past year): Estimated prevalence and prevalence ratio (PR) adjusted for age, sex, race/ethnicity, and smoking status by agricultural group, U.S. residents age 17 and over, 1988-1994

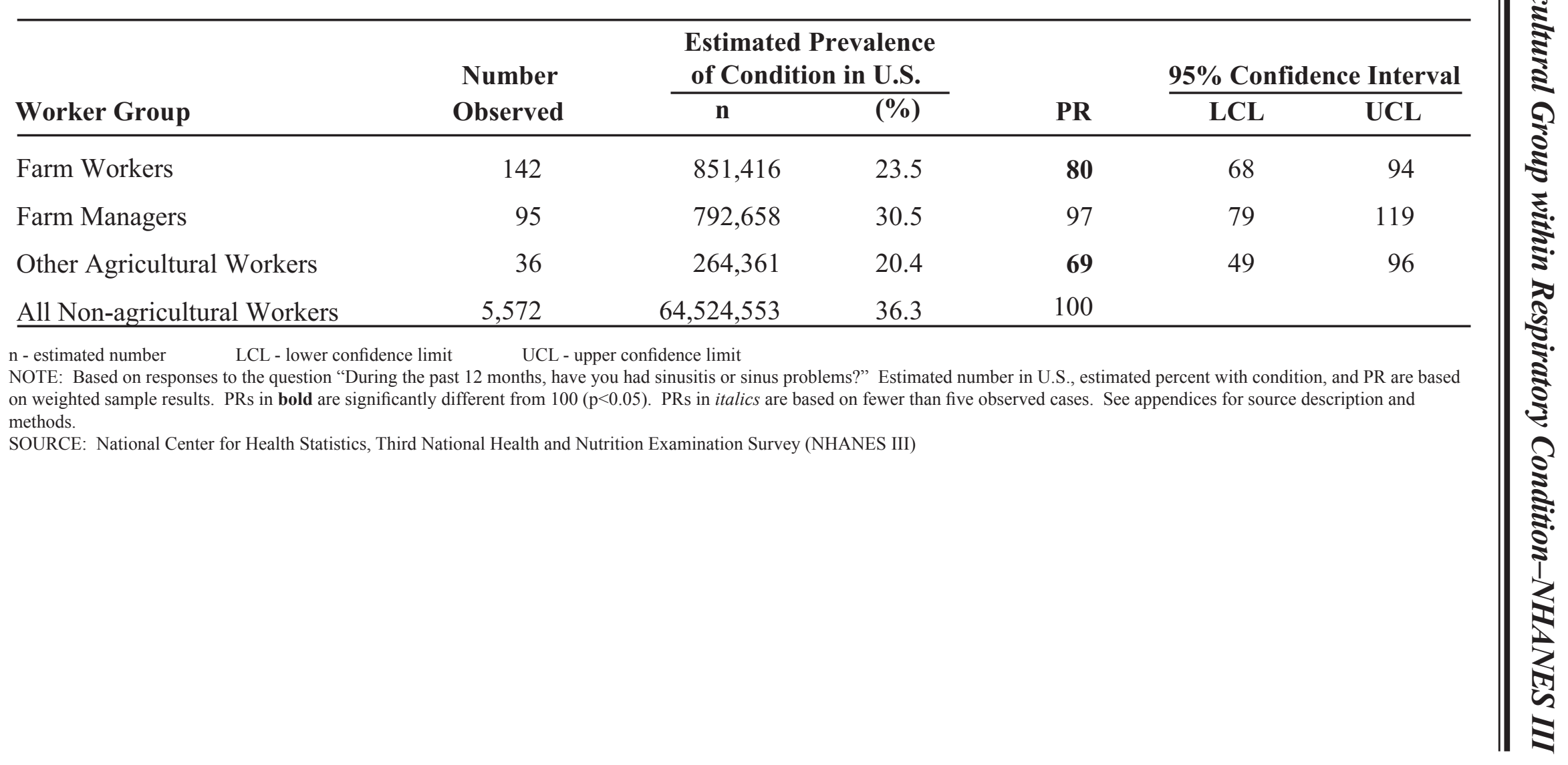


Table 3-14. Pneumonia (past year): Estimated prevalence and prevalence ratio (PR) adjusted for age, sex, race/ethnicity, and smoking status by agricultural group, U.S. residents age 17 and over, 1988-1994

\begin{tabular}{|c|c|c|c|c|c|c|}
\hline \multirow[b]{2}{*}{ Worker Group } & \multirow{2}{*}{$\begin{array}{l}\text { Number } \\
\text { Observed }\end{array}$} & \multicolumn{2}{|c|}{$\begin{array}{l}\text { Estimated Prevalence } \\
\text { of Condition in U.S. }\end{array}$} & \multirow[b]{2}{*}{ PR } & \multicolumn{2}{|c|}{ 95\% Confidence Interval } \\
\hline & & n & $(\%)$ & & LCL & UCL \\
\hline Farm Workers & 17 & 52,159 & 1.4 & 78 & 45 & 125 \\
\hline Farm Managers & 12 & 68,095 & 2.6 & 136 & 70 & 237 \\
\hline Other Agricultural Workers & 6 & 14,449 & 1.1 & 78 & 29 & 170 \\
\hline All Non-agricultural Workers & 374 & $3,851,288$ & 2.2 & 100 & & \\
\hline
\end{tabular}

$n$ - estimated number

LCL - lower confidence limit

UCL - upper confidence limit

NOTE: Based on responses to the question “During the past 12 months, have you had pneumonia?" Estimated number in U.S., estimated percent with condition, and PR are based on weighted sample results. PRs in bold are significantly different from $100(\mathrm{p}<0.05)$. PRs in italics are based on fewer than five observed cases. See appendices for source description and methods. SOURCE: National Center for Health Statistics, Third National Health and Nutrition Examination Survey (NHANES III) 
Table 3-15. Wheezing (past year): Estimated prevalence and prevalence ratio (PR) adjusted for age, sex, race/ethnicity, and smoking status by agricultural group, U.S. residents age 17 and over, 1988-1994

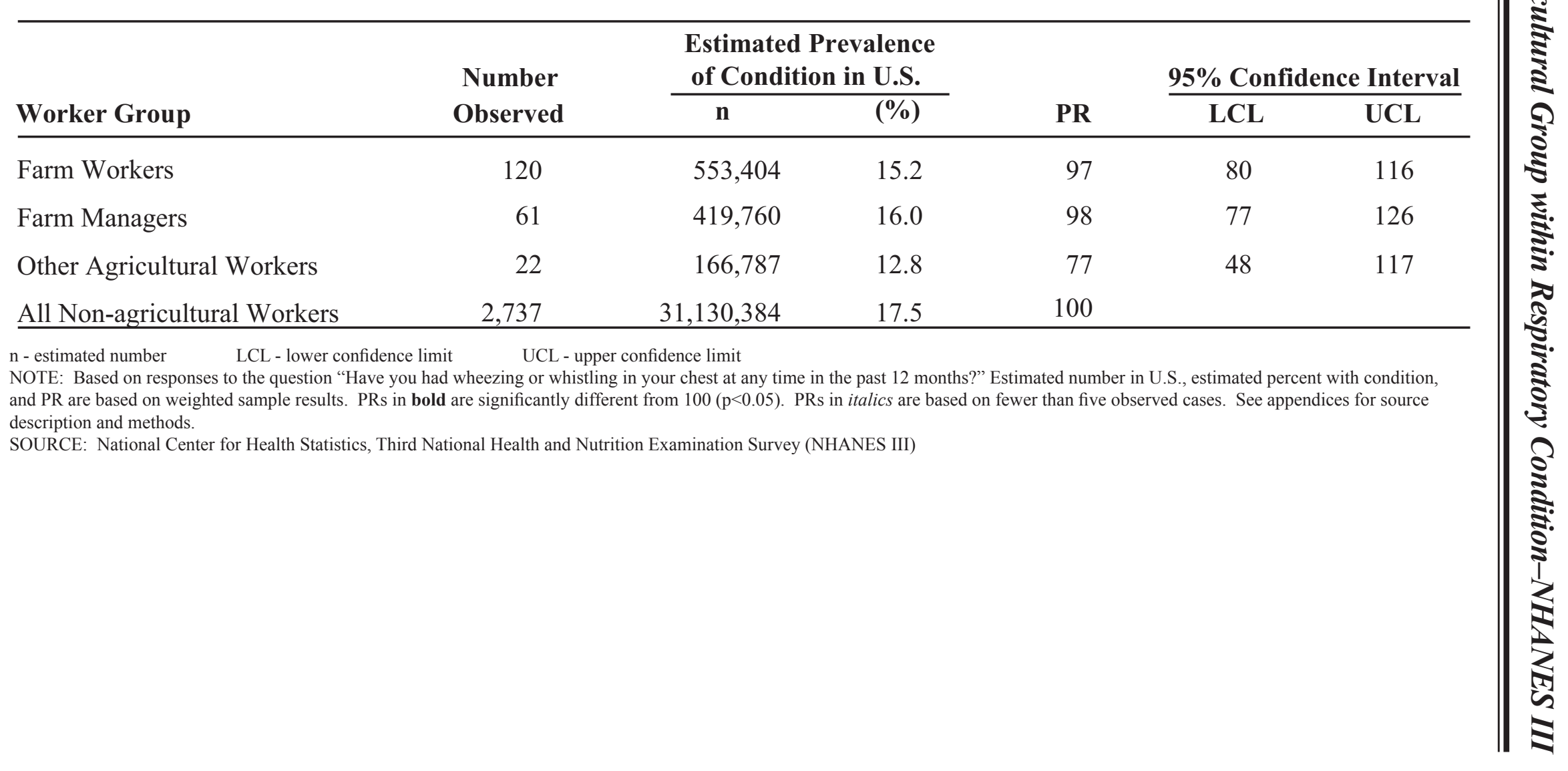


Table 3-16. Asthma (ever): Estimated prevalence and prevalence ratio (PR) adjusted for age, sex, race/ ethnicity, and smoking status by agricultural group, U.S. residents age 17 and over, 1988-1994

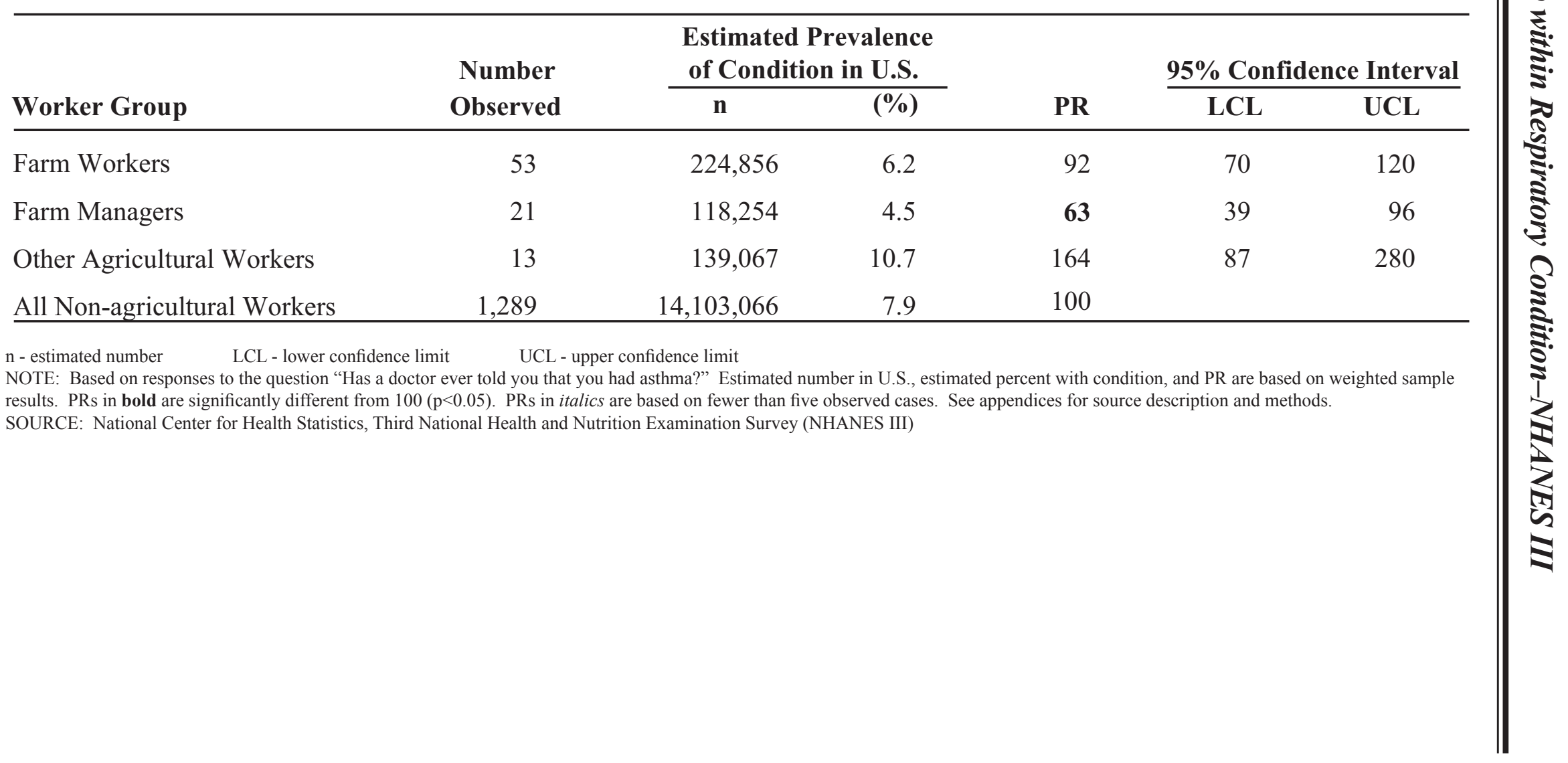


Table 3-17. Chronic bronchitis (ever): Estimated prevalence and prevalence ratio (PR) adjusted for age, sex, race/ethnicity, and smoking status by agricultural group, U.S. residents age 17 and over, 1988-1994

\begin{tabular}{|c|c|c|c|c|c|c|}
\hline \multirow[b]{2}{*}{ Worker Group } & \multirow{2}{*}{$\begin{array}{l}\text { Number } \\
\text { Observed }\end{array}$} & \multicolumn{2}{|c|}{$\begin{array}{l}\text { Estimated Prevalence } \\
\text { of Condition in U.S. }\end{array}$} & \multirow[b]{2}{*}{ PR } & \multicolumn{2}{|c|}{ 95\% Confidence Interval } \\
\hline & & $\mathbf{n}$ & $(\%)$ & & $\mathrm{LCL}$ & UCL \\
\hline Farm Workers & 43 & 170,254 & 4.7 & 97 & 71 & 131 \\
\hline Farm Managers & 20 & 113,407 & 4.3 & 75 & 46 & 116 \\
\hline Other Agricultural Workers & 8 & 67,224 & 5.2 & 120 & 52 & 236 \\
\hline All Non-agricultural Workers & 1,062 & $11,141,371$ & 6.3 & 100 & & \\
\hline
\end{tabular}

n - estimated number

LCL - lower confidence limit

UCL - upper confidence limit

NOTE: Based on responses to the question "Has a doctor ever told you that you had chronic bronchitis?" Estimated number in U.S., estimated percent with condition, and PR are based on weighted sample results. PRs in bold are significantly different from $100(\mathrm{p}<0.05)$. PRs in italics are based on fewer than five observed cases. See appendices for source description and methods. SOURCE: National Center for Health Statistics, Third National Health and Nutrition Examination Survey (NHANES III) 
Table 3-18. Emphysema (ever): Estimated prevalence and prevalence ratio (PR) adjusted for age, sex, race/ethnicity, and smoking status by agricultural group, U.S. residents age 17 and over, 1988-1994

\begin{tabular}{|c|c|c|c|c|c|c|}
\hline \multirow[b]{2}{*}{ Worker Group } & \multirow{2}{*}{$\begin{array}{l}\text { Number } \\
\text { Observed }\end{array}$} & \multicolumn{2}{|c|}{$\begin{array}{l}\text { Estimated Prevalence } \\
\text { of Condition in U.S. }\end{array}$} & \multirow[b]{2}{*}{ PR } & \multicolumn{2}{|c|}{ 95\% Confidence Interval } \\
\hline & & n & $(\%)$ & & LCL & UCL \\
\hline Farm Workers & 20 & 104,616 & 2.9 & 134 & 82 & 207 \\
\hline Farm Managers & 28 & 112,971 & 4.3 & 124 & 83 & 179 \\
\hline Other Agricultural Workers & 5 & 27,826 & 2.1 & 128 & 41 & 299 \\
\hline All Non-agricultural Workers & 375 & $3,401,032$ & 1.9 & 100 & & \\
\hline
\end{tabular}

$\mathrm{n}$ - estimated number

LCL - lower confidence limit

UCL - upper confidence limit

NOTE: Based on responses to the question "Has a doctor ever told you that you had emphysema?" Estimated number in U.S., estimated percent with condition, and PR are based on weighted sample results. PRs in bold are significantly different from $100(\mathrm{p}<0.05)$. PRs in italics are based on fewer than five observed cases. See appendices for source description and methods. SOURCE: National Center for Health Statistics, Third National Health and Nutrition Examination Survey (NHANES III) 
Table 3-19. Hayfever (ever): Estimated prevalence and prevalence ratio (PR) adjusted for age, sex, race/ ethnicity, and smoking status by agricultural group, U.S. residents age 17 and over, 1988-1994

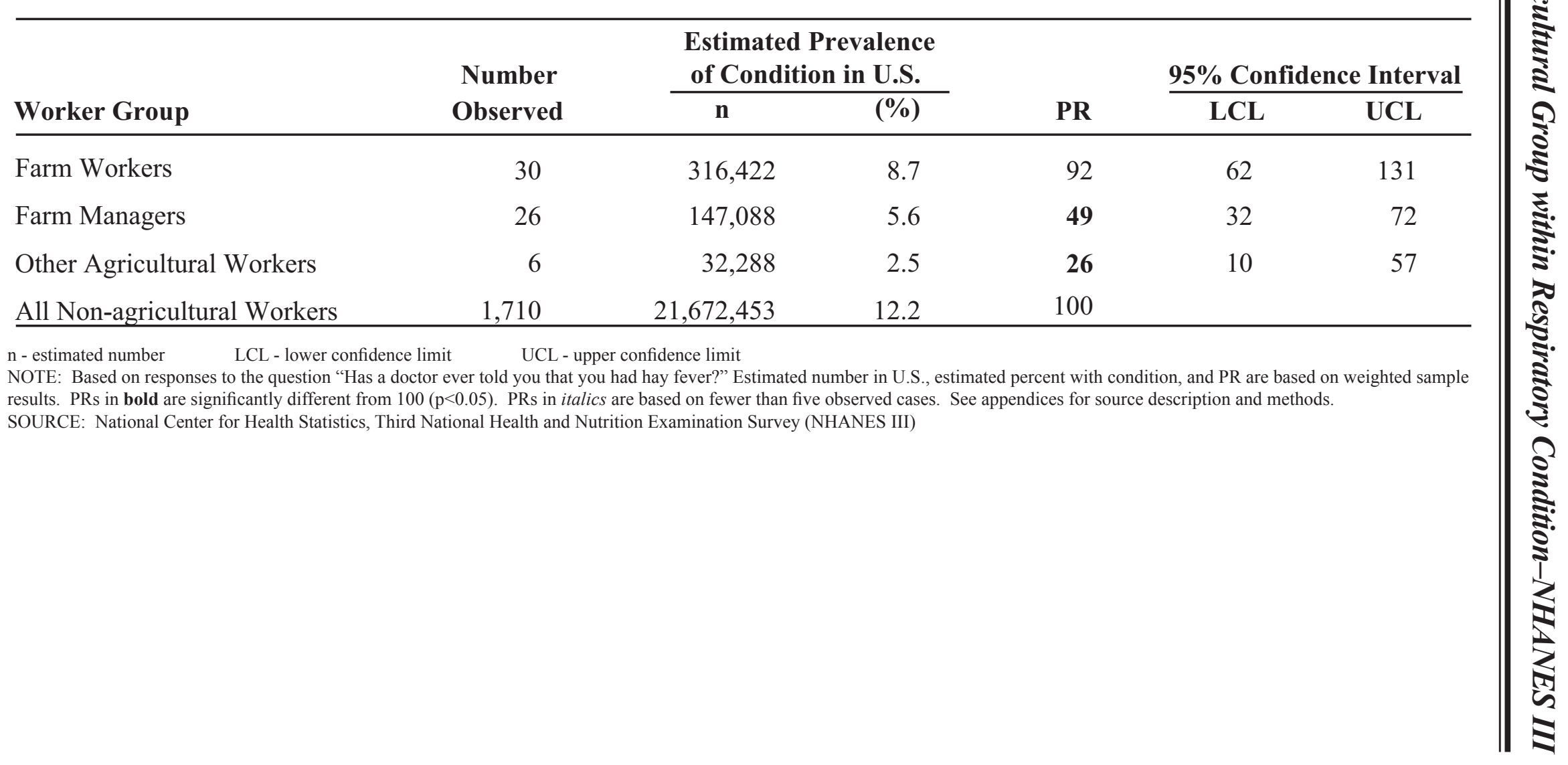


Figure 3-11. Respiratory conditions (current): Prevalence ratio (PR) adjusted for age, sex, race/ethnicity, and smoking status by agricultural group, U.S. residents age 17 and over, 1988-1994

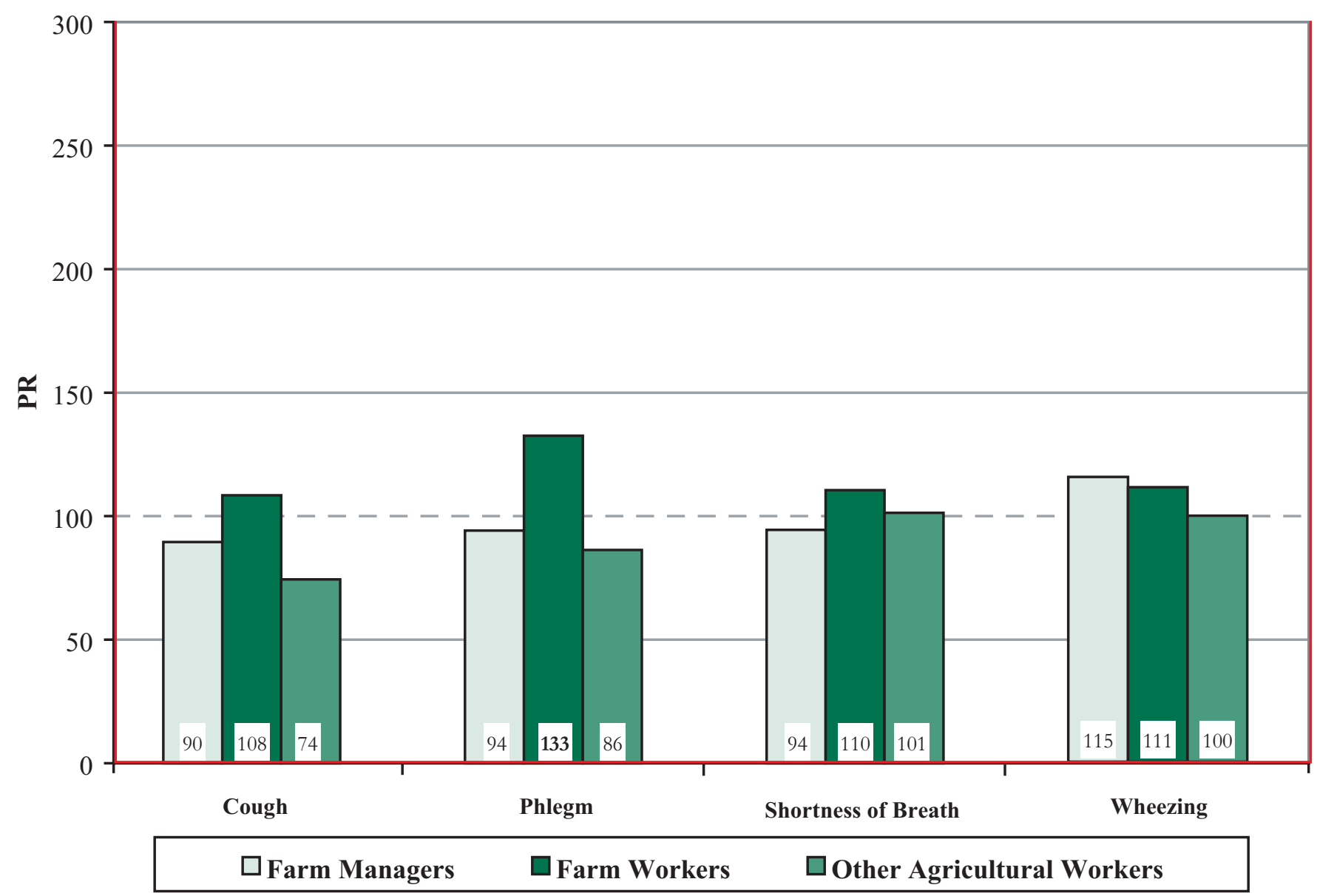

NOTE: Based on responses to the following questions:

"Do you usually cough on most days for 3 consecutive months or more during the year?"

"Do you bring up phlegm on most days for 3 consecutive months or more during the year?"

"Are you troubled by shortness of breath when hurrying on level ground or walking up a slight hill?"

"Apart from when you have a cold, does your chest ever sound wheezy or whistling?"

PRs in bold are significantly different from $100(\mathrm{p}<0.05)$. PRs in italics are based on fewer than five observed cases. See appendices for source description and methods.

SOURCE: National Center for Health Statistics, Third National Health and Nutrition Examination Survey (NHANES III) 
Figure 3-12. Respiratory conditions (past year): Prevalence ratio (PR) adjusted for age, sex, race/ethnicity, and smoking status by agricultural group, U.S. residents age 17 and over, 1988-1994

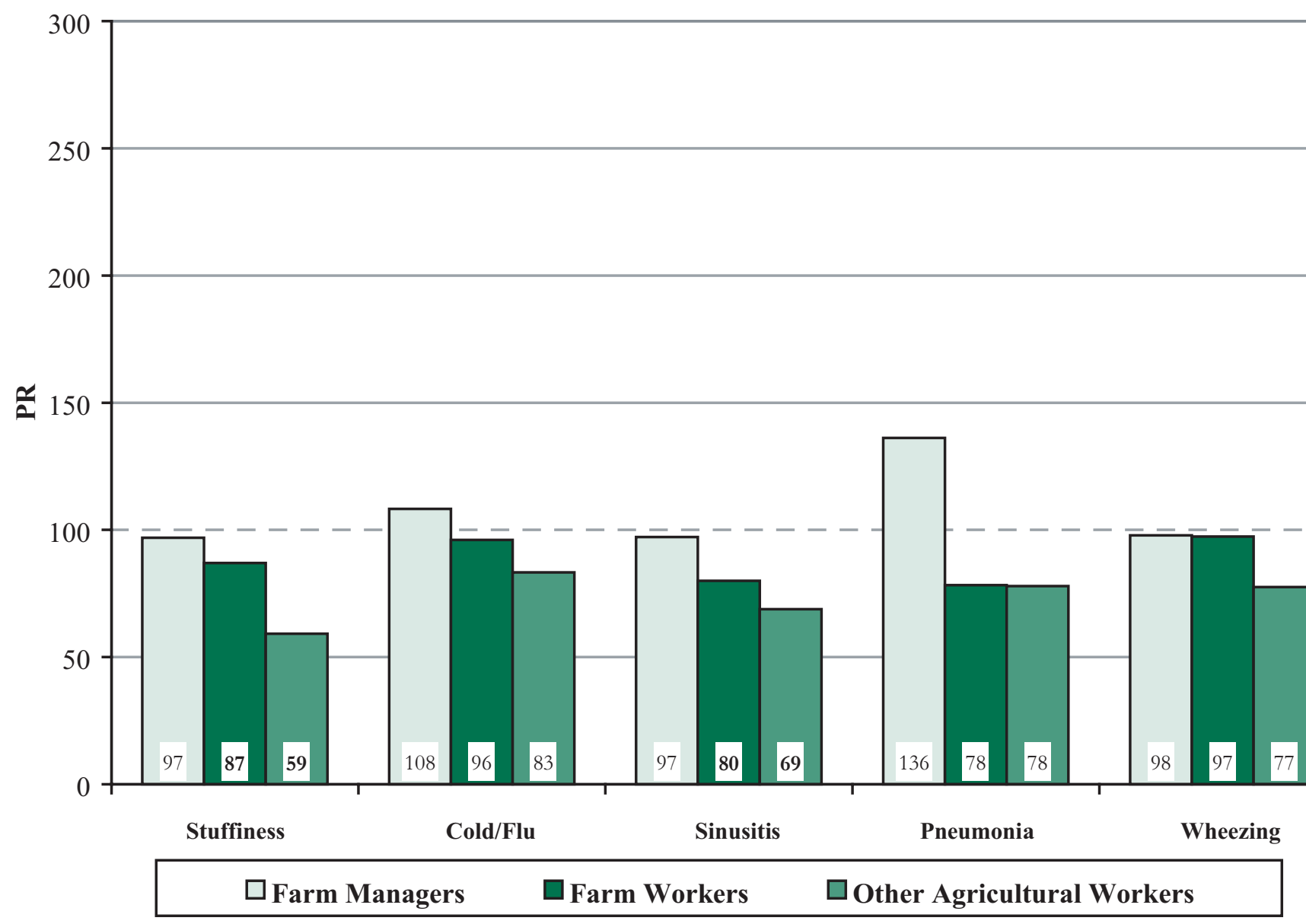

NOTE: Based on responses to the following questions:

"During the past 12 months, have you had any episodes of stuffy, itchy, or runny nose?"

"During the past 12 months, have you had a cold or the flu?"

"During the past 12 months, have you had sinusitis or sinus problems?"

"During the past 12 months, have you had pneumonia?"

"Have you had wheezing or whistling in your chest at any time in the past 12 months?"

PRs in bold are significantly different from $100(\mathrm{p}<0.05)$. PRs in italics are based on fewer than five observed cases. See appendices for source description and methods.

SOURCE: National Center for Health Statistics, Third National Health and Nutrition Examination Survey (NHANES III) 
Figure 3-13. Respiratory conditions (ever): Prevalence ratio (PR) adjusted for age, sex, race/ethnicity, and smoking status by agricultural group, U.S. residents age 17 and over, 1988-1994

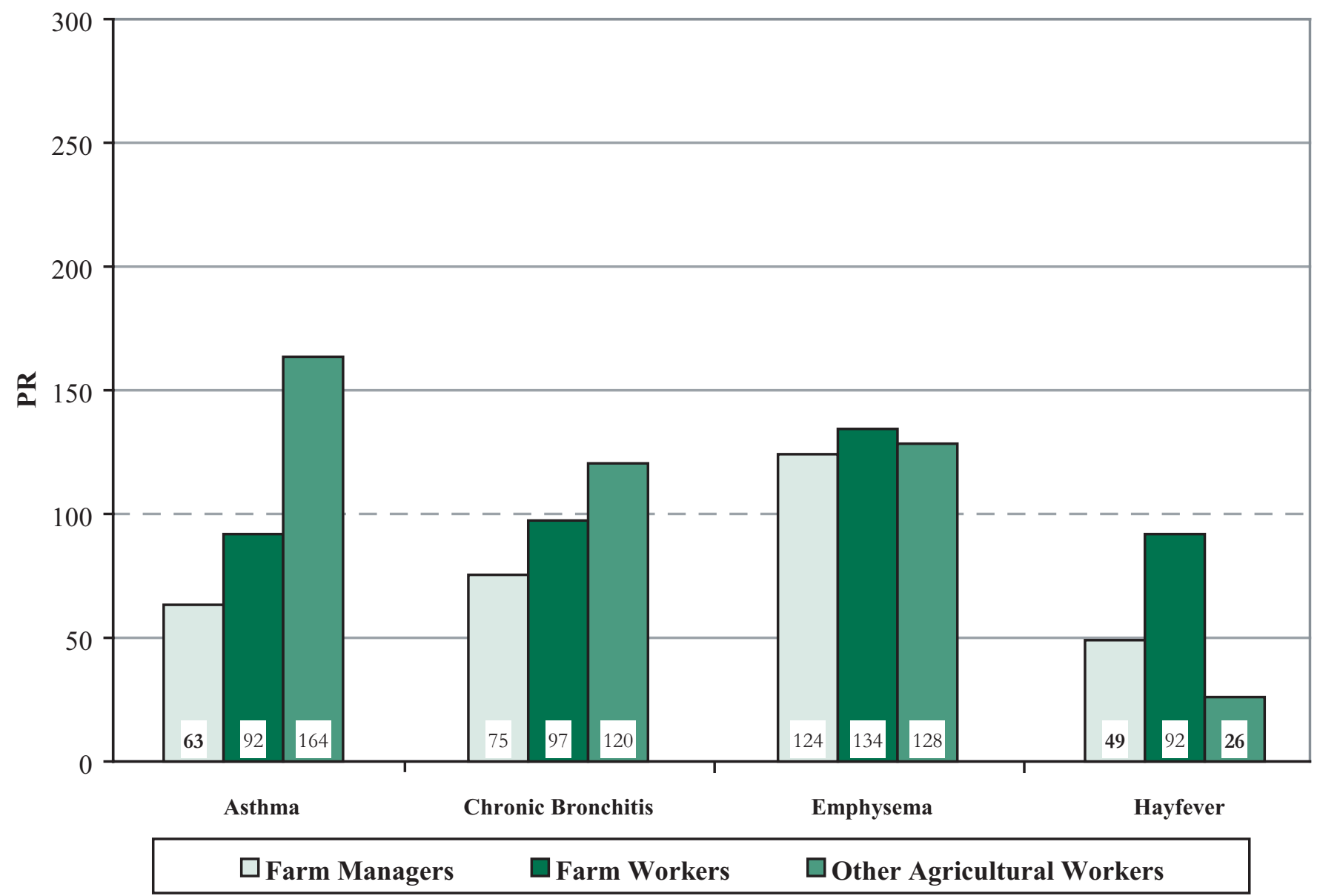

NOTE: Based on responses to the following questions:

"Has a doctor ever told you that you had asthma?"

"Has a doctor ever told you that you had chronic bronchitis?"

"Has a doctor ever told you that you had emphysema?"

"Has a doctor ever told you that you had hay fever?"

PRs in bold are significantly different from $100(\mathrm{p}<0.05)$. PRs in italics are based on fewer than five observed cases. See appendices for source description and methods.

SOURCE: National Center for Health Statistics, Third National Health and Nutrition Examination Survey (NHANES III) 
Figure 3-14. Respiratory conditions (current), farm workers: Prevalence ratio (PR) adjusted for age, race/ethnicity, and smoking status by sex, U.S. residents age 17 and over, 1988-1994

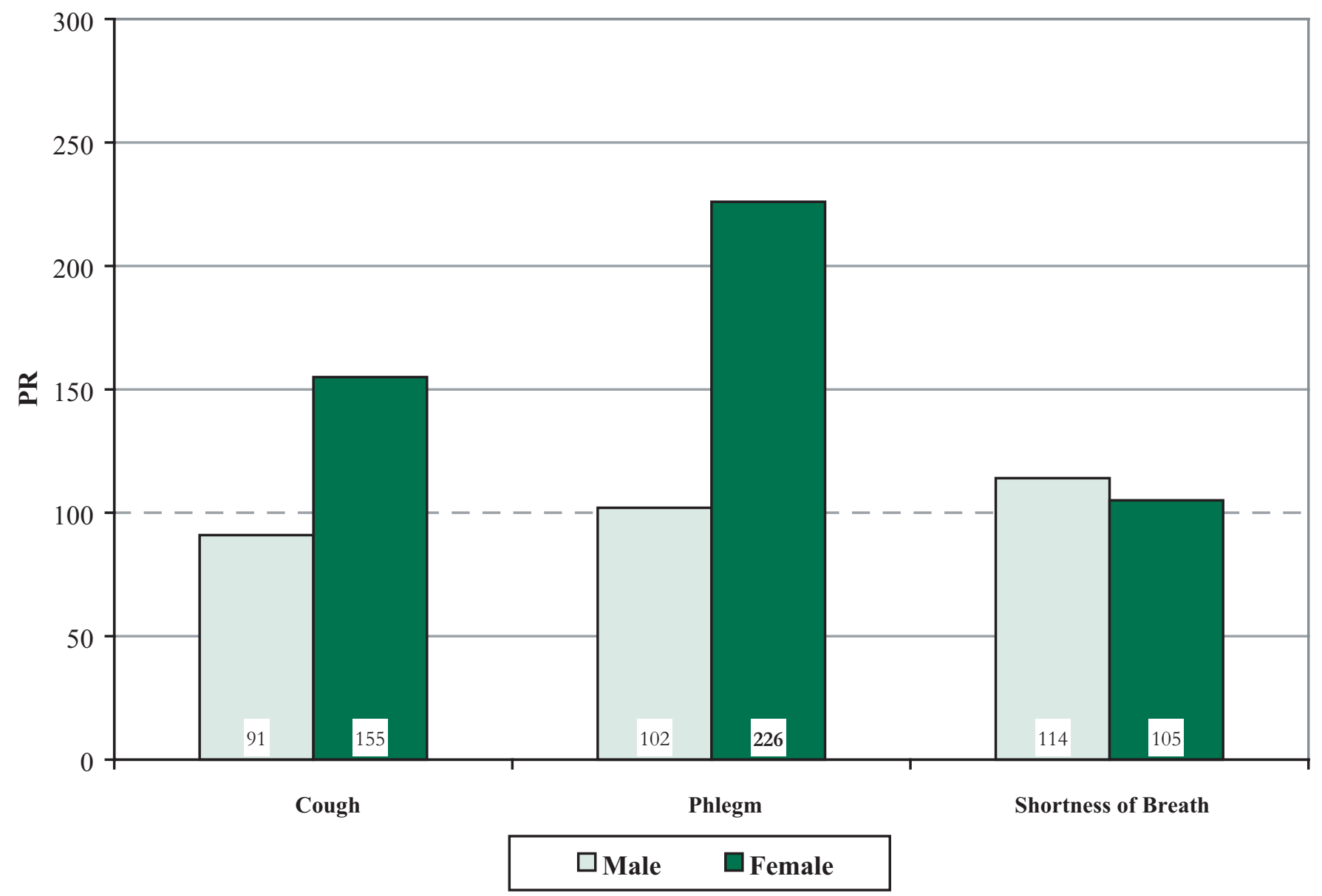

NOTE: Based on responses to the following questions:

"Do you usually cough on most days for 3 consecutive months or more during the year?"

"Do you bring up phlegm on most days for 3 consecutive months or more during the year?"

"Are you troubled by shortness of breath when hurrying on level ground or walking up a slight hill?"

PRs in bold are significantly different from $100(\mathrm{p}<0.05)$. PRs in italics are based on fewer than five observed cases. See appendices for source description and methods.

SOURCE: National Center for Health Statistics, Third National Health and Nutrition Examination Survey (NHANES III) 
Figure 3-15. Respiratory conditions (current), farm managers: Prevalence ratio (PR) adjusted for age, race/ethnicity, and smoking status by sex, U.S. residents age 17 and over, 1988-1994

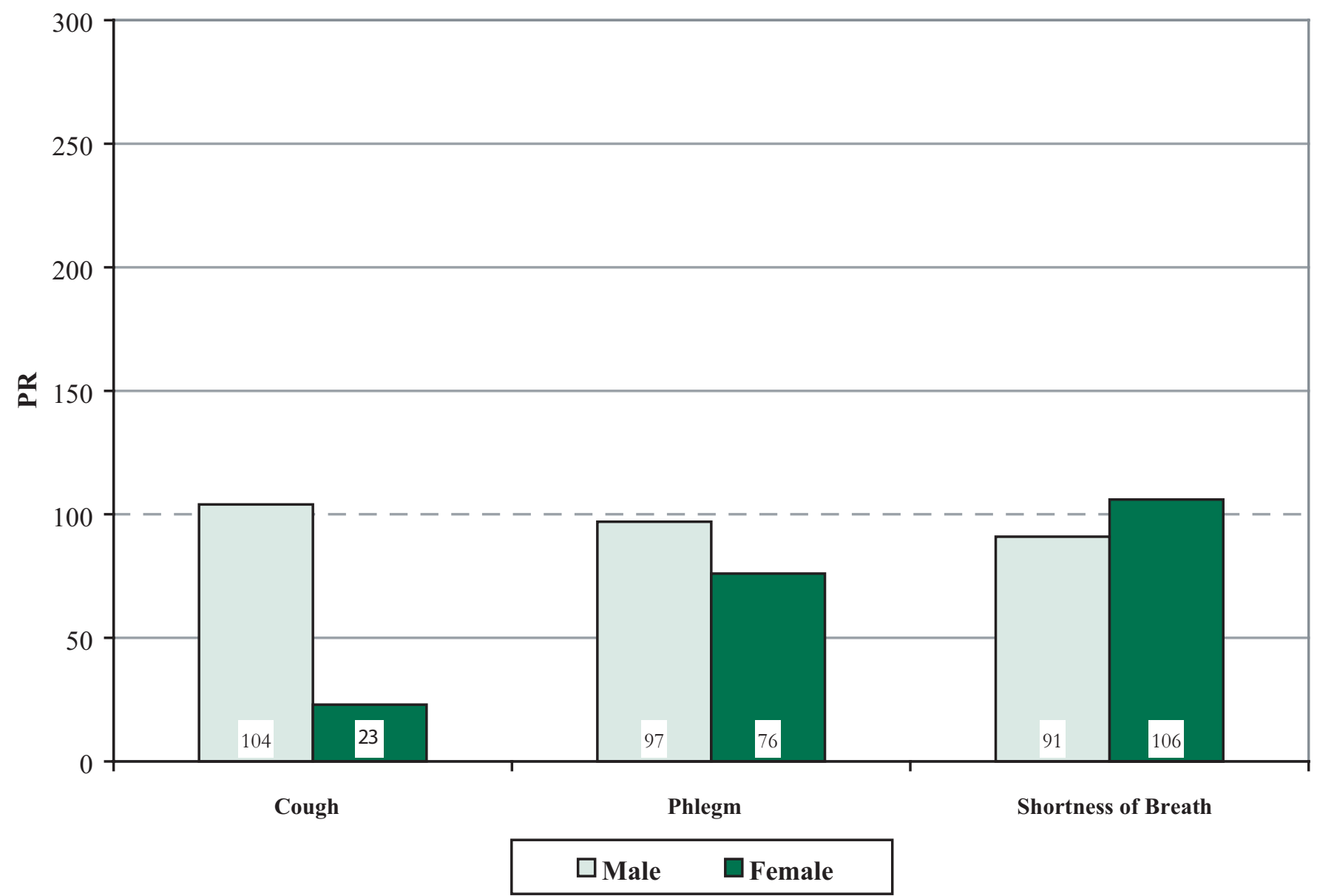

NOTE: Based on responses to the following questions:

"Do you usually cough on most days for 3 consecutive months or more during the year?"

"Do you bring up phlegm on most days for 3 consecutive months or more during the year?"

"Are you troubled by shortness of breath when hurrying on level ground or walking up a slight hill?"

PRs in bold are significantly different from $100(\mathrm{p}<0.05)$. PRs in italics are based on fewer than five observed cases. See appendices for source description and methods.

SOURCE: National Center for Health Statistics, Third National Health and Nutrition Examination Survey (NHANES III) 
Figure 3-16. Respiratory conditions (current), other agricultural workers: Prevalence ratio (PR) adjusted for age, race/ethnicity, and smoking status by sex, U.S. residents age 17 and over, 1988-1994

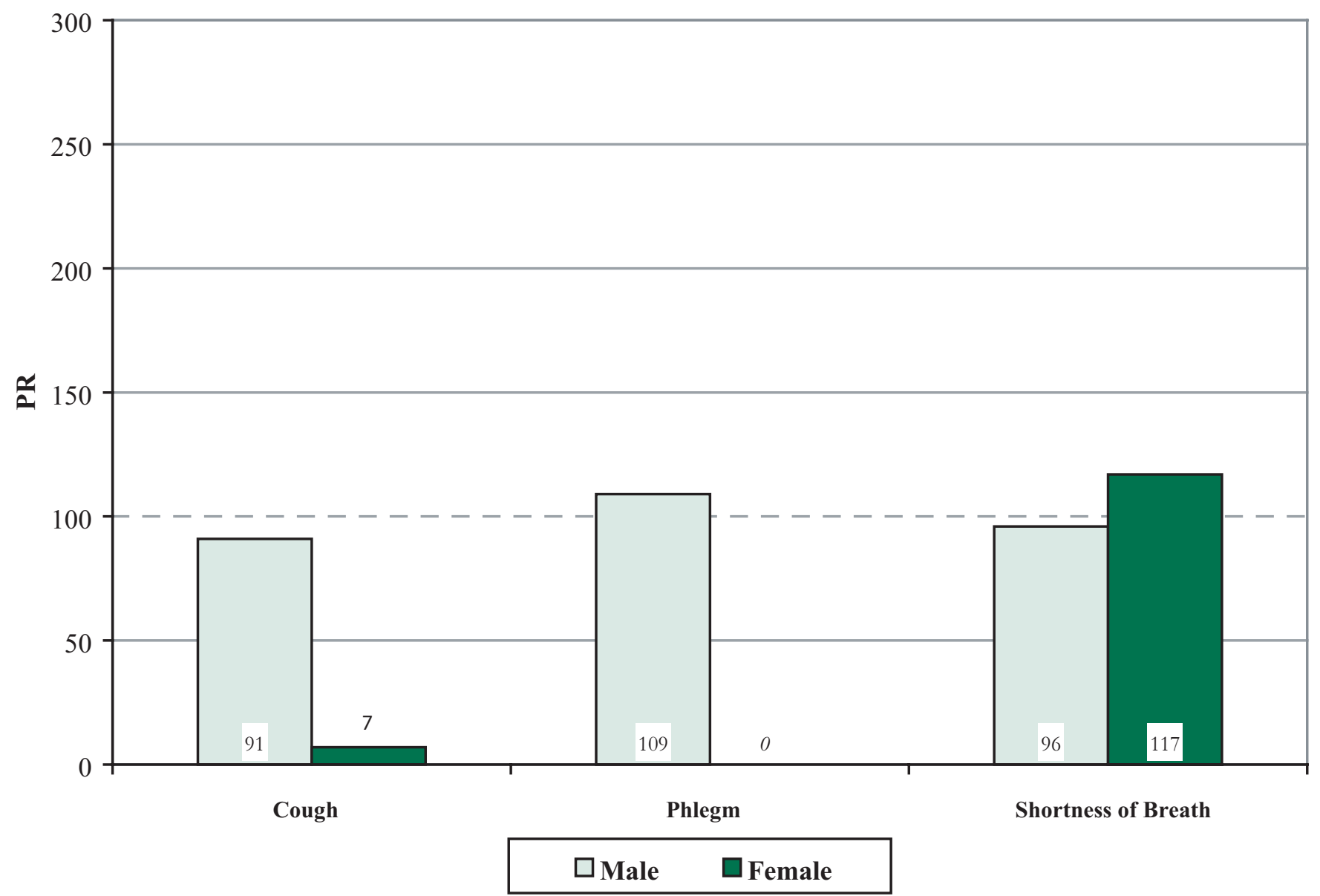

NOTE: Based on responses to the following questions:

"Do you usually cough on most days for 3 consecutive months or more during the year?"

"Do you bring up phlegm on most days for 3 consecutive months or more during the year?"

"Are you troubled by shortness of breath when hurrying on level ground or walking up a slight hill?"

PRs in bold are significantly different from $100(\mathrm{p}<0.05)$. PRs in italics are based on fewer than five observed cases. See appendices for source description and methods.

SOURCE: National Center for Health Statistics, Third National Health and Nutrition Examination Survey (NHANES III) 
Figure 3-17. Respiratory conditions (past year), farm workers: Prevalence ratio (PR) adjusted for age, race/ethnicity, and smoking status by sex, U.S. residents age 17 and over, 1988-1994

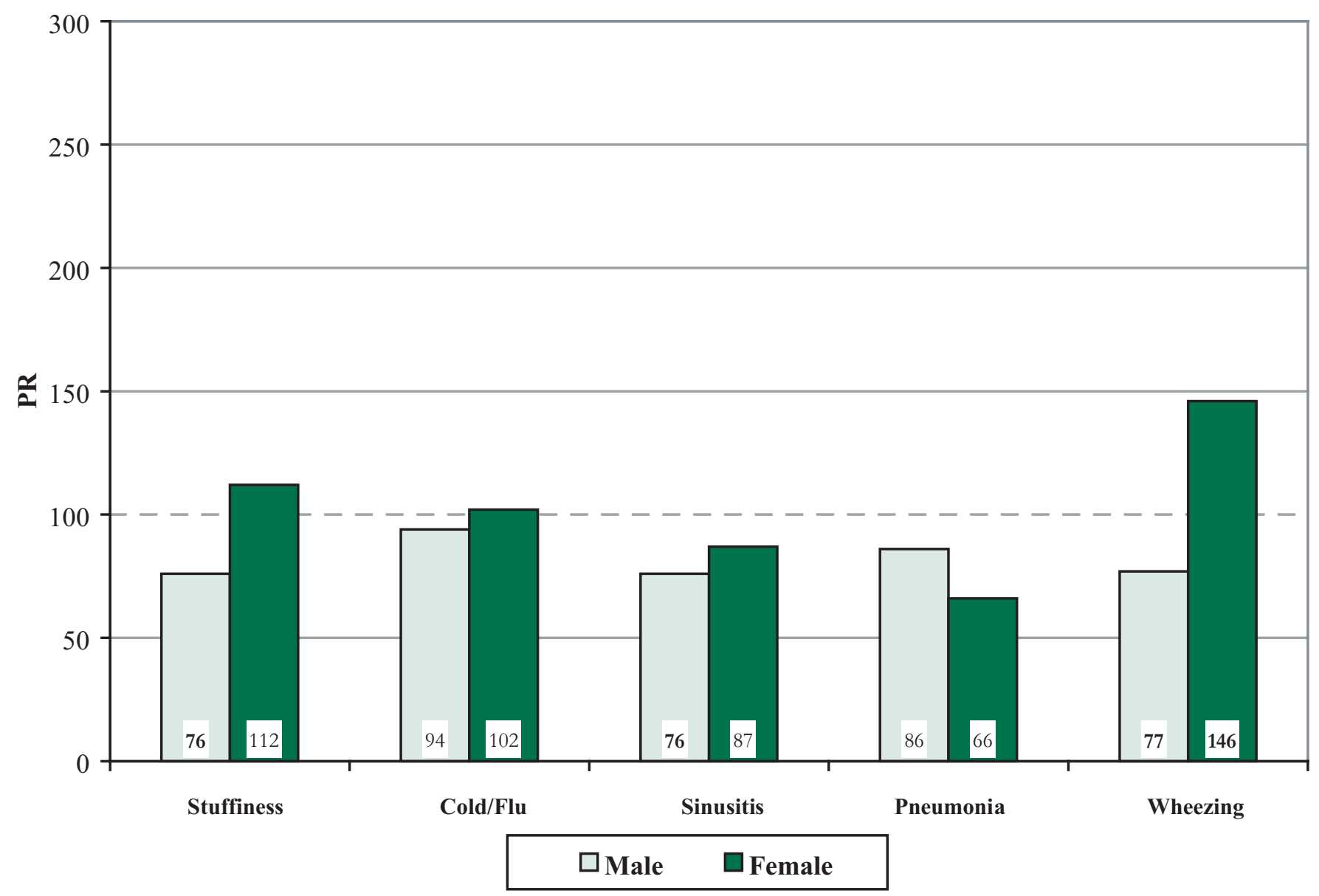

NOTE: Based on responses to the following questions:

"During the past 12 months, have you had any episodes of stuffy, itchy, or runny nose?"

"During the past 12 months, have you had a cold or the flu?"

"During the past 12 months, have you had sinusitis or sinus problems?"

"During the past 12 months, have you had pneumonia?"

"Have you had wheezing or whistling in your chest at any time in the past 12 months?"

PRs in bold are significantly different from $100(\mathrm{p}<0.05)$. PRs in italics are based on fewer than five observed cases. See appendices for source description and methods.

SOURCE: National Center for Health Statistics, Third National Health and Nutrition Examination Survey (NHANES III) 
Figure 3-18. Respiratory conditions (past year), farm managers: Prevalence ratio (PR) adjusted for age, race/ethnicity, and smoking status by sex, U.S. residents age 17 and over, 1988-1994

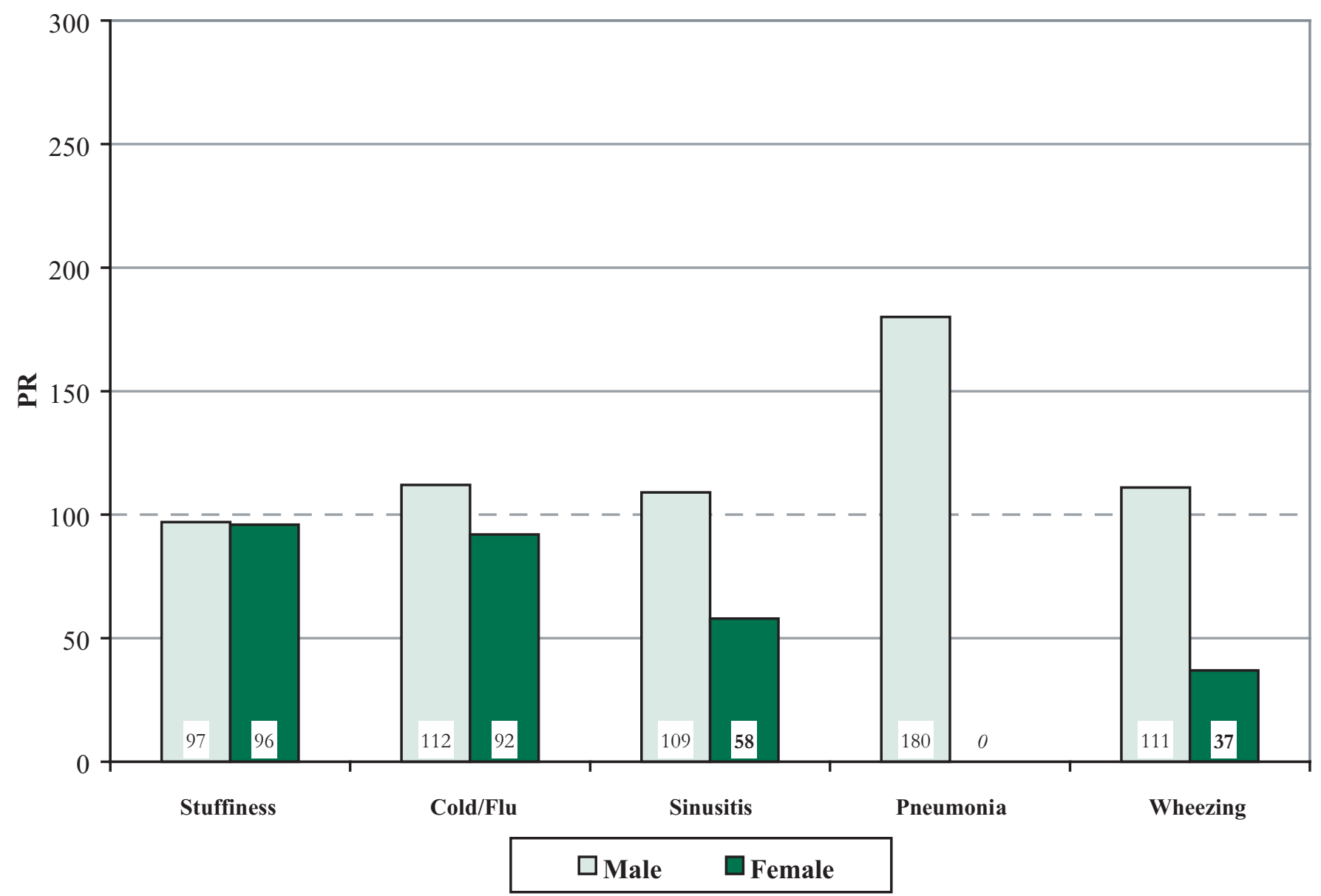

NOTE: Based on responses to the following questions:

"During the past 12 months, have you had any episodes of stuffy, itchy, or runny nose?"

"During the past 12 months, have you had a cold or the flu?"

"During the past 12 months, have you had sinusitis or sinus problems?"

"During the past 12 months, have you had pneumonia?"

"Have you had wheezing or whistling in your chest at any time in the past 12 months?"

PRs in bold are significantly different from $100(\mathrm{p}<0.05)$. PRs in italics are based on fewer than five observed cases. See appendices for source description and methods.

SOURCE: National Center for Health Statistics, Third National Health and Nutrition Examination Survey (NHANES III) 
Figure 3-19. Respiratory conditions (past year), other agricultural workers: Prevalence ratio (PR) adjusted for age, race/ethnicity, and smoking status by sex, U.S. residents age 17 and over, 1988-1994

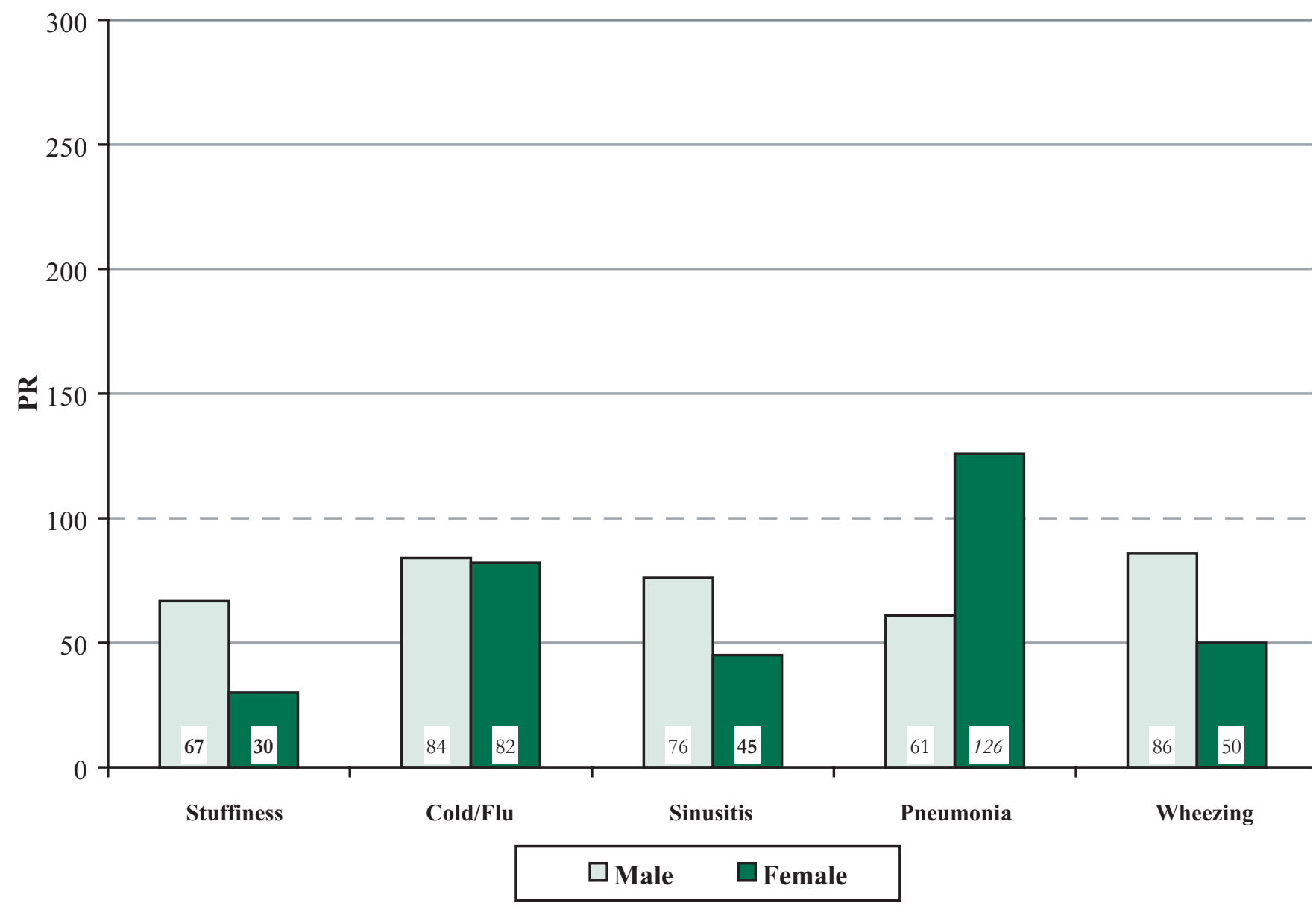

NOTE: Based on responses to the following questions:

"During the past 12 months, have you had any episodes of stuffy, itchy, or runny nose?"

"During the past 12 months, have you had a cold or the flu?"

"During the past 12 months, have you had sinusitis or sinus problems?"

"During the past 12 months, have you had pneumonia?"

"Have you had wheezing or whistling in your chest at any time in the past 12 months?"

PRs in bold are significantly different from $100(\mathrm{p}<0.05)$. PRs in italics are based on fewer than five observed cases. See appendices for source description and methods.

SOURCE: National Center for Health Statistics, Third National Health and Nutrition Examination Survey (NHANES III) 
Figure 3-20. Respiratory conditions (ever), farm workers: Prevalence ratio (PR) adjusted for age, race/ethnicity, and smoking status by sex, U.S. residents age 17 and over, 1988-1994

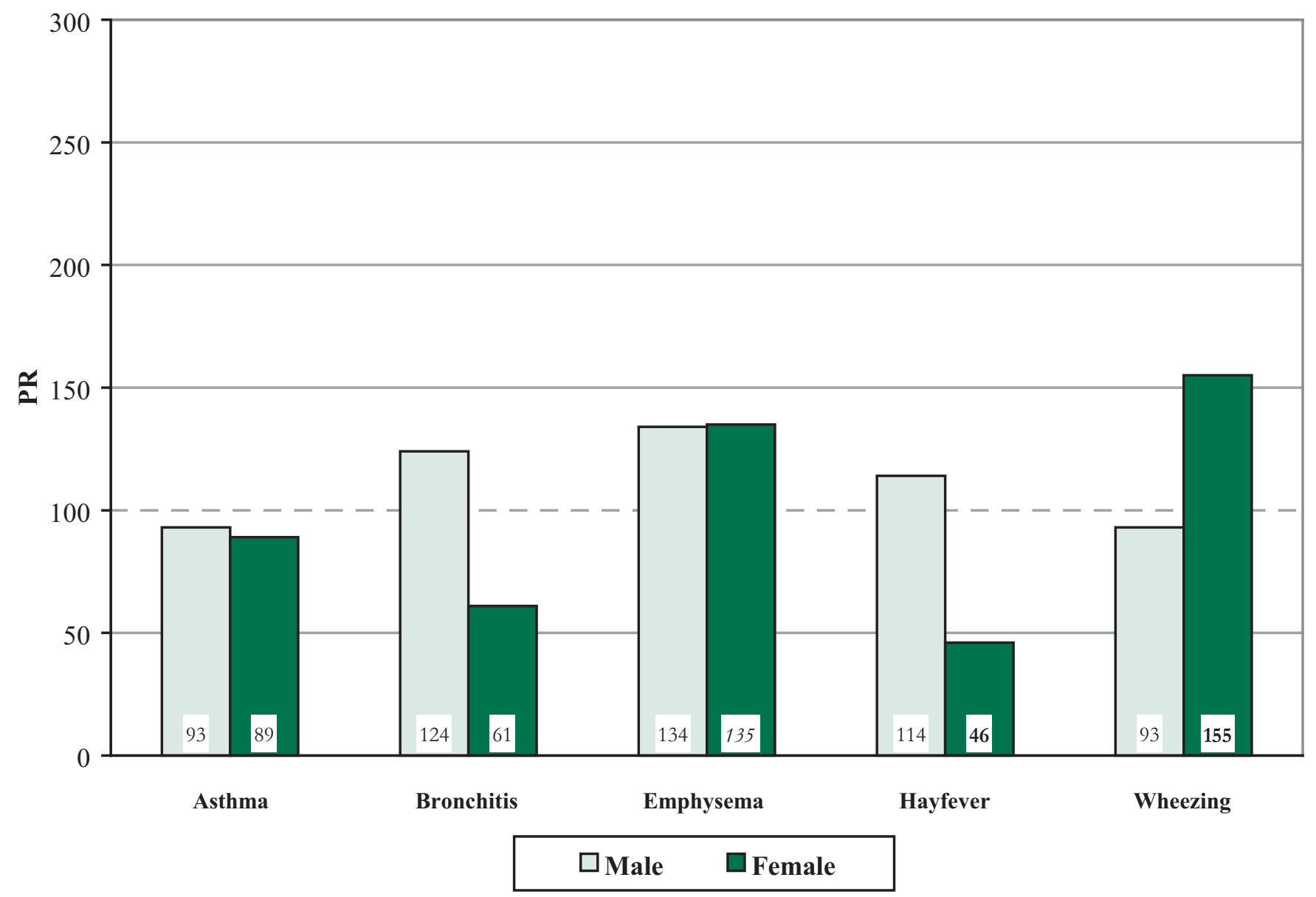

NOTE: Based on responses to the following questions:

"Has a doctor ever told you that you had asthma?"

"Has a doctor ever told you that you had chronic bronchitis?"

"Has a doctor ever told you that you had emphysema?"

"Has a doctor ever told you that you had hay fever?"

"Apart from when you have a cold, does your chest ever sound wheezy or whistling?"

PRs in bold are significantly different from $100(\mathrm{p}<0.05)$. PRs in italics are based on fewer than five observed cases. See appendices for source description and methods.

SOURCE: National Center for Health Statistics, Third National Health and Nutrition Examination Survey (NHANES III) 
Figure 3-21. Respiratory conditions (ever), farm managers: Prevalence ratio (PR) adjusted for age, race/ethnicity, and smoking status by sex, U.S. residents age 17 and over, 1988-1994

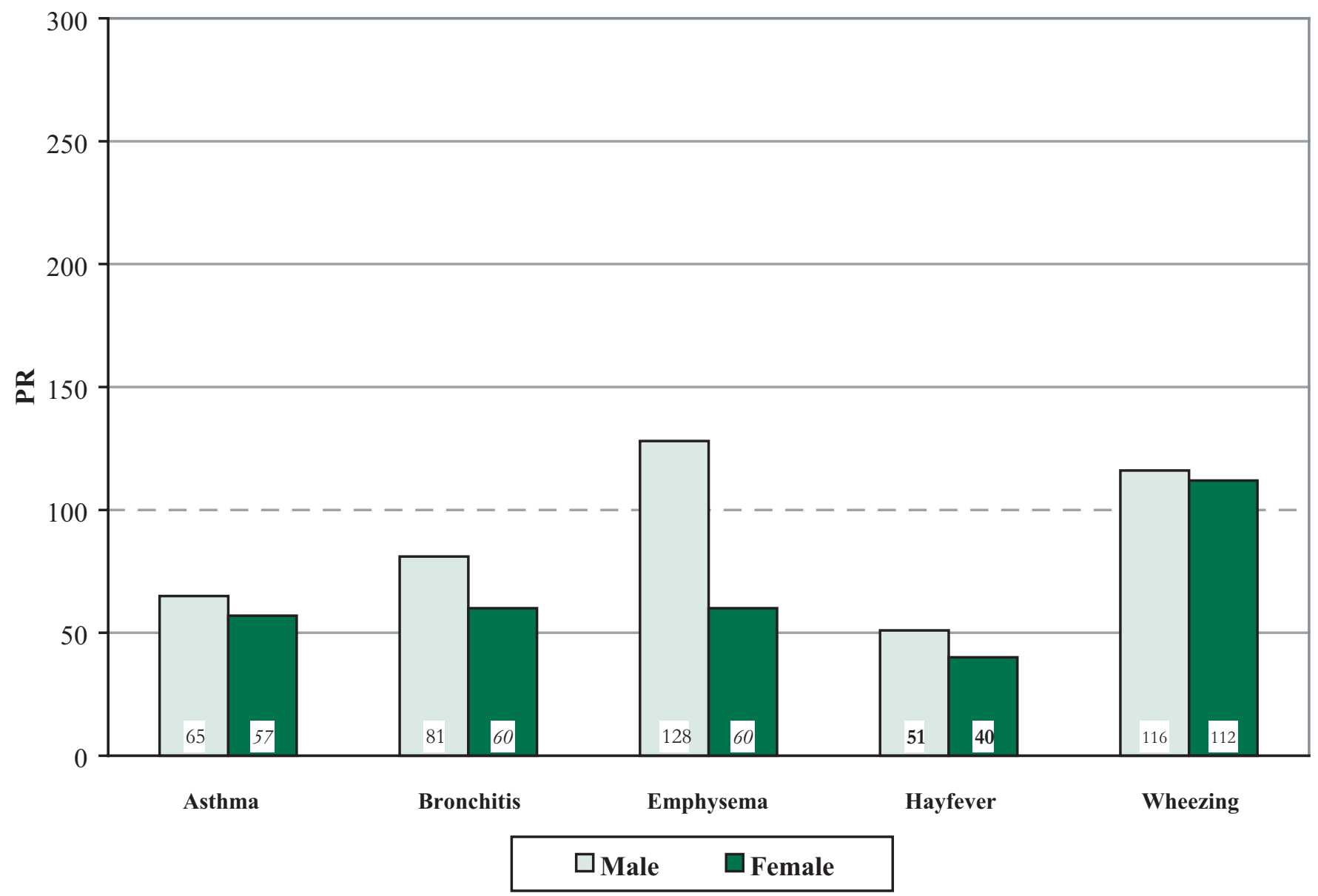

NOTE: Based on responses to the following questions:

"Has a doctor ever told you that you had asthma?"

"Has a doctor ever told you that you had chronic bronchitis?"

"Has a doctor ever told you that you had emphysema?"

"Has a doctor ever told you that you had hay fever?"

"Apart from when you have a cold, does your chest ever sound wheezy or whistling?"

PRs in bold are significantly different from $100(\mathrm{p}<0.05)$. PRs in italics are based on fewer than five observed cases. See appendices for source description and methods.

SOURCE: National Center for Health Statistics, Third National Health and Nutrition Examination Survey (NHANES III) 
Figure 3-22. Respiratory conditions (ever), other agricultural workers: Prevalence ratio (PR) adjusted for age, race/ethnicity, and smoking status by sex, U.S. residents age 17 and over, 1988-1994

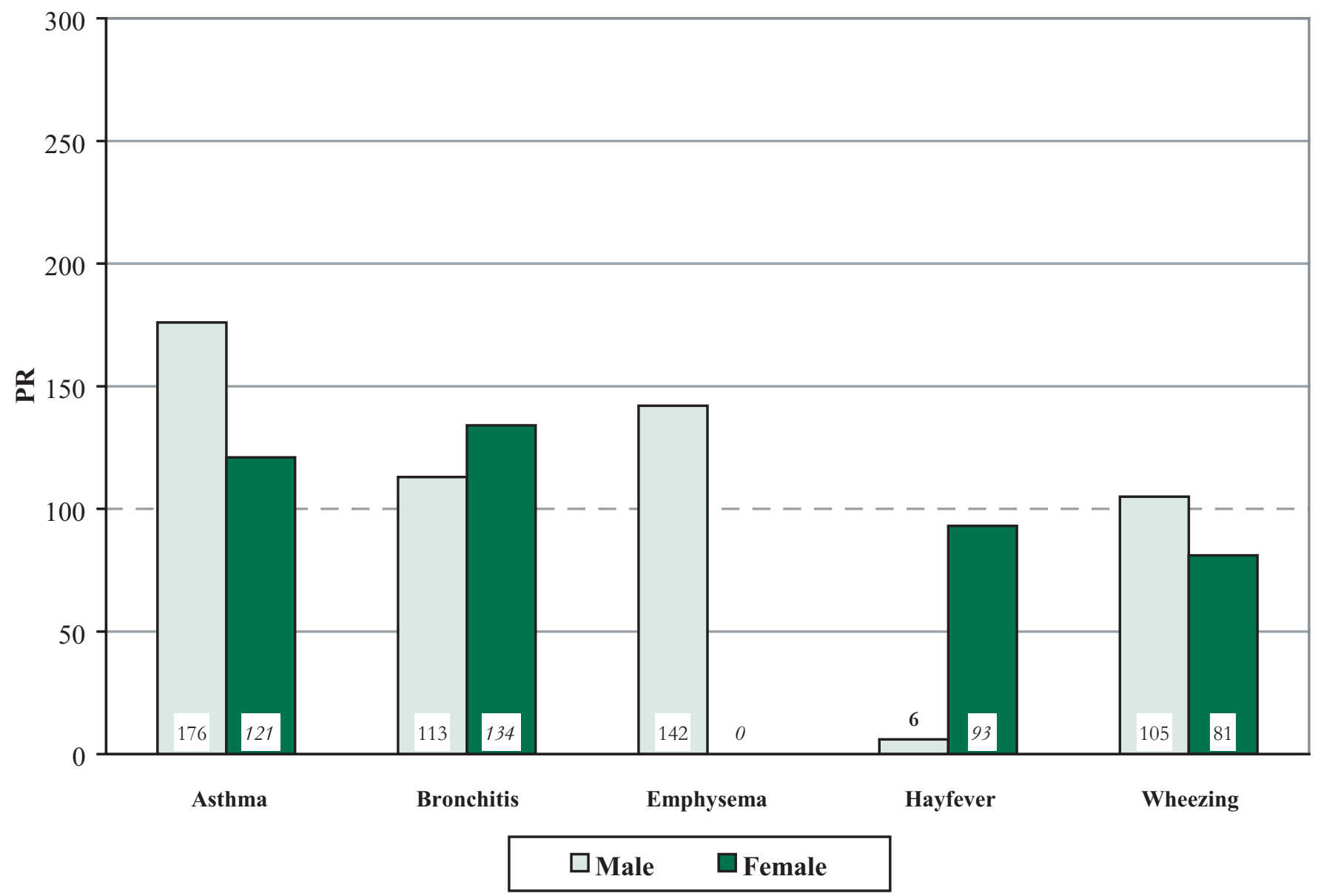

NOTE: Based on responses to the following questions:

"Has a doctor ever told you that you had asthma?"

"Has a doctor ever told you that you had chronic bronchitis?"

"Has a doctor ever told you that you had emphysema?"

"Has a doctor ever told you that you had hay fever?"

"Apart from when you have a cold, does your chest ever sound wheezy or whistling?"

PRs in bold are significantly different from $100(\mathrm{p}<0.05)$. PRs in italics are based on fewer than five observed cases. See appendices for source description and methods.

SOURCE: National Center for Health Statistics, Third National Health and Nutrition Examination Survey (NHANES III) 
Figure 3-23. Respiratory conditions (current), farm workers: Prevalence ratio (PR) adjusted for age, sex, and smoking status by race/ethnicity, U.S. residents age 17 and over, 1988-1994

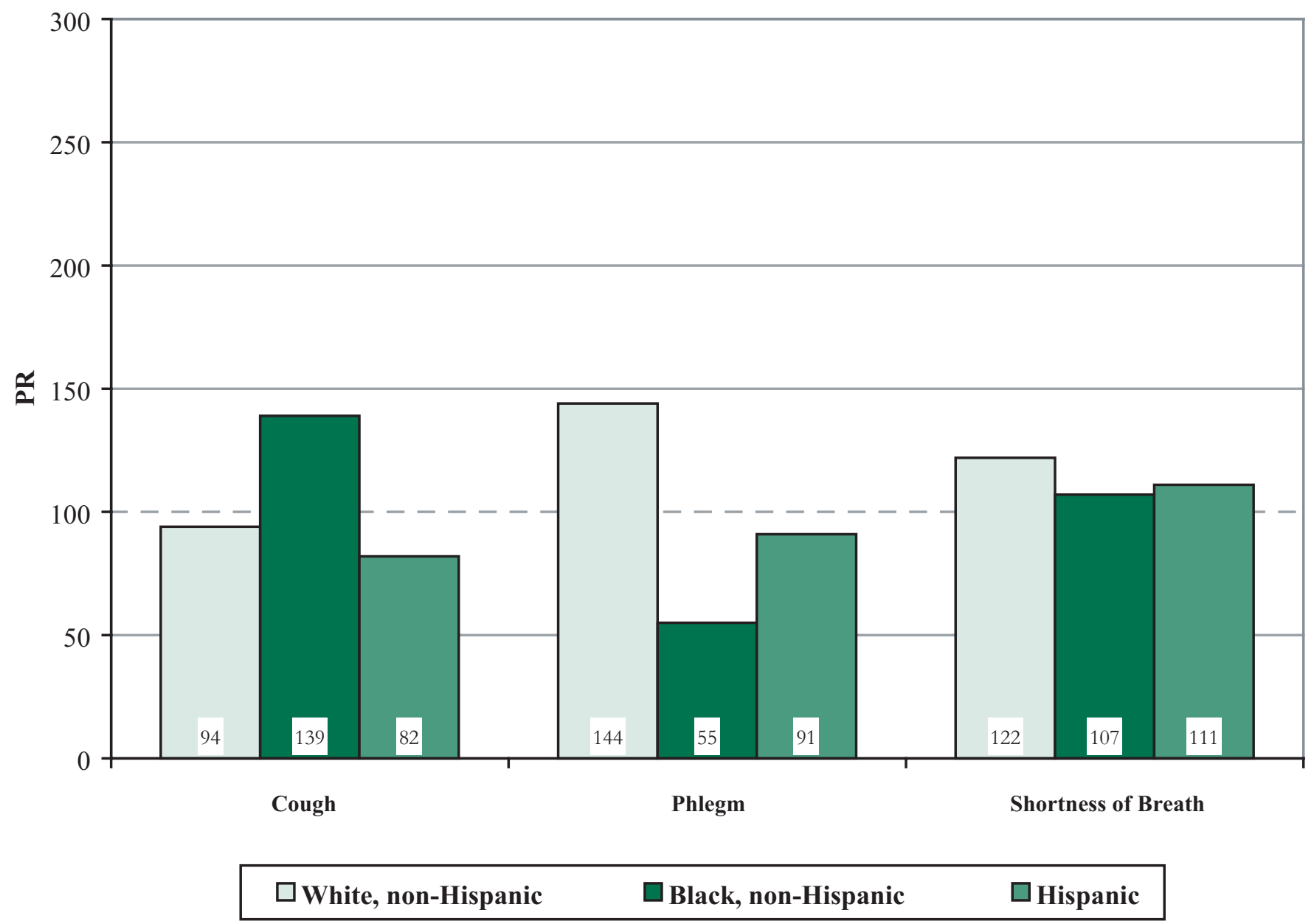

NOTE: Based on responses to the following questions:

"Do you usually cough on most days for 3 consecutive months or more during the year?"

"Do you bring up phlegm on most days for 3 consecutive months or more during the year?"

"Are you troubled by shortness of breath when hurrying on level ground or walking up a slight hill?"

PRs in bold are significantly different from $100(\mathrm{p}<0.05)$. PRs in italics are based on fewer than five observed cases. See appendices for source description and methods.

SOURCE: National Center for Health Statistics, Third National Health and Nutrition Examination Survey (NHANES III) 
Figure 3-24. Respiratory conditions (current), farm managers: Prevalence ratio (PR) adjusted for age, sex, and smoking status by race/ethnicity, U.S. residents age 17 and over, 1988-1994

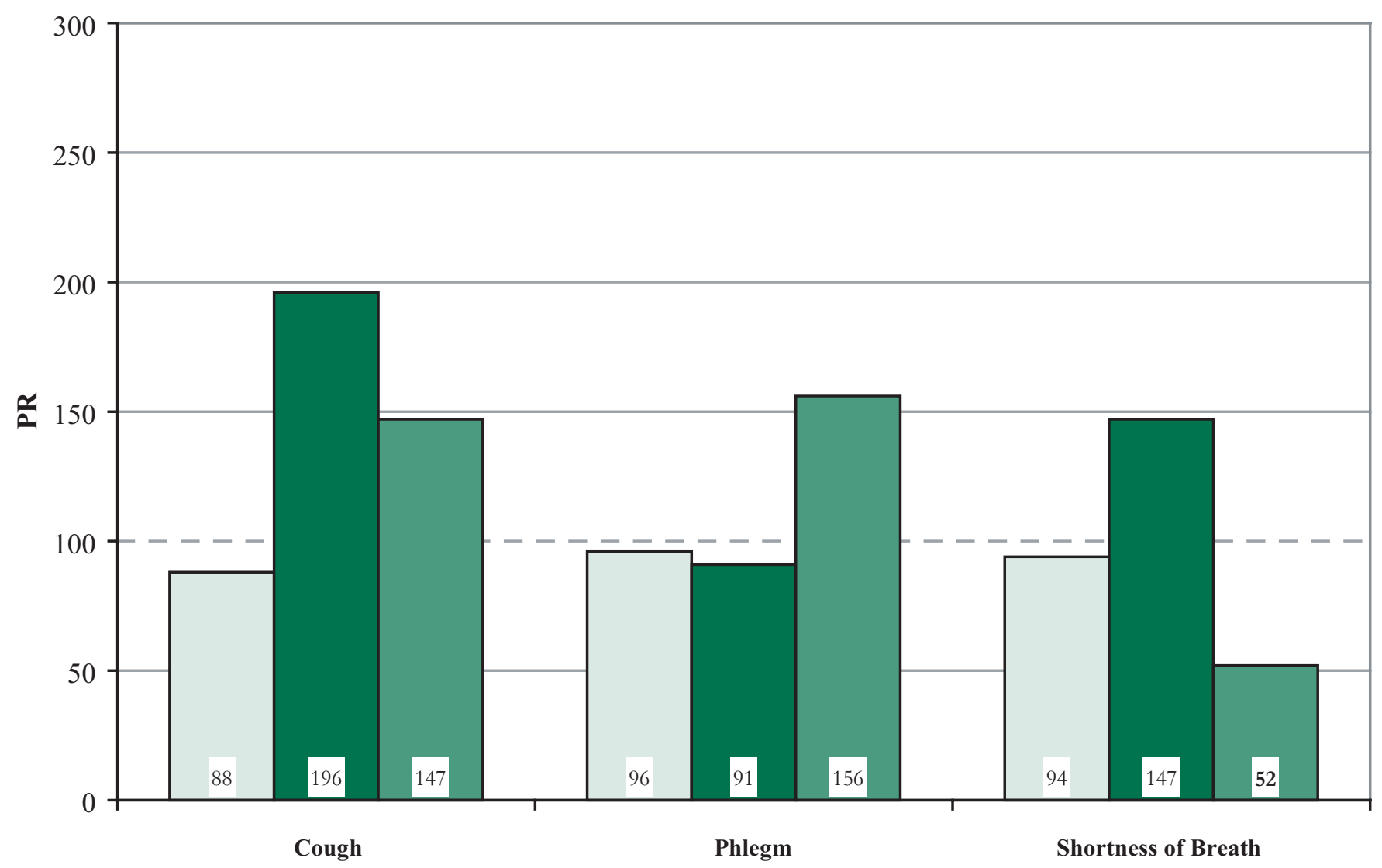

\section{$\square$ White, non-Hispanic $\quad \square$ Black, non-Hispanic $\quad \square$ Hispanic}

NOTE: Based on responses to the following questions:

"Do you usually cough on most days for 3 consecutive months or more during the year?"

"Do you bring up phlegm on most days for 3 consecutive months or more during the year?"

"Are you troubled by shortness of breath when hurrying on level ground or walking up a slight hill?"

PRs in bold are significantly different from $100(\mathrm{p}<0.05)$. PRs in italics are based on fewer than five observed cases. See appendices for source description and methods.

SOURCE: National Center for Health Statistics, Third National Health and Nutrition Examination Survey (NHANES III) 
Figure 3-25. Respiratory conditions (current), other agricultural workers: Prevalence ratio (PR) adjusted for age, sex, and smoking status by race/ethnicity, U.S. residents age 17 and over, 1988-1994

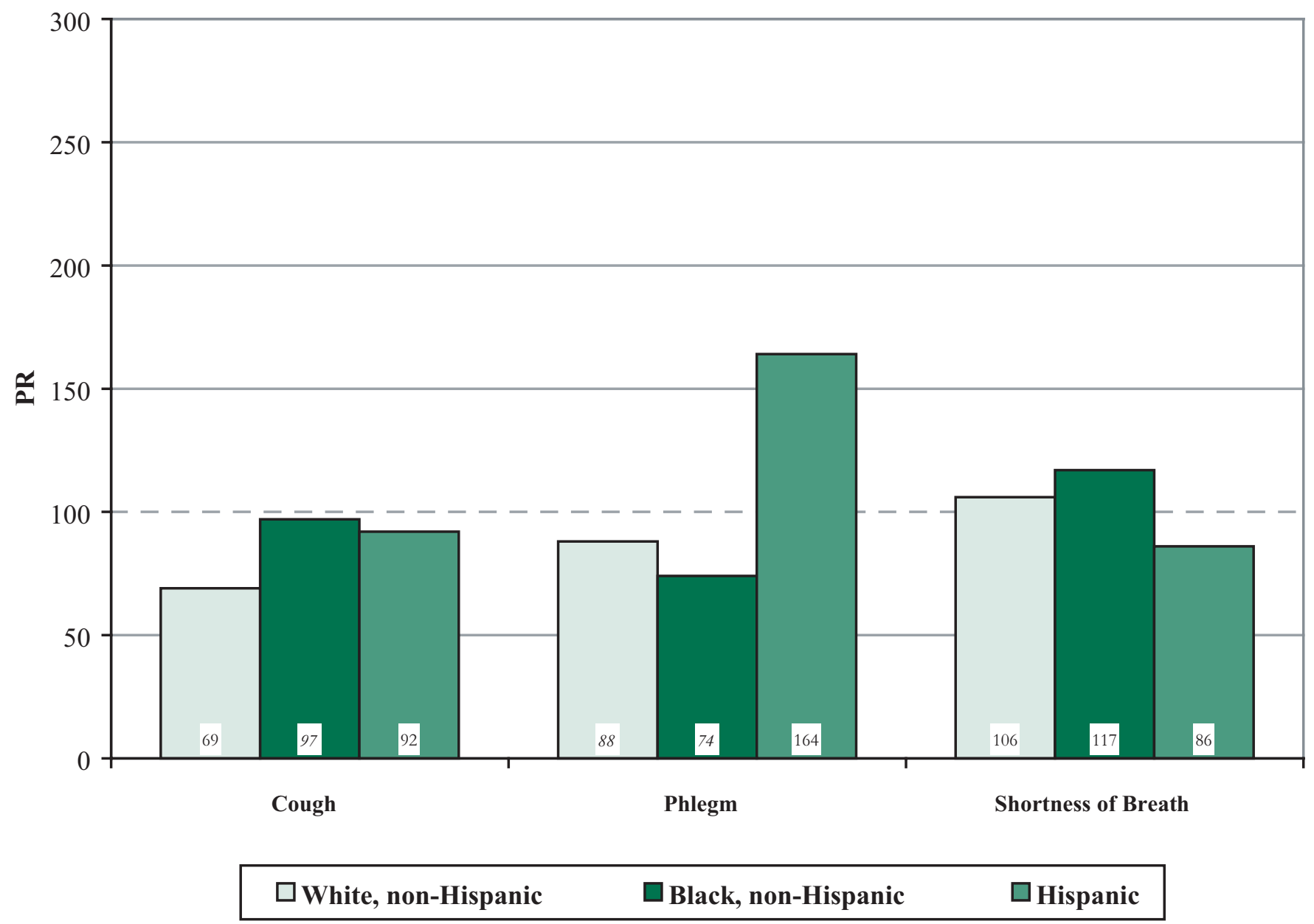

NOTE: Based on responses to the following questions:

"Do you usually cough on most days for 3 consecutive months or more during the year?"

"Do you bring up phlegm on most days for 3 consecutive months or more during the year?"

"Are you troubled by shortness of breath when hurrying on level ground or walking up a slight hill?"

PRs in bold are significantly different from $100(\mathrm{p}<0.05)$. PRs in italics are based on fewer than five observed cases. See appendices for source description and methods.

SOURCE: National Center for Health Statistics, Third National Health and Nutrition Examination Survey (NHANES III) 
Figure 3-26. Respiratory conditions (past year), farm workers: Prevalence ratio (PR) adjusted for age, sex, and smoking status by race/ethnicity, U.S. residents age 17 and over, 1988-1994

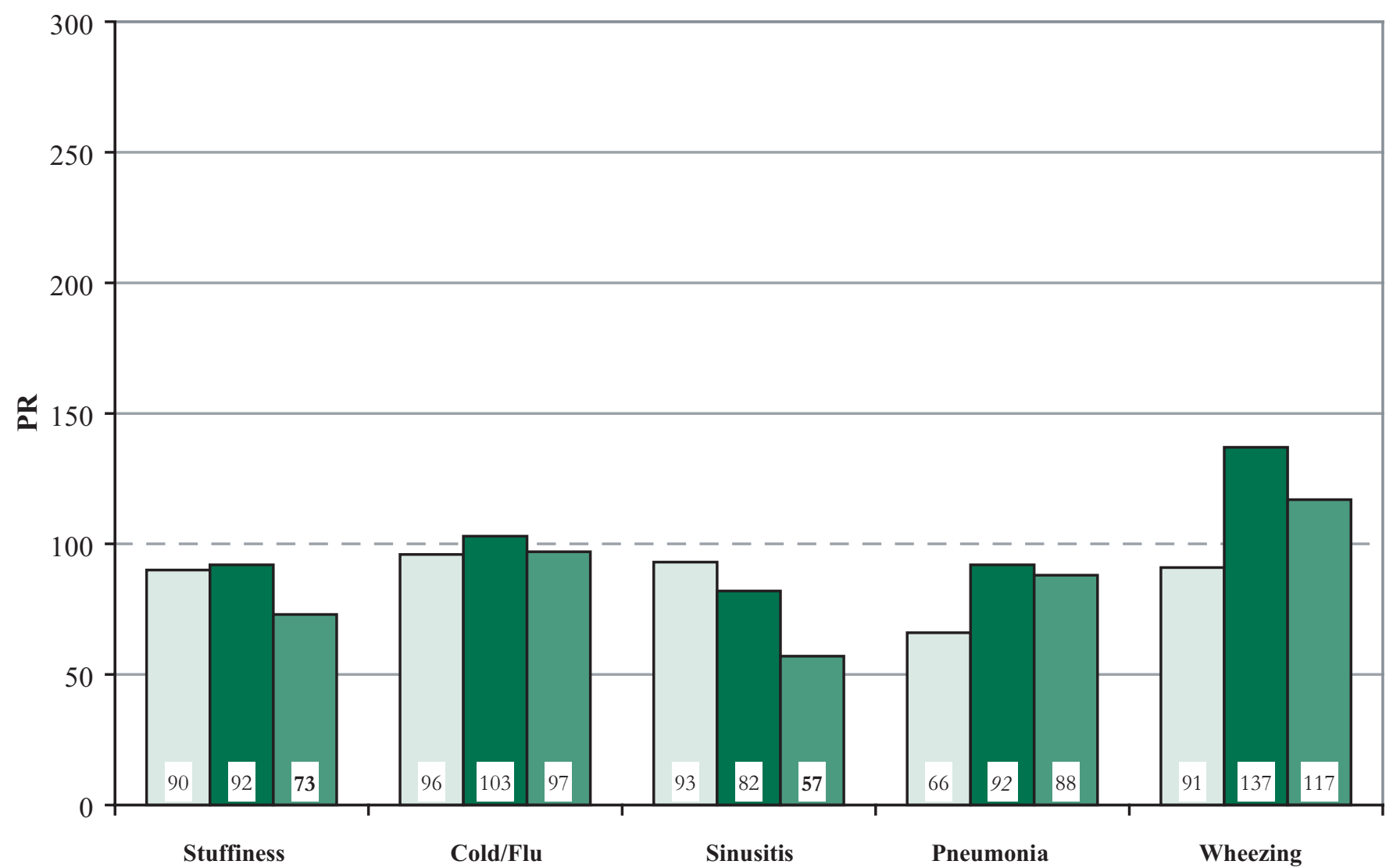

\section{$\square$ White, non-Hispanic \\ Black, non-Hispanic \\ $\square$ Hispanic}

NOTE: Based on responses to the following questions:

"During the past 12 months, have you had any episodes of stuffy, itchy, or runny nose?"

"During the past 12 months, have you had a cold or the flu?"

"During the past 12 months, have you had sinusitis or sinus problems?"

"During the past 12 months, have you had pneumonia?"

"Have you had wheezing or whistling in your chest at any time in the past 12 months?"

PRs in bold are significantly different from $100(\mathrm{p}<0.05)$. PRs in italics are based on fewer than five observed cases. See appendices for source description and methods.

SOURCE: National Center for Health Statistics, Third National Health and Nutrition Examination Survey (NHANES III) 
Figure 3-27. Respiratory conditions (past year), farm managers: Prevalence ratio (PR) adjusted for age, sex, and smoking status by race/ethnicity, U.S. residents age 17 and over, 1988-1994

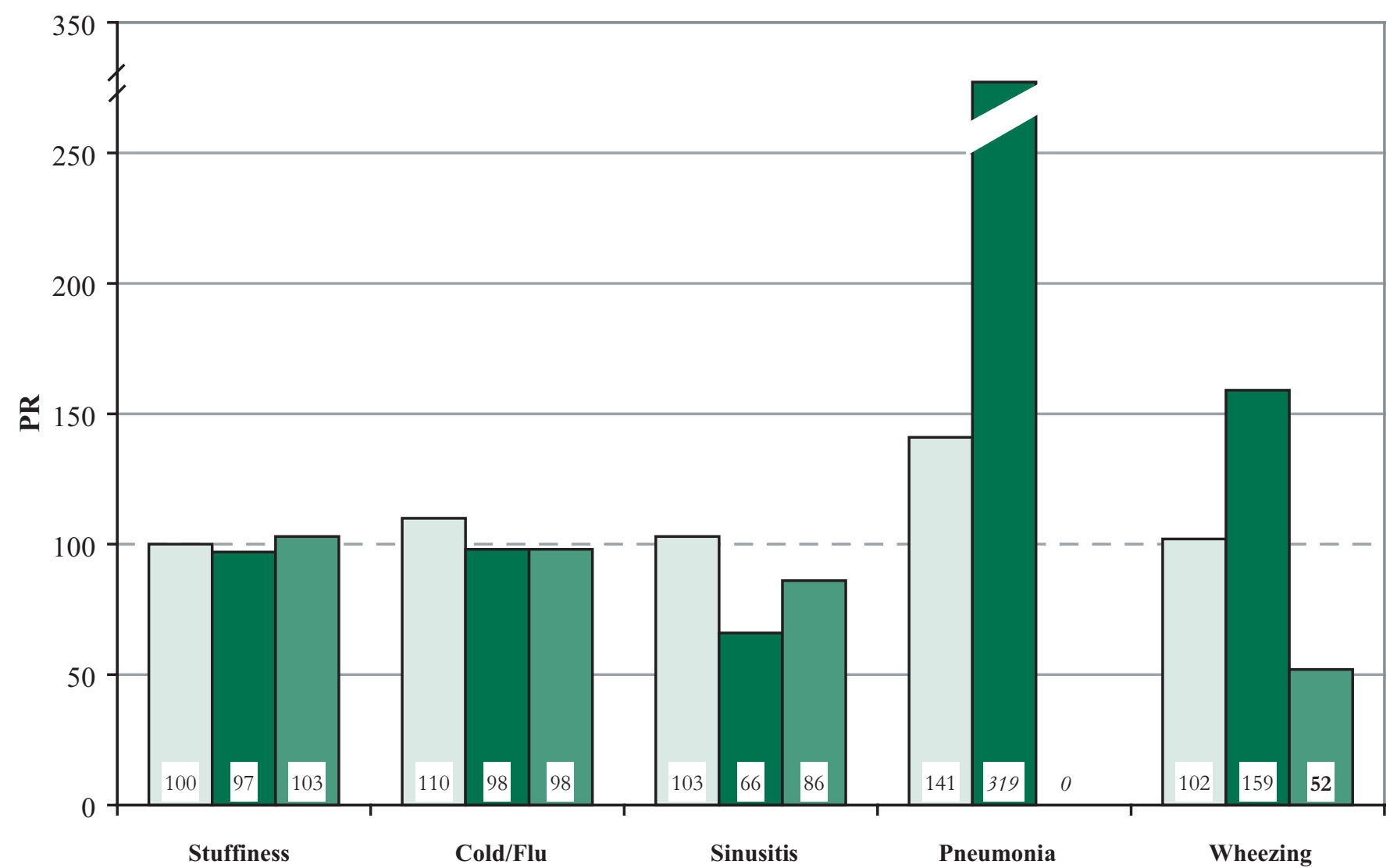

$\square$ White, non-Hispanic

Black, non-Hispanic

Hispanic

NOTE: Based on responses to the following questions:

"During the past 12 months, have you had any episodes of stuffy, itchy, or runny nose?"

"During the past 12 months, have you had a cold or the flu?"

"During the past 12 months, have you had sinusitis or sinus problems?"

"During the past 12 months, have you had pneumonia?"

"Have you had wheezing or whistling in your chest at any time in the past 12 months?"

PRs in bold are significantly different from $100(\mathrm{p}<0.05)$. PRs in italics are based on fewer than five observed cases. See appendices for source description and methods.

SOURCE: National Center for Health Statistics, Third National Health and Nutrition Examination Survey (NHANES III) 
Figure 3-28. Respiratory conditions (past year), other agricultural workers: Prevalence ratio (PR) adjusted for age, sex, and smoking status by race/ethnicity, U.S. residents age 17 and over, 1988-1994

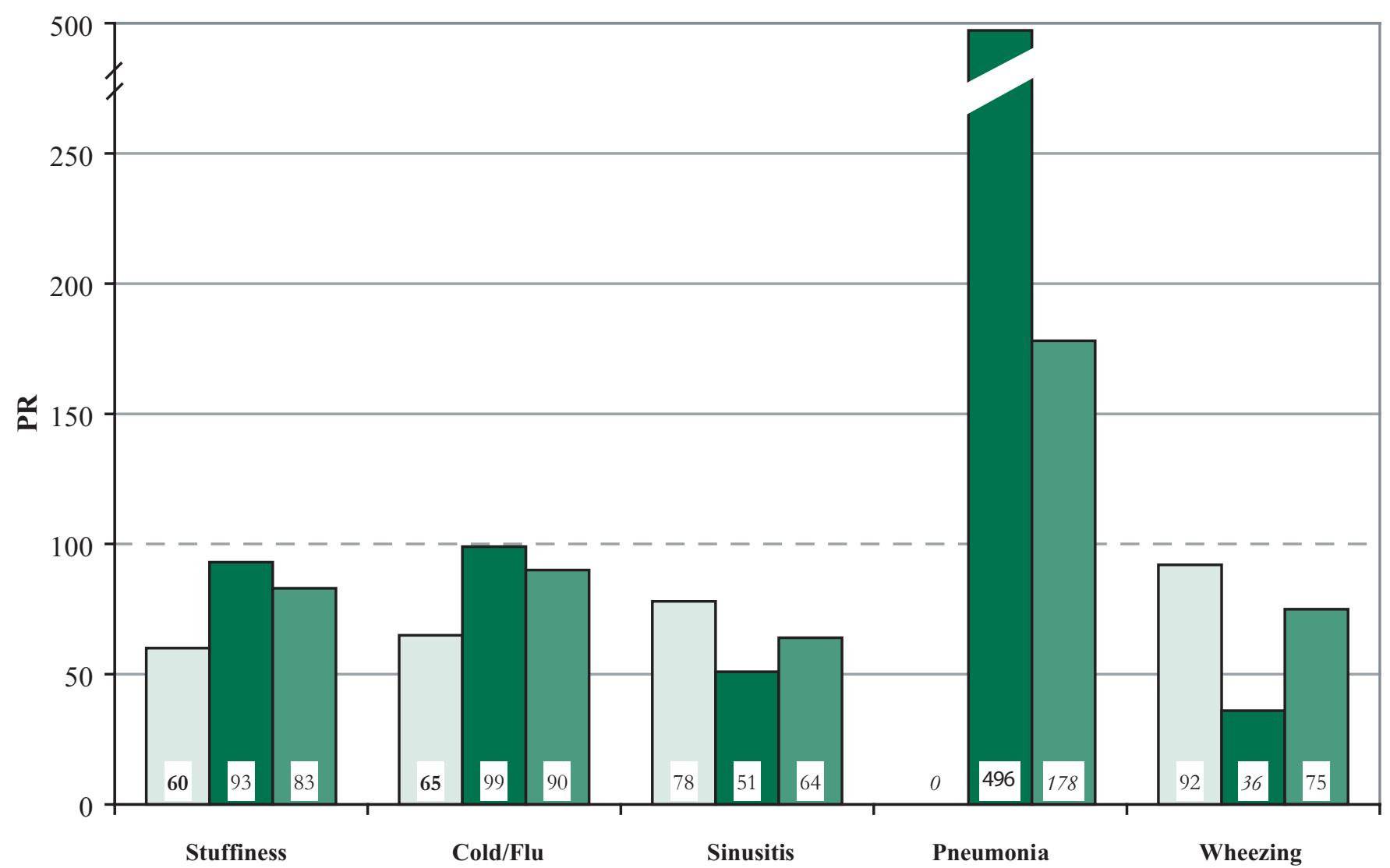

\section{$\square$ White, non-Hispanic $\quad \square$ Black, non-Hispanic $\quad \square$ Hispanic}

NOTE: Based on responses to the following questions:

"During the past 12 months, have you had any episodes of stuffy, itchy, or runny nose?"

"During the past 12 months, have you had a cold or the flu?"

"During the past 12 months, have you had sinusitis or sinus problems?"

"During the past 12 months, have you had pneumonia?"

"Have you had wheezing or whistling in your chest at any time in the past 12 months?"

PRs in bold are significantly different from $100(\mathrm{p}<0.05)$. PRs in italics are based on fewer than five observed cases. See appendices for source description and methods.

SOURCE: National Center for Health Statistics, Third National Health and Nutrition Examination Survey (NHANES III) 
Figure 3-29. Respiratory conditions (ever), farm workers: Prevalence ratio (PR) adjusted for age, sex, and smoking status by race/ethnicity, U.S. residents age 17 and over, 1988-1994

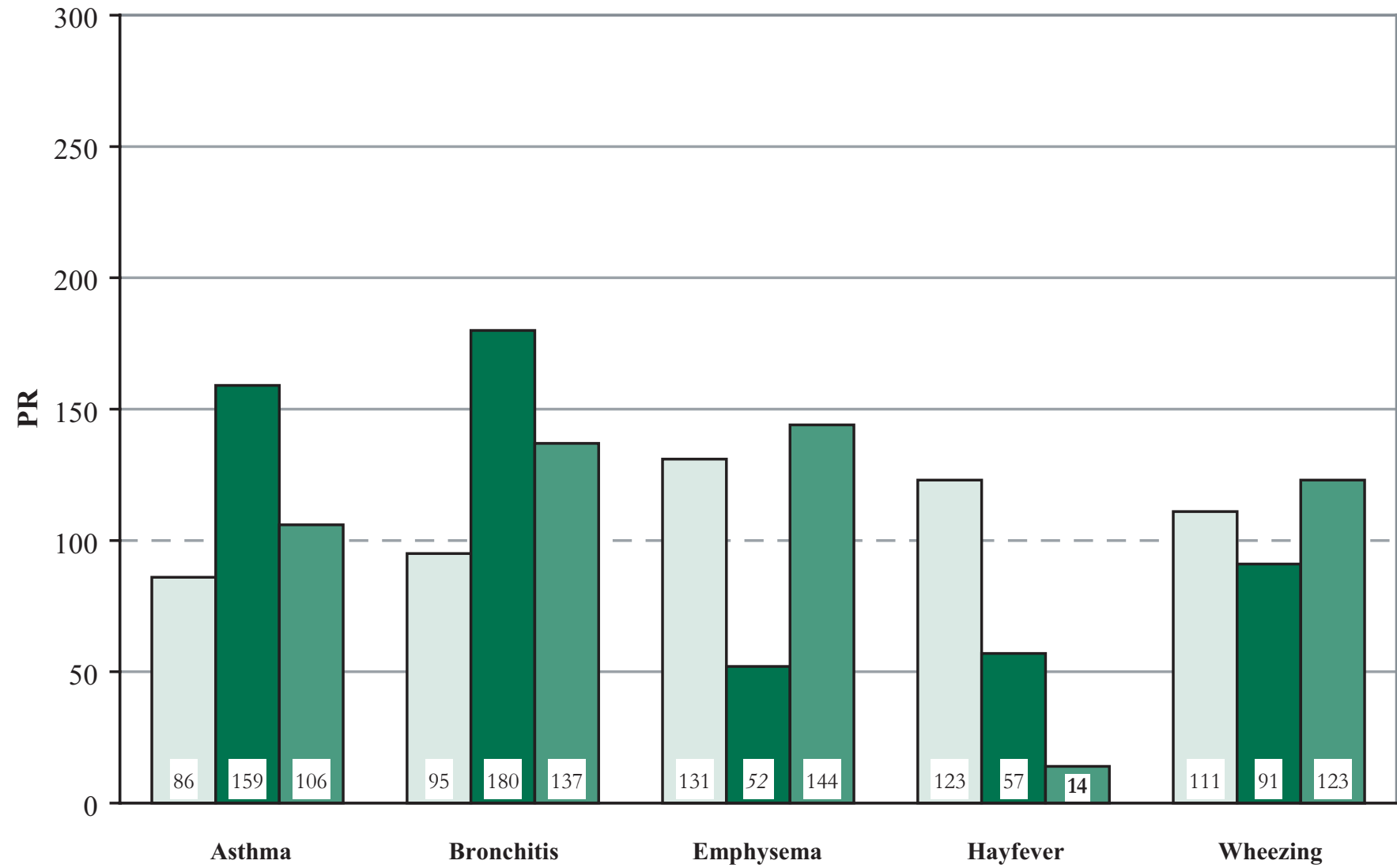

\section{$\square$ White, non-Hispanic \\ Black, non-Hispanic \\ $\square$ Hispanic}

NOTE: Based on responses to the following questions:

"Has a doctor ever told you that you had asthma?"

"Has a doctor ever told you that you had chronic bronchitis?"

"Has a doctor ever told you that you had emphysema?"

"Has a doctor ever told you that you had hay fever?"

"Apart from when you have a cold, does your chest ever sound wheezy or whistling?"

PRs in bold are significantly different from $100(\mathrm{p}<0.05)$. PRs in italics are based on fewer than five observed cases. See appendices for source description and methods.

SOURCE: National Center for Health Statistics, Third National Health and Nutrition Examination Survey (NHANES III) 
Figure 3-30. Respiratory conditions (ever), farm managers: Prevalence ratio (PR) adjusted for age, sex, and smoking status by race/ethnicity, U.S. residents age 17 and over, 1988-1994

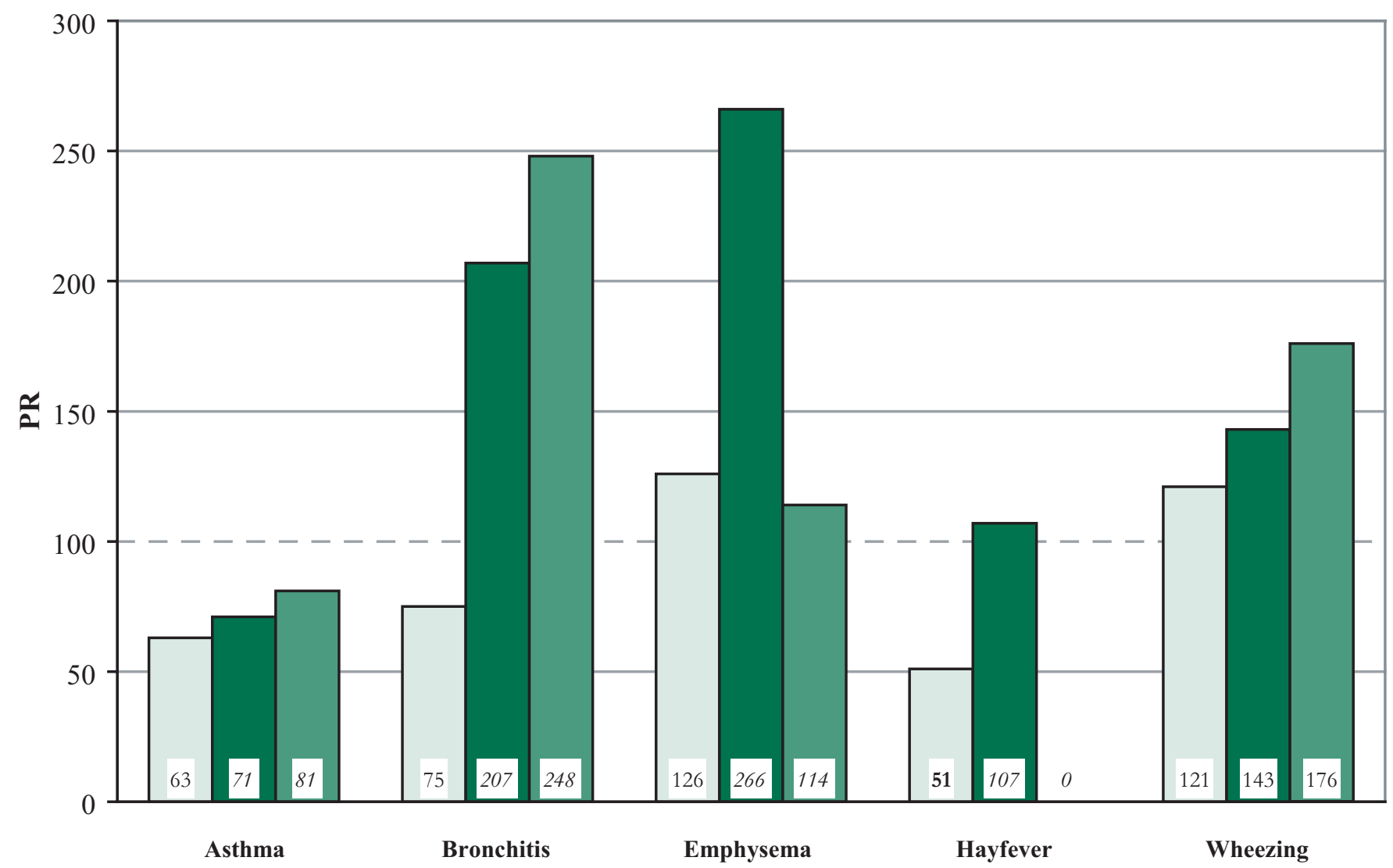

\section{$\square$ White, non-Hispanic \\ Black, non-Hispanic \\ $\square$ Hispanic}

NOTE: Based on responses to the following questions:

"Has a doctor ever told you that you had asthma?"

"Has a doctor ever told you that you had chronic bronchitis?"

"Has a doctor ever told you that you had emphysema?"

"Has a doctor ever told you that you had hay fever?"

"Apart from when you have a cold, does your chest ever sound wheezy or whistling?"

PRs in bold are significantly different from $100(\mathrm{p}<0.05)$. PRs in italics are based on fewer than five observed cases. See appendices for source description and methods.

SOURCE: National Center for Health Statistics, Third National Health and Nutrition Examination Survey (NHANES III) 
Figure 3-31. Respiratory conditions (ever), other agricultural workers: Prevalence ratio (PR) adjusted for age, sex, and smoking status by race/ethnicity, U.S. residents age 17 and over, 1988-1994

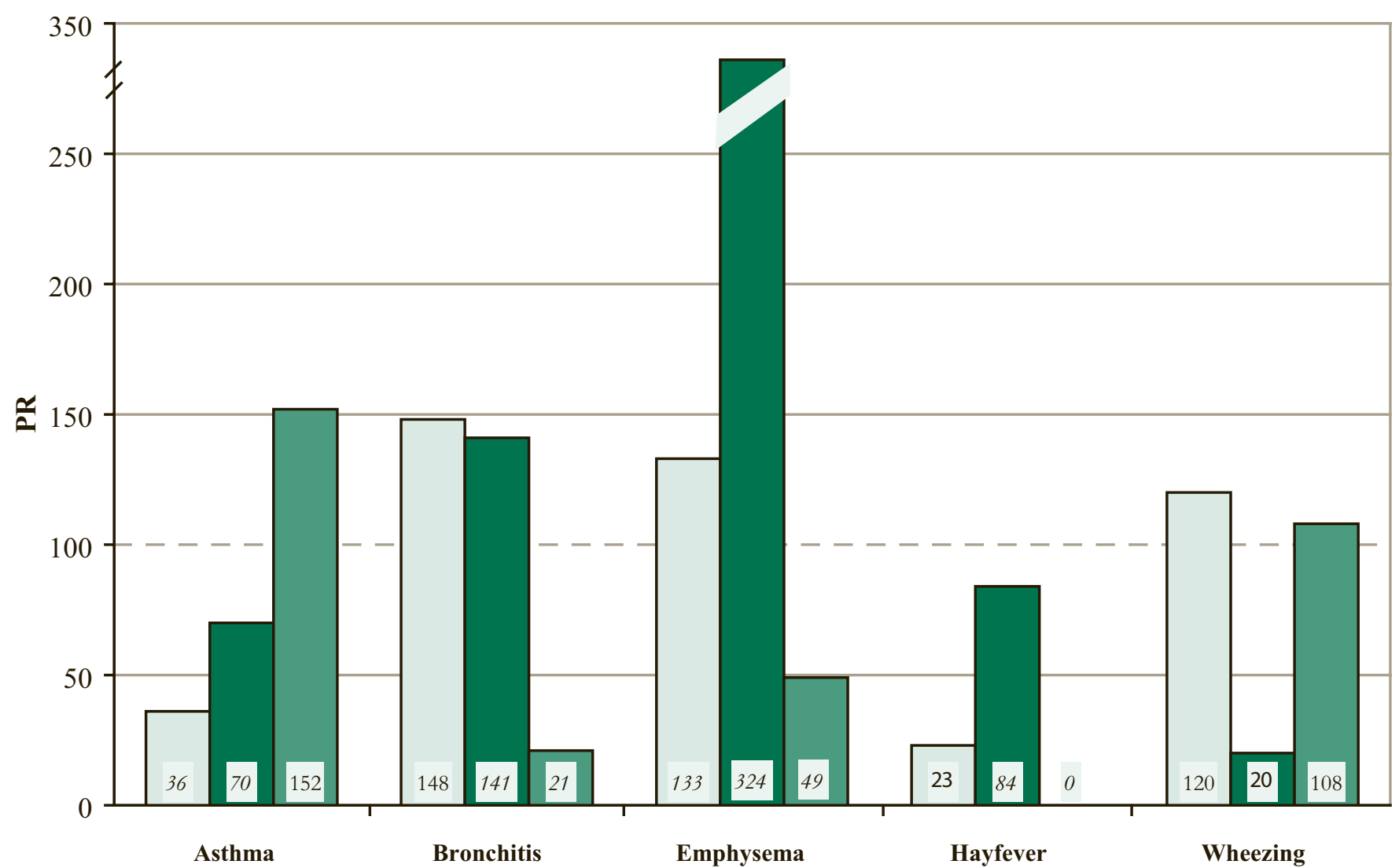

\section{$\square$ White, non-Hispanic \\ Black, non-Hispanic \\ Hispanic}

NOTE: Based on responses to the following questions:

"Has a doctor ever told you that you had asthma?"

"Has a doctor ever told you that you had chronic bronchitis?"

"Has a doctor ever told you that you had emphysema?"

"Has a doctor ever told you that you had hay fever?"

"Apart from when you have a cold, does your chest ever sound wheezy or whistling?"

PRs in bold are significantly different from $100(\mathrm{p}<0.05)$. PRs in italics are based on fewer than five observed cases. See appendices for source description and methods.

SOURCE: National Center for Health Statistics, Third National Health and Nutrition Examination Survey (NHANES III) 
Figure 3-32. Respiratory conditions (current), farm workers: Prevalence ratio (PR) adjusted for age, sex, and race/ethnicity by smoking status, U.S. residents age 17 and over, 1988-1994

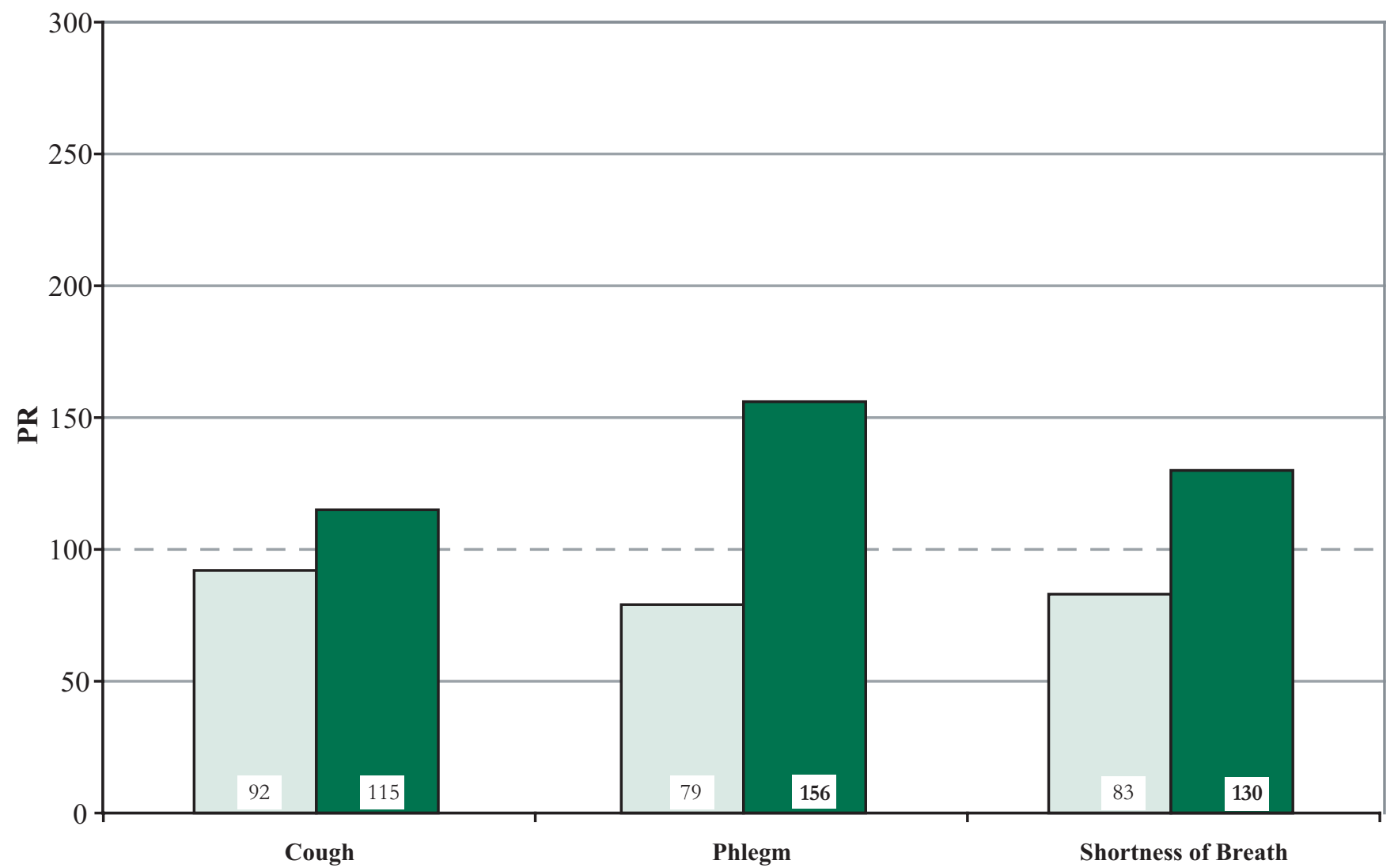

\section{$\square$ Never Smoked $\quad \square$ Ever Smoked}

NOTE: Based on responses to the following questions:

"Do you usually cough on most days for 3 consecutive months or more during the year?"

"Do you bring up phlegm on most days for 3 consecutive months or more during the year?"

"Are you troubled by shortness of breath when hurrying on level ground or walking up a slight hill?"

PRs in bold are significantly different from $100(\mathrm{p}<0.05)$. PRs in italics are based on fewer than five observed cases. See appendices for source description and methods.

SOURCE: National Center for Health Statistics, Third National Health and Nutrition Examination Survey (NHANES III) 
Figure 3-33. Respiratory conditions (current), farm managers: Prevalence ratio (PR) adjusted for age, sex, and race/ethnicity by smoking status, U.S. residents age 17 and over, 1988-1994

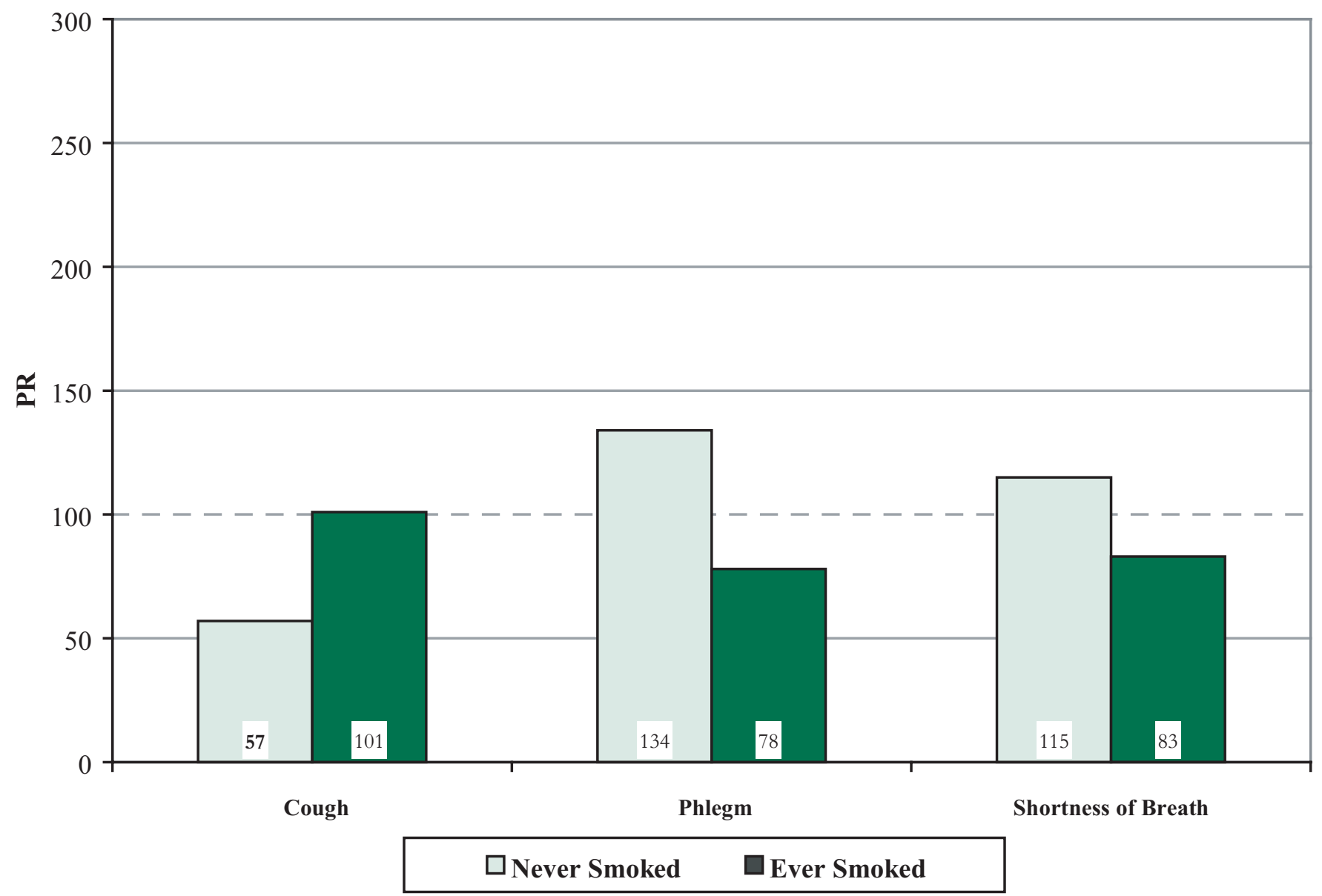

NOTE: Based on responses to the following questions:

"Do you usually cough on most days for 3 consecutive months or more during the year?"

"Do you bring up phlegm on most days for 3 consecutive months or more during the year?"

"Are you troubled by shortness of breath when hurrying on level ground or walking up a slight hill?"

PRs in bold are significantly different from $100(\mathrm{p}<0.05)$. PRs in italics are based on fewer than five observed cases. See appendices for source description and methods.

SOURCE: National Center for Health Statistics, Third National Health and Nutrition Examination Survey (NHANES III) 
Figure 3-34. Respiratory conditions (current), other agricultural workers: Prevalence ratio (PR) adjusted for age, sex, and race/ethnicity by smoking status, U.S. residents age 17 and over, 1988-1994

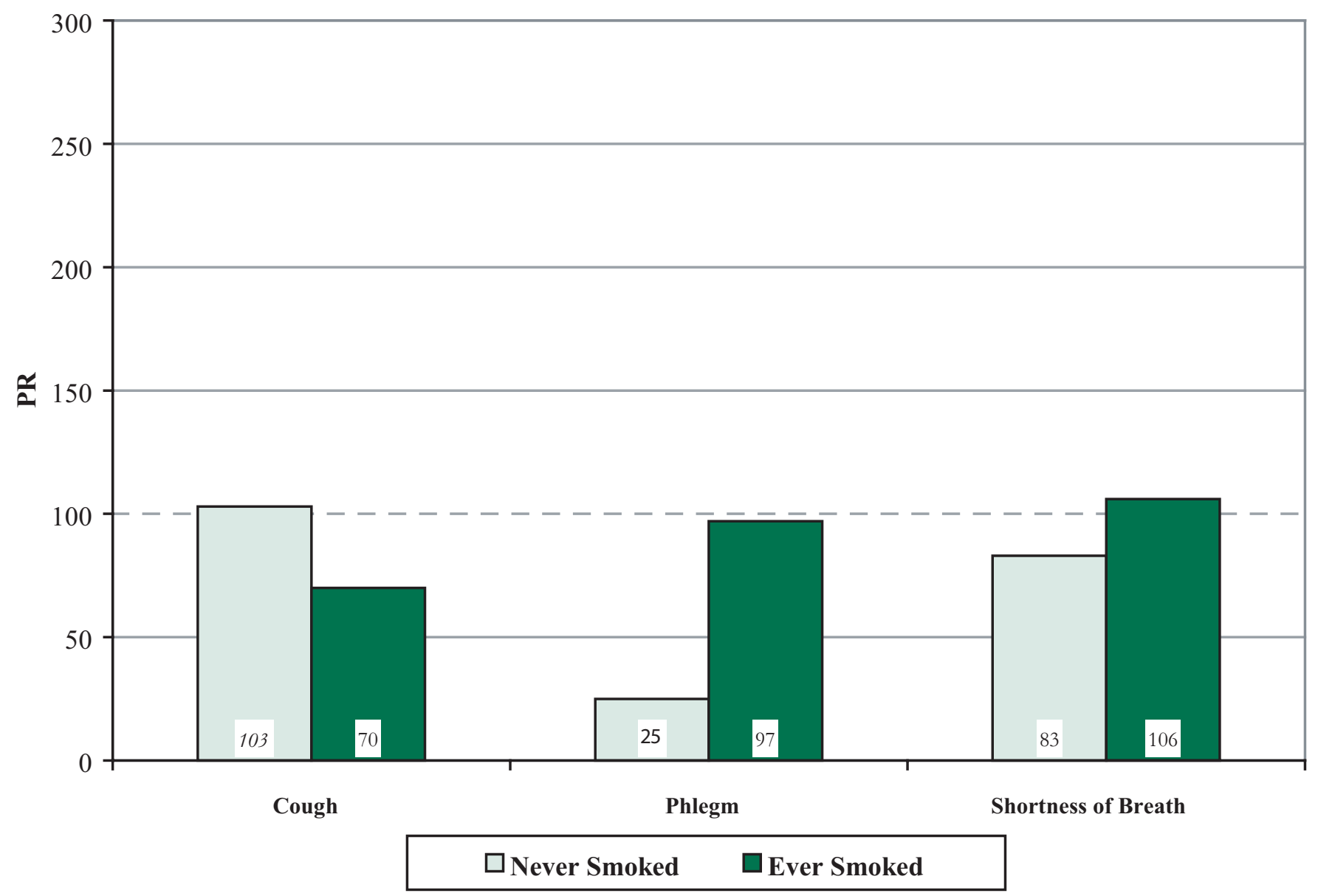

NOTE: Based on responses to the following questions:

"Do you usually cough on most days for 3 consecutive months or more during the year?"

"Do you bring up phlegm on most days for 3 consecutive months or more during the year?"

"Are you troubled by shortness of breath when hurrying on level ground or walking up a slight hill?"

PRs in bold are significantly different from $100(\mathrm{p}<0.05)$. PRs in italics are based on fewer than five observed cases. See appendices for source description and methods.

SOURCE: National Center for Health Statistics, Third National Health and Nutrition Examination Survey (NHANES III) 
Figure 3-35. Respiratory conditions (past year), farm workers: Prevalence ratio (PR) adjusted for age, sex, and race/ethnicity by smoking status, U.S. residents age 17 and over, 1988-1994

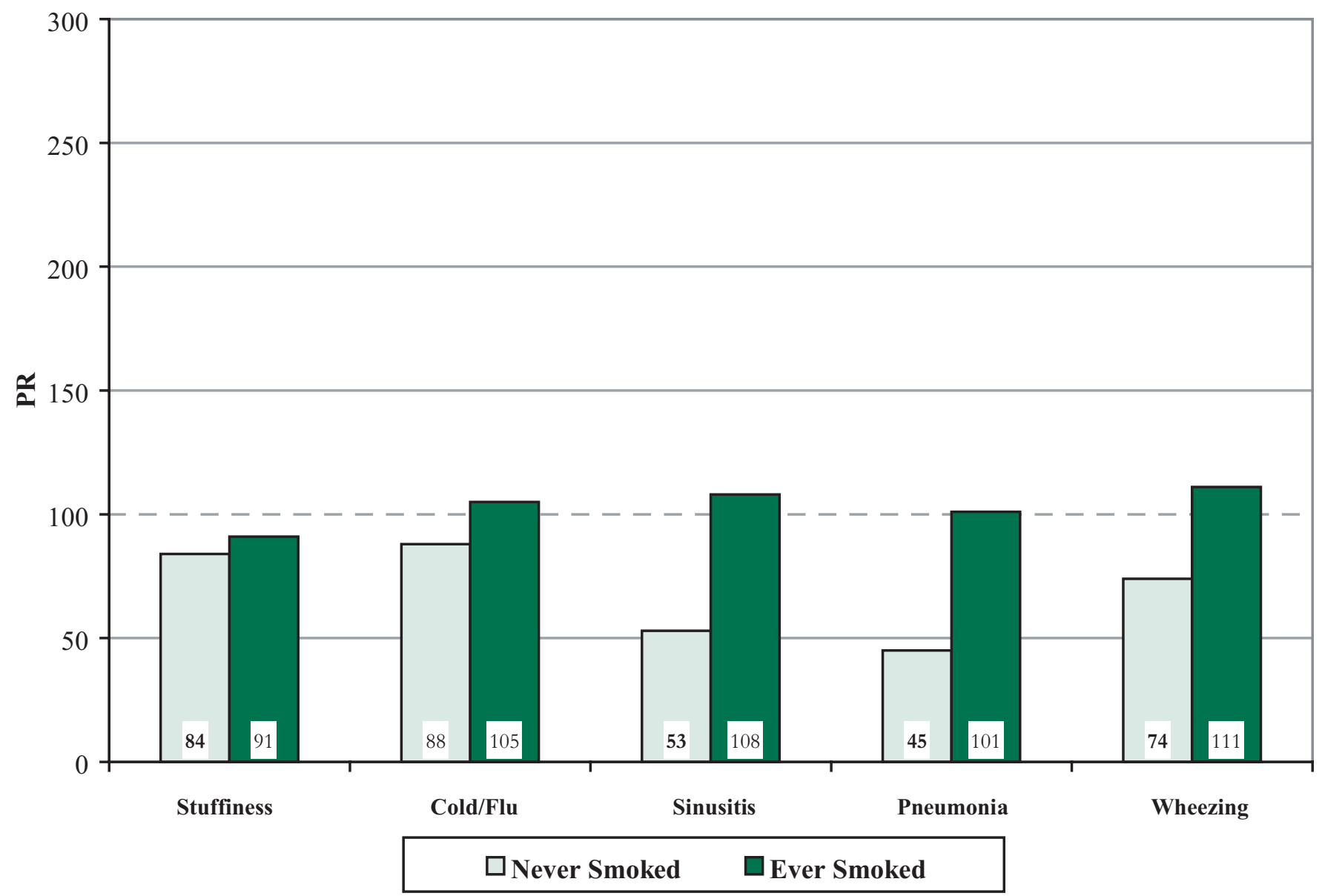

NOTE: Based on responses to the following questions:

"During the past 12 months, have you had any episodes of stuffy, itchy, or runny nose?"

"During the past 12 months, have you had a cold or the flu?"

"During the past 12 months, have you had sinusitis or sinus problems?"

"During the past 12 months, have you had pneumonia?"

"Have you had wheezing or whistling in your chest at any time in the past 12 months?"

PRs in bold are significantly different from $100(\mathrm{p}<0.05)$. PRs in italics are based on fewer than five observed cases. See appendices for source description and methods.

SOURCE: National Center for Health Statistics, Third National Health and Nutrition Examination Survey (NHANES III) 
Figure 3-36. Respiratory conditions (past year), farm managers: Prevalence ratio (PR) adjusted for age, sex, and race/ethnicity by smoking status, U.S. residents age 17 and over, 1988-1994

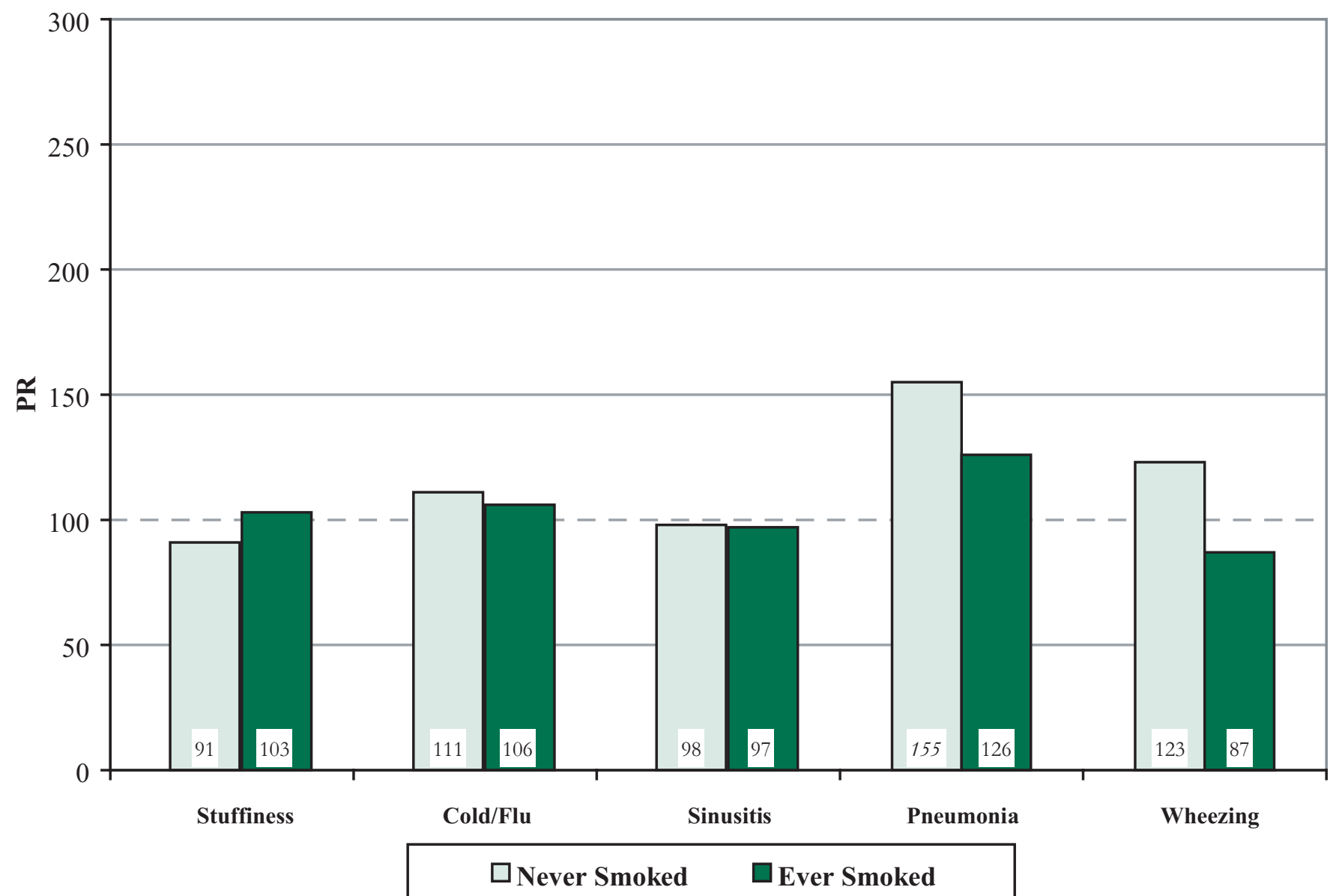

NOTE: Based on responses to the following questions:

"During the past 12 months, have you had any episodes of stuffy, itchy, or runny nose?"

"During the past 12 months, have you had a cold or the flu?"

"During the past 12 months, have you had sinusitis or sinus problems?"

"During the past 12 months, have you had pneumonia?"

"Have you had wheezing or whistling in your chest at any time in the past 12 months?"

PRs in bold are significantly different from $100(\mathrm{p}<0.05)$. PRs in italics are based on fewer than five observed cases. See appendices for source description and methods.

SOURCE: National Center for Health Statistics, Third National Health and Nutrition Examination Survey (NHANES III) 
Figure 3-37. Respiratory conditions (past year), other agricultural workers: Prevalence ratio (PR) adjusted for age, sex, and race/ethnicity by smoking status, U.S. residents age 17 and over, 1988-1994

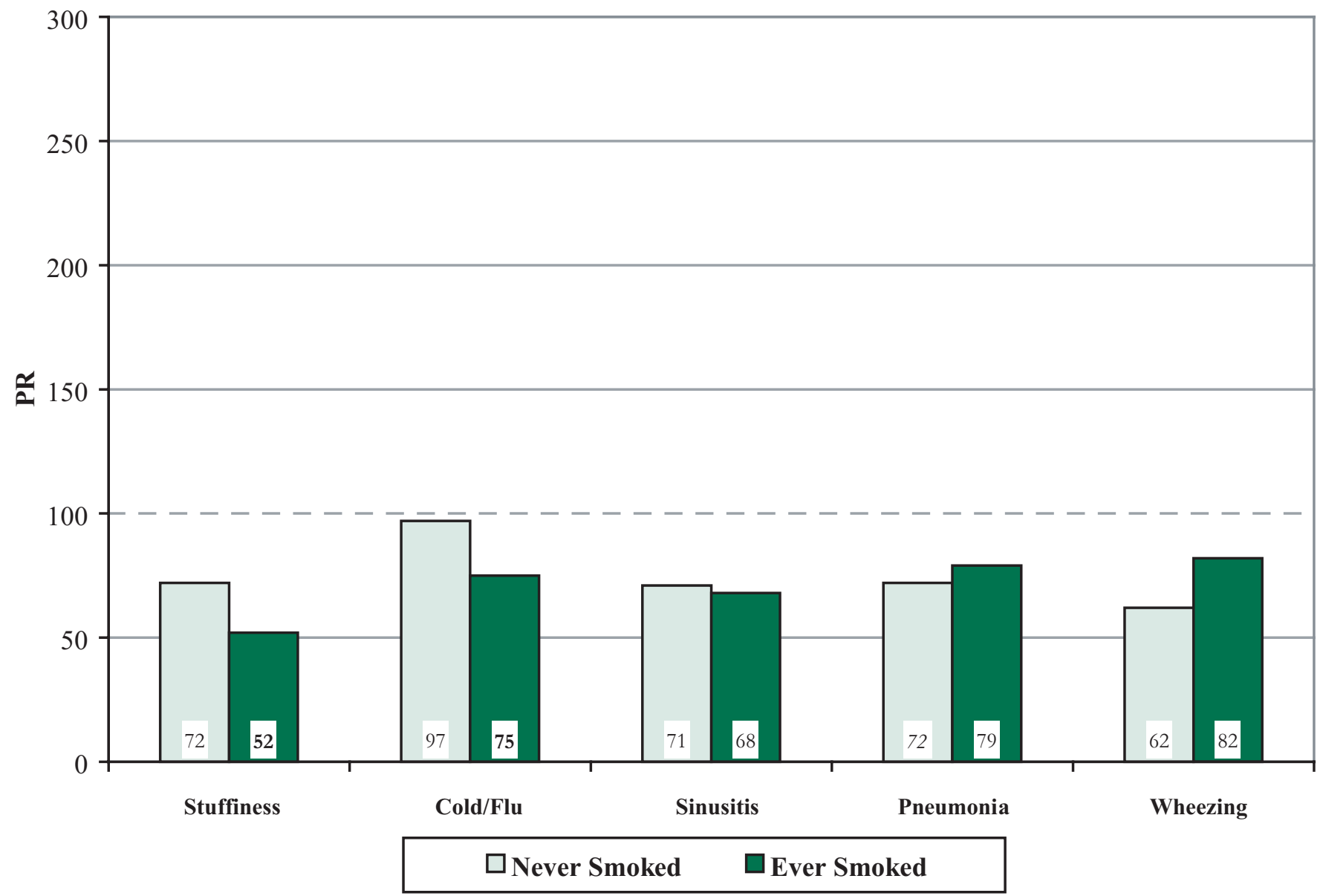

NOTE: Based on responses to the following questions:

"During the past 12 months, have you had any episodes of stuffy, itchy, or runny nose?"

"During the past 12 months, have you had a cold or the flu?"

"During the past 12 months, have you had sinusitis or sinus problems?"

"During the past 12 months, have you had pneumonia?"

"Have you had wheezing or whistling in your chest at any time in the past 12 months?"

PRs in bold are significantly different from $100(\mathrm{p}<0.05)$. PRs in italics are based on fewer than five observed cases. See appendices for source description and methods.

SOURCE: National Center for Health Statistics, Third National Health and Nutrition Examination Survey (NHANES III) 
Figure 3-38. Respiratory conditions (ever), farm workers: Prevalence ratio (PR) adjusted for age, sex, and race/ethnicity by smoking status, U.S. residents age 17 and over, 1988-1994

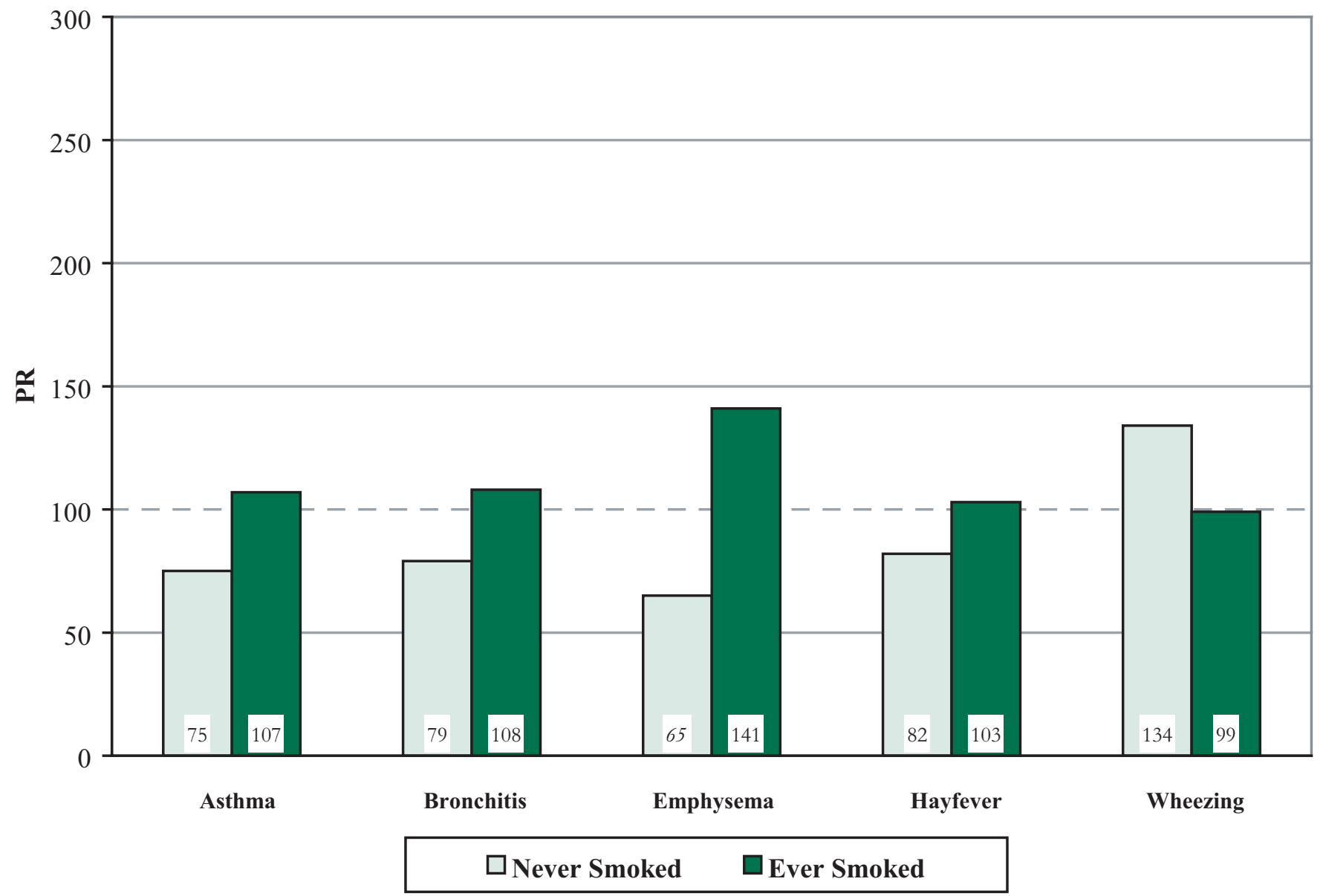

NOTE: Based on responses to the following questions:

"Has a doctor ever told you that you had asthma?"

"Has a doctor ever told you that you had chronic bronchitis?"

"Has a doctor ever told you that you had emphysema?"

"Has a doctor ever told you that you had hay fever?"

"Apart from when you have a cold, does your chest ever sound wheezy or whistling?"

PRs in bold are significantly different from $100(\mathrm{p}<0.05)$. PRs in italics are based on fewer than five observed cases. See appendices for source description and methods.

SOURCE: National Center for Health Statistics, Third National Health and Nutrition Examination Survey (NHANES III) 
Figure 3-39. Respiratory conditions (ever), farm managers: Prevalence ratio (PR) adjusted for age, sex, and race/ethnicity by smoking status, U.S. residents age 17 and over, 1988-1994

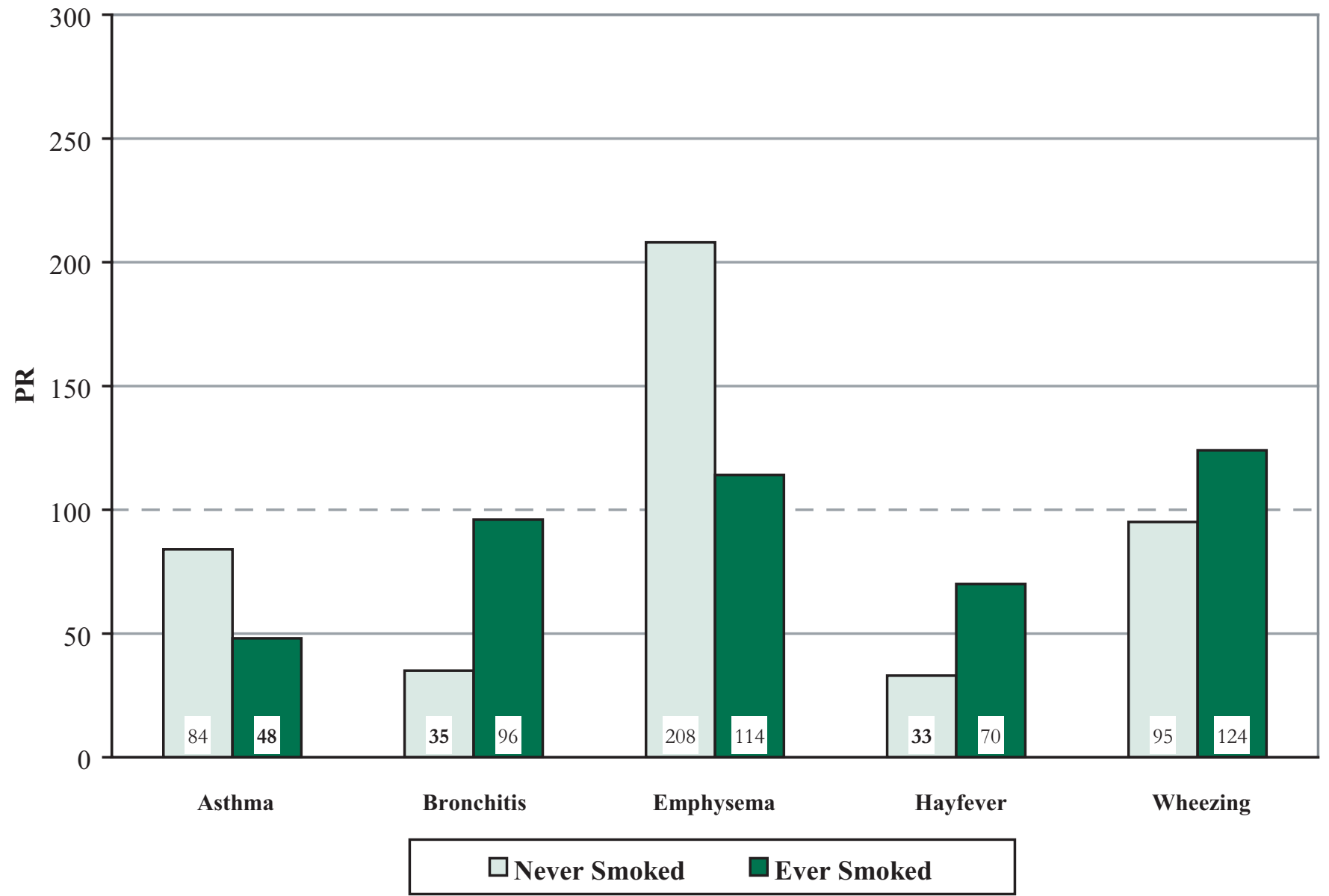

NOTE: Based on responses to the following questions:

"Has a doctor ever told you that you had asthma?"

"Has a doctor ever told you that you had chronic bronchitis?"

"Has a doctor ever told you that you had emphysema?"

"Has a doctor ever told you that you had hay fever?"

"Apart from when you have a cold, does your chest ever sound wheezy or whistling?"

PRs in bold are significantly different from $100(\mathrm{p}<0.05)$. PRs in italics are based on fewer than five observed cases. See appendices for source description and methods.

SOURCE: National Center for Health Statistics, Third National Health and Nutrition Examination Survey (NHANES III) 
Figure 3-40. Respiratory conditions (ever), other agricultural workers: Prevalence ratio (PR) adjusted for age, sex, and race/ethnicity by smoking status, U.S. residents age 17 and over, 1988-1994

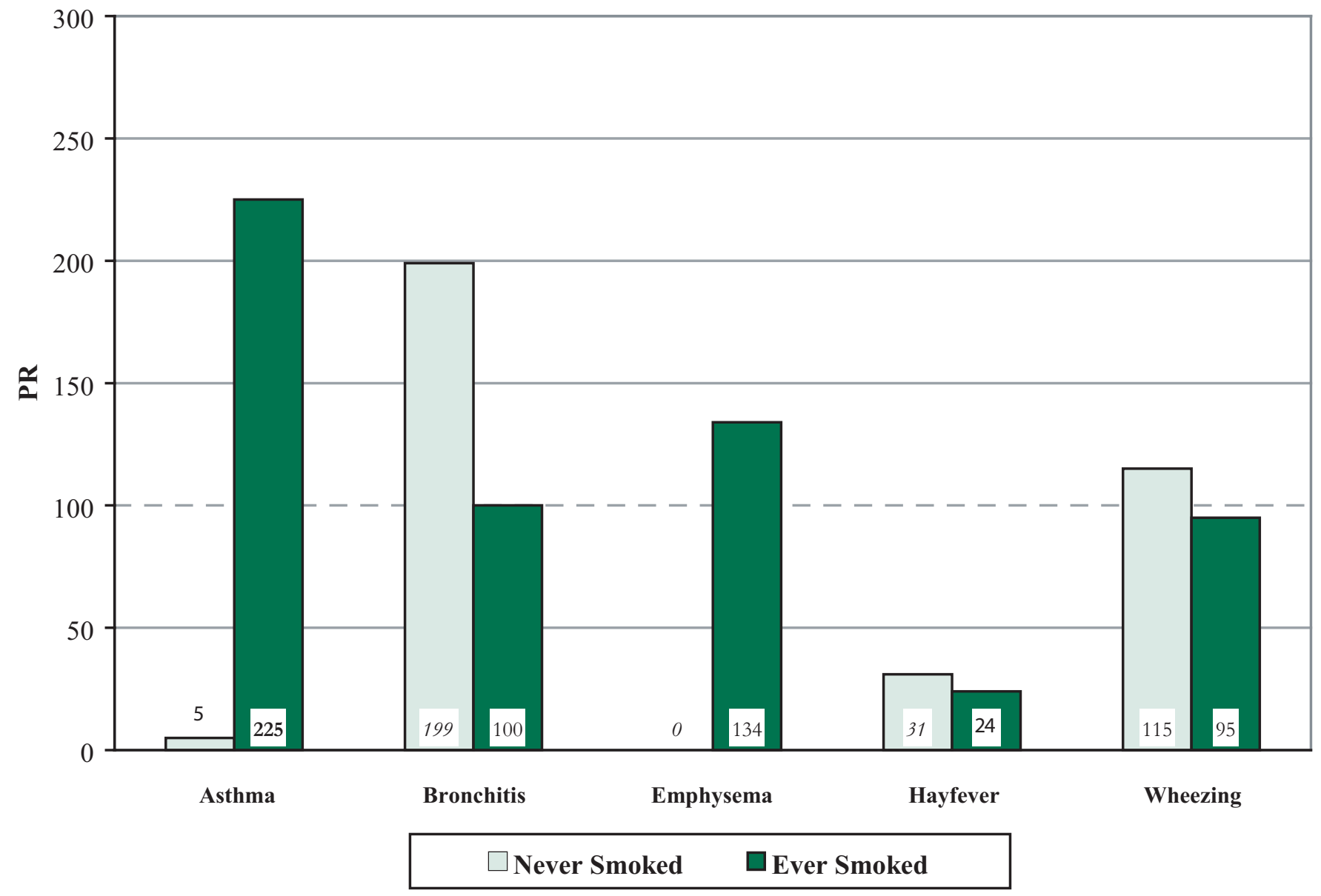

NOTE: Based on responses to the following questions:

"Has a doctor ever told you that you had asthma?"

"Has a doctor ever told you that you had chronic bronchitis?"

"Has a doctor ever told you that you had emphysema?"

"Has a doctor ever told you that you had hay fever?"

"Apart from when you have a cold, does your chest ever sound wheezy or whistling?"

PRs in bold are significantly different from $100(\mathrm{p}<0.05)$. PRs in italics are based on fewer than five observed cases. See appendices for source description and methods.

SOURCE: National Center for Health Statistics, Third National Health and Nutrition Examination Survey (NHANES III) 
Table 3-20. Spirometry: Forced expiratory volume in one second (FEV $)$, forced vital capacity (FVC), and peak expiratory flow (PEF) by agricultural group, U.S. residents age 17 and over, 1988-1994

\begin{tabular}{|c|c|c|c|c|c|c|c|}
\hline \multirow[b]{2}{*}{ Worker Group } & \multirow{2}{*}{$\begin{array}{l}\text { Number } \\
\text { Observed }\end{array}$} & \multicolumn{2}{|c|}{$\mathrm{FEV}_{1}(\mathrm{~L})$} & \multicolumn{2}{|c|}{ FVC (L) } & \multicolumn{2}{|c|}{ PEF (L/sec) } \\
\hline & & Mean & SD & Mean & SD & Mean & SD \\
\hline Farm Workers & 268 & 3.29 & 1.18 & 4.26 & 1.39 & 7.49 & 2.70 \\
\hline Farm Managers & 610 & 3.28 & 1.00 & 4.30 & 1.11 & 7.77 & 2.34 \\
\hline Other Agricultural Workers & 152 & 3.66 & 0.78 & 4.53 & 0.92 & 8.73 & 2.11 \\
\hline All Non-agricultural Workers & 14,811 & 3.22 & 0.97 & 4.07 & 1.14 & 7.60 & 2.32 \\
\hline
\end{tabular}

L - liters $\quad \mathrm{L} / \mathrm{sec}$ - liters per second $\quad$ SD - standard deviation

NOTE: See appendices for source description and methods.

SOURCE: National Center for Health Statistics, Third National Health and Nutrition Examination Survey (NHANES III) 
Table 3-21. Spirometry: Percent predicted forced expiratory volume in one second $\left(\mathrm{FEV}_{1}\right)$, forced vital capacity (FVC), and peak expiratory flow (PEF) by agricultural group, U.S. residents age 17 and over, 1988-1994

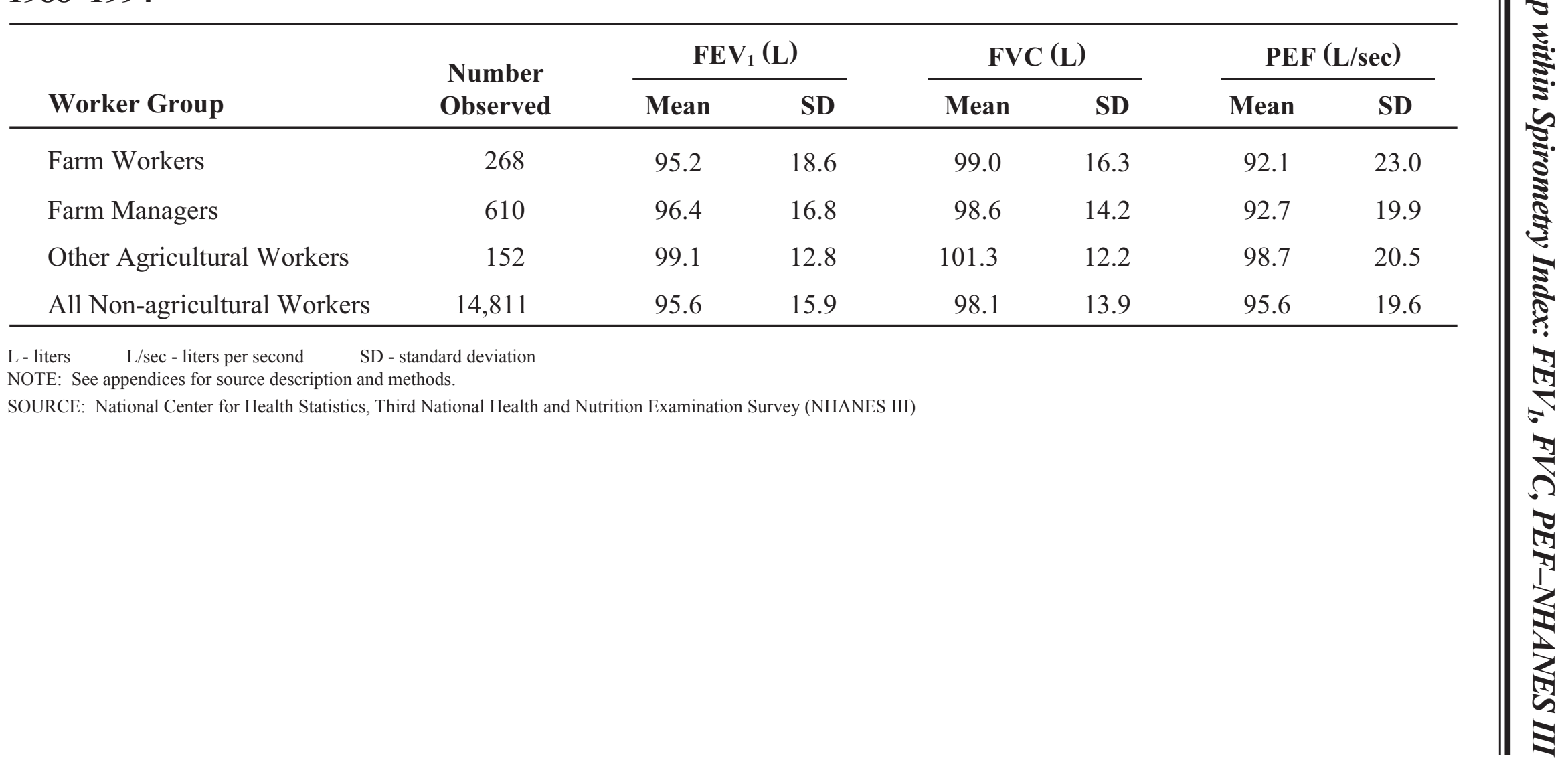


t Table 3-22a. Obstructive abnormality: Estimated prevalence and prevalence ratio (PR) adjusted for age, sex, race/ethnicity, and smoking status by agricultural group, U.S. residents age 17 and over, 1988-1994

\begin{tabular}{|c|c|c|c|c|c|c|}
\hline \multirow[b]{2}{*}{ Worker Group } & \multirow{2}{*}{$\begin{array}{l}\text { Number } \\
\text { Observed }\end{array}$} & \multicolumn{2}{|c|}{$\begin{array}{l}\text { Estimated Prevalence } \\
\text { of Condition in U.S. }\end{array}$} & \multirow[b]{2}{*}{ PR } & \multicolumn{2}{|c|}{$95 \%$ Confidence Interval } \\
\hline & & $\mathbf{n}$ & $(\%)$ & & LCL & $\mathbf{U C L}$ \\
\hline Farm Workers & 110 & 660,225 & 22.5 & 173 & 143 & 209 \\
\hline Farm Managers & 52 & 240,430 & 10.8 & 83 & 63 & 109 \\
\hline Other Agricultural Workers & 19 & 140,157 & 13.1 & 101 & 61 & 158 \\
\hline All Non-agricultural Workers & 1,823 & $20,100,351$ & 13.0 & 100 & & \\
\hline
\end{tabular}

$\mathrm{n}$ - estimated number $\quad$ LCL - lower confidence limit $\quad$ UCL - upper confidence limit

NOTE: Estimated number in U.S., estimated percent with condition, and PR are based on weighted sample results. PRs in bold are significantly different from 100 ( $\mathrm{p}<0.05$ ). PRs in italics are based on fewer than five observed cases. See appendices for source description and methods.

SOURCE: National Center for Health Statistics, Third National Health and Nutrition Examination Survey (NHANES III)

Table 3-22b. Restrictive abnormality: Estimated prevalence and prevalence ratio (PR) adjusted for age, sex, race/ethnicity, and smoking status by agricultural group, U.S. residents age 17 and over, 1988-1994

\begin{tabular}{|c|c|c|c|c|c|c|}
\hline \multirow[b]{2}{*}{ Worker Group } & \multirow{2}{*}{$\begin{array}{l}\text { Number } \\
\text { Observed }\end{array}$} & \multicolumn{2}{|c|}{$\begin{array}{l}\text { Estimated Prevalence } \\
\text { of Condition in U.S. }\end{array}$} & \multirow[b]{2}{*}{ PR } & \multicolumn{2}{|c|}{ 95\% Confidence Interval } \\
\hline & & $\mathbf{n}$ & $(\%)$ & & LCL & $\overline{\mathrm{UCL}}$ \\
\hline Farm Workers & 48 & 165,352 & 5.6 & 80 & 59 & 106 \\
\hline Farm Managers & 20 & 117,745 & 5.3 & 75 & 46 & 116 \\
\hline Other Agricultural Workers & 6 & 27,978 & 2.6 & 37 & 14 & 81 \\
\hline All Non-agricultural Workers & 1,024 & $10,906,623$ & 7.1 & 100 & & \\
\hline
\end{tabular}

n - estimated number $\quad$ LCL - lower confidence limit $\quad$ UCL - upper confidence limit

NOTE: Estimated number in U.S., estimated percent with condition, and PR are based on weighted sample results. PRs in bold are significantly different from 100 ( $\mathrm{p}<0.05$ ). PRs in italics are based on fewer than five observed cases. See appendices for source description and methods.

SOURCE: National Center for Health Statistics, Third National Health and Nutrition Examination Survey (NHANES III) 
Figure 3-41. Percent predicted forced expiratory volume in one second $\left(F_{E} V_{1}\right)$ by agricultural group and sex, U.S. residents age 17 and over, 1988-1994

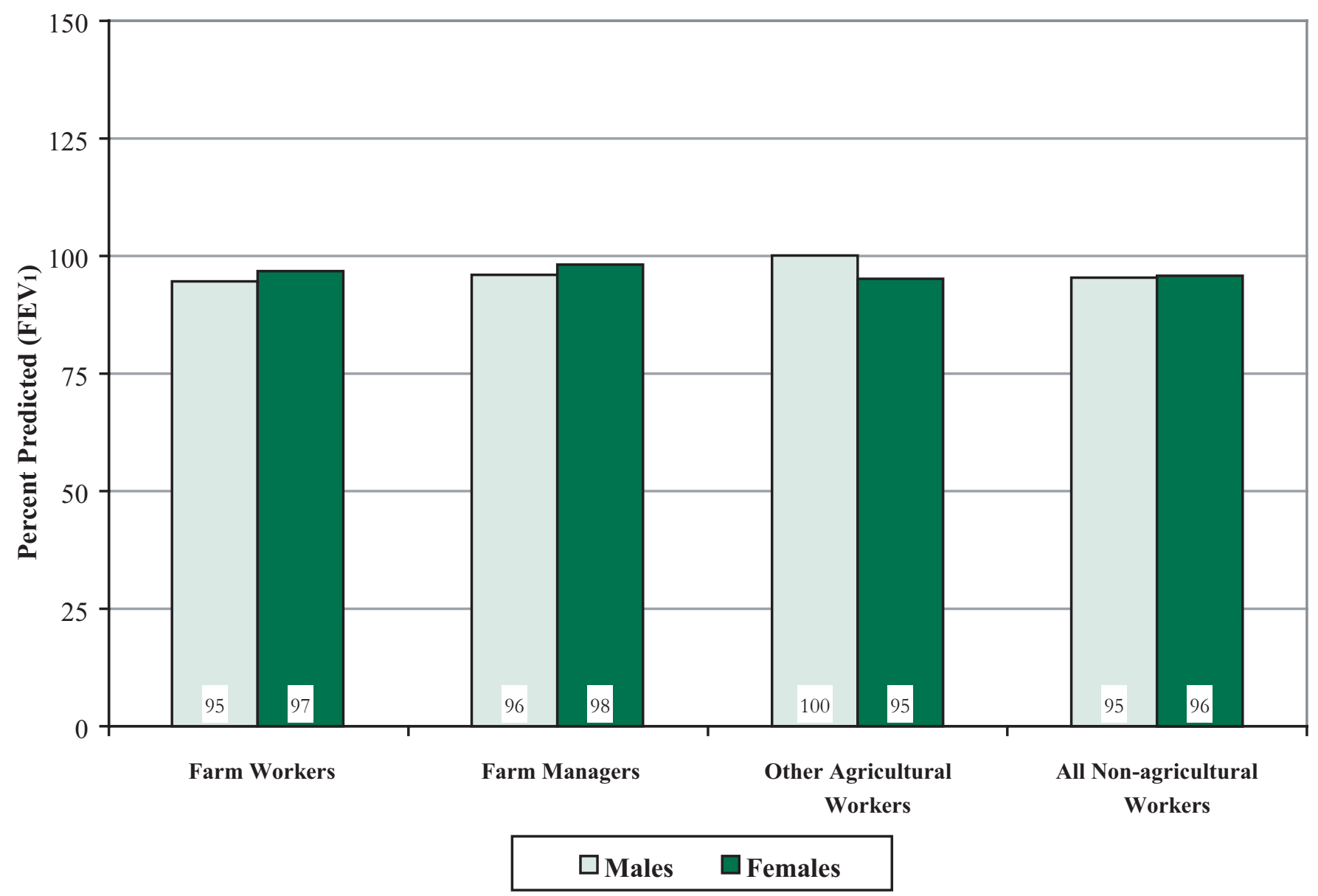

NOTE: See appendices for source description and methods.

SOURCE: National Center for Health Statistics, Third National Health and Nutrition Examination Survey (NHANES III) 
Figure 3-42. Percent predicted forced vital capacity (FVC) by agricultural group and sex, U.S. residents age 17 and over, 1988-1994

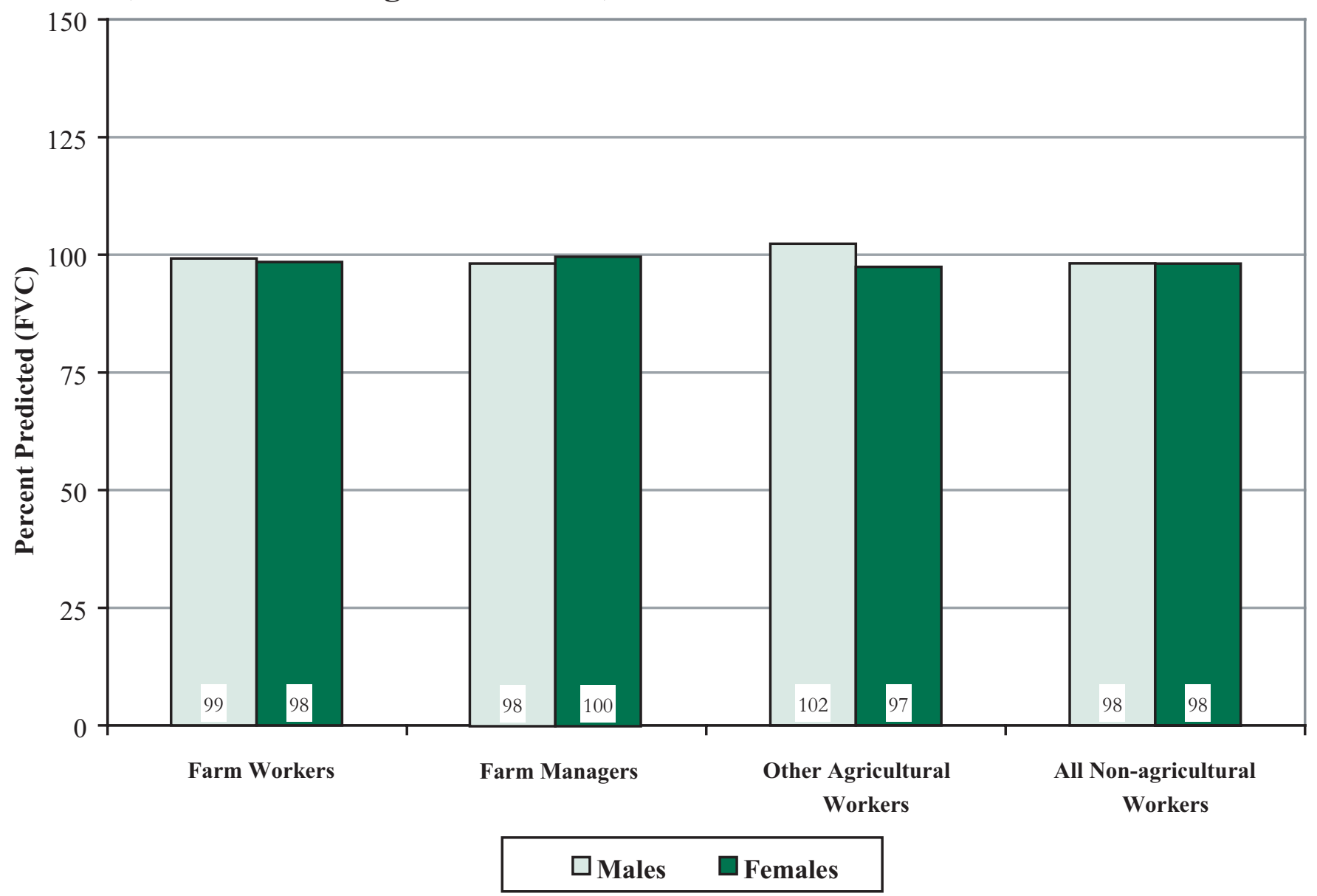

NOTE: See appendices for source description and methods.

SOURCE: National Center for Health Statistics, Third National Health and Nutrition Examination Survey (NHANES III) 
Figure 3-43. Percent predicted peak expiratory flow (PEF) by agricultural group and sex, U.S. residents age 17 and over, 1988-1994

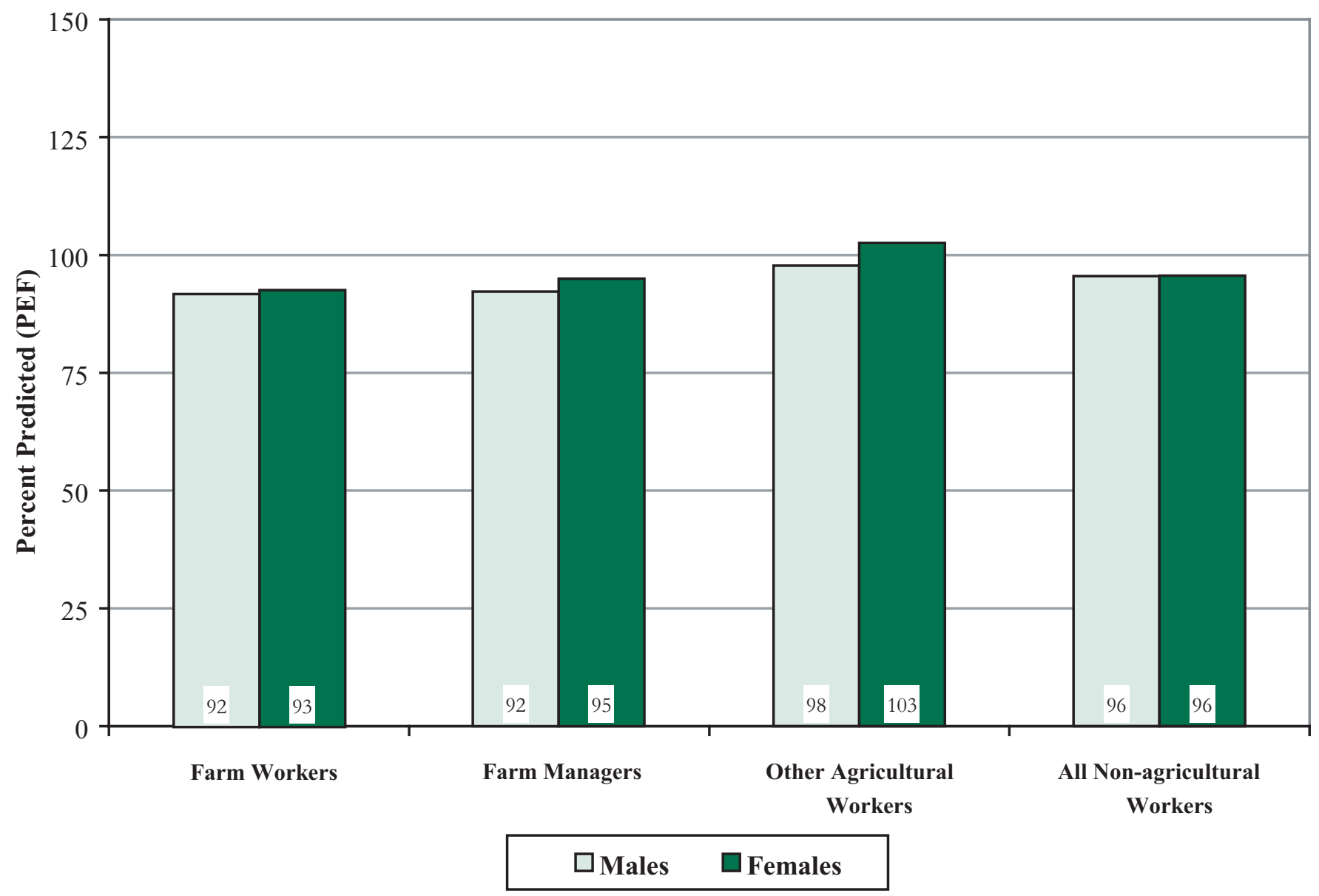

NOTE: See appendices for source description and methods.

SOURCE: National Center for Health Statistics, Third National Health and Nutrition Examination Survey (NHANES III) 
Figure 3-44. Percent predicted forced expiratory volume in one second $\left(F E V_{1}\right)$ by agricultural group and race/ethnicity, U.S. residents age 17 and over, 1988-1994

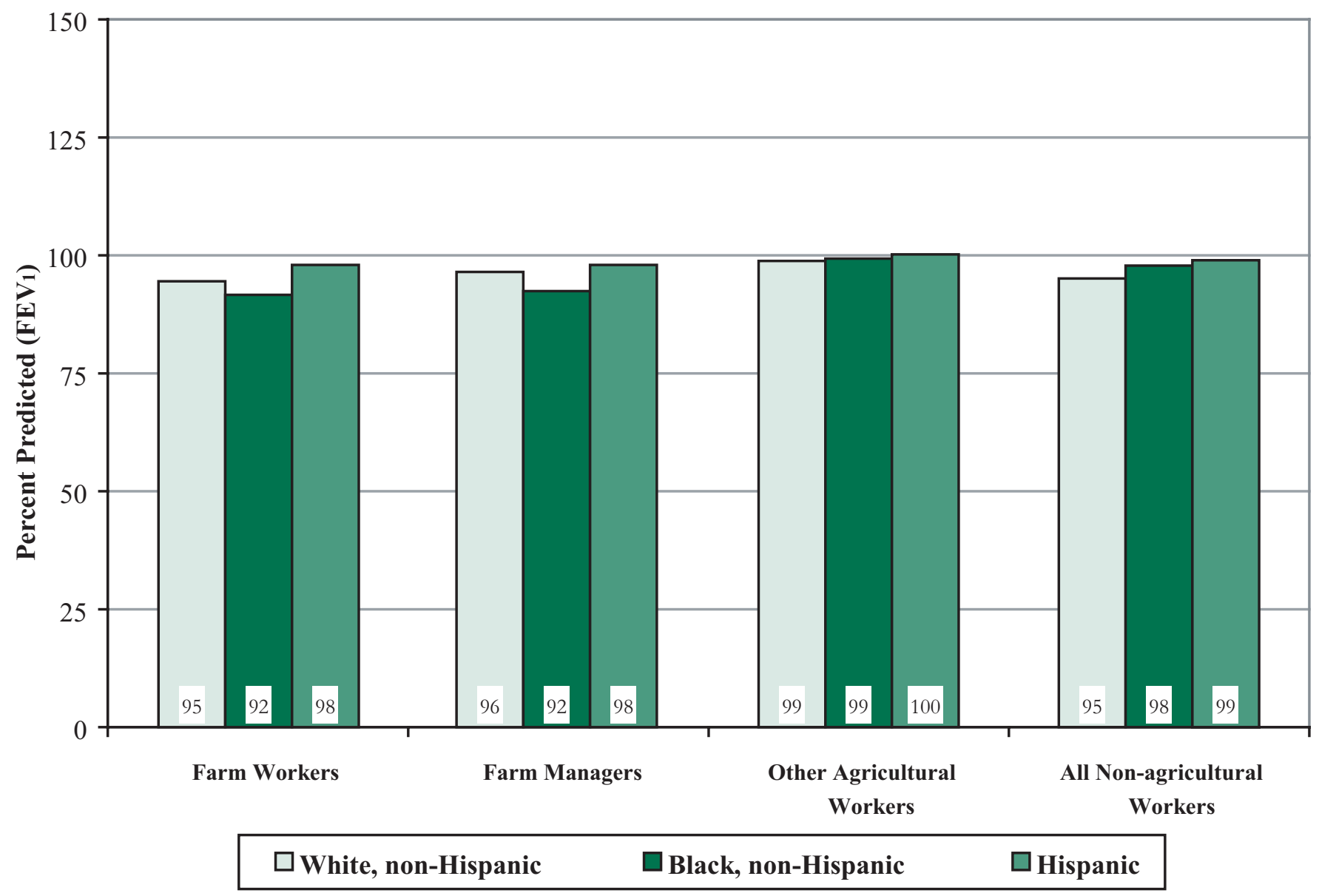

NOTE: See appendices for source description and methods.

SOURCE: National Center for Health Statistics, Third National Health and Nutrition Examination Survey (NHANES III) 
Figure 3-45. Percent predicted forced vital capacity (FVC) by agricultural group and race/ethnicity, U.S. residents age 17 and over, 1988-1994

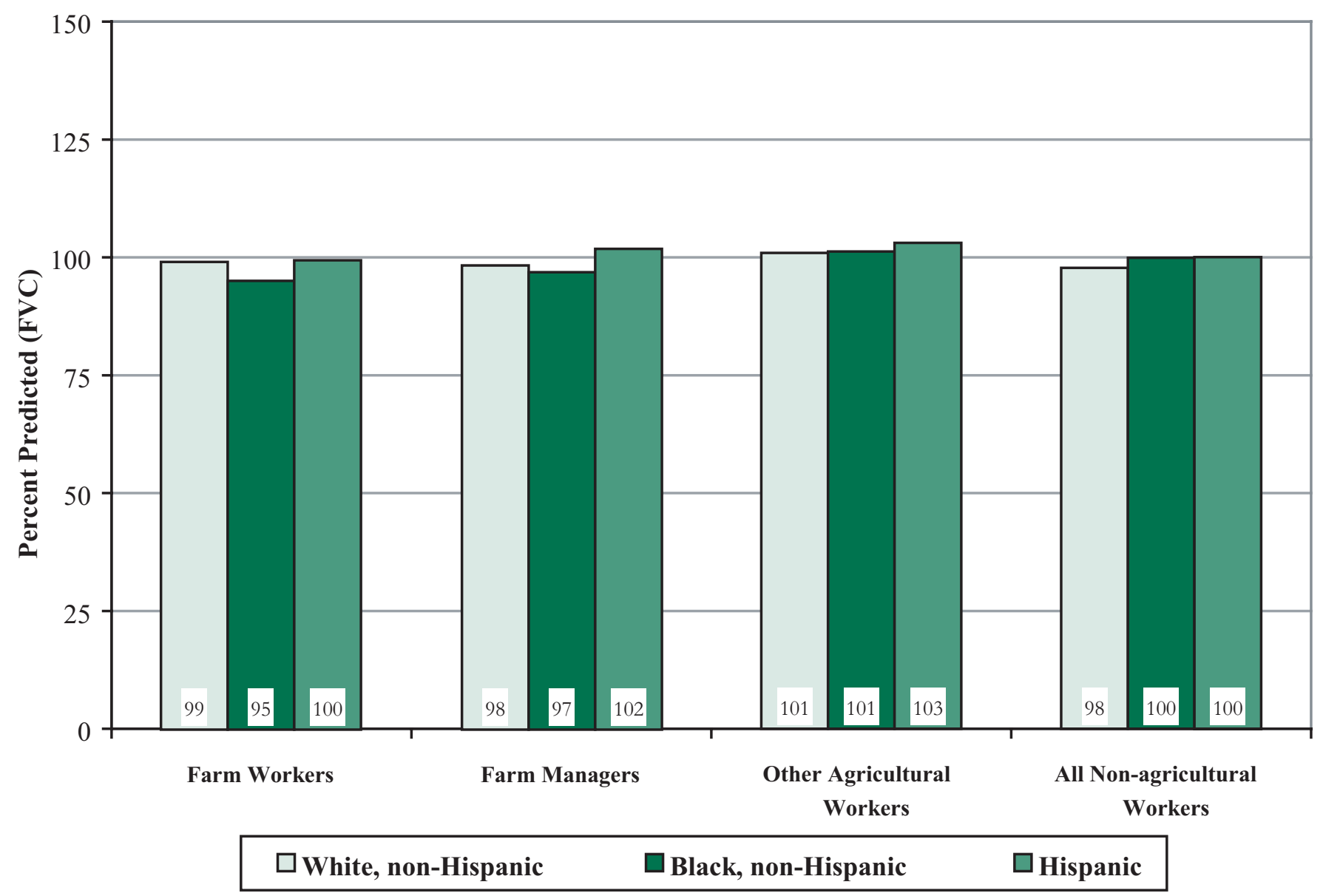

NOTE: See appendices for source description and methods.

SOURCE: National Center for Health Statistics, Third National Health and Nutrition Examination Survey (NHANES III) 
Figure 3-46. Percent predicted peak expiratory flow (PEF) by agricultural group and race/ethnicity, U.S. residents age 17 and over, 1988-1994

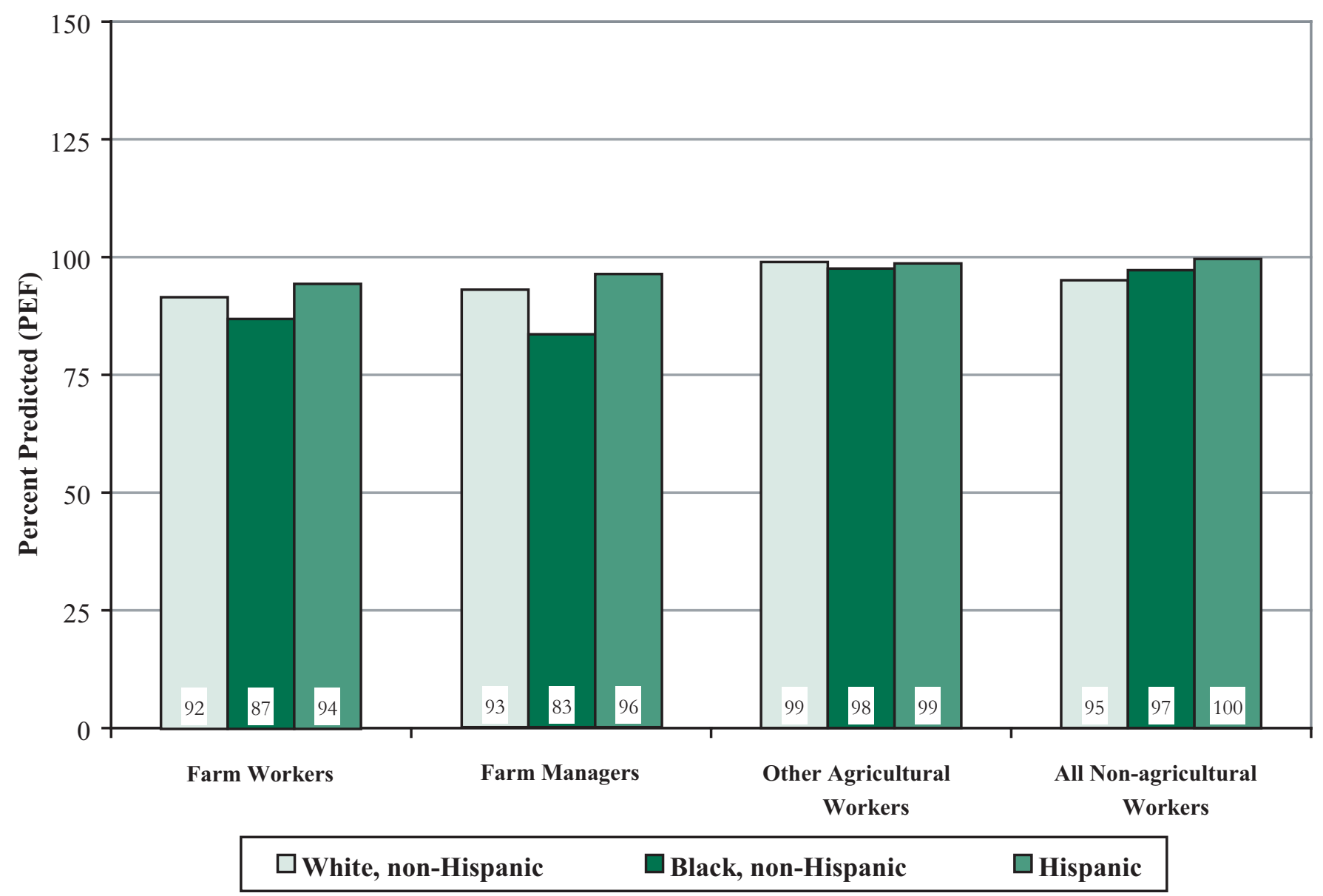

NOTE: See appendices for source description and methods.

SOURCE: National Center for Health Statistics, Third National Health and Nutrition Examination Survey (NHANES III) 
Figure 3-47. Percent predicted forced expiratory volume in one second $\left(\mathrm{FEV}_{1}\right)$ by agricultural group and smoking status, U.S. residents age 17 and over, 1988-1994

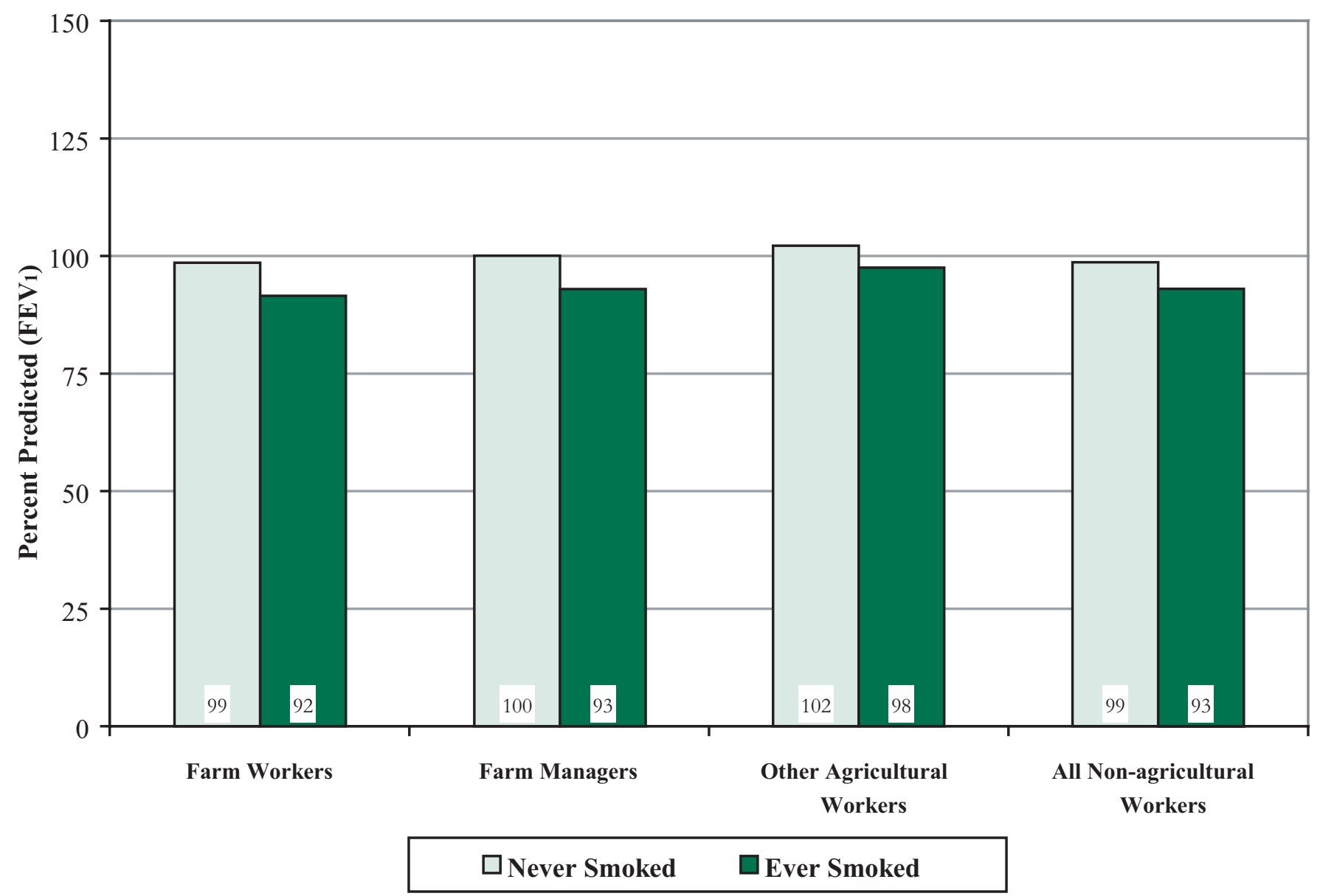

NOTE: See appendices for source description and methods.

SOURCE: National Center for Health Statistics, Third National Health and Nutrition Examination Survey (NHANES III) 
Figure 3-48. Percent predicted forced vital capacity (FVC) by agricultural group and smoking status, U.S. residents age 17 and over, 1988-1994

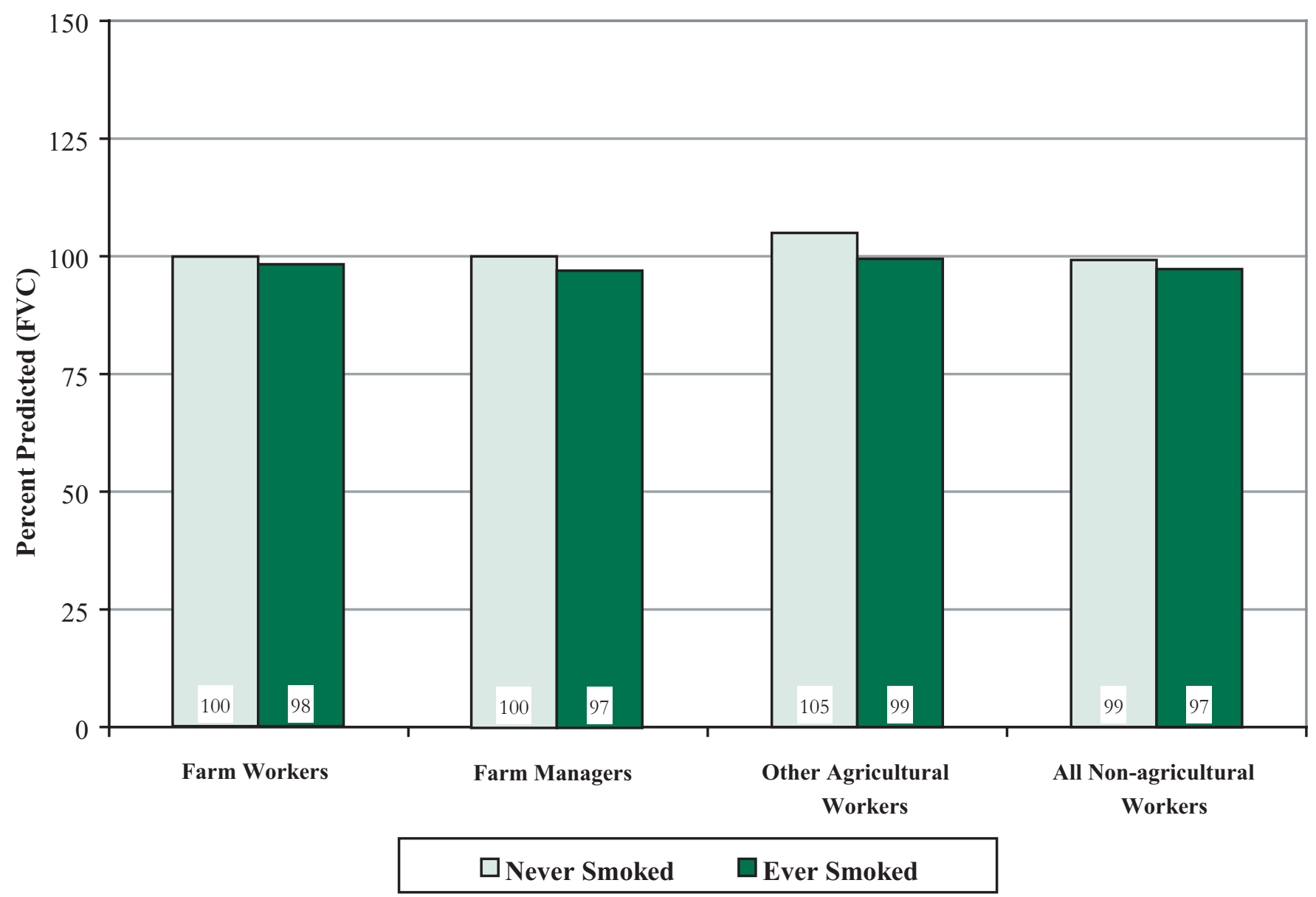

NOTE: See appendices for source description and methods.

SOURCE: National Center for Health Statistics, Third National Health and Nutrition Examination Survey (NHANES III) 
Figure 3-49. Percent predicted peak expiratory flow (PEF) by agricultural group and smoking status, U.S. residents age 17 and over, 1988-1994

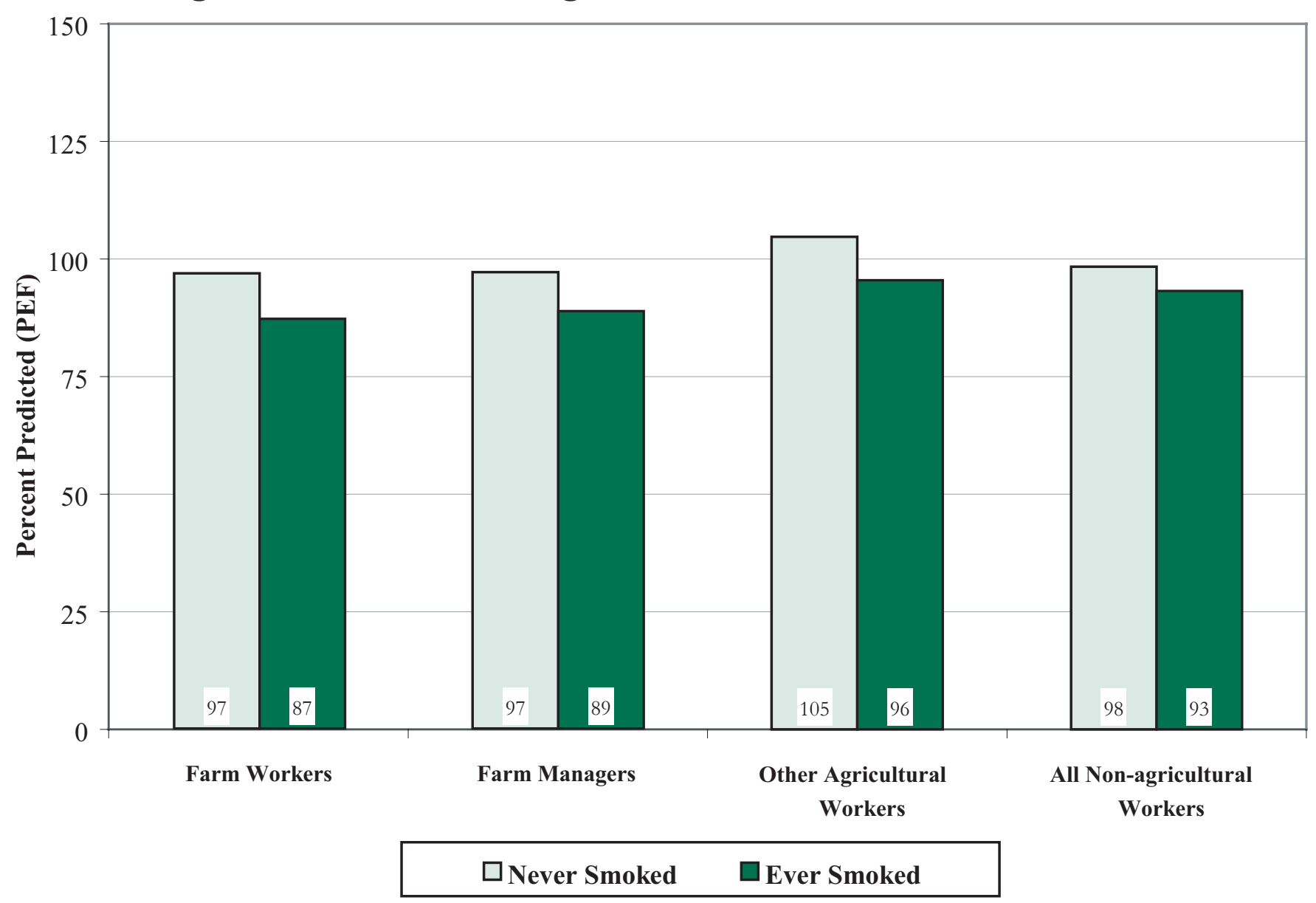

NOTE: See appendices for source description and methods.

SOURCE: National Center for Health Statistics, Third National Health and Nutrition Examination Survey (NHANES III) 
Figure 3-50. Spirometry, obstructive abnormality: Prevalence ratio (PR) adjusted for age and race/ethnicity by agricultural group and sex, U.S. residents age 17 and over, 1988-1994

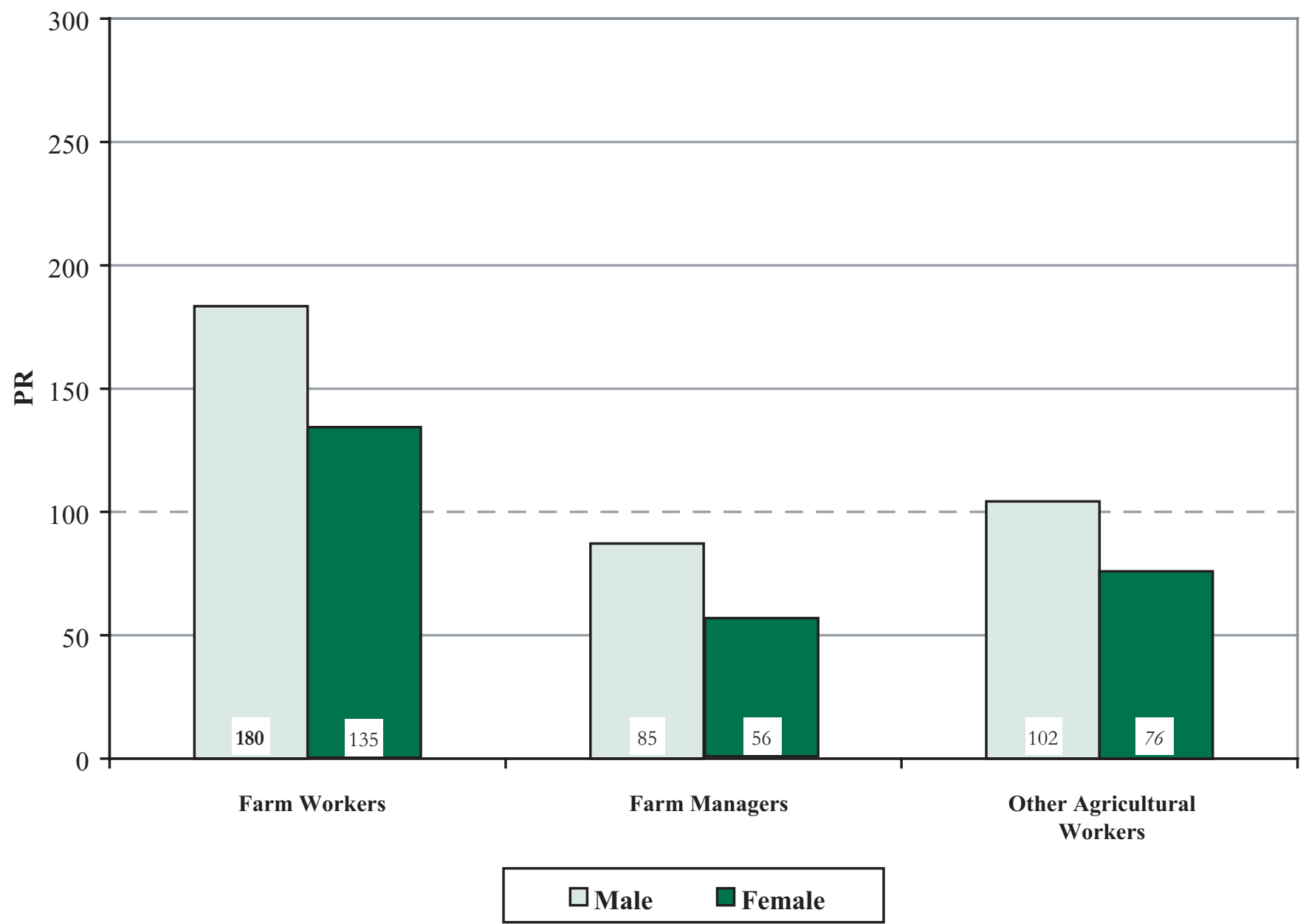

NOTE: PRs in bold are significantly different from $100(\mathrm{p}<0.05)$. PRs in italics are based on fewer than five observed cases. See appendices for source description and methods.

SOURCE: National Center for Health Statistics, Third National Health and Nutrition Examination Survey (NHANES III) 
Figure 3-51. Spirometry, restrictive abnormality: Prevalence ratio (PR) adjusted for age and race/ethnicity by agricultural group and sex, U.S. residents age 17 and over, 1988-1994

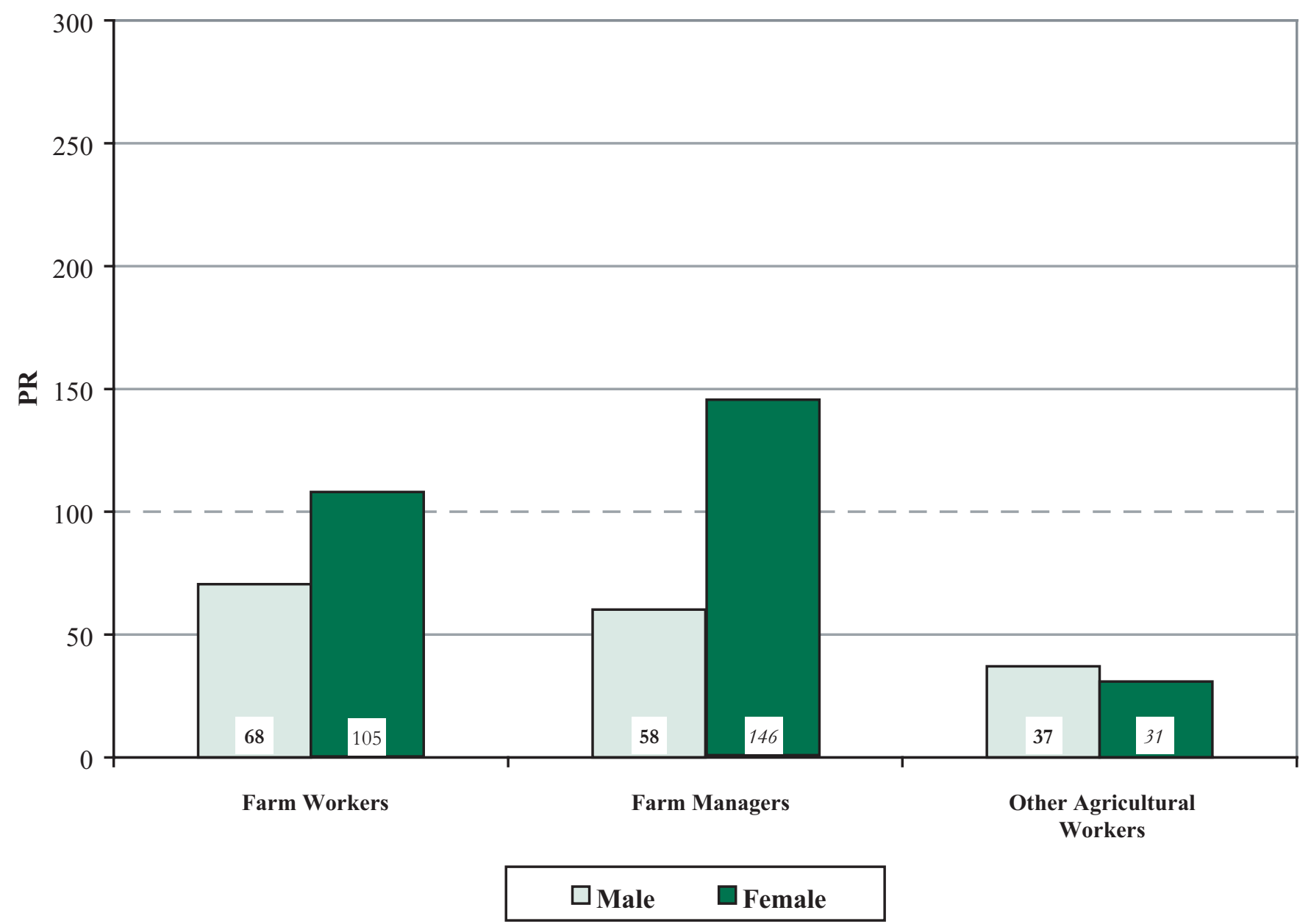

NOTE: PRs in bold are significantly different from $100(\mathrm{p}<0.05)$. PRs in italics are based on fewer than five observed cases. See appendices for source description and methods.

SOURCE: National Center for Health Statistics, Third National Health and Nutrition Examination Survey (NHANES III) 
Figure 3-52. Spirometry, obstructive abnormality: Prevalence ratio (PR) adjusted for age and sex by agricultural group and race/ethnicity, U.S. residents age 17 and over, 1988-1994

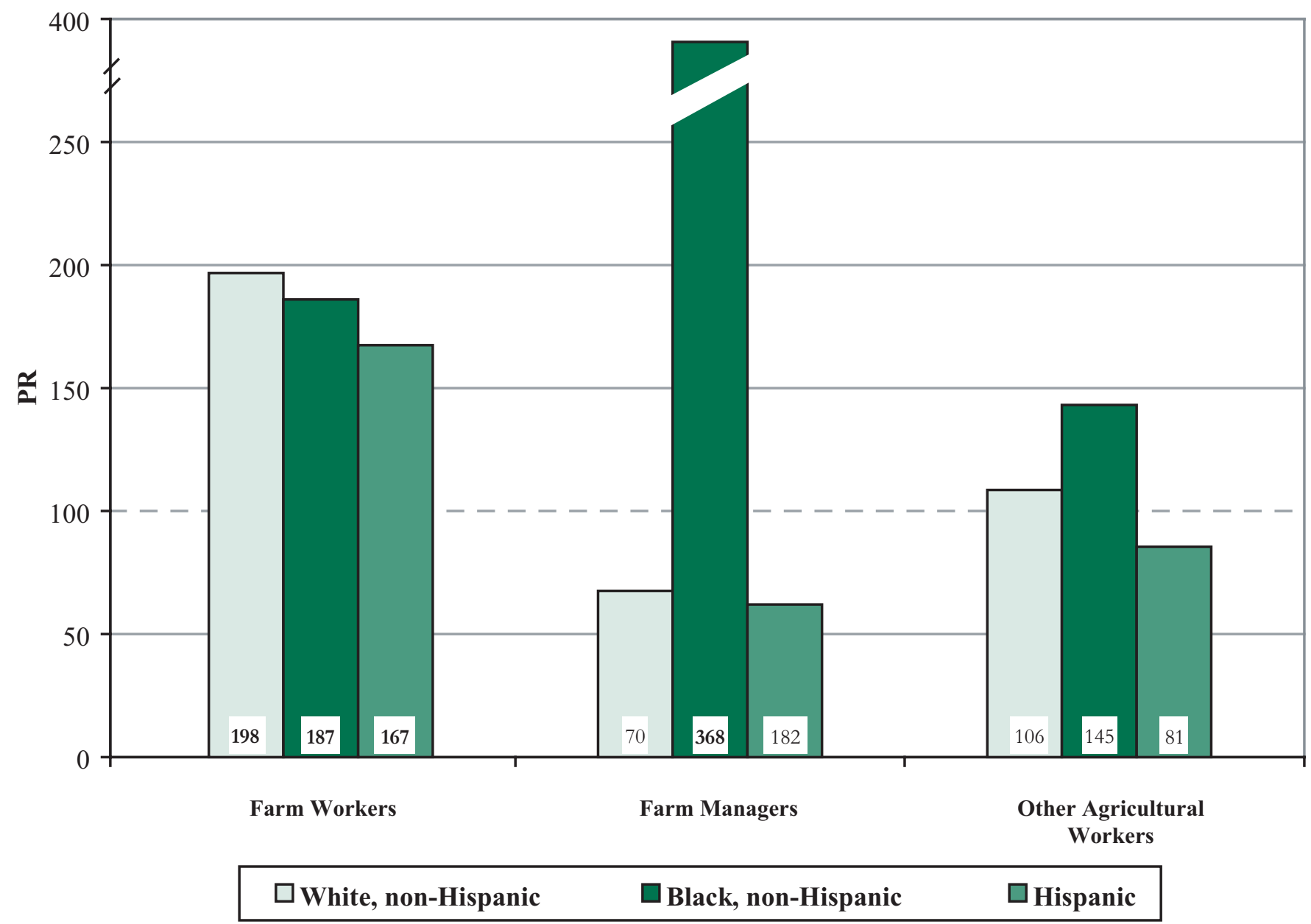

NOTE: PRs in bold are significantly different from $100(\mathrm{p}<0.05)$. PRs in italics are based on fewer than five observed cases. See appendices for source description and methods.

SOURCE: National Center for Health Statistics, Third National Health and Nutrition Examination Survey (NHANES III) 
Figure 3-53. Spirometry, restrictive abnormality: Prevalence ratio (PR) adjusted for age and sex by agricultural group and race/ethnicity, U.S. residents age 17 and over, 1988-1994

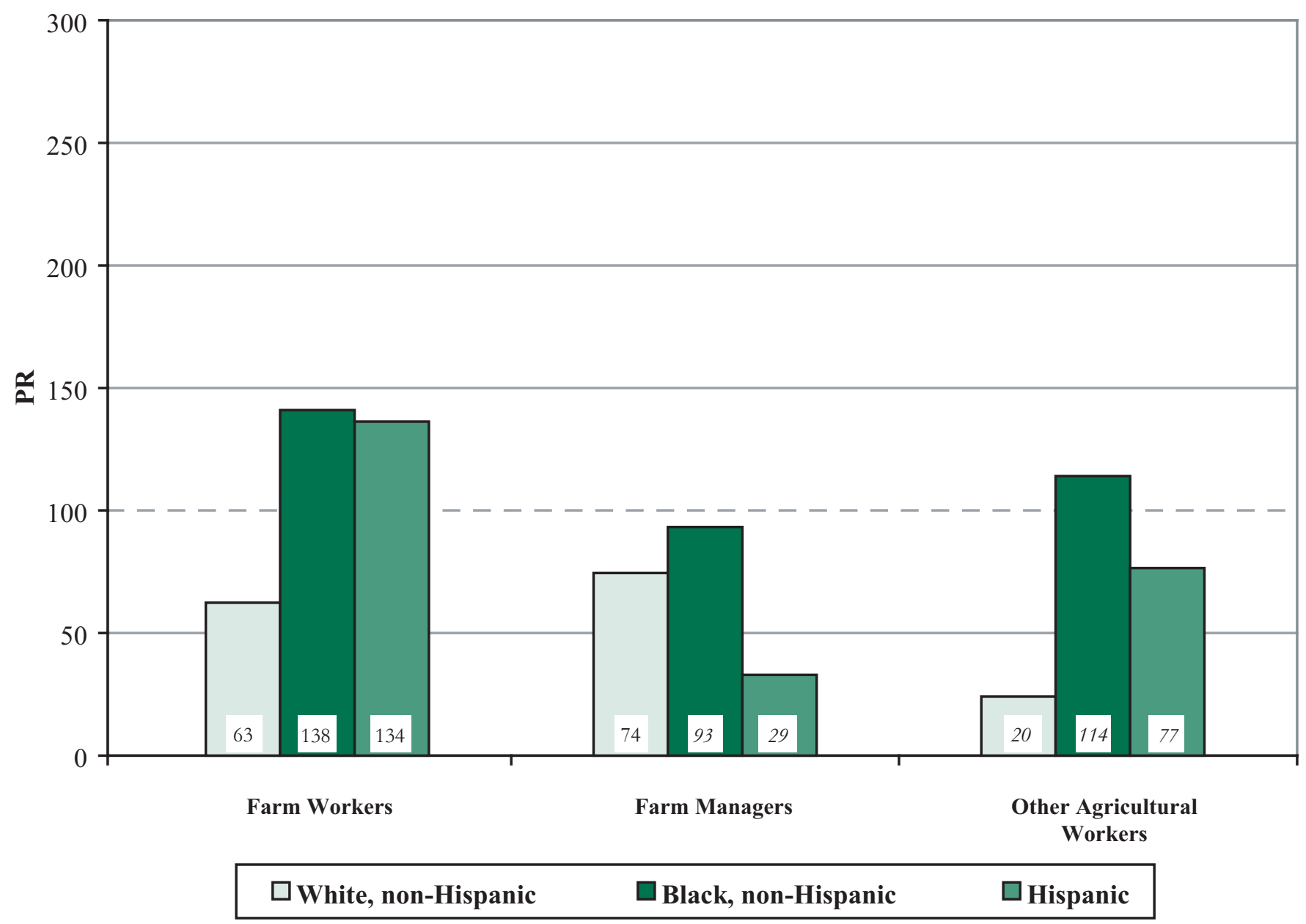

NOTE: PRs in bold are significantly different from $100(\mathrm{p}<0.05)$. PRs in italics are based on fewer than five observed cases. See appendices for source description and methods.

SOURCE: National Center for Health Statistics, Third National Health and Nutrition Examination Survey (NHANES III) 
Figure 3-54. Spirometry, obstructive abnormality: Prevalence ratio (PR) adjusted for age, sex, and race/ethnicity by agricultural group and smoking status, U.S. residents age 17 and over, 1988-1994

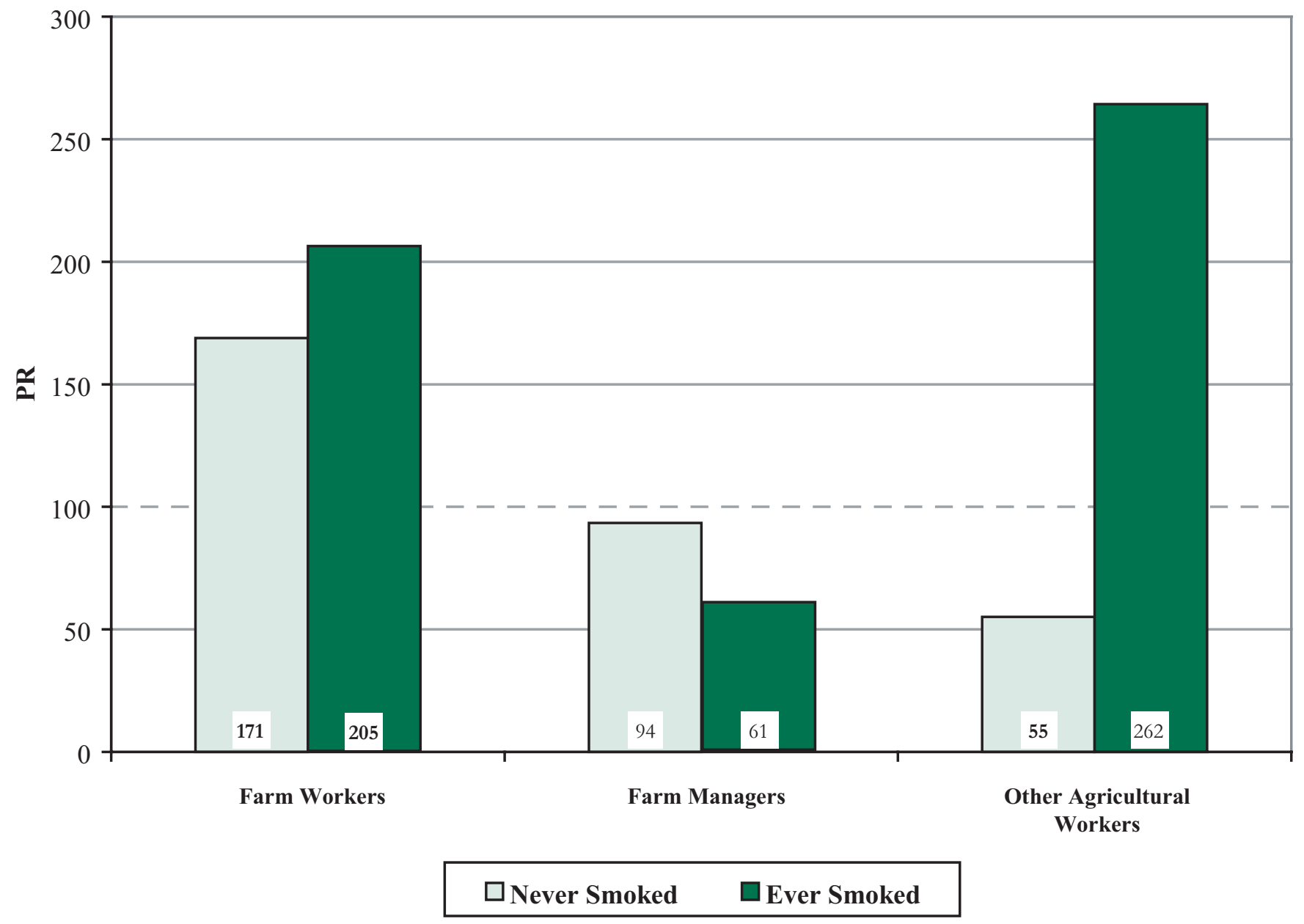

NOTE: PRs in bold are significantly different from $100(\mathrm{p}<0.05)$. PRs in italics are based on fewer than five observed cases. See appendices for source description and methods.

SOURCE: National Center for Health Statistics, Third National Health and Nutrition Examination Survey (NHANES III) 
Figure 3-55. Spirometry, restrictive abnormality: Prevalence ratio (PR) adjusted for age, sex, and race/ethnicity by agricultural group and smoking status, U.S. residents age 17 and over, 1988-1994

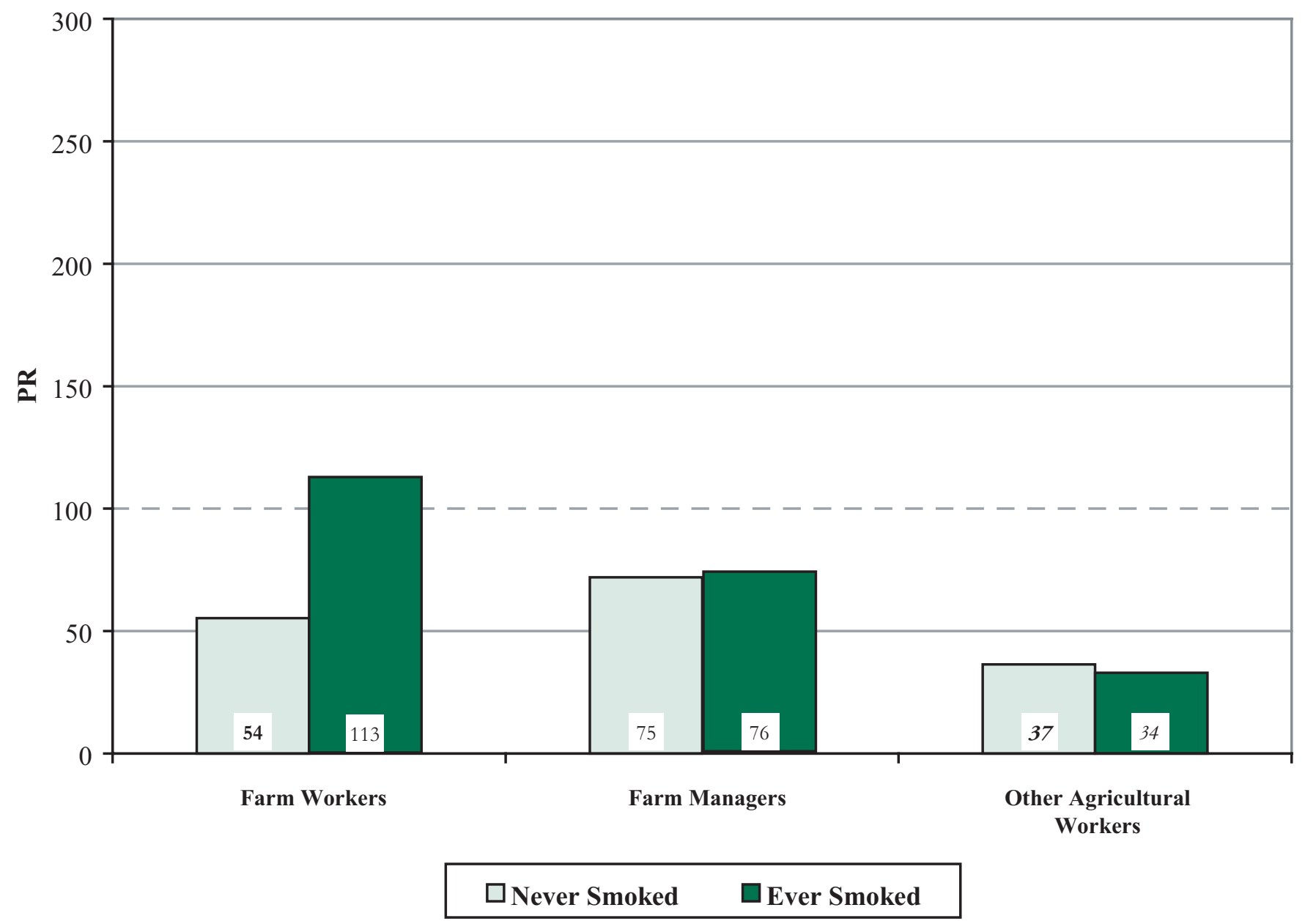

NOTE: PRs in bold are significantly different from $100(\mathrm{p}<0.05)$. PRs in italics are based on fewer than five observed cases. See appendices for source description and methods.

SOURCE: National Center for Health Statistics, Third National Health and Nutrition Examination Survey (NHANES III) 
Table 3-23. Dust diseases of the lung: Estimated incidence per 10,000 workers by agricultural group, 1995-2001

\begin{tabular}{lrrrrrrr}
\hline Agricultural Group & $\mathbf{1 9 9 5}$ & $\mathbf{1 9 9 6}$ & $\mathbf{1 9 9 7}$ & $\mathbf{1 9 9 8}$ & $\mathbf{1 9 9 9}$ & $\mathbf{2 0 0 0}$ & $\mathbf{2 0 0 1}$ \\
\hline Agriculture/Forestry/Fishing* & 0.1 & 0.1 & 0.3 & 0.2 & 0.2 & 0.2 & 0.1 \\
$\quad$ Agricultural Production* & 0.2 & 0.1 & 0.4 & 0.4 & 0.3 & $<0.05$ & 0.2 \\
$\quad$ Agricultural Production, Crops* & 0.2 & 0.1 & 0.3 & 0.3 & 0.5 & $<0.05$ & 0.2 \\
$\quad$ Agricultural Production, Livestock* & 0.2 & 0.1 & 0.7 & 0.9 & $<0.05$ & $<0.05$ & 0.3 \\
Landscape/Horticultural Services & $<0.05$ & $<0.05$ & 0.3 & $<0.05$ & 0.1 & $<0.05$ & $<0.05$ \\
$\quad$ Forestry & $<0.05$ & $<0.05$ & $<0.05$ & $<0.05$ & $<0.05$ & 10.2 & $<0.05$ \\
$\quad$ Fishing/Hunting/Trapping & $<0.05$ & $<0.05$ & 7.8 & $<0.05$ & $<0.05$ & $<0.05$ & $<0.05$ \\
All Private Industry* & 0.3 & 0.4 & 0.3 & 0.2 & 0.2 & 0.2 & 0.1 \\
\hline
\end{tabular}

*Excludes farms with less than 11 employees.

SOURCE: Bureau of Labor Statistics: Survey of Occupational Injuries and Illnesses 
Table 3-24. Respiratory conditions due to toxic agents: Estimated incidence per 10,000 workers by agricultural group, 1995-2001

\begin{tabular}{lrrrrrrr}
\hline Agricultural Group & $\mathbf{1 9 9 5}$ & $\mathbf{1 9 9 6}$ & $\mathbf{1 9 9 7}$ & $\mathbf{1 9 9 8}$ & $\mathbf{1 9 9 9}$ & $\mathbf{2 0 0 0}$ & $\mathbf{2 0 0 1}$ \\
\hline Agriculture/Forestry/Fishing* & 1.4 & 1.7 & 2.7 & 3.7 & 1.8 & 0.8 & 0.4 \\
Agricultural Production* & 1.8 & 1.9 & 3.0 & 4.4 & 1.6 & 1.2 & 0.9 \\
$\quad$ Agricultural Production, Crops* & 1.8 & 1.5 & 3.2 & 2.3 & 1.4 & 0.6 & 0.6 \\
$\quad$ Agricultural Production, Livestock* & 2.1 & 3.1 & 2.1 & 11.2 & 1.9 & 3.0 & 1.7 \\
Landscape/Horticultural Services & 0.5 & $<0.05$ & 0.7 & 2.4 & 1.3 & 0.2 & $<0.05$ \\
Forestry & $<0.05$ & $<0.05$ & $<0.05$ & $<0.05$ & 1.8 & 1.6 & 0.8 \\
Fishing/Hunting/Trapping & 3.3 & 7.4 & 15.7 & 5.4 & $<0.05$ & 2.4 & $<0.05$ \\
All Private Industry* & 3.0 & 2.6 & 2.4 & 2.0 & 1.8 & 1.6 & 1.6 \\
\hline
\end{tabular}

*Excludes farms with less than 11 employees.

SOURCE: Bureau of Labor Statistics: Survey of Occupational Injuries and Illnesses 



\section{Section 4}

Recommendations for Future Studies 



\section{Recommendations for Future Studies}

As noted in the Limitations section of this report, the results in this report are subject to various constraints on their interpretation. The following are recommendations for future study that would help fill gaps and improve data quality.

- For the mortality analysis, increase the number of states having reliable industry and occupation data. The PMR analysis in this report relies on information from only 24 states. These were the states that coded both industry and occupation on death certificates and supplied sufficiently reliable data for analysis. Collectively, these states account for $32 \%$ of the U.S. agricultural worker population. That fraction could be doubled, to about $60 \%$ of the national agricultural worker population, if three additional states-California, Texas, and Floridawere to supply reliable industry and occupation information. Inclusion of further states would progressively increase the representativeness of the findings. Furthermore, the inclusion of additional states would enable more reliable estimation of the PMRs for diseases that are rare.

\section{- Expand temporal coverage of mortality} analyses. Another approach to increasing the reliability of the findings for rare mortality outcomes would be to expand the temporal coverage beyond the range 1988-1998. However, including data for the years 1998 to the present would require reconciling respiratory disease codes across the $9^{\text {th }}$ and $10^{\text {th }} \mathrm{ICD}$ revisions. Because of the transition from the $9^{\text {th }}$ to the $10^{\text {th }}$ revision of the ICD in 1999 , a comparability study on the respiratory diseases would be necessary to evaluate any apparent changes in disease frequency causes as a result of the ICD revision.

- For the morbidity analysis, add further years or cycles of the NHIS or NHANES survey data. Addition of further years from the NHIS and NHANES would enable more reliable estimation of results, particularly when the data are disaggregated by worker group, sex, and race.

- Undertake comprehensive industrial hygiene surveys of worker exposures. Although no exposure databases were identified for application to this report, good exposure data are needed for assessment of work-related respiratory disease for agricultural workers. The best means of filling the gap would be through special-purpose surveys targeting agricultural workers. For the results of such surveys to be meaningful, in terms of the ability to generalize results, they would need to be reasonably broad in coverage-at least statewide, preferably for states with a significant agricultural worker population such as California, Texas or Florida. Regional studies would also be useful. 

Appendices 



\section{Appendix A \\ Sources of Data}

Two main types of data sources were sought for this surveillance report: those that were medicaloutcome related (i.e., mortality, morbidity) and those that were exposure related. However, no major databases of exposure data pertinent to respiratory disease in agricultural workers were identified. As a consequence, this report is restricted to health outcomes only.

\section{Multiple-Cause-of-Death Data}

The National Center for Health Statistics (NCHS) has made available annual multiple-cause-of-death data files for public use since 1968. These files contain records of all deaths in the United States (approximately two million annually) that are reported to state vital statistics offices. Each death record includes codes for up to 20 conditions listed on the death certificate, including both underlying and contributing causes of death in two fields: the entity axis, which preserves diagnostic detail for all listed conditions and their placement on the death certificate; and the record axis, which reorders the codes alphanumerically, removes redundancies, and occasionally combines some associated conditions. Other data include age, race/ethnicity, sex, and state and county of residence at time of death. In addition, usual industry and occupation codes are available for decedents from some states since 1985. NCHS annually determines that certain quality criteria have been met by usual industry and occupation data from individual states (see Appendix D). Multiple-cause-of-death data for 1988-1998 were used in this report.

For more information: http://www.cdc.gov/nchs/ about/major/dvs/mortdata.htm

\section{National Health Interview Survey Data}

NCHS makes available public-use data from the National Health Interview Survey (NHIS), an annual health survey conducted since 1960 . The NHIS is a cross-sectional household interview survey on the health of the U.S. civilian, non- institutionalized population. The main objective of the NHIS is to monitor the health of the population of the United States through the collection and analysis of survey information on a broad range of health topics. NHIS data are collected annually by personal interview from approximately 40,000 households and include about 100,000 persons, with over-sampling of blacks and Hispanics. Through weighting procedures, estimates can be derived that are representative of the target population. The annual response rate of the NHIS is near $90 \%$ of eligible households in the sample. Data from the 1997, 1998 and 1999 surveys were used for this report.

For more information: http://www.cdc.gov/nchs/ nhis.htm

\section{The Third National Health and Nutrition Examination Survey Data}

NCHS makes available public-use data from the third National Health and Nutrition Examination Survey (NHANES III) conducted from 1988 through 1994. NHANES III was designed to provide national estimates of the health and nutritional status of the U.S. civilian, noninstitutionalized population. The NHANES III was a complex, multi-stage, stratified, clustered interview and medical survey of about 5,000 individuals per year, with over-sampling of blacks and Hispanics (and certain other groups). Through weighting procedures, estimates can be derived that are representative of the target population. The NHANES III elicited information on demography, chest symptoms, smoking history, industry and occupation, as well as deriving information on many other medical and health-related variables. Of the 39,695 individuals selected in NHANES III, 33,994 (86\%) were interviewed and 20,492 undertook spirometry.

For more information: http://www.cdc.gov/nchs/ nhanes.htm 
Survey of Occupational Injuries and Illnesses

\section{Data}

The Bureau of Labor Statistics (BLS) Survey of Occupational Injuries and Illnesses (SOII), done in cooperation with participating state agencies, involves data collection by mail from a sample of approximately 250,000 establishments each calendar year. Nearly all industries in the private sector (employers covered by the Occupational Safety and Health Act of 1970) are included. Annual BLS reports of these data incorporate corresponding data from mine operators, provided to BLS by the Mine Safety and Health Administration (MSHA), and from railroad transportation employers, provided to BLS by the Federal Railroad Administration. National estimates of injury and illness incidence rates by industry are developed from the survey data.

Beginning in 1992, the survey was expanded to provide more information on illnesses resulting in days away from work, allowing for more detailed classification of respiratory system diseases. For this report, annual summary data on respiratory illnesses were extracted from BLS annual reports on occupational injuries and illnesses. Data from 1995-2002 SOII surveys were used for this report.

For more information: http://www.bls.gov/iif/ oshsum.htm

\section{Demographic Data}

Statistics on the distribution of agricultural workers by occupation for 1997 and 2002 were obtained from the Bureau of Labor Statistics Current Population Survey.

For more information: ftp://ftp.bls.gov/pub/special. requests/lf/aa97/aat11.txt and ftp://ftp.bls.gov/pub/ special.requests/lf/aa2002/aat11.txt 


\section{Appendix B \\ Methods}

Mortality Analyses of NCHS Multiple-Causeof-Death Data

For this report, the number of deaths for each respiratory condition was defined as either (1) the number of decedents for which the condition was coded as the underlying cause of death, or (2) the number of decedents for which the condition was coded as one of the multiple causes of death (i.e., either the underlying or contributing cause of death). For the years 1988-1998, these numbers were tabulated from the record axis of the NCHS multiple-cause-of-death data files. See Appendix $\mathrm{C}$ for a listing of the ICD-9 codes that were used in this analysis. The tables in Section 2 of this report are based solely on multiple causes of death whereas the figures in Section 2 are based on both underlying cause and multiple causes of death.

Appendix D shows the states and years with industry and occupation data on death certificates that were used for the mortality analysis.

Deaths for the analysis also were restricted to persons 15 years of age or older, appropriate when examining worker populations. Five age categories were used for the analysis: 15-54, 55$64,65-74,75-84$, and 85 years or older. Because the age-at-death distribution is slanted toward older ages, there was a fairly even distribution across the five age categories that were used. Race and ethnicity were combined into a single variable for the analysis, categorized as follows: (1) white, non-Hispanic; (2) black, non-Hispanic; (3) other, non-Hispanic; (4) Hispanic; or (5) unknown race/ ethnicity.

Deaths were tabulated by agricultural groups and by sex, age, and race/ethnicity. The agricultural groups were defined based on industry and occupation codes shown in Appendix E. Six agricultural groups were defined: (1) crop farm workers, (2) livestock farm workers, (3) farm managers, (4) landscape and horticultural workers,
(5) forestry workers, and (6) fishery workers. The remaining non-agricultural workers were used as a comparison group for the analysis.

Combinations of occupation and industry codes that were used to define agricultural groups are listed in Appendix E.

Although most ICD-9 codes used in the analysis clearly are respiratory diseases, a few might be considered only marginally related. The rationale for including the marginally related diseases was as follows:

- Tuberculosis (010-018): Tuberculosis is an infectious disease caused by Mycobacterium tuberculosis. It mainly involves the respiratory tract. Some of the ICD-9 codes explicitly specify other organ systems - for example, code 013 (tuberculosis of the meninges and central nervous system; code 014 (tuberculosis of intestines, peritoneum, and mesenteric glands); code 015 (tuberculosis of bones and joints); code 016 (tuberculosis of genitourinary system); and code 017 (tuberculosis of other organs). However, pulmonary tuberculosis (and other respiratory tuberculosis) predominates in terms of tuberculosis deaths in the United States.

- Mycoses (110-118): Mycoses are fungal infections that can affect various organs, including the lungs and other respiratory organs. Many, but not all of the serious and sometimes fatal mycotic infections do involve the lungs.

- Sarcoidosis (135): Sarcoidosis is a systemic granulomatous inflammatory disease of unknown etiology that typically involves the lungs.

The analysis was accomplished primarily by calculating a proportionate mortality ratio (PMR) for each worker group, for selected respiratory conditions. The PMR is defined as the observed number of deaths with the condition of interest 
(mentioned as either the underlying cause of death or a contributing cause) among all deaths in a specified worker group, divided by the expected number of deaths among those decedents for that condition. For this analysis, PMRs were calculated based on both the underlying cause of death and multiple causes of death (i.e., either underlying or contributing).

For calculating the PMRs, first, deaths from each condition of interest were tabulated by worker group, and for 50 demographic groups (i.e., all combinations of two sex categories, five race/ ethnicity categories, and five age categories) within each worker group. This tabulation was performed separately for each of the years 1988-1998. These results were then summed across years to get totals for all demographic groups within each worker group.

The tabulation of observed deaths was performed separately for the underlying cause of death (at the 3-digit level of detail for ICD-9 codes) and for multiple causes of death. Further tabulations were performed for groupings of the 3-digit ICD codes (see Appendix C for a listing of all groupings).

The expected number of deaths for any worker group, for a specific condition, is the number that would have occurred if that worker group had the same proportion of deaths for that condition as did the comparison group. The expected numbers of deaths were calculated by disease and by demographic group for the six worker groups of interest, by multiplying the total number of observed deaths for each worker group by the fraction of deaths for that disease that occurred in the comparison group. The expected deaths then were summed for each worker group across the 50 demographic groups. The number of observed deaths was divided by the sum of expected deaths and then multiplied by 100 to obtain the PMR. A PMR greater than 100 indicates that there were more deaths associated with the condition in a specified agricultural group than expected.

Lower and upper confidence limits (LCLs and UCLs) for the PMR, at a 95\% level of statistical confidence, were calculated in accordance with a method described by Bailar and Ederer. ${ }^{1}$ The method applies to the ratio of a Poisson variable to its expectation, and is appropriate for this analysis involving diseases for which the fraction of deaths attributable is relatively small. A PMR was considered to be different from 100 at the $95 \%$ level of statistical significance (i.e., $p<0.05$ ) if the $95 \%$ confidence interval did not overlap 100.

\section{Morbidity Analyses of National Health Interview Survey Data}

Because the data from the National Health Interview Survey (NHIS) are based on a sample of the U.S. population, the number of data points can be relatively small when the analysis is restricted to a subgroup such as agricultural workers. Consequently, the three most recent years (19971999) for which NHIS results were available in the form of public-use files were combined in the analysis, to obtain a relatively greater statistical stability.

Weights that are inverse to the probability of selection for each respondent are provided with each yearly NHIS data set to enable development of national estimates from the sample data. These weights were applied separately to each year of data.

The estimates derived from the NHIS data sets concerned the number (and percent) of respondents with specific conditions. More specifically, responses were analyzed for the following questions:

${ }^{1}$ Bailar JC, Ederer F [1964]. Significance factors for the ratio of a Poisson variable to its expectation. Biometrics 20: 639-643. 
- Have you EVER been told by a doctor or other health professional that you had emphysema?

- Have you EVER been told by a doctor or other health professional that you had asthma?

- Have you EVER been told by a doctor or other health professional that you had cancer or a malignancy of any kind? What kind of cancer was it? ... lung?

- During the past 12 months, have you been told by a doctor or other health professional that you had hayfever?

- During the past 12 months, have you been told by a doctor or other health professional that you had sinusitis?

- During the past 12 months, have you been told by a doctor or other health professional that you had chronic bronchitis?

The industry and occupation codes used by NCHS for the NHIS data sets are shown in Appendix F. Three agricultural groups were defined for the NHIS data sets, based on a combination of occupation/ industry codes for a respondent's current job as shown in Appendix F. The remaining respondents were classified as non-agricultural workers. The occupation code of 6 (natural mathematical and computer scientists) was included for the forestry and fishery agricultural group because of relatively high proportion of the respondents were identified in the industry code of 2 (forestry and fisheries).

The number (and percent) of individuals in each worker group of interest with each of the above respiratory conditions was calculated. As with the mortality data, a comparison group (nonagricultural workers) was used as a basis for calculating the expected number of workers with each condition. The expected numbers were calculated separately within each of 80 categories representing combinations of sex (male, female), race/ethnicity (white, non-Hispanic; black, nonHispanic; other, non-Hispanic, Hispanic), age (18-25, 25-34, 35-44, 45-64, 65+), and smoking status (never smoked or ever smoked, based on the question "Have you smoked at least 100 cigarettes in your entire life?"). The observed and expected numbers then were summed across the 80 demographic categories. Prevalence ratios (PRs), or ratios of summed observed to expected numbers, were calculated and then multiplied by 100 to obtain a convenient reference point, and 95\% LCLs and UCLs were calculated according to the method described by Bailar and Ederer ${ }^{1}$ for mortality data. (Strictly speaking, the method may not apply directly for some conditions that are not considered rare, but it should provide an adequate approximation for purposes of screening the results to discount those based on very small numbers of observations). A PR was considered to be different from 100 at the $95 \%$ level of statistical significance (i.e., $p<0.05$ ) if the $95 \%$ confidence interval did not overlap 100.

\section{Morbidity Analyses of the third National Health and Nutritional Examination Survey}

Results from the third National Health and Nutritional Examination Survey (NHANES III) also are based on a statistical sample of the U.S. population, and weights are provided for each respondent in the public-use data files to enable development of national estimates from the sample data. As with the NHIS data, much of the analysis with the NHANES III data set concerned the number (and percent) of respondents with specific conditions that could be considered respiratory in nature. Although the NHANES data set had fewer respondents overall than NHIS (with one round of survey results rather than three available for analysis), there were more questions for NHANES III that concerned respiratory conditions. Responses were analyzed for the following questions:

- Has a doctor ever told you that you had asthma?

- Has a doctor ever told you that you had chronic bronchitis?

- Has a doctor ever told you that you had emphysema?

- Has a doctor ever told you that you had hay fever? 
- Apart from when you have a cold, does your chest ever sound wheezy or whistling?

- Do you usually cough on most days for 3 consecutive months or more during the year?

- Do you bring up phlegm on most days for 3 consecutive months or more during the year?

- Are you troubled by shortness of breath when hurrying on level ground or walking up a slight hill?

- During the past 12 months, have you had any episodes of stuffy, itchy, or runny nose?

- During the past 12 months, have you had a cold or the flu?

- During the past 12 months, have you had sinusitis or sinus problems?

- During the past 12 months, have you had pneumonia?

- Have you had wheezing or whistling in your chest at any time in the past 12 months?

Industry and occupation codes from NHANES III for the worker groups of interest are similar to one another. The occupation code (for longest job held) was used to define three worker groups for the NHANES III data set as shown in Appendix G.

As with the NHIS data, the number (and percent) of individuals with each of the above respiratory conditions was calculated for each worker group, and expected numbers were calculated separately within each of 80 categories for combinations of sex, race/ethnicity, age, and smoking status. The categories used for sex, race/ethnicity, age, and smoking status were the same as those used for the NHIS data sets. Similarly, the question used for determining smoking status for NHANES III participants was the same as that used for the NHIS - "Have you smoked at least 100 cigarettes in your entire life?"

Prevalence ratios (PRs), or ratios of summed observed to expected numbers, were calculated in the same manner as described for the NHIS data and were then multiplied by 100 to obtain a convenient reference point. Similarly, 95\% LCLs and UCLs were calculated according to the method described for mortality data. (Strictly speaking, the method may not apply directly for some conditions that are not considered rare, but it should provide an adequate approximation for purposes of screening the results to discount those based on very small numbers of observations. A PR was considered to be different from 100 at the $95 \%$ level of statistical significance (i.e., $\mathrm{p}<0.05$ ) if the $95 \%$ confidence interval did not overlap 100.

A unique feature of the NHANES data set is the inclusion of spirometry data. The following spirometric parameters were used in the analysis: forced expiratory volume in one second $\left(\mathrm{FEV}_{1}\right)$; forced vital capacity (FVC); and peak expiratory flow (PEF). Expected values for each of these measures were obtained on an individualrespondent basis, using prediction equations developed by Hankinson et al. ${ }^{2}$ These equations provide expected values for each of the three spirometric parameters based on the subject's sex, race/ethnicity, age, and height. Percent predicted ratios were calculated for each subject for each parameter, and resulting distributions were summarized for each worker group (and a comparison group) in terms of the mean and standard deviation of the distribution.

In addition to the summary statistics described above, prevalence ratios based on the fraction of individuals with obstructive or restrictive abnormalities (using the American Thoracic Society criteria ${ }^{3}$ ) were calculated. Individuals with

${ }^{2}$ Hankinson JL, Odencrantz JR, Fedan KB [1999]. Spriometric reference values from a sample of the general U.S. population. Am J Respir Crit Care Med 159: 179-187.

${ }^{3}$ American Thoracic Society Statement [1991]. Lung function testing: Selection of reference values and interpretative strategies. Am Rev Respir Dis 144:1202-1218. 
obstructive abnormalities were defined as those for whom the $\mathrm{FEV}_{1} / \mathrm{FVC}$ ratio was below the lower limit of normal (LLN), again using prediction equations provided by Hankinson et al. ${ }^{2}$ Subjects with restrictive abnormalities were defined as those with an $\mathrm{FEV}_{1} / \mathrm{FVC}$ ratio above the LLN but with an FVC value that was below the LLN.

\section{BLS Data}

Unlike the NHIS and NHANES data, public-use data files are not available for the injury and illness data reported by BLS. Consequently, incidence rates summarized by industry for selected types of illness (dust diseases of the lung and respiratory conditions due to toxic agents) were extracted from BLS reports for the most recent years available: 1995-2001.

\section{Demographic Data}

Estimates extracted from selected BLS web sites or publications were used to develop the demographic statistics for agricultural workers shown in Section 1 of this report.

Statistics on the distribution of agricultural workers by occupation, for the year 2002 (most recent available), were taken from the Current Population Survey. Statistics on the distribution of agricultural workers by the state in which they worked, for the year 2002, also were taken from the Occupational Employment Survey and were retrieved (state by state) from the same web site (Table 1-2).

Statistics on the distribution of agricultural groups by sex and race/ethnicity, for the years 1997 and 2002, were taken from the Current Population Survey (Figures 1-1 through 1-3). 



\section{Appendix $\mathrm{C}$ \\ ICD-9 Codes and Descriptions for Respiratory Diseases \\ Included in the Mortality Analysis}

\begin{tabular}{|c|c|c|c|}
\hline $\begin{array}{l}\text { ICD } \\
\text { Code }\end{array}$ & \multicolumn{3}{|c|}{ ICD } \\
\hline \multicolumn{2}{|c|}{ Tuberculosis (010-018) } & \multicolumn{2}{|c|}{ Other Diseases of Upper Respiratory Tract (cont'd)) } \\
\hline $010^{*}$ & Primary tuberculous infection & 477 & Allergic rhinitis \\
\hline 011 & Pulmonary tuberculosis & 478 & Other diseases of upper respiratory tract \\
\hline 012 & Other respiratory tuberculosis & & \\
\hline \multirow[t]{2}{*}{013} & Tuberculosis of meninges and central nervous & \multicolumn{2}{|c|}{ Pneumonia and Influenza (480-487) } \\
\hline & & 480 & Viral pneumonia \\
\hline 014 & $\begin{array}{l}\text { Tuberculosis of intestines, peritoneum, and } \\
\text { mesenteric glands }\end{array}$ & 481 & Pneumococcal pneumonia \\
\hline 015 & Tuberculosis of bones and joints & 483 & Pneumonia due to other specified organism \\
\hline 016 & Tuberculosis of genitourinary system & 485 & Bronchopneumonia, organism unspecified \\
\hline 017 & Tuberculosis of other organs & 486 & Pneumonia, organism unspecified \\
\hline 018 & Miliary tuberculosis & 487 & Influenza \\
\hline \multicolumn{2}{|c|}{ Mycoses (110-118) } & \multirow{2}{*}{\multicolumn{2}{|c|}{$\begin{array}{l}\text { Chronic Obstructive Pulmonary Disease and Allied } \\
\text { Conditions }(490-496)\end{array}$}} \\
\hline 110 & Dermatophytosis & & \\
\hline 111 & Dermatomycosis, other and unspecified & 490 & Bronchitis, not specified as acute or chronic \\
\hline 112 & Candidiasis & 491 & Chronic bronchitis \\
\hline 114 & Coccidioidomycosis & 492 & Emphysema \\
\hline 115 & Histoplasmosis & 493 & Asthma \\
\hline 116 & Blastomycotic infection & 494 & Bronchiectasis \\
\hline 117 & Other mycoses & 495 & Extrinsic allergic alveolitis (hypersensitivity \\
\hline \multirow[t]{2}{*}{$118^{*}$} & Opportunistic mycoses & & pneumonitis) \\
\hline & Sarcoidosis (135) & 496 & $\begin{array}{l}\text { Chronic airway obstruction, not elsewhere } \\
\text { classified }\end{array}$ \\
\hline & Sarcoidosis & & \\
\hline \multirow{2}{*}{\multicolumn{2}{|c|}{$\begin{array}{l}\text { Malignant Neoplasms of } \\
\text { Trachea/Bronchus/Lung/Pleura (162-163) }\end{array}$}} & \multicolumn{2}{|c|}{$\begin{array}{l}\text { Pneumoconiosis and Other Lung Diseases - External } \\
\text { Agents (500-508) }\end{array}$} \\
\hline & & 500 & Coal workers' pneumoconiosis \\
\hline \multirow{2}{*}{162} & Malignant neoplasm of trachea, bronchus, and & 501 & Asbestosis \\
\hline & Lung & 502 & Pneumoconiosis due to other silica or silicates \\
\hline \multirow[t]{2}{*}{163} & Malignant neoplasm of pleura & 503 & Pneumoconiosis due to other inorganic dust \\
\hline & & 504 & Pneumonopathy due to inhalation of other dust \\
\hline \multicolumn{2}{|c|}{ Acute Respiratory Infections (460-466) } & 505 & Pneumoconiosis, unspecified \\
\hline 460 & Acute nasopharyngitis [common cold] & 506 & Respiratory conditions due to chemical fumes \\
\hline $461^{*}$ & Acute sinusitis & & and vapors \\
\hline 462 & Acute pharyngitis & 507 & Pneumonitis due to solids and liquids \\
\hline 463 & Acute tonsillitis & 508 & Respiratory conditions due to other and \\
\hline 464 & Acute laryngitis and tracheitis & & unspecified external agents \\
\hline 465 & $\begin{array}{l}\text { Acute upper respiratory infections of multiple } \\
\text { or unspecified sites }\end{array}$ & \multicolumn{2}{|c|}{ Other Diseases of Respiratory System (510-519) } \\
\hline \multirow[t]{2}{*}{466} & Acute bronchitis and bronchiolitis & 510 & Empyema \\
\hline & & 511 & Pleurisy \\
\hline \multicolumn{2}{|c|}{ Other Diseases of Upper Respiratory Tract (470-478) } & 512 & Pneumothorax \\
\hline $470^{*}$ & Deflected nasal septum & 513 & Abscess of lung and mediastinum \\
\hline 471 & Nasal polyps & 514 & Pulmonary congestion and hypostasis \\
\hline 472 & Chronic pharyngitis and nasopharyngitis & 515 & Postinflammatory pulmonary fibrosis \\
\hline 473 & Chronic sinusitis & 516 & Other alveolar and parietoalveolar \\
\hline 474 & Chronic disease of tonsils and adenoids & & pneumonopathy \\
\hline 475 & Peritonsillar abscess & 518 & Other diseases of lung \\
\hline $476^{*}$ & Chronic laryngitis and laryngotracheitis & 519 & Other diseases of respiratory system \\
\hline
\end{tabular}

*ICD code had no observed deaths for each of the agricultural groups defined in Appendix D. 



\begin{tabular}{lccccccccccc}
\hline State & 1988 & 1989 & 1990 & 1991 & 1992 & 1993 & 1994 & 1995 & 1996 & 1997 & 1998 \\
\hline Alaska & $\mathrm{X}$ & & & & & & & & & & \\
Colorado & $\mathrm{X}$ & $\mathrm{X}$ & $\mathrm{X}$ & $\mathrm{X}$ & $\mathrm{X}$ & $\mathrm{X}$ & $\mathrm{X}$ & $\mathrm{X}$ & $\mathrm{X}$ & $\mathrm{X}$ & $\mathrm{X}$ \\
Georgia & $\mathrm{X}$ & $\mathrm{X}$ & $\mathrm{X}$ & $\mathrm{X}$ & $\mathrm{X}$ & $\mathrm{X}$ & $\mathrm{X}$ & $\mathrm{X}$ & $\mathrm{X}$ & $\mathrm{X}$ & $\mathrm{X}$ \\
Hawaii & & & & & & $\mathrm{X}$ & $\mathrm{X}$ & $\mathrm{X}$ & $\mathrm{X}$ & & $\mathrm{X}$ \\
Idaho & $\mathrm{X}$ & $\mathrm{X}$ & $\mathrm{X}$ & $\mathrm{X}$ & $\mathrm{X}$ & $\mathrm{X}$ & $\mathrm{X}$ & $\mathrm{X}$ & $\mathrm{X}$ & $\mathrm{X}$ & $\mathrm{X}$ \\
Indiana & $\mathrm{X}$ & $\mathrm{X}$ & $\mathrm{X}$ & $\mathrm{X}$ & $\mathrm{X}$ & $\mathrm{X}$ & & $\mathrm{X}$ & $\mathrm{X}$ & & $\mathrm{X}$ \\
Kansas & $\mathrm{X}$ & $\mathrm{X}$ & $\mathrm{X}$ & $\mathrm{X}$ & $\mathrm{X}$ & $\mathrm{X}$ & $\mathrm{X}$ & $\mathrm{X}$ & $\mathrm{X}$ & $\mathrm{X}$ & $\mathrm{X}$ \\
Kentucky & $\mathrm{X}$ & $\mathrm{X}$ & $\mathrm{X}$ & $\mathrm{X}$ & $\mathrm{X}$ & $\mathrm{X}$ & $\mathrm{X}$ & $\mathrm{X}$ & $\mathrm{X}$ & $\mathrm{X}$ & $\mathrm{X}$ \\
Maine & $\mathrm{X}$ & $\mathrm{X}$ & $\mathrm{X}$ & $\mathrm{X}$ & $\mathrm{X}$ & $\mathrm{X}$ & $\mathrm{X}$ & $\mathrm{X}$ & $\mathrm{X}$ & & $\mathrm{X}$ \\
Nevada & $\mathrm{X}$ & $\mathrm{X}$ & $\mathrm{X}$ & $\mathrm{X}$ & $\mathrm{X}$ & $\mathrm{X}$ & $\mathrm{X}$ & $\mathrm{X}$ & $\mathrm{X}$ & $\mathrm{X}$ & $\mathrm{X}$ \\
New Hampshire & $\mathrm{X}$ & $\mathrm{X}$ & $\mathrm{X}$ & $\mathrm{X}$ & $\mathrm{X}$ & $\mathrm{X}$ & $\mathrm{X}$ & $\mathrm{X}$ & $\mathrm{X}$ & & $\mathrm{X}$ \\
New Jersey & $\mathrm{X}$ & $\mathrm{X}$ & $\mathrm{X}$ & $\mathrm{X}$ & $\mathrm{X}$ & $\mathrm{X}$ & $\mathrm{X}$ & $\mathrm{X}$ & $\mathrm{X}$ & $\mathrm{X}$ & $\mathrm{X}$ \\
New Mexico & $\mathrm{X}$ & $\mathrm{X}$ & $\mathrm{X}$ & $\mathrm{X}$ & $\mathrm{X}$ & $\mathrm{X}$ & $\mathrm{X}$ & $\mathrm{X}$ & $\mathrm{X}$ & $\mathrm{X}$ & $\mathrm{X}$ \\
North Carolina & $\mathrm{X}$ & $\mathrm{X}$ & $\mathrm{X}$ & $\mathrm{X}$ & $\mathrm{X}$ & $\mathrm{X}$ & $\mathrm{X}$ & $\mathrm{X}$ & $\mathrm{X}$ & $\mathrm{X}$ & $\mathrm{X}$ \\
Ohio & $\mathrm{X}$ & $\mathrm{X}$ & $\mathrm{X}$ & $\mathrm{X}$ & $\mathrm{X}$ & $\mathrm{X}$ & & $\mathrm{X}$ & $\mathrm{X}$ & $\mathrm{X}$ & $\mathrm{X}$ \\
Oklahoma & $\mathrm{X}$ & $\mathrm{X}$ & $\mathrm{X}$ & $\mathrm{X}$ & $\mathrm{X}$ & $\mathrm{X}$ & & $\mathrm{X}$ & $\mathrm{X}$ & & \\
Rhode Island & $\mathrm{X}$ & $\mathrm{X}$ & $\mathrm{X}$ & $\mathrm{X}$ & $\mathrm{X}$ & $\mathrm{X}$ & $\mathrm{X}$ & $\mathrm{X}$ & $\mathrm{X}$ & $\mathrm{X}$ & $\mathrm{X}$ \\
South Carolina & $\mathrm{X}$ & $\mathrm{X}$ & $\mathrm{X}$ & $\mathrm{X}$ & $\mathrm{X}$ & $\mathrm{X}$ & $\mathrm{X}$ & $\mathrm{X}$ & $\mathrm{X}$ & $\mathrm{X}$ & $\mathrm{X}$ \\
Tennessee & $\mathrm{X}$ & & & & & & & & & & \\
Utah & $\mathrm{X}$ & $\mathrm{X}$ & $\mathrm{X}$ & $\mathrm{X}$ & $\mathrm{X}$ & $\mathrm{X}$ & $\mathrm{X}$ & $\mathrm{X}$ & $\mathrm{X}$ & $\mathrm{X}$ & $\mathrm{X}$ \\
Vermont & $\mathrm{X}$ & $\mathrm{X}$ & $\mathrm{X}$ & $\mathrm{X}$ & $\mathrm{X}$ & $\mathrm{X}$ & $\mathrm{X}$ & $\mathrm{X}$ & $\mathrm{X}$ & $\mathrm{X}$ & $\mathrm{X}$ \\
Washington & & $\mathrm{X}$ & $\mathrm{X}$ & $\mathrm{X}$ & $\mathrm{X}$ & $\mathrm{X}$ & $\mathrm{X}$ & & & & \\
West Virginia & $\mathrm{X}$ & $\mathrm{X}$ & $\mathrm{X}$ & $\mathrm{X}$ & $\mathrm{X}$ & $\mathrm{X}$ & $\mathrm{X}$ & $\mathrm{X}$ & $\mathrm{X}$ & $\mathrm{X}$ & $\mathrm{X}$ \\
Wisconsin & $\mathrm{X}$ & $\mathrm{X}$ & $\mathrm{X}$ & $\mathrm{X}$ & $\mathrm{X}$ & $\mathrm{X}$ & $\mathrm{X}$ & $\mathrm{X}$ & $\mathrm{X}$ & $\mathrm{X}$ & $\mathrm{X}$ \\
\hline NorE & & & & & & & & & & & \\
\hline
\end{tabular}

NOTE: Upper case ' $\mathrm{X}$ ' means the occupation/industry data coded from state death certificates met NCHS quality criteria; lower case ' $\mathrm{x}$ ' means the data did not meet NCHS quality criteria. Data that did not meet NCHS quality criteria comprised $1.8 \%$ of the total deaths represented in the mortality analysis.

SOURCE: National Center for Health Statiscs multiple cause-of-death data 



\section{Appendix E \\ Agricultural Groups Used in the Mortality Analysis and Their Derivation from the U.S. Bureau of Census Industry and Occupation Codes}

Table E-1. Derivation of agricultural groups from U.S. Bureau of Census industry and occupation codes

\begin{tabular}{|c|c|c|c|c|c|}
\hline \multirow{2}{*}{$\begin{array}{c}\text { Census } \\
\text { Occupation } \\
\text { Code } \\
\text { (See Table E-2.) }\end{array}$} & \multicolumn{5}{|c|}{$\begin{array}{c}\text { Census Industry Code } \\
\text { (See Table E-2.) }\end{array}$} \\
\hline & 010 & 011 & 020 & 031 & 032 \\
\hline 473 & crop farm workers & livestock farm workers & & & \\
\hline 474 & $\begin{array}{l}\text { landscape and } \\
\text { horticultural workers }\end{array}$ & $\begin{array}{l}\text { landscape and } \\
\text { horticultural workers }\end{array}$ & $\begin{array}{l}\text { landscape and } \\
\text { horticultural workers }\end{array}$ & & \\
\hline 475 & farm managers & farm managers & $\begin{array}{l}\text { landscape and } \\
\text { horticultural workers }\end{array}$ & & \\
\hline 476 & $\begin{array}{l}\text { landscape and } \\
\text { horticultural workers }\end{array}$ & $\begin{array}{c}\text { landscape and } \\
\text { horticultural workers }\end{array}$ & $\begin{array}{l}\text { landscape and } \\
\text { horticultural workers }\end{array}$ & & \\
\hline 477 & farm managers & farm managers & & forestry workers & fishery workers \\
\hline 479 & crop farm workers & livestock farm workers & $\begin{array}{l}\text { landscape and } \\
\text { horticultural workers }\end{array}$ & forestry workers & \\
\hline 483 & & livestock farm workers & & & fishery workers \\
\hline 484 & crop farm workers & crop farm workers & $\begin{array}{l}\text { landscape and } \\
\text { horticultural workers }\end{array}$ & & \\
\hline 485 & $\begin{array}{l}\text { landscape and } \\
\text { horticultural workers }\end{array}$ & $\begin{array}{l}\text { landscape and } \\
\text { horticultural workers }\end{array}$ & $\begin{array}{l}\text { landscape and } \\
\text { horticultural workers }\end{array}$ & forestry workers & fishery workers \\
\hline 486 & $\begin{array}{l}\text { landscape and } \\
\text { horticultural workers }\end{array}$ & $\begin{array}{c}\text { landscape and } \\
\text { horticultural workers }\end{array}$ & $\begin{array}{l}\text { landscape and } \\
\text { horticultural workers }\end{array}$ & forestry workers & \\
\hline 494 & & & & forestry workers & \\
\hline 495 & & & & forestry workers & \\
\hline 496 & forestry workers & forestry workers & forestry workers & forestry workers & \\
\hline 497 & & & & & fishery workers \\
\hline 498 & & & & & fishery workers \\
\hline 499 & & & & & fishery workers \\
\hline
\end{tabular}

SOURCE: U.S. Bureau of the Census: Classified Index of Industries and Occupations. 1990 Census of Population and Housing, first edition 
Table E-2. U.S. Bureau of Census industry and occupation codes used in the mortality data analyses

\begin{tabular}{ll}
\multicolumn{1}{c}{ Industry Codes } \\
Agriculture, Forestry, and Fisheries \\
010 & Agricultural production, crops \\
011 & Agricultural production, livestock \\
020 & Landscape and horticultural services \\
031 & Forestry \\
032 & Fishing, hunting, and trapping
\end{tabular}

\section{Occupation Codes}

Farming, Forestry, and Fishing Occupations

473 Farmers, except horticultural

474 Horticultural specialty farmers

475 Managers, farms, except horticultural

476 Managers, horticultural specialty farms

477 Supervisors, farm workers

479 Farm workers

483 Marine life cultivation workers

484 Nursery workers

485 Supervisors, related agricultural occupations

486 Groundskeepers and gardeners, except farm

494 Supervisors, forestry, and logging workers

495 Forestry workers, except logging

496 Timber cutting and logging occupations

497 Captains and other officers, fishing vessels

498 Fishers

499 Hunters and trappers

SOURCE: U.S. Bureau of the Census: Classified Index of Industries and Occupations. 1990 Census of Population and Housing, first edition 


\begin{tabular}{l} 
Appendix F \\
Agricultural Groups Used in the Morbidity Analysis and Their Derivation from \\
the National Health and Interview Survey (NHIS) Industry and Occupation Codes \\
\hline
\end{tabular}

SOURCE: 1997/1998/1999 National Health Interview Surveys, Sample Adult Person Section - Public Use (pdf files, available from www.cdc.gov/nchs/ about/major/nhis/quest_data_related_1997_forward.htm). 



\section{Appendix G \\ Agricultural Groups Used in the Morbidity Analysis and Their Derivation from the Third National Health and Nutrition Examination Survey (NHANES III) Industry and Occupation Codes}

\begin{tabular}{rlcc}
\hline & & \multicolumn{2}{c}{ NHANES III Industry Code } \\
\cline { 3 - 4 } NHANES III Occupation Code & Agricultural Production & Agricultural Services, Forestry, Fishing \\
\hline $\mathbf{2 5}$ & Farm operators, managers, and supervisors & farm managers & \\
$\mathbf{2 6}$ & Farm and nursery workers & farm workers & \\
$\mathbf{2 7}$ & Related agricultural, forestry, fishing & other agricultural workers & other agricultural workers \\
\hline
\end{tabular}

SOURCE: Third National Health and Nutrition Examination Survey, Household Adult and Examination Data File Documentation (http://www.cdc.gov/ nchs/about/major/nhanes/datalink.htm). 




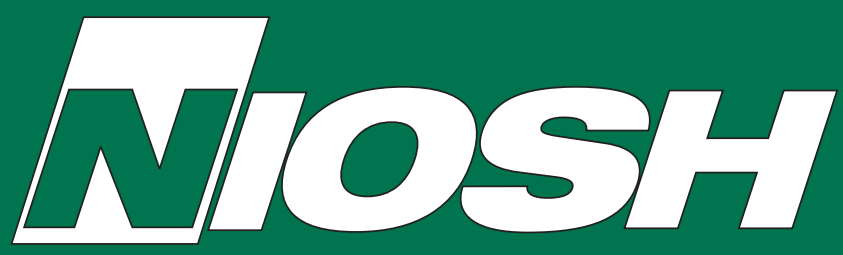

Delivering on the Nation's promise: Safety and health at work for all people through research and prevention

To receive NIOSH documents or more information about occupational safety and health topics, contact NIOSH at

1-800-35-NIOSH (1-800-356-4674)

Fax: (513) 533-8573

E-mail: pubstaft@cdc.gov

or visit the NIOSH Web site at www.cdc.gov/niosh

DHHS (NIOSH) Publication No. 2007-106

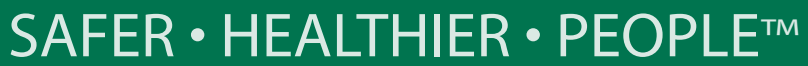

